\title{
Reagent control enables selective and regiodivergent opening of unsymmetrical phenonium ions
}

\author{
Shiyan Xu, Hannah M. Holst, Shelby B. McGuire, and Nicholas J. Race* \\ Department of Chemistry, University of Minnesota Twin Cities, Minneapolis, MN 55455
}

SUPPORTING INFORMATION

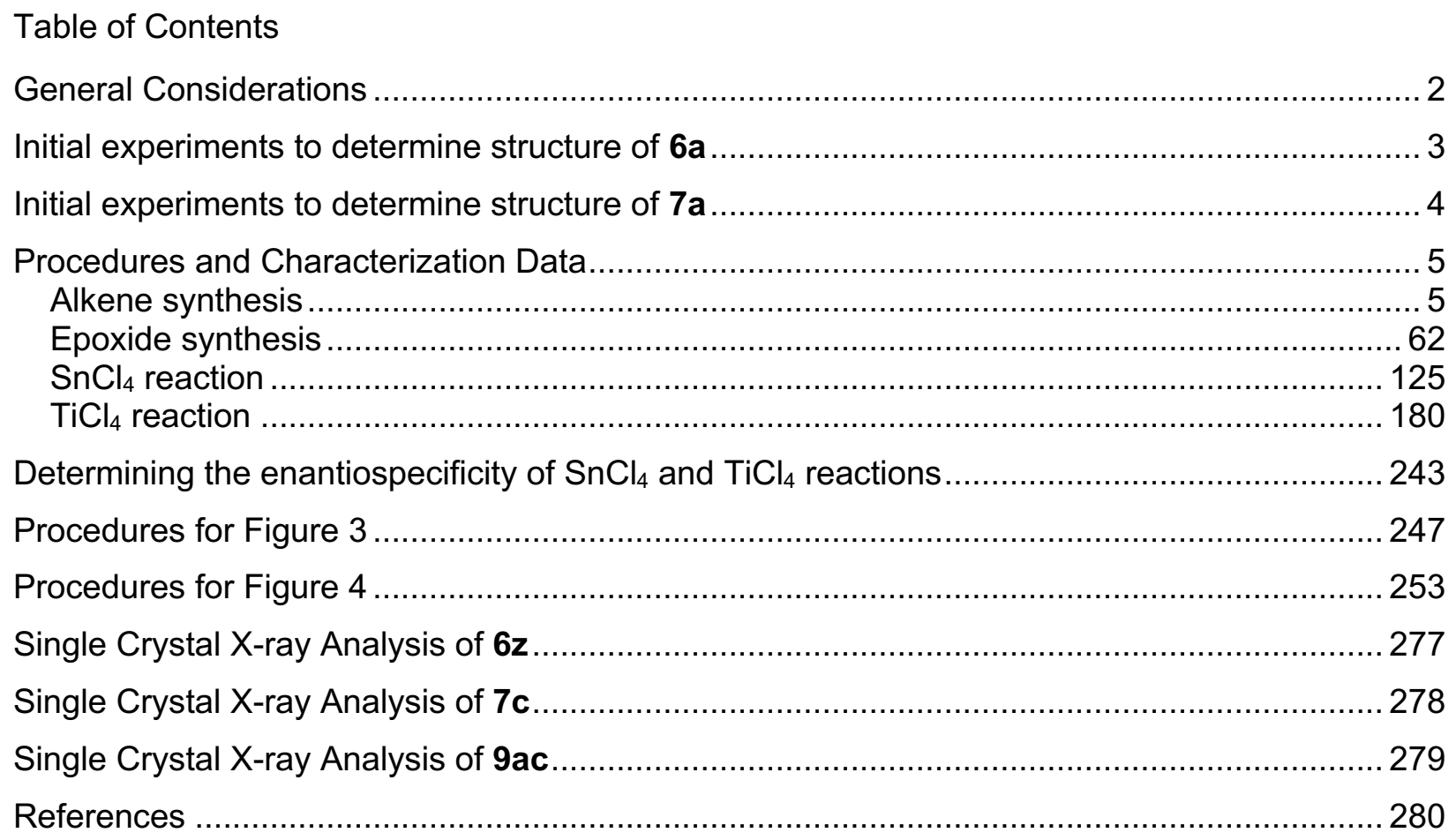




\section{General Considerations}

All reactions were performed using oven-dried or flame-dried glassware equipped with a magnetic stir bar under an atmosphere of argon unless otherwise noted. All reagents were purchased from commercial suppliers and used without further purification. The Shi catalyst (see below) was purchased from Oakwood Chemical. Dichloromethane, tetrahydrofuran, toluene, and acetonitrile were dried by passage through a column of anhydrous alumina using equipment from Pure Process Technology. ${ }^{1} \mathrm{H}$ NMR spectra were obtained in $\mathrm{CDCl}_{3}$ or $\mathrm{C}_{6} \mathrm{D}_{6}$ at $400 \mathrm{MHz}$ or $500 \mathrm{MHz}$. Chemical shifts are reported in ppm and referenced to the $\mathrm{CHCl}_{3}$ singlet at $7.26 \mathrm{ppm}$ or $\mathrm{C}_{6} \mathrm{D}_{6}$ singlet at $7.16 \mathrm{ppm} .{ }^{13} \mathrm{C}\left\{{ }^{1} \mathrm{H}\right\}$ NMR spectra were obtained in $\mathrm{CDCl}_{3}$ or $\mathrm{CD}_{2} \mathrm{Cl}_{2}$ at $101 \mathrm{MHz}$ or 126 $\mathrm{MHz}$ and referenced to the center peak of the $\mathrm{CDCl}_{3}$ triplet at $77.00 \mathrm{ppm}$ or $\mathrm{CD}_{2} \mathrm{Cl}_{2}$ at $54.00 \mathrm{ppm}$. The abbreviations s, d, t, app. h, dd, ddd, dt, and $\mathrm{m}$ stand for the resonance multiplicities singlet, doublet, triplet, apparent heptet, doublet of doublets, doublet of doublet of doublets, doublet of triplets, and multiplet, respectively. Thin-layer chromatography was performed with EMD silica gel $60 \mathrm{~F}_{254}$ plates eluting with solvents indicated, visualized by a $254 \mathrm{~nm}$ UV lamp and stained with potassium permanganate $\left(\mathrm{KMnO}_{4}\right)$ or phosphomolybdic acid (PMA). Flash chromatography was performed using EM reagent silica 60 (230-400 mesh). Infrared (IR) spectra were performed as a film on $\mathrm{NaCl}$ plates on a Nexus $670 \mathrm{FT}-\mathrm{IR}$ and are reported in $\mathrm{cm}^{-1}$. HRMS data were obtained on a Bruker BioTOF II ESI/TOF-MS, an Agilent 7200 GC/QTOF-MS, and a Thermo Orbitrap Velos instrument. Chiral HPLC was performed on an Agilent 1260 Infinity II system. X-ray crystallographic data collection and structure solution were conducted at the X-Ray Crystallographic Laboratory, 192 Kolthoff Hall, Department of Chemistry, University of Minnesota. All calculations were performed using Pentium computers using the current SHELXTL suite of programs. The Bruker-AXS D8 Venture diffractometer was purchased through a grant from NSF/MRI (\#1229400) and the University of Minnesota.

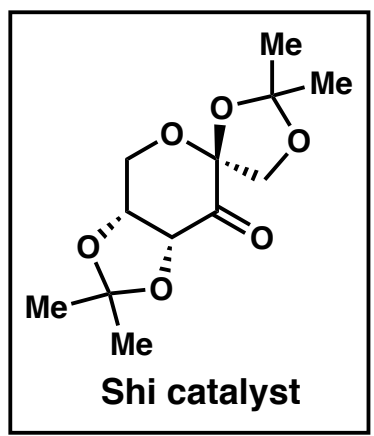




\section{Initial experiments to determine structure of $6 \mathbf{a}$}

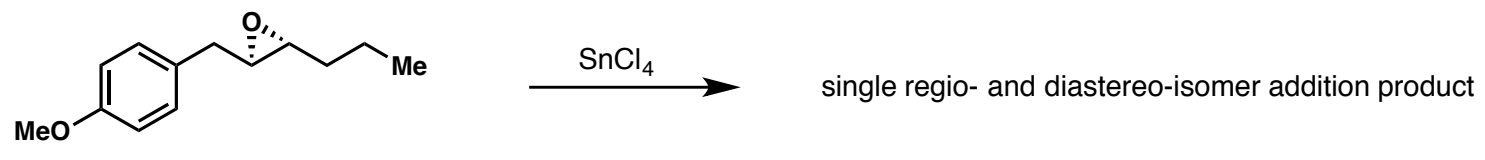

Four possible products:

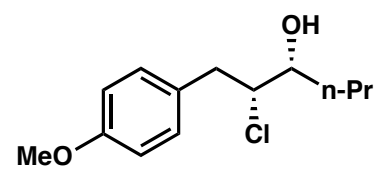

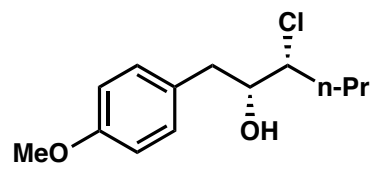<smiles>COc1ccc(CC(Cl)[C@H](O)C(=O)c2ccccc2)cc1</smiles><smiles>COc1ccc(C[C@@H](O)C(Cl)C(C)C)cc1</smiles>

Reaction of epoxide 5a with $\mathrm{SnCl}_{4}$ afforded addition product as a single regio- and diastereoisomer. ${ }^{1} \mathrm{H}$ analysis of the product was not sufficient to determine which of the four possible products had been formed. Product derivatization was used to determine the regioselectivity and stereochemistry.

\section{Regioselectivity}<smiles>COc1ccc(C[C@@H](Cl)[C@H](O)[C@H](Cl)C(=O)O)cc1</smiles><smiles>CCCNC(Cl)[C@H](O)Cc1ccc(OC)cc1</smiles>
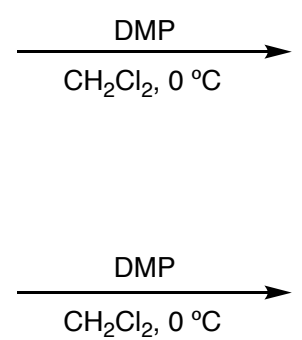<smiles>CCCCC(=O)C(Cl)C(Cl)c1ccc(OC)cc1</smiles><smiles>CCCC(Cl)C(=O)C(Cl)c1ccc(OC)cc1</smiles>

The regioselectivity of addition was confirmed through Dess-Martin oxidation of the secondary alcohol. ${ }^{1} \mathrm{H}$ NMR analysis of the crude ketone was sufficient to determine the benzylic hydrogen exhibited a doublet of doublets which was consistent with oxidation of $\mathbf{A}$. The ${ }^{1} \mathrm{H}$ NMR data was consistent with related compounds in the literature. ${ }^{1}$

\section{Stereochemistry}<smiles>CCCNC(O)[C@H](Cl)Cc1ccc(OC)cc1</smiles>

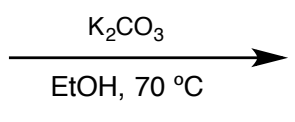<smiles>CCCC1OC1Cc1ccc(OC)cc1</smiles><smiles>CCCNC(O)[C@H](Cl)Cc1ccc(OC)cc1</smiles>

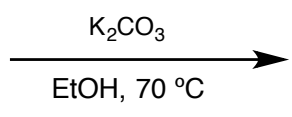<smiles>CCCC1O[C@@H]1Cc1ccc(OC)cc1</smiles>

Having confirmed the site-selectivity of epoxide opening, the relative stereochemistry between the chlorine and the oxygen substituents was determined after base-promoted cyclization of the 
chlorohydrin to a cis epoxide. Formation of the cis-substituted epoxide is consistent with the chlorohydrin having a syn stereochemical relationship.

Conclusion

Based on the above experiments we determined syn-chlorohydrin 6a was formed.

Additional evidence for syn stereochemistry was obtained via x-ray crystallographic analysis of chlorohydrin $6 z$.

\section{Initial experiments to determine structure of $7 a$}

The structure of 7 a was determined after cyclization to the corresponding oxetane 16. See section Procedures for Figure 4 below for more details. 


\section{Procedures and Characterization Data}

\section{Alkene synthesis}

General Procedure A: for the synthesis of allylbenzene derivatives

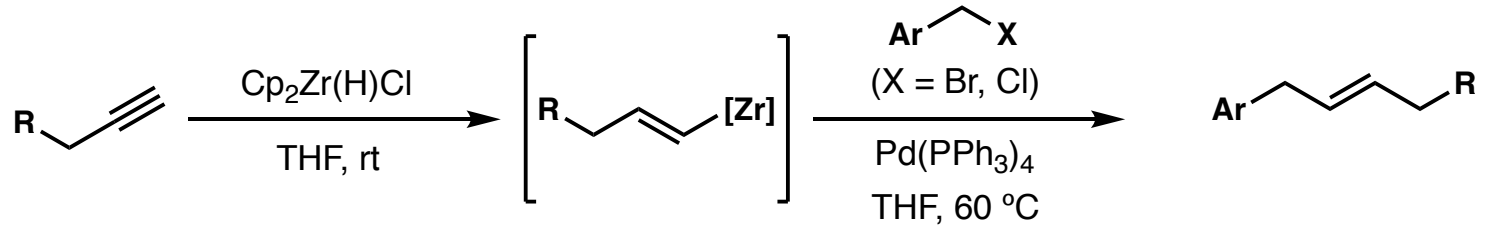

To a solution of Schwartz reagent $(110 \mathrm{mmol} \%)$ in THF $(5 \mathrm{~mL} / \mathrm{mmol}$ alkyne) was added alkyne $(100 \mathrm{~mol} \%)$ dropwise over five minutes. NB. For large scale reactions $(>10 \mathrm{mmol})$ the addition was performed at $0{ }^{\circ} \mathrm{C}$ due to the mild exotherm that occurs during the addition. The reaction mixture was then stirred at room temperature for one hour, after which time the mixture became clear. The reaction was diluted with THF (2 $\mathrm{mL} / \mathrm{mmol}$ alkyne) followed by sequential addition of $\mathrm{Pd}\left(\mathrm{PPh}_{3}\right)_{4}(3 \mathrm{~mol} \%)$ and benzyl halide derivative $(100 \mathrm{~mol} \%)$. The resulting mixture was heated at $60{ }^{\circ} \mathrm{C}$ and monitored by TLC analysis. After the reaction was complete, it was cooled to room temperature and concentrated in vacuo. The residue was taken up in hexane-EtOAc $(9: 1,10$ $\mathrm{mL} / \mathrm{mmol}$ alkyne) and filtered through Celite ${ }^{\circledR}$. The solvent was removed in vacuo and the residue purified by FCC, under the conditions noted, to afford the corresponding allylbenzene derivative. 


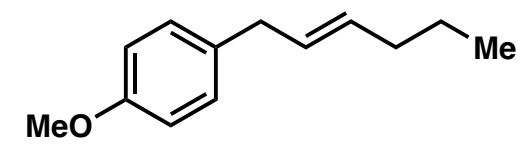

\section{(E)-1-(Hex-2-en-1-yl)-4-methoxybenzene}

General Procedure A: 1-Pentyne $(0.98 \mathrm{~mL}, 10 \mathrm{mmol})$ and 4-methoxybenzyl chloride $(1.4 \mathrm{~mL}, 10$ $\mathrm{mmol})$ were employed. Purification of the residue by FCC (100:1, hexane-EtOAc) afforded the title compound $(1.32 \mathrm{~g}, 69 \%)$ as a colorless oil.

${ }^{1} \mathrm{H}$ NMR $\left(400 \mathrm{MHz}, \mathrm{CDCl}_{3}\right) \delta 7.10(\mathrm{~d}, J=8.6 \mathrm{~Hz}, 2 \mathrm{H}), 6.84(\mathrm{~d}, J=8.6 \mathrm{~Hz}, 2 \mathrm{H}), 5.59-5.44(\mathrm{~m}, 2 \mathrm{H})$, $3.79(\mathrm{~s}, 3 \mathrm{H}), 3.27(2 \mathrm{H}, \mathrm{d}, J=6.0 \mathrm{~Hz}, 2 \mathrm{H}), 2.00$ (tdd, $J=7.4,6.2,0.9 \mathrm{~Hz}, 2 \mathrm{H}$ ), 1.40 (app. hept, $J=$ $7.3 \mathrm{~Hz}), 0.90(\mathrm{t}, \mathrm{J}=7.3 \mathrm{~Hz}, 3 \mathrm{H})$;

${ }^{13} \mathrm{C}$ NMR $\left(101 \mathrm{MHz}, \mathrm{CDCl}_{3}\right) \delta 157.8,133.2,131.5,129.3,113.8,55.3,38.2,34.6,22.6,13.7$;

IR ( $\mathrm{NaCl}$, thin film) 3029, 2957, 2929, 2835, 1611, 1584, 1511, 1464, 1300, 1245, 1175, 1039, $968,818 \mathrm{~cm}^{-1}$

HRMS $\left(\mathrm{El}^{+}\right) \mathrm{m} / \mathrm{z}$ calcd for $\mathrm{C}_{13} \mathrm{H}_{18} \mathrm{O}^{+}(\mathrm{M})^{+}:$190.1352, found 190.1355 . 

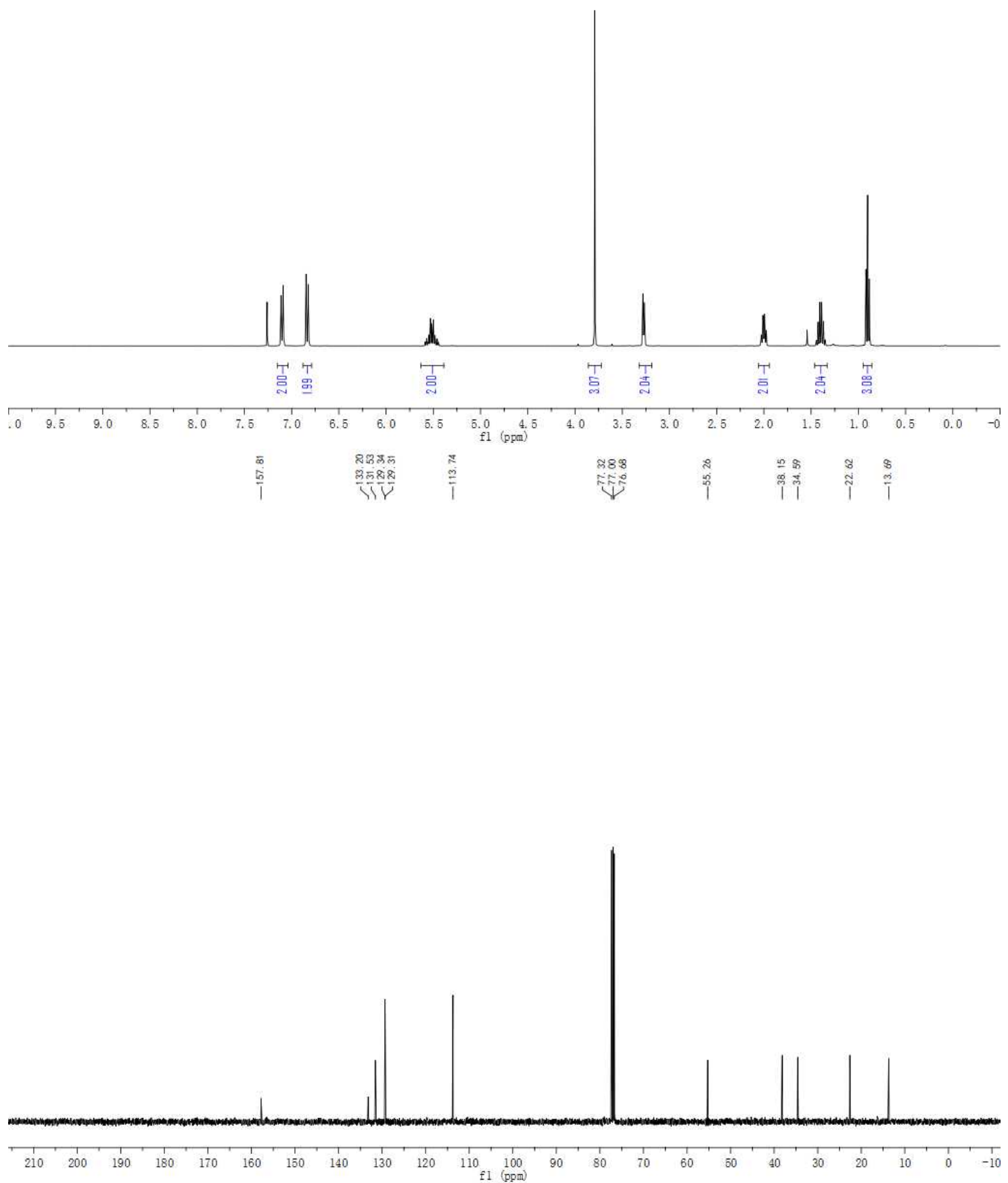


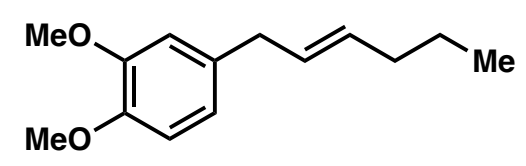

(E)-4-(Hex-2-en-1-yl)-1,2-dimethoxybenzene

General Procedure A: 1-Pentyne $(0.98 \mathrm{~mL}, 10 \mathrm{mmol})$ and 3,4-dimethoxybenzyl bromide ${ }^{2}(2.74 \mathrm{~g}$, $10 \mathrm{mmol})$ were employed. Purification of the residue by FCC (10:1, hexane-EtOAc) afforded the title compound $(2.40 \mathrm{~g}, 88 \%)$ as a colorless oil.

${ }^{1} \mathrm{H}$ NMR $\left(400 \mathrm{MHz}, \mathrm{CDCl}_{3}\right) \delta$ 6.81-6.79 (m, 1H), 6.73-6.71 (m, 2H), 5.59-5.46 (m, 2H), $3.87(\mathrm{~s}$, $3 \mathrm{H}), 3.86(\mathrm{~s}, 3 \mathrm{H}), 3.28(\mathrm{~d}, J=5.7 \mathrm{~Hz}, 2 \mathrm{H}), 2.04-1.99(\mathrm{~m}, 2 \mathrm{H}), 1.40$ (app. hept, $J=7.3 \mathrm{~Hz}, 2 \mathrm{H}$ ), $0.91(\mathrm{t}, J=7.3 \mathrm{~Hz}, 3 \mathrm{H}$ );

${ }^{13} \mathrm{C} \mathrm{NMR}\left(101 \mathrm{MHz}, \mathrm{CDCl}_{3}\right) \delta 148.8,147.2,133.8,131.7,129.2,120.2,111.8,111.2,55.9,55.8$, 38.6, 34.6, 22.6, 13.7;

IR ( $\mathrm{NaCl}$, thin film) 2969, 2872, 1593, 1515, 1464, 1419, 1265, 1235, 1139, $1027 \mathrm{~cm}^{-1}$;

HRMS (ESI-TOF) $\mathrm{m} / \mathrm{z}$ calcd for $\mathrm{C}_{14} \mathrm{H}_{20} \mathrm{NaO}_{2}{ }^{+}(\mathrm{M}+\mathrm{Na})^{+}:$243.1356, found 243.1351. 


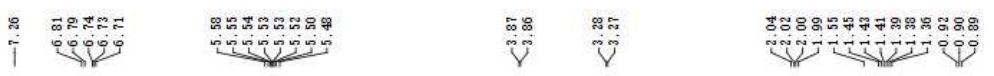
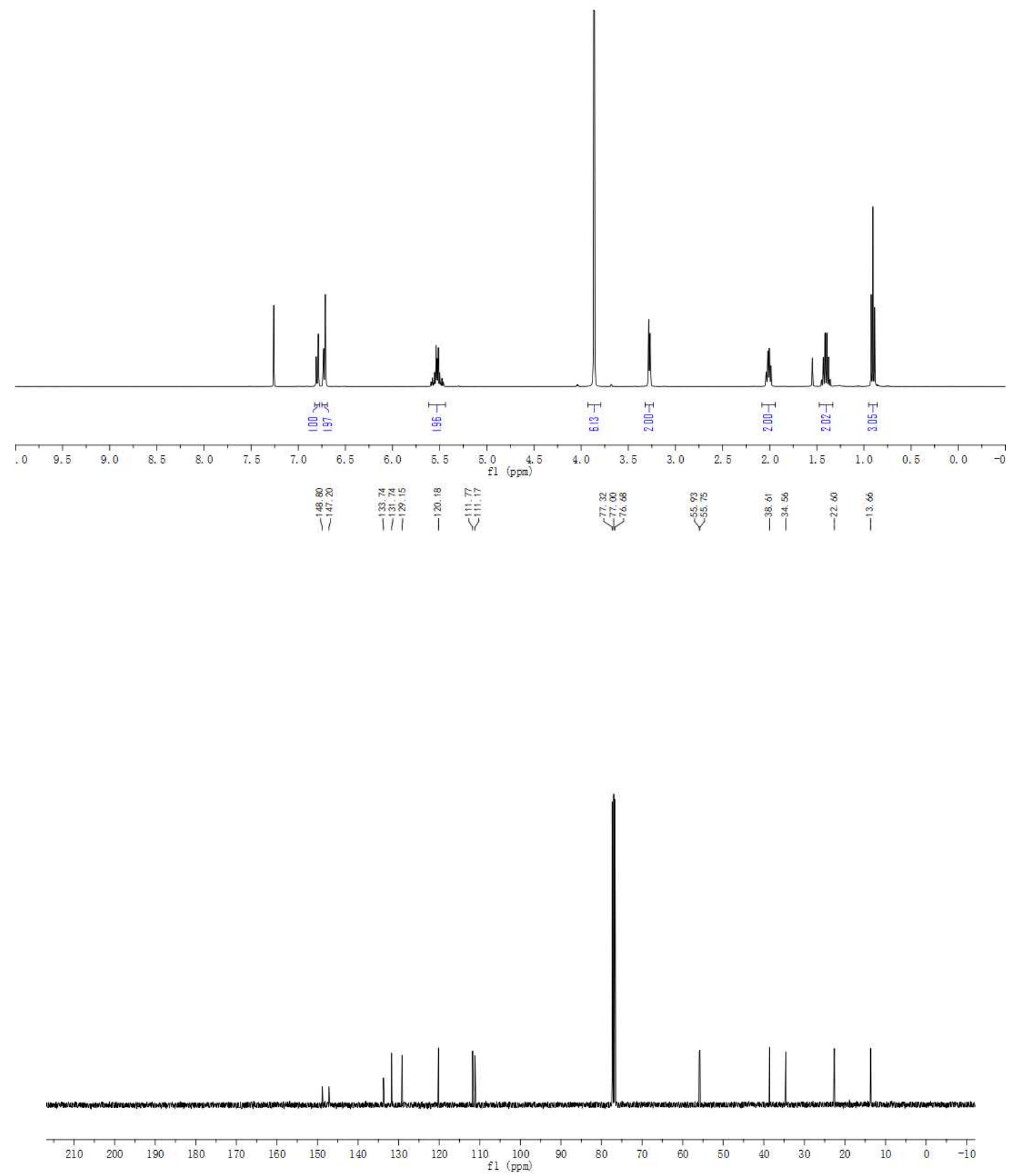


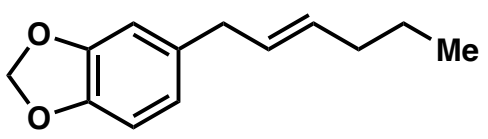

\section{(E)-5-(Hex-2-en-1-yl)benzo[d][1,3]dioxole}

General Procedure A: 1-Pentyne $(0.98 \mathrm{~mL}, 10 \mathrm{mmol})$ and 5-(bromomethyl)benzo[d][1,3]dioxole ${ }^{3}$ $(2.16 \mathrm{~g}, 10 \mathrm{mmol})$ were employed. Purification of the residue by FCC (80:1, hexane-EtOAc) afforded the title compound $(1.38 \mathrm{~g}, 68 \%)$ as a pale yellow oil.

${ }^{1} \mathrm{H}$ NMR $\left(400 \mathrm{MHz}, \mathrm{CDCl}_{3}\right) \delta 6.73(\mathrm{~d}, J=7.9 \mathrm{~Hz}, 1 \mathrm{H}), 6.68(\mathrm{~d}, J=1.7 \mathrm{~Hz}, 1 \mathrm{H}), 6.63(\mathrm{dd}, J=7.9$, $1.7 \mathrm{~Hz}, 1 \mathrm{H}), 5.92(\mathrm{~s}, 2 \mathrm{H}), 5.56-5.44(\mathrm{~m}, 2 \mathrm{H}), 3.24(\mathrm{~d}, J=5.0 \mathrm{~Hz}, 2 \mathrm{H}), 2.02(\mathrm{~m}, 2 \mathrm{H}), 1.40$ (app. hept, $J=7.3 \mathrm{~Hz}, 2 \mathrm{H}), 0.9(\mathrm{t}, J=7.4 \mathrm{~Hz}, 3 \mathrm{H})$;

${ }^{13} \mathrm{C}$ NMR $\left(101 \mathrm{MHz}, \mathrm{CDCl}_{3}\right) \delta 147.5,145.6,135.0,131.8,129.0,121.1,109.0,108.1,100.7,38.7$, 34.6, 22.6, 13.7;

IR ( $\mathrm{NaCl}$, thin film): 2958, 2927, 1608, 1504, 1441, 1378, 1246, 1183, 1042, 968, 858, $807 \mathrm{~cm}^{-1}$; HRMS $\left(\mathrm{El}^{+}\right) \mathrm{m} / \mathrm{z}$ calcd for $\mathrm{C}_{13} \mathrm{H}_{16} \mathrm{O}_{2}{ }^{+}(\mathrm{M})^{+}:$204.1139, found 204.1130. 

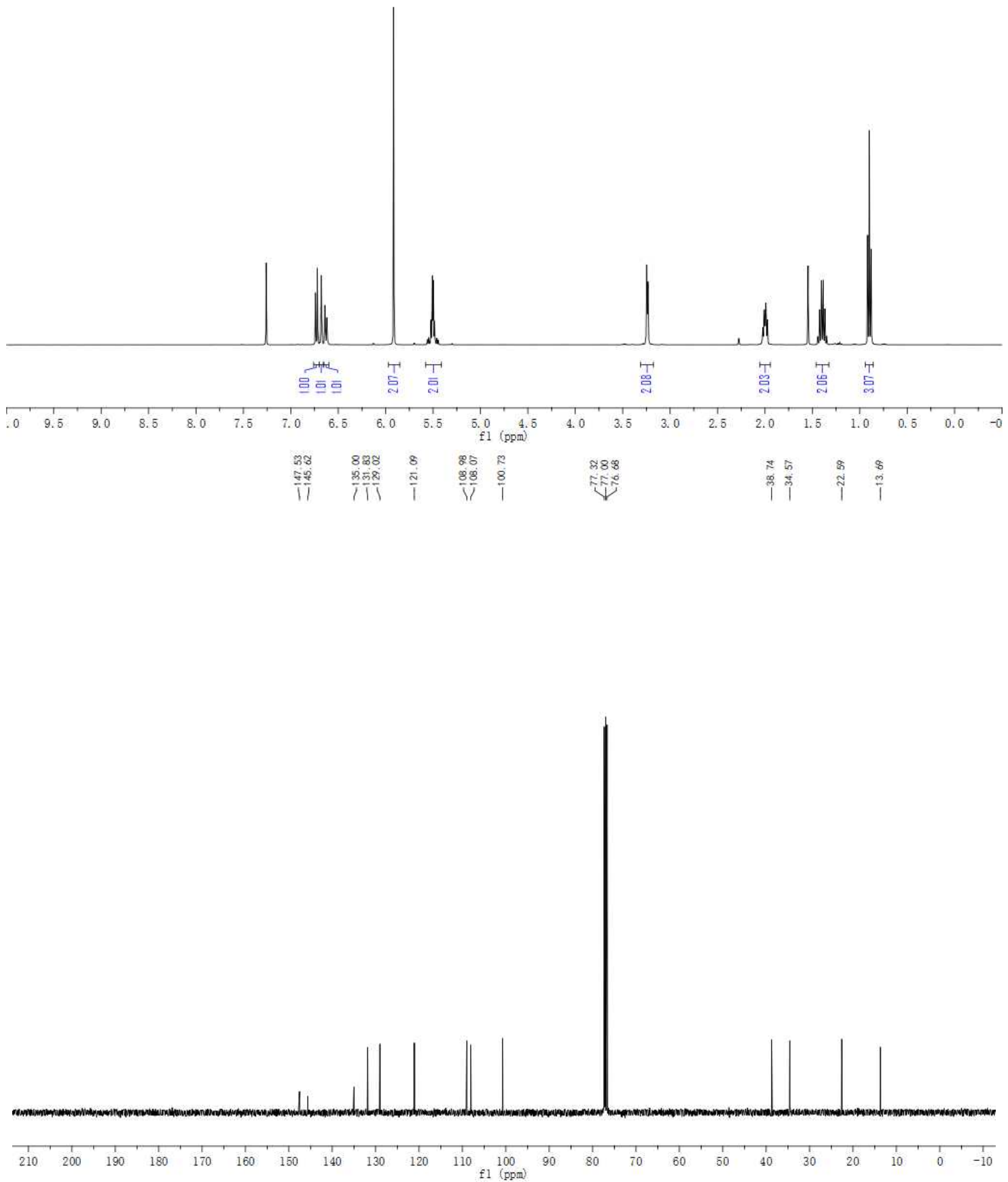


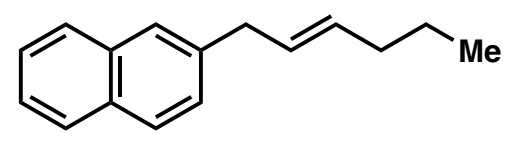

\section{(E)-2-(Hex-2-en-1-yl)naphthalene}

General Procedure A: 1-Pentyne $(0.98 \mathrm{~mL}, 10 \mathrm{mmol})$ and 2-(bromomethyl)naphthalene (3.30 g, $10 \mathrm{mmol}$ ) were employed. Purification of the residue by FCC (100:1, hexane-EtOAc) afforded the title compound $(2.12 \mathrm{~g}, 67 \%)$ as a pale colorless oil.

${ }^{1} \mathrm{H}$ NMR $\left(400 \mathrm{MHz}, \mathrm{CDCl}_{3}\right) \delta$ 7.83-7.77 (m, 3H), 7.62 (br. s, 1H), 7.48-7.40 (m, 2H), 7.34 (dd, $J=$ 8.4, 1.7 Hz, 1H), $5.66(\mathrm{dtt}, J=15.1,6.5,1.2 \mathrm{~Hz}, 1 \mathrm{H}), 5.58(\mathrm{dtt}, J=15.1,6.5,1.2 \mathrm{~Hz}, 1 \mathrm{H}), 3.50$ (app. d, $J=6.5 \mathrm{~Hz}, 2 \mathrm{H}$ ), 2.07-2.02 (m, 2H), 1.43 (app. hept, $J=7.4 \mathrm{~Hz}, 2 \mathrm{H}$ ), 0.92 (t, $J=7.4 \mathrm{~Hz}$, $3 \mathrm{H})$;

${ }^{13} \mathrm{C}$ NMR $\left(101 \mathrm{MHz}, \mathrm{CDCl}_{3}\right) \delta 138.6,133.6,132.2,132.0,128.8,127.8,127.6,127.5,127.4$, $126.4,125.8,125.1,39.2,34.6,22.6,13.7$;

IR ( $\mathrm{NaCl}$, thin film) 3045, 2958, 2871, 1597, 1510, 1455, 1396, 1377, $968 \mathrm{~cm}^{-1}$;

HRMS $\left(\mathrm{EI}^{+}\right) \mathrm{m} / \mathrm{z}$ calcd for $\mathrm{C}_{16} \mathrm{H}_{18}{ }^{+}(\mathrm{M})^{+}:$210.1409, found 210.1401. 

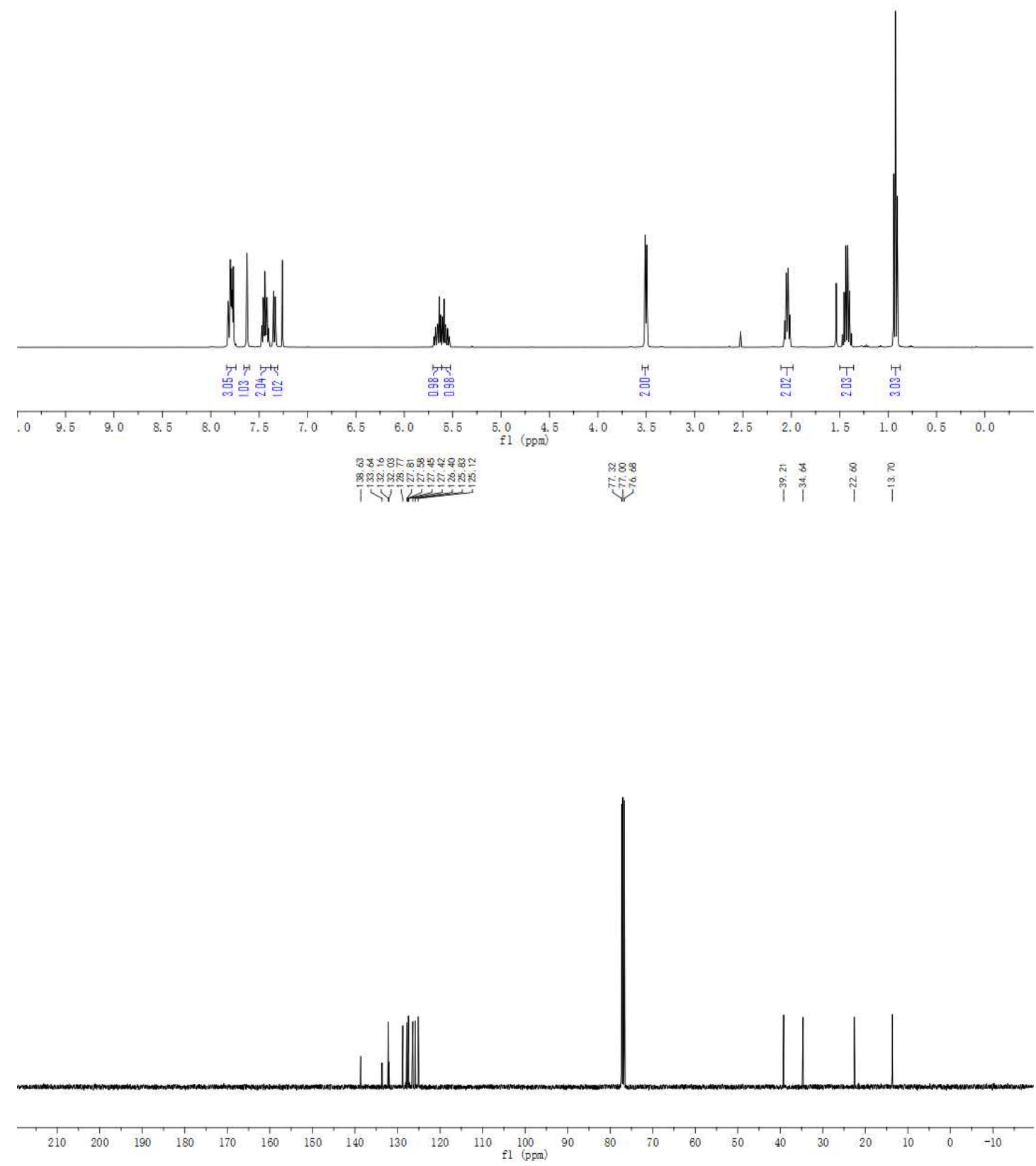


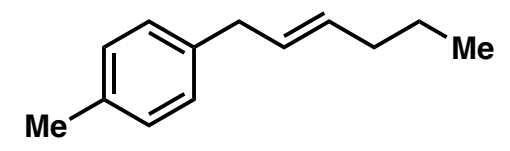

\section{(E)-1- (Hex-2-en-1-yl)-4-methylbenzene}

General Procedure A: 1-Pentyne $(1.0 \mathrm{~mL}, 15 \mathrm{mmol})$ and 4-methylbenzyl bromide $(2.79 \mathrm{~g}, 15$ $\mathrm{mmol})$ were employed. Purification of the residue by FCC (100\% hexane) afforded the title compound $(2.13 \mathrm{~g}, 81 \%)$ as a colorless oil.

${ }^{1} \mathrm{H}$ NMR (400 MHz, $\left.\mathrm{CDCl}_{3}\right)$ ठ 7.11-7.06 (m, 4H), $5.53(\mathrm{~m}, 2 \mathrm{H}), 3.29(\mathrm{~d}, J=5.91 \mathrm{~Hz}, 2 \mathrm{H}), 2.32(\mathrm{~s}$, $3 \mathrm{H}$ ), 2.03-1.97 (m, 2H), 1.40 (app. $\mathrm{h}, J=7.3 \mathrm{~Hz}, 2 \mathrm{H}), 0.90$ (t, $J=7.4 \mathrm{~Hz}, 3 \mathrm{H}$ );

${ }^{13} \mathrm{C}$ NMR $\left(101 \mathrm{MHz}_{\mathrm{CDCl}}\right) \delta 138.2,135.4,131.8,129.3,129.2,128.5,38.8,34.8,22.8,21.1$, $13.8 \mathrm{~cm}^{-1}$;

IR ( $\mathrm{NaCl}$, thin film) 3020, 2958, 2927, 2872, 1514, 1457, 1437, 966, $803 \mathrm{~cm}^{-1}$;

HRMS $\left(\mathrm{EI}^{+}\right) \mathrm{m} / \mathrm{z}$ calcd for $\mathrm{C}_{13} \mathrm{H}_{18}{ }^{+}(\mathrm{M})^{+}:$: 174.1409, found 174.1402. 

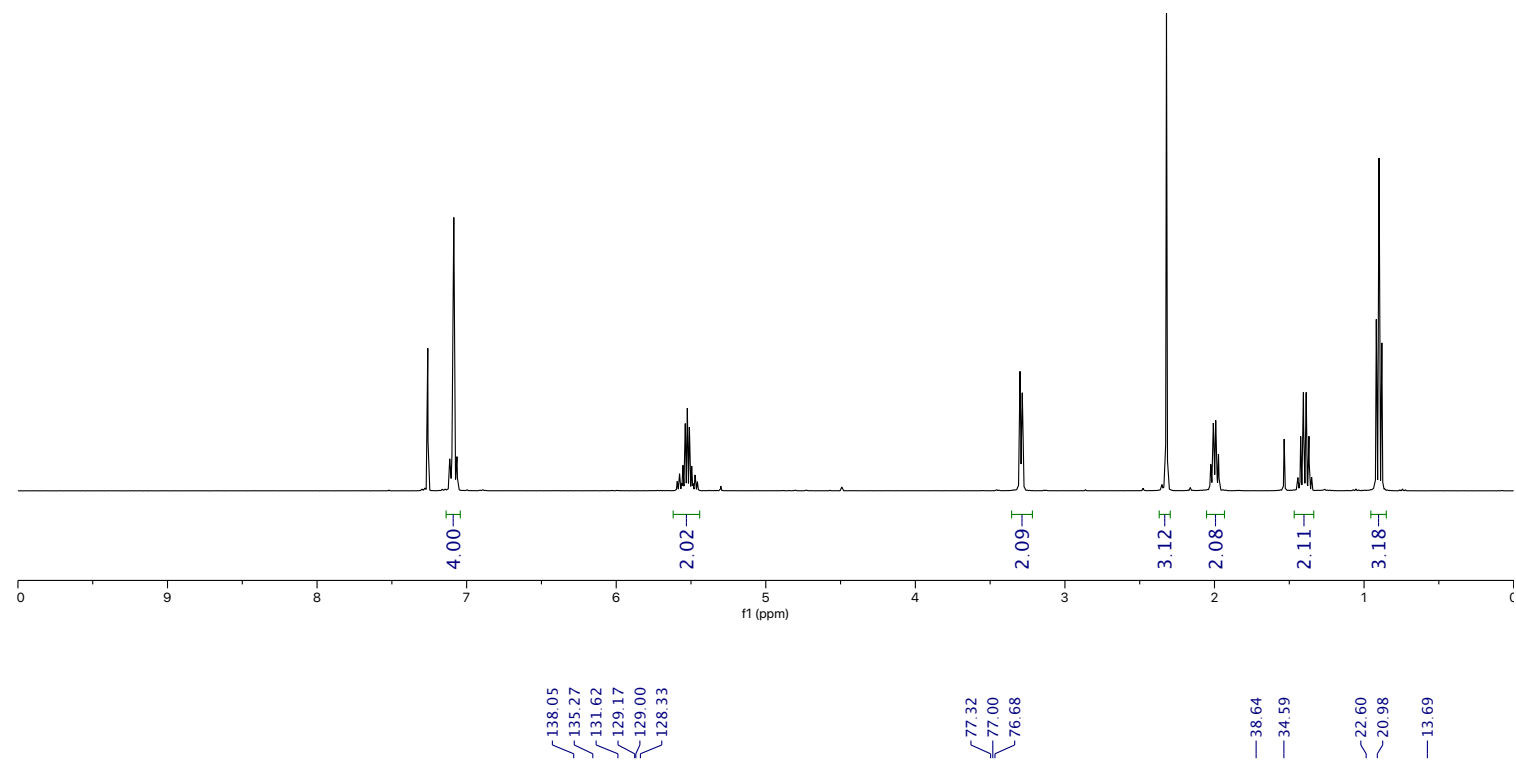

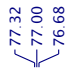

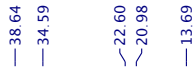

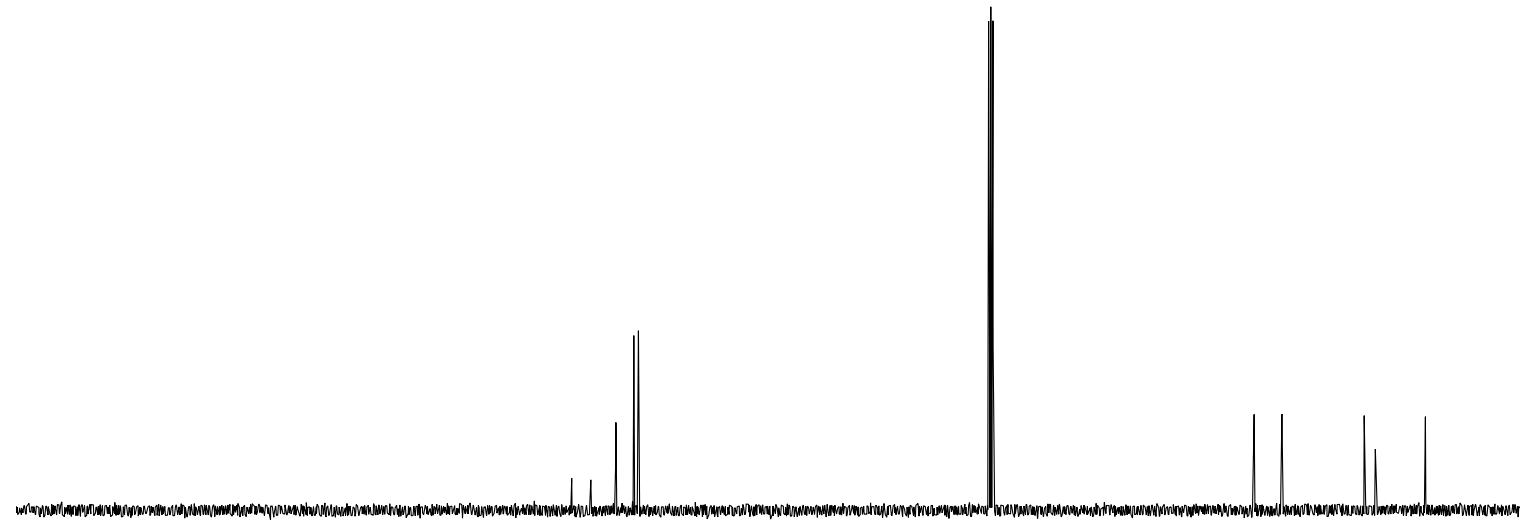




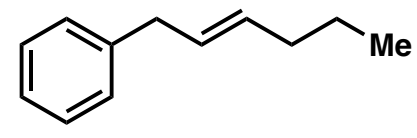

\section{(E)-Hex-2-en-1-ylbenzene}

General Procedure A: 1-Pentyne $(0.67 \mathrm{~mL}, 10 \mathrm{mmol})$ and benzyl bromide $(1.2 \mathrm{~mL}, 10 \mathrm{mmol})$ were employed. Purification of the residue by FCC (100\% hexane) afforded the title compound (1.09 $\mathrm{g}, 66 \%)$ as a colorless oil.

${ }^{1} \mathbf{H}$ NMR $\left(400 \mathrm{MHz}, \mathrm{CDCl}_{3}\right)$ ठ 7.31-7.26 (m, 2H), 7.20-7.17 (m, 3H), 5.61-5.47 (m, 2H), $3.34(\mathrm{~d}, J$ $=6.0 \mathrm{~Hz}, 2 \mathrm{H}), 2.03-1.98(\mathrm{~m}, 2 \mathrm{H}), 1.40($ app. $\mathrm{h}, J=7.4 \mathrm{~Hz}, 2 \mathrm{H}), 0.90$ (t, J = $7.4 \mathrm{~Hz}, 3 \mathrm{H})$;

${ }^{13} \mathrm{C}$ NMR $\left(101 \mathrm{MHz}, \mathrm{CDCl}_{3}\right) \delta$ 141.3, 132.1, 129.0, 128.6, 128.5, 126.0, 39.2, 34.8, 22.8, 13.8;

IR ( $\mathrm{NaCl}$, thin film) 3028, 2959, 2928, 2872, 1604, 1494, 1454, 1436, 1072, 1030, 968, 745, 698 $\mathrm{cm}^{-1}$;

HRMS $\left(\mathrm{El}^{+}\right) \mathrm{m} / \mathrm{z}$ calcd for $\mathrm{C}_{12} \mathrm{H}_{16}{ }^{+}(\mathrm{M})^{+}:$160.1247, found 160.1251. 

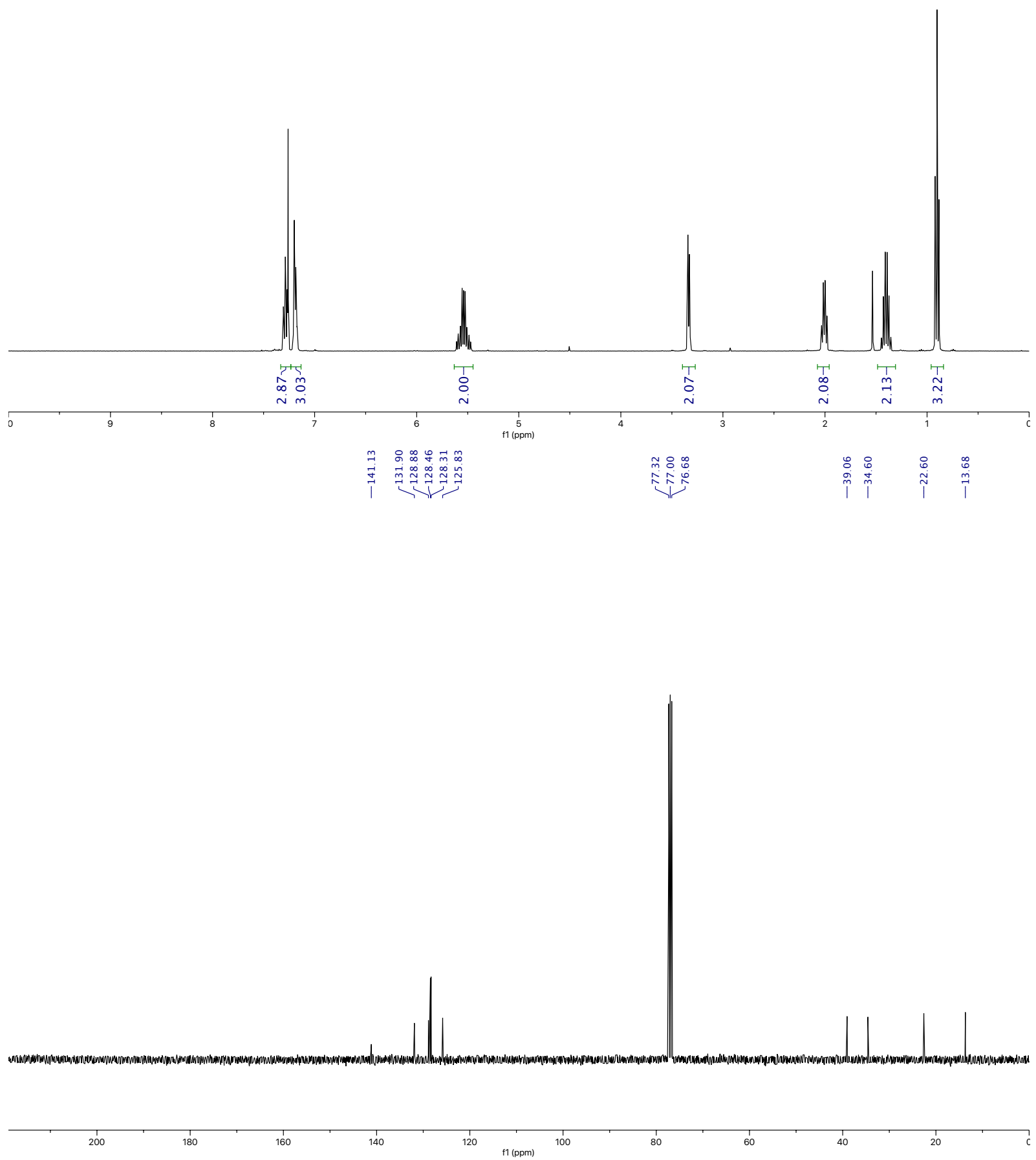


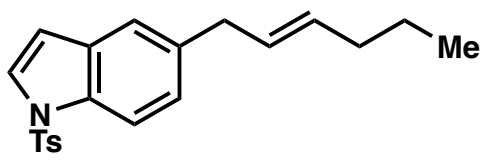

(E)-5-(Hex-2-en-1-yl)-1-tosyl-1H-indole

General Procedure A: 1-Pentyne $(0.54 \mathrm{~mL}, 5.5 \mathrm{mmol})$ and 5-(bromomethyl)-1-tosyl-1H-indole, ${ }^{4}$ (2.0 g, $5.5 \mathrm{mmol})$ were employed. Purification of the residue by FCC (100:1, hexane-EtOAc) afforded the title compound $(1.41 \mathrm{~g}, 74 \%)$ as a pale yellow oil.

${ }^{1} \mathrm{H}$ NMR $\left(400 \mathrm{MHz}, \mathrm{CDCl}_{3}\right) \delta 7.89(\mathrm{~d}, J=8.5 \mathrm{~Hz}, 1 \mathrm{H}), 7.75(\mathrm{~d}, J=8.4 \mathrm{~Hz}, 2 \mathrm{H}), 7.52(\mathrm{~d}, J=3.6$ $\mathrm{Hz}, 1 \mathrm{H}), 7.32-7.31(\mathrm{~m}, 1 \mathrm{H}), 7.22-7.20(\mathrm{~m}, 2 \mathrm{H}), 7.14(\mathrm{dd}, J=8.5,1.7 \mathrm{~Hz}, 1 \mathrm{H}), 6.59(\mathrm{dd}, J=3.7$, $0.8 \mathrm{~Hz}, 1 \mathrm{H}), 5.60-5.47(\mathrm{~m}, 2 \mathrm{H}), 3.38(\mathrm{~d}, J=5.9 \mathrm{~Hz}, 2 \mathrm{H}), 2.33(\mathrm{~s}, 3 \mathrm{H}), 2.03-1.97(\mathrm{~m}, 2 \mathrm{H}), 1.39$ (app. h, $J=7.4 \mathrm{~Hz}, 2 \mathrm{H}$ ), 0.90 (t, $J=7.4 \mathrm{~Hz}, 3 \mathrm{H}$ );

${ }^{13} \mathrm{C}$ NMR $\left(101 \mathrm{MHz}, \mathrm{CDCl}_{3}\right) \delta 144.8,136.2,135.4,133.4,131.9,131.0,129.8,129.0,126.8$, $126.4,125.4,120.7,113.3,108.9,38.9,34.6,22.6,21.5,13.7$;

IR ( $\mathrm{NaCl}$, thin film): 3142, 3029, 2959, 2929, 2872, 1596, 1456, 1372, 1268, 1173, 1093, 995, $812,724 \mathrm{~cm}^{-1}$;

HRMS (ESI-TOF) $m / z$ calcd for $\mathrm{C}_{21} \mathrm{H}_{23} \mathrm{NO}_{2} \mathrm{SNa}^{+}(\mathrm{M}+\mathrm{Na})^{+}: 376.1347$, found 376.1340. 

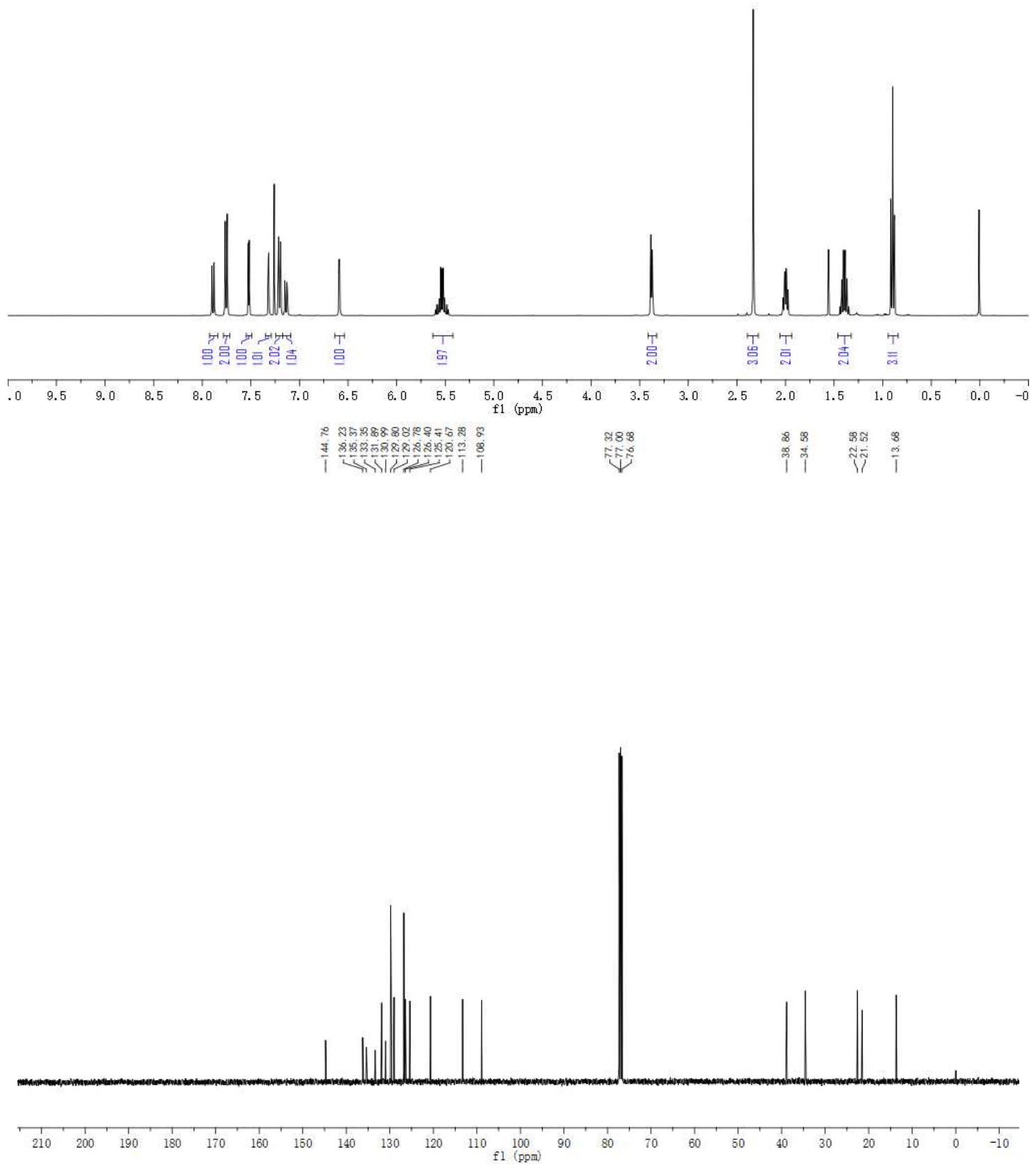
<smiles>CCC/C=C/Cc1ccc(F)cc1</smiles>

\section{(E)-1-Fluoro-4-(hex-2-en-1-yl)benzene}

General Procedure A: 1-Pentyne $(1.2 \mathrm{~mL}, 12 \mathrm{mmol})$ and 4-fluorobenzyl bromide $(1.5 \mathrm{~mL}, 12$ $\mathrm{mmol})$ were employed. Purification of the residue by FCC (100:1, hexane-EtOAc) afforded the title compound $(1.41 \mathrm{~g}, 65 \%)$ as a colorless oil.

${ }^{1} \mathrm{H}$ NMR $\left(400 \mathrm{MHz}, \mathrm{CDCl}_{3}\right)$ ठ 7.15.7-11 (m, 2H), 6.99-6.93 (m, 2H), 5.57-5.45 (m, 2H), 3.30 (d, J $=5.4 \mathrm{~Hz}, 2 \mathrm{H}), 2.03-1.98(\mathrm{~m}, 2 \mathrm{H}), 1.40$ (app. $\mathrm{h}, \mathrm{J}=7.3 \mathrm{~Hz}, 2 \mathrm{H}), 0.90(\mathrm{t}, J=7.4 \mathrm{~Hz}, 3 \mathrm{H})$;

${ }^{13} \mathrm{C} \mathrm{NMR}\left(126 \mathrm{MHz}, \mathrm{CDCl}_{3}\right) \delta 161.3(\mathrm{~d}, J=243.4 \mathrm{~Hz}), 136.7(\mathrm{~d}, J=3.2 \mathrm{~Hz}), 132.1,129.8(\mathrm{~d}, J=$ $7.9 \mathrm{~Hz}), 128.8,116$ (d, $J=21.0 \mathrm{~Hz}), 38.2,34.6,22.6,13.7$;

${ }^{19} \mathrm{~F}\left\{{ }^{1} \mathrm{H}\right\}$ NMR $\left(376 \mathrm{MHz}, \mathrm{CDCl}_{3}\right) \delta-117.9(\mathrm{~s})$;

IR ( $\mathrm{NaCl}$, thin film) 3038, 2960, 2929, 2873, 1604, 1509, 1464, 1222, 1157, 1092, 969, $822 \mathrm{~cm}^{-1}$; HRMS $\left(\mathrm{EI}^{+}\right) \mathrm{m} / \mathrm{z}$ calcd for $\mathrm{C}_{12} \mathrm{H}_{15} \mathrm{~F}^{+}(\mathrm{M})^{+}:$178.1152, found 178.1151 . 

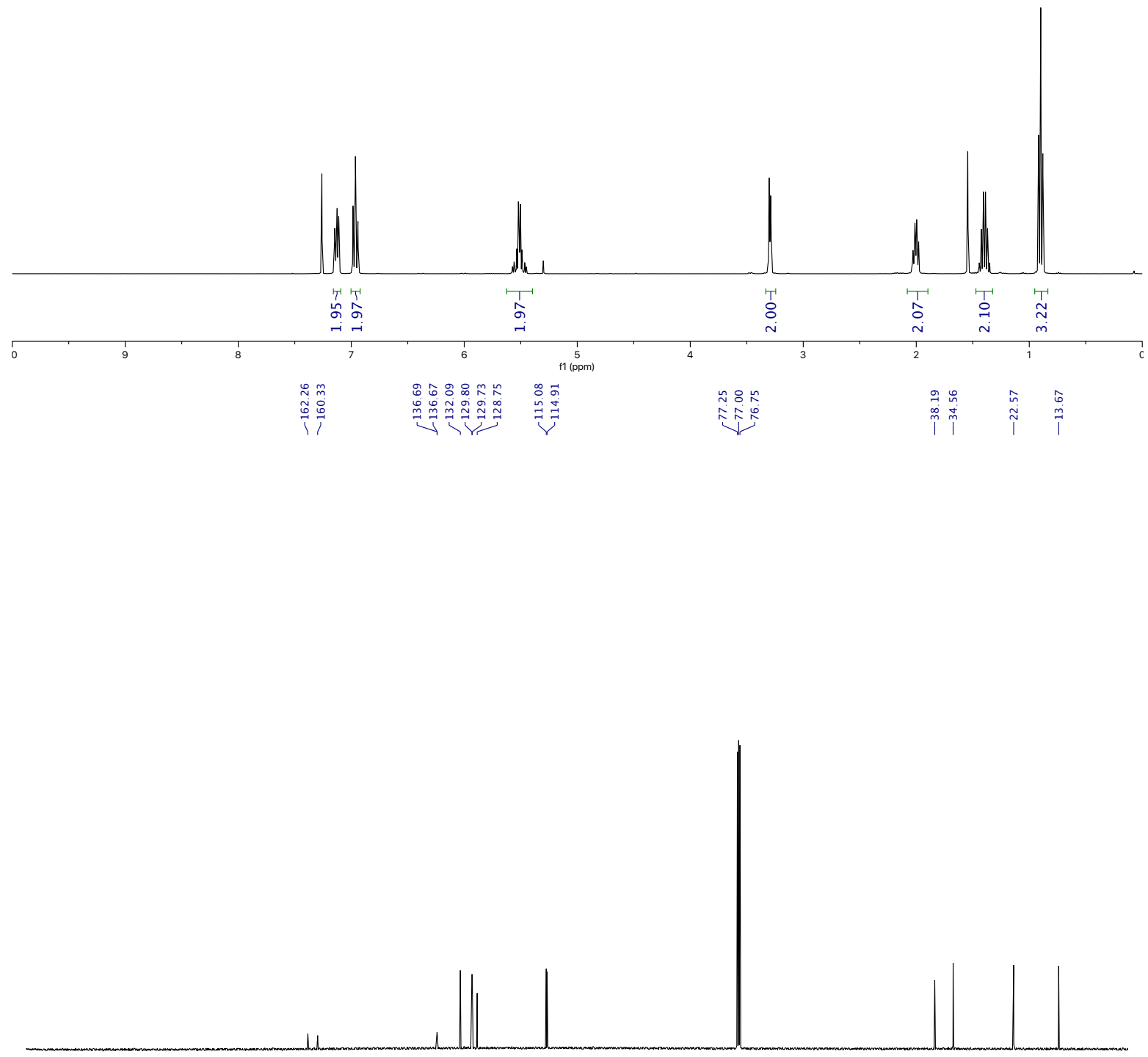

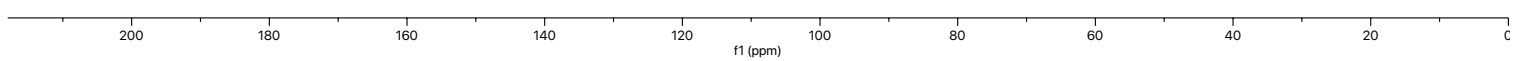


<smiles>CCC/C=C/Cc1ccc(Cl)cc1</smiles>

\section{(E)-1-Chloro-4-(hex-2-en-1-yl)benzene}

General Procedure A: 1-Pentyne $(1.1 \mathrm{~mL}, 11 \mathrm{mmol})$ and 4-chlorobenzyl bromide $(2.25 \mathrm{~g}, 11$ $\mathrm{mmol})$ were employed. Purification of the residue by FCC (100\% pentane) afforded the title compound $(1.41 \mathrm{~g}, 66 \%)$ as a colorless oil.

${ }^{1} \mathrm{H}$ NMR $\left(400 \mathrm{MHz}, \mathrm{CDCl}_{3}\right) \delta 7.25(\mathrm{~d}, J=8.4 \mathrm{~Hz}, 2 \mathrm{H}), 7.11(\mathrm{~d}, J=8.4 \mathrm{~Hz}, 2 \mathrm{H}), 5.56-5.45(\mathrm{~m}, 2 \mathrm{H})$, 3.29 (d, $J=4.8 \mathrm{~Hz}, 2 \mathrm{H}), 2.03-1.98(\mathrm{~m}, 2 \mathrm{H}), 1.39$ (app. h, J = $7.3 \mathrm{~Hz}, 2 \mathrm{H}), 0.90$ (t, $J=7.4 \mathrm{~Hz}, 3 \mathrm{H}$ );

${ }^{13} \mathrm{C}$ NMR $\left(101 \mathrm{MHz}, \mathrm{CDCl}_{3}\right) \delta$ 139.6, 132.4, 131.6, 129.8, 128.4, 128.3, 38.4, 34.6, 22.5, 13.7;

IR ( $\mathrm{NaCl}$, thin film) 3028, 2959, 2928, 2872, 1596, 1491, 1464, 1435, 1378, 1195, 1093, 1016 , 968, $804 \mathrm{~cm}^{-1}$;

HRMS $\left(\mathrm{El}^{+}\right) \mathrm{m} / \mathrm{z}$ calcd for $\mathrm{C}_{12} \mathrm{H}_{15}{ }^{35} \mathrm{Cl}^{+}(\mathrm{M})^{+}:$194.0857, found 194.0862. 

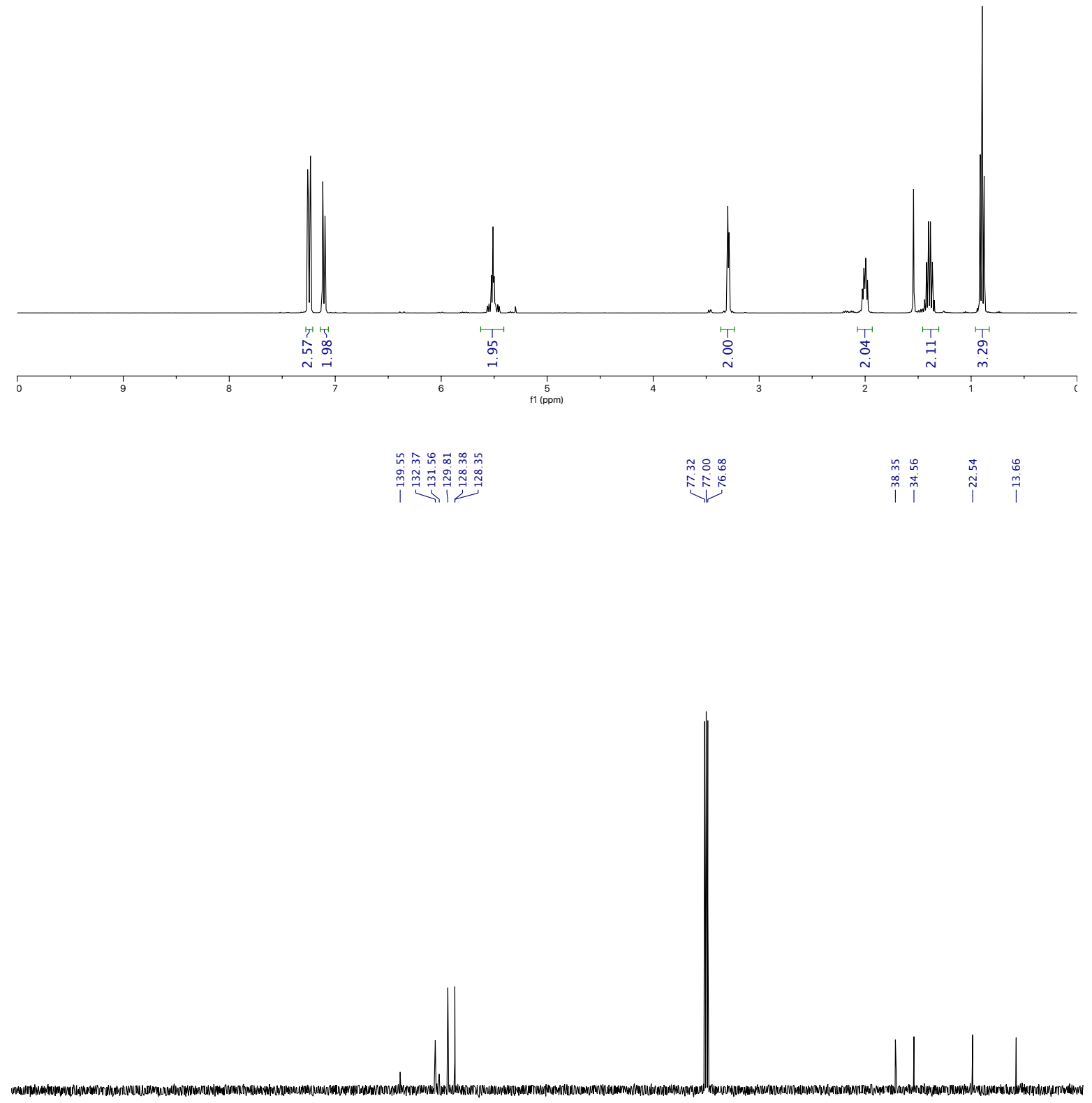

$200+180$

160

140

120

100

80

60

40

${ }_{20}^{1} \cdot c$ 
<smiles>CCC/C=C/Cc1ccc(Br)cc1</smiles>

\section{(E)-1-Bromo-4-(hex-2-en-1-yl)benzene}

General Procedure A: 1-Pentyne $(1.0 \mathrm{~mL}, 10 \mathrm{mmol})$ and 4-bromobenzyl bromide $(2.50 \mathrm{~g}, 10$ $\mathrm{mmol})$ were employed. Purification of the residue by FCC (100\% pentane) afforded the title compound $(1.84 \mathrm{~g}, 77 \%)$ as a colorless oil.

${ }^{1} \mathrm{H}$ NMR $\left(400 \mathrm{MHz}, \mathrm{CDCl}_{3}\right) \delta 7.39(\mathrm{~d}, \mathrm{~J}=8.3 \mathrm{~Hz}, 2 \mathrm{H}), 7.05(\mathrm{~d}, J=8.3 \mathrm{~Hz}, 2 \mathrm{H}), 5.56-5.45(\mathrm{~m}, 2 \mathrm{H})$, 3.27 (d, $J=4.7 \mathrm{~Hz}, 2 \mathrm{H}), 2.03-1.97(\mathrm{~m}, 2 \mathrm{H}), 1.39$ (app. h, $J=7.4 \mathrm{~Hz}, 2 \mathrm{H}), 0.89$, (t, J = 7.3 Hz, 3H);

${ }^{13} \mathrm{C}$ NMR $\left(126 \mathrm{MHz}, \mathrm{CDCl}_{3}\right) \delta 140.1,132.4,131.4,130.3,128.3,119.61,38.4,34.6,22.6,13.7$;

IR ( $\mathrm{NaCl}$, thin film) 3026, 2959, 2927, 2871, 1590, 1487, 1403, 1194, 1071, 1012, 968, $800 \mathrm{~cm}^{-1}$; HRMS $\left(E l^{+}\right) \mathrm{m} / \mathrm{z}$ calcd for $\mathrm{C}_{12} \mathrm{H}_{15}{ }^{79} \mathrm{Br}^{+}(\mathrm{M})^{+}:$238.0352, found 238.0359. 


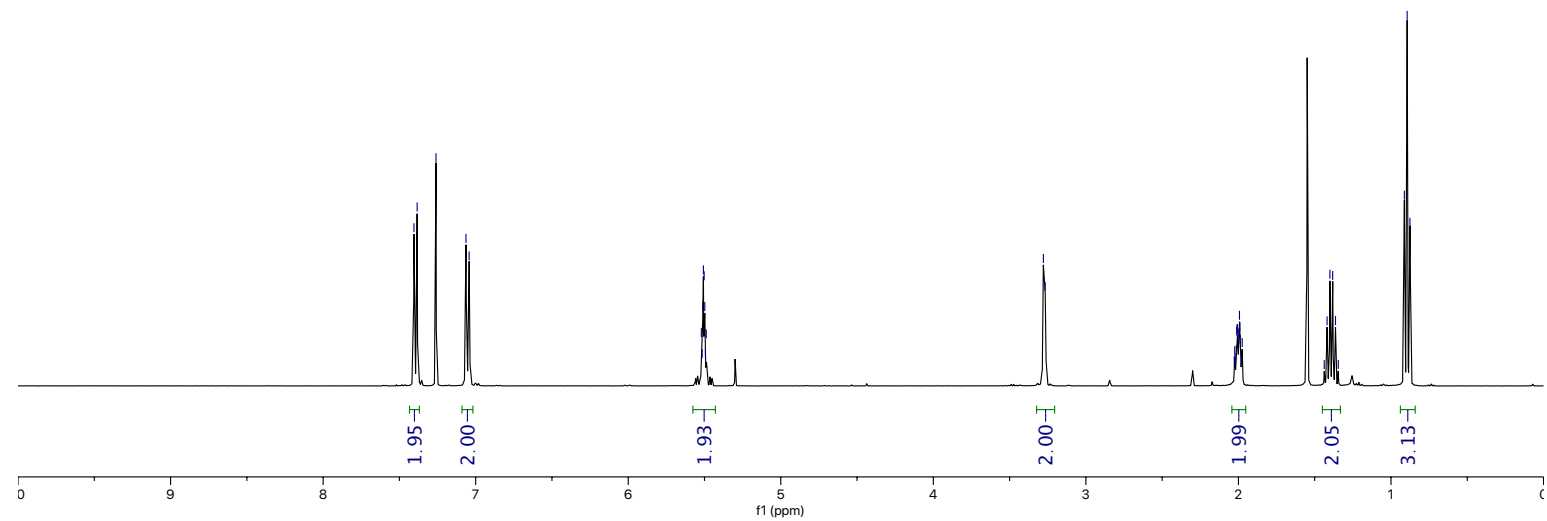

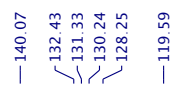

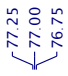

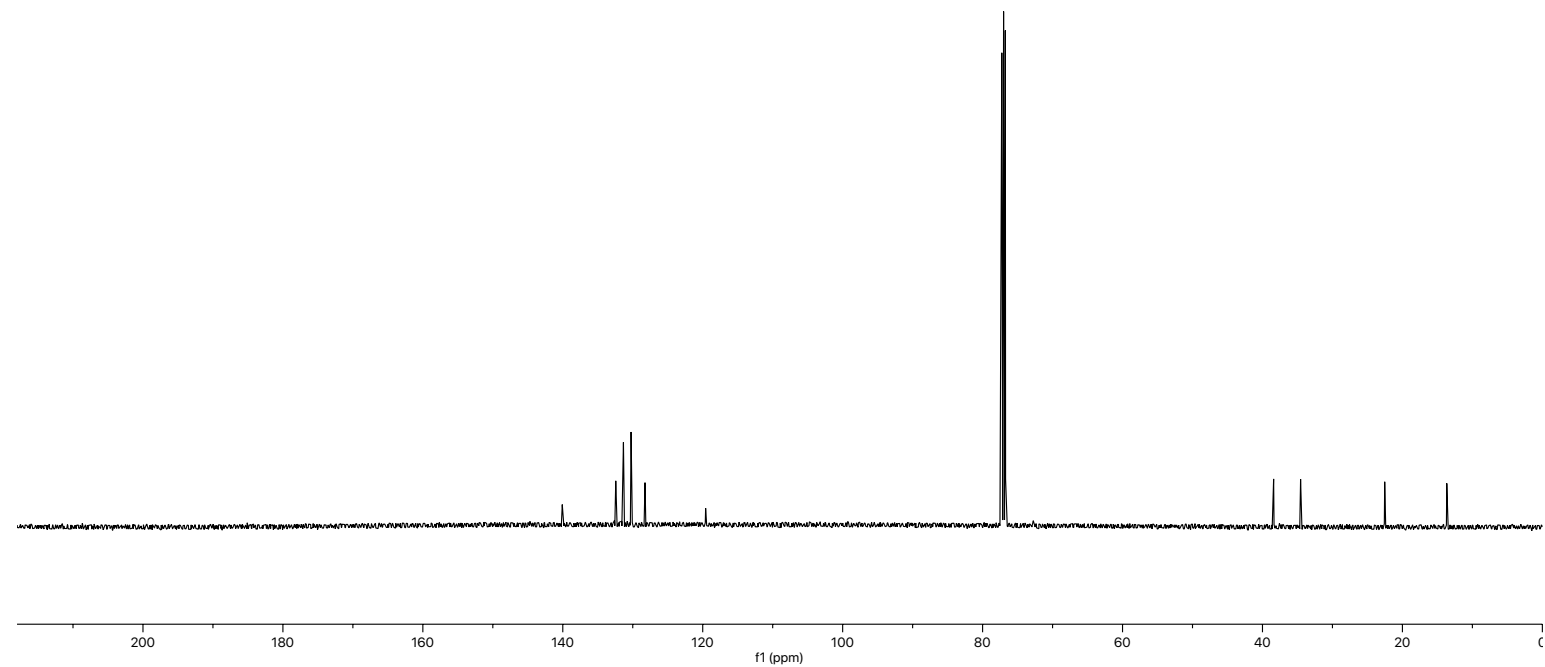


<smiles>CCC/C=C/Cc1ccc(C(F)(F)F)cc1</smiles>

\section{(E)-1-(Hex-2-en-1-yl)-4-(trifluoromethyl)benzene}

General Procedure A: 1-Pentyne (1.3 mL, $13.3 \mathrm{mmol}$ ) and 4-(trifluoromethyl)benzyl bromide (2.66 $\mathrm{g}, 11.1 \mathrm{mmol}$ ) were employed. Purification of the residue by FCC (100\% pentane) afforded the title compound $(1.82 \mathrm{~g}, 72 \%)$ as a colorless oil.

${ }^{1} \mathrm{H}$ NMR $\left(400 \mathrm{MHz}, \mathrm{CDCl}_{3}\right) \delta 7.53(\mathrm{~d}, J=8.0 \mathrm{~Hz}, 2 \mathrm{H}), 7.29(\mathrm{~d}, J=8.1 \mathrm{~Hz}, 2 \mathrm{H}), 5.59-5.49(\mathrm{~m}, 2 \mathrm{H})$, 3.38 (d, $J=4.2 \mathrm{~Hz}, 2 \mathrm{H}$ ), 2.04-1.99 (m, 2H), 1.40 (app. h, J = 7.4 Hz, 2H), 0.90 (t, J = 7.4 Hz, 3H);

${ }^{13} \mathrm{C}$ NMR $\left(125 \mathrm{MHz}, \mathrm{CDCl}_{3}\right)$ 145.3, 132.9, 128.8, 128.2 (q, $\left.J=32.3 \mathrm{~Hz}\right), 128.0,125.2$ (q, $J=3.7$ $\mathrm{Hz}), 124.4$ (q, $J=271.1 \mathrm{~Hz}), 38.8,34.6,22.5,13.7$;

${ }^{19} \mathrm{~F}\left\{{ }^{1} \mathrm{H}\right\}$ NMR $\left(376 \mathrm{MHz}, \mathrm{CDCl}_{3}\right) \delta-62.3(\mathrm{~s})$;

IR ( $\mathrm{NaCl}$, thin film) 2961, 2931, 2875, 1619, 1417, 1325, 1164, 1130, 1067, 1019, 968, 820;

HRMS $\left(\mathrm{El}^{+}\right) \mathrm{m} / \mathrm{z}$ calcd for $\mathrm{C}_{13} \mathrm{H}_{15} \mathrm{~F}_{3}{ }^{+}(\mathrm{M})^{+}:$: 228.1120, found 228.1122 . 

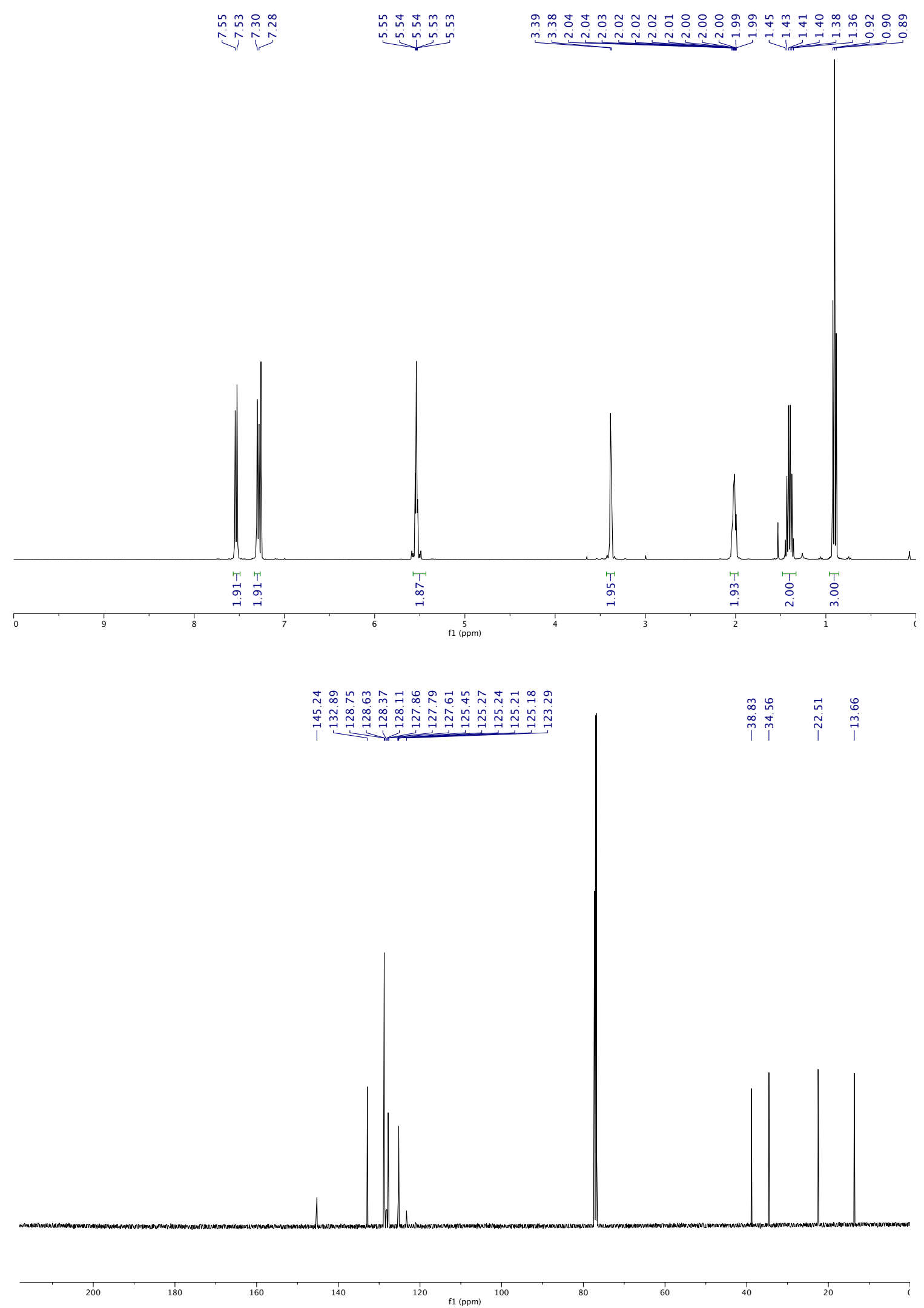


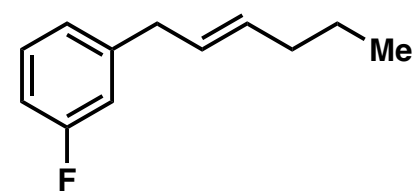

\section{(E)-1-Fluoro-3-(hex-2-en-1-yl)benzene}

General Procedure A: 1-Pentyne $(0.57 \mathrm{~mL}, 5.83 \mathrm{mmol})$ and 3-fluorobenzyl bromide $(0.71 \mathrm{~mL}$, $5.83 \mathrm{mmol})$ were employed. Purification of the residue by FCC (100:1, hexane-EtOAc) afforded the title compound (760 mg, 73\%) as a colorless oil.

${ }^{1} \mathrm{H}$ NMR $\left(400 \mathrm{MHz}, \mathrm{CDCl}_{3}\right) \delta 7.26-7.21(\mathrm{~m}, 1 \mathrm{H}), 6.96(\mathrm{~d}, \mathrm{~J}=7.7 \mathrm{~Hz}, 1 \mathrm{H}), 6.91-6.86(\mathrm{~m}, 2 \mathrm{H})$, 5.59-5.48 (m, 2H), 3.37-3.29 (m, 2H), 2.05-1.99 (m, 2H), 1.41 (app. h, J = 7.4 Hz, 2H), 0.91 (t, J $=7.4 \mathrm{~Hz}, 3 \mathrm{H})$;

${ }^{13} \mathrm{C}$ NMR $\left(101 \mathrm{MHz}, \mathrm{CDCl}_{3}\right) \delta 162.9(\mathrm{~d}, J=245.1 \mathrm{~Hz}), 143.8(\mathrm{~d}, J=7.2 \mathrm{~Hz}), 132.6,129.6(\mathrm{~d}, J=$ 8.4 Hz), 128.0, $124.1(\mathrm{~d}, J=2.7 \mathrm{~Hz}), 115.3(\mathrm{~d}, J=21.0 \mathrm{~Hz}), 112.7(\mathrm{~d}, J=21.0 \mathrm{~Hz}), 38.7(\mathrm{~d}, J=$ $1.8 \mathrm{~Hz}), 34.6,22.5,13.7$;

${ }^{19} \mathrm{~F}\left\{{ }^{1} \mathrm{H}\right\}$ NMR $\left(376 \mathrm{MHz}, \mathrm{CDCl}_{3}\right) \delta-113.9(\mathrm{~s})$;

IR ( $\mathrm{NaCl}$, thin film) 3032, 2960, 2873, 1615, 1487, 1447, 1250, 1137, 968, 945, 868, $799 \mathrm{~cm}^{-1}$; HRMS $\left(\mathrm{EI}^{+}\right) \mathrm{m} / \mathrm{z}$ calcd for $\mathrm{C}_{12} \mathrm{H}_{15} \mathrm{~F}^{+}(\mathrm{M})^{+}:$: 178.1158, found 178.1160 . 

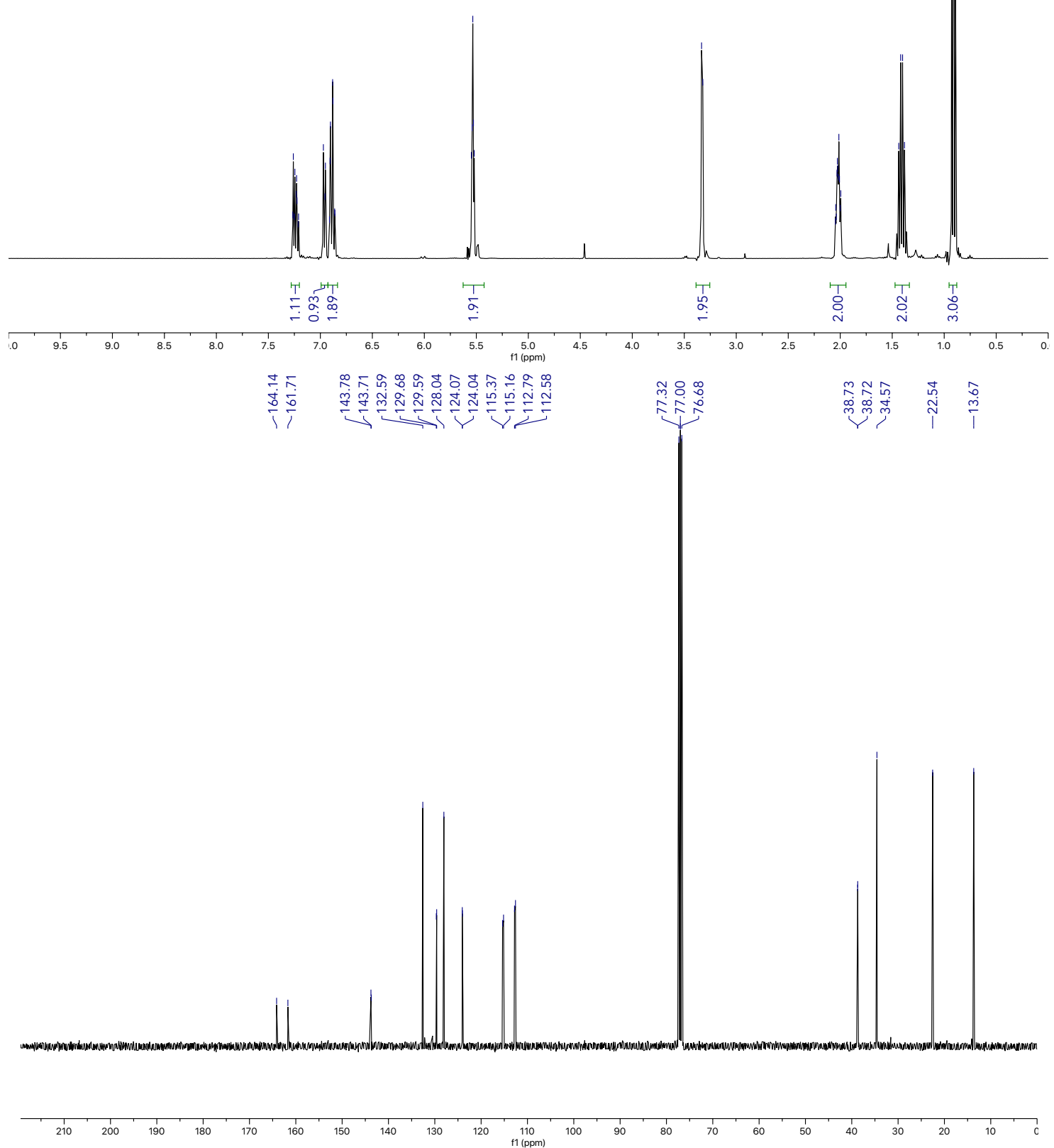
<smiles>CCC/C=C/Cc1cccc(Cl)c1</smiles>

\section{(E)-1-Chloro-3-(hex-2-en-1-yl)benzene}

General Procedure A: 1-Pentyne $(0.98 \mathrm{~mL}, 10 \mathrm{mmol})$ and 3-chlorobenzyl chloride $(1.27 \mathrm{~mL}, 10$ $\mathrm{mmol})$ were employed. Purification of the residue by FCC (100:1, hexane-EtOAc) afforded the title compound $(1.53 \mathrm{~g}, 79 \%)$ as a pale yellow oil.

${ }^{1} \mathrm{H}$ NMR $\left(400 \mathrm{MHz}, \mathrm{CDCl}_{3}\right) \delta$ 7.23-7.15 (3H, m), 7.06 (ddd, $\left.J=7.5,2.0,1.2 \mathrm{~Hz}, 1 \mathrm{H}\right), 5.58-5.48$ (m, 2H), 2.05-1.99 (m, 2H), 1.41 (app. hept, J = 7.4 Hz, 2H), 0.91 (t, J=7.4 Hz, 7.4 Hz, 3H);

${ }^{13} \mathrm{C}$ NMR $\left(101 \mathrm{MHz}, \mathrm{CDCl}_{3}\right) \delta 143.2,134.1,132.7,129.5,128.6,128.0,126.6,38.7,34.6,22.5$ 13.7;

IR (NaCl, thin film): 2959, 2930, 2872, 1596, 1474, 1430, 1200, 1078, 967, $779 \mathrm{~cm}^{-1}$; HRMS $\left(\mathrm{El}^{+}\right) \mathrm{m} / \mathrm{z}$ calcd for $\mathrm{C}_{12} \mathrm{H}_{15}{ }^{35} \mathrm{Cl}^{+}\left(\mathrm{M}^{+}\right)$: 194.0857, found 194.0860. 

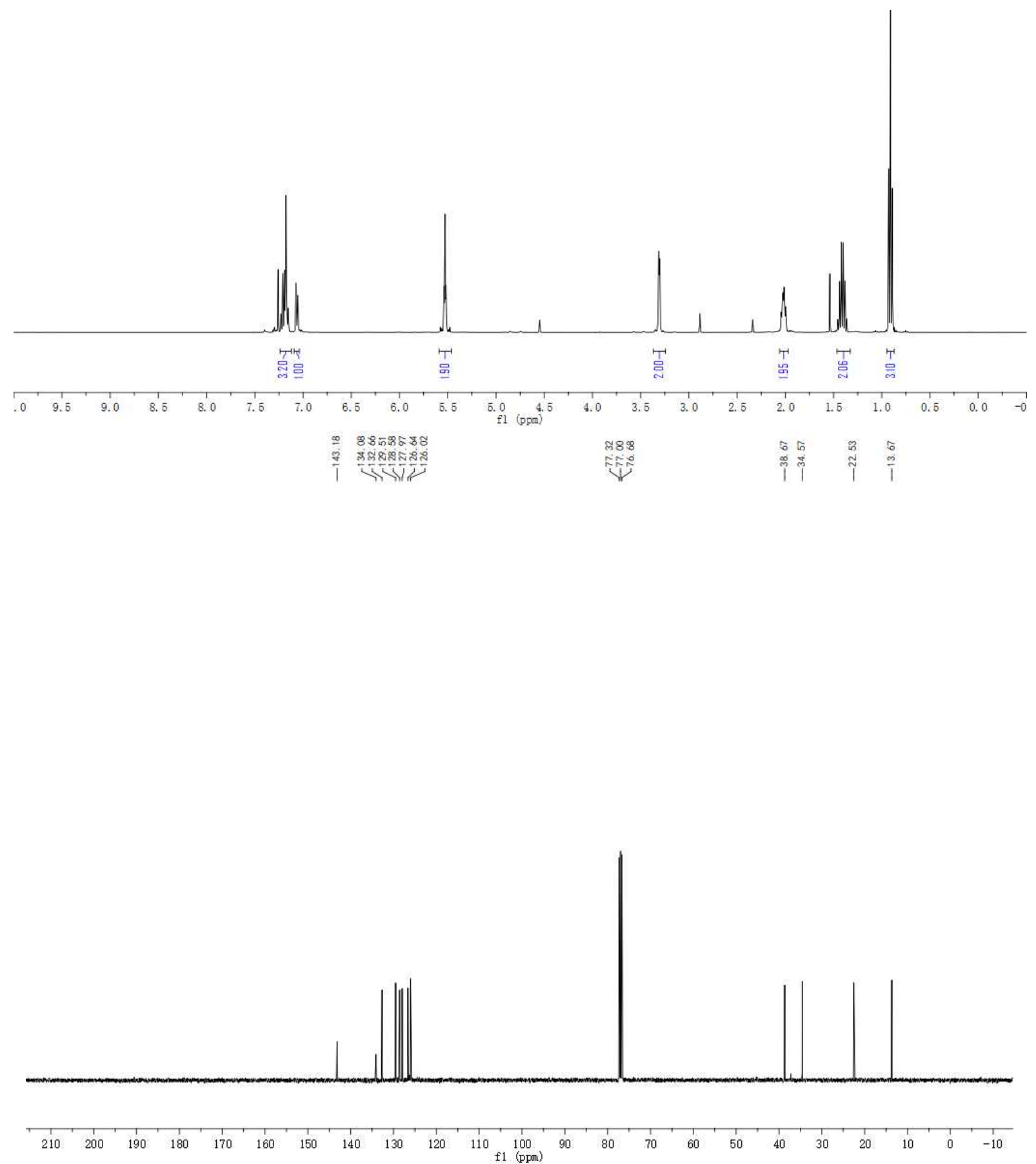
<smiles>CCC/C=C/Cc1cccc(OC)c1</smiles>

\section{(E)-1-(Hex-2-en-1-yl)-3-methoxybenzene}

General Procedure A: 1-Pentyne $(1.0 \mathrm{~mL}, 10 \mathrm{mmol})$ and 3-methoxybenzyl bromide $(0.72 \mathrm{~mL}, 10$ $\mathrm{mmol})$ were employed. Purification of the residue by FCC (100:1, hexane-EtOAc) afforded the title compound $(1.56 \mathrm{~g}, 82 \%)$ as a yellow oil.

${ }^{1} \mathrm{H}$ NMR $\left(400 \mathrm{MHz}, \mathrm{CDCl}_{3}\right) \delta$ 7.22-7.18 (m, 1H), 6.79-6.72 (m, 3H), 5.60-5.48 (m, 2H), $3.80(\mathrm{~s}$, $3 \mathrm{H}), 3.31$ (d, J = 5.5 Hz, 2H), 2.03-1.98 (m, 2H), 1.39 (app. h, $J=7.4 \mathrm{~Hz}, 2 \mathrm{H}$ ), 0.90 (t, J = 7.4 Hz, $3 \mathrm{H})$;

${ }^{13} \mathrm{C}$ NMR $\left(101 \mathrm{MHz}, \mathrm{CDCl}_{3}\right) \delta 159.7,142.8,132.1,129.3,128.7,120.9,114.2,111.2,55.1,39.1$, 34.6, 22.6, 13.7;

IR ( $\mathrm{NaCl}$, thin film) 3028, 2957, 2930, 2835, 1601, 1585, 1489, 1455, 1436, 1260, 1149, 1051, 968, 875, 774, $694 \mathrm{~cm}^{-1}$;

HRMS $\left(\mathrm{El}^{+}\right) \mathrm{m} / \mathrm{z}$ calcd for $\mathrm{C}_{13} \mathrm{H}_{18} \mathrm{O}^{+}(\mathrm{M})^{+}:$190.1352, found 190.1355 . 

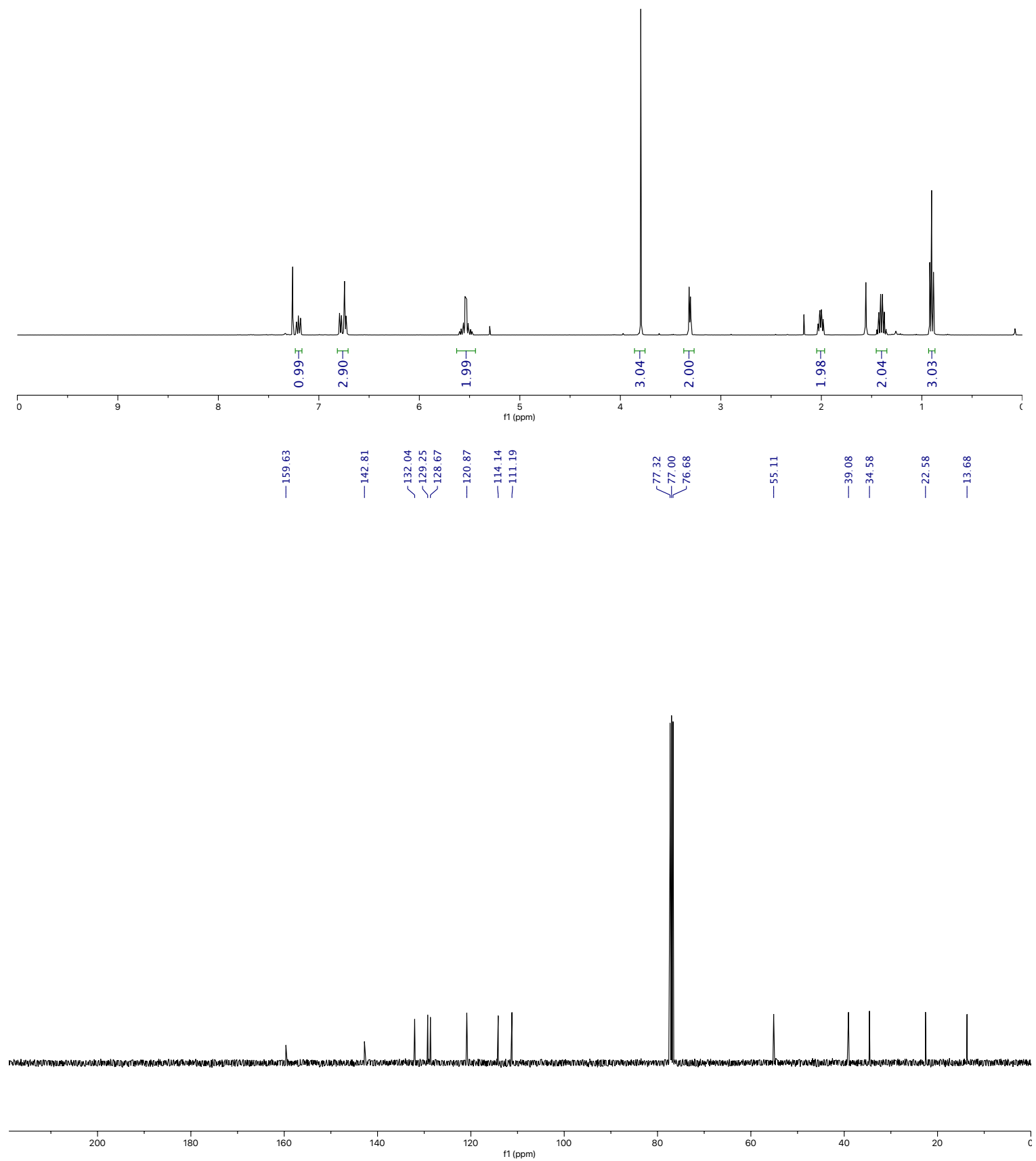


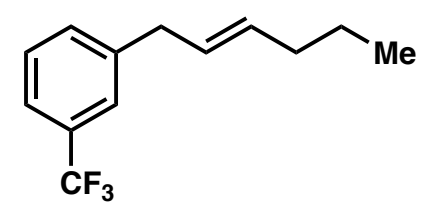

\section{(E)-1-(Hex-2-en-1-yl)-3-(trifluoromethyl)benzene}

General Procedure A: 1-Pentyne $(0.49 \mathrm{~mL}, 5.00 \mathrm{mmol})$ and 3-(trifluoromethyl)benzyl bromide $(0.76 \mathrm{~mL}, 5.00 \mathrm{mmol})$ were employed. Purification of the residue by FCC (100:1, hexane-EtOAc) afforded the title compound ( $869 \mathrm{mg}, 76 \%$ ) as a colorless oil.

${ }^{1} \mathrm{H}$ NMR $\left(400 \mathrm{MHz}, \mathrm{CDCl}_{3}\right)$ ठ 7.46-7.36 (m, 4H), 5.60-5.50 (m, 2H), 3.43-3.35 (m, 2H), 2.07-1.97 (m, 2H), 1.41 (app. h, $J=7.3 \mathrm{~Hz}, 2 \mathrm{H}), 0.91$ (t, $J=7.3 \mathrm{~Hz}, 3 \mathrm{H}$ );

${ }^{13}$ C NMR (101 MHz, $\left.\mathrm{CDCl}_{3}\right) \delta$ 142.0, 132.9, $131.9(\mathrm{q}, J=1.5 \mathrm{~Hz}), 130.6(\mathrm{q}, J=31.9 \mathrm{~Hz}), 128.7$, 127.8, 125.2 (q, $J=3.8 \mathrm{~Hz}$ ), 124.3 (q, $J=272.2 \mathrm{~Hz}$ ), 122.8 (q, $J=3.9 \mathrm{~Hz}$ ), 38.8, 34.6, 22.5, 13.6;

${ }^{19}$ F NMR (376 MHz, $\left.\mathrm{CDCl}_{3}\right) \delta-62.6(\mathrm{~s})$;

IR ( $\mathrm{NaCl}$, thin film) 2961, 2931, 2874, 1449, 1332, 1164, 1127, 1074, 968, 799, 702, $660 \mathrm{~cm}^{-1}$;

HRMS $\left(\mathrm{El}^{+}\right) \mathrm{m} / \mathrm{z}$ calcd for $\mathrm{C}_{13} \mathrm{H}_{15} \mathrm{~F}_{3}{ }^{+}(\mathrm{M})^{+}:$: 228.1126, found 228.1123. 


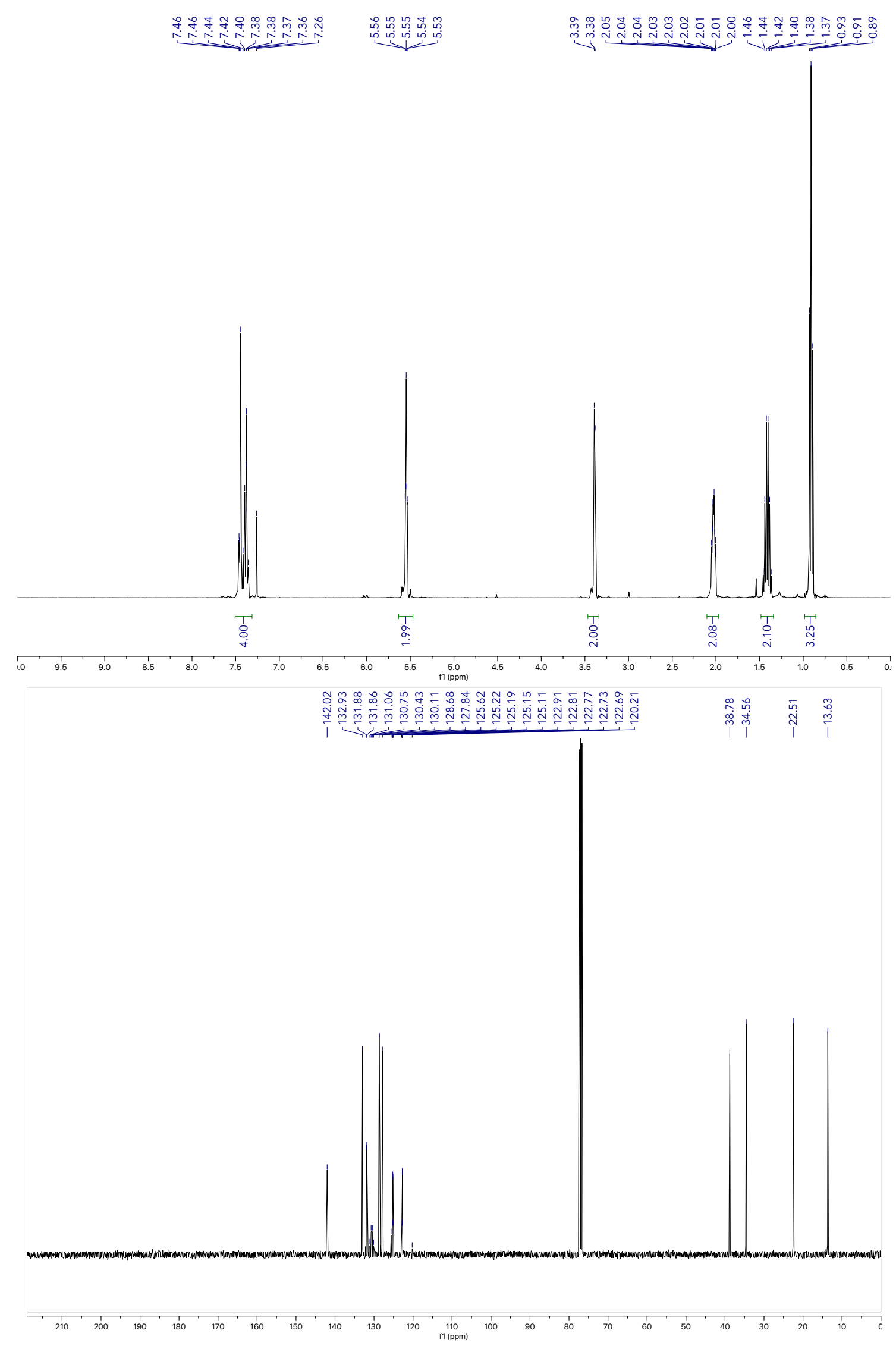




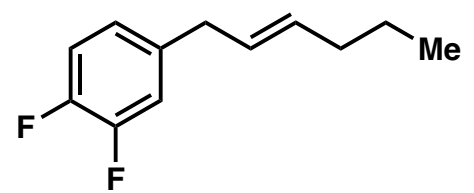

\section{(E)-1,2-Difluoro-4-(hex-2-en-1-yl)benzene}

General Procedure A: 1-Pentyne $(0.57 \mathrm{~mL}, 5.83 \mathrm{mmol})$ and 3,4-difluorobenzyl bromide $(1.20 \mathrm{~g}$, $5.83 \mathrm{mmol}$ ) were employed. Purification of the residue by FCC (100:1, hexane-EtOAc) afforded the title compound $(725 \mathrm{mg}, 64 \%)$ as a colorless oil.

${ }^{1} \mathrm{H}$ NMR $\left(400 \mathrm{MHz}, \mathrm{CDCl}_{3}\right) \delta 7.05$ (dt, $\left.J=10.4,8.3 \mathrm{~Hz}, 1 \mathrm{H}\right), 6.98$ (ddd, $\left.J=11.5,7.7,2.2 \mathrm{~Hz}, 1 \mathrm{H}\right)$, 6.89-6.86 (m, 1H), 5.56-5.46 (m, 2H), 3.32-3.24 (m, 2H), 2.04-1.99 (m, 2H), 1.41 (app. h, J= 7.4 $\mathrm{Hz}, 2 \mathrm{H}), 0.91(\mathrm{t}, \mathrm{J}=7.4 \mathrm{~Hz}, 3 \mathrm{H})$;

${ }^{13} \mathrm{C}$ NMR $\left(101 \mathrm{MHz}, \mathrm{CDCl}_{3}\right) \delta 150.2$ (dd, $\left.J=247.9,12.6 \mathrm{~Hz}\right), 148.8$ (dd, $\left.J=245.7,12.6 \mathrm{~Hz}\right), 138.1$ (dd, $J=5.3,3.8 \mathrm{~Hz}$ ), 132.8, 127.9, 124.1 (dd, $J=6.1,3.5 \mathrm{~Hz}), 117.1(\mathrm{~d}, J=16.9 \mathrm{~Hz}), 116.8(\mathrm{~d}, J$ $=17.0 \mathrm{~Hz}), 38.1$ (d, $J=1.4 \mathrm{~Hz}), 34.5,22.5,13.7$;

${ }^{19} \mathrm{~F}\left\{{ }^{1} \mathrm{H}\right\}$ NMR $\left(376 \mathrm{MHz}, \mathrm{CDCl}_{3}\right) \delta-138.6(\mathrm{~d}, J=21.3 \mathrm{~Hz}, 1 \mathrm{~F}),-142.4(\mathrm{~d}, J=21.3 \mathrm{~Hz}, 1 \mathrm{~F})$;

IR ( $\mathrm{NaCl}$, thin film) 2961, 2931, 2874, 1608, 1519, 1465, 1435, 1284, 1209, 1114, 969, 954, 872, $818,775 \mathrm{~cm}^{-1}$;

HRMS $\left(\mathrm{El}^{+}\right) \mathrm{m} / \mathrm{z}$ calcd for $\mathrm{C}_{12} \mathrm{H}_{14} \mathrm{~F}_{2}{ }^{+}(\mathrm{M})^{+}:$: 196.1064, found 196.1069. 

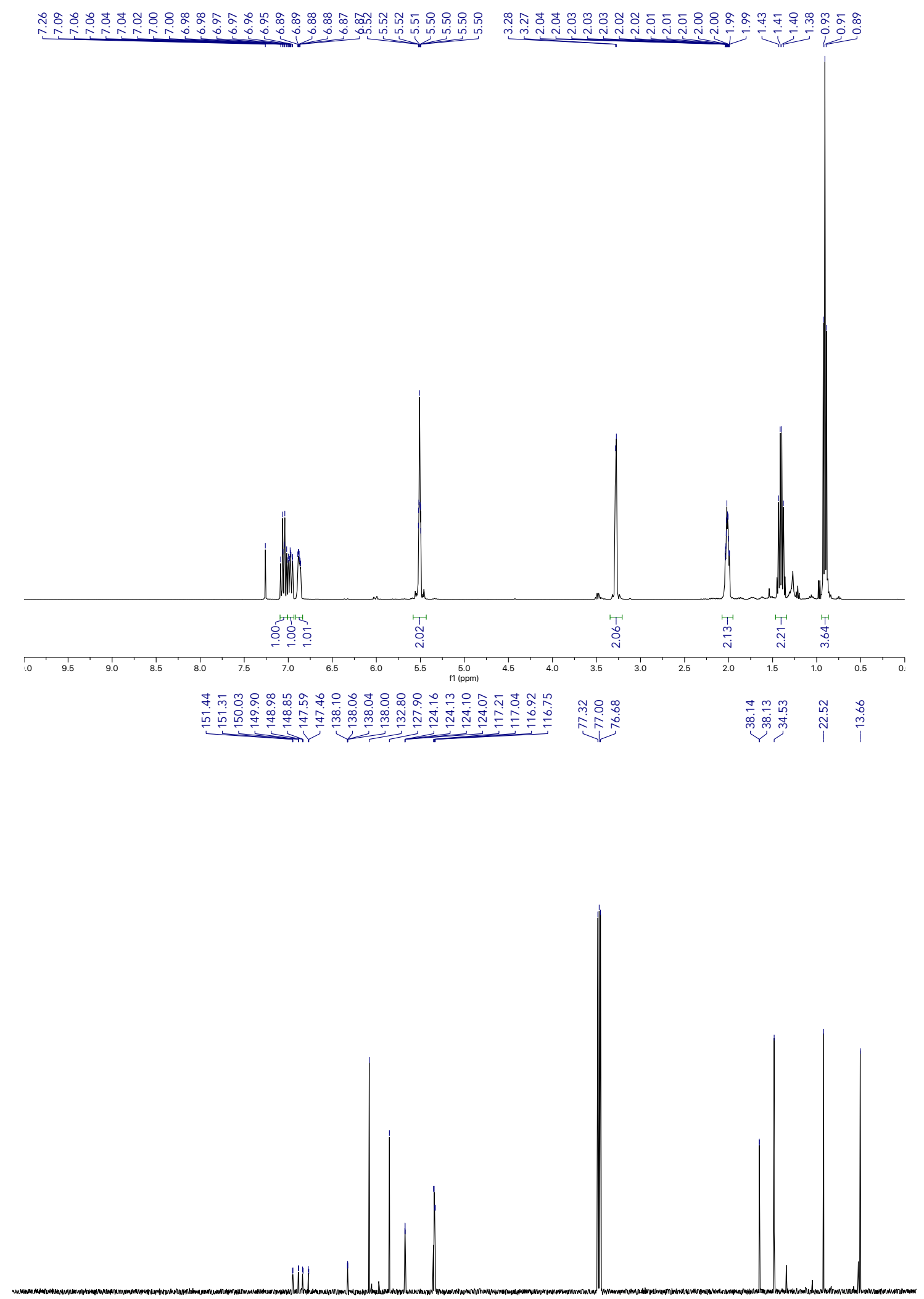

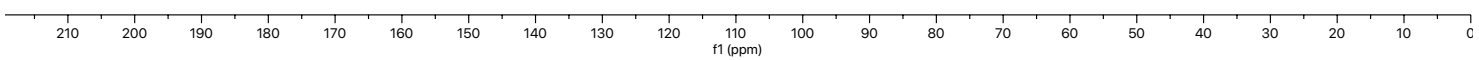




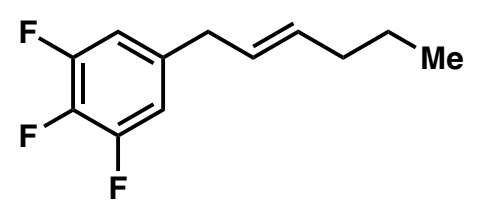

(E)-1,2,3-Trifluoro-5-(hex-2-en-1-yl)benzene

General Procedure A: 1-Pentyne (0.49 mL, $5.00 \mathrm{mmol})$ and 5-(bromomethyl)-1,2,3trifluorobenzene $(0.67 \mathrm{~mL}, 5.00 \mathrm{mmol})$ were employed. Purification of the residue by FCC (100:1, hexane-EtOAc) afforded the title compound $(730 \mathrm{mg}, 68 \%)$ as a colorless oil.

${ }^{1} \mathrm{H}$ NMR (400 MHz, $\left.\mathrm{CDCl}_{3}\right)$ ס 6.82-6.74 (m, 2H), 5.57-5.43 (m, 2H), 3.27-3.25 (m, 2H), 2.05-2.00 (m, 2H), 1.41 (app. h, $J=7.4 \mathrm{~Hz}, 2 \mathrm{H}), 0.91(\mathrm{t}, J=7.4 \mathrm{~Hz}, 3 \mathrm{H})$;

${ }^{13} \mathrm{C}$ NMR (101 MHz, $\left.\mathrm{CDCl}_{3}\right) \delta 151.0$ (ddd, $\left.J=249.1,9.9,4.0 \mathrm{~Hz}\right), 138.1$ (dt, $J=248.3,15.4 \mathrm{~Hz}$ ), 137.4 (td, $J=7.0,4.5 \mathrm{~Hz}$ ), 133.6, 127.0, 112.2 (ddd, $J=15.4,5.5,1.1 \mathrm{~Hz}$ ), 38.2, 34.5, 22.5, 13.6;

${ }^{19} \mathrm{~F}\left\{{ }^{1} \mathrm{H}\right\}$ NMR $\left(376 \mathrm{MHz}, \mathrm{CDCl}_{3}\right) \delta-135.3(\mathrm{~d}, \mathrm{~J}=20.5 \mathrm{~Hz}, 2 \mathrm{~F}),-164.8$ (t, J = 20.5 Hz, 1F);

IR ( $\mathrm{NaCl}$, thin film) 2964, 2935, 2876, 2356, 1621, 1531, 1448, 1353, 1235, 1040, 902, 855, 786 $\mathrm{cm}^{-1}$;

HRMS (EI) $m / z$ calcd for $\mathrm{C}_{12} \mathrm{H}_{13} \mathrm{~F}_{3}(\mathrm{M})^{+}:$214.0964, found 214.0961 . 

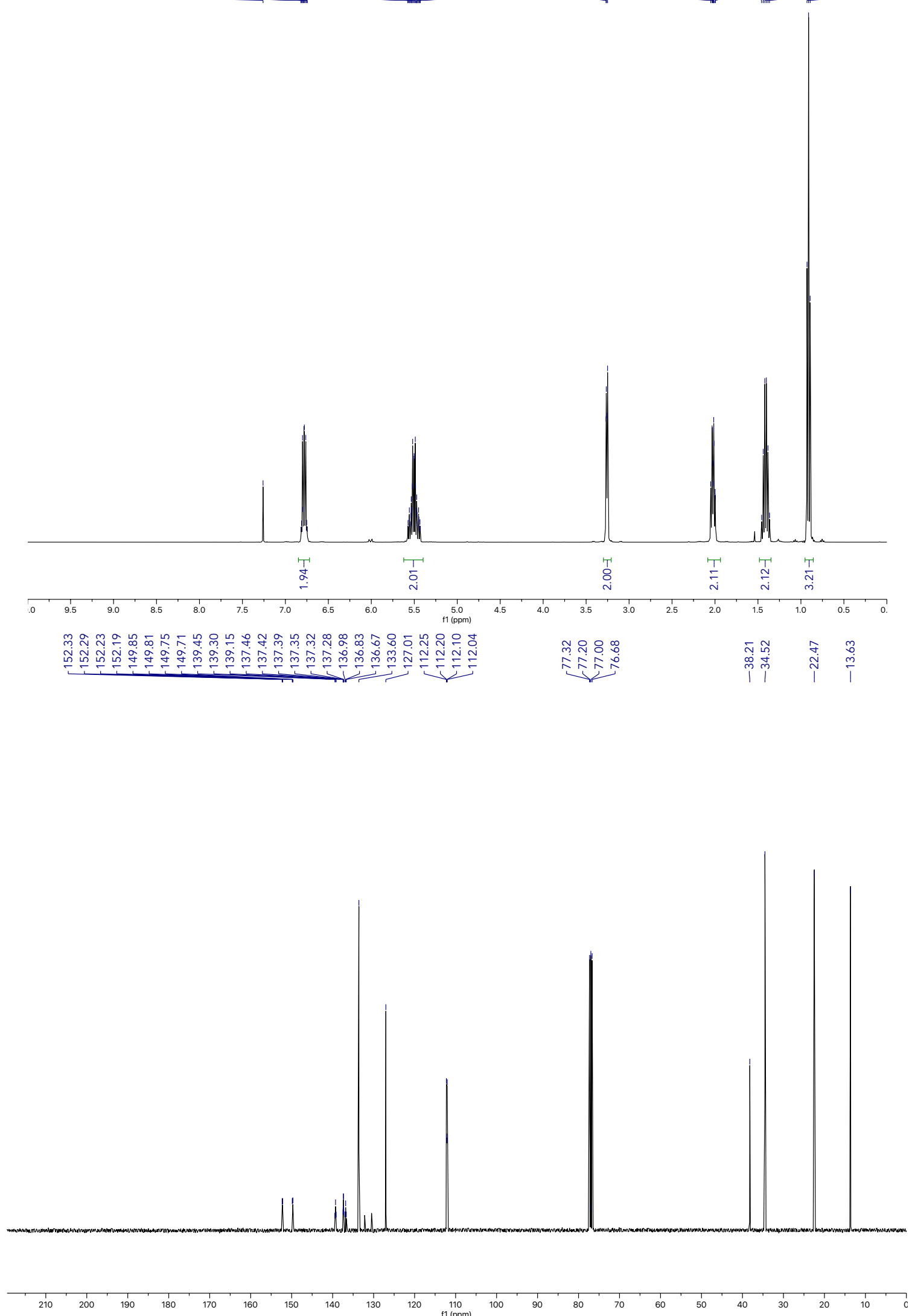
PMP $\widehat{\mathrm{CH}_{4}^{\mathrm{Cl}}}$

\section{(E)-1-(7-Chlorohept-2-en-1-yl)-4-methoxybenzene}

General Procedure A: 6-Chloro-1-hexyne $(2.0 \mathrm{~mL}, 16.4 \mathrm{mmol})$ and 4-methoxybenzyl chloride $(2.20 \mathrm{~mL}, 16.4 \mathrm{mmol})$ were employed. Purification of the residue by FCC (100:1 - 50:1, hexaneEtOAc) afforded the title compound ( $3.63 \mathrm{~g}, 93 \%)$ as a colorless oil.

${ }^{1} \mathrm{H}$ NMR $\left(400 \mathrm{MHz}, \mathrm{CDCl}_{3}\right) \delta 7.09$ (d, J=8.6 Hz, 2H), $6.84(\mathrm{~d}, J=8.6 \mathrm{~Hz}, 2 \mathrm{H}), 5.57$ (dtt, J = 15.1, 6.6, $1.3 \mathrm{~Hz}, 1 \mathrm{H}), 5.46(\mathrm{dtt}, J=15.1,6.6,1.3 \mathrm{~Hz}, 1 \mathrm{H}), 3.79(\mathrm{~s}, 3 \mathrm{H}), 3.54(\mathrm{t}, J=6.7 \mathrm{~Hz}, 2 \mathrm{H}), 3.27$ (d, $J=6.5 \mathrm{~Hz}, 2 \mathrm{H}), 2.09-2.03(\mathrm{~m}, 2 \mathrm{H}), 1.82-1.75(\mathrm{~m}, 2 \mathrm{H}), 1.56-1.49(\mathrm{~m}, 2 \mathrm{H})$;

${ }^{13} \mathrm{C}$ NMR $\left(101 \mathrm{MHz}, \mathrm{CDCl}_{3}\right) \delta 157.9,132.9,130.7,130.0,129.3,113.8,55.3,45.0,38.1,32.1$, 31.7, 26.6;

IR ( $\mathrm{NaCl}$, thin film) 2937, 2865, 1608, 1513, 1463, 1442, 1302, 1248, 1175, 1108, 1034, 970, 830 $\mathrm{cm}^{-1}$;

HRMS $\left(\mathrm{El}^{+}\right) \mathrm{m} / \mathrm{z}$ calcd for $\mathrm{C}_{14} \mathrm{H}_{19}{ }^{35} \mathrm{ClO}^{+}(\mathrm{M})^{+}:$: 238.1119, found 238.1116. 

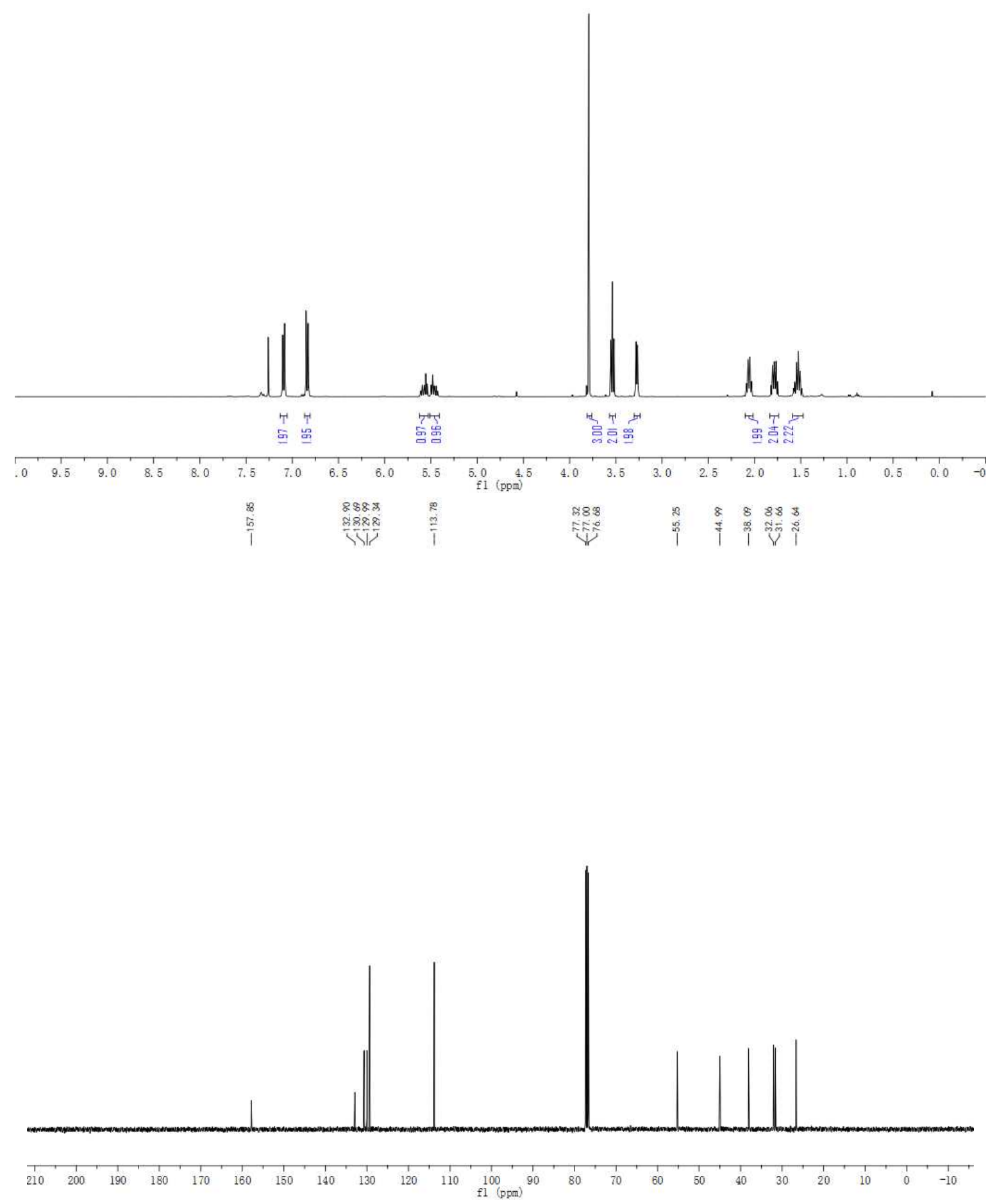


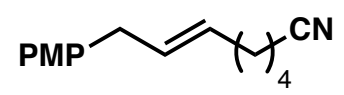

\section{(E)-8-(4-Methoxyphenyl)oct-6-enenitrile}

To a solution of (E)-1-(7-chlorohept-2-en-1-yl)-4-methoxybenzene (1.0 g, $4.2 \mathrm{mmol})$ in DMSO (5 $\mathrm{mL}$ ) was added $\mathrm{NaCN}$. The reaction mixture was heated at $80^{\circ} \mathrm{C}$ for one hour after which time TLC analysis indicated full consumption of starting material. The reaction was cooled to room temperature, diluted with $\mathrm{H}_{2} \mathrm{O}(10 \mathrm{~mL})$ and extracted with diethyl ether $(3 \times 20 \mathrm{~mL})$. The combined organic layers were washed with brine $(10 \mathrm{~mL})$, dried $\left(\mathrm{Na}_{2} \mathrm{SO}_{4}\right)$, filtered and concentrated in vacuo. Purification of the residue by FCC (15:1, hexane-EtOAc) afforded the title compound (745 $\mathrm{mg}, 78 \%)$ as a colorless oil.

${ }^{1} \mathrm{H}$ NMR $\left(400 \mathrm{MHz}, \mathrm{CDCl}_{3}\right) \delta 7.08(\mathrm{~d}, J=8.6 \mathrm{~Hz}, 2 \mathrm{H}), 6.84(\mathrm{~d}, J=8.6 \mathrm{~Hz}, 2 \mathrm{H}), 5.58$ (dtt, $J=15.1$, 6.7, $1.3 \mathrm{~Hz}, 1 \mathrm{H}$ ), 5.44 (dtt, $J=15.1,6.7,1.3 \mathrm{~Hz}, 1 \mathrm{H}), 3.79(\mathrm{~s}, 3 \mathrm{H}), 3.27(\mathrm{~d}, J=6.5 \mathrm{~Hz}, 2 \mathrm{H}), 2.33$ (t, $J=7.1 \mathrm{~Hz}, 2 \mathrm{H}$ ), 2.07 (app. q, $J=7.1 \mathrm{~Hz}, 2 \mathrm{H}), 1.70-1.63(\mathrm{~m}, 2 \mathrm{H}), 1.58-1.50(\mathrm{~m}, 2 \mathrm{H})$;

${ }^{13} \mathrm{C}$ NMR $\left(101 \mathrm{MHz}, \mathrm{CDCl}_{3}\right) \delta 157.9,132.7,130.5,130.0,129.3,119.7,113.8,55.2,38.0,31.5$, 28.3, 24.8, 17.0;

IR ( $\mathrm{NaCl}$, thin film) 2936, 2246, 1609. 1152, 1463, 1300, 1246, 1177, 1109, 1034, 970, $818 \mathrm{~cm}^{-1}$ HRMS $\left(\mathrm{EI}^{+}\right) \mathrm{m} / \mathrm{z}$ calcd for $\mathrm{C}_{15} \mathrm{H}_{19} \mathrm{NO}^{+}(\mathrm{M})^{+}:$229.1461, found 229.1457 . 

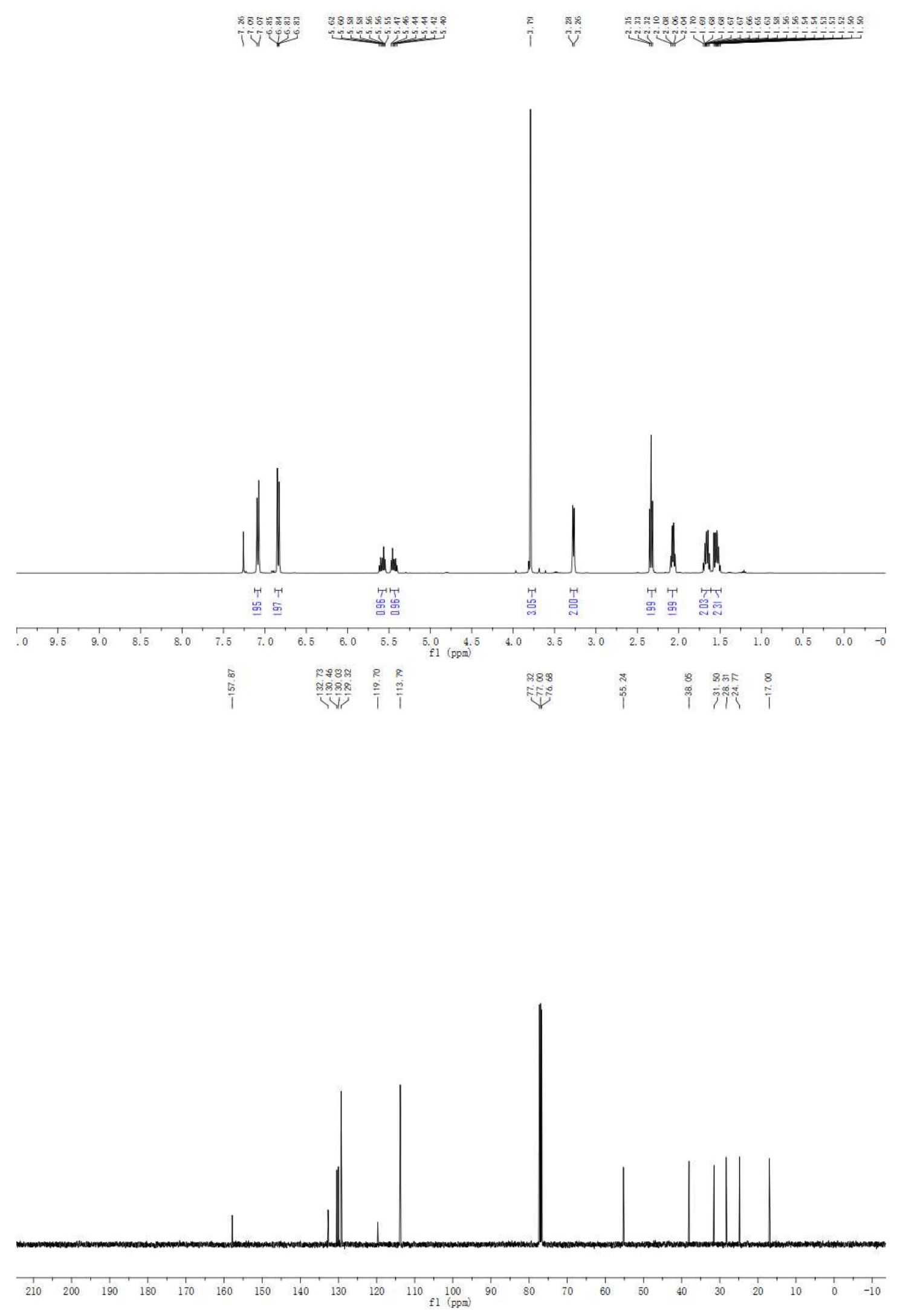
$\mathrm{CHMP}_{4}^{\mathrm{OTS}}$

\section{(E)-7-(4-Methoxyphenyl)hept-5-en-1-yl 4-methylbenzenesulfonate}

General Procedure A: Hex-5-yn-1-yl 4-methylbenzenesulfonate ${ }^{5}$ (350 mg, $1.4 \mathrm{mmol}$ ) and 4methoxybenzyl chloride $(0.19 \mathrm{~mL}, 1.4 \mathrm{mmol})$ were employed. Purification of the residue by FCC (20:1, hexane-EtOAc) afforded the title compound $(288 \mathrm{mg})$ as a colorless oil. This material was contaminated with approximately $5-10 \%$ of an unknown impurity therefore an accurate yield was not determined. The mixture was carried forward into the epoxidation step after which the impurity could be removed.

${ }^{1} \mathrm{H}$ NMR $\left(400 \mathrm{MHz}, \mathrm{CDCl}_{3}\right) \delta 7.78(\mathrm{~d}, J=8.3 \mathrm{~Hz}, 2 \mathrm{H}), 7.33(\mathrm{~d}, J=8.0 \mathrm{~Hz}, 2 \mathrm{H}), 7.06(\mathrm{~d}, J=8.6$ $\mathrm{Hz}, 2 \mathrm{H}), 6.83(\mathrm{~d}, J=8.6 \mathrm{~Hz}, 2 \mathrm{H}), 5.50(\mathrm{dtt}, J=15.1,6.5,1.2 \mathrm{~Hz}, 1 \mathrm{H}), 5.38(\mathrm{dtt}, J=15.1,6.7,1.3$ $\mathrm{Hz}, 1 \mathrm{H}), 4.01(\mathrm{t}, J=6.5 \mathrm{~Hz}, 2 \mathrm{H}), 3.79(\mathrm{~s}, 3 \mathrm{H}), 3.24(\mathrm{~d}, J=6.6 \mathrm{~Hz}, 2 \mathrm{H}), 2.44(\mathrm{~s}, 3 \mathrm{H}), 1.97(\mathrm{q}, J=$ $6.9 \mathrm{~Hz}, 2 \mathrm{H}), 1.72-1.56(\mathrm{~m}, 2 \mathrm{H}), 1.40$ (q, $J=7.7 \mathrm{~Hz}, 2 \mathrm{H})$;

${ }^{13} \mathrm{C}$ NMR $\left(101 \mathrm{MHz}, \mathrm{CDCl}_{3}\right) \delta 157.8,144.6,133.1,132.8,130.4,130.1,129.8,129.3,127.9$, 113.8, 70.4, 55.3, 38.0, 31.6, 28.2, 25.1, 21.6;

IR ( $\mathrm{NaCl}$, thin film) 2934, 2857, 1610, 1512, 1463, 1358, 1246, 1176, 1035, 933, 816, $665 \mathrm{~cm}^{-1}$;

HRMS (ESI-TOF) $\mathrm{m} / \mathrm{z}$ calcd for $\mathrm{C}_{21} \mathrm{H}_{26} \mathrm{O}_{4} \mathrm{SNa}^{+}(\mathrm{M}+\mathrm{Na})^{+}:$397.1449, found 397.1446. 


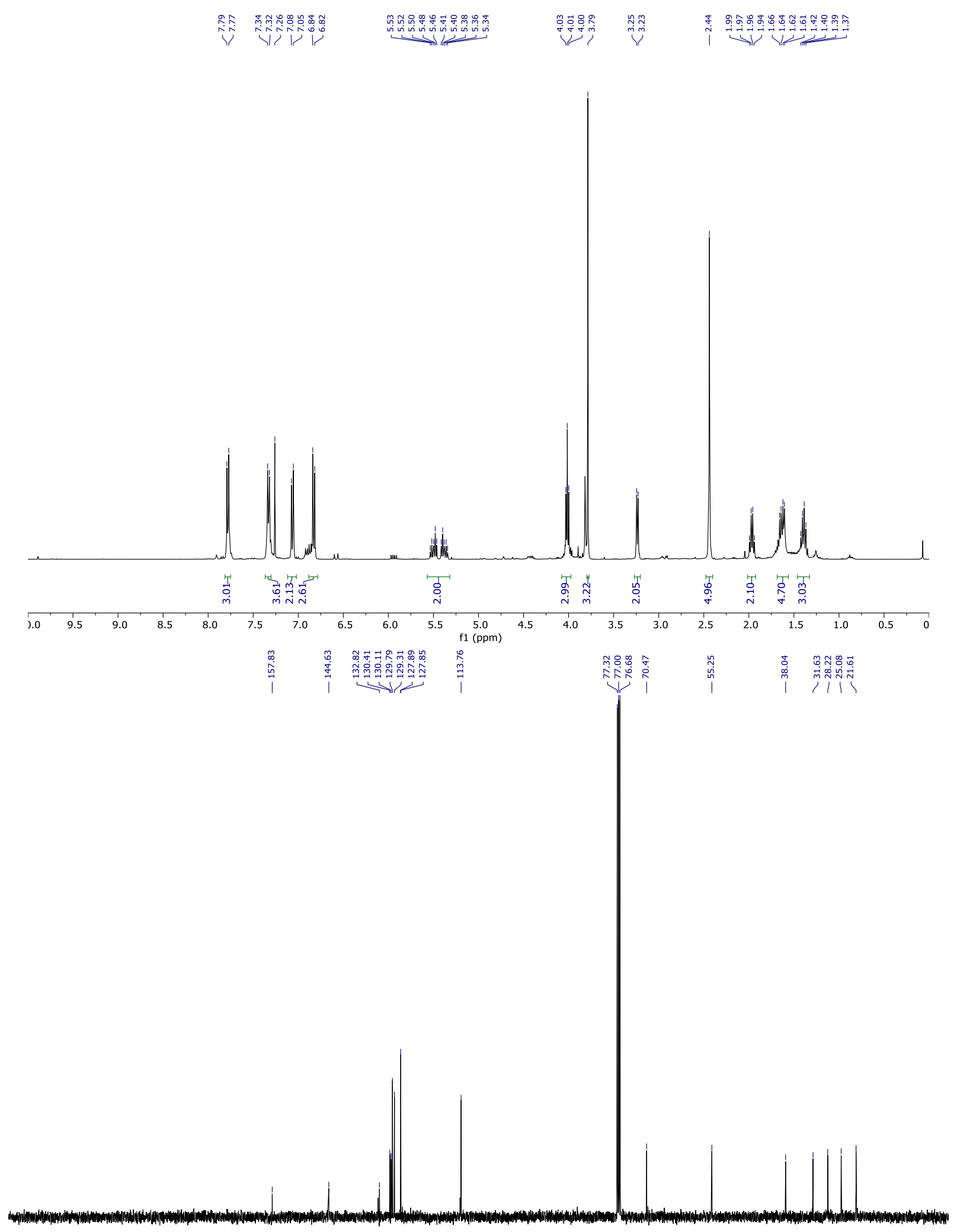

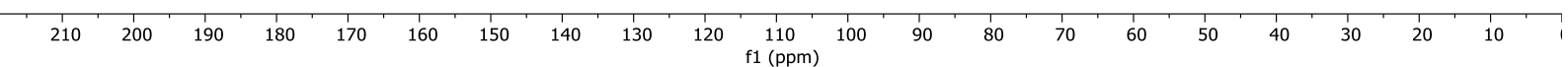


$\widehat{P M P} \widehat{\mathrm{CH}_{4}^{\mathrm{OBn}}}$

\section{(E)-1-(7-(Benzyloxy)hept-2-en-1-yl)-4-methoxybenzene}

General Procedure A: ((Hex-5-yn-1-yloxy)methyl)benzene $(1.5 \mathrm{~g}, 7.9 \mathrm{mmol})$ and 4methoxybenzyl chloride $(1.08 \mathrm{~mL}, 7.9 \mathrm{mmol})$ were employed. Purification of the residue by FCC (20:1, hexane-EtOAc) afforded the title compound ( $813 \mathrm{mg}, 55 \%)$ as a colorless oil.

${ }^{1} \mathrm{H}$ NMR $\left(400 \mathrm{MHz}, \mathrm{CDCl}_{3}\right) \delta 7.51-7.28(\mathrm{~m}, 5 \mathrm{H}), 7.12(\mathrm{~d}, J=8.4 \mathrm{~Hz}, 2 \mathrm{H}), 6.86(\mathrm{~d}, J=8.5 \mathrm{~Hz}$, $2 \mathrm{H}), 5.65-5.44(\mathrm{~m}, 2 \mathrm{H}), 4.53(\mathrm{~s}, 2 \mathrm{H}), 3.81(\mathrm{~s}, 3 \mathrm{H}), 3.50(\mathrm{t}, J=6.5 \mathrm{~Hz}, 2 \mathrm{H}), 3.30(\mathrm{~d}, J=6.2 \mathrm{~Hz}$, 2H), 2.08 (q, $J=7.0 \mathrm{~Hz}, 2 \mathrm{H}), 1.67$ (dt, $J=14.8,6.6 \mathrm{~Hz}, 3 \mathrm{H}), 1.58-1.44(\mathrm{~m}, 2 \mathrm{H})$;

${ }^{13} \mathrm{C}$ NMR $\left(100 \mathrm{MHz}, \mathrm{CDCl}_{3}\right) \delta 157.8,138.6,133.0,131.2,129.5,129.3,128.3,127.6,127.4$, 113.7, 72.8, 70.2, 55.2, 38.1, 32.2, 29.2, 26.0;

IR ( $\mathrm{NaCl}$, thin film) 3029, 2995, 2933, 2856, 1611, 1511, 1454, 1245, 970, 819, 735, $698 \mathrm{~cm}^{-1}$;

HRMS (ESI-TOF) $\mathrm{m} / \mathrm{z}$ calcd for $\mathrm{C}_{21} \mathrm{H}_{26} \mathrm{O}_{2} \mathrm{Na}^{+}(\mathrm{M}+\mathrm{Na})^{+}: 333.1830$, found 333.1823. 

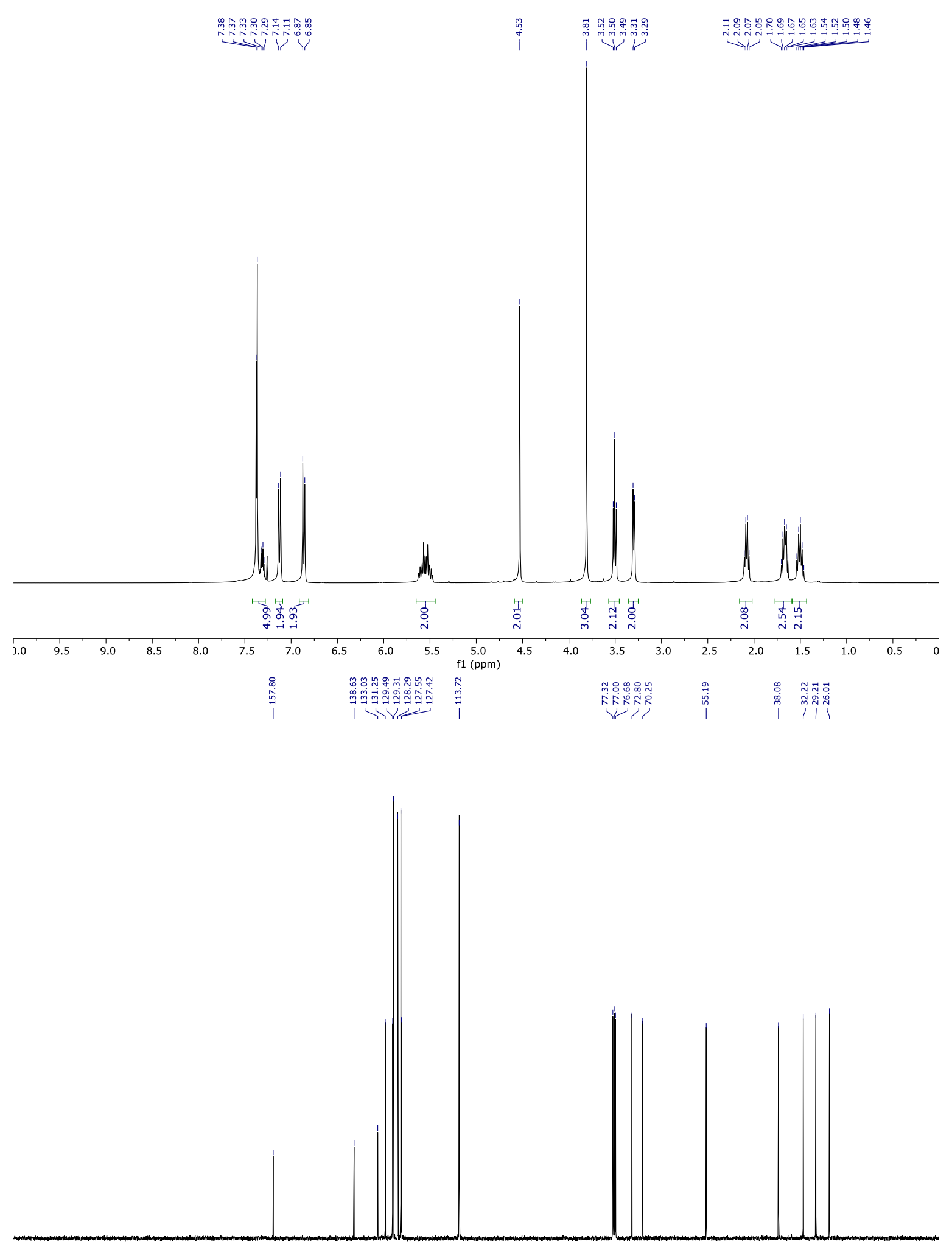
$\widehat{P M P} \widehat{Y_{4}{ }^{\text {OTBS }}}$

(E)-tert-Butyl((7-(4-methoxyphenyl)hept-5-en-1-yl)oxy)dimethylsilane

General Procedure A: tert-Butyl(hex-5-yn-1-yloxy)dimethylsilane ${ }^{6}(2.0 \mathrm{~g}, 9.4 \mathrm{mmol})$ and 4methoxybenzyl chloride $(1.27 \mathrm{~mL}, 9.4 \mathrm{mmol})$ were employed. Purification of the residue by FCC (100:1, hexane-EtOAc) afforded the title compound (813 $\mathrm{mg}, 34 \%)$ as a colorless oil.

${ }^{1} \mathrm{H}$ NMR $\left(400 \mathrm{MHz}, \mathrm{CDCl}_{3}\right) \delta 7.09$ (d, $\left.J=8.5 \mathrm{~Hz}, 2 \mathrm{H}\right), 6.83$ (d, J=8.6 Hz, 2H), 5.51 (qt, J = 15.2, $6.3 \mathrm{~Hz}, 2 \mathrm{H}), 3.79(\mathrm{~s}, 3 \mathrm{H}), 3.61(\mathrm{t}, J=6.5 \mathrm{~Hz}, 2 \mathrm{H}), 3.27(\mathrm{~d}, J=6.2 \mathrm{~Hz}, 2 \mathrm{H}), 2.04(\mathrm{q}, J=6.9 \mathrm{~Hz}$, $2 \mathrm{H}), 1.56-1.48(\mathrm{~m}, 2 \mathrm{H}), 1.46-1.35(\mathrm{~m}, 2 \mathrm{H}), 0.90(\mathrm{~s}, 9 \mathrm{H}), 0.05(\mathrm{~s}, 6 \mathrm{H})$;

${ }^{13} \mathrm{C}$ NMR $\left(100 \mathrm{MHz}, \mathrm{CDCl}_{3}\right) \delta 158.3,129.9,129.5,113.9,62.9,59.0,58.7,55.2,37.6,32.5,31.7$, 29.7, 26.0, 22.3, 18.3, -5.3;

IR ( $\mathrm{NaCl}$, thin film) 3030, 2995, 2930, 2856, 1611, 1511, 1463, 1247, 1039, 836, $776 \mathrm{~cm}^{-1}$;

HRMS (ESI-TOF) $\mathrm{m} / \mathrm{z}$ calcd for $\mathrm{C}_{20} \mathrm{H}_{34} \mathrm{O}_{2} \mathrm{SiNa}^{+}(\mathrm{M}+\mathrm{Na})^{+}:$357.2226, found 357.2240. 


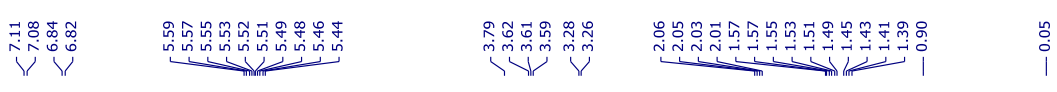
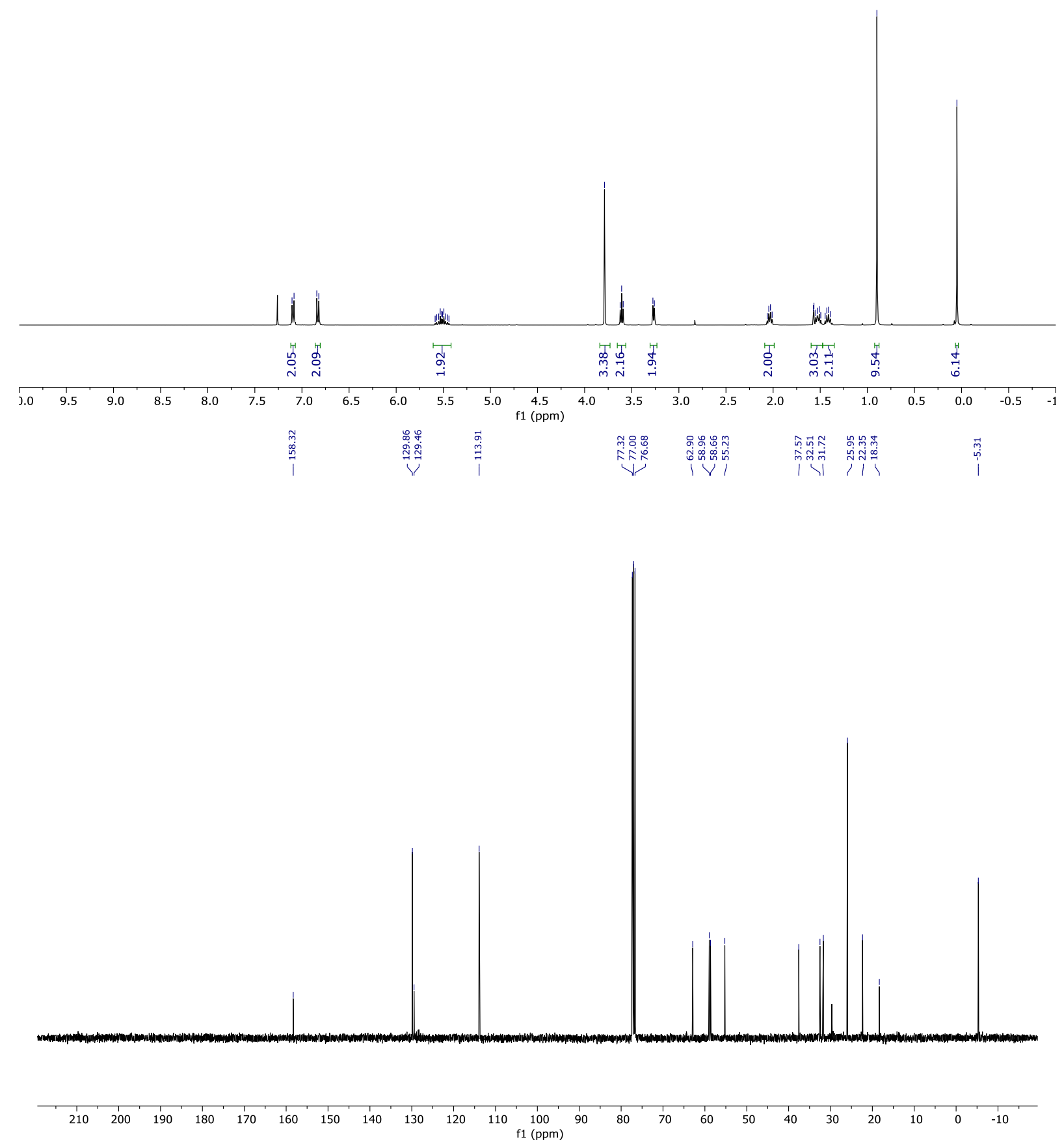
<smiles>C#CCCCCOc1ccccc1</smiles>

\section{(Hex-5-yn-1-yloxy)benzene}

To a solution of 5-hexyn-1-ol $(0.78 \mathrm{~mL}, 7.08 \mathrm{mmol}), \mathrm{PPh}_{3}(2.78 \mathrm{~g}, 10.6 \mathrm{mmol})$, and $\mathrm{PhOH}$ (996 $\mathrm{mg}, 10.6 \mathrm{mmol})$ in THF (35 mL) was added DIAD $(2.10 \mathrm{~mL}, 10.6 \mathrm{mmol})$ dropwise over five minutes. After stirring at room temperature for one hour the mixture was concentrated in vacuo. The residue was re-dissolved in $\mathrm{Et}_{2} \mathrm{O}$-pentane $(1: 1,100 \mathrm{~mL})$ and the $\mathrm{O}=\mathrm{PPh}_{3}$ was removed via Büchner filtration. The solvent was concentrated in vacuo and the purification of the residue by FCC (40:1, hexane-EtOAc) afforded the title compound (930 mg, 76\%) as a pale yellow oil.

${ }^{1} \mathrm{H}$ NMR $\left(400 \mathrm{MHz}, \mathrm{CDCl}_{3}\right)$ ठ 7.30-7.26 (m, 2H), 6.96-6.88 (m, 3H), $3.99(\mathrm{t}, \mathrm{J}=6.3 \mathrm{~Hz}, 2 \mathrm{H}), 2.28$ $(\mathrm{td}, J=7.1,2.7 \mathrm{~Hz}, 2 \mathrm{H}), 1.97(\mathrm{t}, J=2.7 \mathrm{~Hz}, 1 \mathrm{H}), 1.95-1.88(\mathrm{~m}, 2 \mathrm{H}), 1.77-1.70(\mathrm{~m}, 2 \mathrm{H})$;

${ }^{13} \mathrm{C}$ NMR $\left(101 \mathrm{MHz}, \mathrm{CDCl}_{3}\right) \delta 159.0,129.4,120.6,114.5,84.1,68.6,67.1,28.3,25.1,18.2$.

The spectroscopic properties of this compound matched those reported in the literature. ${ }^{7}$<smiles>CC(C)(C=CCC(=O)Oc1ccccc1)Oc1ccccc1</smiles>

\section{(E)-1-Methoxy-4-(7-phenoxyhept-2-en-1-yl)benzene}

General Procedure A: (Hex-5-yn-1-yloxy)benzene (900 mg, $5.17 \mathrm{mmol}$ ) and 4-methoxybenzyl chloride $(0.70 \mathrm{~mL}, 5.17 \mathrm{mmol})$ were employed. Purification of the residue by FCC $(60: 1-20: 1$, hexane-EtOAc) afforded the title compound $(1.08 \mathrm{~g})$ as a colorless oil. This material was contaminated with 4-methoxybenzyl chloride therefore an accurate yield was not determined. The mixture was carried forward into the epoxidation step after which the impurity could be removed.

${ }^{1} \mathrm{H}$ NMR (400 MHz, $\left.\mathrm{CDCl}_{3}\right)$ ठ 7.30-7.26 (m, 2H), 7.10 (d, J = 8.6 Hz, 2H), 6.95-6.88 (m, 3H), 6.84 $(\mathrm{d}, J=6.8 \mathrm{~Hz}, 2 \mathrm{H}), 5.59$ (dtt, $J=15.2,6.5,1.2 \mathrm{~Hz}, 1 \mathrm{H}), 5.50(\mathrm{dtt}, J=15.2,6.4,1.2 \mathrm{~Hz}, 1 \mathrm{H}), 3.96$ $(\mathrm{t}, J=6.5 \mathrm{~Hz}, 2 \mathrm{H}), 3.79(\mathrm{~s}, 3 \mathrm{H}), 3.28(\mathrm{~d}, J=6.4 \mathrm{~Hz}, 2 \mathrm{H}), 2.13-2.08(\mathrm{~m}, 2 \mathrm{H}), 1.84-1.77(\mathrm{~m}, 2 \mathrm{H})$, 1.60-1.52 (2H);

${ }^{13} \mathrm{C}$ NMR $\left(126 \mathrm{MHz}, \mathrm{CDCl}_{3}\right) \delta 159.0,157.8,133.0,131.1,129.8,129.4,129.4,120.5,114.5$, 113.8, 67.7, 55.3, 38.1, 32.2, 28.8, 25.9;

IR ( $\mathrm{NaCl}$, thin film) 2938, 1600, 1586. 1512, 1498, 1245, 1174, 1035, 971, 827, $755 \mathrm{~cm}^{-1}$;

HRMS $\left(\mathrm{EI}^{+}\right) \mathrm{m} / \mathrm{z}$ calcd for $\mathrm{C}_{20} \mathrm{H}_{24} \mathrm{O}_{2}{ }^{+}\left(\mathrm{M}^{+}\right): 296.1771$, found 296.1763. 


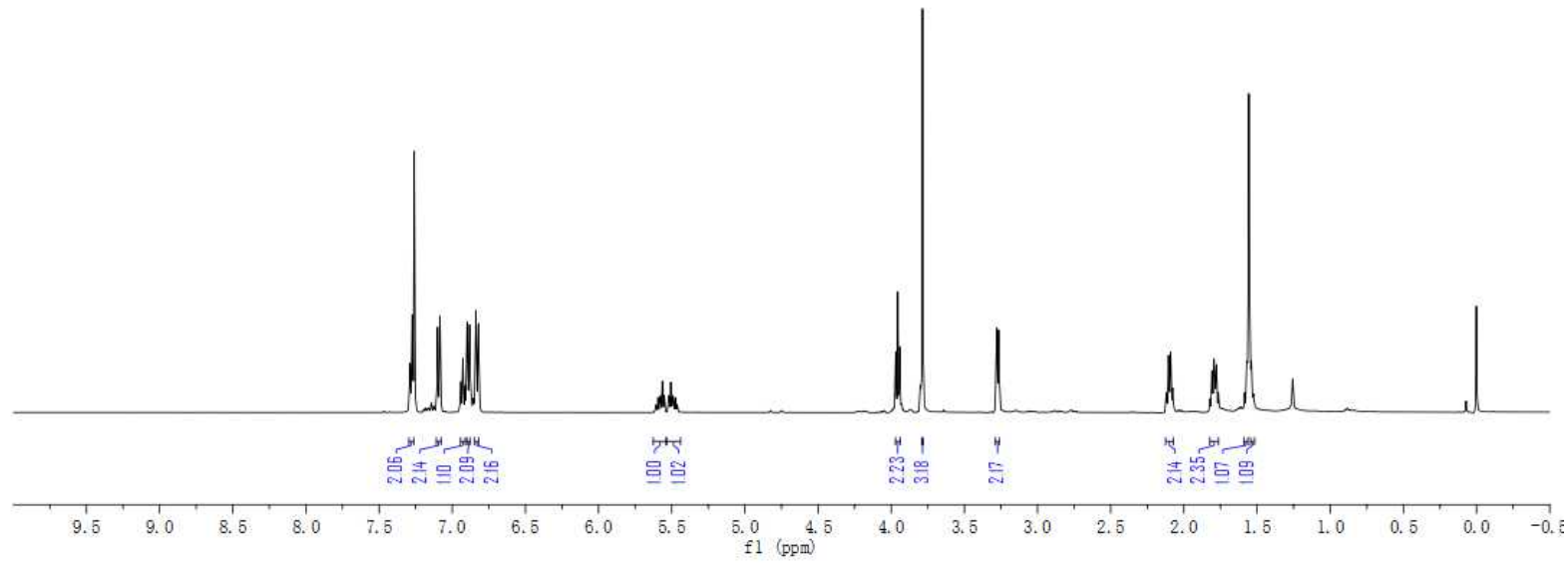

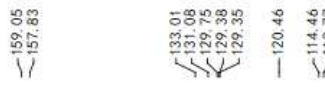

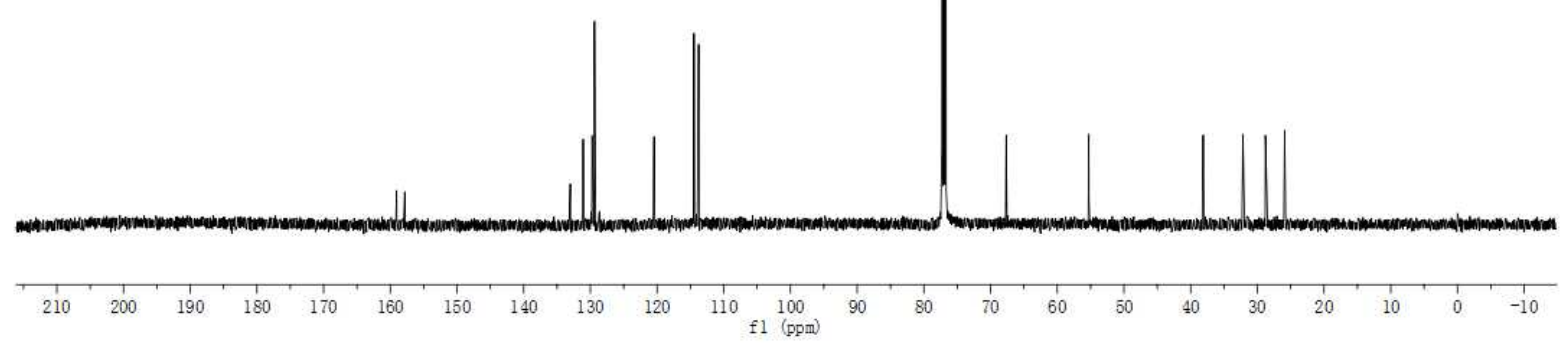


$\widehat{P M P} ح \mathrm{YY}_{4}^{\mathrm{NPhth}}$

\section{(E)-2-(7-(4-Methoxyphenyl)hept-5-en-1-yl)isoindoline-1,3-dione}

General Procedure A: (E)-2-(7-(4-Methoxyphenyl)hept-5-en-1-yl)isoindoline-1,3-dione (1.21 g, $4.98 \mathrm{mmol})$ and 4-methoxybenzyl chloride $(0.67 \mathrm{~mL}, 4.98 \mathrm{mmol})$ were employed. Purification of the residue by FCC (10:1, hexane-EtOAc) afforded the title compound (715 $\mathrm{mg}, 41 \%)$ as a yellow oil.

${ }^{1} \mathrm{H}$ NMR $\left(400 \mathrm{MHz}, \mathrm{CDCl}_{3}\right) \delta 7.84$ (dd, $\left.J=5.4,3.0 \mathrm{~Hz}, 2 \mathrm{H}\right), 7.70$ (dd, $\left.J=5.4,3.0 \mathrm{~Hz}, 2 \mathrm{H}\right), 7.07$ $(\mathrm{d}, J=8.6 \mathrm{~Hz}, 2 \mathrm{H}), 6.82(\mathrm{~d}, J=8.7 \mathrm{~Hz}, 2 \mathrm{H}), 5.69-5.35(\mathrm{~m}, 2 \mathrm{H}), 3.78(\mathrm{~s}, 3 \mathrm{H}), 3.68(\mathrm{t}, J=7.2 \mathrm{~Hz}$, $2 \mathrm{H}$ ), 3.25 (d, $J=6.4 \mathrm{~Hz}, 2 \mathrm{H}$ ), 2.06 (q, J = 6.9 Hz, 2H), 1.69 (app. pent, $J=7.6 \mathrm{~Hz}, 2 \mathrm{H}$ ), 1.42 (app. pent, $J=7.5 \mathrm{~Hz}, 2 \mathrm{H})$;

${ }^{13} \mathrm{C}$ NMR $\left(101 \mathrm{MHz}, \mathrm{CDCl}_{3}\right) \delta 168.4,157.8,133.8,133.0,132.2,130.8,129.9,129.3,123.1$, 113.7, 55.2, 38.1, 37.9, 32.0, 28.1, 26.6;

IR ( $\mathrm{NaCl}$, thin film) 3061, 3030, 2936, 2860, 2839, 1770, 1709, 1609, 1511, 1466, 1438, 1246 , 1036, 820, $720 \mathrm{~cm}^{-1}$;

HRMS (ESI-TOF) $\mathrm{m} / \mathrm{z}$ calcd for $\mathrm{C}_{22} \mathrm{H}_{23} \mathrm{NO}_{3} \mathrm{Na}^{+}(\mathrm{M}+\mathrm{Na})^{+}: 372.1576$, found 372.1578. 

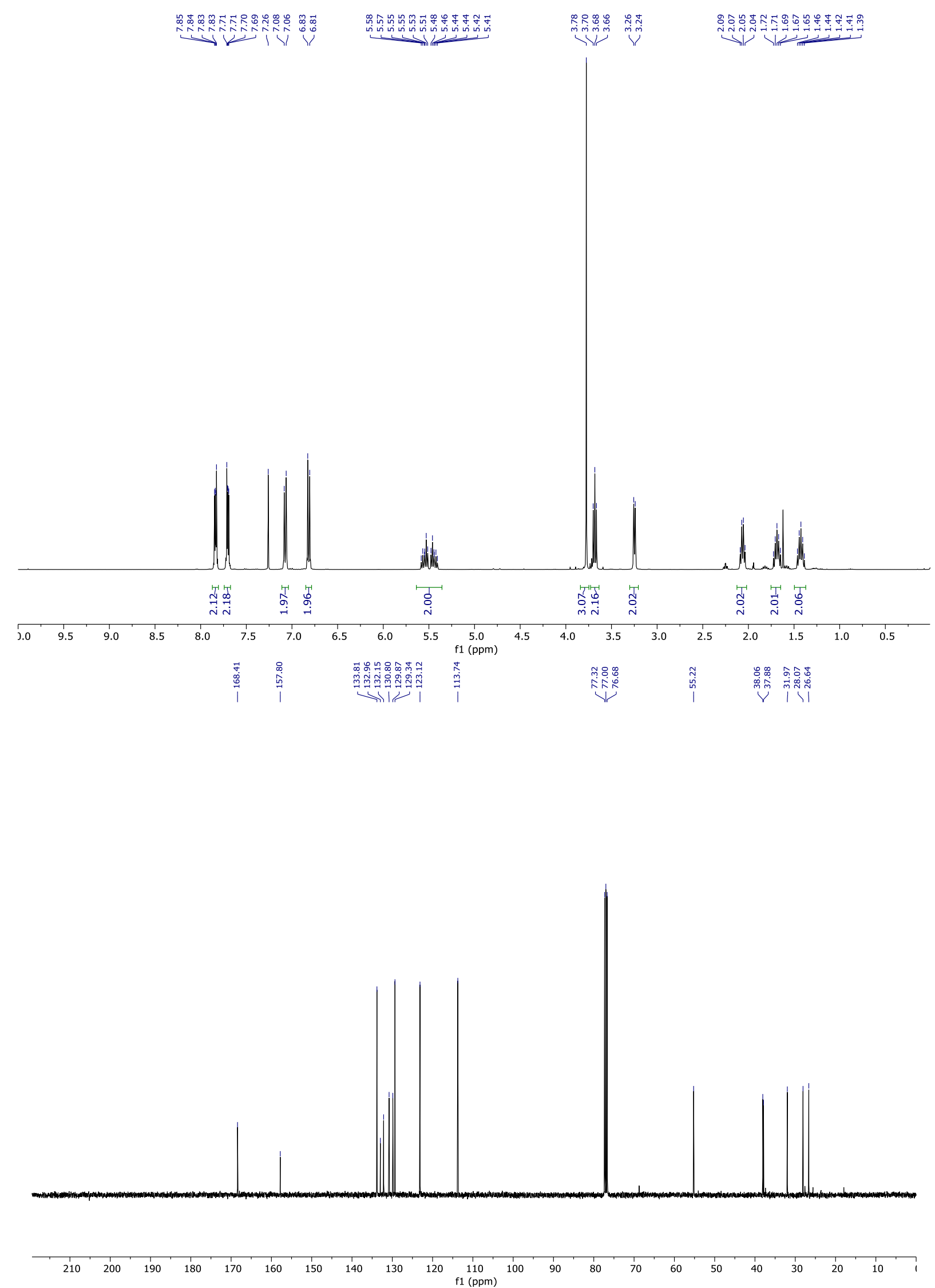
<smiles>COc1ccc(C/C=C/C(C)=O)cc1</smiles>

\section{(E)-5-(4-Methoxyphenyl)pent-3-en-2-one}

To a solution of 4-allylanisole $(500 \mathrm{mg}, 3.37 \mathrm{mmol})$ in $\mathrm{CH}_{2} \mathrm{Cl}_{2}(0.1 \mathrm{M})$ was added methyl vinyl ketone (709 mg, $10.12 \mathrm{mmol}$ ) and Hoveyda-Grubbs $2^{\text {nd }}$ generation catalyst $(105.7 \mathrm{mg}, 5 \mathrm{~mol} \%$ ). The reaction mixture was stirred at room temperature for $18 \mathrm{~h}$. The reaction was then concentrated in vacuo and the crude mixture purified by FCC $(5: 1$, hexane-EtOAc) to afford the title compound ( $371 \mathrm{mg}, 58 \%)$ as a yellow oil.

${ }^{1} \mathrm{H}$ NMR $\left(400 \mathrm{MHz}, \mathrm{CDCl}_{3}\right)$ ठ 7.17-7.02 (m, 2H), $6.91(\mathrm{~s}, 1 \mathrm{H}), 6.88-6.85(\mathrm{~m}, 2 \mathrm{H}), 6.05(\mathrm{~d}, J=15.9$ $\mathrm{Hz}, 1 \mathrm{H}), 3.80$ (s, 3H), 3.48 (dd, $J=6.7,1.6 \mathrm{~Hz}, 2 \mathrm{H}), 2.24(\mathrm{~s}, 3 \mathrm{H})$;

${ }^{13} \mathrm{C}$ NMR $\left(100 \mathrm{MHz}, \mathrm{CDCl}_{3}\right) \delta 198.8,158.6,147.0,131.9,129.9,129.7,114.3,55.4,38.1,27.1$;

IR ( $\mathrm{NaCl}$, thin film) 3003, 2935, 2836, 1696, 1673, 1512, 1361, 1249, 1177, 1033, 981, $826 \mathrm{~cm}^{-1}$;

HRMS (El-TOF) $\mathrm{m} / \mathrm{z}$ calcd for $\mathrm{C}_{12} \mathrm{H}_{14} \mathrm{O}_{2} \mathrm{Na}(\mathrm{M}+\mathrm{Na})^{+}: 213.0886$, found 213.0899 . 


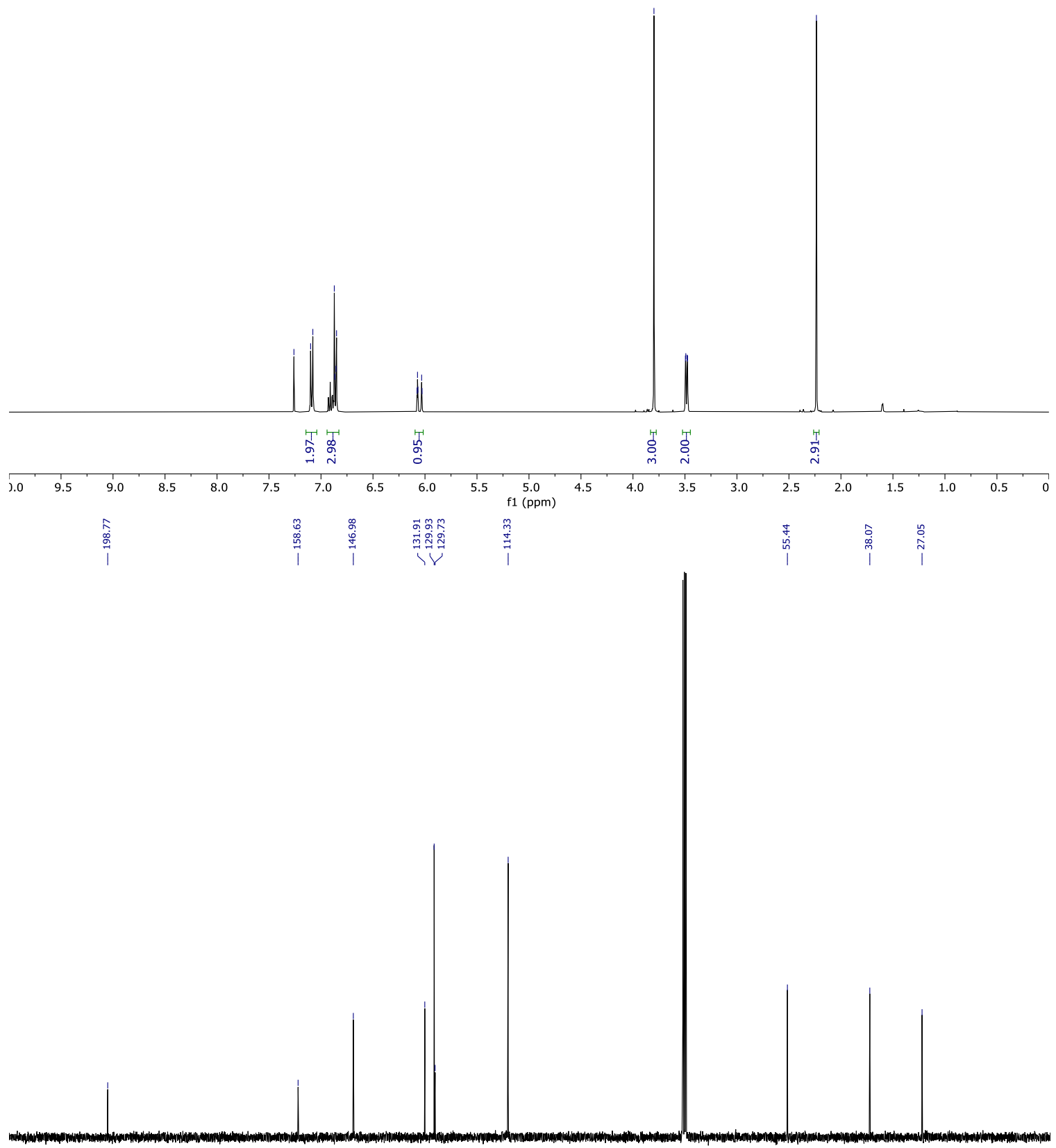

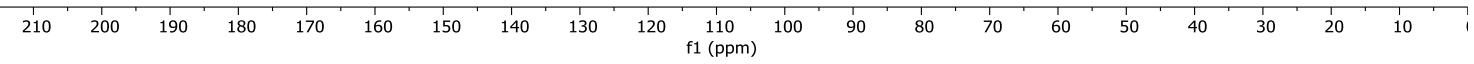


<smiles>COc1ccc(C/C=C/C2CCOCC2)cc1</smiles>

\section{(E)-4-(3-(4-Methoxyphenyl)prop-1-en-1-yl)tetrahydro-2H-pyran}

General Procedure A: 4-Ethynyltetrahydro-2H-pyran ${ }^{8}$ (720 mg, $6.54 \mathrm{mmol}$ ) and 4-methoxybenzyl chloride $(0.86 \mathrm{~mL}, 6.54 \mathrm{mmol})$ were employed. Purification of the residue by FCC $(10: 1-5: 1$, hexane-EtOAc) afforded the title compound (402 mg, 26\%) as a colorless oil.

${ }^{1} \mathrm{H}$ NMR $\left(400 \mathrm{MHz}, \mathrm{CDCl}_{3}\right) \delta 7.11$ (d, $\left.J=8.6 \mathrm{~Hz}, 2 \mathrm{H}\right), 6.86(\mathrm{~d}, J=8.6 \mathrm{~Hz}, 2 \mathrm{H}), 5.58$ (dtd, $J=15.4$, 6.6, $1.1 \mathrm{~Hz}, 1 \mathrm{H}$ ), 5.47 (ddt, $J=15.4,6.3,1.2 \mathrm{~Hz}, 1 \mathrm{H}$ ), 3.98 (ddd, $J=11.7,4.5,2.0 \mathrm{~Hz}, 2 \mathrm{H}$ ), 3.82 $(\mathrm{s}, 3 \mathrm{H}), 3.43(\mathrm{td}, J=11.72 .2 \mathrm{~Hz}, 2 \mathrm{H}), 3.30(\mathrm{~d}, J=6.5 \mathrm{~Hz}, 2 \mathrm{H}), 2.27-2.17(\mathrm{~m}, 1 \mathrm{H}), 1.67-1.61(\mathrm{~m}$, $2 \mathrm{H}), 1.49$ (dtd, $J=13.4,11.7,4.4 \mathrm{~Hz}, 2 \mathrm{H}$ );

${ }^{13} \mathrm{C}$ NMR $\left(101 \mathrm{MHz}_{\mathrm{CDCl}}\right) \delta 157.9,135.7,132.8,129.3,127.8,113.8,67.8,55.2,38.1,37.8$, 32.8;

IR ( $\mathrm{NaCl}$, thin film) 2932, 2838, 1610, 1491, 1300, 1246, 1176, 1128, 1091, $1035 \mathrm{~cm}^{-1}$;

HRMS $\left(\mathrm{El}^{+}\right) \mathrm{m} / \mathrm{z}$ calcd for $\mathrm{C}_{15} \mathrm{H}_{20} \mathrm{O}_{2}{ }^{+}\left(\mathrm{M}^{+}\right): 232.1458$ found 232.1461 . 

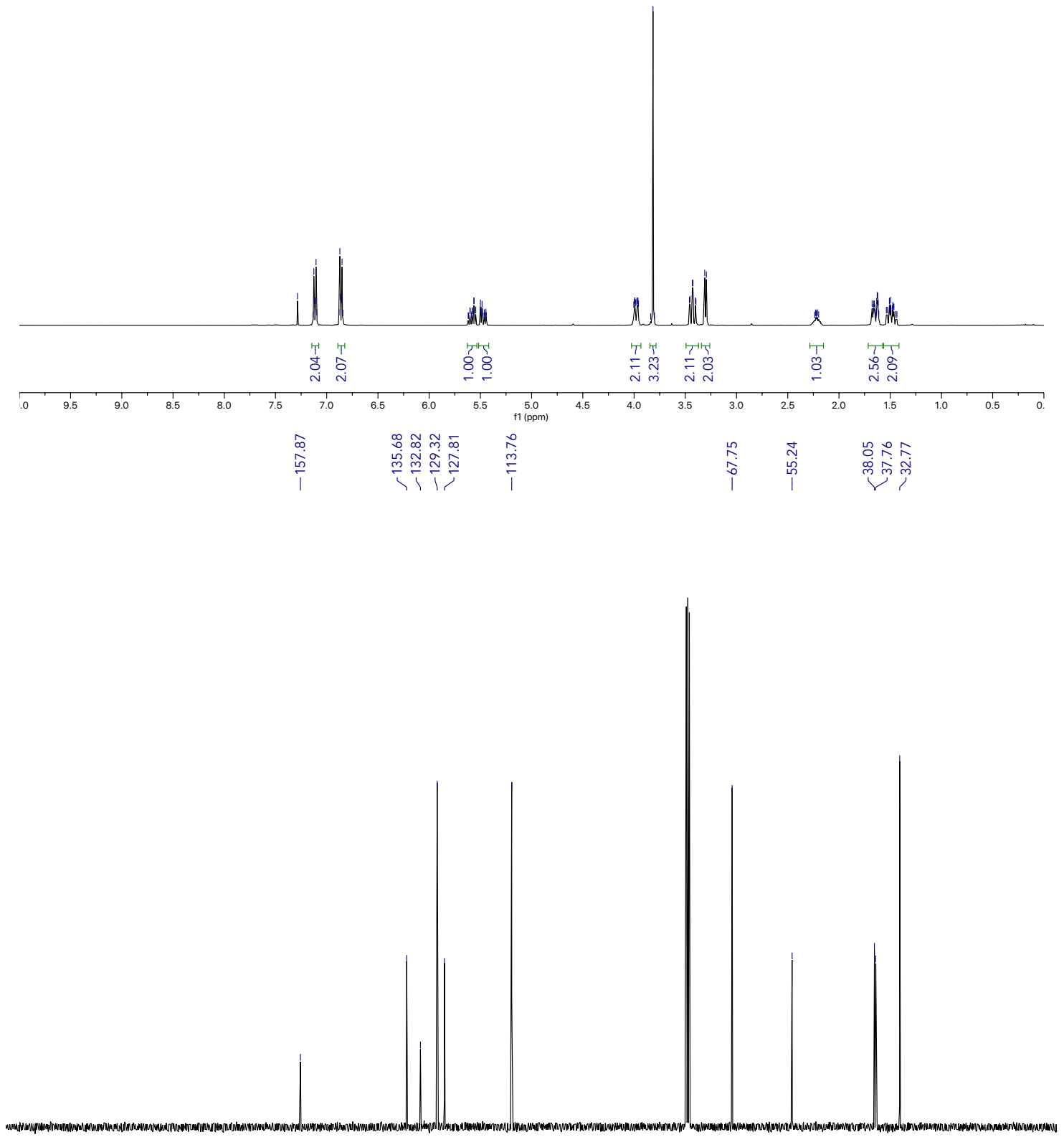

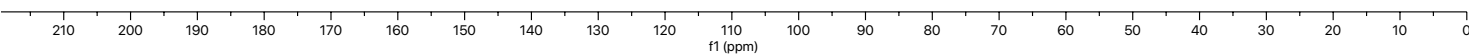


<smiles>COc1ccc(C/C=C/C2CCN(C(=O)O)CC2)cc1</smiles>

\section{tert-Butyl (E)-4-(3-(4-methoxyphenyl)prop-1-en-1-yl)piperidine-1-carboxylate}

General Procedure A: tert-Butyl-4-ethynylpiperidine-1-carboxylate ${ }^{9}(836 \mathrm{mg}, 4.00 \mathrm{mmol})$ and 4-methoxybenzyl chloride $(0.53 \mathrm{~mL}, 4.00 \mathrm{mmol})$ were employed. Purification of the residue by FCC (15:1 - 5:1, hexane-EtOAc) afforded the title compound $(1.07 \mathrm{~g}, 81 \%)$ as a yellow oil.

${ }^{1} \mathrm{H}$ NMR $\left(400 \mathrm{MHz}, \mathrm{CDCl}_{3}\right) \delta 7.08(\mathrm{~d}, J=8.6 \mathrm{H}, 2 \mathrm{H}), 6.83(\mathrm{~d}, J=8.6 \mathrm{~Hz}, 2 \mathrm{H}), 5.55$ (dtd, $J=15.4$, 6.6, $1.1 \mathrm{~Hz}, 1 \mathrm{H}$ ), 5.43 (ddt, $J=15.4,6.5,1.4 \mathrm{~Hz}, 1 \mathrm{H}$ ), 4.06 (br. d, J=13.2 Hz, 2H), 3.79 (s, 3H), $3.26(\mathrm{~d}, J=6.6 \mathrm{~Hz}, 2 \mathrm{H}), 2.76-2.69(\mathrm{~m}, 2 \mathrm{H}), 2.14-2.04(\mathrm{~m}, 1 \mathrm{H}), 1.66$ (br. d, $J=13.1 \mathrm{~Hz}, 2 \mathrm{H}), 1.45$ (s, $9 \mathrm{H}), 1.32-1.22(\mathrm{~m}, 2 \mathrm{H})$;

${ }^{13} \mathrm{C}$ NMR $\left(101 \mathrm{MHz}, \mathrm{CDCl}_{3}\right) \delta 157.9,154.9,135.4,132.8,129.3,128.0,113.8,79.2,55.3,43.6$, $38.8,38.1,31.9,28.5$;

IR ( $\mathrm{NaCl}$, thin film) 3321, 2932, 1693, 1610, 1512, 1422, 1366, 1248, 1167, 1036, $969 \mathrm{~cm}^{-1}$; HRMS (ESI-TOF) $m / z$ calcd for $\mathrm{C}_{20} \mathrm{H}_{29} \mathrm{NO}_{3} \mathrm{Na}^{+}(\mathrm{M}+\mathrm{Na})^{+}: 354.2045$ found 354.2047. 


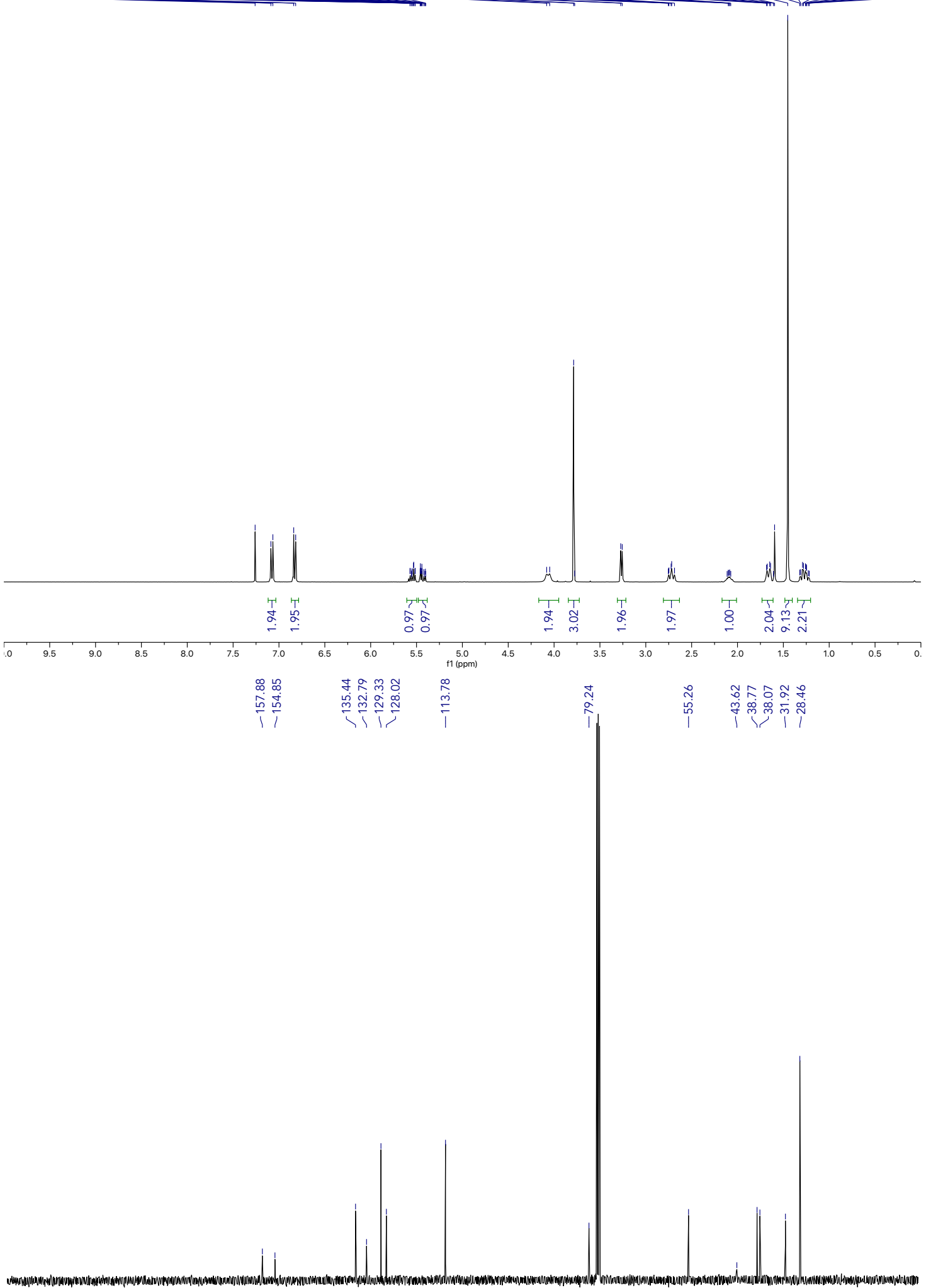


LeOC

\section{tert-Butyl (E)-3-(3-(4-methoxyphenyl)prop-1-en-1-yl)azetidine-1-carboxylate}

General Procedure A: tert-Butyl 3-ethynylazetidine-1-carboxylate ${ }^{10}(1.90 \mathrm{~g}, 8.37 \mathrm{mmol})$ and 4-methoxybenzyl chloride $(1.14 \mathrm{~mL}, 8.37 \mathrm{mmol})$ were employed. Purification of the residue by FCC (15:1 - 5:1, hexane-EtOAc) afforded the title compound (1.25 g, 49\%) as a yellow oil.

${ }^{1} \mathrm{H}$ NMR $\left(400 \mathrm{MHz}, \mathrm{CDCl}_{3}\right) \delta 7.10(\mathrm{~d}, \mathrm{~J}=8.7 \mathrm{~Hz}, 2 \mathrm{H}), 6.86(\mathrm{~d}, \mathrm{~J}=8.7 \mathrm{~Hz}, 2 \mathrm{H}), 5.71-5.61(\mathrm{~m}, 2 \mathrm{H})$, $4.08(\mathrm{t}, J=8.5 \mathrm{~Hz}, 2 \mathrm{H}), 3.81(\mathrm{~s}, 3 \mathrm{H}), 3.73(\mathrm{dd}, J=8.5,6.0 \mathrm{~Hz}, 2 \mathrm{H}), 3.36-3.27(\mathrm{~m}, 2 \mathrm{H}), 3.24-3.15$ $(\mathrm{m}, 1 \mathrm{H}), 1.46(\mathrm{~s}, 9 \mathrm{H})$;

${ }^{13} \mathrm{C}$ NMR $\left(101 \mathrm{MHz}, \mathrm{CDCl}_{3}\right) \delta 158.0,156.3,132.1,131.6,131.1,129.4,113.9,79.3,55.3,37.9$, 31.2, 28.4, $1 \times \mathrm{C}$ not observed due to rotameric nature of Boc group;

IR ( $\mathrm{NaCl}$, thin film) 3295, 2974, 2883, 2836, 1694, 1610, 1513, 1404, 1249, 1146, 1036, 970, $826 \mathrm{~cm}^{-1}$;

HRMS (ESI-TOF) $\mathrm{m} / \mathrm{z}$ calcd for $\mathrm{C}_{17} \mathrm{H}_{23} \mathrm{NO}_{3} \mathrm{Na}^{+}(\mathrm{M}+\mathrm{Na})^{+}: 326.1732$, found 326.1735. 

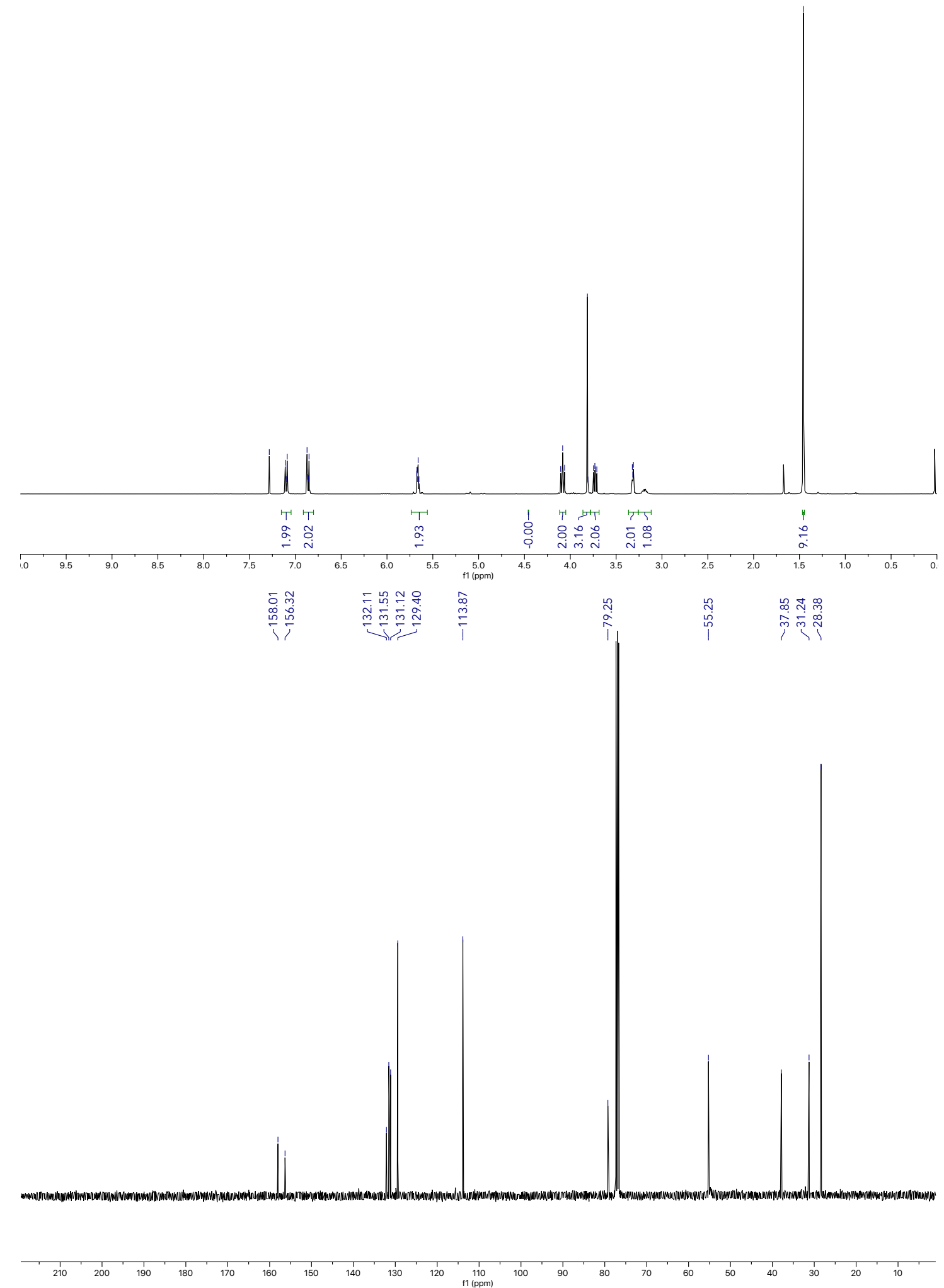


\section{Epoxide synthesis}

\section{General Procedure B: for the epoxidation of allylbenzene derivatives}<smiles>[R]C/C=C/C[Te]</smiles>
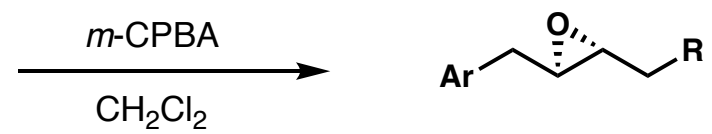

To a solution of allylbenzene derivative $(100 \mathrm{~mol} \%)$ in $\mathrm{CH}_{2} \mathrm{Cl}_{2}(0.3 \mathrm{M})$ was added $m$-CPBA $(75 \%$, $125 \mathrm{~mol} \%$ ) portion-wise over five minutes. The reaction mixture was stirred at room temperature until consumption of starting material occurred, as determined by TLC analysis. The reaction was then quenched by addition of saturated aq. $\mathrm{NaHSO}_{3}(1 \mathrm{~mL} / \mathrm{mmol} m$-CPBA). The mixture was filtered through celite ${ }^{\circledR}$, washing with $\mathrm{Et}_{2} \mathrm{O}$. The filtrate was transferred to a separatory funnel, washed with saturated aq. $\mathrm{NaHCO}_{3}(3 \times 5 \mathrm{~mL} / \mathrm{mmol} m$-CPBA), brine $(5 \mathrm{~mL} / \mathrm{mmol} m$-CPBA), dried $\left(\mathrm{Na}_{2} \mathrm{SO}_{4}\right)$, filtered, and concentrated in vacuo. Purification of the residue by FCC, under the condition noted, afforded the corresponding epoxide.

Note: The epoxides stain better in PMA compared to $\mathrm{KMnO}_{4}$. 
(N)

$\left(2 R^{*}, 3 R^{*}\right)-2-(4-M e t h o x y b e n z y l)-3-p r o p y l o x i r a n e ~( \pm)-5 a$

General Procedure B: (E)-1-(Hex-2-en-1-yl)-4-methoxybenzene (1.08 g, $5.7 \mathrm{mmol}$ ) was employed. Purification of the residue by FCC (5:1, hexane-EtOAc) afforded the title compound $(0.85 \mathrm{~g}, 72 \%)$ as a colorless oil.

${ }^{1} \mathrm{H}$ NMR $\left(400 \mathrm{MHz}, \mathrm{CDCl}_{3}\right) \delta 7.15(\mathrm{~d}, J=8.6 \mathrm{~Hz}, 2 \mathrm{H}), 6.85$ (d, J = 8.6 Hz, 2H), 3.79 (s, 3H), 2.88$2.83(\mathrm{~m}, 2 \mathrm{H}), 2.77-2.71(\mathrm{~m}, 2 \mathrm{H}), 1.55-1.36(\mathrm{~m}, 4 \mathrm{H}), 0.92(\mathrm{t}, J=7.2 \mathrm{~Hz}, 3 \mathrm{H})$;

${ }^{13} \mathrm{C}$ NMR $\left(101 \mathrm{MHz}, \mathrm{CDCl}_{3}\right) \delta$ 158.5, 130.0, 129.7, 114.1, 59.1, 58.7, 55.4, 37.8, 34.1, 19.4, 14.1;

IR ( $\mathrm{NaCl}$, thin film) 2960, 2933, 2873, 2836, 1612, 1524, 1245, 1178, 1036, 933, $822 \mathrm{~cm}^{-1}$;

HRMS (ESI-TOF) $\mathrm{m} / \mathrm{z}$ calcd for $\mathrm{C}_{13} \mathrm{H}_{18} \mathrm{O}_{2} \mathrm{Na}^{+}(\mathrm{M}+\mathrm{Na})^{+}:$229.1204, found 229.1206. 

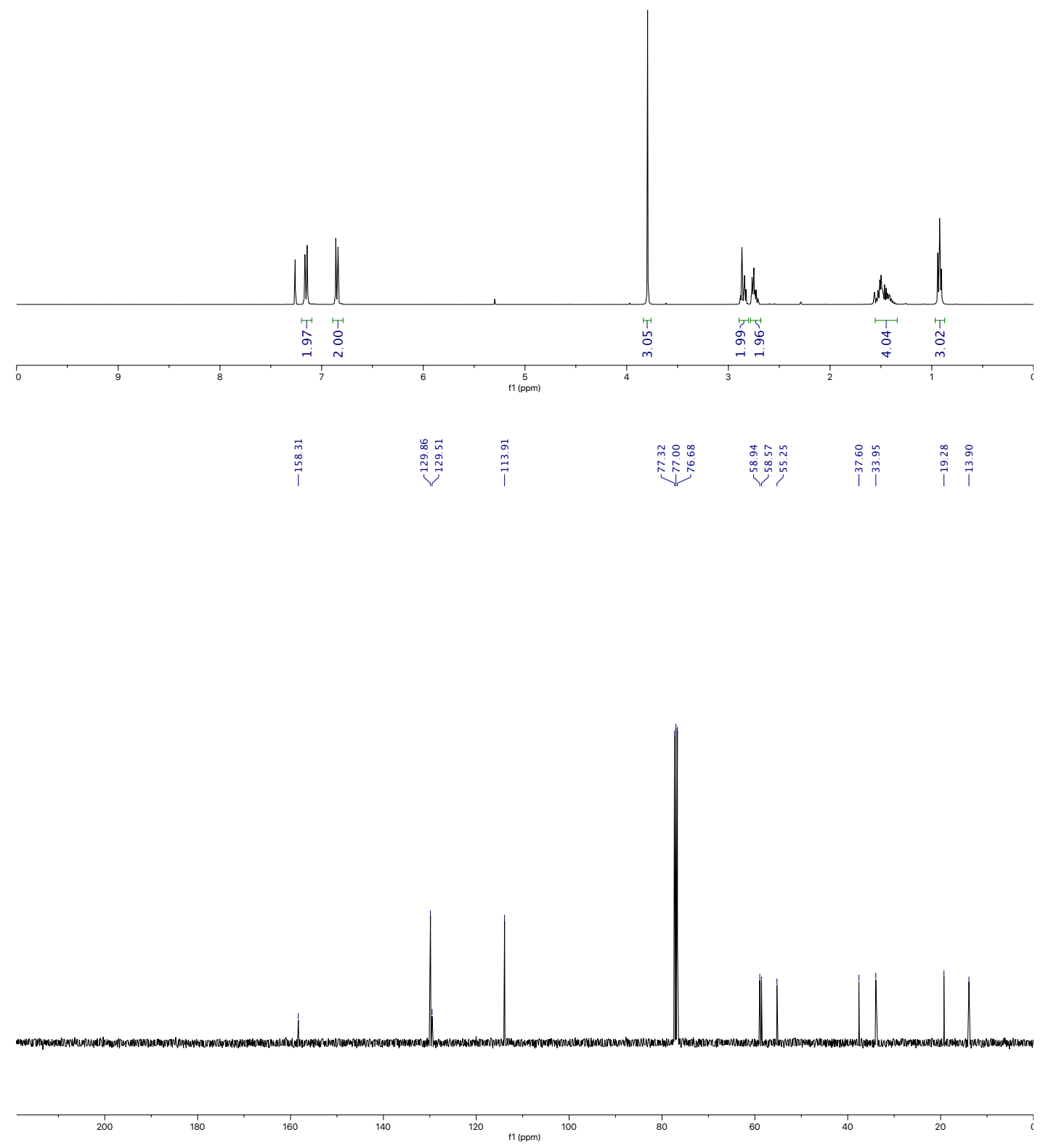
<smiles>CCCC1O[C@@H]1Cc1ccc(OC)c(OC)c1</smiles>

\section{$\left(2 R^{*}, 3 R^{*}\right)-2-(3,4-D i m e t h o x y b e n z y l)-3-p r o p y l o x i r a n e ~( \pm)-5 b$}

General Procedure B: (E)-4-(Hex-2-en-1-yl)-1,2-dimethoxybenzene (1.50 g, $6.52 \mathrm{mmol}$ ) was employed. Purification of the residue by FCC (5:1, hexane-EtOAc) afforded the title compound $(1.12 \mathrm{~g}, 70 \%)$ as a colorless oil.

${ }^{1} \mathrm{H}$ NMR $\left(400 \mathrm{MHz}, \mathrm{CDCl}_{3}\right)$ ठ 6.83-6.80 (m, 1H), 6.78-6.75 (m, 2H), 3.88 (s, 3H), $3.86(\mathrm{~s}, 3 \mathrm{H}), 2.88$ $(\mathrm{td}, J=5.5,2.2 \mathrm{~Hz}, 1 \mathrm{H}), 2.85-2.74(\mathrm{~m}, 3 \mathrm{H}), 1.58-1.36(\mathrm{~m}, 4 \mathrm{H}), 0.93(\mathrm{t}, J=7.1 \mathrm{~Hz}, 3 \mathrm{H})$;

${ }^{13} \mathrm{C}$ NMR $\left(101 \mathrm{MHz}, \mathrm{CDCl}_{3}\right) \delta$ 148.9, 147.7, 130.1, 120.8, 112.1, 111.2, 58.9, 58.6, 55.9, 55.8, $38.1,33.9,19.3,13.9$;

IR ( $\mathrm{NaCl}$, thin film) 2960, 2934, 2873, 1607, 1591, 1517, 1464, 1334, 1263, 1237, 1157, 1141, $1030 \mathrm{~cm}^{-1}$;

HRMS (ESI-TOF) $\mathrm{m} / \mathrm{z}$ calcd for $\mathrm{C}_{14} \mathrm{H}_{20} \mathrm{NaO}_{3}{ }^{+}(\mathrm{M}+\mathrm{Na})^{+}:$259.1305, found 259.1305. 
뿌

i iviog

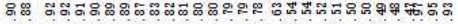

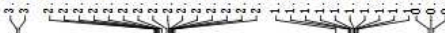
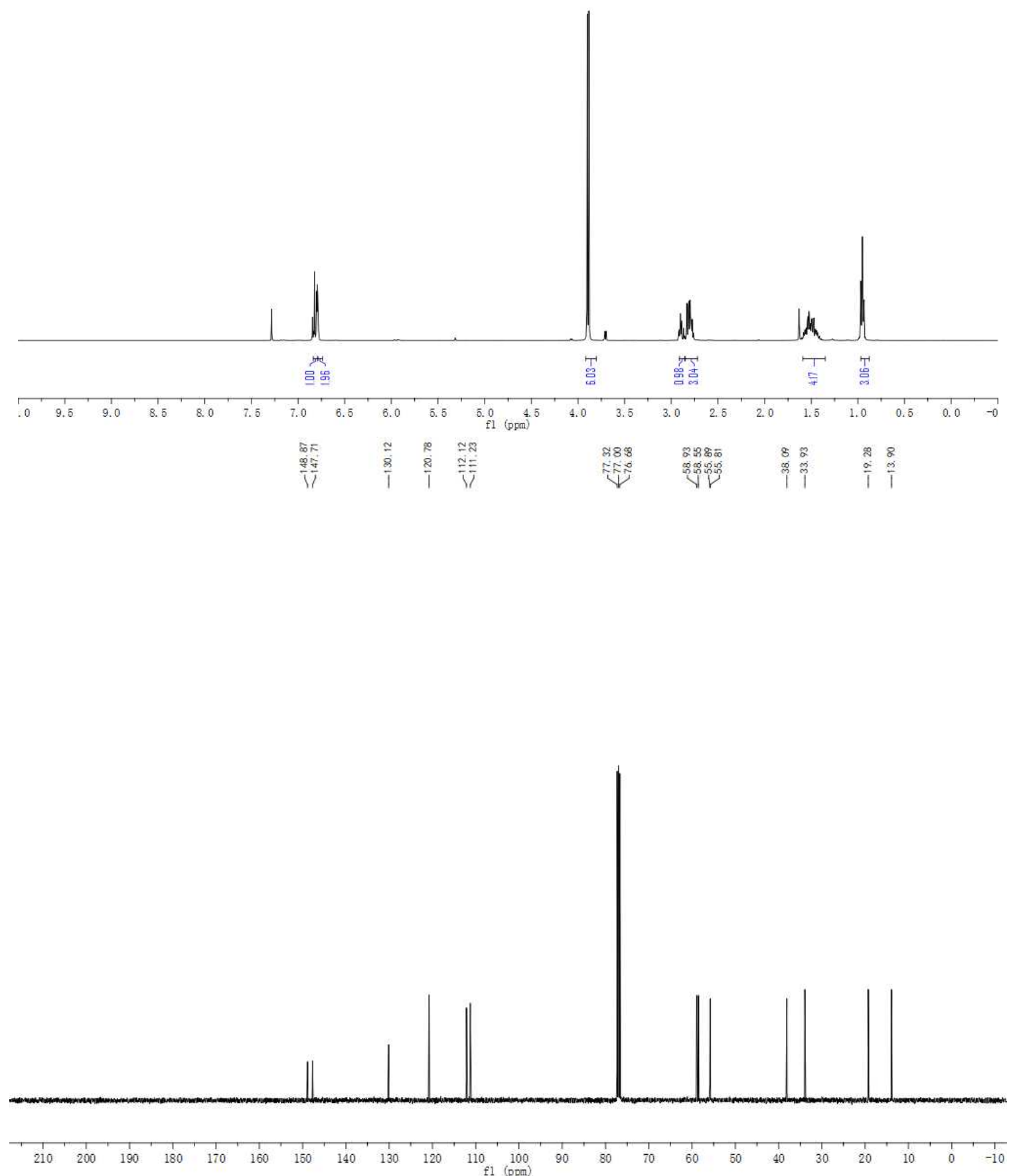
<smiles>CCC[C@@H]1O[C@@H]1Cc1ccc2c(c1)OCO2</smiles>

\section{5-(((2R*, $\left.\left.3 R^{*}\right)-3-P r o p y l o x i r a n-2-y l\right)$ methyl)benzo[d][1,3]dioxole $( \pm)-5 \mathrm{c}$}

General Procedure B: (E)-5-(Hex-2-en-1-yl)benzo[d][1,3]dioxole $(610 \mathrm{mg}, 3.0 \mathrm{mmol})$ was employed. Purification of the residue by FCC (10:1, hexane-EtOAc) afforded the title compound (588 $\mathrm{mg}, 89 \%)$ as a colorless oil.

${ }^{1} \mathrm{H}$ NMR $\left(400 \mathrm{MHz} \mathrm{CDCl}_{3}\right) \delta$ 6.76-6.73 (m, 2H), 6.69-6.67 (m, 1H), $5.93(\mathrm{~s}, 2 \mathrm{H})$, 2.87-2.70 (m, $4 \mathrm{H}), 1.54-1.36(\mathrm{~m}, 4 \mathrm{H}), 0.93(\mathrm{t}, J=7.1 \mathrm{~Hz}, 3 \mathrm{H})$;

${ }^{13} \mathrm{C}$ NMR $\left(101 \mathrm{MHz}, \mathrm{CDCl}_{3}\right) \delta 147.7,146.2,131.2,121.8,109.4,108.3,100.9,58.8,58.6,38.2$, 33.9, 19.3, 13.9;

IR ( $\mathrm{NaCl}$, thin film) 2962, 2932, 2874, 1503, 1490, 1443, 1247, 1040, 932, $810 \mathrm{~cm}^{-1}$;

HRMS $\left(\mathrm{EI}^{+}\right) \mathrm{m} / \mathrm{z}$ calcd for $\mathrm{C}_{13} \mathrm{H}_{16} \mathrm{O}_{3}{ }^{+}(\mathrm{M})^{+}:$220.1086, found 220.1079 . 

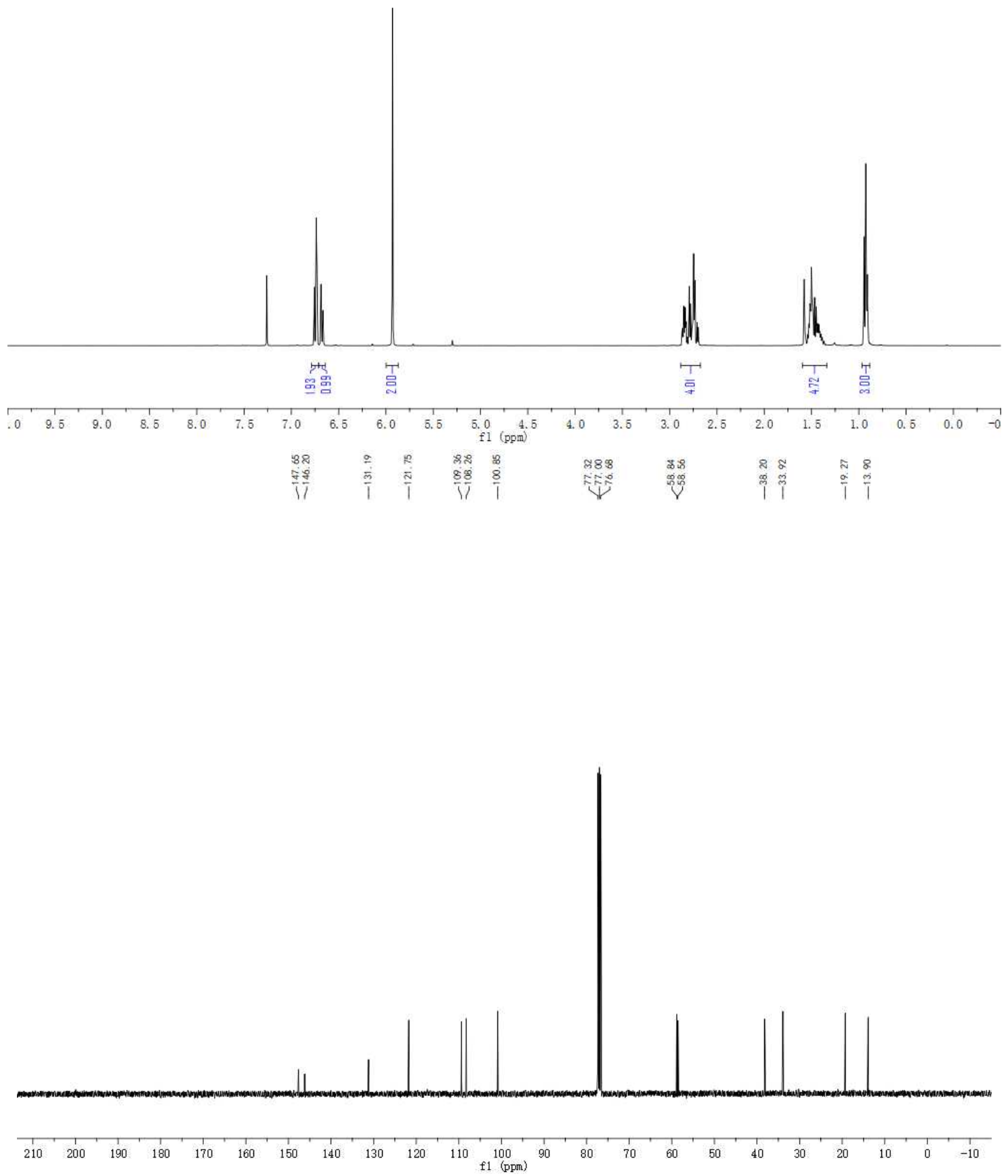
<smiles>CCCC1OC1Cc1ccc2ccccc2c1</smiles>

$\left(2 R^{*}, 3 R^{*}\right)-2-($ Naphthalen-2-ylmethyl)-3-propyloxirane $( \pm)-5 \mathrm{~d}$

General Procedure B: (E)-2-(Hex-2-en-1-yl)naphthalene (630 mg, 3.00) was employed. Purification of the residue by FCC (20:1, hexane-EtOAc) afforded the title compound (540 mg, $80 \%$ ) as a colorless oil.

${ }^{1} \mathrm{H}$ NMR $\left(400 \mathrm{MHz}, \mathrm{CDCl}_{3}\right)$ ठ 7.84-7.80 (m, 3H), 7.70 (br. s, 1H), 7.50-7.43 (m, 2H), 7.39 (dd, $J=$ 8.4, $1.8 \mathrm{~Hz}, 1 \mathrm{H}), 3.11-3.05(\mathrm{~m}, 1 \mathrm{H}), 3.02-2.96(\mathrm{~m}, 2 \mathrm{H}), 2.85-2.82(\mathrm{~m}, 1 \mathrm{H}), 1.58-1.39(\mathrm{~m}, 4 \mathrm{H}), 0.94$ (t, $J=7.2 \mathrm{~Hz}, 3 \mathrm{H}$ );

${ }^{13} \mathrm{C}$ NMR $\left(101 \mathrm{MHz}, \mathrm{CDCl}_{3}\right) \delta 135.0133 .5,132.3,128.1,127.6,127.5,127.4,127.2,126.0,125.5$, 58.7, 58.6, 38.6, 33.9, 19.3, 13.9;

IR ( $\mathrm{NaCl}$, thin film) 3053, 2960, 2931, 2872, 1633, 1601, 1509, 1464, 1366, 1270, $1245 \mathrm{~cm}^{-1}$ HRMS $\left(\mathrm{El}^{+}\right) \mathrm{m} / \mathrm{z}$ calcd for $\mathrm{C}_{16} \mathrm{H}_{18} \mathrm{O}^{+}(\mathrm{M})^{+}:$:226.1352, found 226.1344 . 

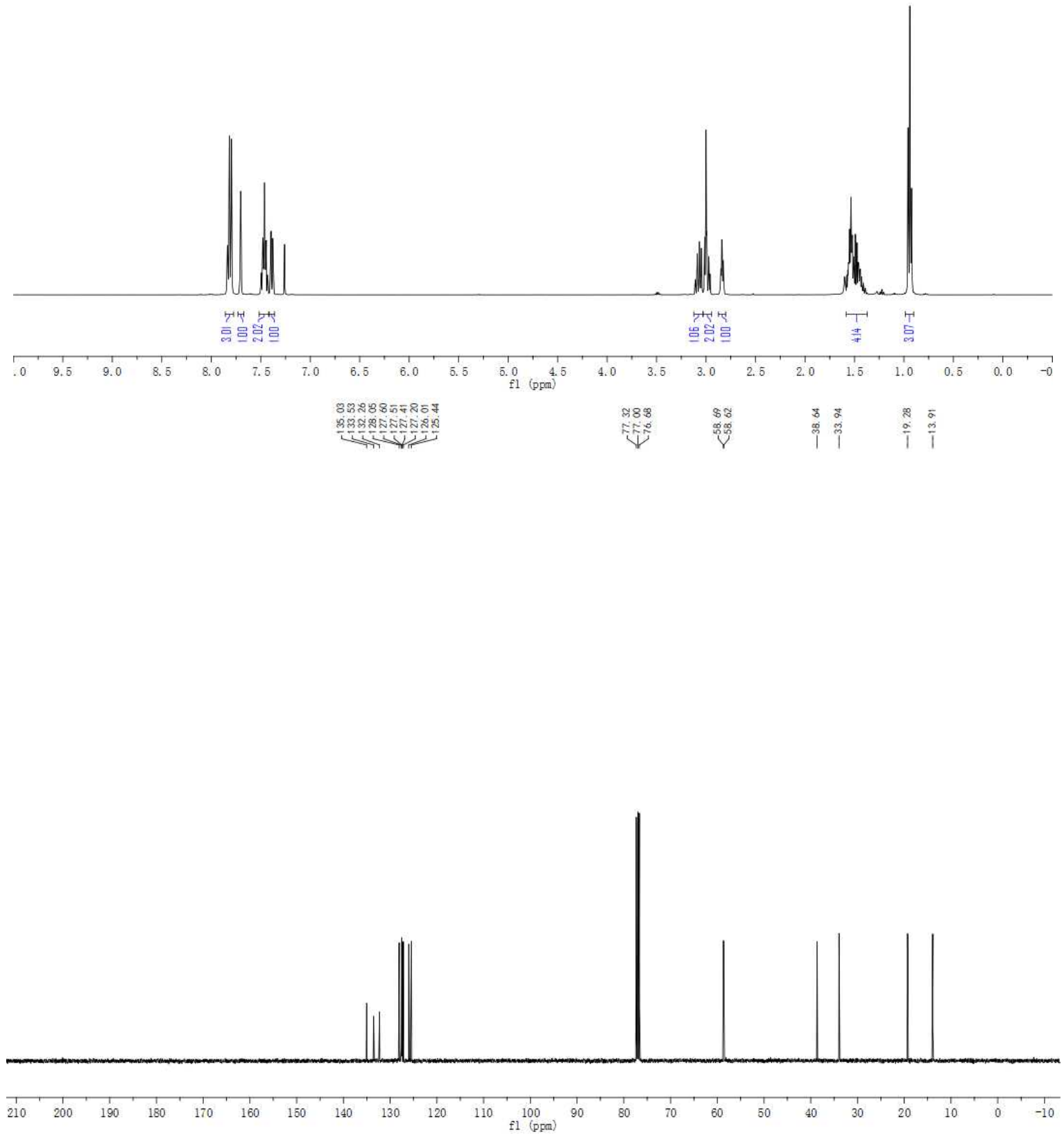
<smiles>CCCC1OC1Cc1ccc(C)cc1</smiles>

$\left(2 R^{\star}, 3 R^{\star}\right)-2-(4-M e t h y l b e n z y l)-3-p r o p y l o x i r a n e ~( \pm)-5 e$

General Procedure B: $(E)-1-(H e x-2-e n-1-y l)-4-m e t h y l b e n z e n e ~(1.60 \mathrm{~g}, 9.2 \mathrm{mmol})$ was employed. Purification of the residue by FCC $(5: 1$, hexane-EtOAc) afforded the title compound $(1.47 \mathrm{~g}, 84 \%)$ as a colorless oil.

${ }^{1} \mathrm{H}$ NMR $\left(400 \mathrm{MHz}, \mathrm{CDCl}_{3}\right) \delta 7.12(\mathrm{~s}, 4 \mathrm{H}), 2.91-2.86(\mathrm{~m}, 2 \mathrm{H}), 2.78-2.71(\mathrm{~m}, 2 \mathrm{H}), 2.33(\mathrm{~s}, 3 \mathrm{H})$, 1.54-1.37 (m, 4H), $0.92(\mathrm{t}, J=7.2 \mathrm{~Hz}, 3 \mathrm{H})$;

${ }^{13} \mathrm{C}$ NMR $\left(101 \mathrm{MHz}, \mathrm{CDCl}_{3}\right) \delta$ 136.0, 134.4, 129.2, 128.8, 58.8, 58.6, 38.1, 34.0, 21.0, 19.3, 13.9;

IR ( $\mathrm{NaCl}$, thin film) 2961, 2931, 2873, 1515, 1458, 1380, 1121, 931, $805 \mathrm{~cm}^{-1}$;

HRMS (ESI-TOF) $m / z$ calcd for $\mathrm{C}_{13} \mathrm{H}_{18} \mathrm{ONa}^{+}(\mathrm{M}+\mathrm{Na})^{+}: 213.1255$, found 213.1250. 

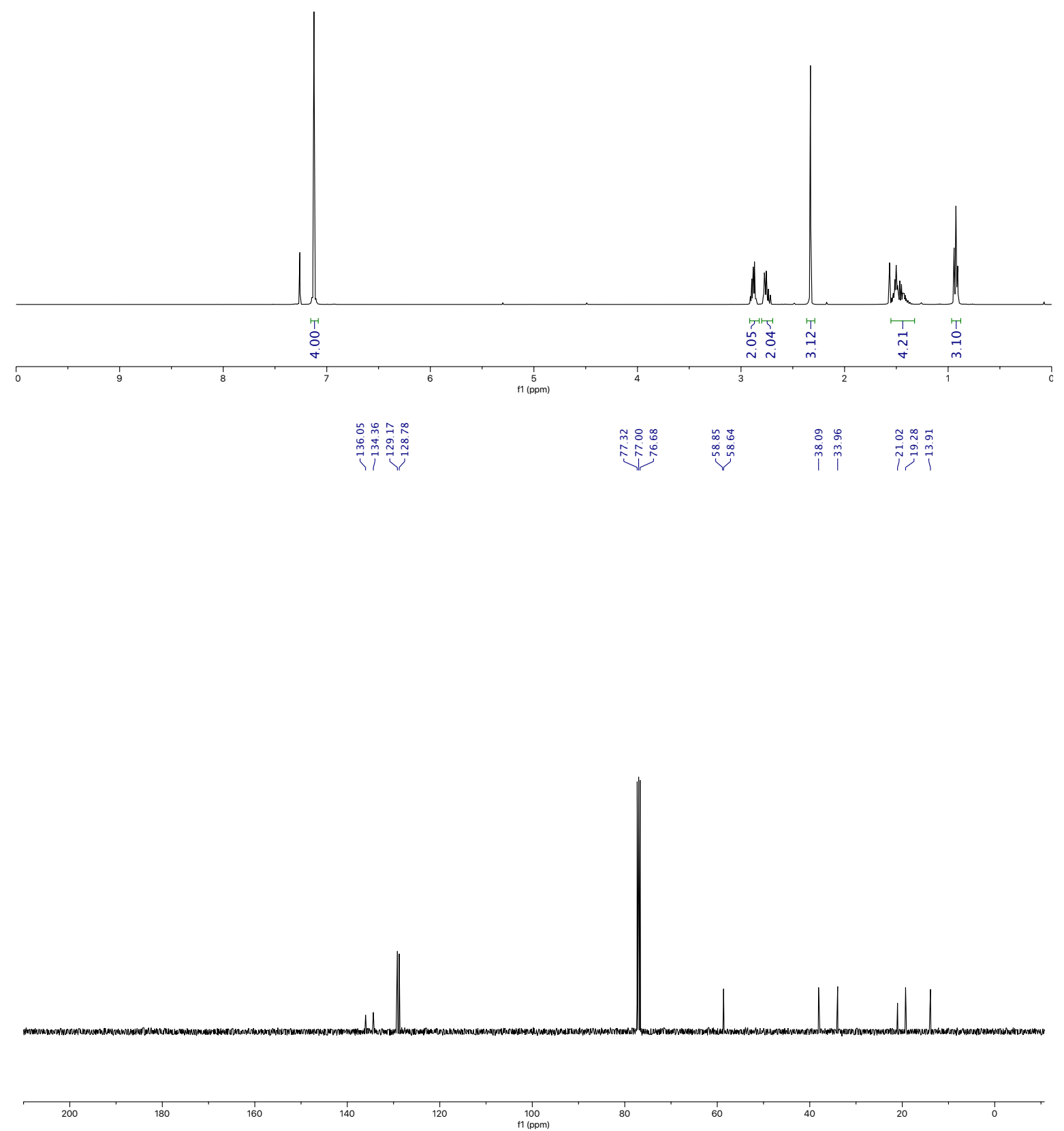
<smiles>CCCC1OC1Cc1ccccc1</smiles>

$\left(2 R^{*}, 3 R^{*}\right)-2-B e n z y l-3-p r o p y l o x i r a n e( \pm)-5 f$

General Procedure B: $(E)$-Hex-2-en-1-ylbenzene $(1.09 \mathrm{~g}, 6.8 \mathrm{mmol})$ was employed. Purification of the residue by FCC $(5: 1$, hexane-EtOAc) afforded the title compound $(0.84 \mathrm{~g}, 70 \%)$ as a colorless oil.

${ }^{1} \mathrm{H}$ NMR $\left(400 \mathrm{MHz}, \mathrm{CDCl}_{3}\right)$ ठ 7.33-7.29 (m, 2H), 7.25-7.22 (m, 3H), 2.94-2.89 (m, 2H), 2.83-2.76 (m, 2H), 1.55-1.37, (m, 4H), 0.92 (t, J=7.2 Hz, 3H);

${ }^{13} \mathrm{C}$ NMR $\left(101 \mathrm{MHz}, \mathrm{CDCl}_{3}\right) \delta 137.5,128.9,128.5,126.5,58.7,58.6,38.5,33.9,19.3,13.9$;

IR ( $\mathrm{NaCl}$, thin film) 3029, 2961, 2932, 2873, 1605, 1495, 1454, 1381, 1103, 1066, 1032, 936, 748 $\mathrm{cm}^{-1}$;

HRMS $\left(\mathrm{El}^{+}\right) \mathrm{m} / \mathrm{z}$ calcd for $\mathrm{C}_{12} \mathrm{H}_{16} \mathrm{O}^{+}(\mathrm{M})^{+}:$176.1196, found 176.1196 . 

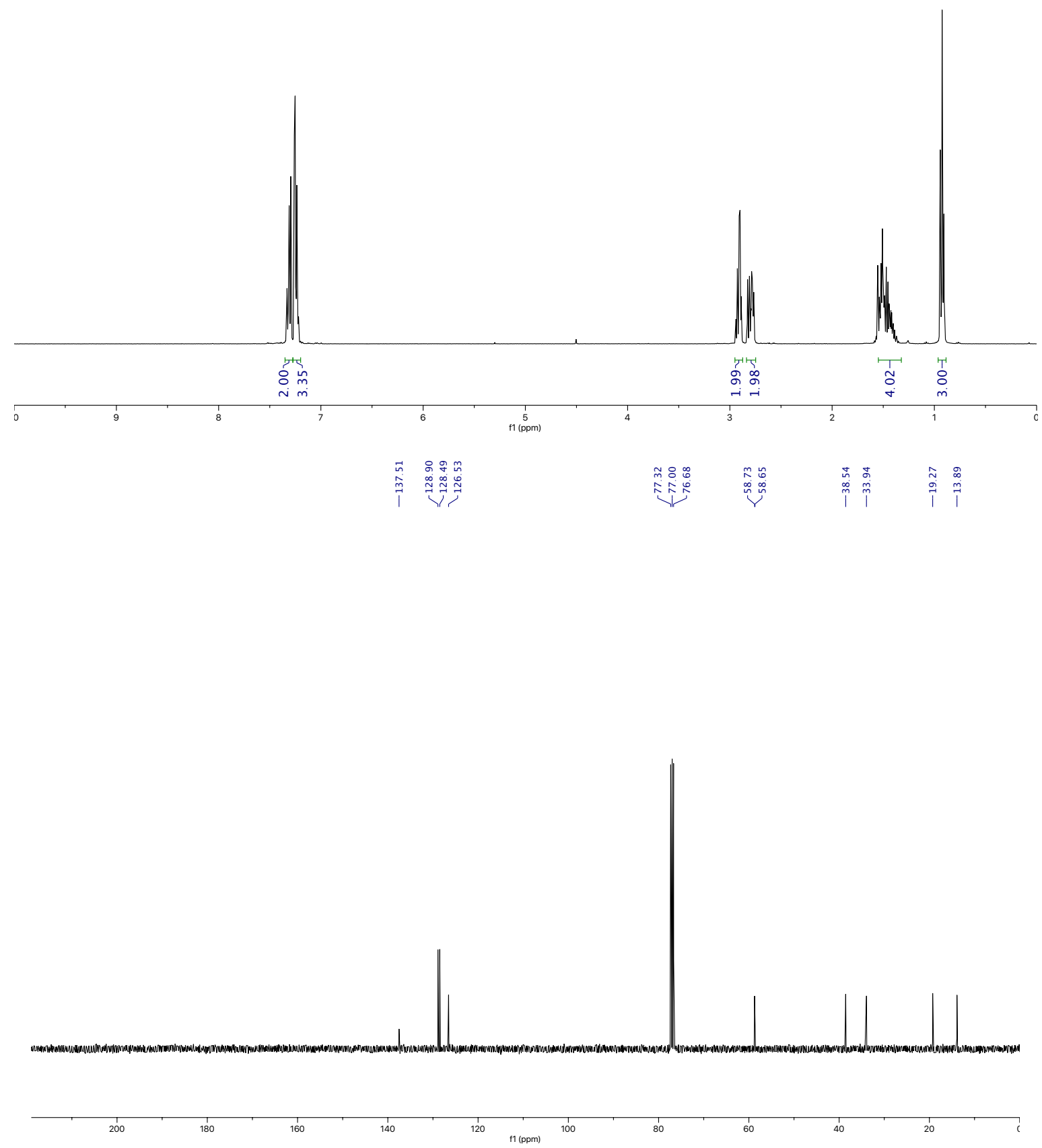


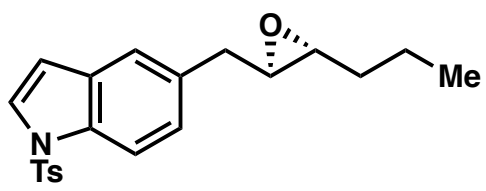

5-(((2R*,3R*)-3-Propyloxiran-2-yl)methyl)-1-tosyl-1H-indole ( \pm )-5g

General Procedure B: (E)-5-(Hex-2-en-1-yl)-1-tosyl-1H-indole (500 mg, $1.42 \mathrm{mmol}$ ) was employed.

Purification of the residue by FCC (5:1, hexane-EtOAc) afforded the title compound (312 mg, $60 \%)$ as a colorless oil.

${ }^{1} \mathrm{H}$ NMR $\left(400 \mathrm{MHz}, \mathrm{CDCl}_{3}\right) \delta 7.91(\mathrm{dt}, J=8.6,0.8 \mathrm{~Hz}, 1 \mathrm{H}), 7.75(\mathrm{~d}, J=8.4 \mathrm{~Hz}, 2 \mathrm{H}), 7.53(\mathrm{~d}, J=$ $3.6 \mathrm{~Hz}, 1 \mathrm{H}), 7.39(\mathrm{~d}, J=1.6 \mathrm{~Hz}, 1 \mathrm{H}), 7.22-7.17(\mathrm{~m}, 3 \mathrm{H}), 6.60(\mathrm{dd}, J=3.7,0.8 \mathrm{~Hz}, 1 \mathrm{H}), 2.89$ (s, $3 \mathrm{H}), 2.77(\mathrm{td}, J=5.6,1.4 \mathrm{~Hz}, 1 \mathrm{H}), 2.33(\mathrm{~s}, 3 \mathrm{H}), 1.53-1.35(\mathrm{~m}, 4 \mathrm{H}), 0.91(\mathrm{t}, J=7.2 \mathrm{~Hz}, 3 \mathrm{H})$;

${ }^{13} \mathrm{C}$ NMR $\left(101 \mathrm{MHz}, \mathrm{CDCl}_{3}\right) \delta$ 144.9, 135.3, 133.7, 132.6 ,131.1, 129.8, 126.8, 126.6, 125.6, $121.3,113.5,108.9,58.9,58.7,38.3,33.9,21.5,19.3,13.9$;

IR ( $\mathrm{NaCl}$, thin film): 2962, 2931, 2873, 1596, 1461, 1372, 1268, 1173, 1092, 995, 812, $724 \mathrm{~cm}^{-1}$; HRMS (ESI-TOF) $\mathrm{m} / \mathrm{z}$ calcd for $\mathrm{C}_{21} \mathrm{H}_{23} \mathrm{NO}_{3} \mathrm{SNa}^{+}(\mathrm{M}+\mathrm{Na})^{+}: 392.1296$ found 392.1304 . 

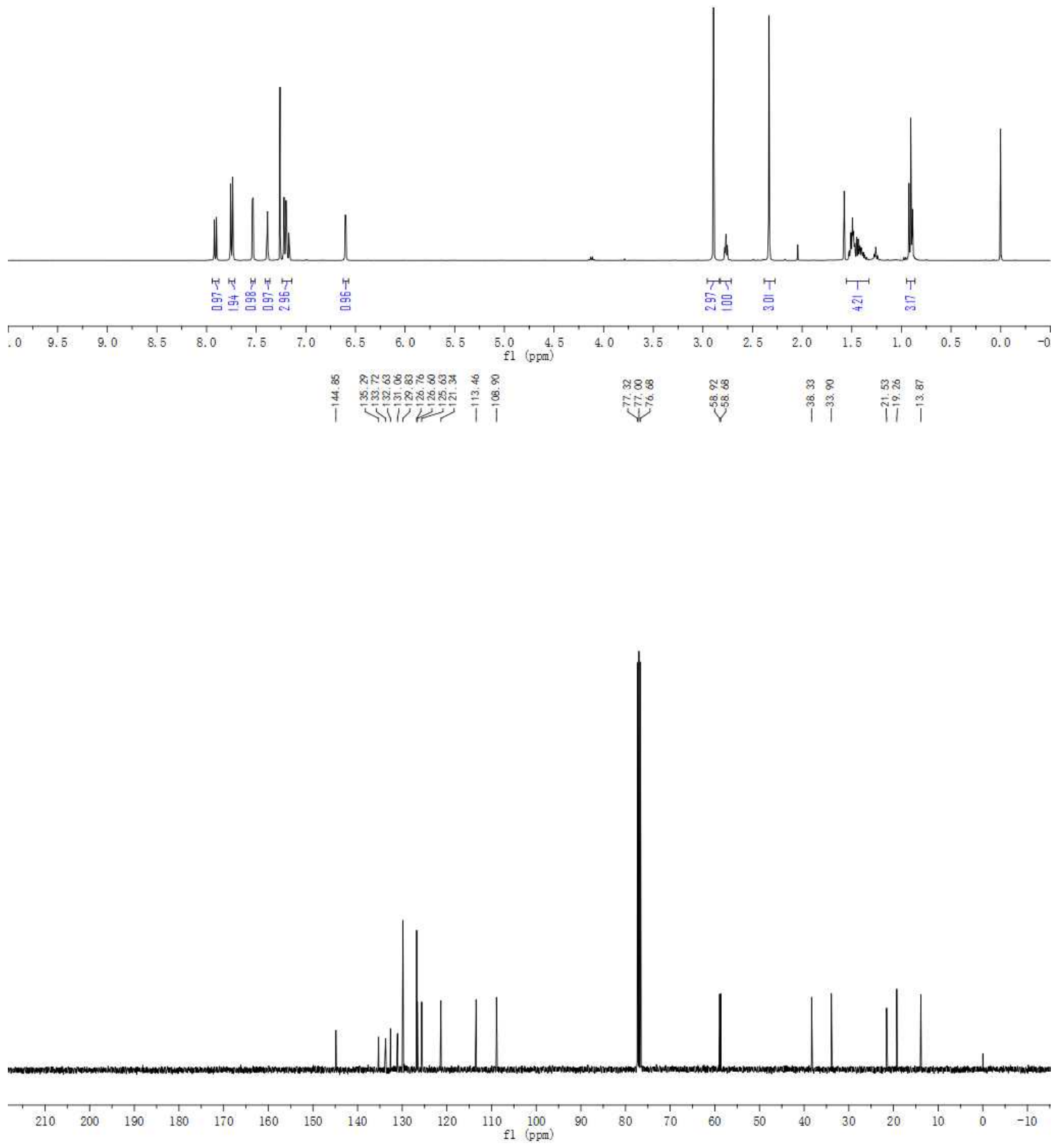
<smiles>CCCC1O[C@@H]1Cc1ccc(F)cc1</smiles>

$\left(2 R^{*}, 3 R^{*}\right)-2-(4-F l u o r o b e n z y l)-3-p r o p y l o x i r a n e ~( \pm)-5 h$

General Procedure B: (E)-1-Fluoro-4-(hex-2-en-1-yl)benzene (500 mg, $2.8 \mathrm{mmol}$ ) was employed. Purification of the residue by FCC (5:1, hexane-EtOAc) afforded the title compound (488 $\mathrm{mg}$, $90 \%$ ) as a colorless oil.

${ }^{1} \mathrm{H}$ NMR $\left(400 \mathrm{MHz}, \mathrm{CDCl}_{3}\right) \delta 7.20$ (dd, $\left.J=8.4,5.6 \mathrm{~Hz}, 2 \mathrm{H}\right), 6.99$ (app. $\left.\mathrm{t}, J=8.7 \mathrm{~Hz}, 2 \mathrm{H}\right), 2.89$ $2.85(\mathrm{~m}, 1 \mathrm{H}), 2.83-2.81(\mathrm{~m}, 2 \mathrm{H}), 2.76-2.73(\mathrm{~m}, 1 \mathrm{H}), 1.53-1.35(\mathrm{~m}, 4 \mathrm{H}), 0.92(\mathrm{t}, J=7.1 \mathrm{~Hz}, 3 \mathrm{H})$;

${ }^{13} \mathrm{C}$ NMR (101 MHz, $\left.\mathrm{CDCl}_{3}\right) \delta 161.8(\mathrm{~d}, \mathrm{~J}=244.3 \mathrm{~Hz}), 133.2(\mathrm{~d}, \mathrm{~J}=3.3 \mathrm{~Hz}), 130.3(\mathrm{~d}, \mathrm{~J}=8.0 \mathrm{~Hz})$, $115.3(\mathrm{~d}, J=21.2 \mathrm{~Hz}), 58.7$ (d, $J=1.3 \mathrm{~Hz}), 58.5,37.7,33.9,19.3,13.9$;

${ }^{19} \mathrm{~F}\left\{{ }^{1} \mathrm{H}\right\}$ NMR $\left(376 \mathrm{MHz}, \mathrm{CDCl}_{3}\right) \delta-116.7(\mathrm{~s})$;

IR ( $\mathrm{NaCl}$, thin film) 2963, 2874, 1606, 1511, 1465, 1223, 1158, 1099, 1017, 927, 825, $754 \mathrm{~cm}^{-1}$;

HRMS $\left(\mathrm{El}^{+}\right) \mathrm{m} / \mathrm{z}$ calcd for $\mathrm{C}_{12} \mathrm{H}_{15} \mathrm{FO}^{+}(\mathrm{M})^{+}:$194.1101, found 194.1101. 


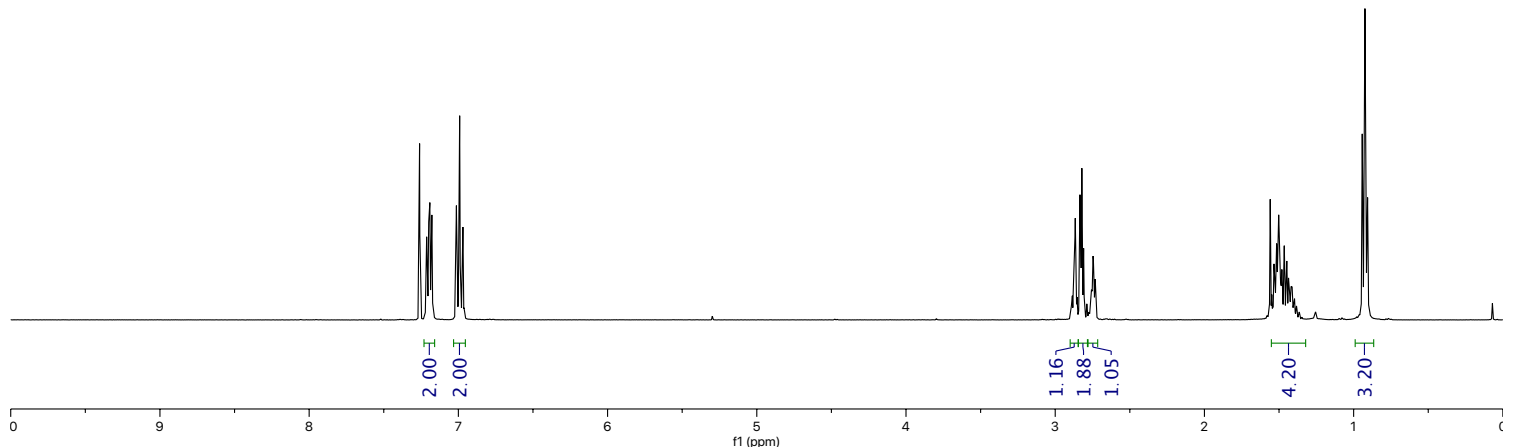

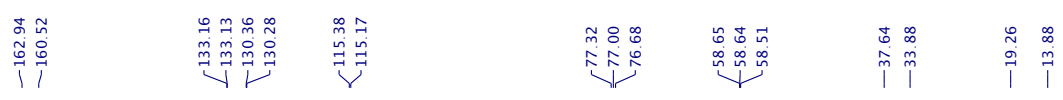

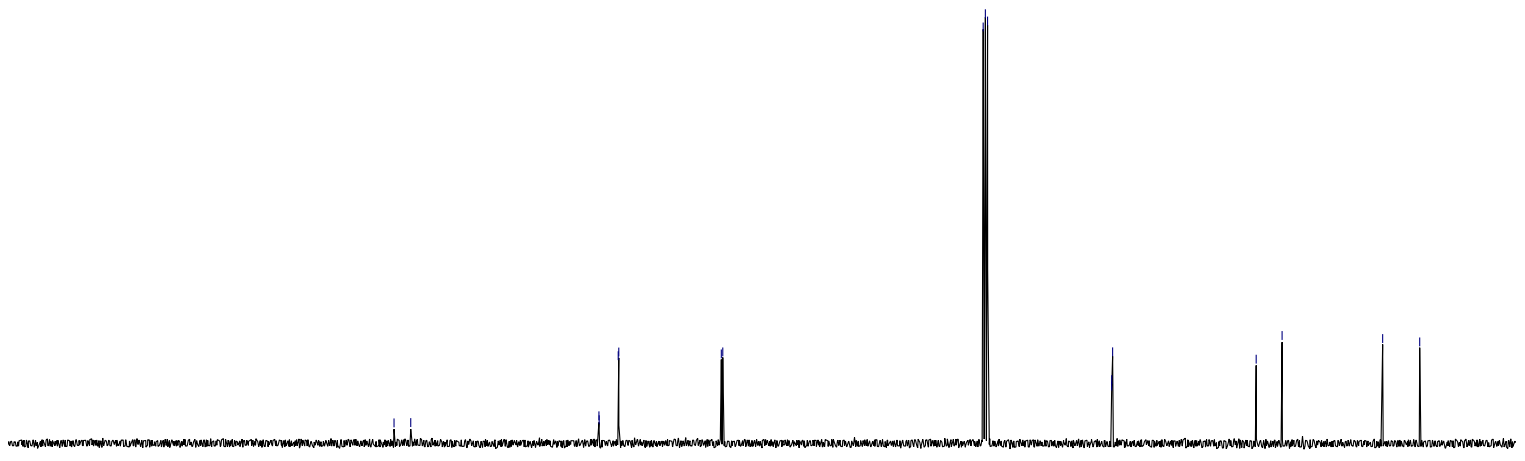

$200-180$

180

160

140

${ }_{120}^{120}+11(\mathrm{pom})$

${ }^{1} 0$

60

40

20 
<smiles>CCCC1OC1Cc1ccc(Cl)cc1</smiles>

\section{$\left(2 R^{*}, 3 R^{*}\right)-2-(4-C h l o r o b e n z y l)-3-p r o p y l o x i r a n e( \pm)-5 i$}

General Procedure B: (E)-1-Chloro-4-(hex-2-en-1-yl)benzene (500 mg, $2.6 \mathrm{mmol})$ was employed. Purification of the residue by FCC (5:1, hexane-EtOAc) afforded the title compound (493 mg, $91 \%)$ as a pale yellow oil.

${ }^{1} \mathrm{H}$ NMR $\left(400 \mathrm{MHz}, \mathrm{CDCl}_{3}\right) \delta 7.28(\mathrm{~d}, J=8.4 \mathrm{~Hz}, 2 \mathrm{H}), 7.17(\mathrm{~d}, J=8.4 \mathrm{~Hz}, 2 \mathrm{H}), 2.89-2.86(\mathrm{~m}, 1 \mathrm{H})$, 2.83-2.81 (m, 2H), 2.76-2.72 (m, 1H), 1.54-1.35 (m, 4H), 0.93 (t, J = 7.2 Hz, 3H);

${ }^{13} \mathrm{C}$ NMR $\left(126 \mathrm{MHz}, \mathrm{CDCl}_{3}\right) \delta 136.0,132.4,130.3,128.6,58.5,58.4,37.8,33.9,19.3,13.9$;

IR $\left(\mathrm{NaCl}\right.$, think film) 2961, 2932, 2873, 1492, 1465, 1093, 1016, 932, $803 \mathrm{~cm}^{-1}$;

HRMS $\left(\mathrm{El}^{+}\right) \mathrm{m} / \mathrm{z}$ calcd for $\mathrm{C}_{12} \mathrm{H}_{15}{ }^{35} \mathrm{ClO}^{+}(\mathrm{M})^{+}: 210.0806$ found 210.0809 . 


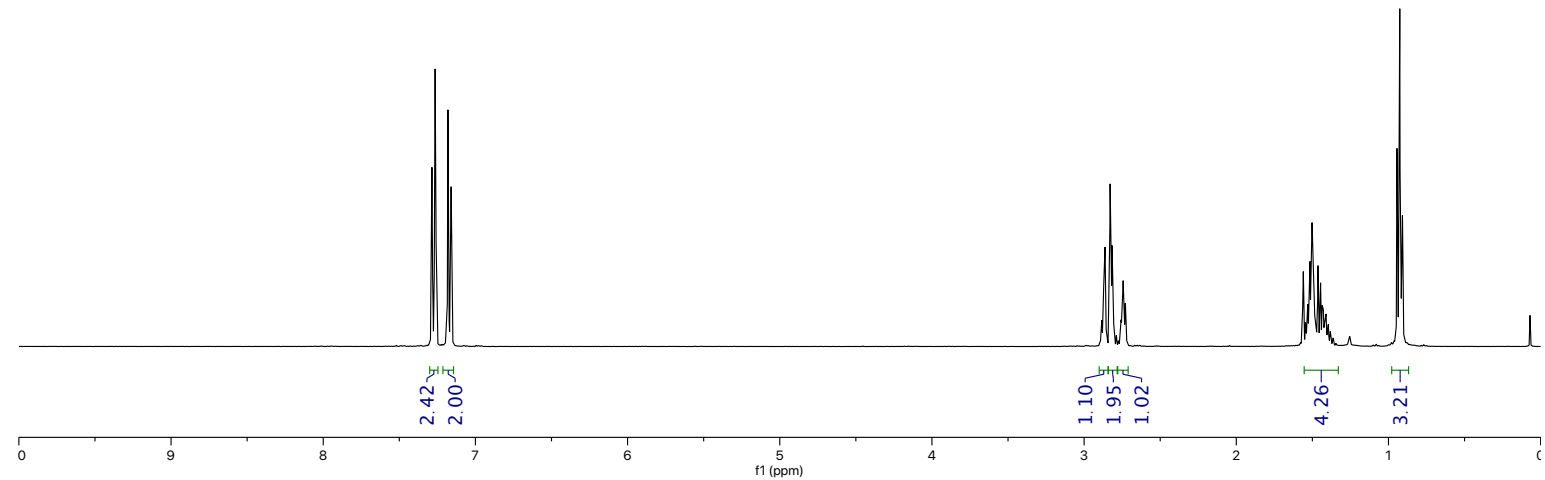

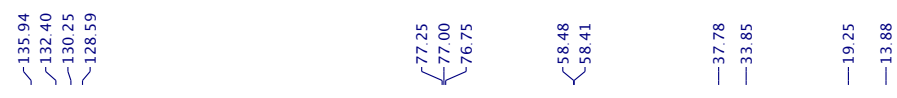

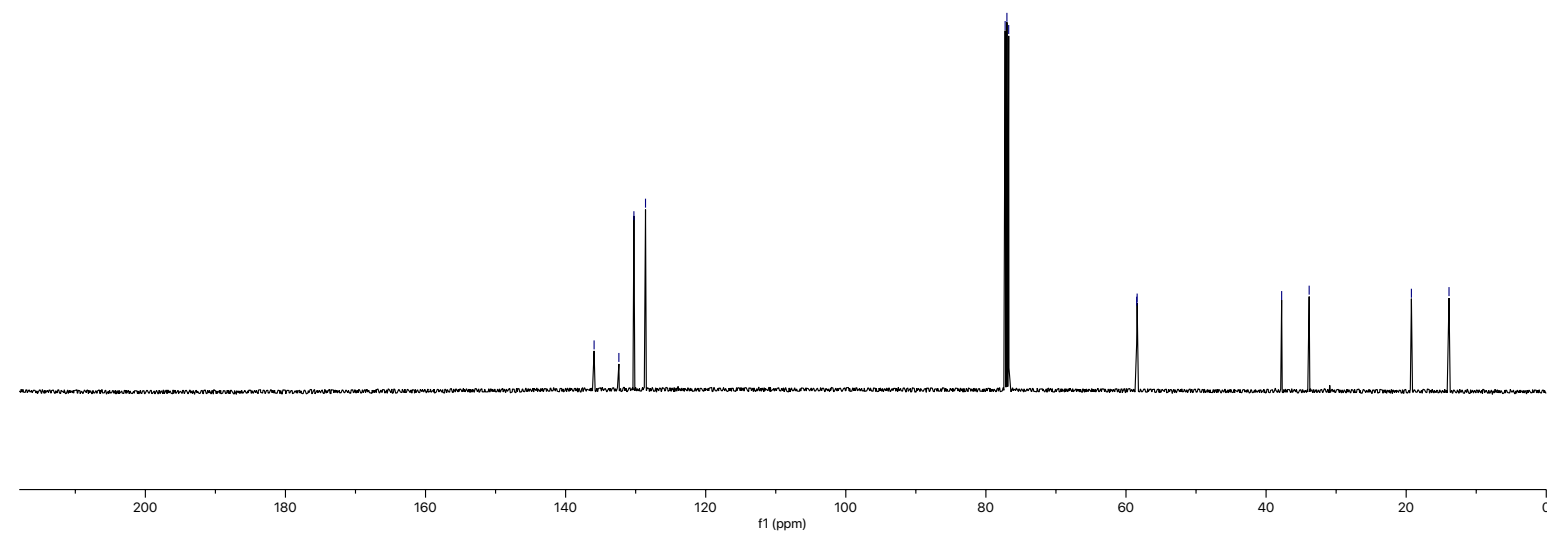


<smiles>CCCC1O[C@@H]1Cc1ccc(Br)cc1</smiles>

$\left(2 R^{*}, 3 R^{*}\right)-2-(4-B r o m o b e n z y l)-3-p r o p y l o x i r a n e ~( \pm)-5 j$

General Procedure B: (E)-1-Bromo-4-(hex-2-en-1-yl)benzene (300 mg, $1.68 \mathrm{mmol}$ ) was employed. Purification of the residue by FCC (20:1, hexane-EtOAc) afforded the title compound (207 $\mathrm{mg}, 80 \%)$ as a pale yellow oil.

${ }^{1} \mathrm{H}$ NMR (400 MHz, $\left.\mathrm{CDCl}_{3}\right) \delta 7.43(\mathrm{~d}, \mathrm{~J}=8.4,2 \mathrm{H}), 7.12$ (d, $\left.J=8.3,2 \mathrm{H}\right), 2.87$ (ddd, $J=5.4,5.4$, $2.1 \mathrm{~Hz}, 1 \mathrm{H}), 2.81-2.79(\mathrm{~m}, 2 \mathrm{H}), 2.74(\mathrm{ddd}, J=5.8,5.5,2.2 \mathrm{~Hz}, 1 \mathrm{H}), 1.53-1.37(\mathrm{~m}, 4 \mathrm{H}), 0.93(\mathrm{t}, J$ $=7.2 \mathrm{~Hz}, 3 \mathrm{H})$;

${ }^{13} \mathrm{C}$ NMR $\left(126 \mathrm{MHz}, \mathrm{CDCl}_{3}\right) \delta 136.5,131.6,130.7,120.5,58.5,58.4,37.9,33.9,19.3,13.9$;

IR ( $\mathrm{NaCl}$, thin film) 2960, 2931, 2873, 1488, 1463, 1072, 1012, 932, $798.08 \mathrm{~cm}^{-1}$;

HRMS $\left(\mathrm{El}^{+}\right) \mathrm{m} / \mathrm{z}$ calcd for $\mathrm{C}_{12} \mathrm{H}_{15}{ }^{79} \mathrm{BrO}^{+}(\mathrm{M})^{+}:$: 254.0301, found 254.0296. 


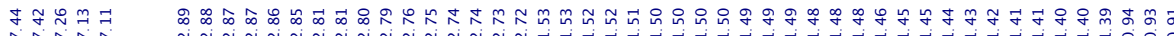

Níti
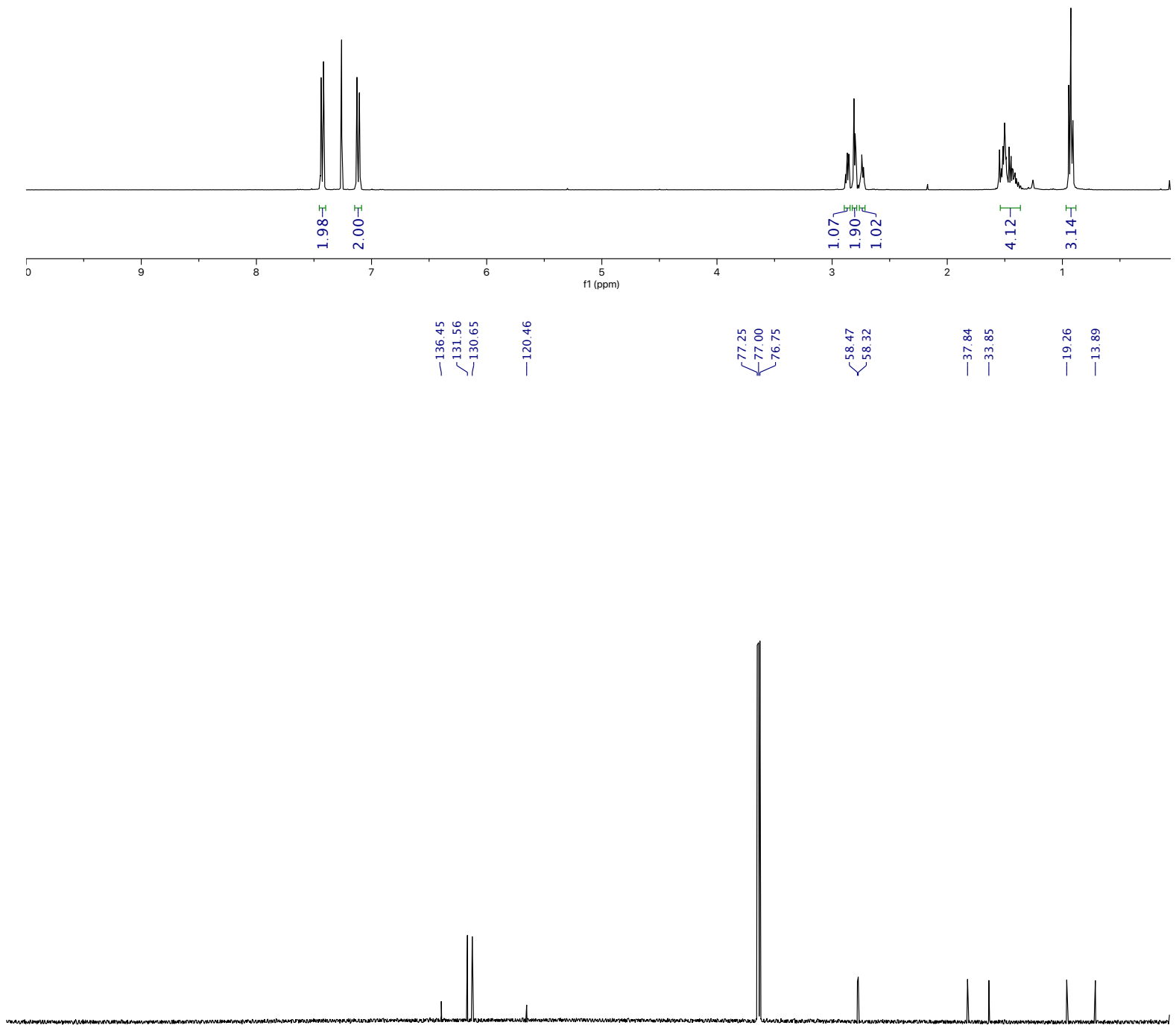

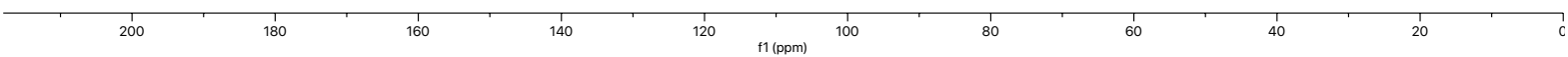


<smiles>CCCC1OC1Cc1ccc(C(F)(F)F)cc1</smiles>

$\left(2 R^{*}, 3 R^{*}\right)$-2-Propyl-3-(4-(trifluoromethyl)benzyl)oxirane $( \pm)-5 \mathrm{k}$

General Procedure B: (E)-1-(Hex-2-en-1-yl)-4-(trifluoromethyl)benzene (500 mg, $2.2 \mathrm{mmol}$ ) was employed. Purification of the residue by FCC (5:1, hexane-EtOAc) afforded the title compound (471 $\mathrm{mg}, 88 \%)$ as a pale yellow oil.

${ }^{1} \mathrm{H}$ NMR $\left(400 \mathrm{MHz}, \mathrm{CDCl}_{3}\right) \delta 7.57(\mathrm{~d}, J=8.0,2 \mathrm{H}), 7.36(\mathrm{~d}, J=8.0,2 \mathrm{H}), 3.00-2.88(\mathrm{~m}, 3 \mathrm{H}), 2.76$ $(\mathrm{t}, J=5.5,1 \mathrm{H}), 1.55-1.36(\mathrm{~m}, 4 \mathrm{H}), 0.93(\mathrm{t}, J=7.2,3 \mathrm{H})$;

${ }^{13}$ C NMR (125 MHz, $\left.\mathrm{CDCl}_{3}\right) \delta$ 141.7, 129.3, 129.0 (q, J = 32.4 Hz), 125.4 (q, J = $\left.3.9 \mathrm{~Hz}\right), 124.2$ (q, $J=270.5 \mathrm{~Hz}$ ), 58.5, 58.2, 38.3, 33.8, 19.3, 13.9;

${ }^{19} \mathrm{~F}\left\{{ }^{1} \mathrm{H}\right\}$ NMR $\left(376 \mathrm{MHz}, \mathrm{CDCl}_{3}\right) \delta-62.4(\mathrm{~s})$;

IR ( $\mathrm{NaCl}$, thin film) 2964, 2935, 2876, 1620, 1419, 1327, 1165, 1124, 1068, 1020, $821 \mathrm{~cm}^{-1}$;

HRMS $\left(\mathrm{Cl}^{+}\right) \mathrm{m} / \mathrm{z}$ calcd for $\mathrm{C}_{13} \mathrm{H}_{15} \mathrm{~F}_{3} \mathrm{ONH}_{4}{ }^{+}\left(\mathrm{M}+\mathrm{NH}_{4}\right)^{+}:$262.1413, found 262.1410. 

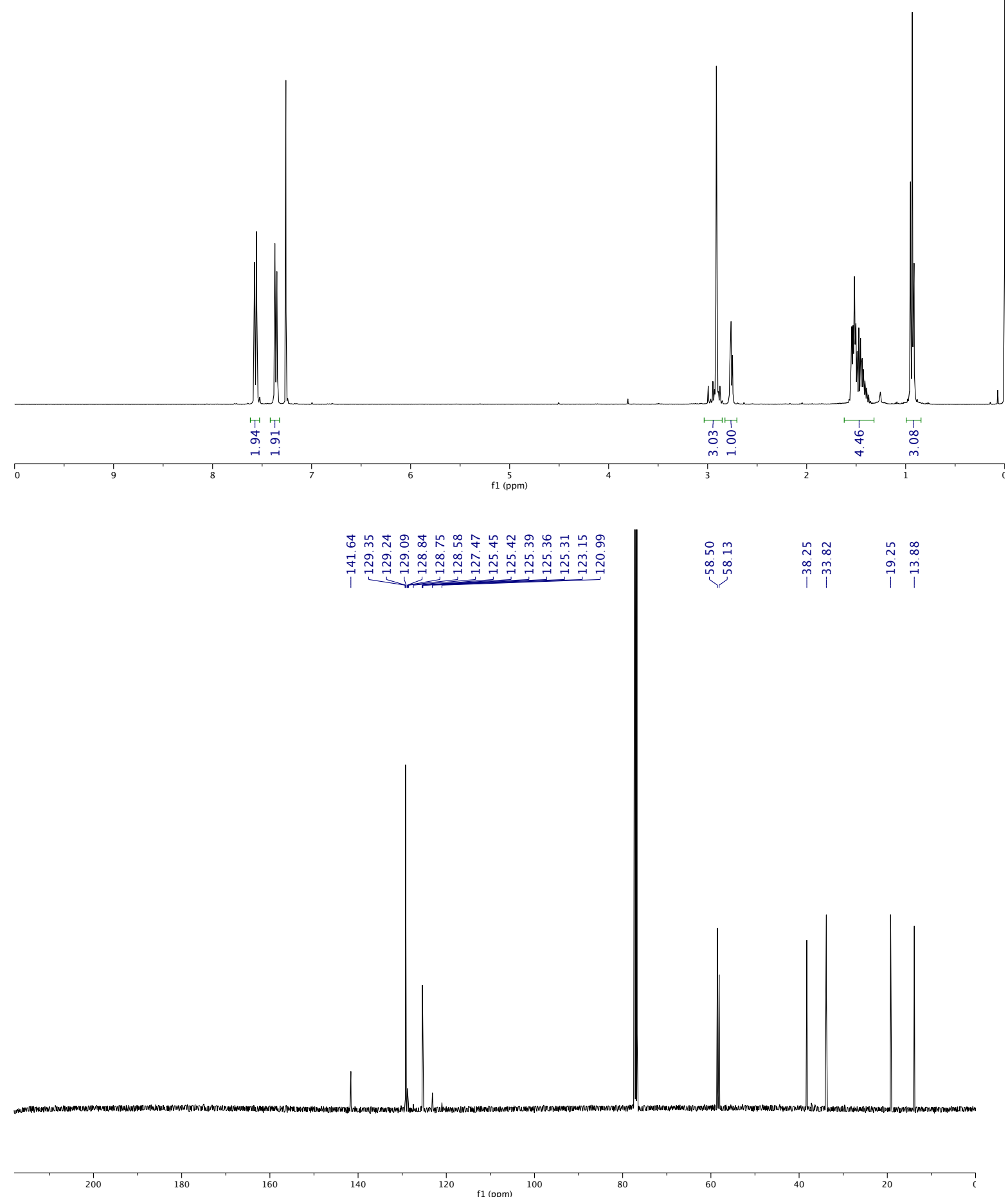


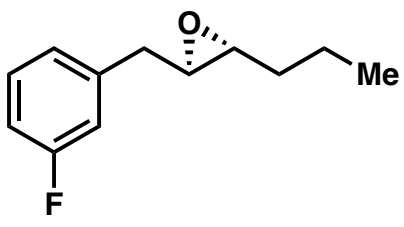

\section{$\left(2 R^{*}, 3 R^{\star}\right)-2-(3-$ Fluorobenzyl)-3-propyloxirane $( \pm)-5 I$}

General Procedure B: (E)-1-Fluoro-3-(hex-2-en-1-yl)benzene (730 mg, $4.10 \mathrm{mmol}$ ) was employed. Purification of the residue by FCC (20:1, hexane-EtOAc) afforded the title compound (620 mg, 78\%) as a colorless oil.

${ }^{1} \mathrm{H}$ NMR $\left(400 \mathrm{MHz}, \mathrm{CDCl}_{3}\right) \delta$ 7.29-7.24 (m, 1H), 7.01 (d, J = 7.6 Hz, 1H), 6.98-6.91 (m, 2H), 2.92$2.81(\mathrm{~m}, 3 \mathrm{H}), 2.80-2.75(\mathrm{~m}, 1 \mathrm{H}), 1.56-1.38(\mathrm{~m}, 4 \mathrm{H}), 0.93(\mathrm{t}, J=7.4 \mathrm{~Hz}, 3 \mathrm{H})$;

${ }^{13} \mathrm{C}$ NMR (101 MHz, $\left.\mathrm{CDCl}_{3}\right) \delta 162.8$ (d, $\left.J=245.8 \mathrm{~Hz}\right), 140.0$ (d, J = 7.3 Hz), 129.9 (d, J = 8.3 Hz), $124.5(\mathrm{~d}, J=2.9 \mathrm{~Hz}), 115.8(\mathrm{~d}, J=21.1 \mathrm{~Hz}), 113.4(\mathrm{~d}, J=21.0 \mathrm{~Hz}), 58.5,58.3,38.2(\mathrm{~d}, J=1.8$ $\mathrm{Hz}), 33.8,19.2,13.9$;

${ }^{19} \mathrm{~F}\left\{{ }^{1} \mathrm{H}\right\}$ NMR $\left(376 \mathrm{MHz}, \mathrm{CDCl}_{3}\right) \delta-113.4(\mathrm{~s})$;

IR ( $\mathrm{NaCl}$, thin film) 2961, 2874, 2359, 2341, 1616, 1590, 1488, 1449, 1248, 1141, 948, 893, 868, $780 \mathrm{~cm}^{-1}$;

HRMS $\left(\mathrm{EI}^{+}\right) \mathrm{m} / \mathrm{z}$ calcd for $\mathrm{C}_{12} \mathrm{H}_{15} \mathrm{FO}^{+}(\mathrm{M})^{+}:$194.1107, found 194.1103. 

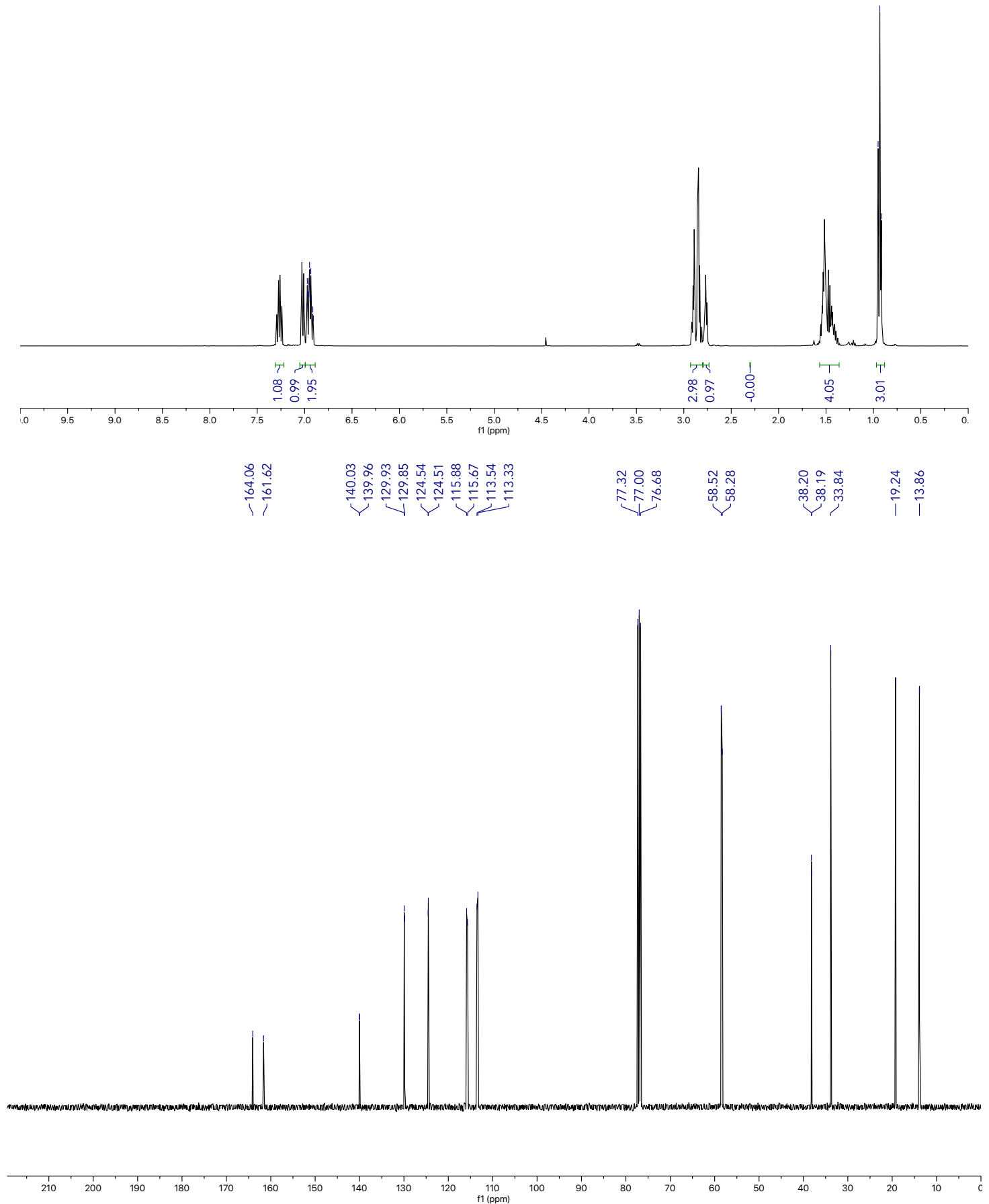
<smiles>CCC[C@@H]1O[C@@H]1Cc1cccc(Cl)c1</smiles>

\section{$\left(2 R^{*}, 3 R^{*}\right)-2-(3-C h l o r o b e n z y l)-3-p r o p y l o x i r a n e( \pm)-5 m$}

General Procedure B: (E)-1-Chloro-3-(hex-2-en-1-yl)benzene (500 mg, $2.57 \mathrm{mmol}$ ) was employed. Purification of the residue by FCC (20:1, hexane-EtOAc) afforded the title compound (413 $\mathrm{mg}, 80 \%)$ as a colorless oil.

${ }^{1} \mathrm{H}$ NMR $\left(400 \mathrm{MHz}, \mathrm{CDCl}_{3}\right) \delta$ 7.25-7.20 (m, 3H), 7.14-7.11 (m, 1H), 2.89 (ddd, J = 5.5, 5.4, 2.1 $\mathrm{Hz}, 1 \mathrm{H}), 2.86-2.79(\mathrm{~m}, 2 \mathrm{H}), 2.76(\mathrm{ddd}, J=5.7,5.5,2.2 \mathrm{~Hz}, 1 \mathrm{H}), 1.56-1.37(\mathrm{~m}, 4 \mathrm{H}), 0.93(\mathrm{t}, J=7.2$ $\mathrm{Hz}, 3 \mathrm{H})$;

${ }^{13} \mathrm{C}$ NMR $\left(101 \mathrm{MHz}, \mathrm{CDCl}_{3}\right) \delta 139.5,134.3,129.7,129.0,127.1,126.8,58.5,58.3,38.1,33.8$, 19.2, 13.9;

IR (NaCl, thin film): 2962, 2932, 2873, 1645, 1598, 1475, 1430, 1080, 937, 867, $781 \mathrm{~cm}^{-1}$;

HRMS $\left(\mathrm{Cl}^{+}\right) \mathrm{m} / \mathrm{z}$ calcd for $\mathrm{C}_{12} \mathrm{H}_{15}{ }^{35} \mathrm{CINH}_{4}{ }^{+}\left(\mathrm{M}+\mathrm{NH}_{4}\right)^{+}:$228.1150, found 228.1149. 

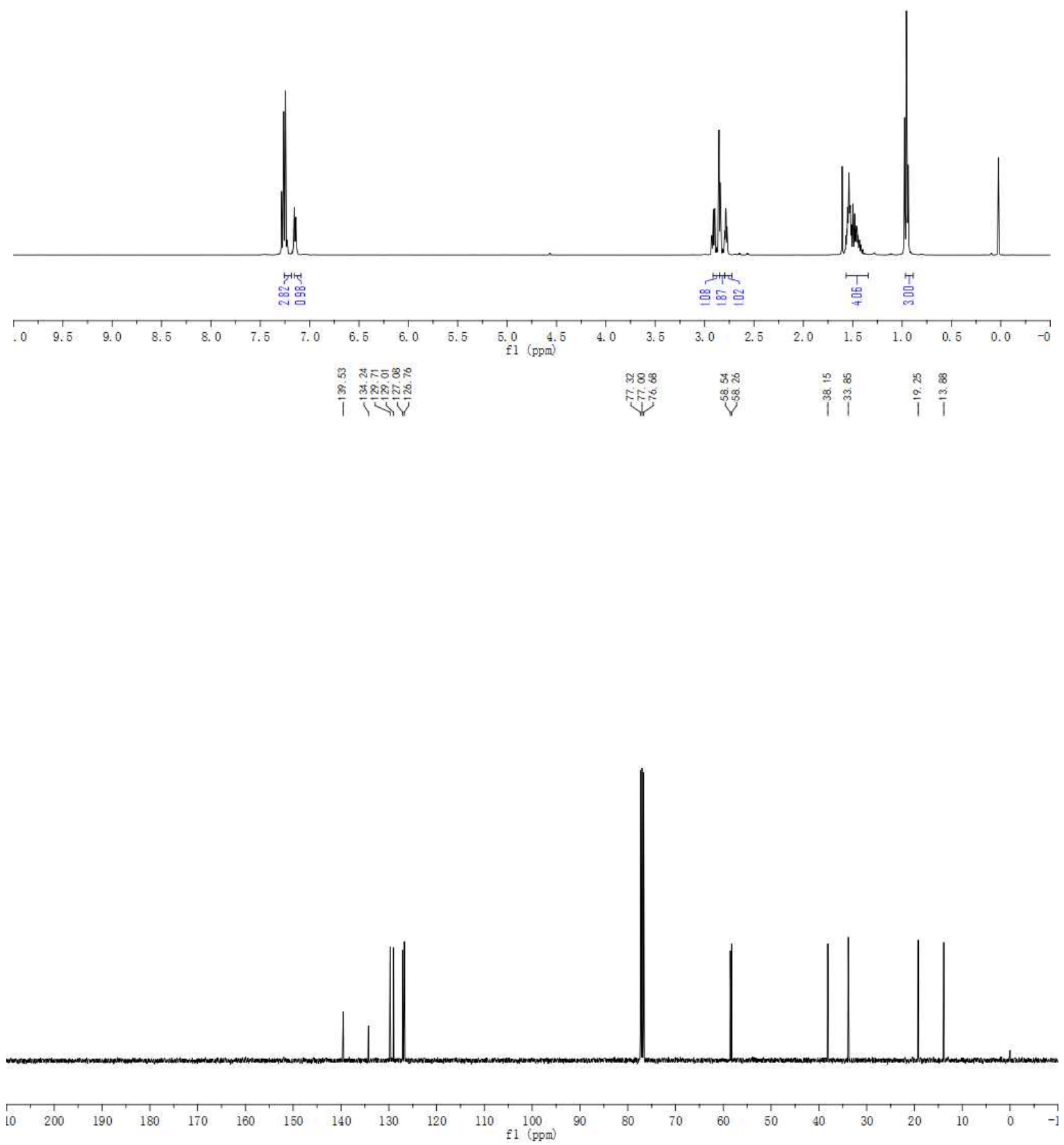
<smiles>CCCC1OC1Cc1cccc(OC)c1</smiles>

$\left(2 R^{*}, 3 R^{*}\right)-2-(3-M e t h o x y b e n z y l)-3-p r o p y l o x i r a n e ~( \pm)-5 n$

General Procedure B: (E)-1-(Hex-2-en-1-yl)-3-methoxybenzene (453 mg, $2.4 \mathrm{mmol}$ ) was employed. Purification of the residue by FCC (5:1, hexane-EtOAc) afforded the title compound (399 $\mathrm{mg}, 81 \%)$ as a pale yellow oil.

${ }^{1} \mathrm{H}$ NMR $\left(400 \mathrm{MHz} \mathrm{CDCl}_{3}\right) \delta$ 7.26-7.21 (m, 1H), 6.84-6.77 (m, 3H), $3.81(\mathrm{~s}, 3 \mathrm{H})$, 2.92-2.68 (m, $2 \mathrm{H}), 2.80-2.74(\mathrm{~m}, 2 \mathrm{H}), 1.54-1.37(\mathrm{~m}, 4 \mathrm{H}), 0.93$, (t, $J=7.3 \mathrm{~Hz}, 3 \mathrm{H})$;

${ }^{13} \mathrm{C}$ NMR $\left(126 \mathrm{MHz}_{\mathrm{CDCl}}\right) \delta 159.7,139.1,129.5,121.3,114.6,112.0,58.7,58.7,55.2,38.6$, 34.0, 19.3, 13.9;

IR ( $\mathrm{NaCl}$, thin film) 3028, 2959, 2928, 2872, 1604, 1494, 1454, 1072, 1030, $968 \mathrm{~cm}^{-1}$;

HRMS (ESI-TOF) $\mathrm{m} / \mathrm{z}$ calcd for $\mathrm{C}_{13} \mathrm{H}_{18} \mathrm{O}_{2} \mathrm{Na}^{+}(\mathrm{M}+\mathrm{Na})^{+}:$229.1204, found 229.1202. 

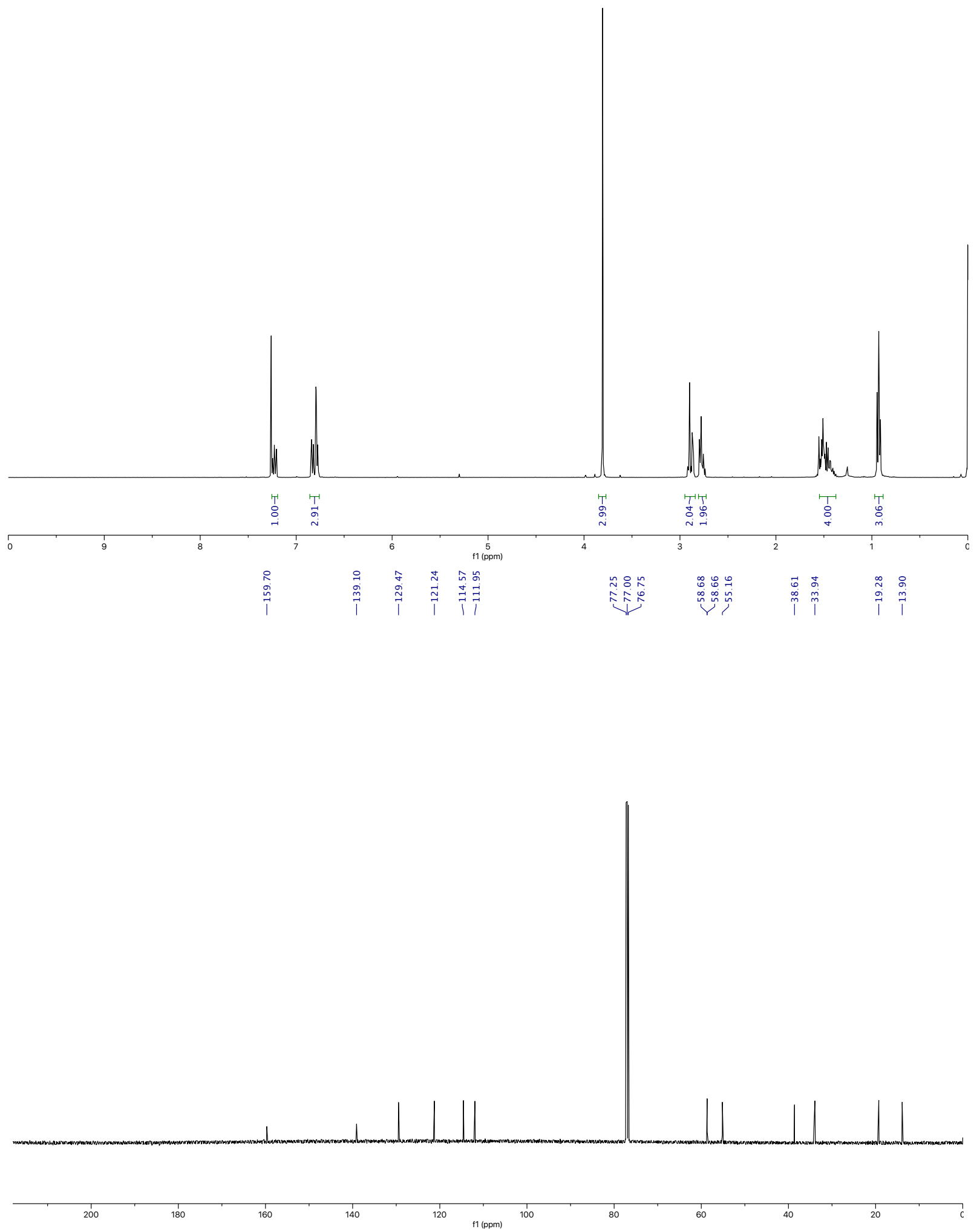


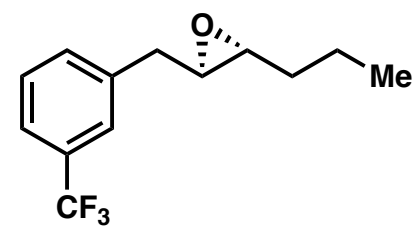

$\left(2 R^{*}, 3 R^{*}\right)-2-$ Propyl-3-(3-(trifluoromethyl)benzyl)oxirane $( \pm)$-5o

General Procedure B: (E)-1-(Hex-2-en-1-yl)-3-(trifluoromethyl)benzene (840 mg, $3.68 \mathrm{mmol}$ ) was employed. Purification of the residue by FCC (20:1, hexane-EtOAc) afforded the title compound (724 mg, 81\%) as a colorless oil.

${ }^{1} \mathrm{H}$ NMR $\left(400 \mathrm{MHz}, \mathrm{CDCl}_{3}\right) \delta 7.50(\mathrm{~m}, 2 \mathrm{H}), 7.45-7.40(\mathrm{~m}, 2 \mathrm{H})$, 2.98-2.85 (m, 3H), 2.79-2.76 (m, $1 \mathrm{H}), 1.61-1.37(\mathrm{~m}, 4 \mathrm{H}), 0.93(\mathrm{t}, J=7.2 \mathrm{~Hz}, 3 \mathrm{H})$;

${ }^{13} \mathrm{C}$ NMR (101 MHz, $\left.\mathrm{CDCl}_{3}\right) \delta 138.5,132.3$ (q, $\left.J=1.2 \mathrm{~Hz}\right), 130.8(\mathrm{q}, J=32.1 \mathrm{~Hz}), 128.9,125.6$ (q, $J=3.8 \mathrm{~Hz}), 124.1$ (q, $J=272.3 \mathrm{~Hz}), 123.5$ (q, $J=3.9 \mathrm{~Hz}), 58.5,58.2$, 38.3, 33.8, 19.2, 13.9;

${ }^{19} \mathrm{~F} \mathrm{NMR}\left(376 \mathrm{MHz}, \mathrm{CDCl}_{3}\right) \delta-62.6(\mathrm{~s})$;

IR ( $\mathrm{NaCl}$, thin film) 2965, 2935, 2876, 1493, 1450, 1333, 1198, 1128, 1075, 909, 800, $703 \mathrm{~cm}^{-1}$;

HRMS $\left(\mathrm{El}^{+}\right) \mathrm{m} / \mathrm{z}$ calcd for $\mathrm{C}_{13} \mathrm{H}_{15} \mathrm{~F}_{3} \mathrm{O}^{+}(\mathrm{M})^{+}:$244.1075, found 244.1072. 

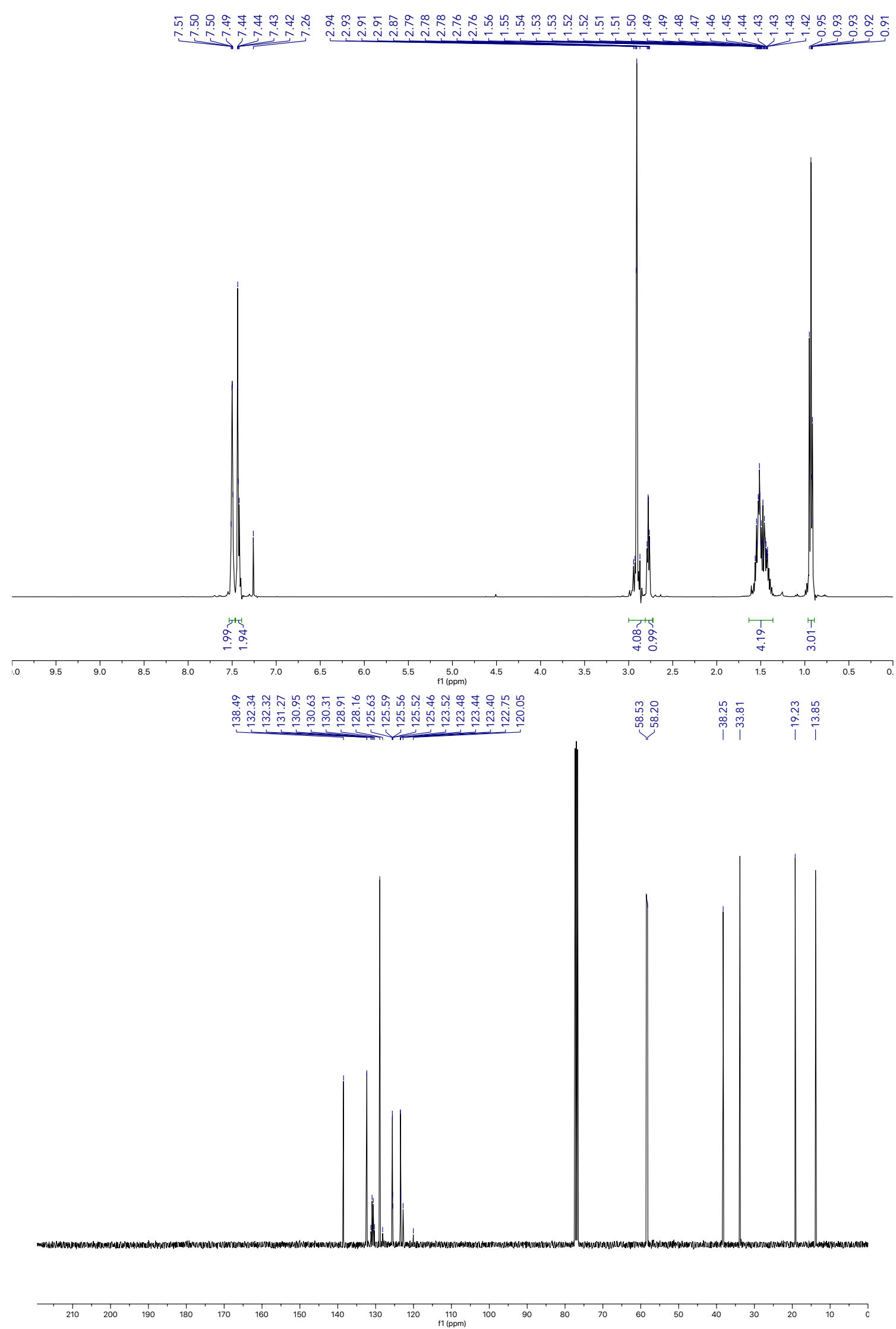


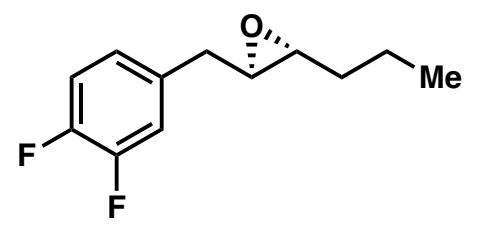

\section{$\left(2 R^{*}, 3 R^{*}\right)-2-(3,4-D i f l u o r o b e n z y l)-3-p r o p y l o x i r a n e ~( \pm)-5 p$}

General Procedure B: (E)-1,2-Difluoro-4-(hex-2-en-1-yl)benzene $(690 \mathrm{mg}, 3.52 \mathrm{mmol}$ ) was employed. Purification of the residue by FCC (20:1, hexane-EtOAc) afforded the title compound (560 $\mathrm{mg}, 75 \%)$ as a pale yellow oil.

${ }^{1} \mathrm{H}$ NMR $\left(400 \mathrm{MHz}, \mathrm{CDCl}_{3}\right)$ ठ 7.11-7.03 (m, 2H), 6.96-6.92 (m, 1H), 2.88-2.72 (m, 4H), 1.55-1.38 (m, 4H), $0.93(\mathrm{t}, J=7.2 \mathrm{~Hz}, 3 \mathrm{H})$;

${ }^{13} \mathrm{C}$ NMR $\left(101 \mathrm{MHz}, \mathrm{CDCl}_{3}\right) \delta 150.2(\mathrm{dd}, J=248.1,12.8 \mathrm{~Hz}), 149.3(\mathrm{dd}, J=246.7,12.7 \mathrm{~Hz}), 134.5$ (dd, $J=5.6,4.0 \mathrm{~Hz}$ ), 124.8 (dd, $J=6.2,3.6 \mathrm{~Hz}), 117.7(\mathrm{~d}, J=17.1 \mathrm{~Hz}), 117.1(\mathrm{~d}, J=17.1 \mathrm{~Hz}$ ), 58.4, $58.2(\mathrm{~d}, J=1.4 \mathrm{~Hz}), 37.6(\mathrm{~d}, J=1.3 \mathrm{~Hz}), 33.8,19.2$, 13.9;

${ }^{19} \mathrm{~F}\left\{{ }^{1} \mathrm{H}\right\}$ NMR $\left(376 \mathrm{MHz}, \mathrm{CDCl}_{3}\right) \delta-138.0(\mathrm{~d}, J=21.5 \mathrm{~Hz}, 1 \mathrm{~F}),-141.2(\mathrm{~d}, J=21.2 \mathrm{~Hz}, 1 \mathrm{~F})$;

IR ( $\mathrm{NaCl}$, thin film) 2964, 2934, 2875, 1609, 1519, 1464, 1434, 1283, 1211, 1118, 954, 815, 779 $\mathrm{cm}^{-1}$;

HRMS $\left(\mathrm{EI}^{+}\right) \mathrm{m} / \mathrm{z}$ calcd for $\mathrm{C}_{12} \mathrm{H}_{14} \mathrm{~F}_{2} \mathrm{O}^{+}(\mathrm{M})^{+}:$212.1013, found 212.1006. 

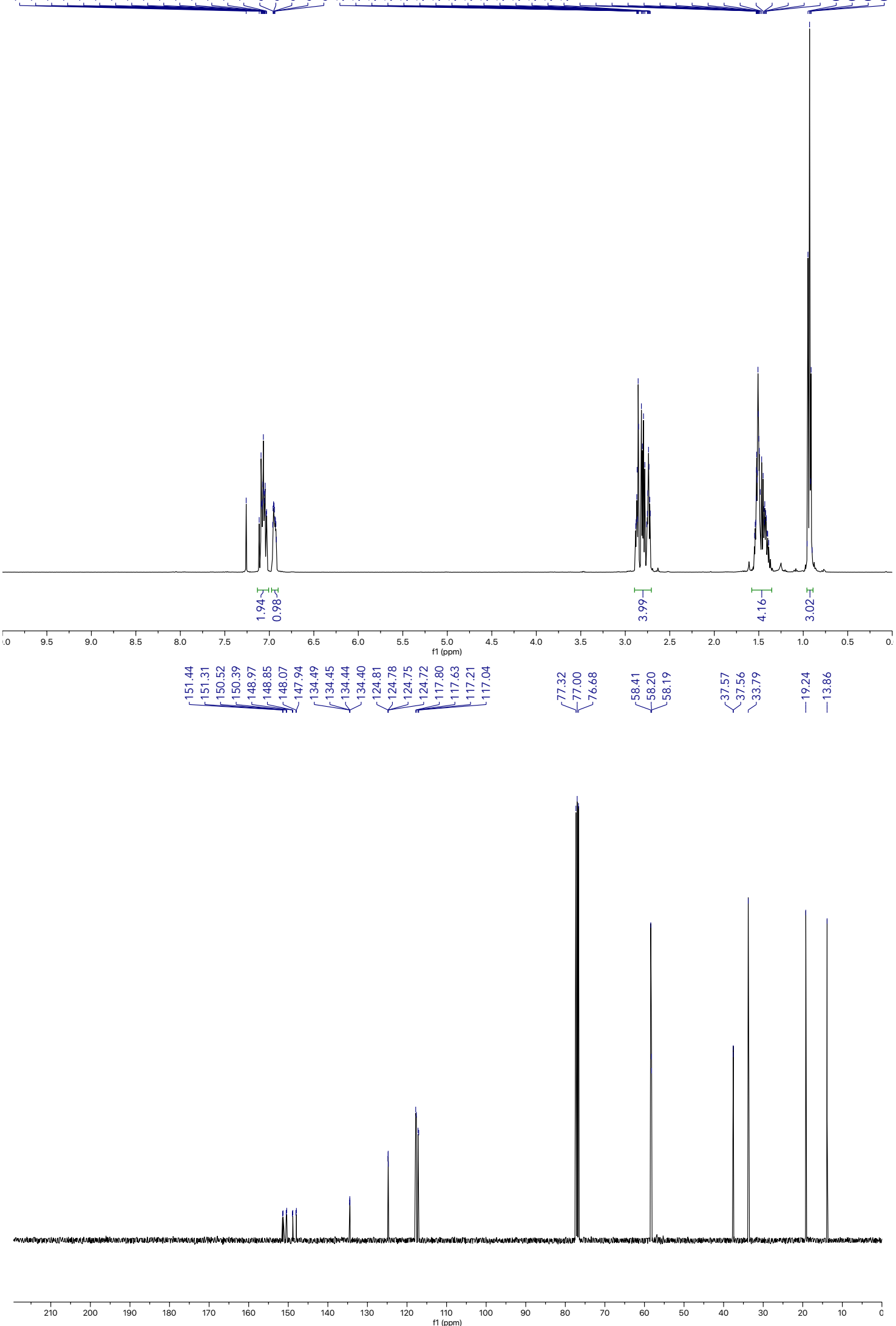


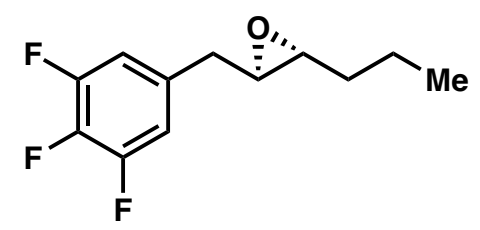

$\left(2 R^{\star}, 3 R^{\star}\right)$-2-Propyl-3-(3,4,5-trifluorobenzyl)oxirane ( \pm )-5ac

General Procedure B: (E)-1,2,3-Trifluoro-5-(hex-2-en-1-yl)benzene (500 mg, $2.34 \mathrm{mmol}$ ) was employed. Purification of the residue by FCC (20:1, hexane-EtOAc) afforded the title compound $(415 \mathrm{mg}, 77 \%)$ as a colorless oil.

${ }^{1} \mathrm{H}$ NMR $\left(400 \mathrm{MHz}, \mathrm{CDCl}_{3}\right)$ ठ 6.90-6.83 (m, 2H), 2.87-2.82 (m, 2H), 2.74-2.68 (m, 2H), 1.55-1.38 $(\mathrm{m}, 4 \mathrm{H}), 0.94(\mathrm{t}, J=7.2 \mathrm{~Hz}, 3 \mathrm{H})$;

${ }^{13} \mathrm{C}$ NMR (101 MHz, $\left.\mathrm{CDCl}_{3}\right) \delta 151.1$ (ddd, $\left.J=249.7,9.9,4.1 \mathrm{~Hz}\right), 138.6$ (dt, $J=249.8,15.2 \mathrm{~Hz}$ ), 133.8 (td, $J=7.4,4.7 \mathrm{~Hz}$ ), 112.9 (ddd, $J=15.4,5.7,1.1 \mathrm{~Hz}), 58.4,57.8,37.7,33.7,19.2,13.9$;

${ }^{19} \mathrm{~F}\left\{{ }^{1} \mathrm{H}\right\}$ NMR $\left(376 \mathrm{MHz}, \mathrm{CDCl}_{3}\right) \delta-134.7$ (d, J = 20.5 Hz, 2F), -163.6 (t, J=20.2 Hz, 1F);

IR ( $\mathrm{NaCl}$, thin film) 2962, 2932, 2874, 2359, 2341, 1621, 1530, 1448, 1349, 1234, 1043, 970, 847, $779 \mathrm{~cm}^{-1}$;

HRMS $\left(\mathrm{Cl}^{+}\right) \mathrm{m} / \mathrm{z}$ calcd for $\mathrm{C}_{12} \mathrm{H}_{13} \mathrm{~F}_{3} \mathrm{OH}^{+}(\mathrm{M}+\mathrm{H})^{+}:$: 231.0991, found 231.0991 . 

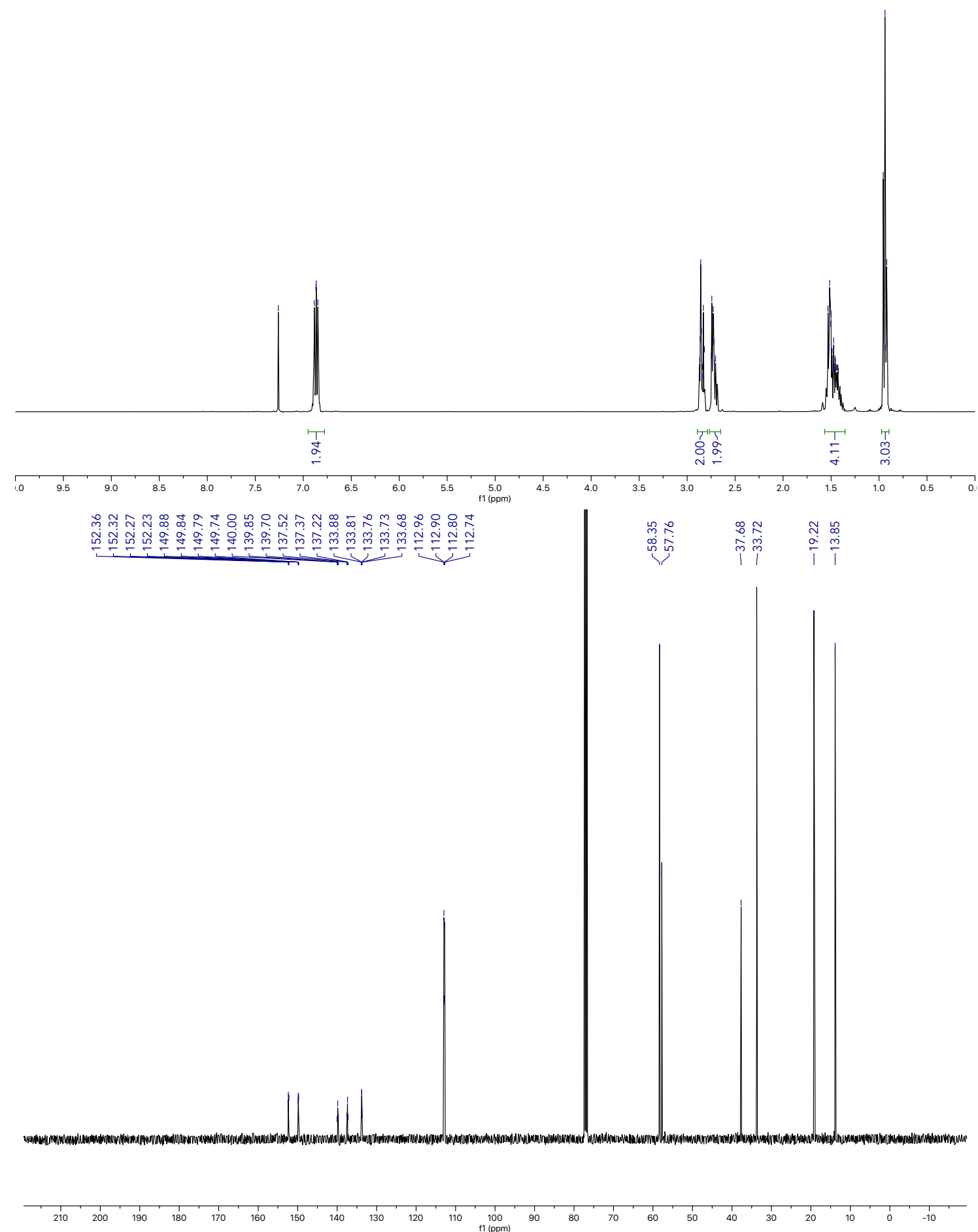


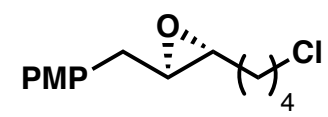

$\left(2 R^{*}, 3 R^{\star}\right)-2-(4-C h l o r o b u t y l)-3-(4-m e t h o x y b e n z y l) o x i r a n e( \pm)-5 q$

General Procedure B: (E)-1-(7-Chlorohept-2-en-1-yl)-4-methoxybenzene (714 mg, $3.00 \mathrm{mmol})$ was employed. Purification of the residue by FCC (15:1, hexane-EtOAc) afforded the title compound (700 $\mathrm{mg}, 92 \%)$ as a colorless oil.

${ }^{1} \mathrm{H}$ NMR $\left(400 \mathrm{MHz}, \mathrm{CDCl}_{3}\right) \delta 7.27(\mathrm{~d}, J=8.6 \mathrm{~Hz}, 2 \mathrm{H}), 6.88(\mathrm{~d}, J=8.6 \mathrm{~Hz}, 2 \mathrm{H}), 3.82(\mathrm{~s}, 3 \mathrm{H}), 3.54$ $(\mathrm{t}, J=6.6,2 \mathrm{H}), 2.92-2.86(\mathrm{~m}, 2 \mathrm{H}), 2.79-2.73(\mathrm{~m}, 2 \mathrm{H}), 1.84-1.77(\mathrm{~m}, 2 \mathrm{H}), 1.66-1.52(\mathrm{~m}, 4 \mathrm{H})$;

${ }^{13} \mathrm{C}$ NMR $\left(101 \mathrm{MHz}, \mathrm{CDCl}_{3}\right) \delta 158.4,129.9,129.3,114.0,58.9,58.3,55.3,44.7$ 37.5, 32.2, 31.1, 23.4;

IR ( $\mathrm{NaCl}$, thin film): 2936, 2862, 2836,1614, 1515, 1455, 1249, 1177, 1034, 921, $820 \mathrm{~cm}^{-1}$;

HRMS $\left(\mathrm{El}^{+}\right) \mathrm{m} / \mathrm{z}$ calcd for $\mathrm{C}_{14} \mathrm{H}_{19}{ }^{35} \mathrm{ClO}_{2}{ }^{+}(\mathrm{M})^{+}: 254.1074$, found 254.1066. 


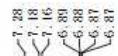

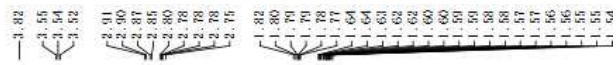
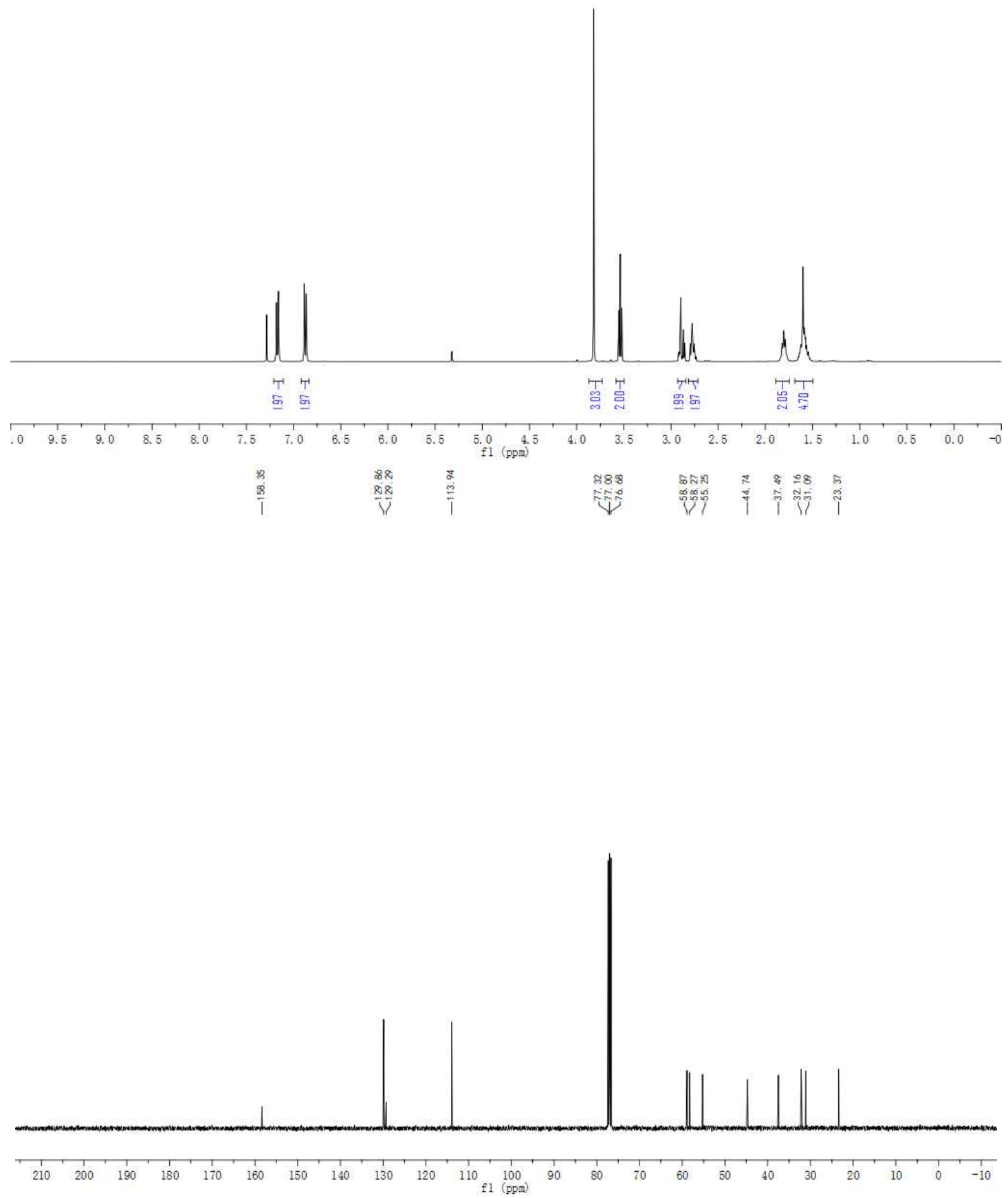

S98 
<smiles>CC(C#N)C1O[C@H]1CCP=N</smiles>

\section{5-((2R*,3R*)-3-(4-methoxybenzyl)oxiran-2-yl)pentanenitrile $( \pm)-5 \mathrm{r}$}

General Procedure B: (E)-8-(4-Methoxyphenyl)oct-6-enenitrile (500 mg, $2.22 \mathrm{mmol}$ ) was employed. Purification of the residue by $\operatorname{FCC}\left(2: 1\right.$, hexane-EtOAc $\left.+1 \% \mathrm{Et}_{3} \mathrm{~N}\right)$ afforded the title compound (450 $\mathrm{mg}, 84 \%)$ as a colorless oil.

${ }^{1} \mathrm{H}$ NMR $\left(400 \mathrm{MHz}, \mathrm{CDCl}_{3}\right) \delta 7.14(\mathrm{~d}, J=8.6 \mathrm{~Hz}, 2 \mathrm{H}), 6.85(\mathrm{~d}, J=8.6 \mathrm{~Hz}, 2 \mathrm{H}), 3.79(\mathrm{~s}, 3 \mathrm{H}), 2.90-$ $2.83(\mathrm{~m}, 2 \mathrm{H}), 2.77-2.71(\mathrm{~m}, 2 \mathrm{H}), 2.33(\mathrm{t}, J=6.9 \mathrm{~Hz}, 2 \mathrm{H}), 1.70-1.48(\mathrm{~m}, 6 \mathrm{H})$;

${ }^{13} \mathrm{C}$ NMR $\left(101 \mathrm{MHz}, \mathrm{CDCl}_{3}\right) \delta 158.4,129.9,129.2,119.5,114.0,58.8,58.0,55.2,37.4,31.0,25.2$, 25.0, 17.1;

IR ( $\mathrm{NaCl}$, thin film) 2937, 2836, 1612, 1584, 1513, 1463, 1302, 1247, 1178, 1112, 1035, 920, 820 $\mathrm{cm}^{-1}$

HRMS (El $\left.{ }^{+}\right) m / z$ calcd for $\mathrm{C}_{15} \mathrm{H}_{19} \mathrm{NO}_{2}{ }^{+}(\mathrm{M})^{+}:$245.1411, found 245.1410. 

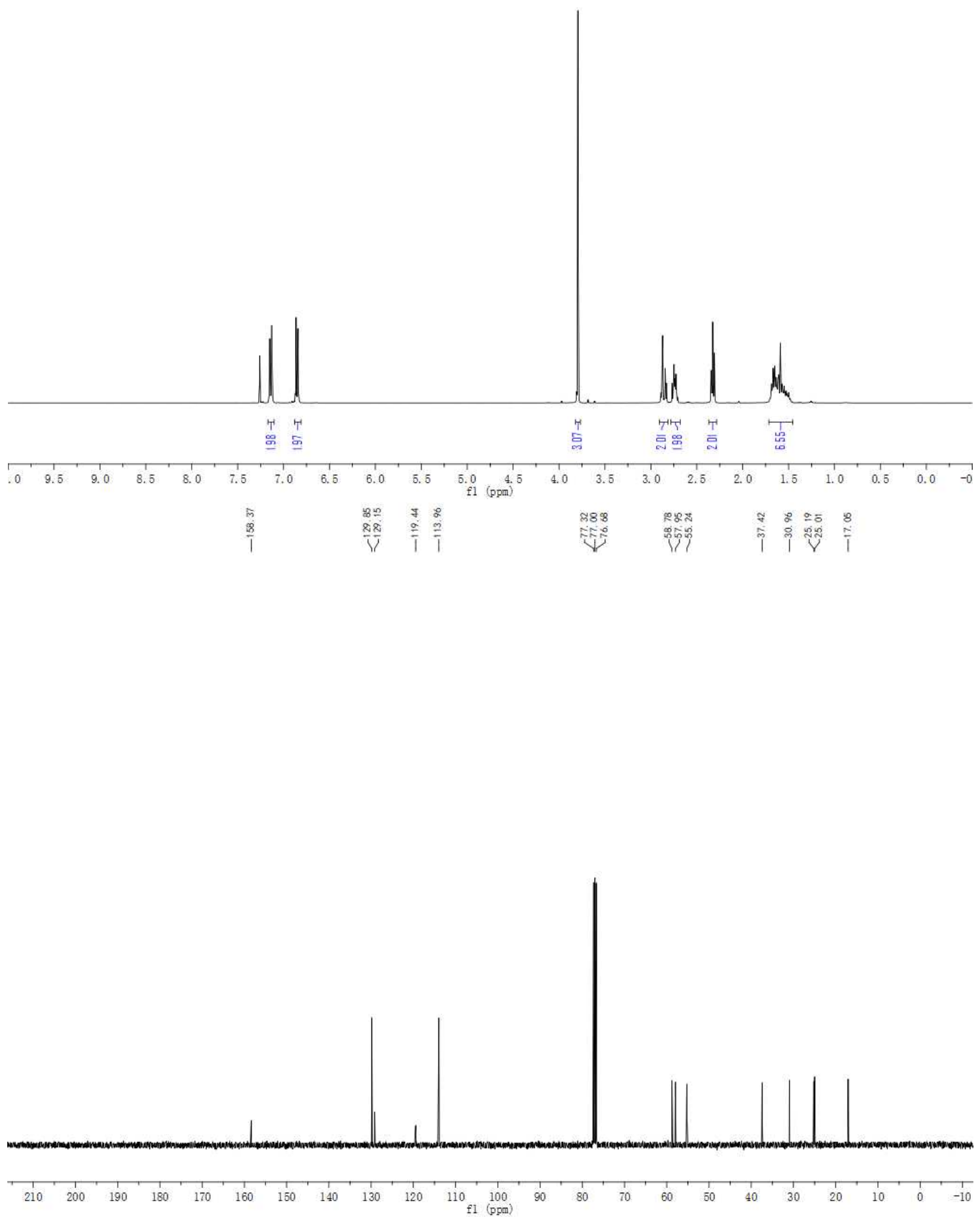


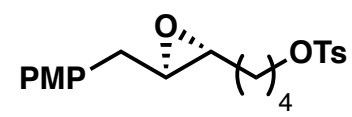

\section{4-((2R*,3R*)-3-(4-Methoxybenzyl)oxiran-2-yl)butyl 4-methylbenzenesulfonate $( \pm)-5 \mathrm{~s}$}

General Procedure B: (E)-7-(4-Methoxyphenyl)hept-5-en-1-yl 4-methylbenzenesulfonate (200 $\mathrm{mg}, 0.53 \mathrm{mmol})$ was employed. Purification of the residue by FCC (5:1, hexane-EtOAc) afforded the title compound ( $86 \mathrm{mg}, 42 \%$ ) as a colorless oil.

${ }^{1} \mathrm{H}$ NMR $\left(400 \mathrm{MHz}, \mathrm{CDCl}_{3}\right) \delta 7.77(\mathrm{~d}, J=8.3 \mathrm{~Hz}, 2 \mathrm{H}), 7.33(\mathrm{~d}, J=8.1 \mathrm{~Hz}, 2 \mathrm{H}), 7.13(\mathrm{~d}, J=8.6$ $\mathrm{Hz}, 2 \mathrm{H}), 6.85(\mathrm{~d}, J=8.6 \mathrm{~Hz}, 2 \mathrm{H}), 4.00(\mathrm{t}, J=6.3 \mathrm{~Hz}, 2 \mathrm{H}), 3.79(\mathrm{~s}, 3 \mathrm{H}), 2.88-2.77$ (m, 2H), 2.78$2.65(\mathrm{~m}, 2 \mathrm{H}), 2.44(\mathrm{~s}, 3 \mathrm{H}), 1.74-1.36(\mathrm{~m}, 6 \mathrm{H})$;

${ }^{13} \mathrm{C}$ NMR $\left(101 \mathrm{MHz}, \mathrm{CDCl}_{3}\right) \delta 158.4,144.7,133.1,129.8,129.8,129.2,127.8,113.9,70.2,58.8$, 58.1, 55.2, 37.4, 31.1, 28.5, 22.0, 21.6;

IR ( $\mathrm{NaCl}$, thin film) 3029, 2995, 2934, 2856, 1610, 1584, 1510, 1454, 1247, 1175, 818, $736 \mathrm{~cm}^{-1}$;

HRMS (ESI-TOF) $\mathrm{m} / \mathrm{z}$ calcd for $\mathrm{C}_{21} \mathrm{H}_{26} \mathrm{O}_{5} \mathrm{SNa}^{+}(\mathrm{M}+\mathrm{Na})^{+}:$413.1399, found 413.1409. 

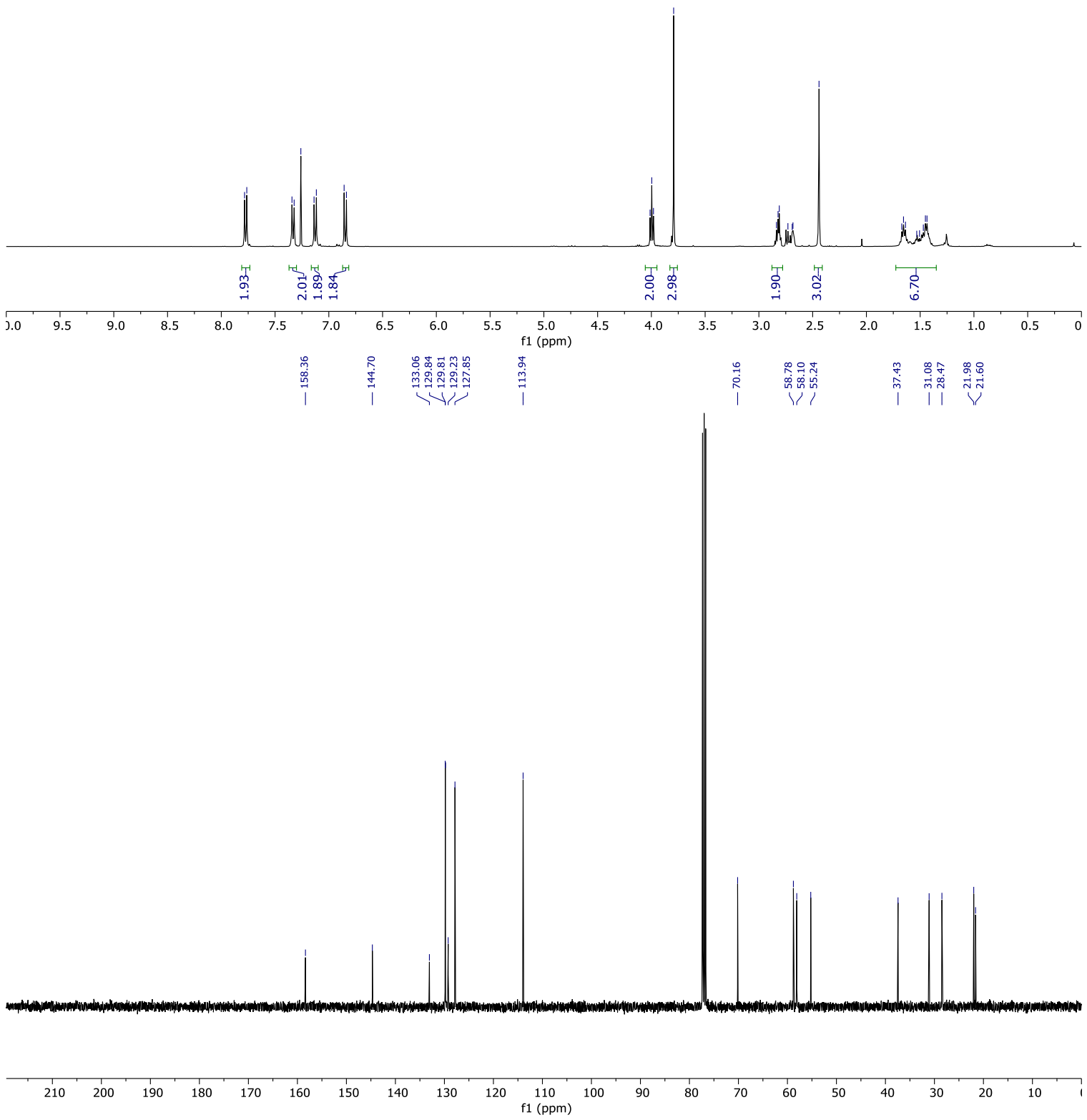


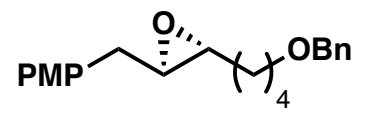

\section{$\left(2 R^{\star}, 3 R^{\star}\right)-2-(4-(B e n z y l o x y)$ butyl)-3-(4-methoxybenzyl)oxirane $( \pm)-5 t$}

General Procedure B: (E)-1-(7-(Benzyloxy)hept-2-en-1-yl)-4-methoxybenzene (700 mg, 2.24 $\mathrm{mmol})$ was employed. Purification of the residue by FCC $(5: 1$, hexane-EtOAc) afforded the title compound $(716 \mathrm{mg}, 98 \%)$ as a colorless oil.

${ }^{1} \mathrm{H}$ NMR $\left(400 \mathrm{MHz}, \mathrm{CDCl}_{3}\right) \delta 7.33(\mathrm{~s}, 5 \mathrm{H}), 7.15(\mathrm{~d}, \mathrm{~J}=8.6 \mathrm{~Hz}, 2 \mathrm{H}), 6.85(\mathrm{~d}, J=8.7 \mathrm{~Hz}, 2 \mathrm{H}), 4.49$ $(\mathrm{s}, 2 \mathrm{H}), 3.79(\mathrm{~s}, 3 \mathrm{H}), 3.46(\mathrm{t}, J=6.3 \mathrm{~Hz}, 2 \mathrm{H}), 2.91-2.80(\mathrm{~m}, 2 \mathrm{H}), 2.80-2.69(\mathrm{~m}, 2 \mathrm{H}), 1.69-1.61(\mathrm{~m}$, 2H), 1.53 (dd, $J=11.7,6.4 \mathrm{~Hz}, 4 \mathrm{H})$;

${ }^{13} \mathrm{C}$ NMR $\left(100 \mathrm{MHz}, \mathrm{CDCl}_{3}\right) \delta 158.3,138.5,129.9,129.4,128.3,127.6,127.5,113.9,72.9,70.0$, $58.9,58.5,55.2,37.5,31.7,29.4,22.7$;

IR ( $\mathrm{NaCl}$, thin film) 2932, 2856, 1555, 1511, 1249, 1105, 1035, $742 \mathrm{~cm}^{-1}$;

HRMS (ESI-TOF) $\mathrm{m} / \mathrm{z}$ calcd for $\mathrm{C}_{21} \mathrm{H}_{26} \mathrm{O}_{3} \mathrm{Na}^{+}(\mathrm{M}+\mathrm{Na})^{+}: 349.1780$, found 349.1787 

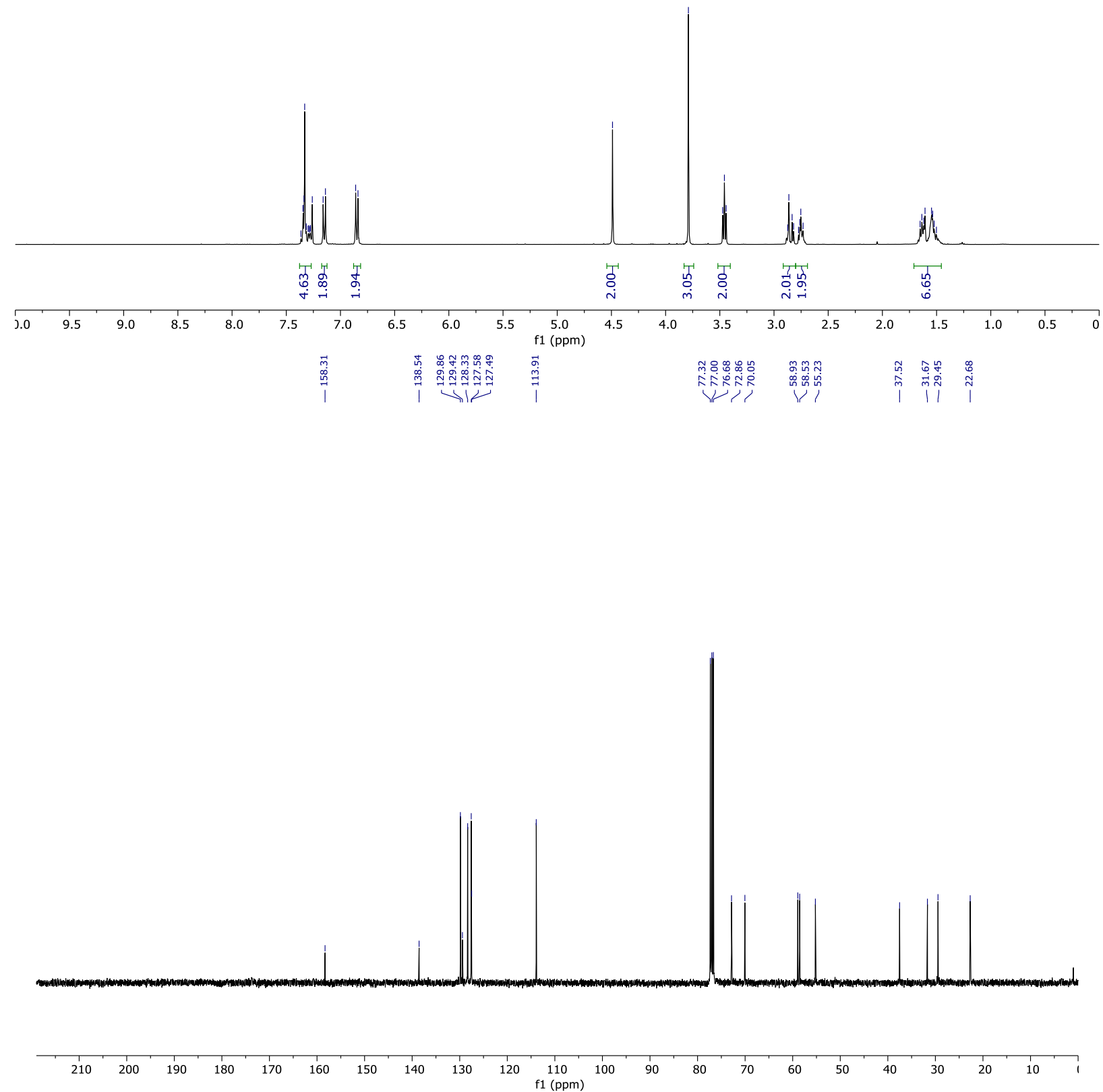


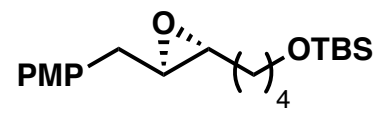

tert-Butyl(4-((2R*,3R*)-3-(4-methoxybenzyl)oxiran-2-yl)butoxy)dimethylsilane ( \pm )-5u

General Procedure B: (E)-tert-Butyl((7-(4-methoxyphenyl)hept-5-en-1-yl)oxy)dimethylsilane (700 $\mathrm{mg}, 2.09 \mathrm{mmol}$ ) was employed. Purification of the residue by FCC (20:1, hexane-EtOAc) afforded the title compound (129 $\mathrm{mg}, 52 \%$ ) as a colorless oil.

${ }^{1} \mathrm{H}$ NMR $\left(400 \mathrm{MHz}, \mathrm{CDCl}_{3}\right) \delta 7.15(\mathrm{~d}, J=8.6 \mathrm{~Hz}, 2 \mathrm{H}), 6.85(\mathrm{~d}, J=8.6 \mathrm{~Hz}, 2 \mathrm{H}), 3.79(\mathrm{~s}, 3 \mathrm{H}), 3.59$ $(\mathrm{t}, J=6.0 \mathrm{~Hz}, 2 \mathrm{H}), 2.91-2.80(\mathrm{~m}, 2 \mathrm{H}), 2.78-2.69(\mathrm{~m}, 2 \mathrm{H}), 1.56-1.39(\mathrm{~m}, 6 \mathrm{H}), 0.89(\mathrm{~s}, 10 \mathrm{H}), 0.04$ (s, 6H);

${ }^{13} \mathrm{C}$ NMR $\left(100 \mathrm{MHz}, \mathrm{CDCl}_{3}\right) \delta 158.5,130.0,129.6,114.1,63.1,59.1,58.8,55.4,37.7,32.7,31.9$, 26.1, 22.5, 18.5, -5.2;

IR ( $\mathrm{NaCl}$, thin film) 2930, 2857, 1613, 1513, 1463, 1248, $8363 \mathrm{~cm}^{-1}$;

HRMS (ESI-TOF) $\mathrm{m} / \mathrm{z}$ calcd for $\mathrm{C}_{20} \mathrm{H}_{34} \mathrm{O}_{3} \mathrm{SiNa}^{+}(\mathrm{M}+\mathrm{Na})^{+}:$373.2175, found 373.2192. 

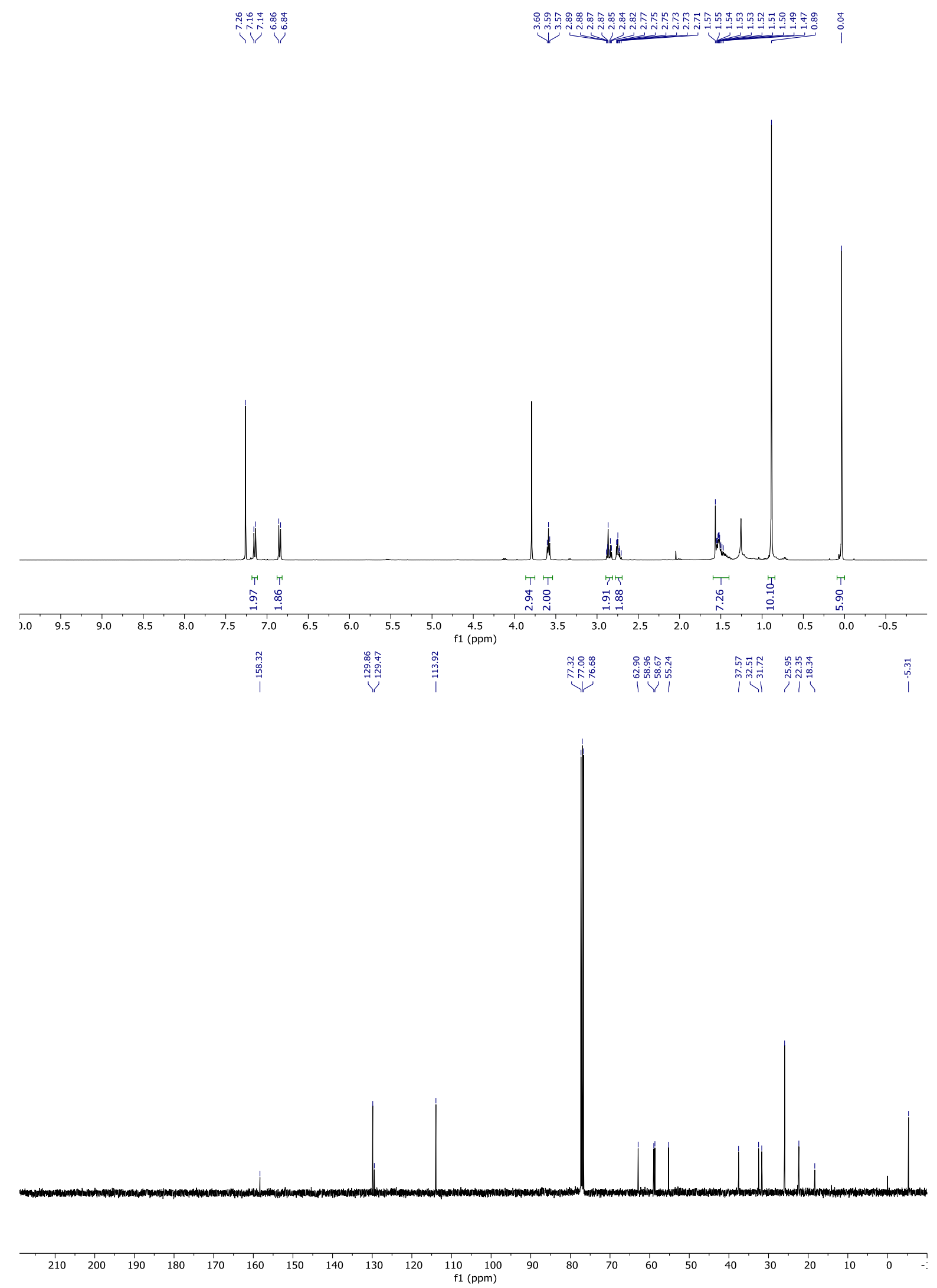


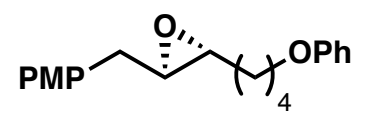

\section{$\left(2 R^{*}, 3 R^{*}\right)-2-(4-M e t h o x y b e n z y l)-3-(4-p h e n o x y b u t y l) o x i r a n e( \pm)-5 \mathrm{v}$}

General Procedure B: (E)-1-Methoxy-4-(7-phenoxyhept-2-en-1-yl)benzene (1.04 g, $3.51 \mathrm{mmol})$ was employed. Purification of the residue by FCC $\left(15: 1\right.$, hexane-EtOAc $\left.+1 \% \mathrm{Et}_{3} \mathrm{~N}\right)$ afforded the title compound (530 $\mathrm{mg}, 48 \%$ ) as a colorless oil.

${ }^{1} \mathrm{H}$ NMR $\left(400 \mathrm{MHz}, \mathrm{CDCl}_{3}\right) \delta 7.28-7.26(\mathrm{~m}, 2 \mathrm{H}), 7.16(\mathrm{~d}, J=8.6 \mathrm{~Hz}, 2 \mathrm{H}), 6.94(\mathrm{tt}, J=7.4,1.1 \mathrm{~Hz}$, $1 \mathrm{H}), 6.90-6.84(\mathrm{~m}, 4 \mathrm{H}), 3.94(\mathrm{t}, J=6.3 \mathrm{~Hz}, 2 \mathrm{H}), 3.94(\mathrm{t}, J=6.3 \mathrm{~Hz}, 2 \mathrm{H}), 3.79(\mathrm{~s}, 3 \mathrm{H}), 2.92-2.84$ $(\mathrm{m}, 2 \mathrm{H}), 2.89-2.73(\mathrm{~m}, 2 \mathrm{H}), 1.84-1.79(\mathrm{~m}, 2 \mathrm{H}), 1.66-1.56(\mathrm{~m}, 4 \mathrm{H})$;

${ }^{13} \mathrm{C}$ NMR $\left(101 \mathrm{MHz}, \mathrm{CDCl}_{3}\right) \delta 159.0,158.3,129.9,129.4(2 \mathrm{C}), 120.5,114.4,113.9,67.4,58.9$, 58.5, 55.2 37.5, 31.6, 29.0, 22.6;

IR ( $\mathrm{NaCl}$, thin film) 2938, 2865, 1600, 1586, 1513, 1497, 1301, 1247, 1301, 1247, 1176, 1035, $820 \mathrm{~cm}^{-1}$

HRMS (ESI-TOF) $m / z$ calcd for $\mathrm{C}_{20} \mathrm{H}_{24} \mathrm{O}_{3} \mathrm{Na}^{+}(\mathrm{M}+\mathrm{Na})^{+}:$335.1623, found 335.1607. 

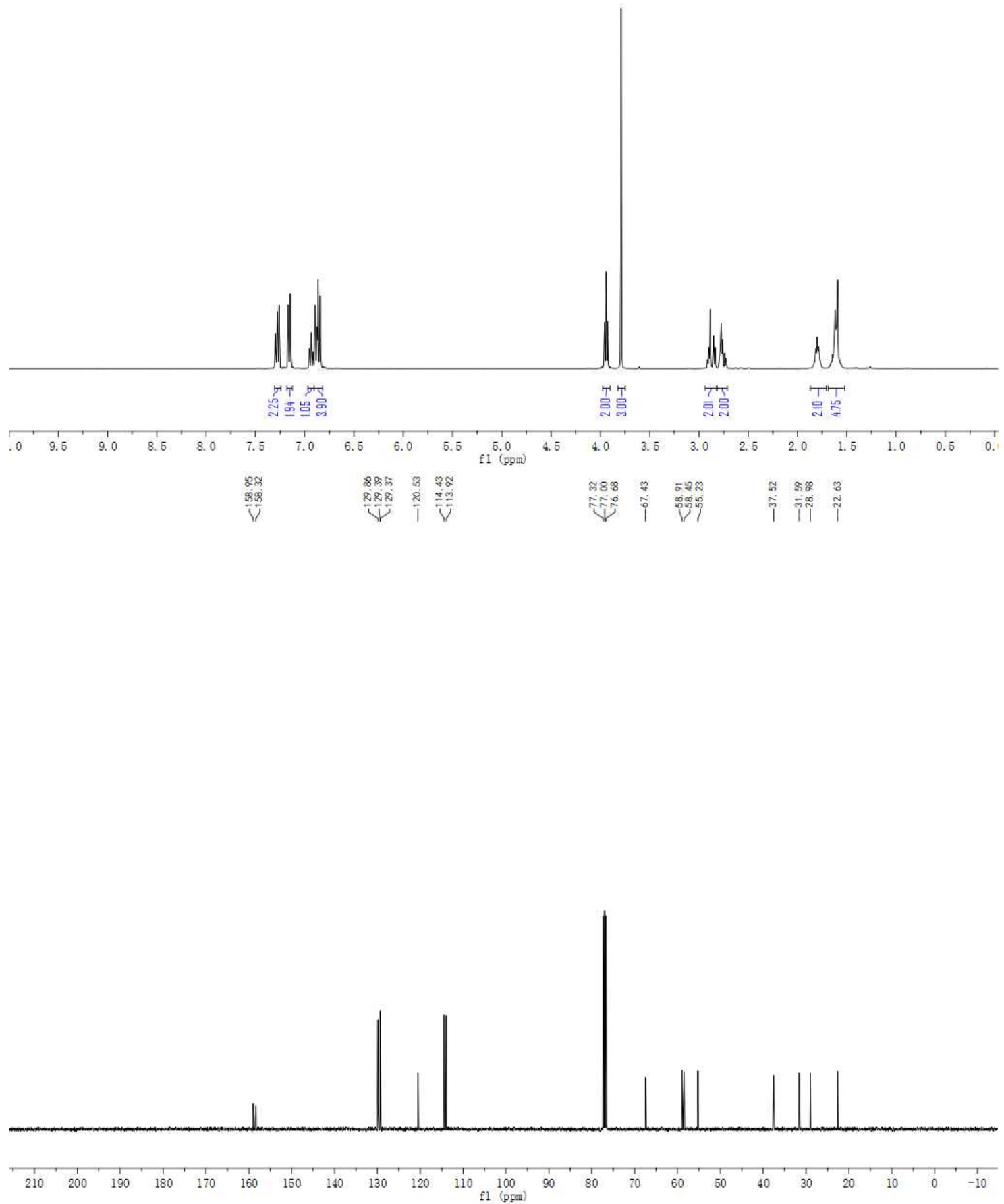


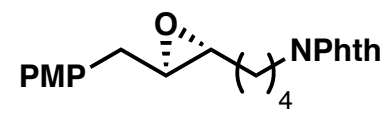

\section{2-(4-((2R*, $\left.\left.\left.3 R^{*}\right)-3-(4-M e t h o x y b e n z y l) o x i r a n-2-y l\right) b u t y l\right) i s o i n d o l i n e-1,3-d i o n e ~( \pm)-5 w$}

General Procedure B: (E)-2-(7-(4-Methoxyphenyl)hept-5-en-1-yl)isoindoline-1,3-dione (200 mg, $0.53 \mathrm{mmol})$ was employed. Purification of the residue by FCC (5:1, hexane-EtOAc) afforded the title compound ( $86 \mathrm{mg}, 42 \%)$ as a colorless oil.

${ }^{1} \mathrm{H}$ NMR $\left(500 \mathrm{MHz}, \mathrm{CDCl}_{3}\right) \delta 7.83(\mathrm{dd}, J=5.1,3.0 \mathrm{~Hz}, 2 \mathrm{H}), 7.70(\mathrm{dd}, J=5.0,3.0 \mathrm{~Hz}, 2 \mathrm{H}), 7.12$ $(\mathrm{d}, J=8.4 \mathrm{~Hz}, 2 \mathrm{H}), 6.82(\mathrm{~d}, J=8.4 \mathrm{~Hz}, 2 \mathrm{H}), 3.77(\mathrm{~s}, 3 \mathrm{H}), 3.66(\mathrm{t}, J=7.2 \mathrm{~Hz}, 2 \mathrm{H}), 2.93-2.78(\mathrm{~m}$, 2H), $2.72(\mathrm{dd}, J=13.6,5.3 \mathrm{~Hz}, 2 \mathrm{H}), 1.71-1.66(\mathrm{~m}, 2 \mathrm{H}), 1.61-1.54(\mathrm{~m}, 2 \mathrm{H}), 1.53-1.41(\mathrm{~m}, 2 \mathrm{H})$;

${ }^{13} \mathrm{C}$ NMR $\left(126 \mathrm{MHz}, \mathrm{CDCl}_{3}\right) \delta 168.3,158.3,133.8,132.1,129.8,129.3,123.1,113.9,58.9,58.3$, $55.2,37.7,37.5,31.4,28.2,23.3$;

IR $\left(\mathrm{NaCl}\right.$, thin film) 2936, 2856, 1773, 1711, 1249, 1035, 826, $723 \mathrm{~cm}^{-1}$;

HRMS (ESI-TOF) $m / z$ calcd for $\mathrm{C}_{22} \mathrm{H}_{23} \mathrm{NO}_{4} \mathrm{Na}^{+}(\mathrm{M}+\mathrm{Na})^{+}: 388.1525$, found 388.1516. 

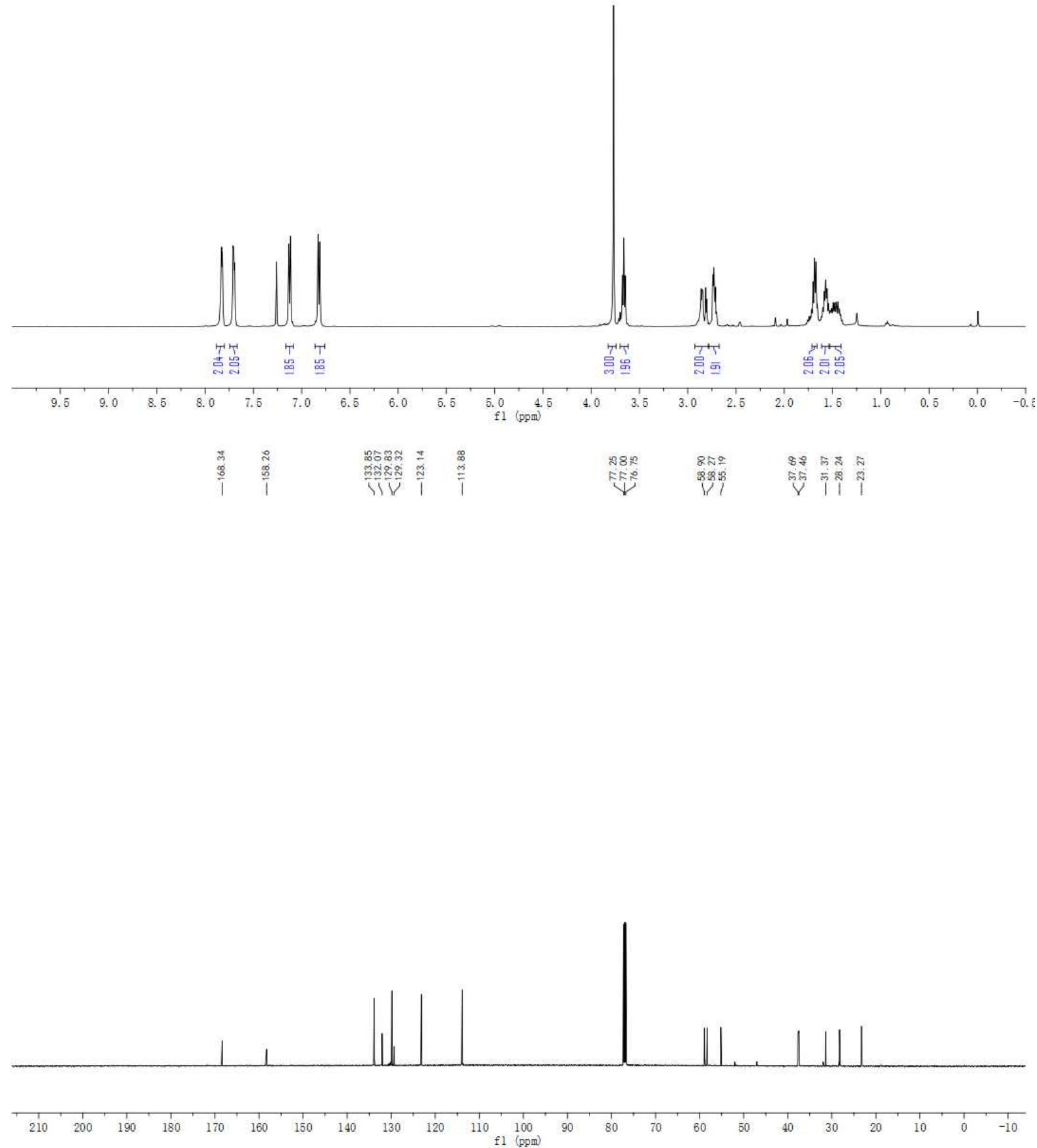
<smiles>CC(C)C1OC1CCN(C)S(=O)(=O)c1ccccc1</smiles>

\section{$\left(2 R^{*}, 3 R^{*}\right)-2-(4-M e t h o x y b e n z y l)-3-(4-(p h e n y l s u l f o n y l) b u t y l) o x i r a n e ~( \pm)-5 x$}

To a solution of $(E)$-1-(7-chlorohept-2-en-1-yl)-4-methoxybenzene (238 mg, $1.00 \mathrm{mmol})$ in DMF $(10 \mathrm{~mL})$ was added $\mathrm{NaSO}_{2} \mathrm{Ph}(180 \mathrm{mg}, 1.10 \mathrm{mmol})$ and $\mathrm{Nal}(15 \mathrm{mg}, 0.10 \mathrm{mmol})$. The reaction mixture was stirred at $40{ }^{\circ} \mathrm{C}$ for 12 hours. The solution was then quenched by pouring into saturated aq. $\mathrm{NH}_{4} \mathrm{Cl}$ solution $(20 \mathrm{~mL})$. The solution was extracted with $\mathrm{Et}_{2} \mathrm{O}(4 \times 10 \mathrm{~mL})$ and the combined organic layers were washed with water $(3 \times 1 \mathrm{~mL})$ and brine $(5 \mathrm{~mL})$. The organic layer was dried $\left(\mathrm{MgSO}_{4}\right)$ and concentrated in vacuo. The resulting crude residue was dissolved in $\mathrm{CH}_{2} \mathrm{Cl}_{2}(5 \mathrm{~mL})$ and cooled to $0{ }^{\circ} \mathrm{C}$. m-CPBA $(75 \%, 230 \mathrm{mg}, 1.00 \mathrm{mmol})$ was added and the reaction mixture was allowed to warm to room temperature and stirred for 4 hours. The reaction was quenched by addition of saturated aq. $\mathrm{NaHSO}_{3}(2 \mathrm{~mL})$ and filtered through Celite ${ }^{\circledR}$, washing with $\mathrm{Et}_{2} \mathrm{O}(10 \mathrm{~mL})$. The filtrate was washed with saturated aq. $\mathrm{NaHCO}_{3}(3 \times 5 \mathrm{~mL})$, dried $\left(\mathrm{Na}_{2} \mathrm{SO}_{4}\right)$, filtered, and concentrated in vacuo. Purification of the residue by FCC (10:1, hexane-EtOAc) afforded the title compound ( $240 \mathrm{mg}, 70 \%$ yield over 2 steps) as a light yellow oil.

${ }^{1} \mathrm{H}$ NMR $\left(500 \mathrm{MHz}, \mathrm{CDCl}_{3}\right) \delta 7.89(\mathrm{~d}, J=7.6 \mathrm{~Hz}, 2 \mathrm{H}), 7.65(\mathrm{t}, J=7.4 \mathrm{~Hz}, 1 \mathrm{H}), 7.56(\mathrm{t}, J=7.7 \mathrm{~Hz}$, $2 \mathrm{H}), 7.11(\mathrm{~d}, J=8.5 \mathrm{~Hz}, 2 \mathrm{H}), 6.84(\mathrm{~d}, J=8.5 \mathrm{~Hz}, 2 \mathrm{H}), 3.79(\mathrm{~s}, 3 \mathrm{H}), 3.11-3.03(\mathrm{~m}, 2 \mathrm{H}), 2.85-2.78$ $(\mathrm{m}, 2 \mathrm{H}), 2.75-2.66(\mathrm{~m}, 2 \mathrm{H}), 1.79-1.69(\mathrm{~m}, 2 \mathrm{H}), 1.59-1.53(\mathrm{~m}, 1 \mathrm{H}), 1.52-1.41(\mathrm{~m}, 3 \mathrm{H})$;

${ }^{13} \mathrm{C}$ NMR $\left(126 \mathrm{MHz}, \mathrm{CDCl}_{3}\right) \delta 158.3,139.1,133.7,129.8,129.3,129.2,128.0,113.9,58.8,57.8$, 56.0, 55.2, 37.7, 31.2, 24.7, 22.4;

IR ( $\mathrm{NaCl}$, thin film) 2959, 2931, 2873, 1645, 1512, 1245, 1143, 750, $689 \mathrm{~cm}^{-1}$;

HRMS $\left(\mathrm{EI}^{+}\right) \mathrm{m} / \mathrm{z}$ calcd for $\mathrm{C}_{20} \mathrm{H}_{24} \mathrm{O}_{4} \mathrm{~S}^{+}(\mathrm{M})^{+}: 360.1395$, found 360.1396 . 

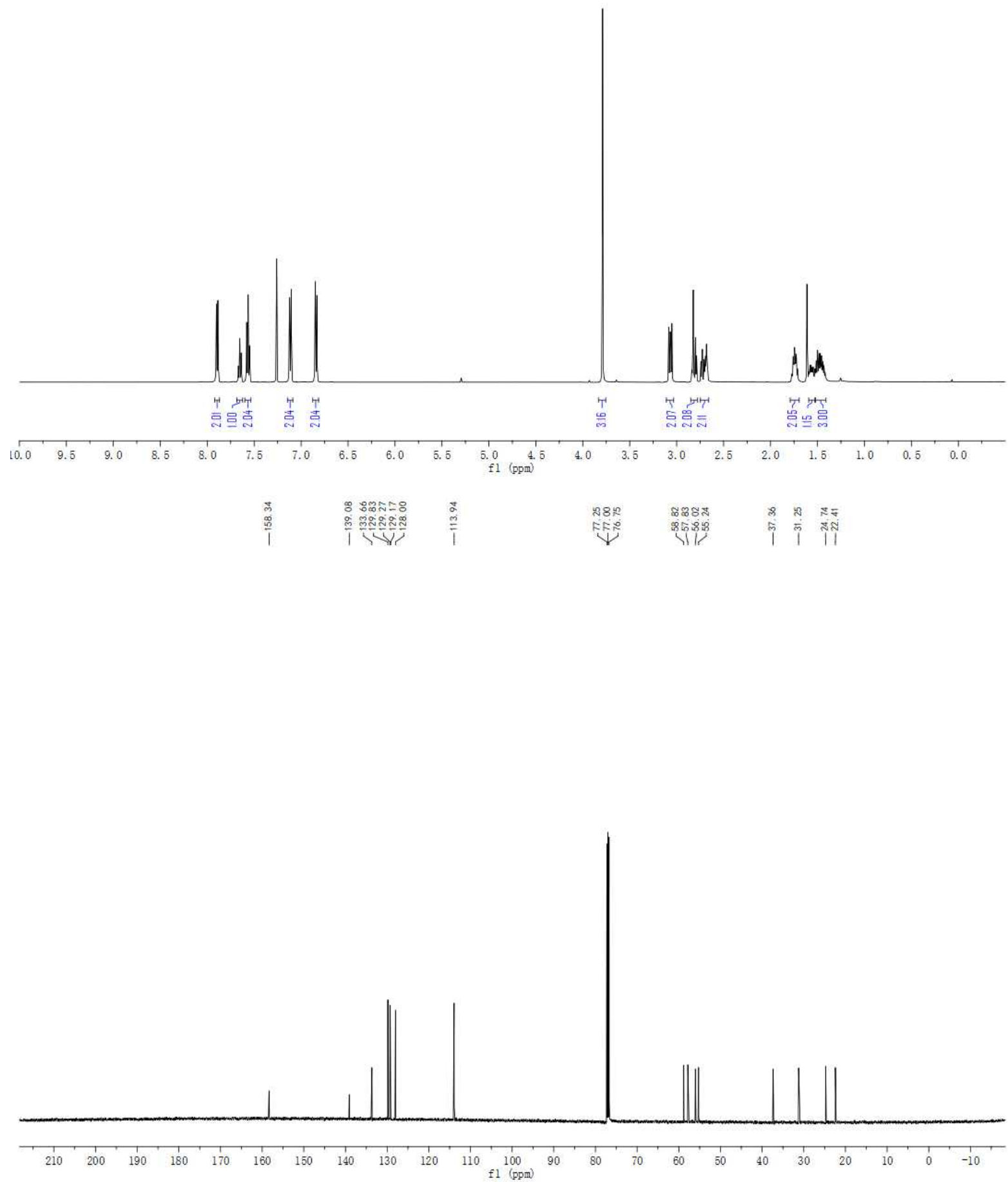
<smiles>COc1ccc(C[C@@H]2O[C@H]2C(C)=O)cc1</smiles>

\section{1-((2S*,3R*)-3-(4-Methoxybenzyl)oxiran-2-yl)ethan-1-one $( \pm)-5 y$}

To a solution of $(E)-5$-(4-Methoxyphenyl)pent-3-en-2-one (150 mg, $0.79 \mathrm{mmol})$ in $\mathrm{MeOH}(0.2 \mathrm{M})$ was added $\mathrm{H}_{2} \mathrm{O}_{2}(30 \%, 0.241 \mathrm{~mL}, 2.13 \mathrm{mmol})$ at room temperature. $\mathrm{NaHCO}_{3}$ (33 $\left.\mathrm{mg}, 0.39 \mathrm{mmol}\right)$ was added and the mixture was stirred overnight. The reaction was quenched with $\mathrm{Na}_{2} \mathrm{SO}_{4}$ and extracted with DCM. The reaction was then concentrated in vacuo and the crude mixture purified by FCC (4:1, hexane-EtOAc) afforded the title compound $(115 \mathrm{mg}, 70 \%)$ as a colorless oil.

${ }^{1} \mathrm{H}$ NMR $\left(400 \mathrm{MHz}, \mathrm{CDCl}_{3}\right) \delta 7.15(\mathrm{~d}, J=8.6 \mathrm{~Hz}, 2 \mathrm{H}), 6.86(\mathrm{~d}, J=8.6 \mathrm{~Hz}, 2 \mathrm{H}), 3.80(\mathrm{~s}, 3 \mathrm{H}), 3.28$ (ddd, $J=5.5,4.7,2.0 \mathrm{~Hz}, 1 \mathrm{H}$ ), 3.22 (d, $J=1.9 \mathrm{~Hz}, 1 \mathrm{H}$ ), 2.94 (dd, $J=14.8,4.7 \mathrm{~Hz}, 1 \mathrm{H}$ ), 2.88 (dd, $J=14.8,5.6 \mathrm{~Hz}), 2.04(\mathrm{~s}, 3 \mathrm{H})$;

${ }^{13} \mathrm{C}$ NMR $\left(100 \mathrm{MHz}, \mathrm{CDCl}_{3}\right) \delta 205.9,158.9,130.2,127.8,114.3,59.7,58.2,55.4,37.2,24.5$;

IR ( $\mathrm{NaCl}$, thin film) 3000, 2936, 2836, 1709, 1612, 1513, 1464, 1249, 1179, $1033 \mathrm{~cm}^{-1}$;

HRMS (El-Orbitrap) $\mathrm{m} / \mathrm{z}$ calcd for $\mathrm{C}_{12} \mathrm{H}_{15} \mathrm{O}_{3}{ }^{+}(\mathrm{M}+\mathrm{H})^{+} 207.1016$ found 207.1009 . 

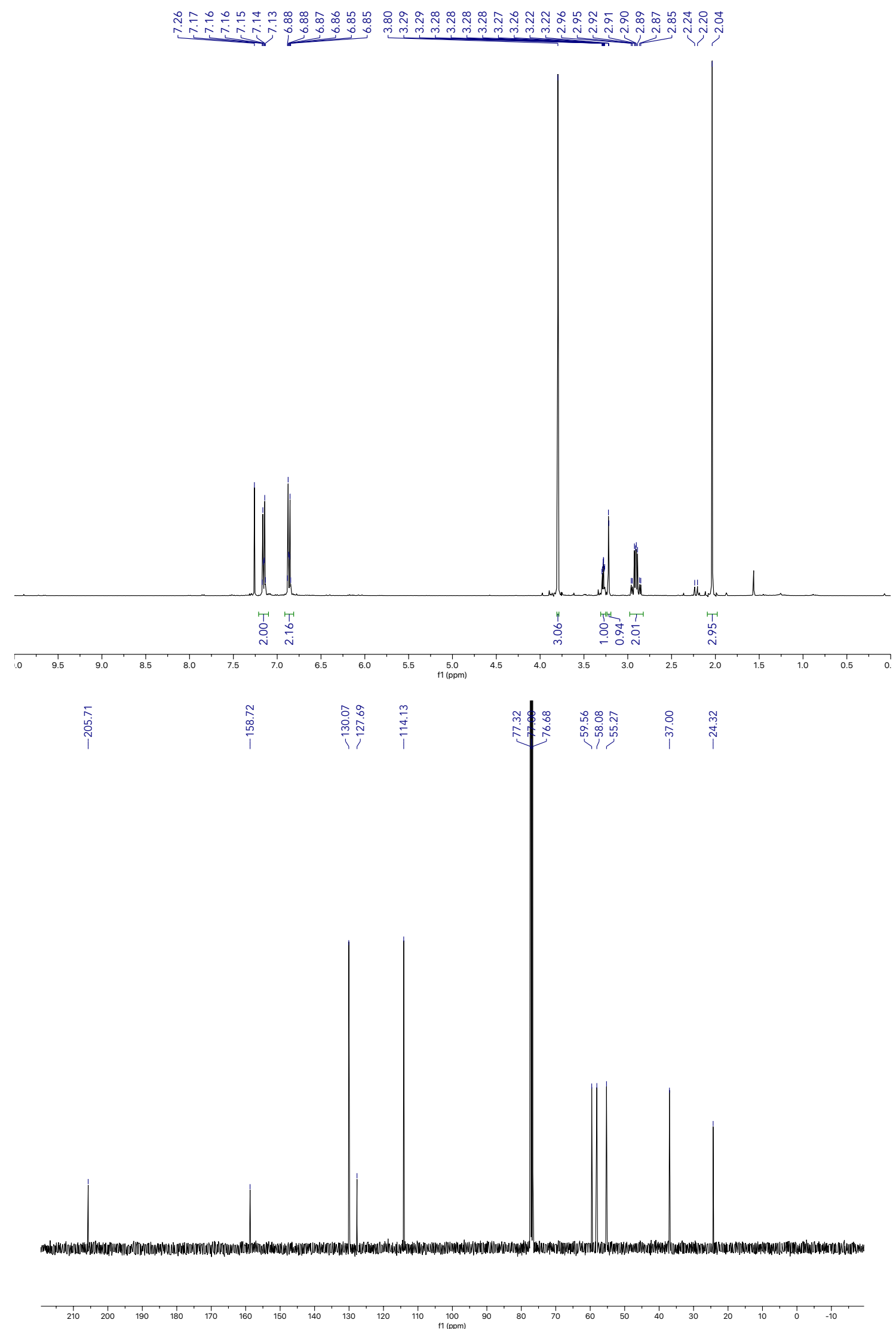
<smiles>COc1ccc(CC2OC2C2CCOCC2)cc1</smiles>

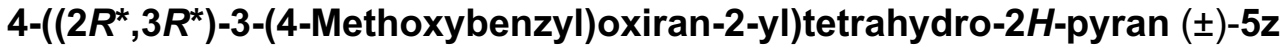

General Procedure B: (E)-4-(3-(4-Methoxyphenyl)prop-1-en-1-yl)tetrahydro-2H-pyran (402 mg, $1.73 \mathrm{mmol})$ was employed. Purification of the residue by FCC $(4: 1-3: 1$, hexane-EtOAc) afforded the title compound (341 $\mathrm{mg}, 79 \%$ ) as a colorless oil.

${ }^{1} \mathrm{H}$ NMR $\left(400 \mathrm{MHz}, \mathrm{CDCl}_{3}\right) \delta 7.16(\mathrm{~d}, J=8.6 \mathrm{~Hz}, 2 \mathrm{H}), 6.87(\mathrm{~d}, J=8.6 \mathrm{~Hz}, 2 \mathrm{H}), 4.01-3.95(\mathrm{~m}, 2 \mathrm{H})$, $3.82(\mathrm{~s}, 3 \mathrm{H}), 3.40-3.34(\mathrm{~m}, 2 \mathrm{H}), 2.97(\mathrm{td}, J=5.6,2.2 \mathrm{~Hz}, 1 \mathrm{H}), 2.90(\mathrm{dd}, J=14.3,5.5 \mathrm{~Hz}, 1 \mathrm{H}), 2.74$ (dd, $J=14.3,5.7 \mathrm{~Hz}, 1 \mathrm{H}), 2.66-2.63(\mathrm{~m}, 1 \mathrm{H}), 1.75-1.71(\mathrm{~m}, 1 \mathrm{H}), 1.55-1.47(\mathrm{~m}, 4 \mathrm{H})$;

${ }^{13} \mathrm{C} \mathrm{NMR}\left(101 \mathrm{MHz}, \mathrm{CDCl}_{3}\right) \delta 158.4,129.8,129.2,114.0,67.4,67.3,62.2,57.6,55.2,37.6,37.2$, 29.3, 28.7;

IR ( $\mathrm{NaCl}$, thin film) 2934, 2839, 1612. 1513. 1465, 1302, 1247, 1178, 1141, 1094, $983 \mathrm{~cm}^{-1}$; HRMS (ESI-TOF) $\mathrm{m} / \mathrm{z}$ calcd for $\mathrm{C}_{15} \mathrm{H}_{20} \mathrm{O}_{3} \mathrm{Na}^{+}(\mathrm{M}+\mathrm{Na})^{+}:$271.1310, found 271.1304. 


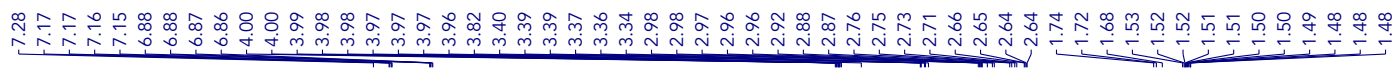

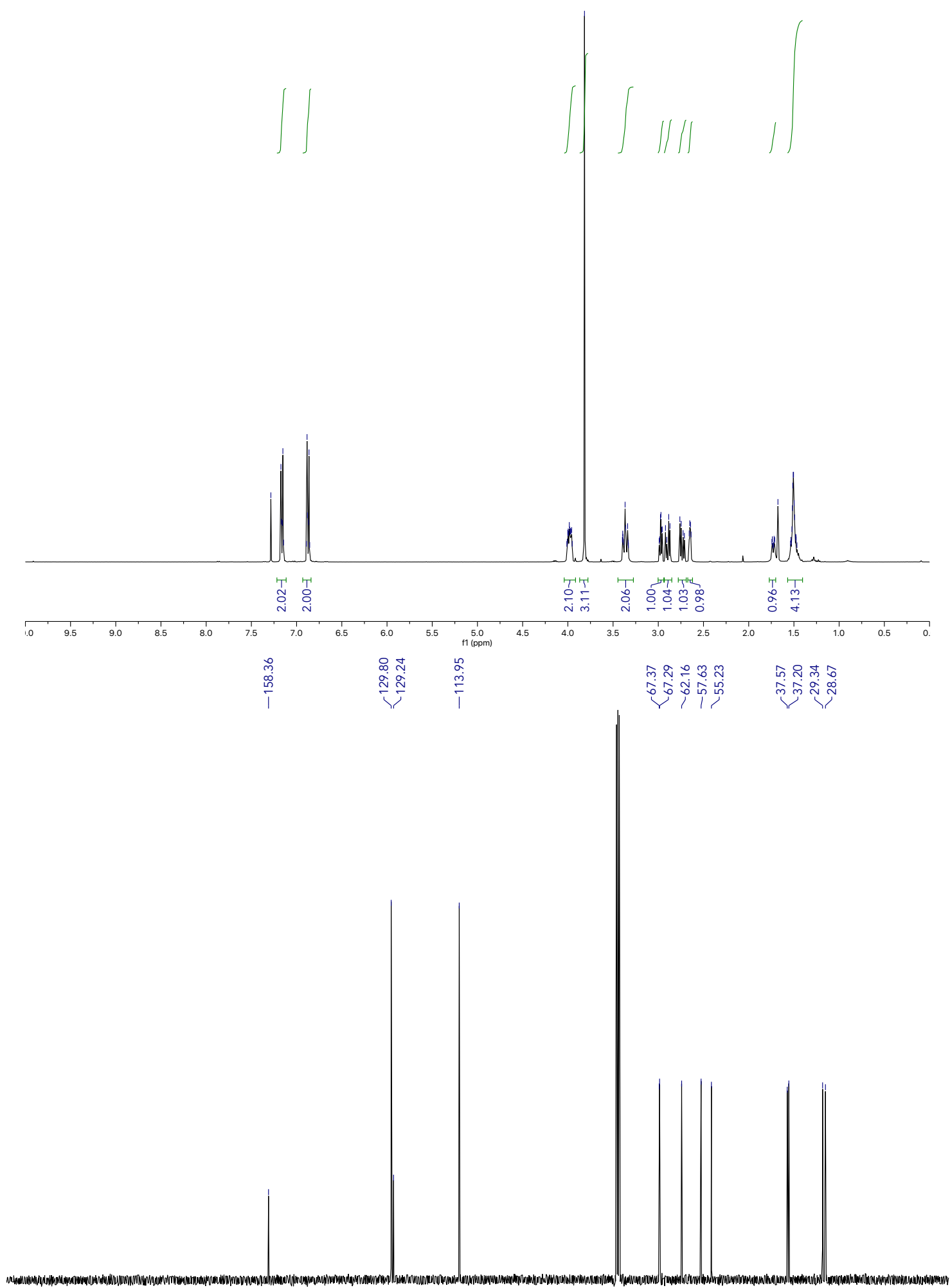

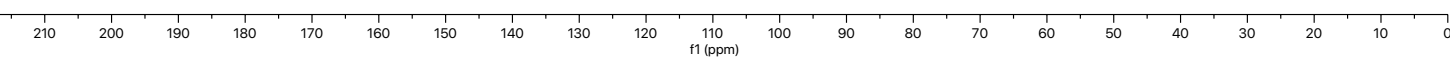


<smiles>COc1ccc(CC2OC2C2CCN(C(=O)OCc3ccccc3)CC2)cc1</smiles>

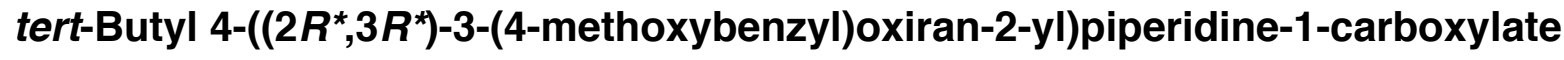
$( \pm)-5$ aa-Boc

General Procedure B: (E)-4-(3-(4-Methoxyphenyl)prop-1-en-1-yl)tetrahydro-2H-pyran (402 mg, $1.73 \mathrm{mmol})$ was employed. Purification of the residue by FCC $(4: 1-3: 1$, hexane-EtOAc) afforded the title compound ( $341 \mathrm{mg}, 79 \%$ ) as a colorless oil.

${ }^{1} \mathrm{H}$ NMR $\left(400 \mathrm{MHz}, \mathrm{CDCl}_{3}\right) \delta 7.12(\mathrm{~d}, J=8.6 \mathrm{~Hz}, 2 \mathrm{H}), 6.84(\mathrm{~d}, J=8.6 \mathrm{~Hz}, 2 \mathrm{H}), 4.14-4.03(\mathrm{~m}, 2 \mathrm{H})$, $3.78(\mathrm{~s}, 3 \mathrm{H}), 2.95-2.84(\mathrm{~m}, 2 \mathrm{H}), 2.72-2.58(\mathrm{~m}, 2 \mathrm{H}), 1.79-1.74(\mathrm{~m}, 1 \mathrm{H}), 1.55(\mathrm{~d}, J=12.6 \mathrm{~Hz}, 1 \mathrm{H})$, $1.44(\mathrm{~s}, 9 \mathrm{H}), 1.39-1.19(\mathrm{~m}, 3 \mathrm{H})$;

${ }^{13} \mathrm{C}$ NMR $\left(101 \mathrm{MHz}, \mathrm{CDCl}_{3}\right) \delta 158.3,154.7,129.8,129.2,113.9,79.4,62.0,57.7,55.2,43.4$ (broad), 38.3, 37.5, 28.6, 28.4, 27.9;

IR ( $\mathrm{NaCl}$, thin film) 2937, 2932, 2853, 1692, 1612, 1513, 1423, 1247, 1156, 1036, 973, $869 \mathrm{~cm}^{-1}$; HRMS (ESI-TOF) $\mathrm{m} / \mathrm{z}$ calcd for $\mathrm{C}_{20} \mathrm{H}_{29} \mathrm{NO}_{4} \mathrm{Na}^{+}(\mathrm{M}+\mathrm{Na})^{+}: 370.1994$, found 370.1998 ; 


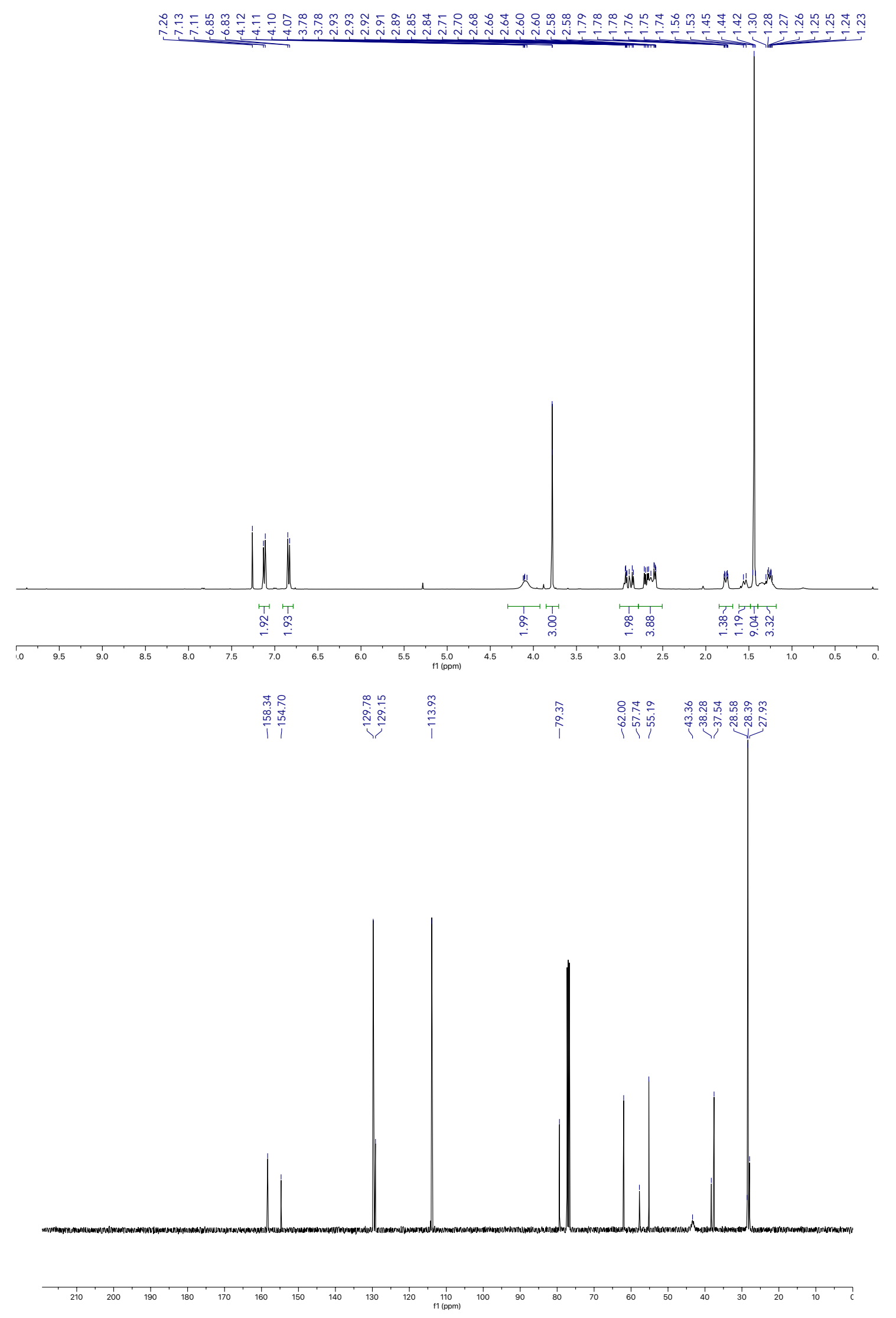


<smiles>COc1ccc(CC2OC2C2CC[N+](S)(S)CC2)cc1</smiles>

\section{4-((2R*, $\left.\left.3 R^{*}\right)-3-(4-M e t h o x y b e n z y l) o x i r a n-2-y l\right)-1-t o s y l p i p e r i d i n e ~( \pm)-5 a a-T s$}

To a solution of tert-Butyl (E)-4-(3-(4-methoxyphenyl)prop-1-en-1-yl)piperidine-1-carboxylate (728 mg, $2.2 \mathrm{mmol})$ in $\mathrm{CH}_{2} \mathrm{Cl}_{2}(20 \mathrm{~mL})$ was added with $\mathrm{CF}_{3} \mathrm{COOH}(251 \mathrm{mg}, 2.20 \mathrm{mmol})$. The reaction mixture was stirred at room temperature for $8 \mathrm{~h}$. The mixture was concentrated in vacuo and the crude mixture was re-dissolved in $\mathrm{CH}_{2} \mathrm{Cl}_{2}(20 \mathrm{~mL}) . \mathrm{Et}_{3} \mathrm{~N}(0.42 \mathrm{~mL}, 3.00 \mathrm{mmol})$ and $\mathrm{TsCl} \cdot \mathrm{H}_{2} \mathrm{O}(420 \mathrm{mg}, 2.20 \mathrm{mmol})$ were added sequentially. The mixture was stirred at room temperature for 6 hours after which it was filtrated through celite ${ }^{\circledR}$ and the filtrate was concentrated in vacuo. The crude residue was re-dissolved in $\mathrm{CH}_{2} \mathrm{Cl}_{2}(20 \mathrm{~mL})$ and cooled to $0{ }^{\circ} \mathrm{C}$. $m$ CPBA $(75 \%, 573 \mathrm{mg}, 2.50 \mathrm{mmol})$ was added and the reaction was allowed to warm to room temperature and stirred for 4 hours. The reaction was quenched by addition of saturated aq. $\mathrm{NaHSO}_{3}(2 \mathrm{~mL})$ and filtered through Celite ${ }^{\circledR}$, washing with $\mathrm{Et}_{2} \mathrm{O}(10 \mathrm{~mL})$. The filtrate was washed with saturated aq. $\mathrm{NaHCO}_{3}(3 \times 5 \mathrm{~mL})$, dried $\left(\mathrm{Na}_{2} \mathrm{SO}_{4}\right)$, filtered, and concentrated in vacuo. Purification of the residue by FCC (3:1, hexane-EtOAc) afforded the title compound (552 $\mathrm{mg}, 68 \%$ yield over 3 steps) as a light yellow oil.

${ }^{1} \mathrm{H}$ NMR $\left(500 \mathrm{MHz}, \mathrm{CDCl}_{3}\right) \delta 7.62(\mathrm{~d}, J=8.2 \mathrm{~Hz}, 2 \mathrm{H}), 7.30(\mathrm{~d}, J=8.0 \mathrm{~Hz}, 2 \mathrm{H}), 7.10(\mathrm{~d}, J=8.5$ $\mathrm{Hz}, 2 \mathrm{H}), 6.84(\mathrm{~d}, J=8.5 \mathrm{~Hz}, 2 \mathrm{H}), 3.79(\mathrm{~s}, 5 \mathrm{H}), 2.88-2.80(\mathrm{~m}, 2 \mathrm{H}), 2.72-2.64(\mathrm{~m}, 1 \mathrm{H}), 2.57(\mathrm{~d}, J=$ $6.6 \mathrm{~Hz}, 1 \mathrm{H}), 2.42(\mathrm{~s}, 3 \mathrm{H}), 2.24-2.15(\mathrm{~m}, 2 \mathrm{H}), 1.85(\mathrm{~d}, J=13.4 \mathrm{~Hz}, 1 \mathrm{H}), 1.62(\mathrm{~d}, J=13.2 \mathrm{~Hz}, 1 \mathrm{H})$, 1.52-1.41 (m, 2H), 1.17-1.12 (m, $1 \mathrm{H})$;

${ }^{13} \mathrm{C} \mathrm{NMR}\left(126 \mathrm{MHz}, \mathrm{CDCl}_{3}\right) \delta 158.4,143.5,133.1,129.8,129.6,129.0,127.6,114.0,61.5,57.8$, 55.2, 45.8, 45.7, 37.5, 37.5, 28.2, 27.4, 21.5;

IR ( $\mathrm{NaCl}$, thin film) 2929, 2873, 1652, 1612, 1513, 1336, 1247, 1167, 1094, 930, $814 \mathrm{~cm}^{-1}$;

HRMS $\left(\mathrm{El}^{+}\right) \mathrm{m} / \mathrm{z}$ calcd for $\mathrm{C}_{22} \mathrm{H}_{27} \mathrm{NO}_{4} \mathrm{~S}^{+}(\mathrm{M})^{+}:$401.1661, found 401.1662. 

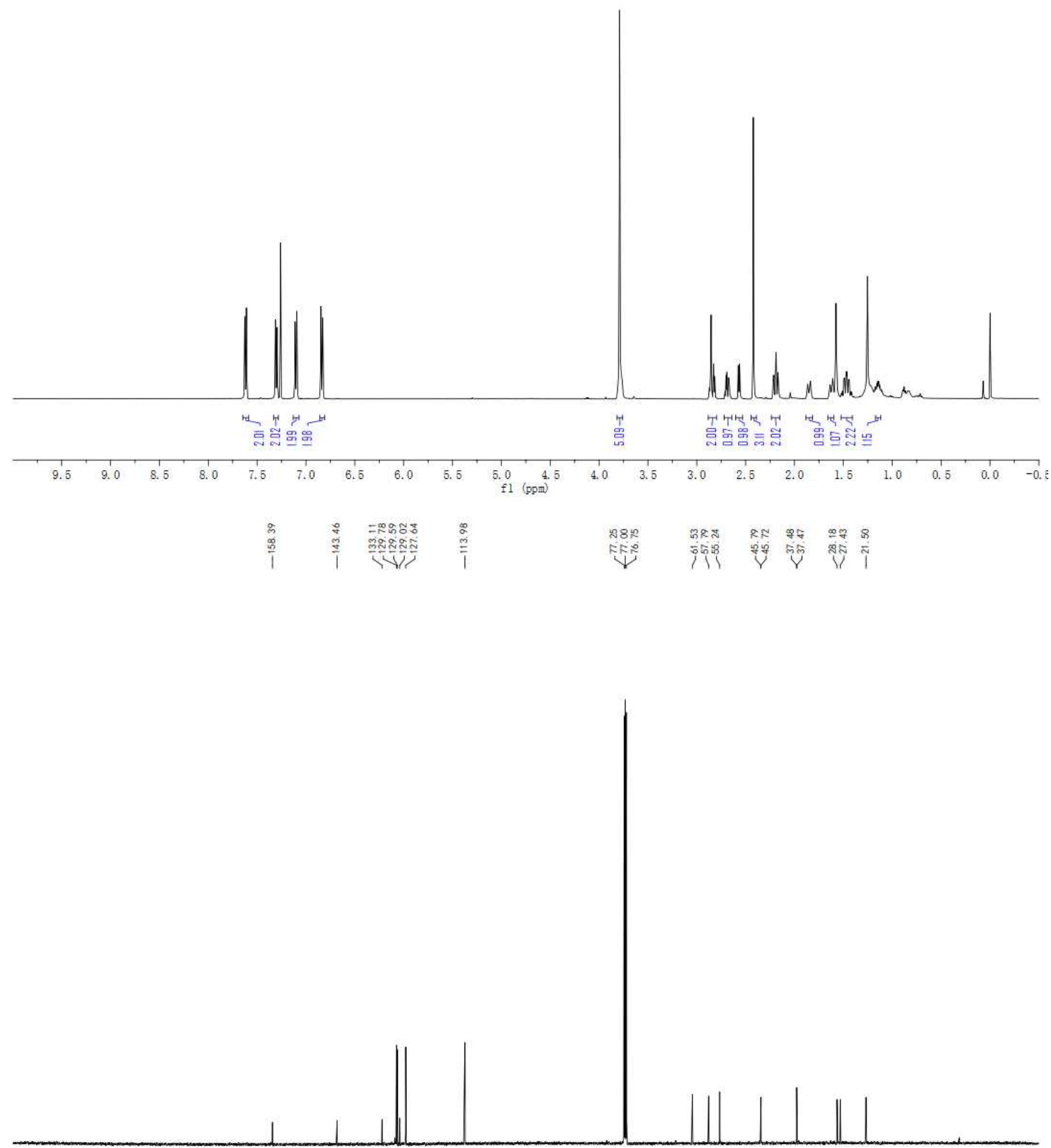

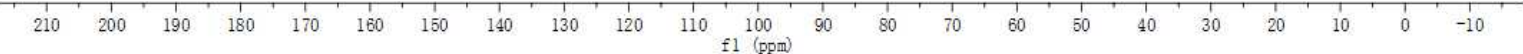


<smiles>COc1ccc(C[C@@H]2O[C@H]2C2CN(C(C)(C)C)C2)cc1</smiles>

tert-Butyl 3-((2R,3R)-3-(4-methoxybenzyl)oxiran-2-yl)azetidine-1-carboxylate ( \pm )-5ab-Boc

General Procedure B: tert-Butyl (E)-3-(3-(4-methoxyphenyl)prop-1-en-1-yl)azetidine-1carboxylate $(150 \mathrm{mg}, 0.50 \mathrm{mmol})$ was employed. Purification of the residue by FCC $(4: 1-3: 1$, hexane-EtOAc) afforded the title compound (99 $\mathrm{mg}, 62 \%)$ as a colorless oil.

${ }^{1} \mathrm{H}$ NMR $\left(400 \mathrm{MHz}, \mathrm{CDCl}_{3}\right) \delta 7.12(\mathrm{~d}, J=8.6 \mathrm{~Hz}, 2 \mathrm{H}), 6.84(\mathrm{~d}, J=8.6 \mathrm{~Hz}, 2 \mathrm{H}), 4.14-4.03(\mathrm{~m}, 2 \mathrm{H})$, $3.78(\mathrm{~s}, 3 \mathrm{H}), 2.95-2.84(\mathrm{~m}, 2 \mathrm{H}), 2.72-2.58(\mathrm{~m}, 2 \mathrm{H}), 1.79-1.74(\mathrm{~m}, 1 \mathrm{H}), 1.55(\mathrm{~d}, J=12.6 \mathrm{~Hz}, 1 \mathrm{H})$, $1.44(\mathrm{~s}, 9 \mathrm{H}), 1.39-1.19(\mathrm{~m}, 3 \mathrm{H})$;

${ }^{13} \mathrm{C}$ NMR $\left(101 \mathrm{MHz}, \mathrm{CDCl}_{3}\right) \delta 158.5,156.3,129.9,128.8,114.0,79.5,59.5,57.4,55.3,50.8$ (broad), 37.1, 29.6, 28.4;

IR (thin film) 2974, 1074, 1612, 1513, 1400, 1366, 1248, 1136, 1035, $820 \mathrm{~cm}^{-1}$;

HRMS (ESI-TOF) $\mathrm{m} / \mathrm{z}$ calcd for $\mathrm{C}_{17} \mathrm{H}_{23} \mathrm{NO}_{4} \mathrm{Na}^{+}(\mathrm{M}+\mathrm{Na})^{+}: 342.1681$, found 342.1684. 

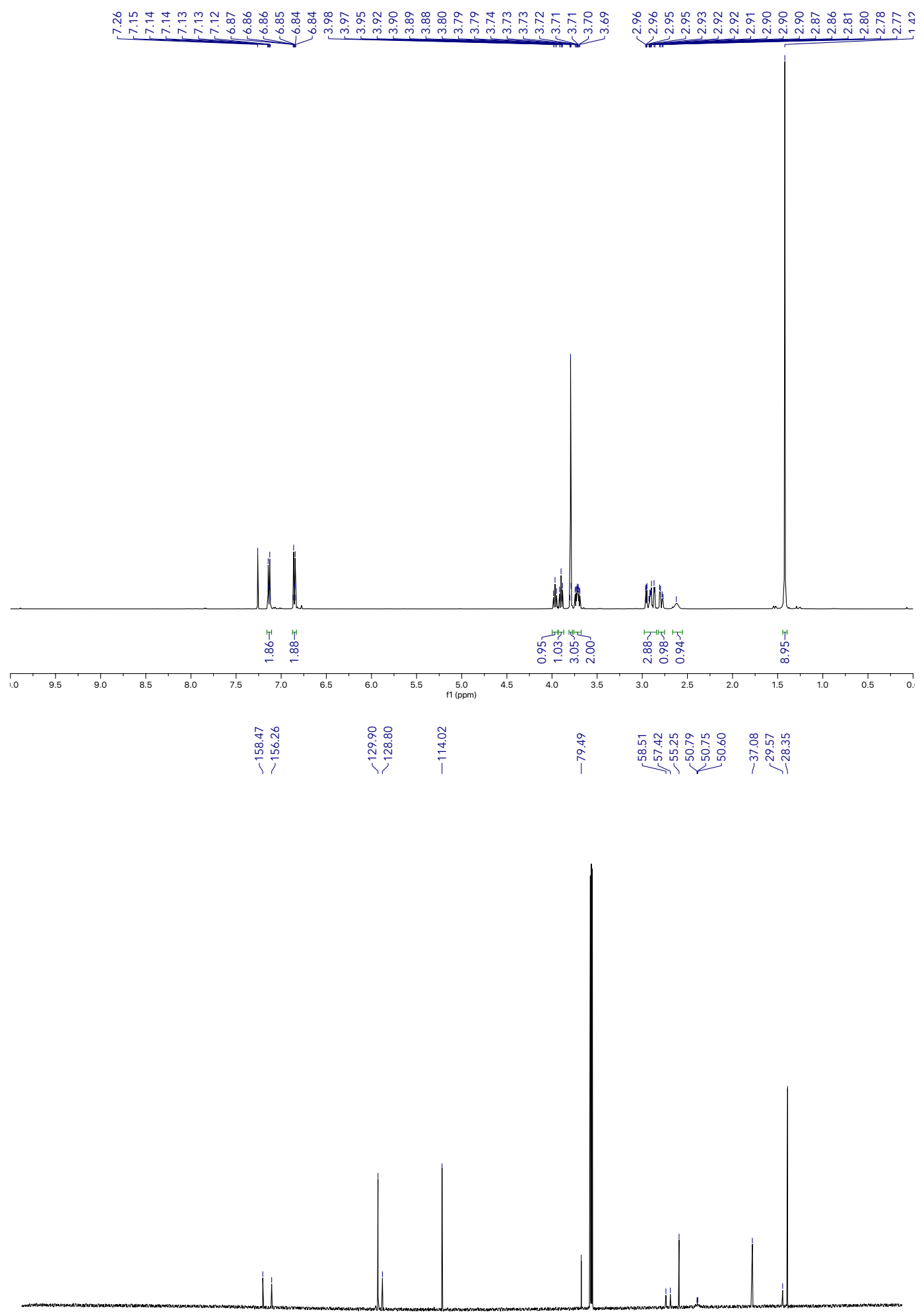
<smiles>COc1ccc(CC2OC2C2C[N+]3(S)CC23)cc1</smiles>

\section{3-((2R*,3R)*-3-(4-Methoxybenzyl)oxiran-2-yl)-1-tosylazetidine ( \pm$)-5 \mathrm{ab}-\mathrm{Ts}$}

To a solution of tert-butyl (E)-3-(3-(4-methoxyphenyl)prop-1-en-1-yl)azetidine-1-carboxylate (303 $\mathrm{mg}, 1.00 \mathrm{mmol})$ in $\mathrm{CH}_{2} \mathrm{Cl}_{2}(10 \mathrm{~mL})$ was added with $\mathrm{CF}_{3} \mathrm{COOH}(225 \mathrm{mg}, 1.10 \mathrm{mmol})$ and the reaction was stirred at room temperature for 8 hours. The mixture was concentrated and the crude residue re-dissolved in $\mathrm{CH}_{2} \mathrm{Cl}_{2}(10 \mathrm{~mL})$. $\mathrm{Et}_{3} \mathrm{~N}(151 \mathrm{mg}, 1.50 \mathrm{mmol})$ and $\mathrm{TsCl}(210 \mathrm{mg}, 1.10 \mathrm{mmol})$ were added sequentially. The mixture was stirred at room temperature for 6 hours. After this time the mixture was filtrated through celite $\AA$ and concentrated in vacuo. The crude residue was redissolved in $\mathrm{CH}_{2} \mathrm{Cl}_{2}(10 \mathrm{~mL})$ and cooled to $0{ }^{\circ} \mathrm{C} . \mathrm{m}$-CPBA $(75 \%, 286 \mathrm{mg}, 1.25 \mathrm{mmol})$ was added and the reaction was allowed to warm to room temperature and stirred for a further 4 hours. The reaction was quenched by addition of saturated aq. $\mathrm{NaHSO}_{3}(5 \mathrm{~mL})$ and the mixture was filtered through celite $囚$. The filtrate was washed with saturated aq. $\mathrm{NaHCO}_{3}(3 \times 5 \mathrm{~mL})$, dried $\left(\mathrm{Na}_{2} \mathrm{SO}_{4}\right)$, filtered, and concentrated in vacuo. Purification of the residue by FCC $(3: 1$, hexane-EtOAc) afforded the title compound (227 mg, 61\% yield over 3 steps) as a light yellow oil.

${ }^{1} \mathrm{H}$ NMR $\left(500 \mathrm{MHz}, \mathrm{CDCl}_{3}\right) \delta 7.69(\mathrm{~d}, J=7.9 \mathrm{~Hz}, 2 \mathrm{H}), 7.36(\mathrm{~d}, J=7.8 \mathrm{~Hz}, 2 \mathrm{H}), 7.05$ (d, J = 8.0 $\mathrm{Hz}, 2 \mathrm{H}), 6.83(\mathrm{~d}, J=8.1 \mathrm{~Hz}, 2 \mathrm{H}), 3.84-3.71(\mathrm{~m}, 5 \mathrm{H}), 3.53(\mathrm{dd}, J=14.0,7.0 \mathrm{~Hz}, 2 \mathrm{H}), 2.80-2.66$ $(\mathrm{m}, 4 \mathrm{H}), 2.51$ (dd, $J=13.4,6.8 \mathrm{~Hz}, 1 \mathrm{H}), 2.44$ (s, 3H);

${ }^{13} \mathrm{C}$ NMR $\left(126 \mathrm{MHz}, \mathrm{CDCl}_{3}\right) \delta 158.4,144.1,131.2,129.7,129.7,128.5,128.3,113.9,57.4,57.1$, $55.2,52.1,52.0,36.8,29.2,21.5$;

IR (thin film): 2960, 2328, 1612, 1598, 1512, 1344, 1247, 1160, 1040, 816, $721 \mathrm{~cm}^{-1}$;

HRMS (ESI-TOF) $\mathrm{m} / \mathrm{z}$ calcd for $\mathrm{C}_{20} \mathrm{H}_{23} \mathrm{NNaO}_{4} \mathrm{~S}^{+}(\mathrm{M}+\mathrm{Na})^{+}: 396.1240$, found 396.1246. 

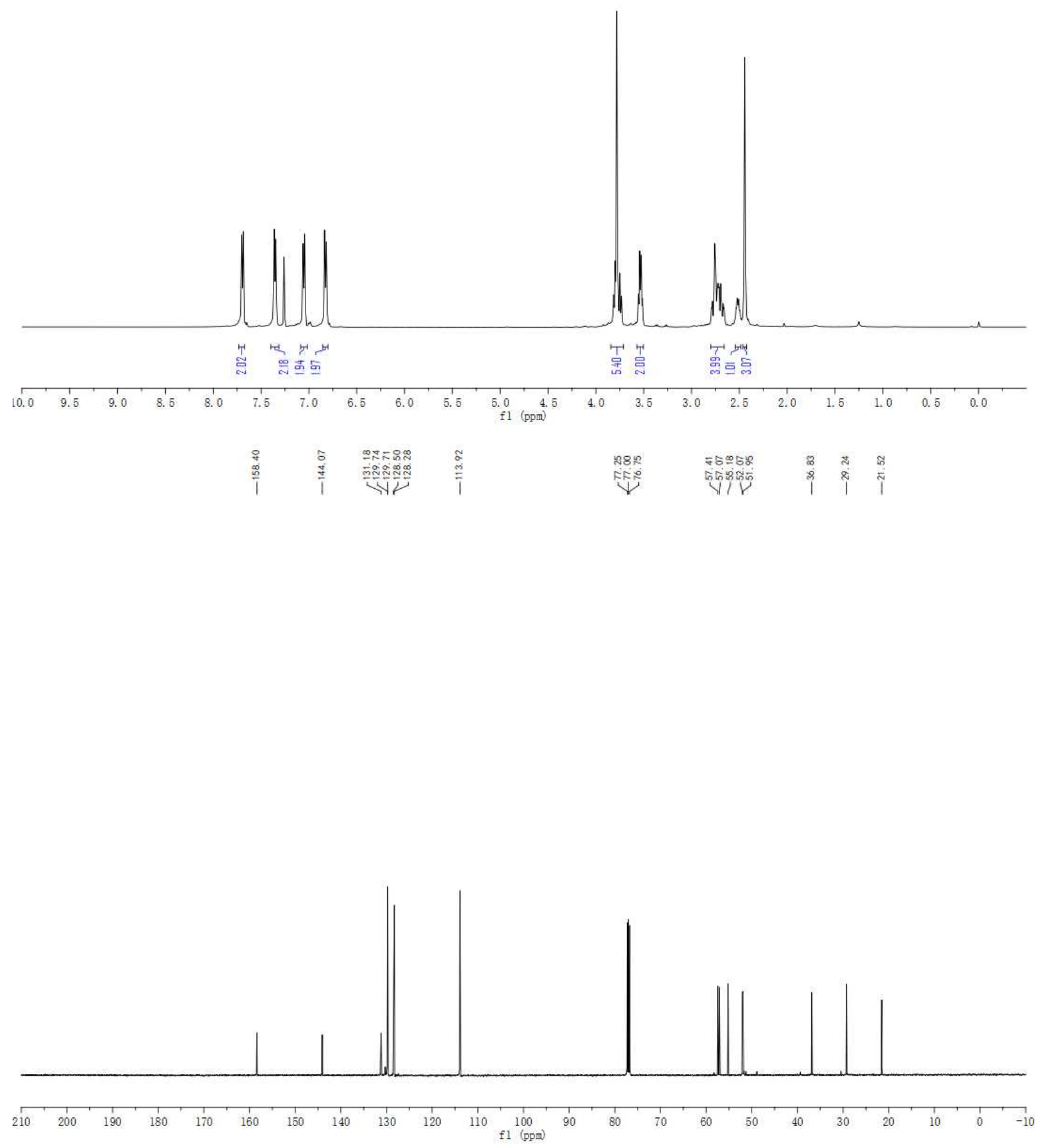


\section{$\mathrm{SnCl}_{4}$ reaction}

\section{General Procedure C: for the $\mathrm{SnCl}_{4}$ ring opening of epoxides}<smiles>[R]C[C@@H]1O[C@@H]1C[13CH3]</smiles>

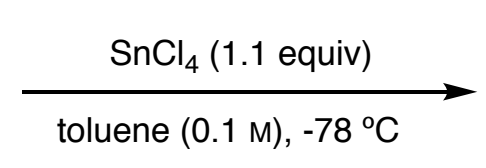<smiles>[R]C[C@@H](O)[C@@H](Cl)CBr</smiles>

To an oven-dried reaction tube, charged with a stir bar, was added the epoxide derivative $(0.10$ $\mathrm{mmol})$. The tube was sealed with a septum and purged with argon after which anhydrous toluene $(1 \mathrm{~mL})$ was added. The reaction mixture was cooled to $-78{ }^{\circ} \mathrm{C}$ and $\mathrm{SnCl}_{4}(1 \mathrm{M}$ in heptane, 0.11 $\mathrm{mL}, 0.11 \mathrm{mmol}$ ) was added dropwise via syringe over five minutes. The reaction was stirred this temperature for six hours and then slowly warmed to room temperature. The mixture was quenched by pouring into saturated aq. $\mathrm{NaHCO}_{3}(1 \mathrm{~mL})$. The solution was filtered through celite ${ }^{\circledR}$ and concentrated in vacuo. Purification of the residue by FCC, under the conditions noted, afforded the pure chlorohydrin. 


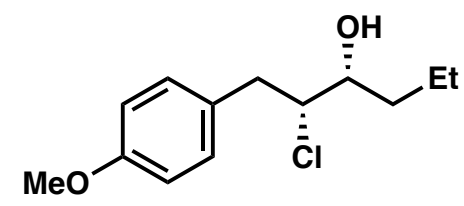

$\left(2 R^{*}, 3 R^{*}\right)-2-C h l o r o-1-(4-m e t h o x y p h e n y l) h e x a n-3-o l ~( \pm)-6 a$

General Procedure C: Epoxide $( \pm)-5 a(21 \mathrm{mg}, 0.10 \mathrm{mmol})$ was employed. Purification of the residue by FCC (10:1, hexane-EtOAc) afforded the title compound (22 $\mathrm{mg}, 90 \%)$ as a colorless oil.

${ }^{1} \mathrm{H}$ NMR $\left(400 \mathrm{MHz}, \mathrm{CDCl}_{3}\right) \delta$ 7.18-7.14 (m, 2H), 6.88-6.84 (m, 2H), $4.06(\mathrm{td}, J=7.4,2.5 \mathrm{~Hz}, 1 \mathrm{H})$, $3.80(\mathrm{~s}, 3 \mathrm{H}), 3.67-3.59(\mathrm{~m}, 1 \mathrm{H}), 3.17(\mathrm{dd}, J=14.1,7.0 \mathrm{~Hz}, 1 \mathrm{H}), 3.03(\mathrm{dd}, J=14.1,7.8 \mathrm{~Hz}, 1 \mathrm{H})$, $1.77(\mathrm{~d}, J=8.3 \mathrm{~Hz}, 1 \mathrm{H}), 1.63(\mathrm{ddt}, J=9.3,4.4,2.3 \mathrm{~Hz}, 1 \mathrm{H}), 1.56-1.41(\mathrm{~m}, 2 \mathrm{H}), 1.40-1.30(\mathrm{~m}, 1 \mathrm{H})$, $0.91(\mathrm{t}, J=7.2 \mathrm{~Hz}, 3 \mathrm{H})$;

${ }^{13} \mathrm{C}$ NMR $\left(101 \mathrm{MHz}, \mathrm{CDCl}_{3}\right) \delta$ 158.4, 130.3, 129.7, 113.9, 71.7, 68.9, 55.2, 40.5, 37.3, 18.8, 13.9;

IR ( $\mathrm{NaCl}$, thin film) 3451, 2957, 2930, 2871, 1614, 1514, 1455, 1301, 1180, 1034, $820 \mathrm{~cm}^{-1}$;

HRMS (ESI-TOF) $\mathrm{m} / \mathrm{z}$ calcd for $\mathrm{C}_{13} \mathrm{H}_{19}{ }^{35} \mathrm{CINaO}_{2}{ }^{+}(\mathrm{M}+\mathrm{Na})^{+}:$265.0966, found 265.0964. 


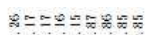

inis

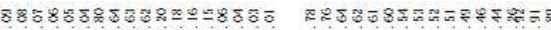

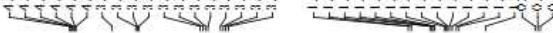

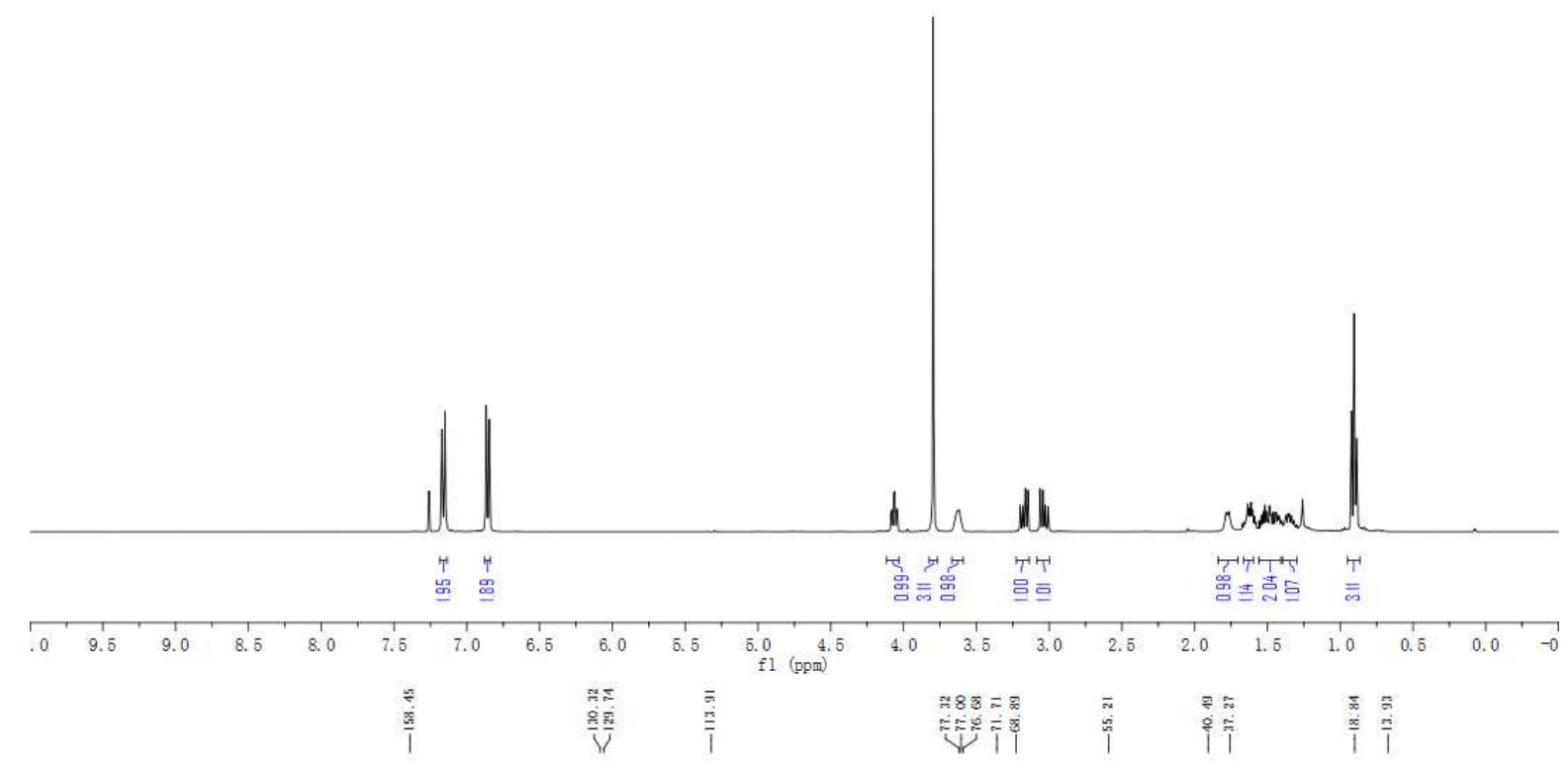


$\overbrace{M e O}^{(1)}$

$\left(2 R^{*}, 3 R^{*}\right)$-2-Bromo-1-(4-methoxyphenyl)hexan-3-ol $( \pm)-6 \mathrm{a}_{\mathrm{Br}}$

General Procedure C: Epoxide $( \pm)-5 a(21 \mathrm{mg}, 0.10 \mathrm{mmol})$ was employed. In a modification to the general procedure $\mathrm{SnBr}_{4}(1 \mathrm{M}$ solution in toluene, $0.11 \mathrm{~mL}, 0.11 \mathrm{mmol}$ ) was employed. Purification of the residue by FCC (10:1, hexane-EtOAc) afforded the title compound ( $26 \mathrm{mg}, 91 \%$ yield) as a colorless oil.

${ }^{1} \mathrm{H}$ NMR $\left(500 \mathrm{MHz}, \mathrm{CDCl}_{3}\right) \delta 7.15(\mathrm{~d}, \mathrm{~J}=8.4 \mathrm{~Hz}, 2 \mathrm{H}), 6.85(\mathrm{~d}, J=8.5 \mathrm{~Hz}, 2 \mathrm{H}), 4.21$ (td, J = 7.5, $2.0 \mathrm{~Hz}, 1 \mathrm{H}$ ), $3.80(\mathrm{~s}, 3 \mathrm{H}), 3.42(\mathrm{~s}, 1 \mathrm{H}), 3.30$ (dd, $J=14.2,7.6 \mathrm{~Hz}, 1 \mathrm{H}), 3.17$ (dd, J = 14.2, $7.6 \mathrm{~Hz}$, $1 \mathrm{H}), 1.71(\mathrm{~d}, J=9.3 \mathrm{~Hz}, 1 \mathrm{H}), 1.66-1.60(\mathrm{~m}, 1 \mathrm{H}), 1.49(\mathrm{ddd}, J=13.9,9.1,4.1 \mathrm{~Hz}, 1 \mathrm{H}), 1.45-1.39$ $(\mathrm{m}, 1 \mathrm{H}), 1.37-1.31(\mathrm{~m}, 1 \mathrm{H}), 0.90(\mathrm{t}, \mathrm{J}=7.3 \mathrm{~Hz}, 3 \mathrm{H})$;

${ }^{13} \mathrm{C}$ NMR $\left(126 \mathrm{MHz}, \mathrm{CDCl}_{3}\right) \delta$ 158.5, 130.4, 130.2, 113.9, 71.4, 64.7, 55.2, 41.4, 38.7, 18.8, 13.9;

IR ( $\mathrm{NaCl}$, thin film) 3593, 3440, 2958, 2931, 1650, 1613, 1513, 1249, 1147, 1085, $749 \mathrm{~cm}^{-1}$;

HRMS (ESI-TOF) $m / z$ calcd for $\mathrm{C}_{13} \mathrm{H}_{19}{ }^{79} \mathrm{BrNaO}_{2}{ }^{+}(\mathrm{M}+\mathrm{Na})^{+}:$309.0466, found 309.0468. 

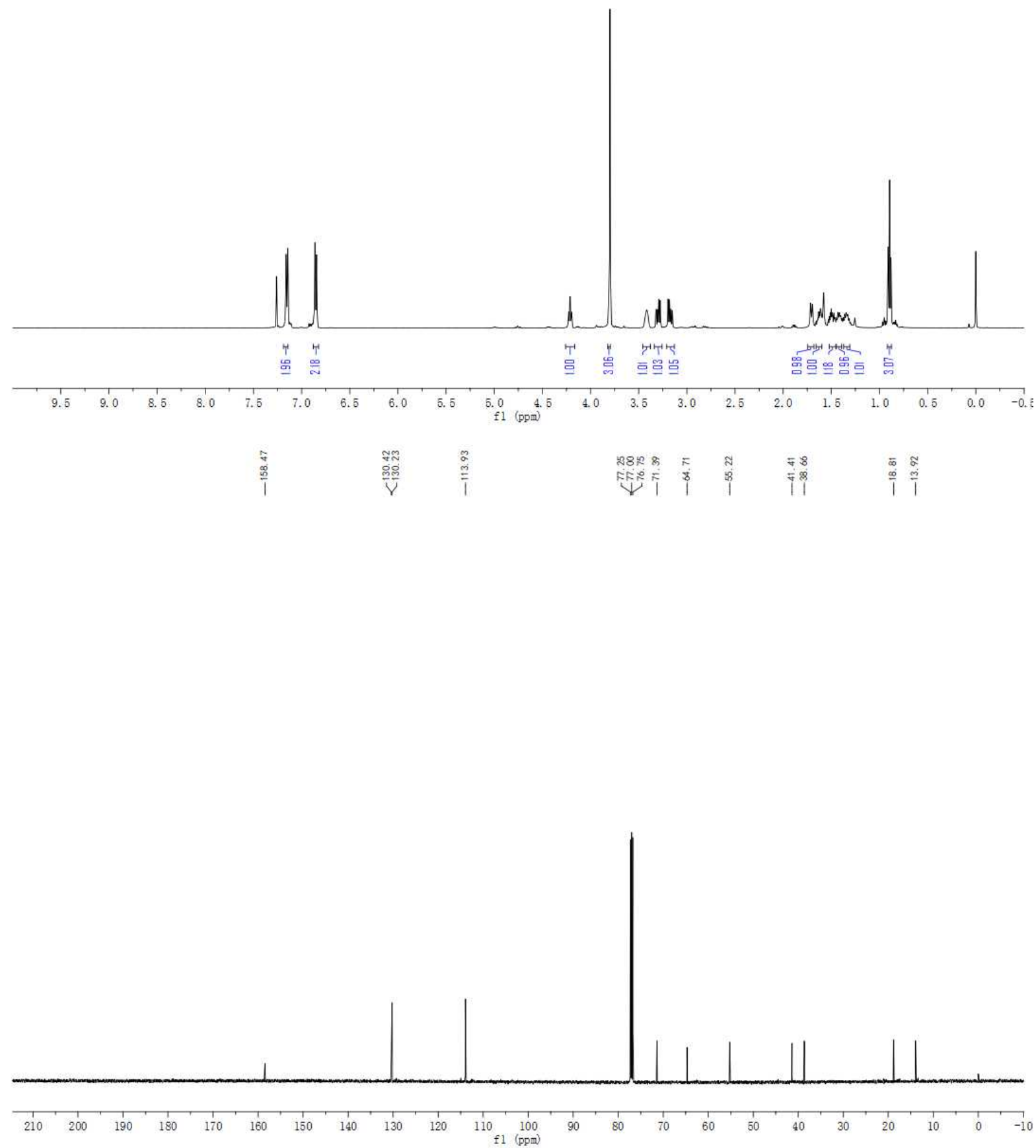
<smiles>CCC[C@H](O)[C@H](Cl)Cc1ccc(OC)c(OC)c1</smiles>

\section{$\left(2 R^{*}, 3 R^{*}\right)$-2-Chloro-1-(3,4-dimethoxyphenyl)hexan-3-ol ( \pm )-6b}

General Procedure C: Epoxide $( \pm)-5 b(22 \mathrm{mg}, 0.10 \mathrm{mmol})$ was employed. Purification of the residue by (10:1, hexane-EtOAc) afforded the title compound (22 $\mathrm{mg}, 80 \%)$ as a colorless oil.

${ }^{1} \mathrm{H}$ NMR (500 MHz, $\left.\mathrm{CDCl}_{3}\right)$ ठ 6.86-6.74 (m, 3H), 4.08 (td, $\left.J=7.4,2.5 \mathrm{~Hz}, 1 \mathrm{H}\right), 3.88(\mathrm{~s}, 3 \mathrm{H}), 3.87$ (s, 3H), 3.64 (dddd, $J=8.6,7.3,4.6,2.4 \mathrm{~Hz}, 1 \mathrm{H}$ ), 3.17 (dd, $J=14.0,7.1 \mathrm{~Hz}, 1 \mathrm{H}), 3.04$ (dd, $J=$ 14.1, 7.7 Hz, 1H), 1.72 (d, $J=9.2 \mathrm{~Hz}, 1 \mathrm{H}$ ), 1.63 (dddd, $J=12.8,9.5,8.2,4.8 \mathrm{~Hz}, 1 \mathrm{H}), 1.57-1.40$ $(\mathrm{m}, 2 \mathrm{H}), 1.36$ (dddd, $J=13.2,10.3,6.6,4.9 \mathrm{~Hz}, 1 \mathrm{H}), 0.91(\mathrm{t}, J=7.3 \mathrm{~Hz}, 3 \mathrm{H})$;

${ }^{13} \mathrm{C}$ NMR $\left(126 \mathrm{MHz}_{\mathrm{CDCl}}\right) \delta 148.8,147.9,130.2,121.4,112.5,111.2,71.7,68.8,55.9,55.9$, 41.0, 37.3, 18.9, 13.9;

IR ( $\mathrm{NaCl}$, thin film) 3489, 2958, 2933, 2872, 1591, 1516, 1464, 1264, 1236, 1142, 1028, $693 \mathrm{~cm}^{-1}$;

HRMS (ESI-TOF) $\mathrm{m} / \mathrm{z}$ calcd for $\mathrm{C}_{14} \mathrm{H}_{21}{ }^{35} \mathrm{CINaO}_{3}{ }^{+}(\mathrm{M}+\mathrm{Na})^{+}:$295.1077, found 295.1079. 

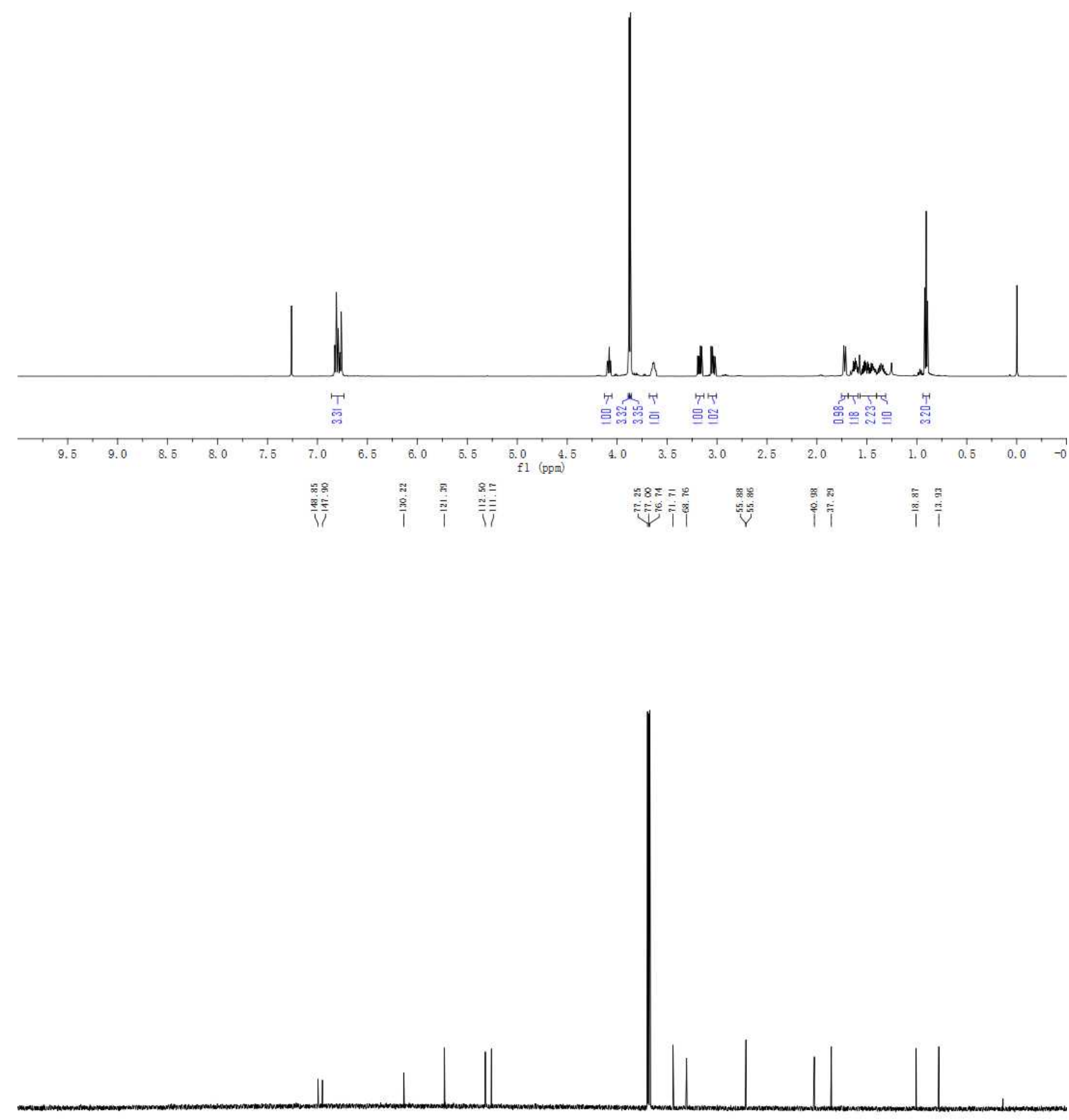

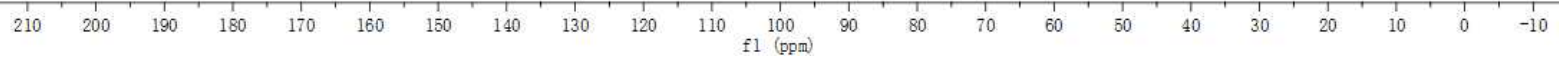


<smiles>CCC[C@@H](O)[C@H](Cl)Cc1ccc2c(c1)OCO2</smiles>

\section{$\left(2 R^{*}, 3 R^{*}\right)-1-(B e n z o[d][1,3]$ dioxol-5-yl)-2-chlorohexan-3-ol ( \pm$)-6 \mathrm{c}$}

General Procedure C: Epoxide $( \pm)-5 \mathrm{c}(24 \mathrm{mg}, 0.10 \mathrm{mmol})$ was employed. Purification of the residue by FCC (5:1, hexane-EtOAc) afforded the title compound (23 mg, 91\%) as a colorless oil.

${ }^{1} \mathrm{H}$ NMR $\left(400 \mathrm{MHz}, \mathrm{CDCl}_{3}\right) \delta$ 6.79-6.66 (m, 3H), $5.94(\mathrm{~s}, 2 \mathrm{H}), 4.04(\mathrm{td}, J=7.4,2.5 \mathrm{~Hz}, 1 \mathrm{H}), 3.63$ $(\mathrm{q}, J=5.7 \mathrm{~Hz}, 1 \mathrm{H}), 3.14(\mathrm{dd}, J=14.1,7.0 \mathrm{~Hz}, 1 \mathrm{H}), 3.00(\mathrm{dd}, J=14.0,7.8 \mathrm{~Hz}, 1 \mathrm{H}), 1.73(\mathrm{~d}, J=$ $8.4 \mathrm{~Hz}, 1 \mathrm{H}), 1.66-1.57(\mathrm{~m}, 1 \mathrm{H}), 1.55-1.42(\mathrm{~m}, 2 \mathrm{H}), 1.36$ (dddd, $J=14.4,9.9,5.1,2.5 \mathrm{~Hz}, 1 \mathrm{H})$, $0.91(\mathrm{t}, J=7.2 \mathrm{~Hz}, 3 \mathrm{H})$;

${ }^{13} \mathrm{C}$ NMR $\left(101 \mathrm{MHz}, \mathrm{CDCl}_{3}\right) \delta 147.7,146.4,131.4,122.4,109.6,108.3,100.9,71.7,68.7,41.0$, $37.3,18.8,13.9$;

IR ( $\mathrm{NaCl}$, thin film) 3452, 3406, 3384, 2957, 2933, 2873, 2360, 1504, 1445, 1250, 1039, $817 \mathrm{~cm}^{-1}$; HRMS (ESI-TOF) $\mathrm{m} / \mathrm{z}$ calcd for $\mathrm{C}_{13} \mathrm{H}_{17}{ }^{35} \mathrm{CINaO}_{3}{ }^{+}(\mathrm{M}+\mathrm{Na})^{+}:$279.0758, found 279.0753 . 

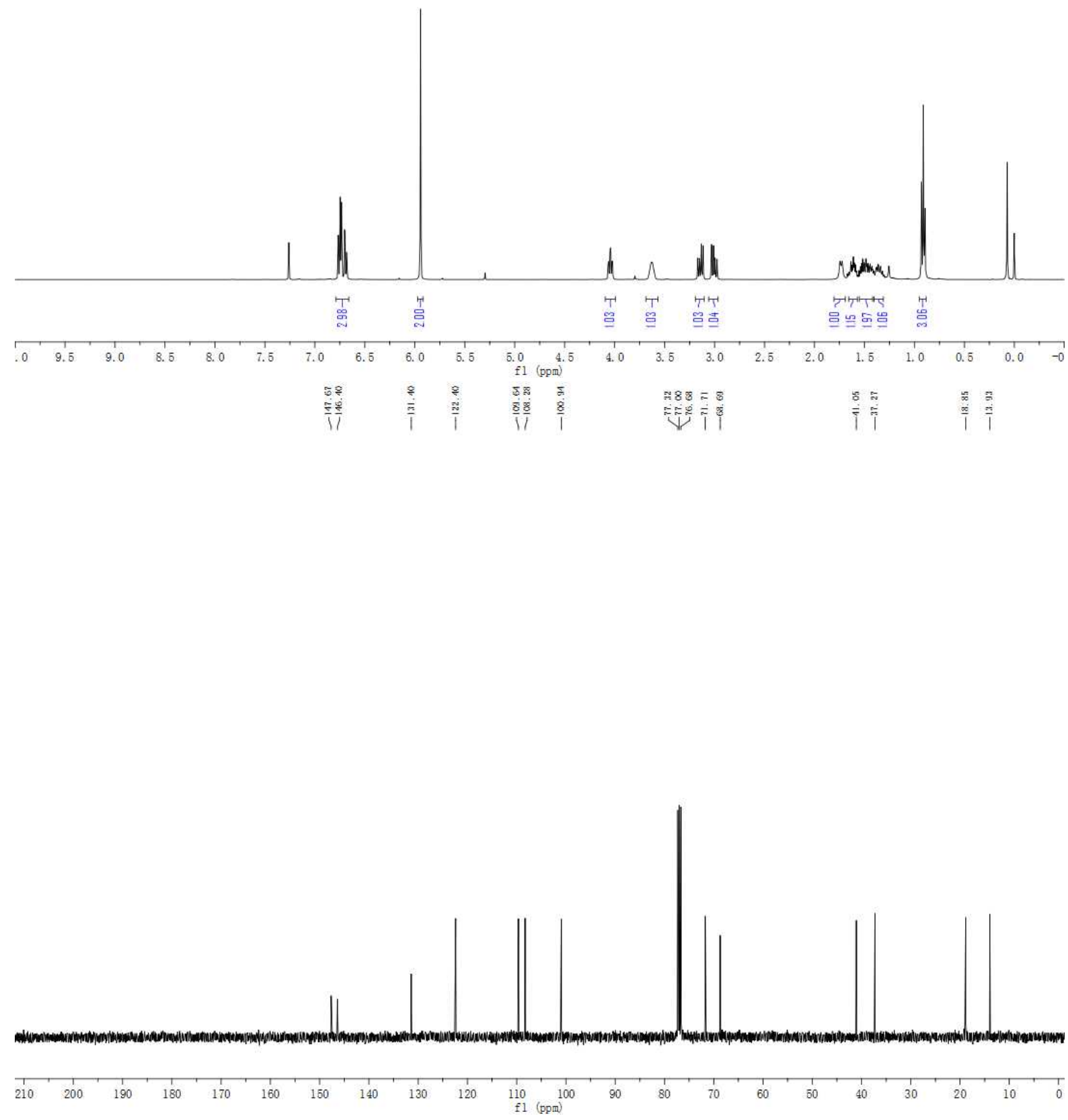
<smiles>CCC[C@@H](O)[C@H](Cl)Cc1ccc2ccccc2c1</smiles>

$\left(2 R^{*}, 3 R^{*}\right)-2-C h l o r o-1-($ naphthalen-2-yl)hexan-3-ol $( \pm)-6 \mathrm{~d}$

General Procedure C: Epoxide $( \pm)-5 d(23 \mathrm{mg}, 0.10 \mathrm{mmol})$ was employed. Purification of the residue by FCC (10:1, hexane-EtOAc) afforded the title compound ( $23 \mathrm{mg}, 86 \%)$ as a colorless oil.

${ }^{1} \mathrm{H}$ NMR $\left(500 \mathrm{MHz}, \mathrm{CDCl}_{3}\right) \delta 7.82(\mathrm{dd}, J=9.1,7.5 \mathrm{~Hz}, 3 \mathrm{H}), 7.71(\mathrm{~d}, J=1.8 \mathrm{~Hz}, 1 \mathrm{H}), 7.51-7.44$ $(\mathrm{m}, 2 \mathrm{H}), 7.38(\mathrm{dd}, J=8.4,1.8 \mathrm{~Hz}, 1 \mathrm{H}), 4.23(\mathrm{td}, J=7.4,2.5 \mathrm{~Hz}, 1 \mathrm{H}), 3.68$ (tdd, $J=8.4,4.7,2.5$ $\mathrm{Hz}, 1 \mathrm{H}), 3.41(\mathrm{dd}, J=13.9,7.0 \mathrm{~Hz}, 1 \mathrm{H}), 3.27(\mathrm{dd}, J=14.0,7.8 \mathrm{~Hz}, 1 \mathrm{H}), 1.81(\mathrm{~d}, J=9.2 \mathrm{~Hz}, 1 \mathrm{H})$, 1.65 (dddd, $J=13.2,9.9,8.3,5.0 \mathrm{~Hz}, 1 \mathrm{H}$ ), 1.54 (dddd, $J=13.5,10.3,7.8,4.8 \mathrm{~Hz}, 1 \mathrm{H}$ ), $1.51-1.42$ $(\mathrm{m}, 1 \mathrm{H}), 1.42-1.31(\mathrm{~m}, 1 \mathrm{H}), 0.91(\mathrm{t}, J=7.3 \mathrm{~Hz}, 3 \mathrm{H})$;

${ }^{13} \mathrm{C}$ NMR $\left(126 \mathrm{MHz}, \mathrm{CDCl}_{3}\right) \delta 135.2,133.5,132.4,128.2,128.0,127.6,127.6,127.4,126.1$, 125.7, 71.9, 68.5, 41.5, 37.3, 18.9, 14.0;

IR ( $\mathrm{NaCl}$, thin film) 3564, 3418, 3054, 2959, 2932, 2872, 1601, 1510, 1380, 1272, 1074, 818, $756 \mathrm{~cm}^{-1}$;

HRMS (ESI-TOF) $\mathrm{m} / \mathrm{z}$ calcd for $\mathrm{C}_{16} \mathrm{H}_{19}{ }^{35} \mathrm{CINaO}^{+}(\mathrm{M}+\mathrm{Na})^{+}:$285.1022, found 285.1024. 

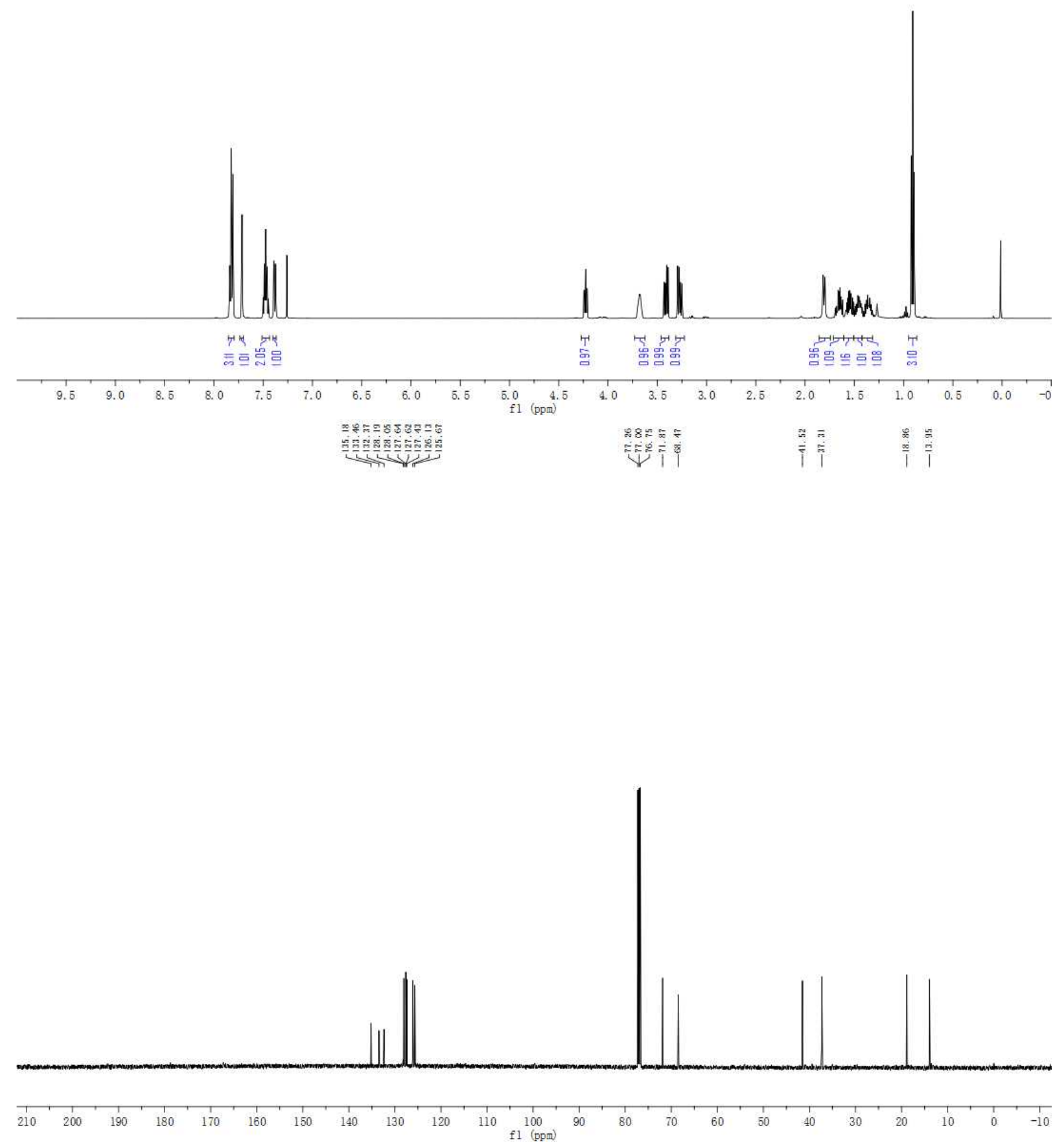
<smiles>CC[C@H](O)[C@H](Cl)Cc1ccc(C)cc1</smiles>

$\left(2 R^{*}, 3 R^{*}\right)-2-C h l o r o-1-(p$-tolyl)hexan-3-ol $( \pm)-6 e$

General Procedure C: Epoxide $( \pm)-5 e(19 \mathrm{mg}, 0.10 \mathrm{mmol})$ was employed. Purification of the residue by FCC (10:1, hexane-EtOAc) afforded the title compound (18 $\mathrm{mg}, 82 \%)$ as a colorless oil.

${ }^{1} \mathrm{H}$ NMR $\left(500 \mathrm{MHz}, \mathrm{CDCl}_{3}\right) \delta 7.13(\mathrm{~s}, 4 \mathrm{H}), 4.09$ (ddd, $\left.J=7.9,6.9,2.6 \mathrm{~Hz}, 1 \mathrm{H}\right), 3.64$ (ddd, $J=7.8$, 4.6, $2.5 \mathrm{~Hz}, 1 \mathrm{H}), 3.19$ (dd, J = 14.0, $6.9 \mathrm{~Hz}, 1 \mathrm{H}), 3.06$ (dd, J = 14.0, 7.9 Hz, 1H), $2.34(\mathrm{~s}, 3 \mathrm{H}), 1.75$ $(\mathrm{s}, 1 \mathrm{H}), 1.62(\mathrm{ddt}, J=9.4,8.0,4.7 \mathrm{~Hz}, 1 \mathrm{H}), 1.55-1.42(\mathrm{~m}, 2 \mathrm{H}), 1.39-1.31(\mathrm{~m}, 1 \mathrm{H}), 0.91(\mathrm{t}, J=7.3$ $\mathrm{Hz}, 3 \mathrm{H})$;

${ }^{13} \mathrm{C}$ NMR $\left(126 \mathrm{MHz} \mathrm{CDCl}_{3}\right) \delta 136.4,134.6,129.2,129.2,71.9,68.8,40.9,37.3,21.1,18.8,13.9$;

IR ( $\mathrm{NaCl}$, thin film) 3429, 3376, 3348, 3022, 2960, 2930, 2871, 2365, 2357, 2343, 1653, 1516 , 1126, $726 \mathrm{~cm}^{-1}$;

HRMS (ESI-TOF) $\mathrm{m} / \mathrm{z}$ calcd for $\mathrm{C}_{13} \mathrm{H}_{19}{ }^{35} \mathrm{CINaO}^{+}(\mathrm{M}+\mathrm{Na})^{+}:$249.1022, found 249.1012. 

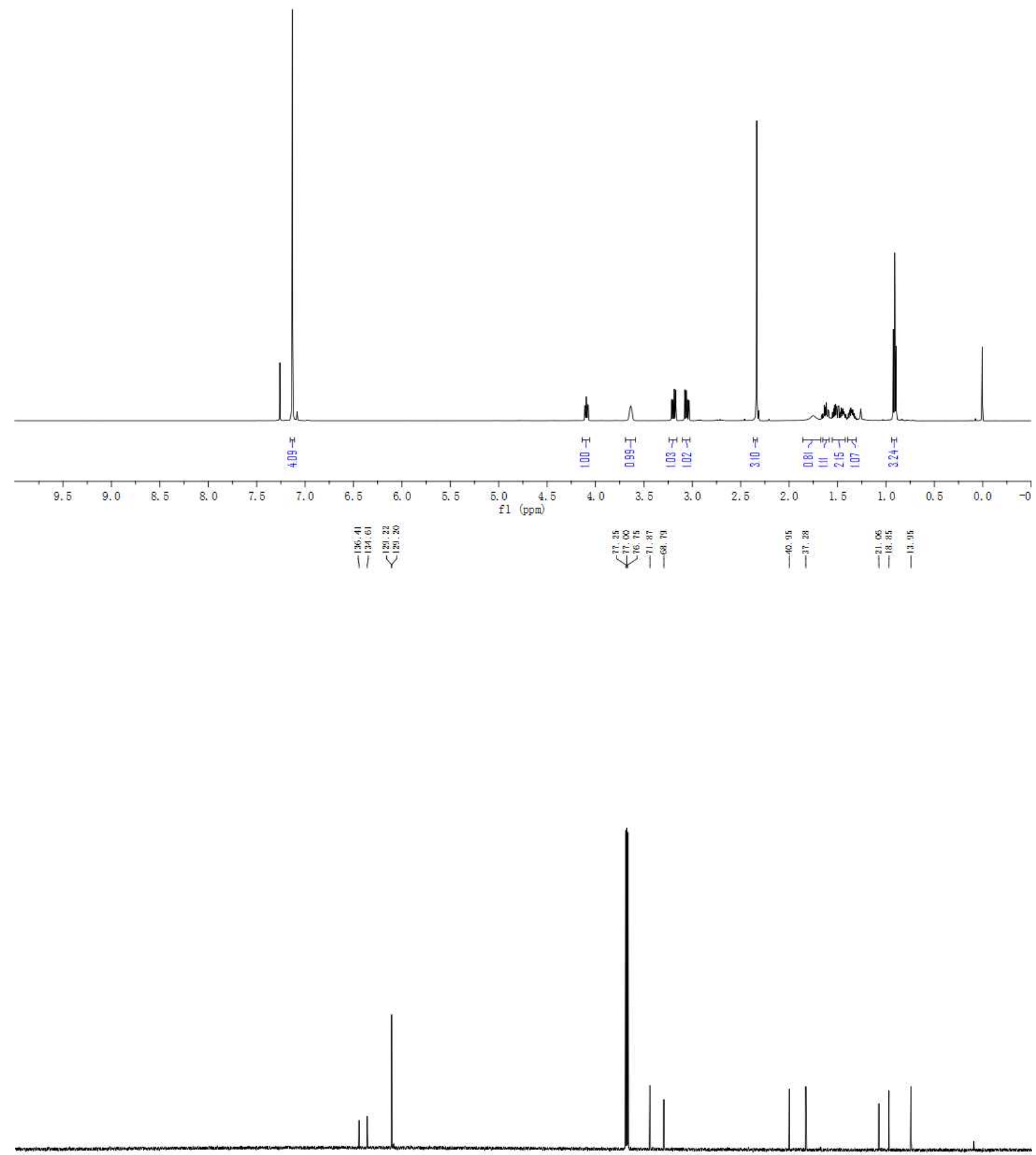

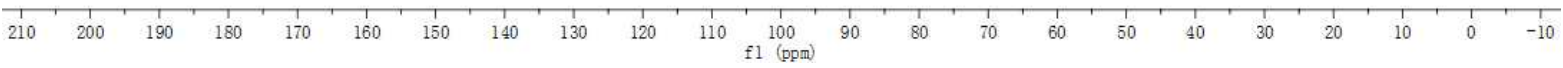


<smiles>CCC[C@@H](O)[C@H](Cl)Cc1ccccc1</smiles>

$\left(2 R^{*}, 3 R^{*}\right)$-2-Chloro-1-phenylhexan-3-ol ( \pm )-6f

General Procedure C: Epoxide $( \pm)-5 f(18 \mathrm{mg}, 0.10 \mathrm{mmol})$ was employed. Purification of the residue by FCC (10:1, hexane-EtOAc) afforded the title compound (18 $\mathrm{mg}, 87 \%)$ as a colorless oil.

${ }^{1} \mathrm{H}$ NMR $\left(500 \mathrm{MHz}, \mathrm{CDCl}_{3}\right) \delta 7.32(\mathrm{dd}, J=8.3,6.3 \mathrm{~Hz}, 2 \mathrm{H}), 7.27-7.23(\mathrm{~m}, 3 \mathrm{H}), 4.11$ (ddd, $J=7.9$, 6.9, $2.6 \mathrm{~Hz}, 1 \mathrm{H}), 3.63(\mathrm{dp}, J=11.5,3.2 \mathrm{~Hz}, 1 \mathrm{H}), 3.23(\mathrm{dd}, J=13.9,6.9 \mathrm{~Hz}, 1 \mathrm{H}), 3.10(\mathrm{dd}, J=$ 14.0, 7.9 Hz, 1H), $1.74(\mathrm{~d}, J=9.1 \mathrm{~Hz}, 1 \mathrm{H}), 1.63(\mathrm{dtd}, J=9.8,5.0,2.4 \mathrm{~Hz}, 1 \mathrm{H}), 1.55-1.49(\mathrm{~m}, 1 \mathrm{H})$, 1.45 (dddd, $J=12.6,9.9,7.2,5.0 \mathrm{~Hz}, 1 \mathrm{H}), 1.41-1.32(\mathrm{~m}, 1 \mathrm{H}), 0.91(\mathrm{t}, J=7.3 \mathrm{~Hz}, 3 \mathrm{H})$;

${ }^{13} \mathrm{C}$ NMR $\left(126 \mathrm{MHz}_{\mathrm{CDCl}}\right)$ $\delta$ 137.7, 129.3, 128.5, 126.8, 71.9, 68.6, 41.4, 37.3, 18.8, 13.9;

IR ( $\mathrm{NaCl}$, thin film) 3506, 3464, 3403, 3030, 2958, 2930, 2874, 2360, 1491, 1454, 1033, $945 \mathrm{~cm}^{-1}$; HRMS (ESI-TOF) $m / z$ calcd for $\mathrm{C}_{12} \mathrm{H}_{17}{ }^{35} \mathrm{CINaO}^{+}(\mathrm{M}+\mathrm{Na})^{+}:$235.0866, found 235.0869. 

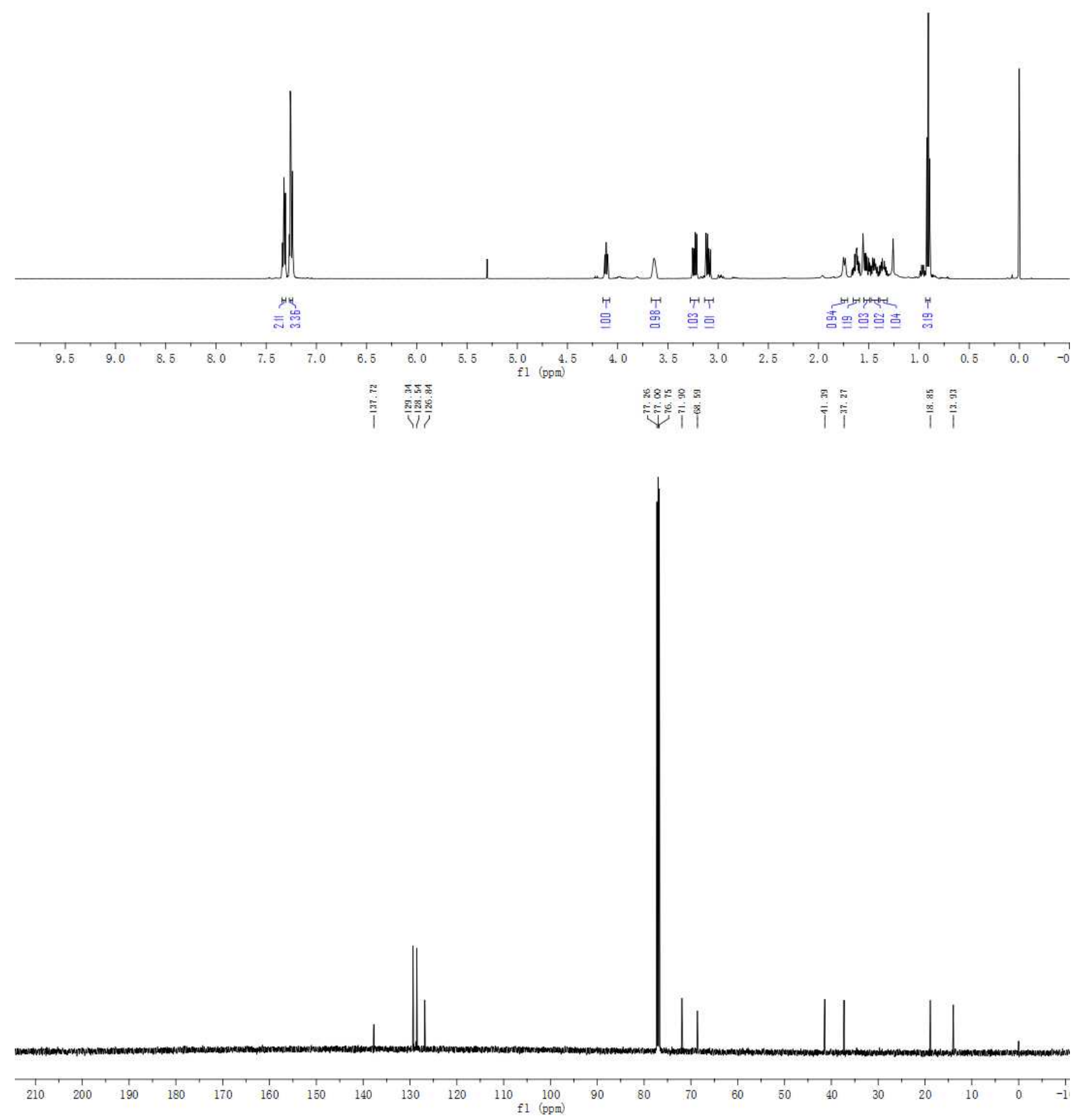
<smiles>CC[C@H](O)[C@@H](Cl)Cc1ccc2c(ccn2[As])c1</smiles>

\section{$\left(2 R^{*}, 3 R^{*}\right)-2-C h l o r o-1-(1-t o s y l-1 H$-indol-5-yl)hexan-3-ol $( \pm)-6 \mathrm{~g}$}

General Procedure C: Epoxide $( \pm)-5 \mathrm{~g}(37 \mathrm{mg}, 0.10 \mathrm{mmol})$ was employed. Purification of the residue by FCC (3:1, hexane-EtOAc) afforded the title compound (37 mg, 91\%) as a colorless oil.

${ }^{1} \mathrm{H}$ NMR $\left(500 \mathrm{MHz}, \mathrm{CDCl}_{3}\right) \delta 7.92(\mathrm{~d}, J=8.5 \mathrm{~Hz}, 1 \mathrm{H}), 7.76(\mathrm{~d}, J=8.3 \mathrm{~Hz}, 2 \mathrm{H}), 7.55(\mathrm{~d}, J=3.6$ $\mathrm{Hz}, 1 \mathrm{H}), 7.40(\mathrm{~s}, 1 \mathrm{H}), 7.22(\mathrm{~d}, J=8.2 \mathrm{~Hz}, 2 \mathrm{H}), 7.19-7.16(\mathrm{~m}, 1 \mathrm{H}), 6.61(\mathrm{~d}, J=3.6 \mathrm{~Hz}, 1 \mathrm{H}), 4.10$ (td, $J=7.5,2.3 \mathrm{~Hz}, 1 \mathrm{H}), 3.60(\mathrm{~s}, 1 \mathrm{H}), 3.28(\mathrm{dd}, J=14.0,7.0 \mathrm{~Hz}, 1 \mathrm{H}), 3.14(\mathrm{dd}, J=14.0,7.8 \mathrm{~Hz}$, $1 \mathrm{H}), 2.34(\mathrm{~s}, 3 \mathrm{H}), 1.75(\mathrm{~d}, J=8.9 \mathrm{~Hz}, 1 \mathrm{H}), 1.67-1.60(\mathrm{~m}, 1 \mathrm{H}), 1.52-1.38(\mathrm{~m}, 2 \mathrm{H}), 1.37-1.29(\mathrm{~m}$, $1 \mathrm{H}), 0.88(\mathrm{t}, J=7.3 \mathrm{~Hz}, 3 \mathrm{H})$;

${ }^{13} \mathrm{C}$ NMR $\left(126 \mathrm{MHz}_{\mathrm{CDCl}}\right) \delta$ 144.9, 135.3, 133.8, 132.8, 131.0, 129.9, 126.8, 126.7, 125.9, 122.0, 113.5, 108.8, 71.7, 68.8, 41.2, 37.3, 21.5, 18.8, 13.9;

IR ( $\mathrm{NaCl}$, thin film) 3443, 2959, 1645, 1597, 1462, 1371, 1268, 1188, 1130, 1093, $995 \mathrm{~cm}^{-1}$;

HRMS (ESI-TOF) $\mathrm{m} / \mathrm{z}$ calcd for $\mathrm{C}_{21} \mathrm{H}_{24}{ }^{35} \mathrm{CINNaO}_{3} \mathrm{~S}^{+}(\mathrm{M}+\mathrm{Na})^{+}:$428.1058, found 428.1052. 

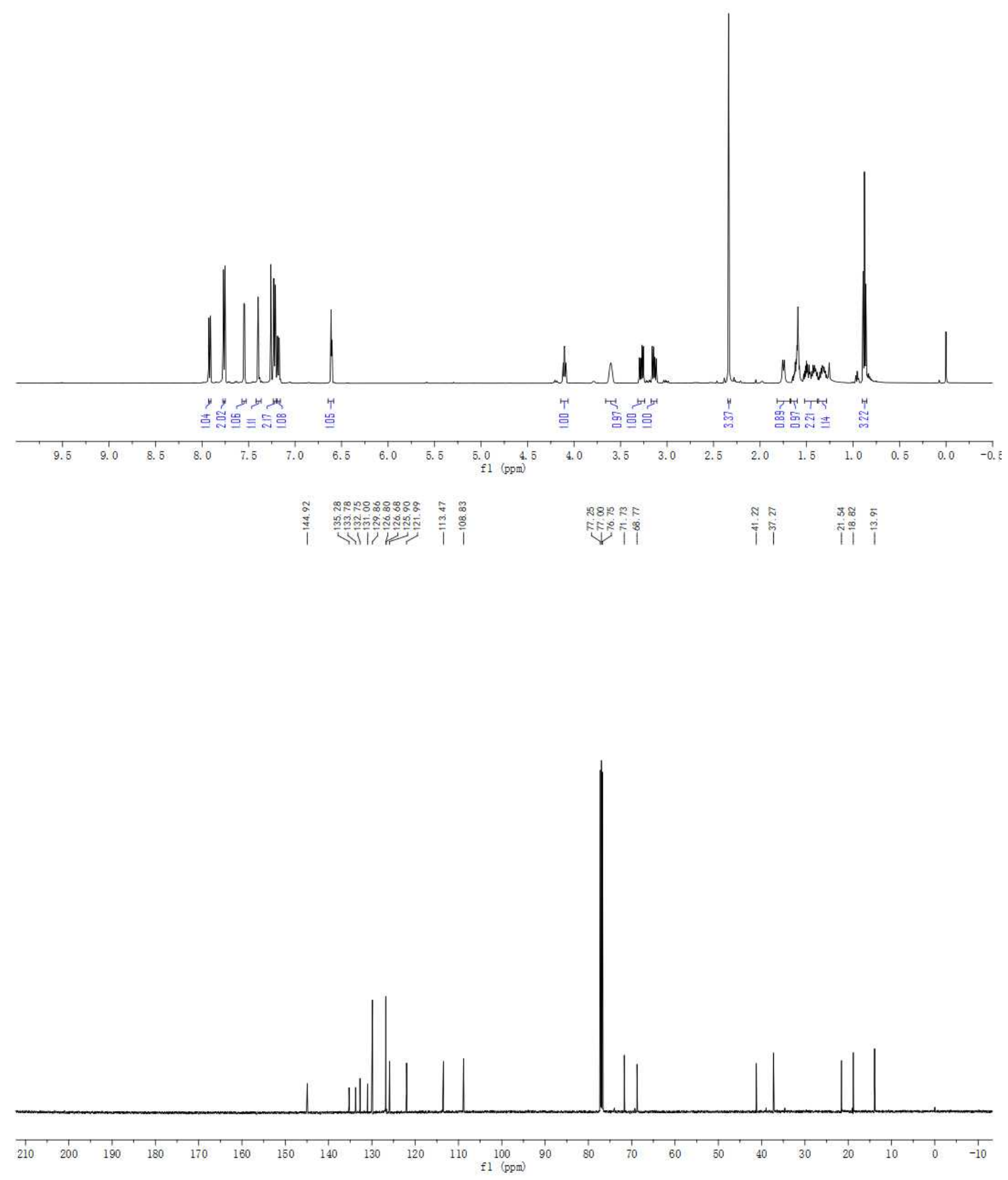
$\overbrace{1}^{c}$

$\left(2 R^{*}, 3 R^{*}\right)-2-C h l o r o-1-(4-f l u o r o p h e n y l) h e x a n-3-o l ~( \pm)-6 h$

General Procedure C: Epoxide $( \pm)-5 \mathrm{~h}(20 \mathrm{mg}, 0.10 \mathrm{mmol})$ was employed. Purification of the residue by FCC (10:1, hexane-EtOAc) afforded the title compound (15 $\mathrm{mg}, 65 \%)$ as a colorless oil.

${ }^{1} \mathrm{H}$ NMR $\left(500 \mathrm{MHz}, \mathrm{CDCl}_{3}\right) \delta 7.20(\mathrm{dd}, J=8.4,5.5 \mathrm{~Hz}, 2 \mathrm{H}), 7.00(\mathrm{t}, J=8.6 \mathrm{~Hz}, 2 \mathrm{H}), 4.11-4.00(\mathrm{~m}$, $1 \mathrm{H}), 3.68-3.56(\mathrm{~m}, 1 \mathrm{H}), 3.20(\mathrm{dd}, J=14.1,6.7 \mathrm{~Hz}, 1 \mathrm{H}), 3.06(\mathrm{dd}, J=14.1,8.1 \mathrm{~Hz}, 1 \mathrm{H}), 1.73(\mathrm{~d}, J$ $=9.1 \mathrm{~Hz}, 1 \mathrm{H}), 1.66-1.59(\mathrm{~m}, 1 \mathrm{H}), 1.541 .42(\mathrm{~m}, 2 \mathrm{H}), 1.40-1.32(\mathrm{~m}, 1 \mathrm{H}), 0.91(\mathrm{t}, J=7.3 \mathrm{~Hz}, 3 \mathrm{H})$;

${ }^{13} \mathrm{C}$ NMR (125 MHz, $\left.\mathrm{CDCl}_{3}\right) \delta 161.8(\mathrm{~d}, J=245.1 \mathrm{~Hz}), 133.4(\mathrm{~d}, J=3.3 \mathrm{~Hz}), 130.8(\mathrm{~d}, J=8.0 \mathrm{~Hz})$, $115.4(\mathrm{~d}, J=21.3 \mathrm{~Hz}), 71.9,68.5,40.5,37.2,18.8,13.9$;

${ }^{19} \mathrm{~F}\left\{{ }^{1} \mathrm{H}\right\}$ NMR $\left(470 \mathrm{MHz}, \mathrm{CDCl}_{3}\right) \delta-116.0(\mathrm{~s})$;

IR ( $\mathrm{NaCl}$, thin film) 3422, 2960, 2933, 2873, 1639, 1604, 1511, 1244, 826, $749 \mathrm{~cm}^{-1}$;

HRMS (ESI-TOF) $\mathrm{m} / \mathrm{z}$ calcd for $\mathrm{C}_{12} \mathrm{H}_{16}{ }^{35} \mathrm{CIFNaO}^{+}(\mathrm{M}+\mathrm{Na})^{+}:$253.0771, found 253.0765. 
<smiles>CCC[C@H](O)[C@H](Cl)Cc1ccc(Cl)cc1</smiles>

$\left(2 R^{*}, 3 R^{*}\right)-2-C h l o r o-1-(4-c h l o r o p h e n y l) h e x a n-3-o l ~( \pm)-6 \mathrm{i}$

General Procedure C: Epoxide $( \pm)-5 i(21 \mathrm{mg}, 0.10 \mathrm{mmol})$ was employed. Purification of the residue by FCC (10:1, hexane-EtOAc) afforded the title compound (15 mg, 61\%) as a colorless oil.

${ }^{1} \mathrm{H}$ NMR $\left(500 \mathrm{MHz}, \mathrm{CDCl}_{3}\right) \delta 7.29(\mathrm{~d}, J=8.0 \mathrm{~Hz}, 2 \mathrm{H}), 7.18(\mathrm{~d}, J=8.0 \mathrm{~Hz}, 2 \mathrm{H}), 4.11-4.02(\mathrm{~m}, 1 \mathrm{H})$, $3.62(\mathrm{dq}, J=10.5,6.3,4.7 \mathrm{~Hz}, 1 \mathrm{H}), 3.19(\mathrm{dd}, J=14.1,6.6 \mathrm{~Hz}, 1 \mathrm{H}), 3.06(\mathrm{dd}, J=14.1,8.2 \mathrm{~Hz}$, $1 \mathrm{H}), 1.73(\mathrm{~d}, J=9.1 \mathrm{~Hz}, 1 \mathrm{H}), 1.62(\mathrm{tt}, J=8.6,4.6 \mathrm{~Hz}, 1 \mathrm{H}), 1.52$ (ddd, $J=8.9,8.3,4.9 \mathrm{~Hz}, 1 \mathrm{H}$ ), 1.48-1.40 (m, 1H), 1.35 (ddd, $J=13.4,11.5,6.6 \mathrm{~Hz}, 1 \mathrm{H}), 0.92$ (t, $J=7.2 \mathrm{~Hz}, 3 \mathrm{H}$ );

${ }^{13} \mathrm{C}$ NMR $\left(126 \mathrm{MHz}, \mathrm{CDCl}_{3}\right) \delta 136.2,132.7,130.7,128.7,72.0,68.2,40.7,37.2,18.8,13.9$;

IR ( $\mathrm{NaCl}$, thin film) 3566, 3406, 2960, 2931, 2872, 2359, 2343, 1493, 1465, 1409, 1380, 1094, 1016, 806, $691 \mathrm{~cm}^{-1}$;

HRMS (ESI-TOF) $\mathrm{m} / \mathrm{z}$ calcd for $\mathrm{C}_{12} \mathrm{H}_{16}{ }^{35} \mathrm{Cl}_{2} \mathrm{NaO}^{+}(\mathrm{M}+\mathrm{Na})^{+}:$269.0476, found 269.0481. 
$8 x: 2=$

पiि

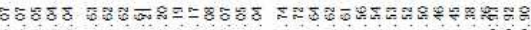

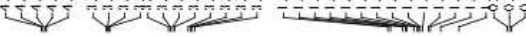
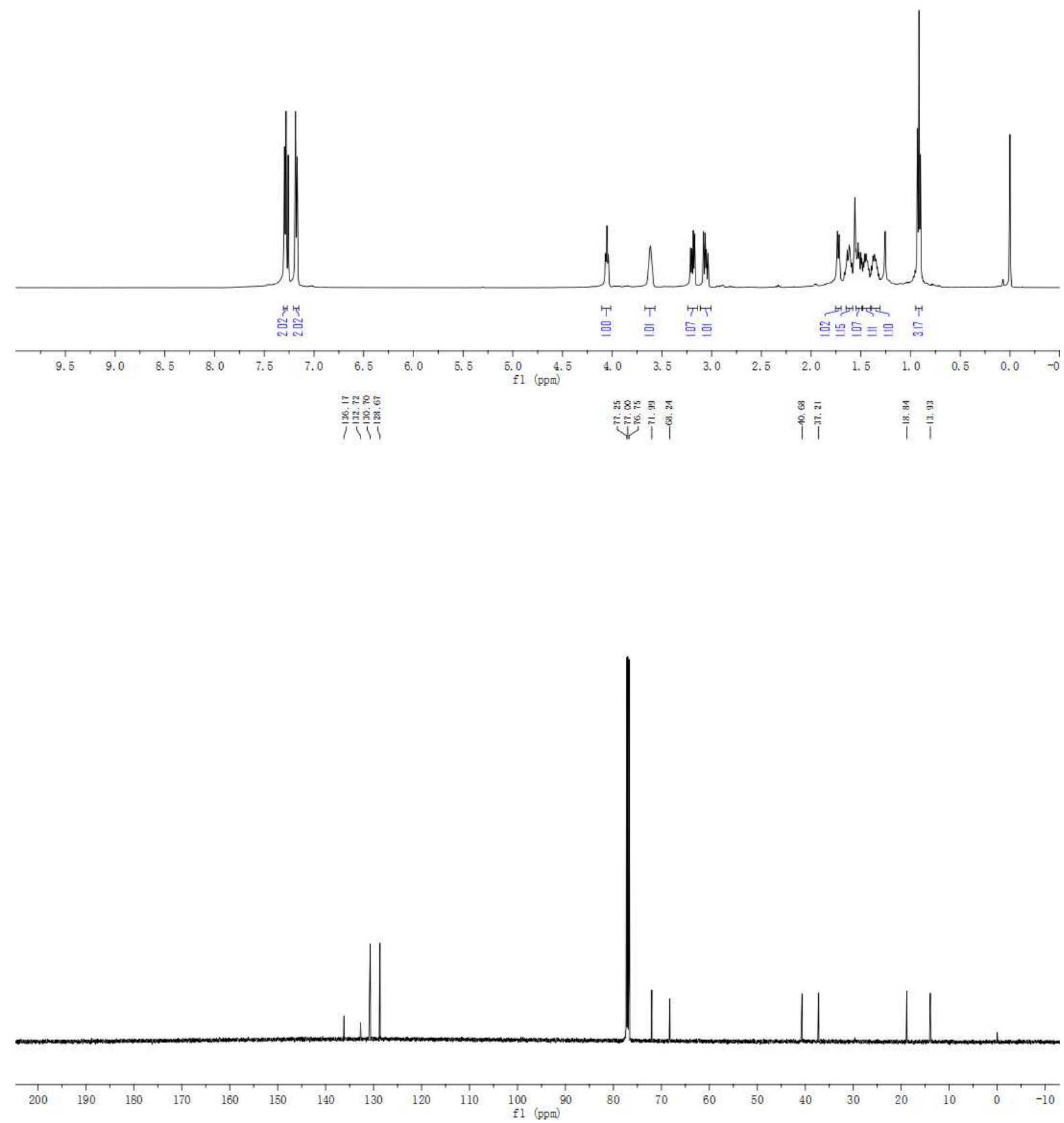
<smiles>CC[C@H](O)[C@@H](Cl)Cc1ccc(Br)cc1</smiles>

$\left(2 R^{*}, 3 R^{*}\right)-1-(4-B r o m o p h e n y l)-2-c h l o r o h e x a n-3-o l ~( \pm)-6 \mathrm{j}$

General Procedure C: Epoxide $( \pm)-5 \mathrm{j}(25 \mathrm{mg}, 0.10 \mathrm{mmol})$ was employed. Purification of the residue by FCC (10:1, hexane-EtOAc) afforded the title compound ( $20 \mathrm{mg}, 69 \%)$ as a colorless oil.

${ }^{1} \mathrm{H}$ NMR $\left(500 \mathrm{MHz}, \mathrm{CDCl}_{3}\right) \delta 7.44(\mathrm{~d}, J=8.3 \mathrm{~Hz}, 2 \mathrm{H}), 7.12(\mathrm{~d}, J=8.2 \mathrm{~Hz}, 2 \mathrm{H}), 4.08-4.00(\mathrm{~m}, 1 \mathrm{H})$, $3.61(\mathrm{~s}, 1 \mathrm{H}), 3.18(\mathrm{dd}, J=14.1,6.5 \mathrm{~Hz}, 1 \mathrm{H}), 3.04(\mathrm{dd}, J=14.1,8.2 \mathrm{~Hz}, 1 \mathrm{H}), 1.73(\mathrm{~d}, J=8.7 \mathrm{~Hz}$, $1 \mathrm{H}), 1.63$ (ddd, $J=13.2,6.8,3.6 \mathrm{~Hz}, 1 \mathrm{H}), 1.54-1.42(\mathrm{~m}, 2 \mathrm{H}), 1.36$ (ddd, $J=13.1,9.6,6.9 \mathrm{~Hz}, 1 \mathrm{H})$, $0.92(\mathrm{t}, \mathrm{J}=7.3 \mathrm{~Hz}, 3 \mathrm{H})$.

${ }^{13} \mathrm{C}$ NMR $\left(126 \mathrm{MHz}, \mathrm{CDCl}_{3}\right) \delta 136.7,131.6,131.1,120.8,72.0,68.1,40.7,37.2,18.8,13.9$.

IR ( $\mathrm{NaCl}$, thin film) 3406, 2959, 2930, 2872, 1489, 1405, 1072, 1012, $606 \mathrm{~cm}^{-1}$;

HRMS $\left(E I^{+}\right) \mathrm{m} / z$ calcd for $\mathrm{C}_{12} \mathrm{H}_{16}{ }^{79} \mathrm{Br}^{35} \mathrm{ClO}^{+}(\mathrm{M})^{+}:$290.0073, found 290.0064 . 

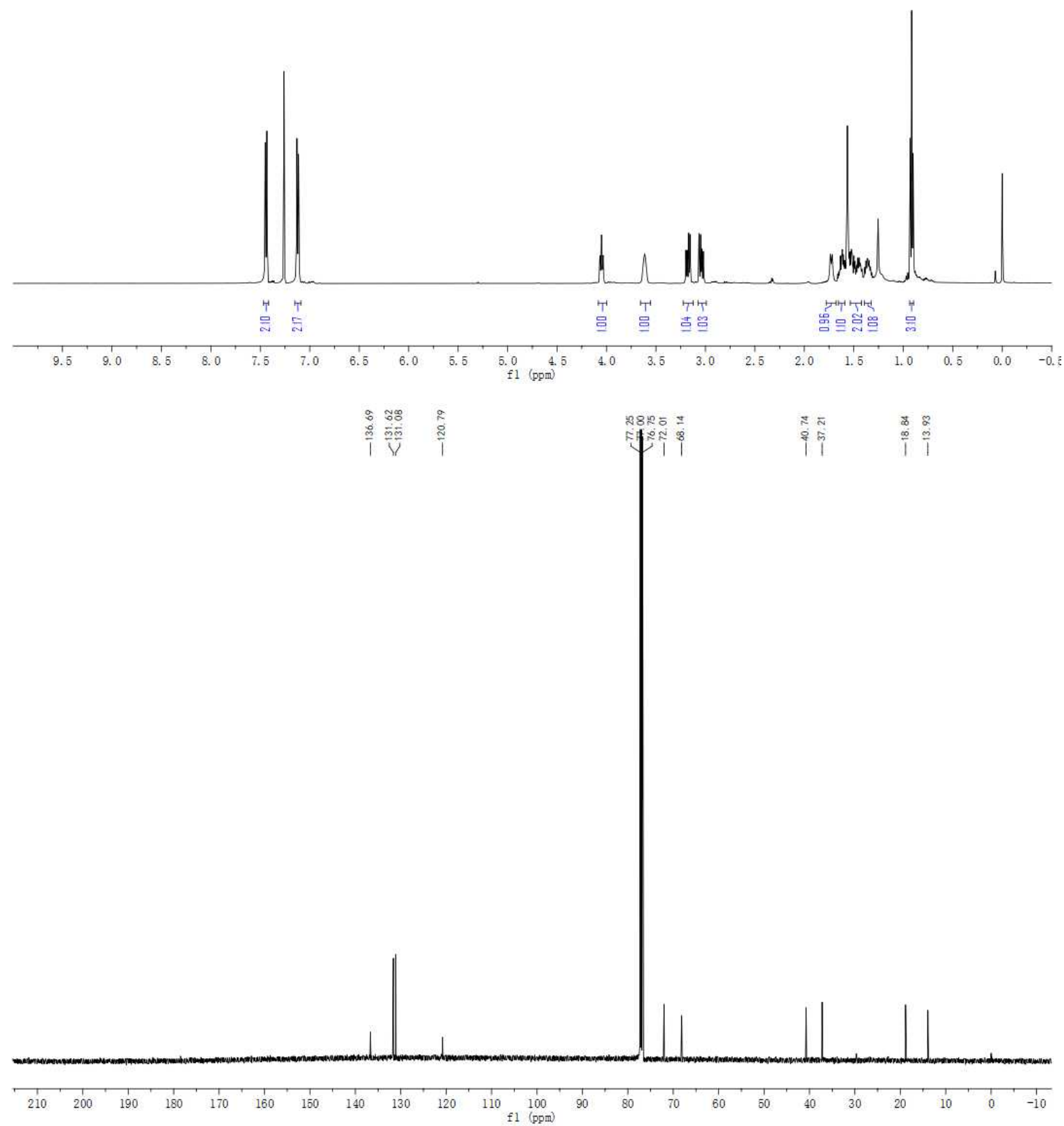
<smiles>CC[C@H](O)[C@H](Cl)Cc1ccc(C(F)(F)F)cc1</smiles>

\section{$\left(2 R^{*}, 3 R^{*}\right)$-2-Chloro-1-(4-(trifluoromethyl)phenyl)hexan-3-ol ( \pm )-6k}

General Procedure C: Epoxide $( \pm)-5 k(24 \mathrm{mg}, 0.10 \mathrm{mmol})$ was employed. Purification of the residue by FCC (5:1, hexane-EtOAc) afforded the title compound (14 mg, 50\%) as a colorless oil.

${ }^{1} \mathrm{H}$ NMR $\left(500 \mathrm{MHz}, \mathrm{CDCl}_{3}\right) \delta 7.58(\mathrm{~d}, J=7.9 \mathrm{~Hz}, 2 \mathrm{H}), 7.36(\mathrm{~d}, J=7.8 \mathrm{~Hz}, 2 \mathrm{H}), 4.21-4.10(\mathrm{~m}$, $1 \mathrm{H}), 3.83(\mathrm{~s}, 1 \mathrm{H}), 3.31-3.17(\mathrm{~m}, 1 \mathrm{H}), 3.01(\mathrm{dd}, J=14.4,10.3 \mathrm{~Hz}, 1 \mathrm{H}), 1.98(\mathrm{~d}, J=4.9 \mathrm{~Hz}, 1 \mathrm{H})$, $1.67-1.58(\mathrm{~m}, 3 \mathrm{H}), 1.43(\mathrm{dt}, J=13.5,6.3 \mathrm{~Hz}, 1 \mathrm{H}), 0.98(\mathrm{t}, J=6.6 \mathrm{~Hz}, 3 \mathrm{H})$;

${ }^{13} \mathrm{C}$ NMR (126 MHz, $\mathrm{CDCl}_{3}$ ) $\delta$ 142.0, 129.7, 129.1 (q, $\left.J=32.3 \mathrm{~Hz}\right), 125.4$ (q, $\left.J=3.7 \mathrm{~Hz}\right), 74.2$, 68.1, 38.6, 35.0, 19.0, 14.0;

${ }^{19} \mathrm{~F}\left\{{ }^{1} \mathrm{H}\right\}$ NMR $\left(471 \mathrm{MHz}, \mathrm{CDCl}_{3}\right) \delta-62.45$;

IR (thin film) 3421, 2962, 2876, 1620, 1532, 1419, 1326, 1163, 1124, 1068, 1020, $729 \mathrm{~cm}^{-1}$;

HRMS (ESI-TOF) $\mathrm{m} / \mathrm{z}$ calcd for $\mathrm{C}_{13} \mathrm{H}_{16}{ }^{35} \mathrm{CIF}_{3} \mathrm{NaO}^{+}(\mathrm{M}+\mathrm{Na})^{+}: 303.0734$, found 303.0729 . 

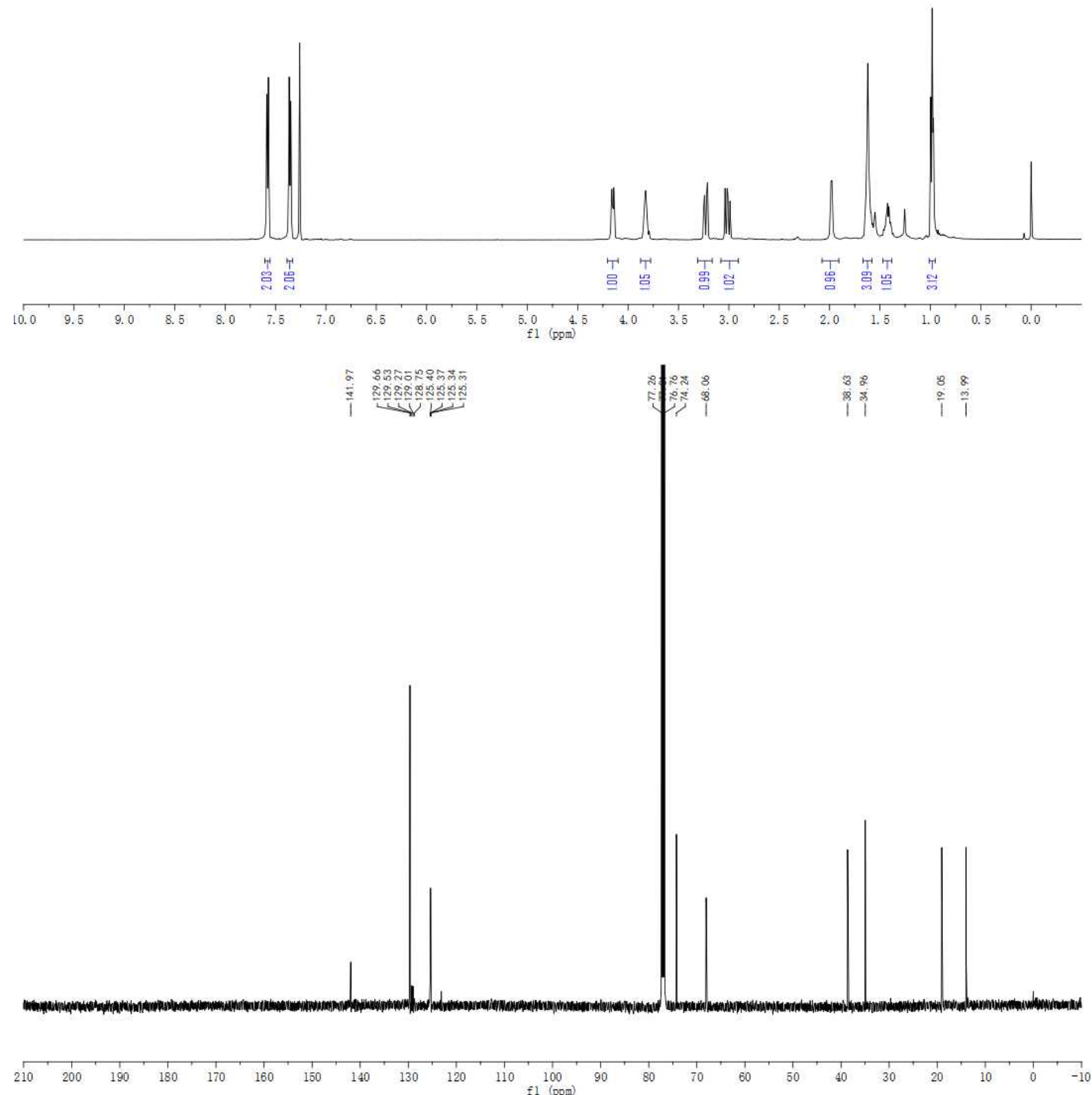
<smiles>CCC[C@@H](O)[C@H](Cl)Cc1cccc(F)c1</smiles>

\section{$\left(2 R^{*}, 3 R^{*}\right)-2-C h l o r o-1-(3-f l u o r o p h e n y l) h e x a n-3-o l ~( \pm)-6 I$}

General Procedure C: Epoxide $( \pm)-5 \mathrm{I}(20 \mathrm{mg}, 0.10 \mathrm{mmol})$ was employed. Purification of the residue by FCC (20:1, hexane-EtOAc) afforded the title compound (17 $\mathrm{mg}, 74 \%)$ as a colorless oil.

${ }^{1} \mathrm{H}$ NMR $\left(500 \mathrm{MHz}, \mathrm{CDCl}_{3}\right) \delta 7.31-7.26(\mathrm{~m}, 1 \mathrm{H}), 7.02(\mathrm{~d}, J=7.6 \mathrm{~Hz}, 1 \mathrm{H}), 6.95(\mathrm{t}, J=8.5 \mathrm{~Hz}, 2 \mathrm{H})$, 4.09 (t, $J=7.3 \mathrm{~Hz}, 1 \mathrm{H}$ ), $3.63(\mathrm{~s}, 1 \mathrm{H}), 3.22$ (dd, $J=14.0,6.5 \mathrm{~Hz}, 1 \mathrm{H}), 3.09$ (dd, $J=14.0,8.2 \mathrm{~Hz}$, $1 \mathrm{H}), 1.72(\mathrm{~d}, J=8.8 \mathrm{~Hz}, 1 \mathrm{H}), 1.63(\mathrm{dt}, J=17.0,6.5 \mathrm{~Hz}, 1 \mathrm{H}), 1.53(\mathrm{dd}, J=11.7,6.6 \mathrm{~Hz}, 1 \mathrm{H}), 1.49-$ $1.42(\mathrm{~m}, 1 \mathrm{H}), 1.37(\mathrm{dt}, J=15.1,6.6 \mathrm{~Hz}, 1 \mathrm{H}), 0.92(\mathrm{t}, J=7.1 \mathrm{~Hz}, 3 \mathrm{H})$;

${ }^{13} \mathrm{C}$ NMR (126 MHz, $\left.\mathrm{CDCl}_{3}\right) \delta 162.8$ (d, J = 246.0 Hz), 140.2 (d, J = 7.4 Hz), 125.0 (d, J = 8.3 Hz), 123.0 (d, $J=2.9 \mathrm{~Hz}), 116.2$ (d, $J=21.2 \mathrm{~Hz}), 113.8$ (d, $J=21.0 \mathrm{~Hz}$ ), 72.0, 68.0, 41.1, 37.2, 18.8, 13.9 ;

${ }^{19}$ F NMR $\left(471 \mathrm{MHz}, \mathrm{CDCl}_{3}\right) \delta-113.2(\mathrm{~s})$;

IR ( $\mathrm{NaCl}$, thin film) 3416, 2960, 2934, 2874, 1748, 1614, 1590, 1489, 1451, 1254, 1142, 954, 788 $\mathrm{cm}^{-1}$;

HRMS (ESI-TOF) $\mathrm{m} / \mathrm{z}$ calcd for $\mathrm{C}_{12} \mathrm{H}_{16}{ }^{35} \mathrm{CIFNaO}^{+}(\mathrm{M}+\mathrm{Na})^{+}:$253.0766, found 253.0760. 


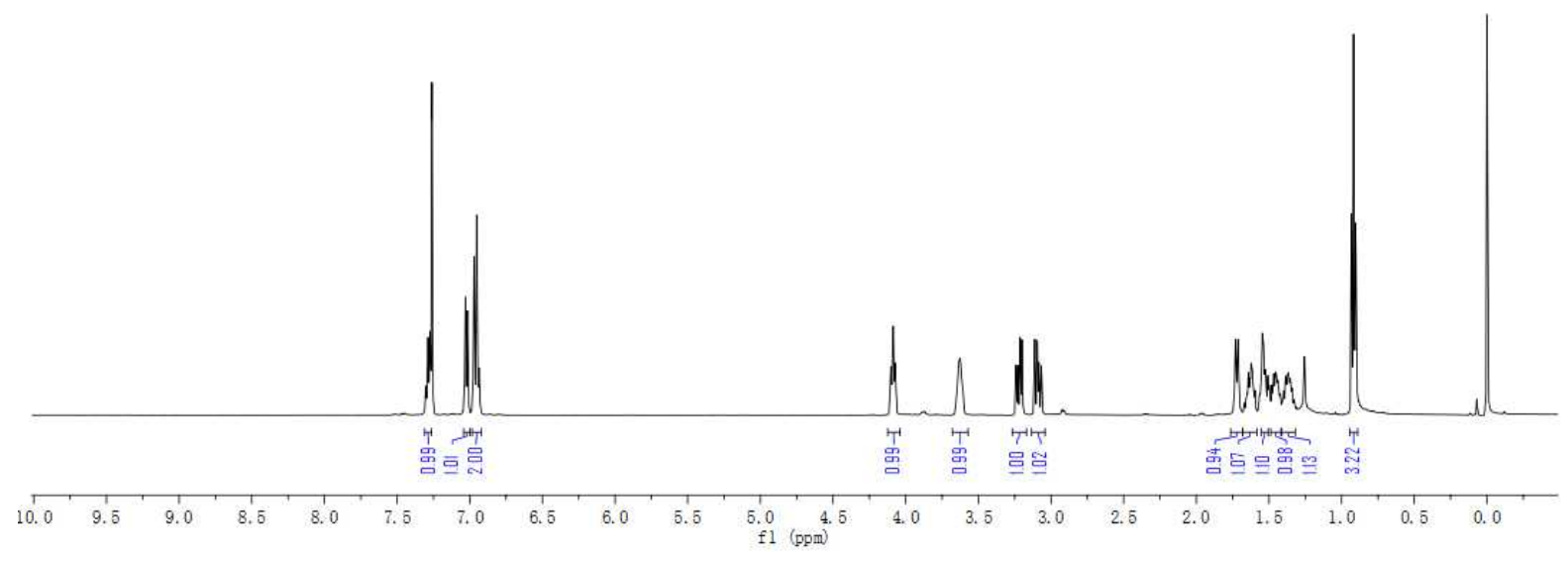

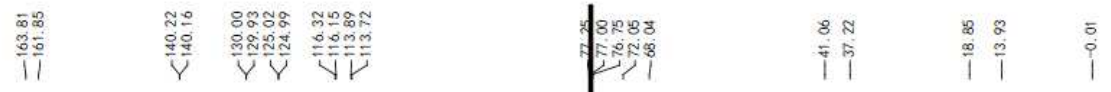

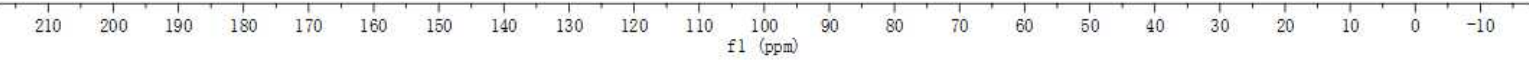


<smiles>CCC[C@@H](O)[C@H](Cl)Cc1cccc(Cl)c1</smiles>

$\left(2 R^{*}, 3 R^{*}\right)-2-C h l o r o-1-(3-c h l o r o p h e n y l) h e x a n-3-o l ~( \pm)-6 m$

General Procedure C: Epoxide $( \pm)-5 \mathrm{~m}(21 \mathrm{mg}, 0.10 \mathrm{mmol})$ was employed. Purification of the residue by FCC (20:1, hexane-EtOAc) afforded the title compound (18 $\mathrm{mg}, 73 \%)$ as a colorless oil.

${ }^{1} \mathrm{H}$ NMR $\left(500 \mathrm{MHz}, \mathrm{CDCl}_{3}\right) \delta 7.24(\mathrm{~d}, J=5.5 \mathrm{~Hz}, 3 \mathrm{H}), 7.13(\mathrm{~d}, J=6.1 \mathrm{~Hz}, 1 \mathrm{H}), 4.11-4.03(\mathrm{~m}, 1 \mathrm{H})$, 3.63 (s, 1H), 3.20 (dd, $J=14.1,6.4 \mathrm{~Hz}, 1 \mathrm{H}$ ), 3.07 (dd, $J=14.1,8.3 \mathrm{~Hz}, 1 \mathrm{H}), 1.72$ (d, $J=9.1 \mathrm{~Hz}$, $1 \mathrm{H}), 1.63(\mathrm{dt}, J=8.5,3.9 \mathrm{~Hz}, 1 \mathrm{H}), 1.53-1.42(\mathrm{~m}, 2 \mathrm{H}), 1.36$ (ddd, $J=16.2,9.9,4.8 \mathrm{~Hz}, 1 \mathrm{H}), 0.92$ (t, $J=7.3 \mathrm{~Hz}, 3 \mathrm{H})$;

${ }^{13} \mathrm{C}$ NMR $\left(126 \mathrm{MHz}_{\mathrm{CDCl}}\right)$ $\delta$ 139.7, 134.3, 129.8, 129.4, 127.6, 127.1, 72.1, 68.0, 41.0, 37.2, 18.8, 13.9;

IR ( $\mathrm{NaCl}$, thin film) 3418, 3308, 2960, 2360, 2341, 1704, 1599, 1429, 1264, 1085, $751 \mathrm{~cm}^{-1}$;

HRMS (ESI-TOF) $\mathrm{m} / \mathrm{z}$ calcd for $\mathrm{C}_{12} \mathrm{H}_{16}{ }^{35} \mathrm{Cl}_{2} \mathrm{NaO}^{+}(\mathrm{M}+\mathrm{Na})^{+}:$269.0470, found 269.0471. 


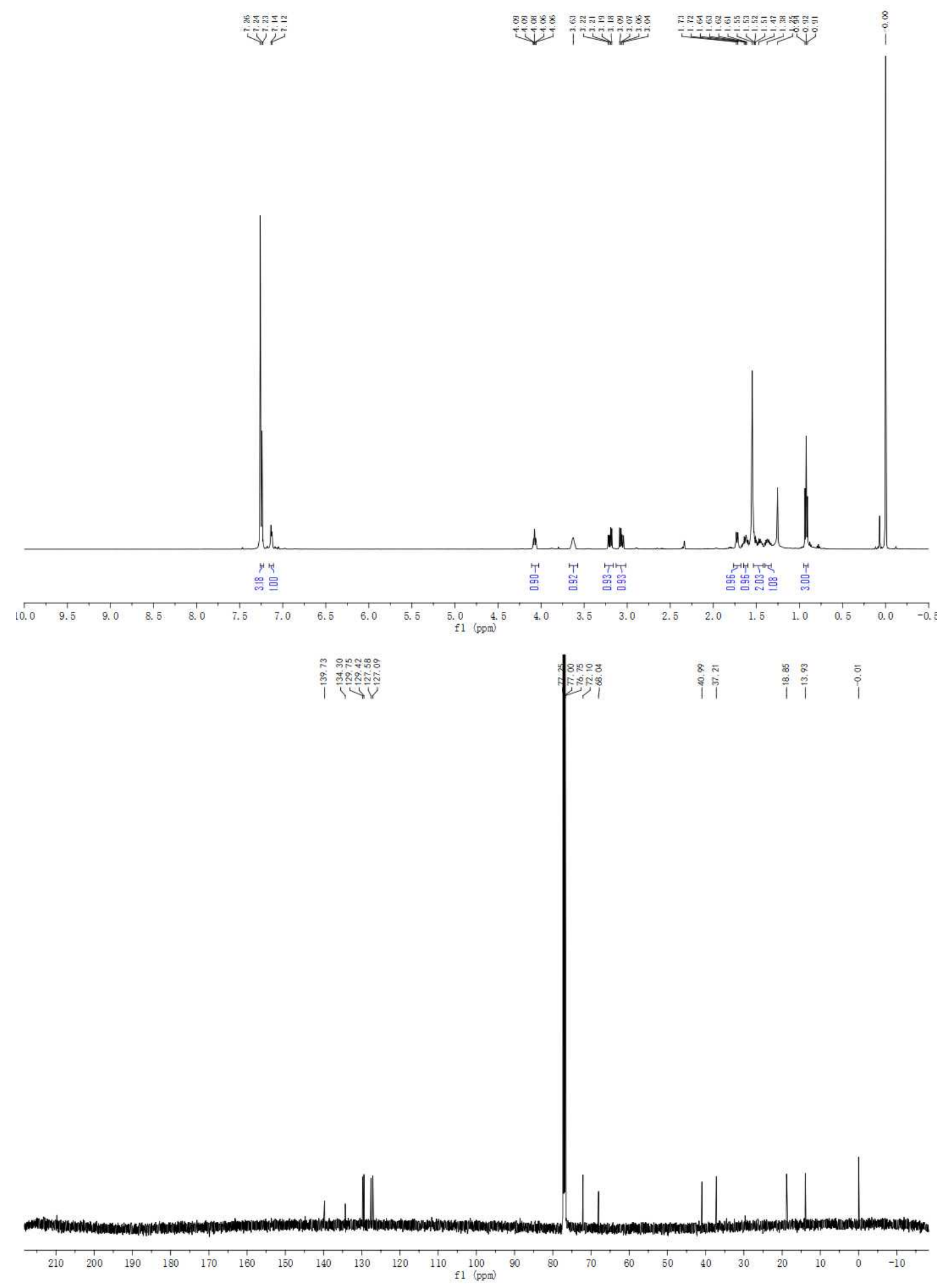


<smiles>CCC[C@H](O)[C@H](Cl)Cc1cccc(OC)c1</smiles>

\section{$\left(2 R^{*}, 3 R^{*}\right)$-2-Chloro-1-(3-methoxyphenyl)hexan-3-ol ( \pm )-6n}

General Procedure C: Epoxide $( \pm)-5 \mathrm{n}(21 \mathrm{mg}, 0.10 \mathrm{mmol})$ was employed. Purification of the residue by FCC (10:1, hexane-EtOAc) afforded the title compound (20 $\mathrm{mg}, 83 \%)$ as a colorless oil.

${ }^{1} \mathrm{H}$ NMR $\left(500 \mathrm{MHz}, \mathrm{CDCl}_{3}\right) \delta 7.24(\mathrm{t}, J=8.1 \mathrm{~Hz}, 1 \mathrm{H}), 6.82(\mathrm{dd}, J=18.2,7.3 \mathrm{~Hz}, 3 \mathrm{H}), 4.12(\mathrm{td}, J$ $=7.6,2.4 \mathrm{~Hz}, 1 \mathrm{H}), 3.81(\mathrm{~s}, 3 \mathrm{H}), 3.67-3.61(\mathrm{~m}, 1 \mathrm{H}), 3.20(\mathrm{dd}, J=14.0,6.9 \mathrm{~Hz}, 1 \mathrm{H}), 3.07(\mathrm{dd}, J=$ $14.0,7.9 \mathrm{~Hz}, 1 \mathrm{H}), 1.75(\mathrm{~d}, J=9.0 \mathrm{~Hz}, 1 \mathrm{H}), 1.65-1.60(\mathrm{~m}, 1 \mathrm{H}), 1.55-1.43(\mathrm{~m}, 2 \mathrm{H}), 1.36(\mathrm{ddd}, J=$ 13.1, 9.6, $6.9 \mathrm{~Hz}, 1 \mathrm{H}), 0.91(\mathrm{t}, J=7.3 \mathrm{~Hz}, 3 \mathrm{H})$;

${ }^{13} \mathrm{C}$ NMR $\left(126 \mathrm{MHz}_{\mathrm{CDCl}}\right) \delta$ 159.7, 139.3, 129.5, 121.6, 115.1, 112.1, 71.9, 68.4, 55.2, 41.4, $37.3,18.9,13.9$;

IR ( $\mathrm{NaCl}$, thin film) 3584, 3445, 2957, 2929, 2873, 1602, 1585, 1491, 1261, 1041, $749 \mathrm{~cm}^{-1}$;

HRMS $\left(\mathrm{EI}^{+}\right) \mathrm{m} / \mathrm{z}$ calcd for $\mathrm{C}_{13} \mathrm{H}_{19}{ }^{35} \mathrm{ClO}_{2}{ }^{+}(\mathrm{M})^{+}:$242.1074, found 242.1082. 

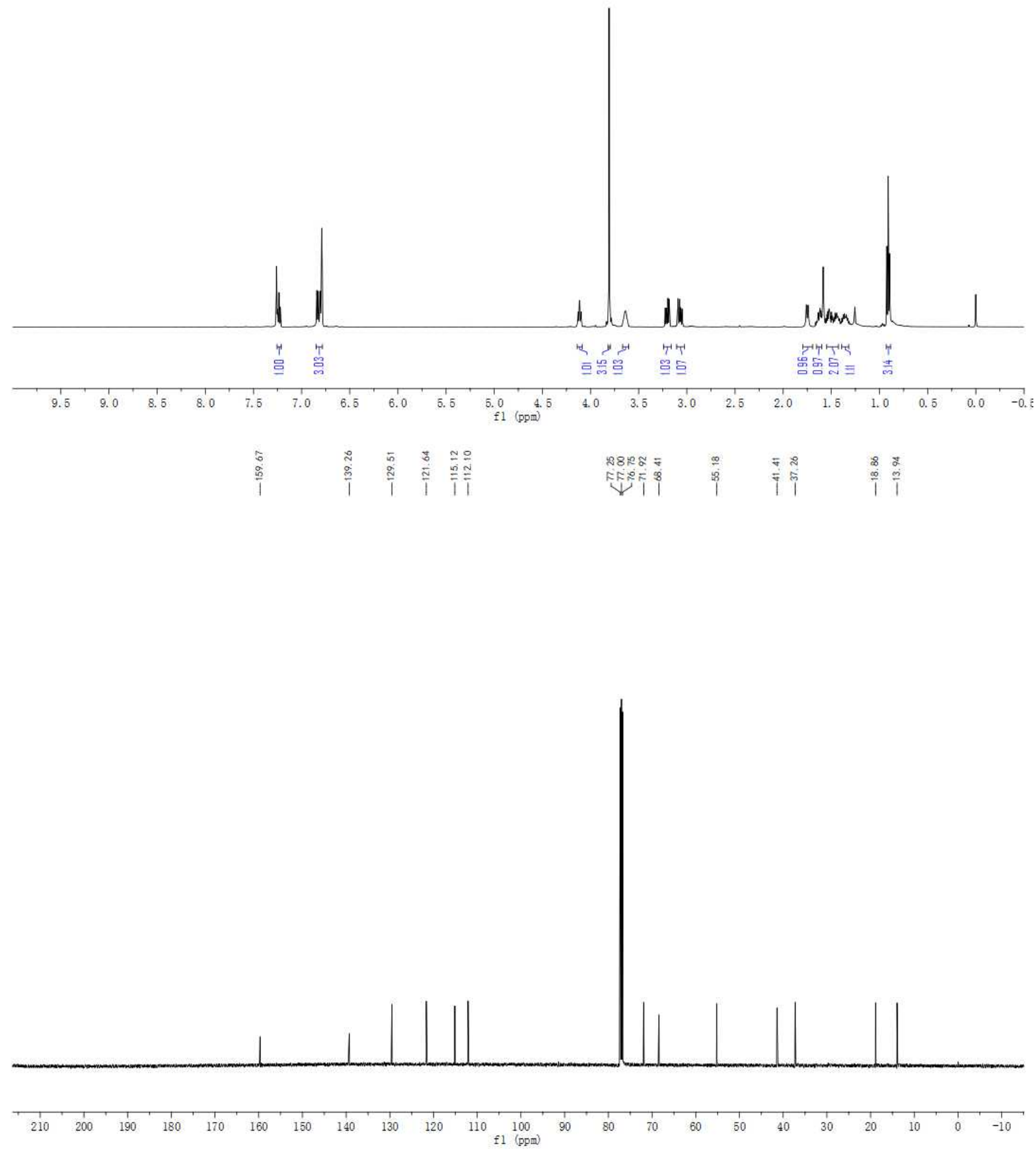


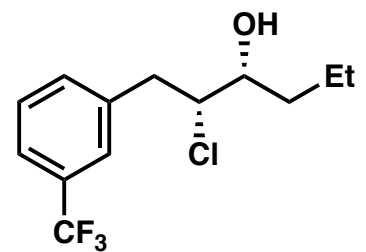

$\left(2 R^{*}, 3 R^{*}\right)$-2-Chloro-1-(3-(trifluoromethyl)phenyl)hexan-3-ol ( \pm )-6o

General Procedure C: Epoxide $( \pm)-50(24 \mathrm{mg}, 0.10 \mathrm{mmol})$ was employed. Purification of the residue by FCC (20:1, hexane-EtOAc) afforded the title compound (18 $\mathrm{mg}, 64 \%)$ as a colorless oil.

${ }^{1} \mathrm{H}$ NMR $\left(500 \mathrm{MHz}, \mathrm{CDCl}_{3}\right) \delta 7.51(\mathrm{~d}, J=11.7 \mathrm{~Hz}, 2 \mathrm{H}), 7.44(\mathrm{~d}, J=3.8 \mathrm{~Hz}, 2 \mathrm{H}), 4.09$ (t, $J=7.2$ $\mathrm{Hz}, 1 \mathrm{H}), 3.64(\mathrm{~s}, 1 \mathrm{H}), 3.28(\mathrm{dd}, J=14.1,6.0 \mathrm{~Hz}, 1 \mathrm{H}), 3.15(\mathrm{dd}, J=14.1,8.7 \mathrm{~Hz}, 1 \mathrm{H}), 1.74(\mathrm{~d}, J=$ $8.9 \mathrm{~Hz}, 1 \mathrm{H}), 1.68-1.60(\mathrm{~m}, 1 \mathrm{H}), 1.57(\mathrm{dd}, J=9.8,5.1 \mathrm{~Hz}, 1 \mathrm{H}), 1.48(\mathrm{dd}, J=14.7,7.2 \mathrm{~Hz}, 1 \mathrm{H})$, $1.43-1.34(\mathrm{~m}, 1 \mathrm{H}), 0.93(\mathrm{t}, J=7.2 \mathrm{~Hz}, 3 \mathrm{H})$;

${ }^{13} \mathrm{C}$ NMR (126 MHz, $\left.\mathrm{CDCl}_{3}\right) \delta 138.7,132.8,130.9$ (q, J = 32.2 Hz), 128.9, 126.0 (q, J = 3.6 Hz), 123.8 (q, J = 4.0 Hz), 72.3, 68.0, 41.1, 37.2, 18.8, 13.9.; The $\mathrm{Ar}_{\underline{C}} \mathrm{~F}_{3}$ carbon was not observed due to its weak intensity.

${ }^{19} \mathrm{~F}$ NMR $\left(471 \mathrm{MHz}, \mathrm{CDCl}_{3}\right) \delta-62.6(\mathrm{~s})$;

IR ( $\mathrm{NaCl}$, thin film) 3419, 2960, 2924, 2856, 2360, 2341, 1684, 1653, 1559, 1506, 1456, 1330, 1128, 1074, $618 \mathrm{~cm}^{-1}$;

HRMS (ESI-TOF) $\mathrm{m} / \mathrm{z}$ calcd for $\mathrm{C}_{13} \mathrm{H}_{16}{ }^{35} \mathrm{CIF}_{3} \mathrm{NaO}^{+}(\mathrm{M}+\mathrm{Na})^{+}: 303.0734$, found 303.0736 . 


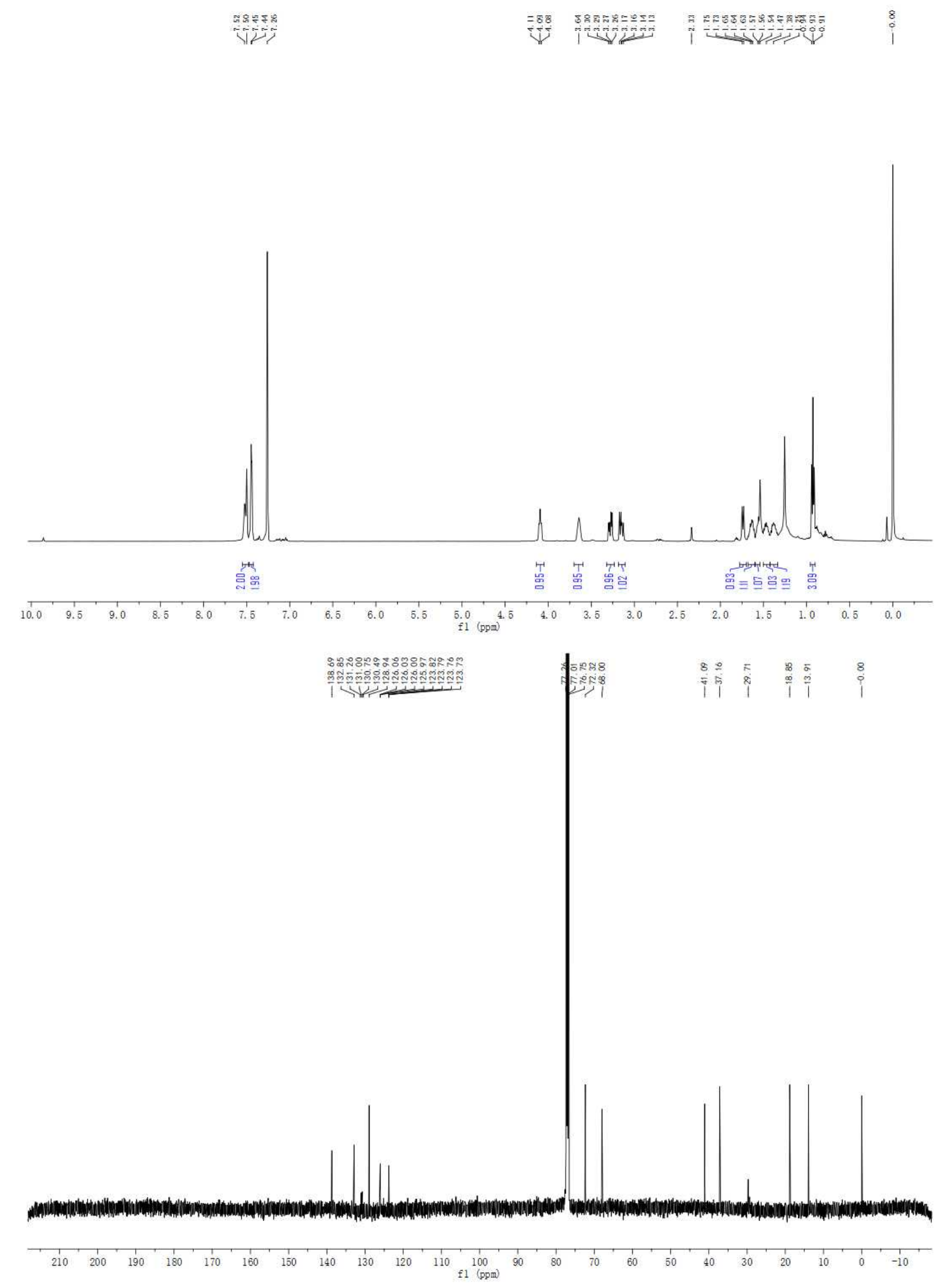


<smiles>CCC[C@@H](O)[C@H](Cl)Cc1ccc(F)c(F)c1</smiles>

\section{$\left(2 R^{*}, 3 R^{*}\right)-2-C h l o r o-1-(3,4-d i f l u o r o p h e n y l) h e x a n-3-o l ~( \pm)-6 p$}

General Procedure C: Epoxide $( \pm)-5 p(21 \mathrm{mg}, 0.10 \mathrm{mmol})$ was employed. Purification of the residue by FCC (20:1, hexane-EtOAc) afforded the title compound (18 $\mathrm{mg}, 72 \%)$ as a colorless oil.

${ }^{1} \mathrm{H}$ NMR $\left(500 \mathrm{MHz}, \mathrm{CDCl}_{3}\right) \delta$ 7.14-7.04 (m, 2H), $6.96(\mathrm{~s}, 1 \mathrm{H}), 4.03(\mathrm{t}, \mathrm{J}=7.2 \mathrm{~Hz}, 1 \mathrm{H}), 3.62(\mathrm{~s}$, $1 \mathrm{H}), 3.17(\mathrm{dd}, J=14.2,6.3 \mathrm{~Hz}, 1 \mathrm{H}), 3.04(\mathrm{dd}, J=14.1,8.4 \mathrm{~Hz}, 1 \mathrm{H}), 1.71(\mathrm{~d}, J=9.0 \mathrm{~Hz}, 1 \mathrm{H})$, $1.67-1.59(\mathrm{~m}, 1 \mathrm{H}), 1.52(\mathrm{dd}, J=13.7,5.1 \mathrm{~Hz}, 1 \mathrm{H}), 1.49-1.42(\mathrm{~m}, 1 \mathrm{H}), 1.36(\mathrm{dd}, J=14.4,7.6 \mathrm{~Hz}$, $1 \mathrm{H}), 0.92(\mathrm{t}, \mathrm{J}=7.2 \mathrm{~Hz}, 3 \mathrm{H})$;

${ }^{13} \mathrm{C}$ NMR (126 MHz, $\left.\mathrm{CDCl}_{3}\right) \delta 150.2$ (dd, $\left.J=248.4,12.7 \mathrm{~Hz}\right), 149.4$ (dd, $\left.J=247.3,12.4 \mathrm{~Hz}\right), 134.7$ (dd, $J=5.5,4.1 \mathrm{~Hz}), 125.3$ (dd, $J=6.1,3.6 \mathrm{~Hz}), 118.2$ (d, $J=17.0 \mathrm{~Hz}), 117.2$ (d, $J=17.0 \mathrm{~Hz}$ ), 72.1, 68.0, 40.5, 37.2, 18.8, 13.9;

${ }^{19} \mathrm{~F}\left\{{ }^{1} \mathrm{H}\right\}$ NMR $\left(376 \mathrm{MHz}, \mathrm{CDCl}_{3}\right) \delta-137.7$ (d, J = 21.2 Hz, $\left.1 \mathrm{~F}\right),-140.50$ (d, J = 21.3 Hz, 1F); IR ( $\mathrm{NaCl}$, thin film) 3392, 2958, 2359, 2340, 1614, 1519, 1451, 1287, 1142, 954, $788 \mathrm{~cm}^{-1}$; HRMS (ESI-TOF) $\mathrm{m} / \mathrm{z}$ calcd for $\mathrm{C}_{12} \mathrm{H}_{15}{ }^{35} \mathrm{CIF}_{2} \mathrm{NaO}^{+}(\mathrm{M}+\mathrm{Na})^{+}:$271.0672, found 271.0673 . 


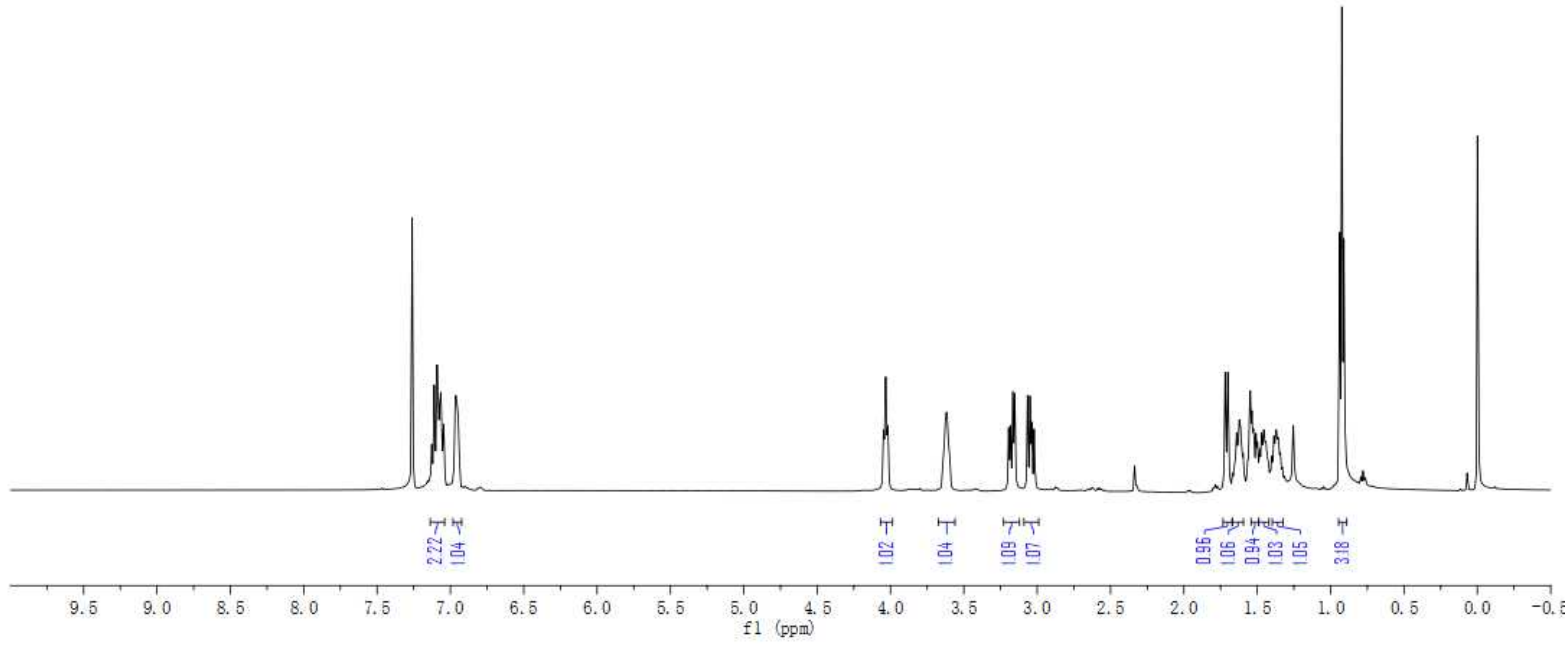

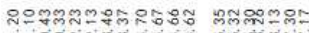

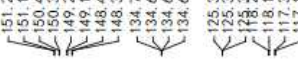
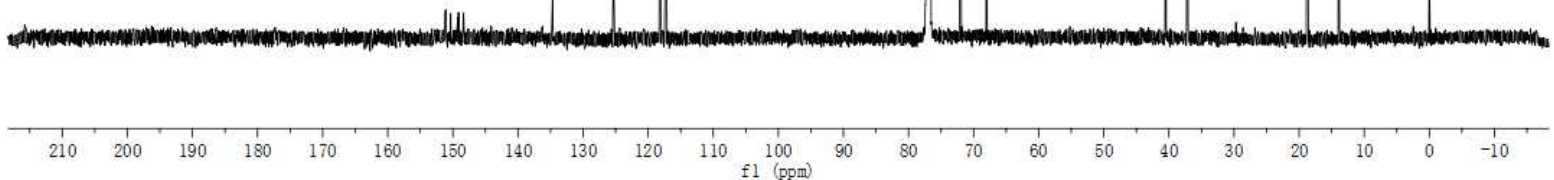
<smiles>CC(C)(Cl)C(O)C(Cl)[C@H](Cl)CP</smiles>

$\left(2 R^{*}, 3 R^{*}\right)-2,7-D i c h l o r o-1-(4-m e t h o x y p h e n y l) h e p t a n-3-o l ~( \pm)-6 q$

General Procedure C: Epoxide $( \pm)-5 q(24 \mathrm{mg}, 0.10 \mathrm{mmol})$ was employed. Purification of the residue by FCC (10:1, hexane-EtOAc) afforded the title compound (27 $\mathrm{mg}, 93 \%)$ as a colorless oil.

${ }^{1} \mathrm{H}$ NMR $\left(500 \mathrm{MHz}, \mathrm{CDCl}_{3}\right) \delta 7.17-7.15(\mathrm{~m}, 2 \mathrm{H}), 6.88-6.85(\mathrm{~m}, 2 \mathrm{H}), 4.07(\mathrm{td}, J=7.4,2.5 \mathrm{~Hz}, 1 \mathrm{H})$, $3.80(\mathrm{~s}, 3 \mathrm{H}), 3.65-3.58(\mathrm{~m}, 1 \mathrm{H}), 3.52(\mathrm{t}, J=6.6 \mathrm{~Hz}, 2 \mathrm{H}), 3.17$ (dd, $J=14.1,7.2 \mathrm{~Hz}, 1 \mathrm{H}), 3.04$ (dd, $J=14.1,7.7 \mathrm{~Hz}, 1 \mathrm{H}), 1.84-1.71(\mathrm{~m}, 3 \mathrm{H}), 1.68-1.55(\mathrm{~m}, 3 \mathrm{H}), 1.48$ (dddd, $J=13.8,11.0,7.9,6.7$ $\mathrm{Hz}, 1 \mathrm{H})$;

${ }^{13} \mathrm{C}$ NMR $\left(126 \mathrm{MHz} \mathrm{CDCl}_{3}\right) \delta 158.5,130.3,129.5,114.0,71.7,68.8,55.2,44.8,40.5,34.4,32.3$, 23.0;

IR (NaCl, thin film) 3485, 3450, 2999, 2860, 2362, 2329, 1613, 1515, 1454, 1301, 1248, 1178, 1033, $786 \mathrm{~cm}^{-1}$;

HRMS (ESI-TOF) $\mathrm{m} / \mathrm{z}$ calcd for $\mathrm{C}_{14} \mathrm{H}_{20}{ }^{35} \mathrm{Cl}_{2} \mathrm{NaO}_{2}{ }^{+}(\mathrm{M}+\mathrm{Na})^{+}: 313.0738$, found 313.0730 . 

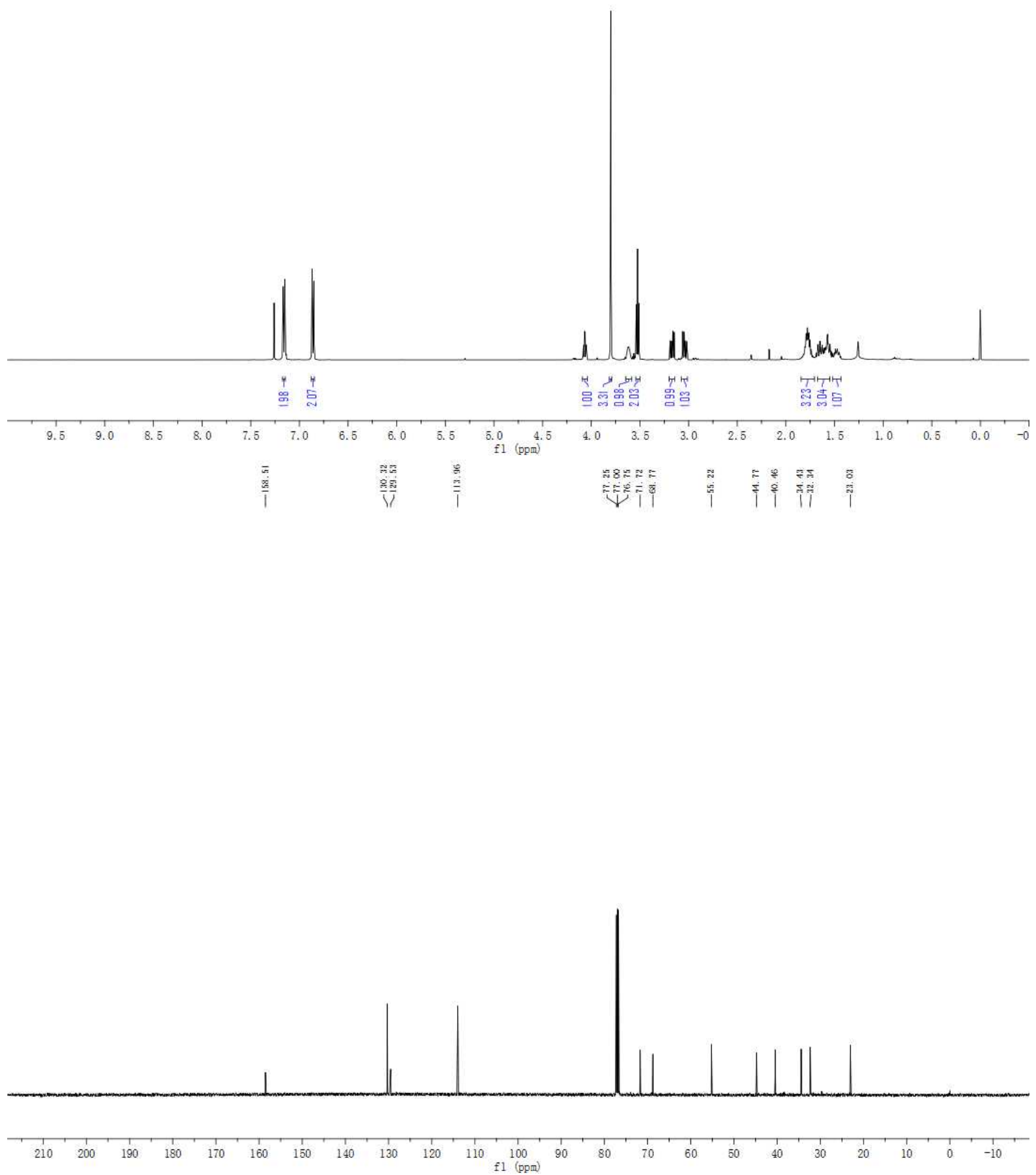


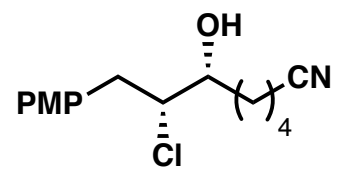

$\left(6 R^{*}, 7 R^{*}\right)-7-C h l o r o-6-h y d r o x y-8-(4-m e t h o x y p h e n y l) o c t a n e n i t r i l e ~( \pm)-6 r$

General Procedure C: Epoxide $( \pm)-5 \mathrm{r}(24 \mathrm{mg}, 0.10 \mathrm{mmol})$ was employed. Purification of the residue by FCC (10:1, hexane-EtOAc) afforded the title compound ( $25 \mathrm{mg}, 87 \%)$ as a colorless oil.

${ }^{1} \mathrm{H}$ NMR $\left(500 \mathrm{MHz}, \mathrm{CDCl}_{3}\right) \delta 7.15(\mathrm{~d}, J=8.6 \mathrm{~Hz}, 2 \mathrm{H}), 6.86(\mathrm{~d}, J=8.6 \mathrm{~Hz}, 2 \mathrm{H}), 4.05$ (td, $J=7.4$, $2.4 \mathrm{~Hz}, 1 \mathrm{H}), 3.80(\mathrm{~s}, 3 \mathrm{H}), 3.64-3.57(\mathrm{~m}, 1 \mathrm{H}), 3.16(\mathrm{dd}, J=14.1,7.3 \mathrm{~Hz}, 1 \mathrm{H}), 3.04(\mathrm{dd}, J=14.1$, $7.6 \mathrm{~Hz}, 1 \mathrm{H}), 2.34(\mathrm{t}, J=6.9 \mathrm{~Hz}, 2 \mathrm{H}), 1.80(\mathrm{~d}, J=9.3 \mathrm{~Hz}, 1 \mathrm{H}), 1.69-1.62(\mathrm{~m}, 3 \mathrm{H}), 1.61-1.44(\mathrm{~m}$, $3 \mathrm{H})$;

${ }^{13} \mathrm{C}$ NMR $\left(126 \mathrm{MHz} \mathrm{CDCl}_{3}\right) \delta 158.6,130.3,129.4,119.5,114.0,71.5,68.7,55.2,40.4,34.4,25.2$, 24.9, 17.1;

IR (NaCl, thin film) 3566, 3485, 2937, 2868, 2359, 2248, 1612, 1513, 1462, 1300, 1247, 1178, 1109, 1036, 836, $666 \mathrm{~cm}^{-1}$;

HRMS (ESI-TOF) $\mathrm{m} / \mathrm{z}$ calcd for $\mathrm{C}_{15} \mathrm{H}_{20}{ }^{35} \mathrm{CINNaO}_{2}{ }^{+}(\mathrm{M}+\mathrm{Na})^{+}:$304.1080, found 304.1073. 

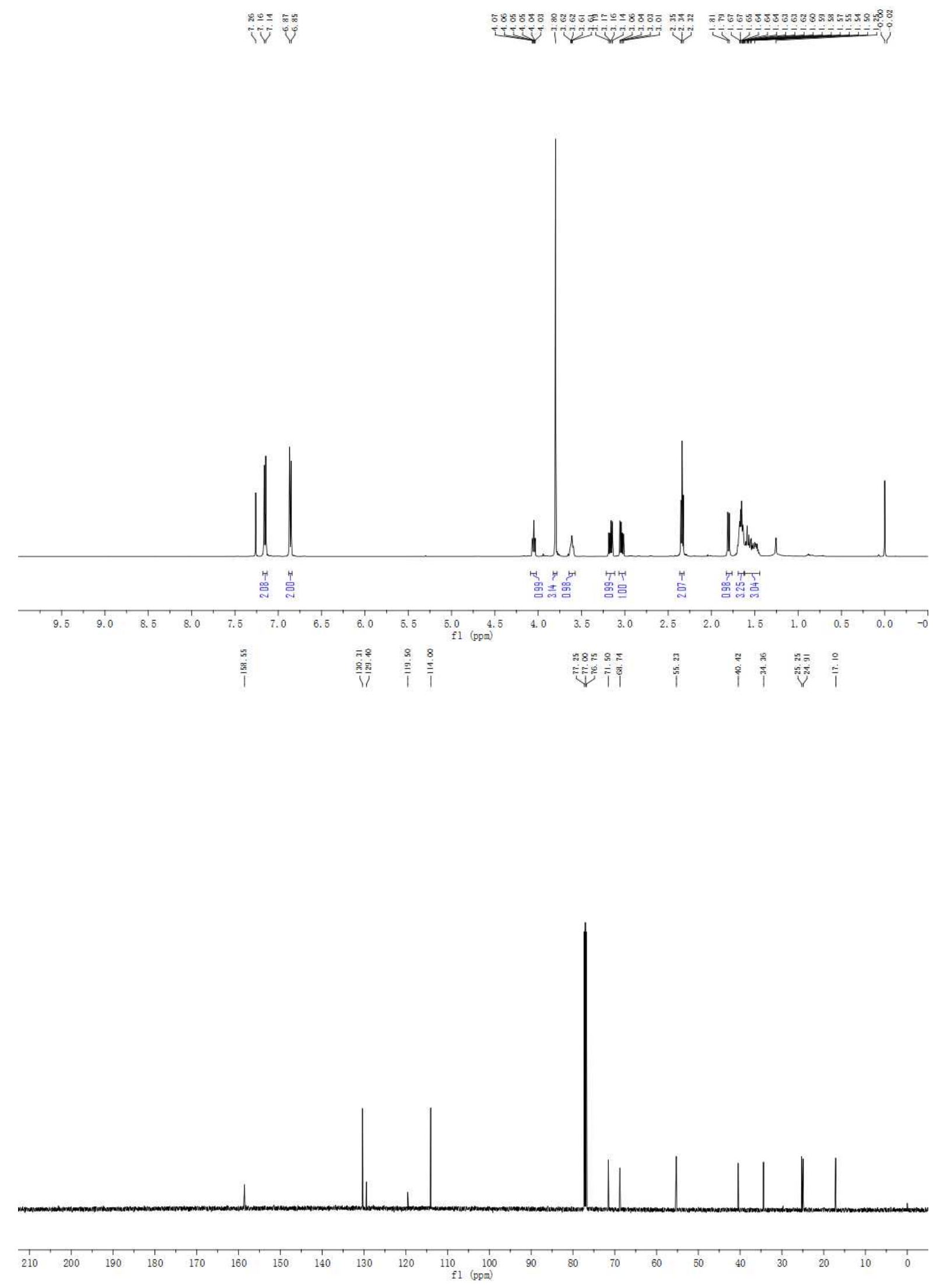


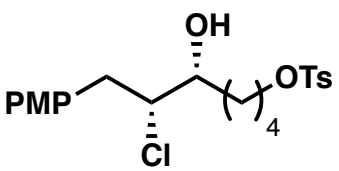

$\left(5 R^{*}, 6 R^{*}\right)-6-C h l o r o-5-h y d r o x y-7-(4-m e t h o x y p h e n y l) h e p t y l$ 4-methylbenzenesulfonate $( \pm)-6 \mathrm{~s}$

General Procedure C: Epoxide $( \pm)-5 \mathrm{~s}(39 \mathrm{mg}, 0.10 \mathrm{mmol})$ was employed. Purification of the residue by FCC (10:1, hexane-EtOAc) afforded the title compound (41 $\mathrm{mg}, 96 \%)$ as a colorless oil.

${ }^{1}$ H NMR (400 MHz, $\left.\mathrm{CDCl}_{3}\right) \delta 7.77$ (d, J = 7.8 Hz, 2H), $7.33(\mathrm{~d}, J=8.0 \mathrm{~Hz}, 2 \mathrm{H}), 7.14$ (d, J = 8.3 $\mathrm{Hz}, 2 \mathrm{H}), 6.85(\mathrm{~d}, J=8.1 \mathrm{~Hz}, 2 \mathrm{H}), 4.01(\mathrm{t}, J=6.1 \mathrm{~Hz}, 3 \mathrm{H}), 3.80(\mathrm{~s}, 3 \mathrm{H}), 3.55(\mathrm{~s}, 1 \mathrm{H}), 3.14(\mathrm{dd}, J=$ 14.0, 7.1 Hz, 1H), 3.01 (dd, $J=14.0,7.7 \mathrm{~Hz}, 1 \mathrm{H}), 2.44$ (s, 3H), $1.73(\mathrm{~d}, J=8.6 \mathrm{~Hz}, 1 \mathrm{H}), 1.69-1.60$ $(\mathrm{m}, 2 \mathrm{H}), 1.57-1.53(\mathrm{~m}, 1 \mathrm{H}), 1.51-1.42(\mathrm{~m}, 2 \mathrm{H}), 1.39-1.32(\mathrm{~m}, 1 \mathrm{H})$;

${ }^{13} \mathrm{C}$ NMR $\left(101 \mathrm{MHz}, \mathrm{CDCl}_{3}\right) \delta 158.5,144.7,133.1,130.3,129.8,129.5,127.9,114.0,71.6,70.2$, 68.8, 55.2, 40.4, 34.4, 28.7, 21.7, 21.6;

IR ( $\mathrm{NaCl}$, thin film) 3521, 2935, 2853, 1612, 1514, 1355, 1248, 1176, 1034, 932, 817, $749 \mathrm{~cm}^{-1}$;

HRMS (ESI-TOF) $\mathrm{m} / \mathrm{z}$ calcd for $\mathrm{C}_{21} \mathrm{H}_{27}{ }^{35} \mathrm{CINaO}_{5} \mathrm{~S}^{+}(\mathrm{M}+\mathrm{Na})^{+}: 449.1160$, found 449.1165 . 

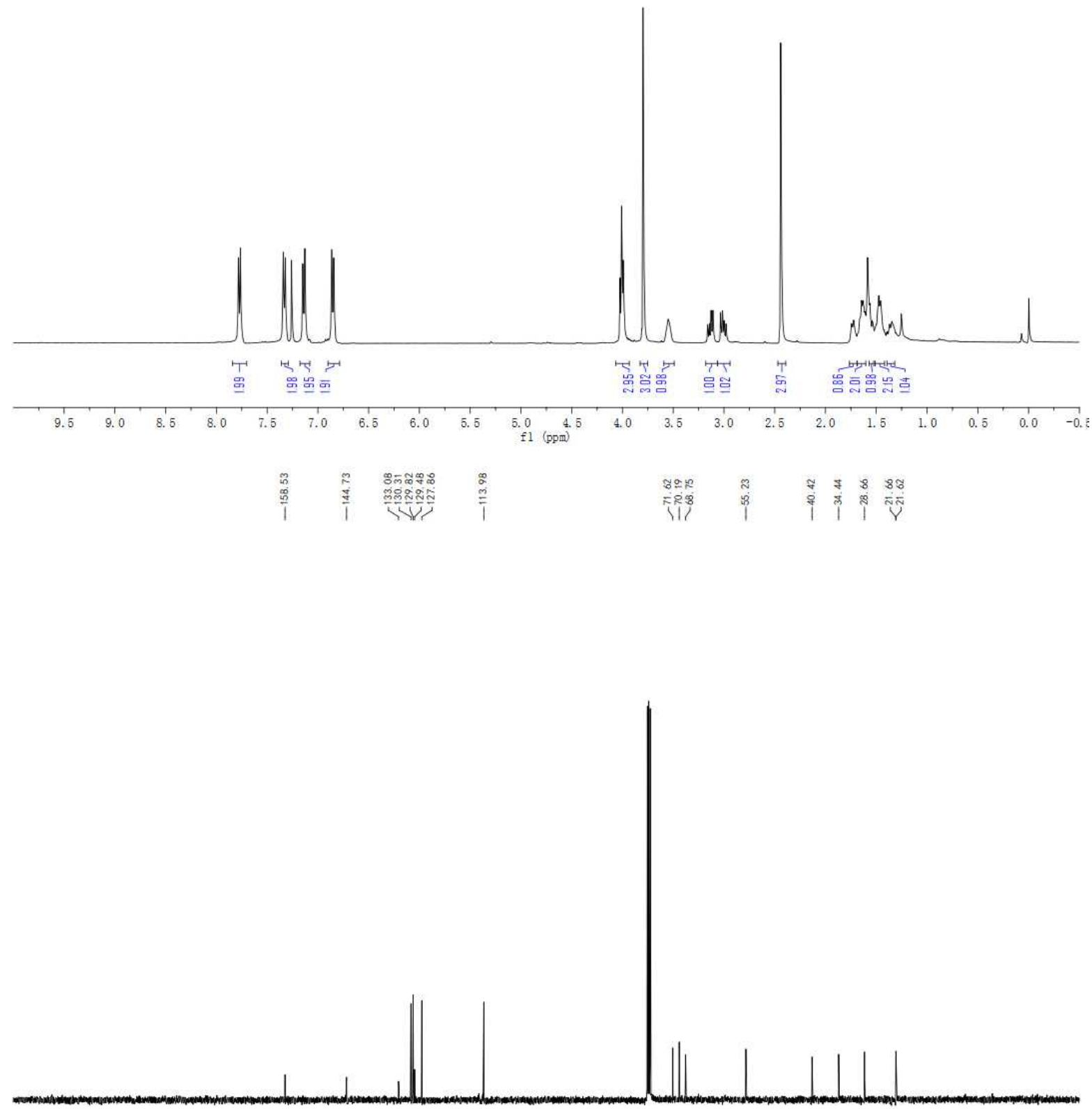

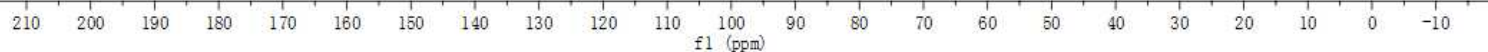


$\overbrace{\text { PMP }}^{\sum_{\bar{c}=1}^{\mathrm{OH}}} \mathrm{H}_{4}^{\mathrm{OTBS}}$

$\left(2 R^{*}, 3 R^{*}\right)-7-(($ tert-Butyldimethylsilyl)oxy)-2-chloro-1-(4-methoxyphenyl)heptan-3-ol ( \pm )-6u

General Procedure C: Epoxide $( \pm)-5 \mathrm{u}(35 \mathrm{mg}, 0.10 \mathrm{mmol})$ was employed. In a modification to the general procedure the reaction was quenched at $0{ }^{\circ} \mathrm{C}$. Purification of the residue by FCC $(5: 1$, hexane-EtOAc) afforded the title compound (36 mg, 93\%) as a colorless oil.

${ }^{1} \mathrm{H}$ NMR $\left(500 \mathrm{MHz}, \mathrm{CDCl}_{3}\right) \delta 7.16(\mathrm{~d}, \mathrm{~J}=8.3 \mathrm{~Hz}, 2 \mathrm{H}), 6.85(\mathrm{~d}, J=8.3 \mathrm{~Hz}, 2 \mathrm{H}), 4.06(\mathrm{t}, J=7.2 \mathrm{~Hz}$, $1 \mathrm{H}), 3.80(\mathrm{~s}, 3 \mathrm{H}), 3.60(\mathrm{t}, J=6.1 \mathrm{~Hz}, 3 \mathrm{H}), 3.17(\mathrm{dd}, J=14.1,7.0 \mathrm{~Hz}, 1 \mathrm{H}), 3.03(\mathrm{dd}, J=14.0,7.8$ $\mathrm{Hz}, 1 \mathrm{H}), 1.72(\mathrm{~m}, 1 \mathrm{H}), 1.68-1.34(\mathrm{~m}, 6 \mathrm{H}), 0.88(\mathrm{~s}, 9 \mathrm{H}), 0.04(\mathrm{~s}, 6 \mathrm{H})$;

${ }^{13} \mathrm{C} \mathrm{NMR}\left(126 \mathrm{MHz}, \mathrm{CDCl}_{3}\right) \delta 158.5,130.3,129.7,113.9,72.0,68.8,63.0,55.2,40.5,34.9,32.5$, 26.0, 22.0, 18.4, -5.3;

IR (thin film): 3434, 2933, 2856, 2358, 2341, 1653, 1514, 1473, 1301, 1252, 1107, 1041, 820, 772 $\mathrm{cm}^{-1}$;

HRMS (ESI-TOF) $m / z$ calcd for $\mathrm{C}_{20} \mathrm{H}_{35}{ }^{35} \mathrm{CINaO}_{3} \mathrm{Si}^{+}(\mathrm{M}+\mathrm{Na})^{+}:$409.1936, found 409.1937. 


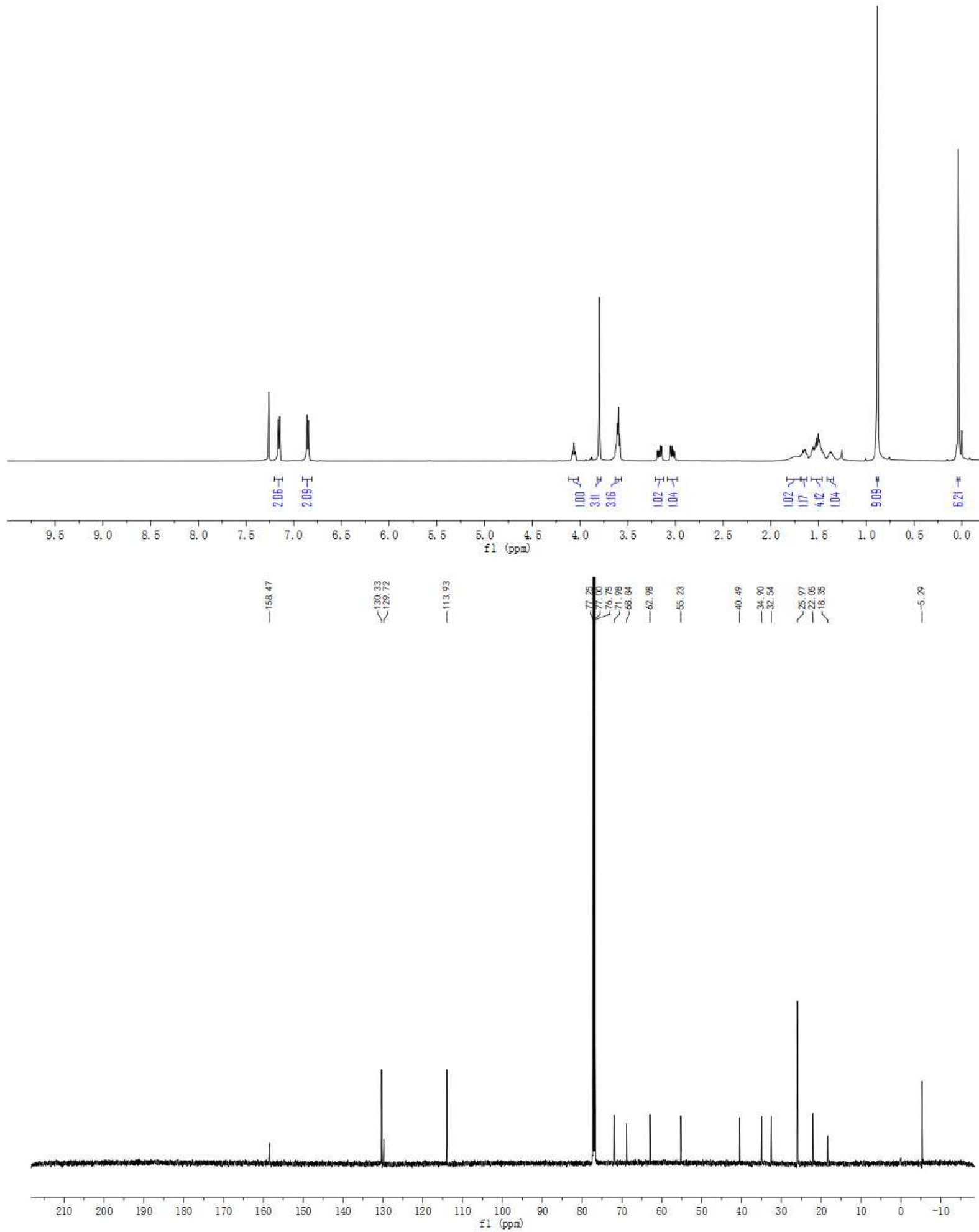


$\overbrace{\substack{\vdots \\ \bar{c} \mathbf{l}}}^{\stackrel{O}{O H}} Y_{4}^{O P h}$

$\left(2 R^{*}, 3 R^{*}\right)-2-C h l o r o-1-(4-m e t h o x y p h e n y l)-7-p h e n o x y h e p t a n-3-o l ~( \pm)-6 \mathrm{v}$

General Procedure C: Epoxide $( \pm)-5 v(31 \mathrm{mg}, 0.10 \mathrm{mmol})$ was employed. Purification of the residue by FCC (10:1, hexane-EtOAc) afforded the title compound (31 $\mathrm{mg}, 89 \%)$ as a colorless oil.

${ }^{1} \mathrm{H}$ NMR $\left(400 \mathrm{MHz}, \mathrm{CDCl}_{3}\right) \delta$ 7.30-7.25 (m, 2H), $7.16(\mathrm{~d}, J=8.2 \mathrm{~Hz}, 2 \mathrm{H}), 6.96-6.90(\mathrm{~m}, 1 \mathrm{H}), 6.87$ $(\mathrm{t}, J=8.3 \mathrm{~Hz}, 4 \mathrm{H}), 4.08(\mathrm{td}, J=7.5,2.6 \mathrm{~Hz}, 1 \mathrm{H}), 3.95(\mathrm{t}, J=6.1 \mathrm{~Hz}, 2 \mathrm{H}), 3.80(\mathrm{~s}, 3 \mathrm{H}), 3.67-3.60$ $(\mathrm{m}, 1 \mathrm{H}), 3.18(\mathrm{dd}, J=14.1,7.1 \mathrm{~Hz}, 1 \mathrm{H}), 3.04(\mathrm{dd}, J=14.0,7.7 \mathrm{~Hz}, 1 \mathrm{H}), 1.85-1.68(\mathrm{~m}, 4 \mathrm{H}), 1.63-$ $1.50(\mathrm{~m}, 3 \mathrm{H})$;

${ }^{13} \mathrm{C}$ NMR $\left(101 \mathrm{MHz}, \mathrm{CDCl}_{3}\right) \delta 158.9,158.5,130.3,129.6,129.4,120.6,114.4,114.0,71.9,68.8$, $67.5,55.2,40.5,34.9,29.1,22.4$;

IR (NaCl, thin film) 3448, 2924, 2854, 1600, 1513, 1496, 1246, 1078, 1032, $754 \mathrm{~cm}^{-1}$;

HRMS (ESI-TOF) $\mathrm{m} / \mathrm{z}$ calcd for $\mathrm{C}_{20} \mathrm{H}_{25}{ }^{35} \mathrm{CINaO}_{3}{ }^{+}(\mathrm{M}+\mathrm{Na})^{+}:$371.1390, found 371.1393. 

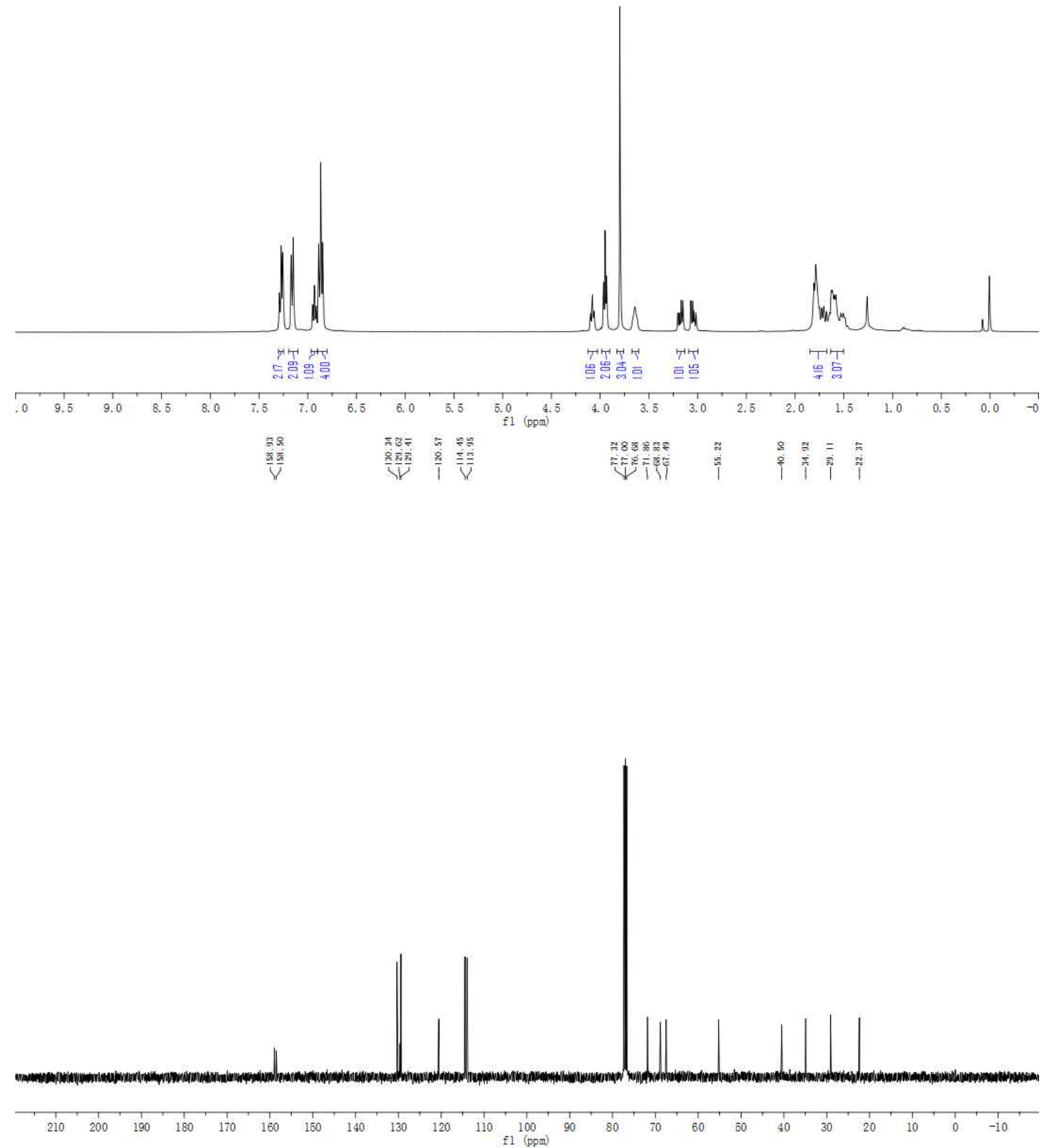


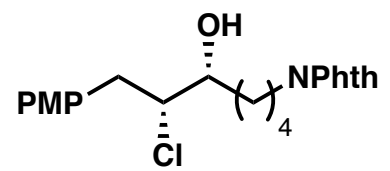

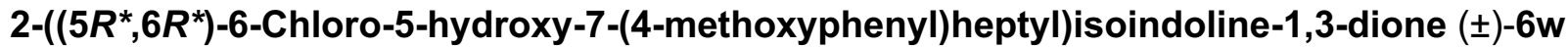

General Procedure C: Epoxide $( \pm)-5 \mathrm{w}(36.5 \mathrm{mg}, 0.10 \mathrm{mmol})$ was employed. Purification of the residue by FCC (5:1, hexane-EtOAc) afforded the title compound (37 $\mathrm{mg}, 92 \%)$ as a colorless oil.

${ }^{1} \mathrm{H}$ NMR $\left(500 \mathrm{MHz}, \mathrm{CDCl}_{3}\right) \delta 7.83(\mathrm{dd}, J=5.2,3.2 \mathrm{~Hz}, 2 \mathrm{H}), 7.70(\mathrm{dd}, J=5.2,3.1 \mathrm{~Hz}, 2 \mathrm{H}), 7.14$ $(\mathrm{d}, J=8.4 \mathrm{~Hz}, 2 \mathrm{H}), 6.84(\mathrm{~d}, J=8.4 \mathrm{~Hz}, 2 \mathrm{H}), 4.04(\mathrm{td}, J=7.4,2.2 \mathrm{~Hz}, 1 \mathrm{H}), 3.78(\mathrm{~s}, 3 \mathrm{H}), 3.68(\mathrm{t}, J$ $=7.1 \mathrm{~Hz}, 2 \mathrm{H}$ ), $3.59(\mathrm{~s}, 1 \mathrm{H}), 3.15(\mathrm{dd}, J=14.1,7.0 \mathrm{~Hz}, 1 \mathrm{H}), 3.01(\mathrm{dd}, J=14.1,7.8 \mathrm{~Hz}, 1 \mathrm{H}), 1.85$ (d, $J=8.8 \mathrm{~Hz}, 1 \mathrm{H}), 1.71-1.65(\mathrm{~m}, 3 \mathrm{H}), 1.60-1.48(\mathrm{~m}, 2 \mathrm{H}), 1.37$ (ddd, $J=21.9,14.6,5.6 \mathrm{~Hz}, 1 \mathrm{H})$;

${ }^{13} \mathrm{C} \mathrm{NMR}\left(126 \mathrm{MHz}, \mathrm{CDCl}_{3}\right) \delta 168.4,158.4,133.9,132.1,130.3,129.6,123.2,113.9,71.8,68.8$, 55.2, 40.4, 37.6, 34.5, 28.4, 22.9;

IR ( $\mathrm{NaCl}$, thin film) 3478, 2942, 1770, 1708, 1514, 1397, 1247, 1032, $721 \mathrm{~cm}^{-1}$;

HRMS (ESI-TOF) $\mathrm{m} / \mathrm{z}$ calcd for $\mathrm{C}_{22} \mathrm{H}_{24}{ }^{35} \mathrm{CINNaO}_{4}{ }^{+}(\mathrm{M}+\mathrm{Na})^{+}:$424.1286, found 424.1293. 

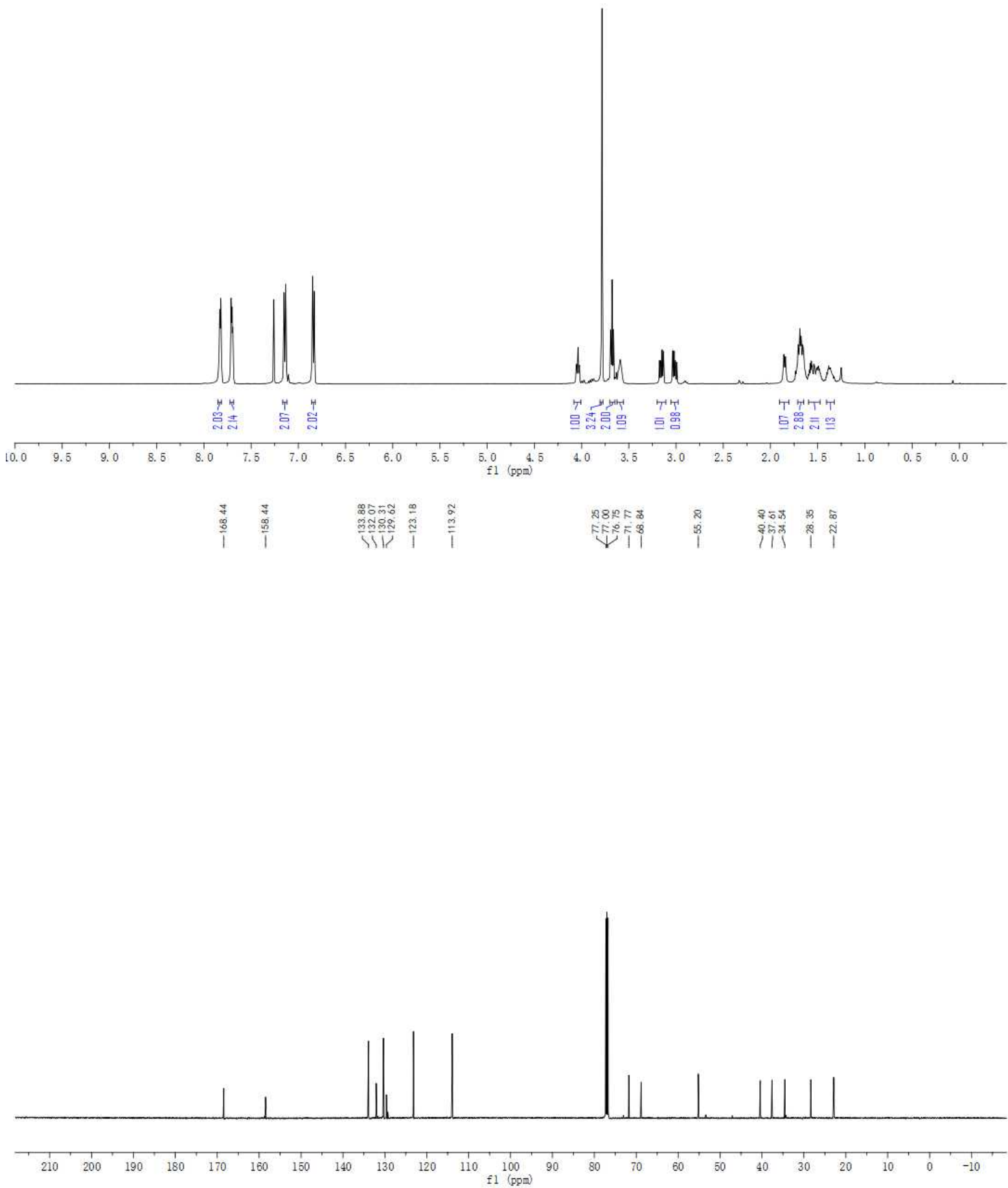


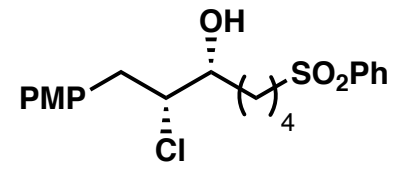

\section{$\left(2 R^{*}, 3 R^{*}\right)-2-C h l o r o-1-(4-m e t h o x y p h e n y l)-7-(p h e n y l s u l f o n y l) h e p t a n-3-o l ~( \pm)-6 x$}

General Procedure C: Epoxide $( \pm)-5 x(36 \mathrm{mg}, 0.10 \mathrm{mmol})$ was employed. Purification of the residue by FCC (5:1, hexane-EtOAc) afforded the title compound (37 $\mathrm{mg}, 93 \%)$ as a colorless oil.

${ }^{1} \mathrm{H}$ NMR $\left(500 \mathrm{MHz}, \mathrm{CDCl}_{3}\right) \delta 7.88(\mathrm{~d}, \mathrm{~J}=8.0 \mathrm{~Hz}, 2 \mathrm{H}), 7.65(\mathrm{t}, J=7.3 \mathrm{~Hz}, 1 \mathrm{H}), 7.56(\mathrm{t}, J=7.6 \mathrm{~Hz}$, $2 \mathrm{H}), 7.13(\mathrm{~d}, J=8.3 \mathrm{~Hz}, 2 \mathrm{H}), 6.84(\mathrm{~d}, J=8.4 \mathrm{~Hz}, 2 \mathrm{H}), 4.00(\mathrm{td}, J=7.3,1.9 \mathrm{~Hz}, 1 \mathrm{H}), 3.79(\mathrm{~s}, 3 \mathrm{H})$, 3.55 (s, 1H), 3.13 (dd, $J=14.1,7.1 \mathrm{~Hz}, 1 \mathrm{H}$ ), 3.07 (t, $J=7.9 \mathrm{~Hz}, 2 \mathrm{H}), 2.99$ (dd, $J=14.2,7.7 \mathrm{~Hz}$, $1 \mathrm{H}), 1.86(\mathrm{~d}, J=8.6 \mathrm{~Hz}, 1 \mathrm{H}), 1.72(\mathrm{dt}, J=13.5,6.7 \mathrm{~Hz}, 2 \mathrm{H}), 1.63-1.51(\mathrm{~m}, 2 \mathrm{H}), 1.43-1.35(\mathrm{~m}$, $1 \mathrm{H})$;

${ }^{13} \mathrm{C}$ NMR $\left(126 \mathrm{MHz}, \mathrm{CDCl}_{3}\right) \delta 158.5,139.0,133.6,130.3,129.4,129.2,128.0,113.9,71.5,68.7$, 56.0, 55.2, 40.3, 34.5, 24.4, 22.6;

IR ( $\mathrm{NaCl}$, thin film) 3481, 2949, 1611, 1514, 1455, 1302, 1247, 1147, 1085, $749 \mathrm{~cm}^{-1}$;

HRMS (ESI-TOF) $\mathrm{m} / \mathrm{z}$ calcd for $\mathrm{C}_{20} \mathrm{H}_{25}{ }^{35} \mathrm{CINaO}_{4} \mathrm{~S}^{+}(\mathrm{M}+\mathrm{Na})^{+}: 419.1060$, found 419.1058 . 

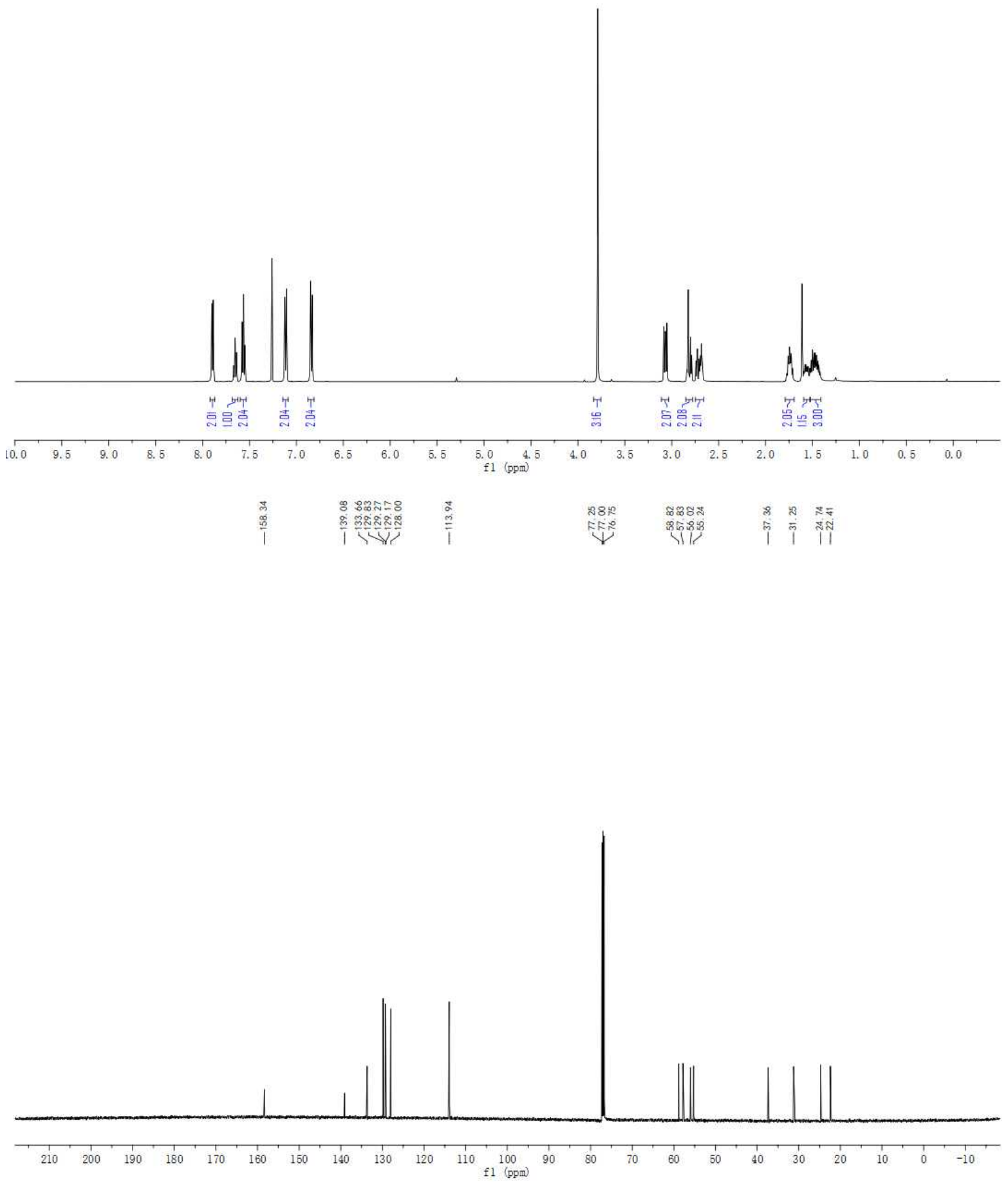
<smiles>O[C@@H](C1CCOCC1)[C@H](Cl)C[PbH]</smiles>

$\left(1 R^{*}, 2 R^{*}\right)-2-C h l o r o-3-(4-m e t h o x y p h e n y l)-1$-(tetrahydro-2H-pyran-4-yl)propan-1-ol ( \pm )-6z

General Procedure C: Epoxide $( \pm)-5 z(25 \mathrm{mg}, 0.10 \mathrm{mmol})$ was employed. Purification of the residue by FCC (2:1, hexane-EtOAc) afforded the title compound ( $27 \mathrm{mg}, 95 \%)$ as a colorless solid

${ }^{1} \mathrm{H}$ NMR $\left(500 \mathrm{MHz}, \mathrm{CDCl}_{3}\right) \delta 7.15(\mathrm{~d}, J=8.6 \mathrm{~Hz}, 2 \mathrm{H}), 6.86(\mathrm{~d}, J=8.6 \mathrm{~Hz}, 2 \mathrm{H}), 4.21$ (td, $J=7.5$, $1.4 \mathrm{~Hz}, 1 \mathrm{H}), 3.99$ (dd, $J=11.5,3.7 \mathrm{~Hz}, 1 \mathrm{H}), 3.94(\mathrm{dd}, J=11.3,4.1 \mathrm{~Hz}, 1 \mathrm{H}), 3.80(\mathrm{~s}, 3 \mathrm{H}), 3.35$ (t, $J=11.8 \mathrm{~Hz}, 2 \mathrm{H}), 3.22(\mathrm{dd}, J=14.1,4.9 \mathrm{~Hz}, 1 \mathrm{H}), 3.12(\mathrm{tt}, J=14.0,6.9 \mathrm{~Hz}, 2 \mathrm{H}), 1.97-1.89(\mathrm{~m}$, $1 \mathrm{H}), 1.85-1.76(\mathrm{~m}, 1 \mathrm{H}), 1.74(\mathrm{t}, J=7.1 \mathrm{~Hz}, 1 \mathrm{H}), 1.49(\mathrm{dd}, J=12.8,1.7 \mathrm{~Hz}, 1 \mathrm{H}), 1.34(\mathrm{ddd}, J=$ 25.2, 12.3, $4.6 \mathrm{~Hz}, 1 \mathrm{H}), 1.21-1.13(\mathrm{~m}, 1 \mathrm{H})$;

${ }^{13} \mathrm{C}$ NMR $\left(126 \mathrm{MHz}, \mathrm{CDCl}_{3}\right) \delta 158.5,130.2,129.4,114.0,75.6,67.8,67.2,65.3,55.2,40.7,39.1$, 29.6, 28.5;

IR ( $\mathrm{NaCl}$, thin film) 3415, 2925, 2851, 1611, 1513, 1455, 1247, 1089, $820 \mathrm{~cm}^{-1}$;

HRMS (ESI-TOF) $m / z$ calcd for $\mathrm{C}_{15} \mathrm{H}_{21}{ }^{35} \mathrm{CINaO}_{3}{ }^{+}(\mathrm{M}+\mathrm{Na})^{+}:$307.1077, found 307.1077. 


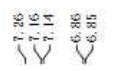

MANA

iriritis
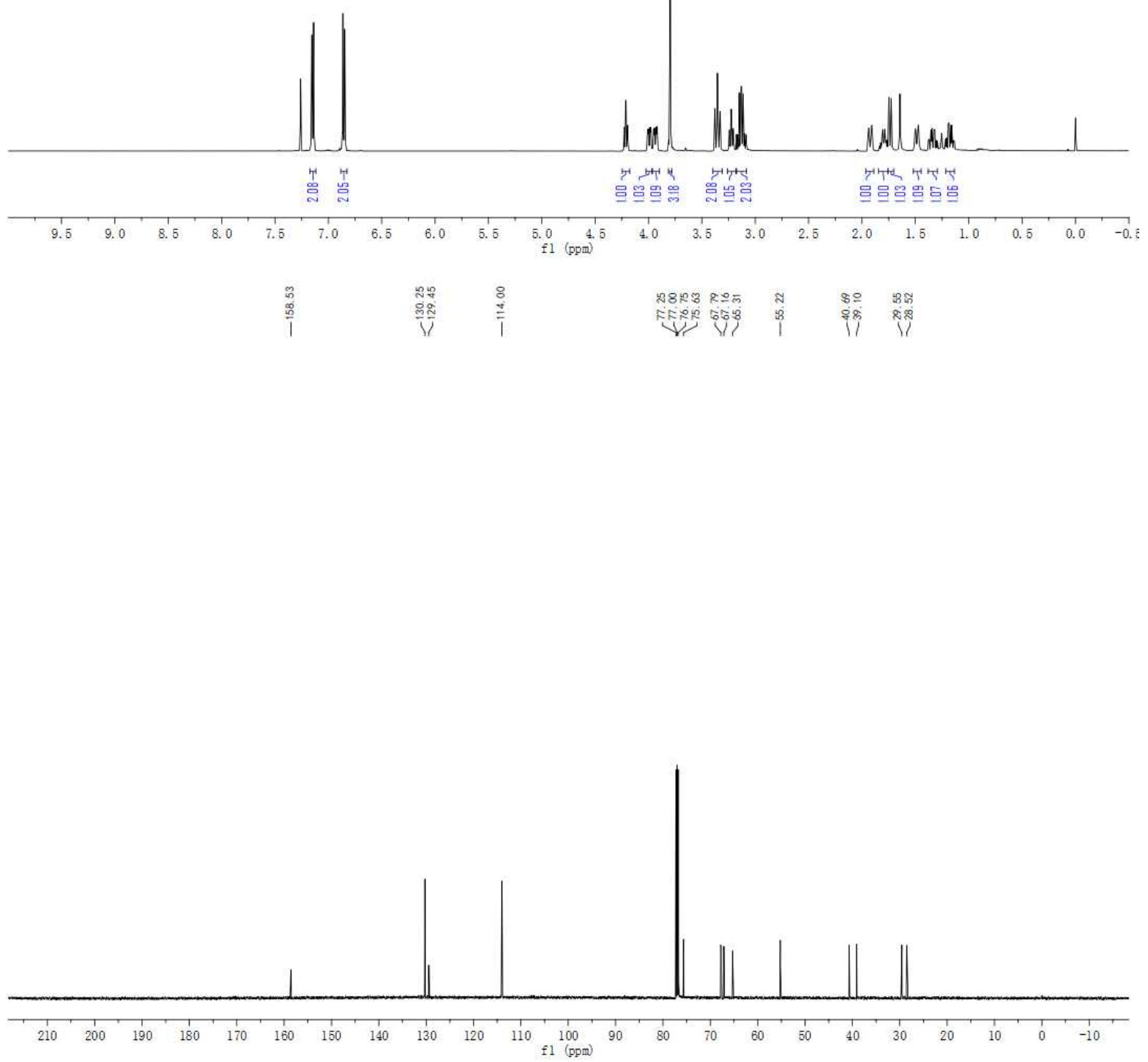

S175 
<smiles>O[C@@H](C1CC[N+](S)(S)CC1)[C@H](Cl)CP</smiles>

\section{$\left(1 R^{*}, 2 R^{*}\right)-2-C h l o r o-3-(4-m e t h o x y p h e n y l)-1-(1-t o s y l p i p e r i d i n-4-y l) p r o p a n-1-o l ~( \pm)-6 a a$}

General Procedure C: Epoxide ( \pm )-5aa-Ts (40 mg, $0.10 \mathrm{mmol}$ ) was employed. Purification of the residue by FCC (2:1, hexane-EtOAc) afforded the title compound ( $40 \mathrm{mg}, 92 \%)$ as a colorless oil.

${ }^{1} \mathrm{H}$ NMR $\left(500 \mathrm{MHz}, \mathrm{CDCl}_{3}\right) \delta 7.61(\mathrm{~d}, J=8.2 \mathrm{~Hz}, 2 \mathrm{H}), 7.31(\mathrm{~d}, J=8.1 \mathrm{~Hz}, 2 \mathrm{H}), 7.12(\mathrm{~d}, J=8.5$ $\mathrm{Hz}, 2 \mathrm{H}), 6.84(\mathrm{~d}, J=8.5 \mathrm{~Hz}, 2 \mathrm{H}), 4.16(\mathrm{dd}, J=8.0,7.0 \mathrm{~Hz}, 1 \mathrm{H}), 3.82-3.76(\mathrm{~m}, 5 \mathrm{H}), 3.20(\mathrm{t}, J=8.3$ $\mathrm{Hz}, 1 \mathrm{H}), 3.13-3.03(\mathrm{~m}, 2 \mathrm{H}), 2.42(\mathrm{~s}, 3 \mathrm{H}), 2.19-2.13(\mathrm{~m}, 2 \mathrm{H}), 2.08(\mathrm{~d}, J=13.0 \mathrm{~Hz}, 1 \mathrm{H}), 1.72(\mathrm{~d}, J$ $=9.7 \mathrm{~Hz}, 1 \mathrm{H}), 1.64(\mathrm{~s}, 1 \mathrm{H}), 1.46(\mathrm{ddd}, J=11.8,10.1,6.3 \mathrm{~Hz}, 1 \mathrm{H}), 1.34(\mathrm{dt}, J=12.2,8.2 \mathrm{~Hz}, 1 \mathrm{H})$, 1.24-1.17 (m, 1H);

${ }^{13} \mathrm{C} \mathrm{NMR}\left(126 \mathrm{MHz}, \mathrm{CDCl}_{3}\right) \delta 158.6,143.5,132.8,130.2,129.6,129.2,127.7,114.0,75.0,65.5$, 55.2, 46.2, 45.8, 40.6, 39.4, 28.2, 27.2, 21.5;

IR ( $\mathrm{NaCl}$, thin film) 3672, 3579, 3503, 2922, 2927, 2359, 2323, 1613, 1519, 1464, 1238, 1035, $818 \mathrm{~cm}^{-1}$;

HRMS (ESI-TOF) $\mathrm{m} / \mathrm{z}$ calcd for $\mathrm{C}_{22} \mathrm{H}_{28}{ }^{35} \mathrm{CINNaO}_{4} \mathrm{~S}^{+}(\mathrm{M}+\mathrm{Na})^{+}:$460.1320, found 460.1326. 

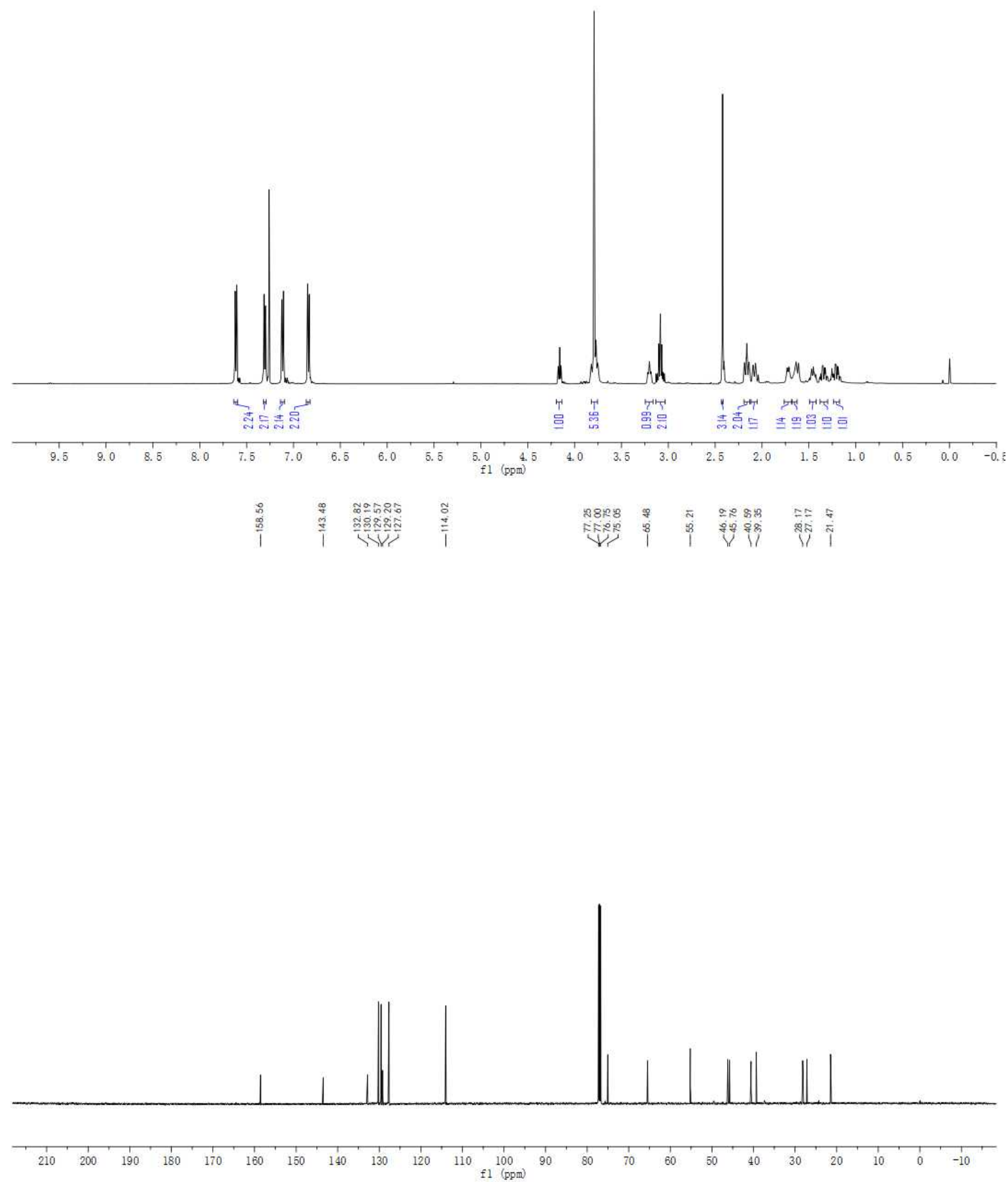
<smiles>O[C@H](C1CN(S)C1)[C@H](Cl)C[Pb]</smiles>

\section{$\left(1 R^{*}, 2 R^{*}\right)-2-C h l o r o-3-(4-m e t h o x y p h e n y l)-1-(1-t o s y l a z e t i d i n-3-y l) p r o p a n-1-o l ~( \pm)-6 a b$}

General Procedure C: Epoxide ( \pm )-5ab-Ts (37 mg, $0.10 \mathrm{mmol}$ ) was employed. Purification of the residue by FCC (5:1, hexane-EtOAc) afforded the title compound (37 $\mathrm{mg}, 90 \%)$ as a colorless oil.

${ }^{1} \mathrm{H}$ NMR $\left(500 \mathrm{MHz}, \mathrm{CDCl}_{3}\right) \delta 7.67(\mathrm{~d}, J=7.9 \mathrm{~Hz}, 2 \mathrm{H}), 7.36(\mathrm{~d}, J=7.9 \mathrm{~Hz}, 2 \mathrm{H}), 7.00(\mathrm{~d}, J=8.3$ $\mathrm{Hz}, 2 \mathrm{H}), 6.84(\mathrm{~d}, J=8.4 \mathrm{~Hz}, 2 \mathrm{H}), 3.88(\mathrm{dd}, J=11.3,4.6 \mathrm{~Hz}, 1 \mathrm{H}), 3.83-3.78(\mathrm{~m}, 4 \mathrm{H}), 3.73-3.64$ $(\mathrm{m}, 3 \mathrm{H}), 3.38(\mathrm{t}, J=8.3 \mathrm{~Hz}, 1 \mathrm{H}), 3.28(\mathrm{t}, J=7.5 \mathrm{~Hz}, 1 \mathrm{H}), 2.71(\mathrm{dd}, J=12.3,8.0 \mathrm{~Hz}, 1 \mathrm{H}), 2.48-$ $2.41(\mathrm{~m}, 4 \mathrm{H}), 1.99(\mathrm{~s}, 1 \mathrm{H})$;

${ }^{13} \mathrm{C} \mathrm{NMR}\left(126 \mathrm{MHz}, \mathrm{CDCl}_{3}\right) \delta 159.1,144.0,131.5,130.2,129.7,129.0,128.3,114.2,73.4,55.2$, 52.3, 51.8, 51.6, 46.5, 32.5, 21.6;

IR (thin film): 3502, 2959, 2361, 2329, 1611, 1514, 1457, 1333, 1249, 1158, 999, 818, $722 \mathrm{~cm}^{-1}$; HRMS (ESI-TOF) $\mathrm{m} / \mathrm{z}$ calcd for $\mathrm{C}_{20} \mathrm{H}_{24}{ }^{35} \mathrm{CINNaO}_{4} \mathrm{~S}^{+}(\mathrm{M}+\mathrm{Na})^{+}:$432.1007, found 432.1011. 


$$
\text { I }
$$




\section{$\mathrm{TiCl}_{4}$ reaction}

General Procedure D: for the $\mathrm{TiCl}_{4}$ ring opening of epoxides<smiles>[R]C[C@@H]1O[C@@H]1C[17O]</smiles>
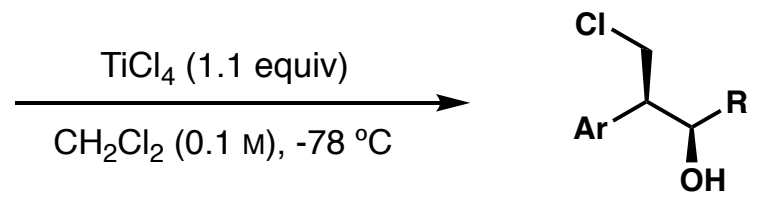

To an oven-dried reaction tube, charged with a stir bar, was added the epoxide derivative (100 mol\%). The tube was sealed with a septum and purged with argon after which anhydrous $\mathrm{CH}_{2} \mathrm{Cl}_{2}$ $\left(0.1 \mathrm{M}\right.$ total reaction concentration) was added. The reaction mixture was cooled to $-78{ }^{\circ} \mathrm{C}$ and $\mathrm{TiCl}_{4}\left(0.5 \mathrm{M}\right.$ in $\mathrm{CH}_{2} \mathrm{Cl}_{2}, 120 \mathrm{~mol} \%$ ) was added dropwise via syringe over five minutes. The reaction was stirred at this temperature for 10 minutes and quenched with saturated aq. $\mathrm{NaHCO}_{3}(2 \mathrm{~mL})$. The solution was filtered through celite ${ }^{\circledR}$, washed with water $(2 \times 5 \mathrm{~mL})$ and brine $(5 \mathrm{~mL})$, dried with $\mathrm{MgSO}_{4}$, and concentrated in vacuo. Purification of the residue by $\mathrm{FCC}$, under the conditions noted, afforded the pure alcohol. 
<smiles>COc1ccc([C@H](CCl)[C@H](O)C(C)C)cc1</smiles>

$\left(2 R^{*}, 3 R^{*}\right)-1-C h l o r o-2-(4-m e t h o x y p h e n y l) h e x a n-3-o l ~( \pm)-7 a$

General Procedure D: Epoxide $( \pm)-5 a(190 \mathrm{mg}, 0.93 \mathrm{mmol})$ was employed. In a modification to the general procedure $\mathrm{TiCl}_{4}(2.1 \mathrm{~mL}, 1.0 \mathrm{mmol})$ was added neat. Purification of the residue by FCC (3:1, hexane-EtOAc) afforded the title compound $(0.22 \mathrm{~g}, 96 \%)$ as a pale yellow oil.

${ }^{1} \mathrm{H}$ NMR $\left(500 \mathrm{MHz}, \mathrm{CDCl}_{3}\right) \delta 7.16(\mathrm{~d}, J=8.5 \mathrm{~Hz}, 2 \mathrm{H}), 6.91(\mathrm{~d}, J=8.6 \mathrm{~Hz}, 2 \mathrm{H}), 4.02$ (dd, $J=10.9$, $4.2 \mathrm{~Hz}, 1 \mathrm{H}), 3.93(\mathrm{dd}, J=10.9,8.2 \mathrm{~Hz}, 1 \mathrm{H}), 3.92-3.90(\mathrm{~m}, 1 \mathrm{H}), 3.83(\mathrm{~s}, 3 \mathrm{H}), 2.95$ (app. td, $J=$ 8.1, $4.2 \mathrm{~Hz}, 1 \mathrm{H}), 1.63$ (br. s, 1H), 1.54-1.46 (m, 1H), 1.41-1.27 (m, 3H), 0.88, (t, J = 6.9 Hz, 3H);

${ }^{13} \mathrm{C}$ NMR (125 MHz, $\left.\mathrm{CDCl}_{3}\right) \delta$ 158.7, 131.9, 129.4, 114.0, 73.3, 55.2, 53.4, 46.9, 37.2, 18.8, 13.9;

IR ( $\mathrm{NaCl}$, thin film) 3421, 2958, 2872, 1611, 1514, 1464, 1301, 1249, 1180, 1035, $830 \mathrm{~cm}^{-1}$;

HRMS (ESI-TOF) $\mathrm{m} / \mathrm{z}$ calcd for $\mathrm{C}_{13} \mathrm{H}_{19}{ }^{35} \mathrm{ClO}_{2} \mathrm{Na}^{+}(\mathrm{M}+\mathrm{Na})^{+}:$265.0971, found 265.0964. 

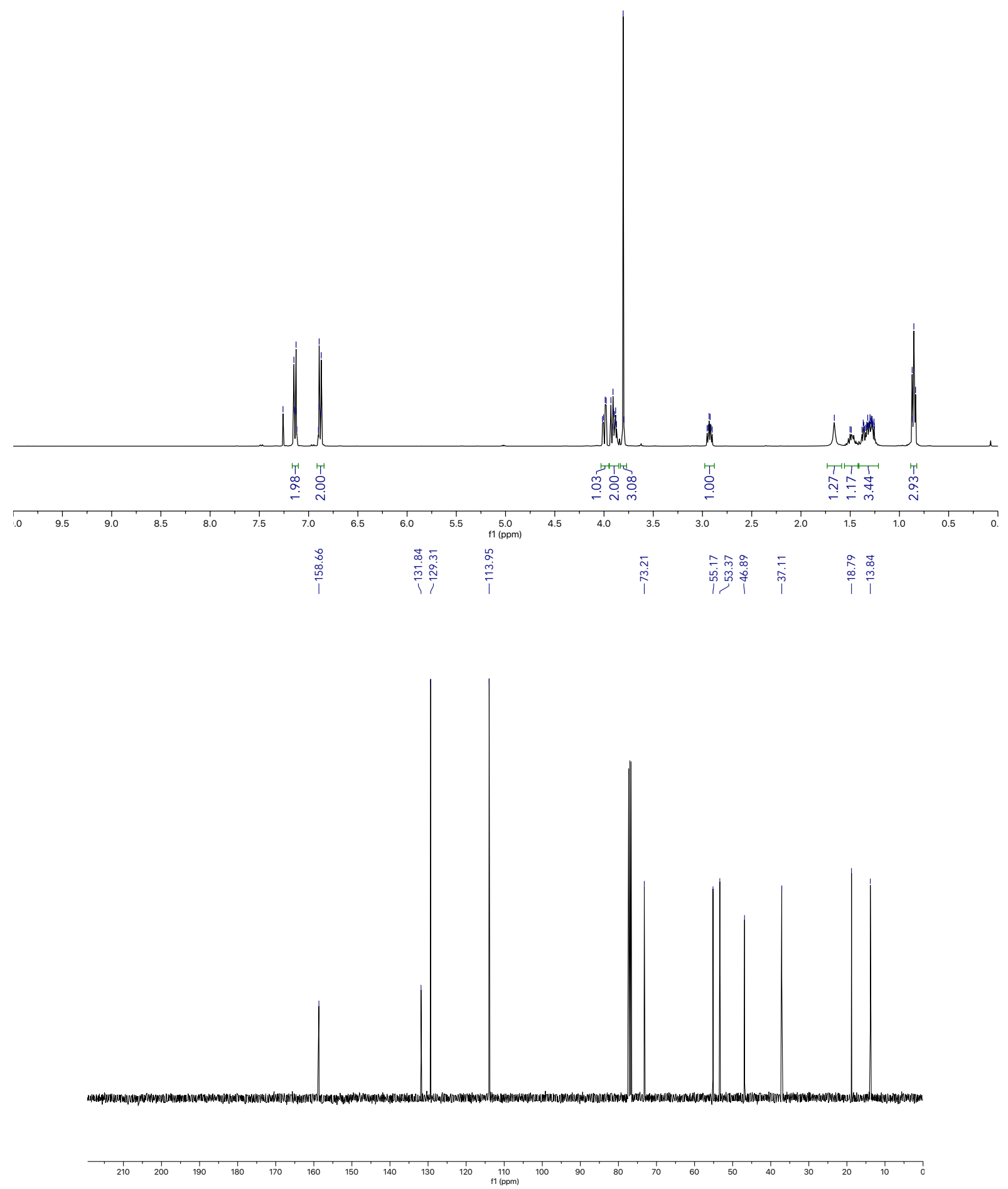
<smiles>COc1ccc([C@H](CBr)[C@H](O)C(C)C)cc1</smiles>

$\left(2 R^{*}, 3 R^{*}\right)$-1-Bromo-2-(4-methoxyphenyl)hexan-3-ol $( \pm)-7 \mathrm{a}_{\mathrm{Br}}$

General Procedure D: Epoxide ( \pm )-5a (190 mg, $0.93 \mathrm{mmol})$ was employed. In a modification to the general procedure $\mathrm{TiBr}_{4}$ was employed in place of $\mathrm{TiCl}_{4}$. Purification of the residue by $\mathrm{FCC}$ (3:1, hexane-EtOAc) afforded the title compound (26 mg, 91\%) as a pale colorless oil.

${ }^{1} \mathrm{H}$ NMR $\left(500 \mathrm{MHz} \mathrm{CDCl}_{3}\right) \delta 7.12(\mathrm{~d}, J=8.3 \mathrm{~Hz}, 2 \mathrm{H}), 6.88(\mathrm{~d}, J=8.3 \mathrm{~Hz}, 2 \mathrm{H}), 3.9-3.85(\mathrm{~m}, 2 \mathrm{H})$, $3.81(\mathrm{~s}, 3 \mathrm{H}), 3.74(\mathrm{t}, J=9.4 \mathrm{~Hz}, 1 \mathrm{H}), 2.96(\mathrm{td}, J=8.3,4.0 \mathrm{~Hz}, 1 \mathrm{H}), 1.61(\mathrm{~s}, 1 \mathrm{H}), 1.48(\mathrm{dd}, J=$ 16.5, 7.7 Hz, 1H), 1.36 (dd, $J=15.0,5.2 \mathrm{~Hz}, 1 \mathrm{H}), 1.32-1.26(\mathrm{~m}, 2 \mathrm{H}), 0.85(\mathrm{t}, J=6.8 \mathrm{~Hz}, 3 \mathrm{H})$;

${ }^{13} \mathrm{C}$ NMR (126 MHz, $\mathrm{CDCl}_{3}$ ) $\delta$ 158.7, 132.2, 129.2, 114.0, 74.0, 55.2, 53.4 37.1, 36.3, 18.8, 13.9;

IR (thin film): 3400, 2958, 2934, 2872, 2360, 2341, 1612, 1514, 1464, 1248, 1180, 1032, 820, 669 $\mathrm{cm}^{-1}$;

HRMS (ESI-TOF) $m / z$ calcd for $\mathrm{C}_{13} \mathrm{H}_{19}{ }^{79} \mathrm{BrNaO}_{2}{ }^{+}(\mathrm{M}+\mathrm{Na})^{+}: 309.0461$, found 309.0460 . 

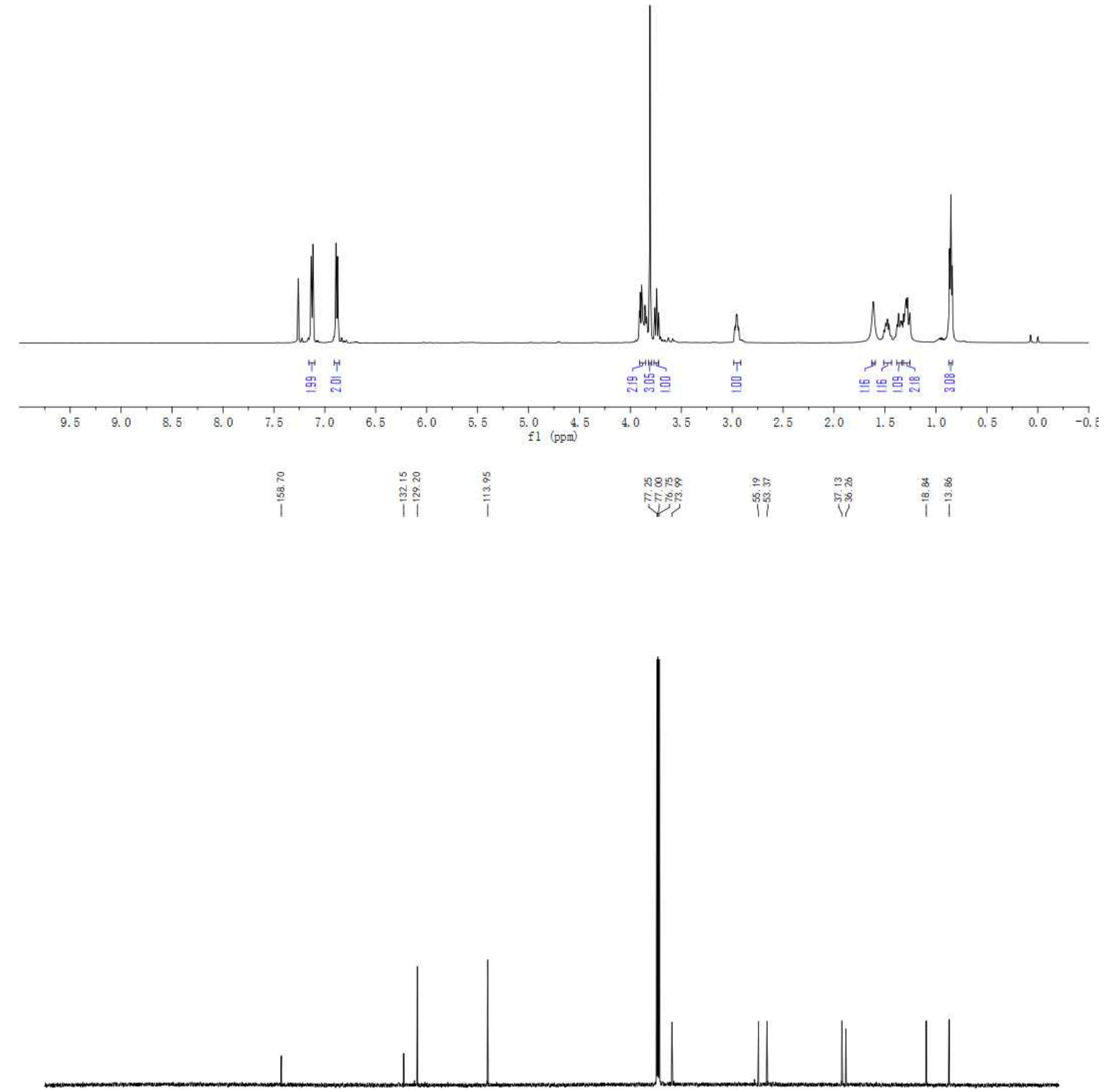

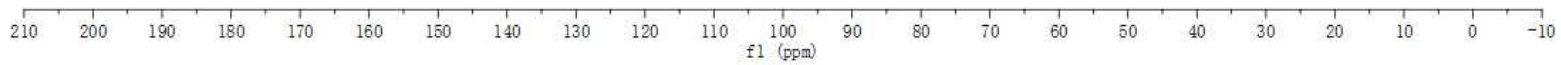


<smiles>COc1ccc([C@@H](CCl)[C@H](O)C(C)C)cc1OC</smiles>

\section{$\left(2 R^{*}, 3 R^{\star}\right)$-1-Chloro-2-(3,4-dimethoxyphenyl)hexan-3-ol ( \pm )-7b}

General Procedure D: Epoxide $( \pm)-5 b(35 \mathrm{mg}, 0.15 \mathrm{mmol})$ was employed. Purification of the residue by FCC (4:1, hexane-EtOAc) afforded the title compound (33 $\mathrm{mg}, 91 \%)$ as a colorless solid.

${ }^{1} \mathrm{H}$ NMR $\left(400 \mathrm{MHz}, \mathrm{CDCl}_{3}\right) \delta 6.84(\mathrm{~d}, J=8.1 \mathrm{~Hz}, 1 \mathrm{H}), 6.77-6.73(\mathrm{~m}, 2 \mathrm{H}), 4.00(\mathrm{dd}, J=10.9,4.2$ $\mathrm{Hz}, 1 \mathrm{H}$ ), 3.94-3.86 (m, 8H), 2.90 (ddd, $J=4.2,4.0,4.0 \mathrm{~Hz}, 1 \mathrm{H}), 1.71$ (br. s, 1H), 1.54-1.44 (m, $1 \mathrm{H}), 1.39-1.25(\mathrm{~m}, 3 \mathrm{H}), 0.86(\mathrm{t}, J=7.0 \mathrm{~Hz}, 3 \mathrm{H})$;

${ }^{13} \mathrm{C}$ NMR $\left(100 \mathrm{MHz}, \mathrm{CDCl}_{3}\right) \delta 148.9,148.1,132.4,120.4,111.6,111.2,73.2,55.9,55.8,53.7$, $46.8,37.2,18.8,13.8$;

IR ( $\mathrm{NaCl}$, thin film) 3423, 2961, 2870, 1609, 1512, 1464, 1302, 1239, 1182, 1035, $838 \mathrm{~cm}^{-1}$;

HRMS (ESI-TOF) $\mathrm{m} / \mathrm{z}$ calcd for $\mathrm{C} 14 \mathrm{H} 21 \mathrm{ClO}_{3} \mathrm{Na}(\mathrm{M}+\mathrm{Na})^{+}:$295.1077, found 295.1079. 


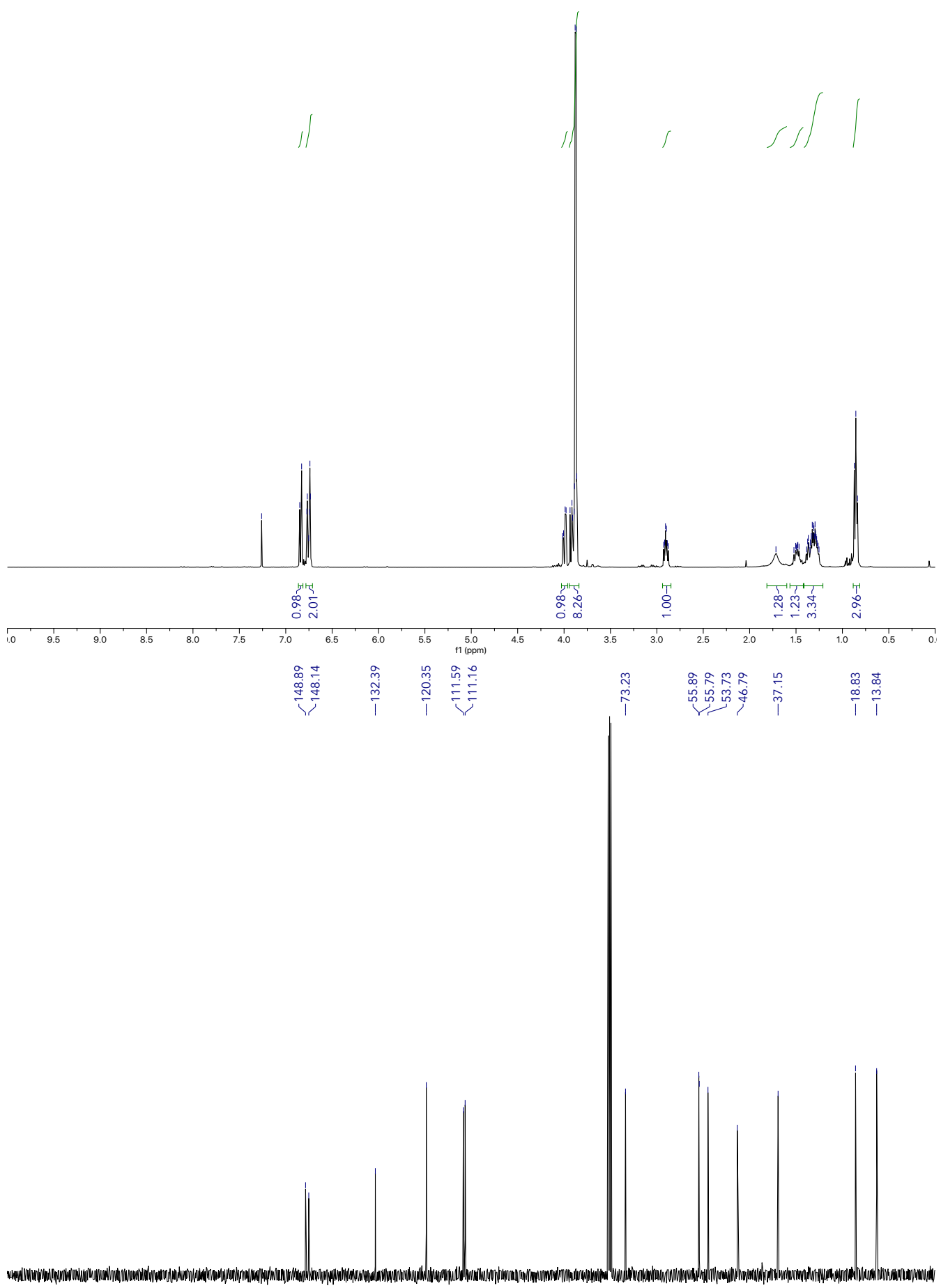

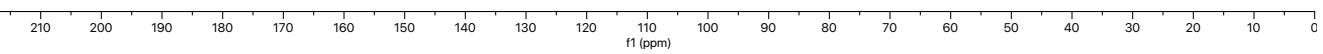


<smiles>CCCC(O)[C@@H](CCl)c1ccc2c(c1)OCO2</smiles>

$\left(2 R^{*}, 3 R^{*}\right)-2-(B e n z o[d][1,3]$ dioxol-5-yl)-1-chlorohexan-3-ol ( \pm )-7c

General Procedure D: Epoxide $( \pm)-5 \mathrm{c}(110 \mathrm{mg}, 0.50 \mathrm{mmol})$ was employed. Purification of the residue by FCC (3:1, hexane-EtOAc) afforded the title compound (124 $\mathrm{mg}, 97 \%)$ as a colorless solid.

${ }^{1} \mathrm{H}$ NMR $\left(400 \mathrm{MHz}, \mathrm{CDCl}_{3}\right) \delta 6.78(\mathrm{~d}, J=7.9 \mathrm{~Hz}, 1 \mathrm{H}), 6.73(\mathrm{~d}, J=1.8 \mathrm{~Hz}, 1 \mathrm{H}), 6.66(\mathrm{dd}, J=7.9$, $1.8 \mathrm{~Hz}, 1 \mathrm{H}), 5.95(\mathrm{~s}, 2 \mathrm{H}), 3.99(\mathrm{dd}, J=10.9,4.1 \mathrm{~Hz}, 1 \mathrm{H}), 3.89-3.82(\mathrm{~m}, 2 \mathrm{H}), 2.88$ (ddd, $J=4.1$, 4.1, $4.1 \mathrm{~Hz}, 1 \mathrm{H}), 1.79$ (br. s, $1 \mathrm{H}), 1.55-1.43(\mathrm{~m}, 1 \mathrm{H}), 1.39-1.23(\mathrm{~m}, 3 \mathrm{H}), 0.86(\mathrm{t}, J=7.1 \mathrm{~Hz}, 3 \mathrm{H})$;

${ }^{13} \mathrm{C}$ NMR $\left(100 \mathrm{MHz}, \mathrm{CDCl}_{3}\right) \delta 147.8,146.6,133.6,121.7,108.4,108.3,101.0,73.2,53.9,46.8$, 37.2, 18.8, 13.8;

IR (NaCl, thin film): 3399, 2959, 2934, 2874, 1488, 1442, 1323, 1245, 1121, 1040, $934 \mathrm{~cm}^{-1}$; HRMS $\left(\mathrm{Cl}^{+}\right) \mathrm{m} / \mathrm{z}$ calcd for $\mathrm{C}_{13} \mathrm{H}_{17}{ }^{35} \mathrm{ClO}_{3} \mathrm{NH}_{4}{ }^{+}\left(\mathrm{M}+\mathrm{NH}_{4}\right)^{+}:$: 274.1204, found 274.1206. 

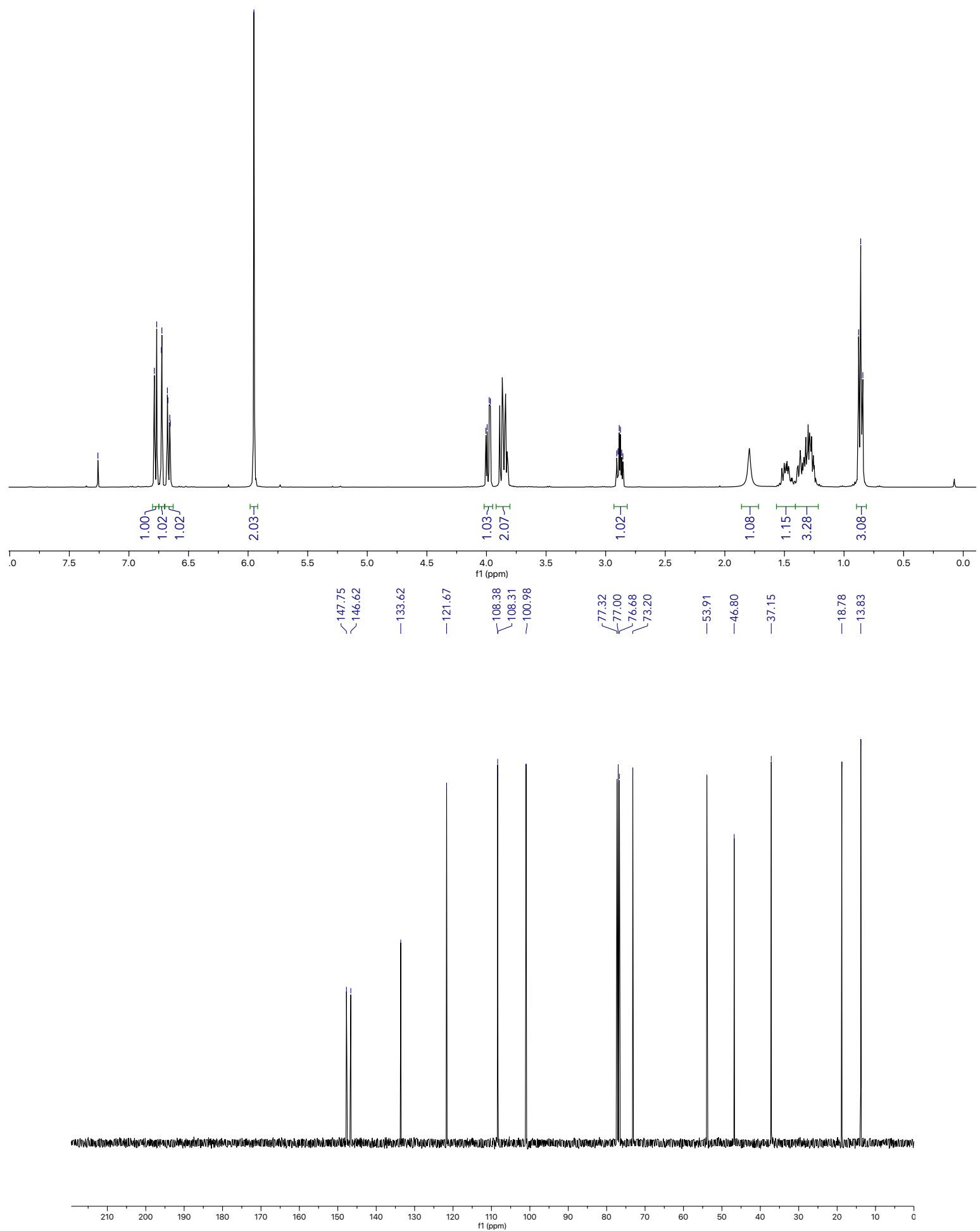
<smiles>O[C@H](CCl)[C@H](CCl)c1ccc2ccccc2c1</smiles>

$\left(2 R^{*}, 3 R^{*}\right)-1-C h l o r o-2-($ naphthalen-2-yl)hexan-3-ol $( \pm)-7 \mathrm{~d}$

General Procedure D: Epoxide $( \pm)-5 \mathbf{d}(113 \mathrm{mg}, 0.50 \mathrm{mmol})$ was employed. Purification of the residue by $\mathrm{FCC}(3: 1$, hexane-EtOAc) afforded the title compound (126 $\mathrm{mg}, 96 \%)$ as a colorless oil.

${ }^{1} \mathrm{H}$ NMR (400 MHz, CDCl $\left.{ }_{3}\right)$ ס 7.86-7.84 (m, 3H), 7.70 (br. s, 1H), 7.53-7.46 (m, 2H), 7.37 (dd, $J=$ 8.5, $1.8 \mathrm{~Hz}, 1 \mathrm{H}), 4.13(\mathrm{dd}, J=11.0,4.2 \mathrm{~Hz}, 1 \mathrm{H}), 4.07-4.01(\mathrm{~m}, 2 \mathrm{H}), 3.16(\mathrm{ddd}, J=4.2,4.1,4.1$ $\mathrm{Hz}, 1 \mathrm{H}), 1.76$ (br. s, 1H), 1.58-1.46 (m, 1H), 1.44-1.28 (m, 3H), 0.85 (t, J = 7.1 Hz, 3H);

${ }^{13} \mathrm{C} \mathrm{NMR}\left(100 \mathrm{MHz}, \mathrm{CDCl}_{3}\right) \delta 137.4,133.4,132.7,128.3,127.8,127.6,127.5,126.2(2 \mathrm{C}), 125.8$, 73.3, 54.5, 46.6, 37.3, 18.8, 13.8;

IR ( $\mathrm{NaCl}$, thin film) 3400, 2958, 2872, 1508, 1454, 1271, 1120, 1019, 934, 857, $748 \mathrm{~cm}^{-1}$; HRMS $\left(\mathrm{Cl}^{+}\right) \mathrm{m} / \mathrm{z}$ calcd for $\mathrm{C}_{16} \mathrm{H}_{19}{ }^{35} \mathrm{ClONH}_{4}{ }^{+}\left(\mathrm{M}+\mathrm{NH}_{4}\right)^{+}:$: 280.1463, found 280.1463. 


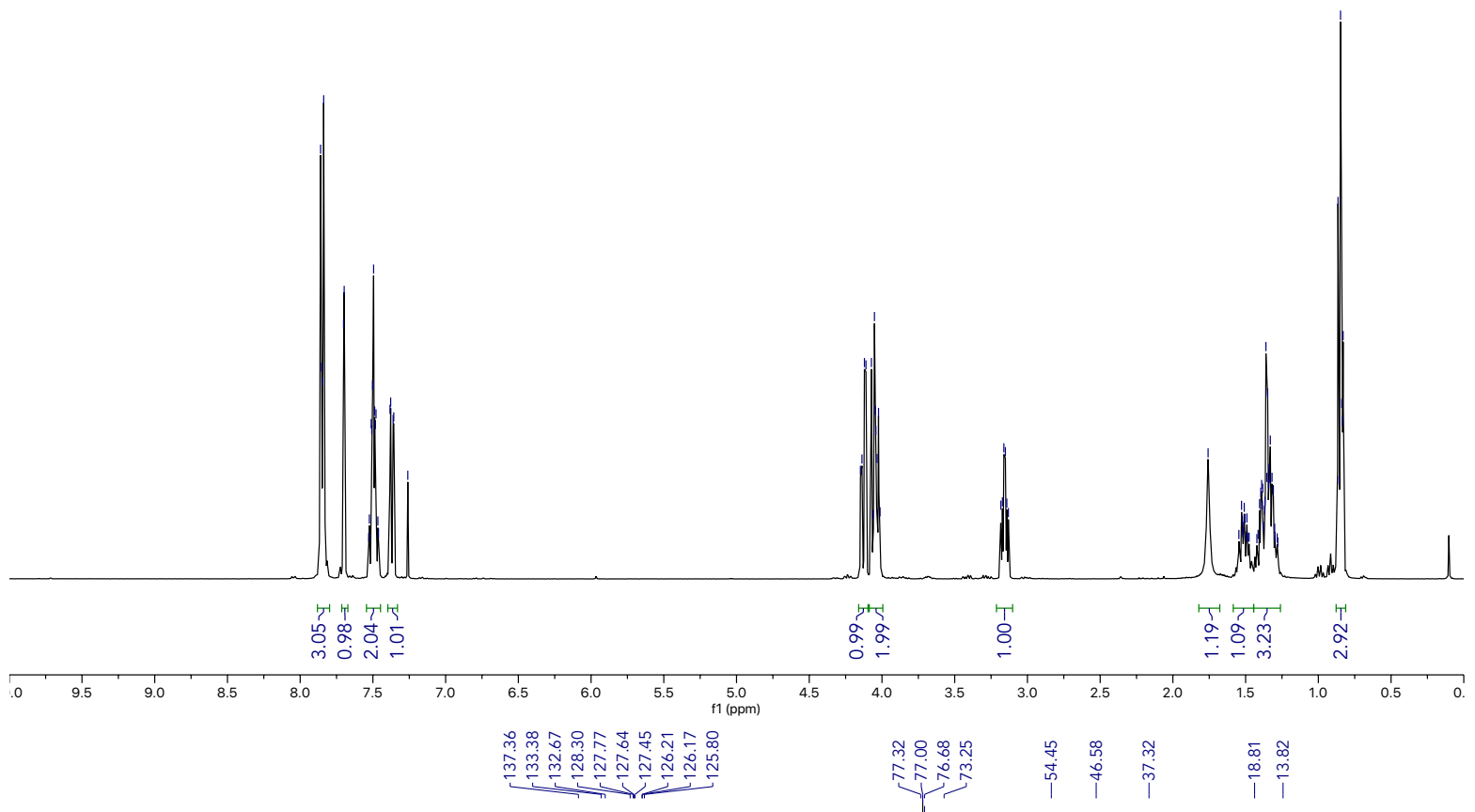

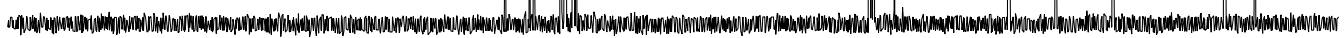

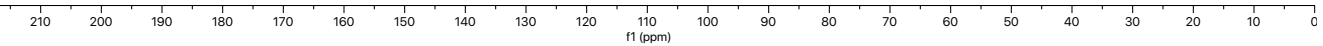


<smiles>CCCC(O)[C@H](CCl)c1ccc(C)cc1</smiles>

$\left(2 R^{\star}, 3 R^{\star}\right)$-1-Chloro-2-(p-tolyl)hexan-3-ol ( \pm -7e

General Procedure D: Epoxide $( \pm)-5 e(47 \mathrm{mg}, 0.25 \mathrm{mmol})$ was employed. Purification of the residue by FCC (5:1, hexane-EtOAc) afforded the title compound (54 mg, 96\%) as a colorless oil.

${ }^{1} \mathrm{H}$ NMR $\left(400 \mathrm{MHz}, \mathrm{CDCl}_{3}\right) \delta 7.16(\mathrm{~d}, J=8.0 \mathrm{~Hz}, 2 \mathrm{H}), 7.10$ (d, J = 8.2 Hz, 2H), 4.01 (dd, J = 10.9, $4.3 \mathrm{~Hz}, 1 \mathrm{H}), 3.92(\mathrm{dd}, J=10.9,8.3 \mathrm{~Hz}, 1 \mathrm{H}), 3.92-3.88(\mathrm{~m}, 1 \mathrm{H}), 2.95(\operatorname{app} . \mathrm{td}, J=8.0,4.3 \mathrm{~Hz}, 1 \mathrm{H})$, $2.34(\mathrm{~s}, 3 \mathrm{H}), 1.60$ (br. s, $1 \mathrm{H}), 1.52-1.43(\mathrm{~m}, 1 \mathrm{H}), 1.40-1.25(\mathrm{~m}, 3 \mathrm{H}), 0.85(\mathrm{t}, J=7.0 \mathrm{~Hz}, 3 \mathrm{H})$;

${ }^{13} \mathrm{C}$ NMR $\left(100 \mathrm{MHz}_{\mathrm{CDCl}}\right)$ $)$ 136.9, 136.7, 129.1, 128.2, 73.2, 53.8, 46.7, 37.1, 21.1, 18.8, 13.8;

IR ( $\mathrm{NaCl}$, thin film) 3366, 3022, 2958, 2872, 2930, 1515, 1456, 1380, 1117, 1021, $816 \mathrm{~cm}^{-1}$;

HRMS (ESI-TOF) $\mathrm{m} / \mathrm{z}$ calcd for $\mathrm{C}_{13} \mathrm{H}_{19}{ }^{35} \mathrm{ClONa}^{+}(\mathrm{M}+\mathrm{Na})^{+}:$249.1022, found 249.1038. 


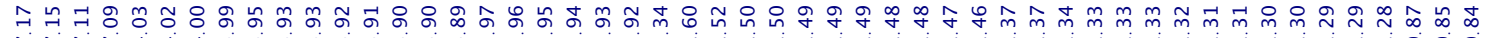

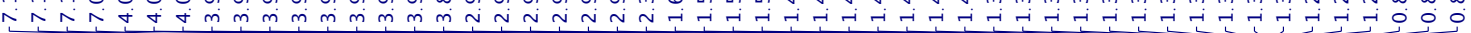
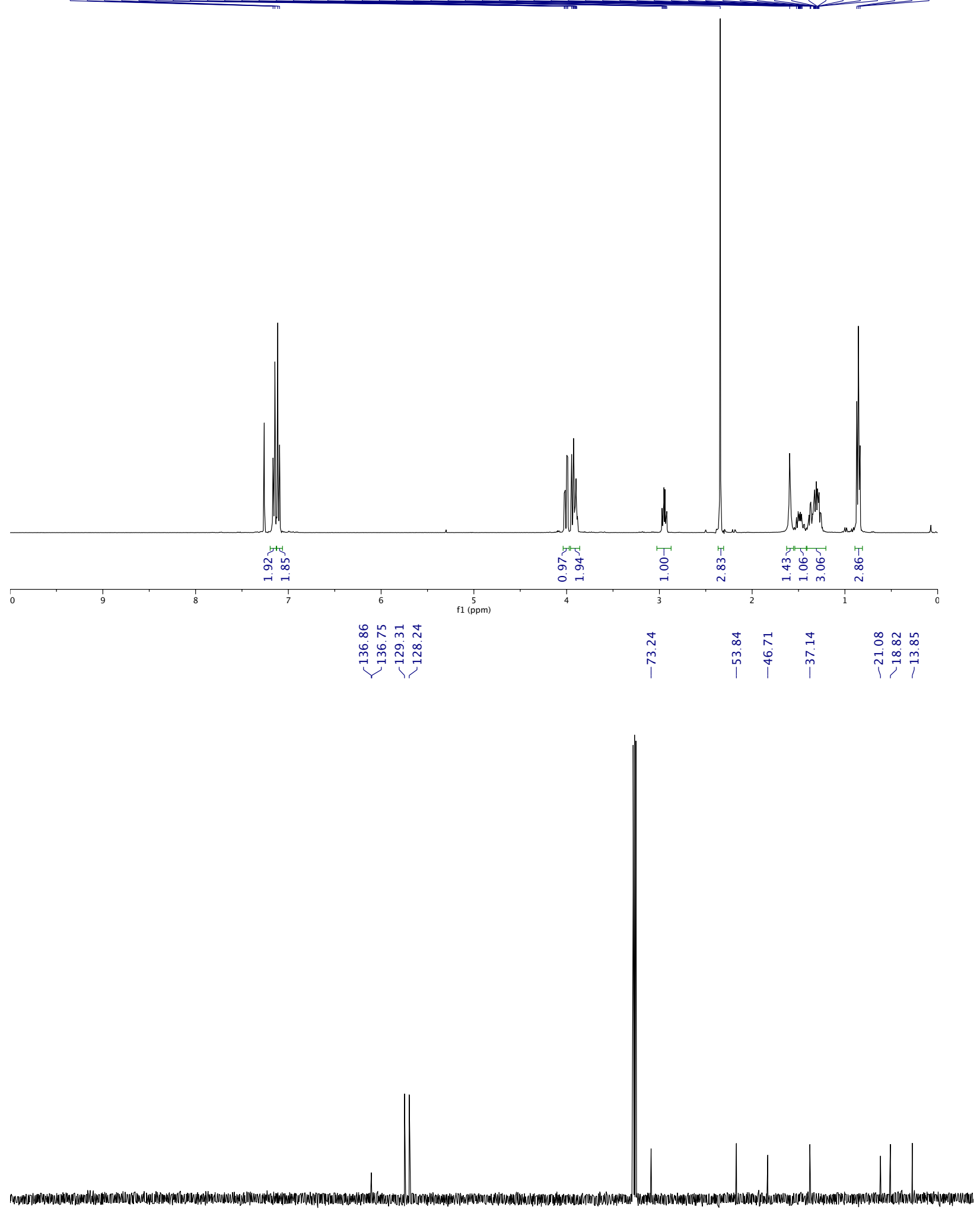

200

180

160

140

${ }_{120} \underset{\mathrm{f} 1(\mathrm{ppm})}{ } \quad 100$

80

60

40

20 
<smiles>O[C@H](CCl)[C@H](CCl)c1ccccc1</smiles>

$\left(2 R^{*}, 3 R^{*}\right)$-1-Chloro-2-phenylhexan-3-ol $( \pm)-7 f$

General Procedure D: Epoxide $( \pm)-5 f(44 \mathrm{mg}, 0.25 \mathrm{mmol})$ was employed. Purification of the residue by FCC (5:1, hexane-EtOAc) afforded the title compound (50 mg, 94\%) as a colorless oil.

${ }^{1}$ H NMR (400 MHz, $\left.\mathrm{CDCl}_{3}\right)$ ठ 7.37-7.33 (m, 2H), 7.30-7.28 (m, 1H), 7.23-7.21 (m, 2H), 4.03 (dd, $J=10.9,4.3 \mathrm{~Hz}, 1 \mathrm{H}), 3.94(\mathrm{dd}, J=11.0,8.3 \mathrm{~Hz}, 1 \mathrm{H}), 3.94-3.91(\mathrm{~m}, 1 \mathrm{H}), 2.98$ (app. td, $J=8.0$, $4.2 \mathrm{~Hz}, 1 \mathrm{H}), 1.62$ (br. s, 1H), 1.52-1.45 (m, 1H), 1.39-1.24 (m, 3H), $0.85(\mathrm{t}, J=7.0 \mathrm{~Hz}, 3 \mathrm{H})$;

${ }^{13} \mathrm{C}$ NMR $\left(100 \mathrm{MHz}, \mathrm{CDCl}_{3}\right) \delta 139.9,128.6,128.4,127.3,73.2,54.3,46.6,37.2,18.8,12.9$;

IR (NaCl, thin film) 3397, 3029, 2958, 2934, 2872, 1495, 1455, 1121, 1030, $700 \mathrm{~cm}^{-1}$;

HRMS (ESI-TOF) $\mathrm{m} / \mathrm{z}$ calcd for $\mathrm{C}_{12} \mathrm{H}_{17}{ }^{35} \mathrm{ClONa}^{+}(\mathrm{M}+\mathrm{Na})^{+}:$235.0866, found 235.0860. 


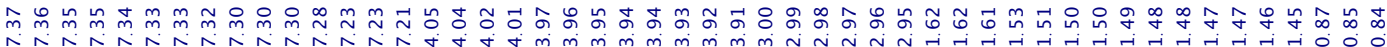

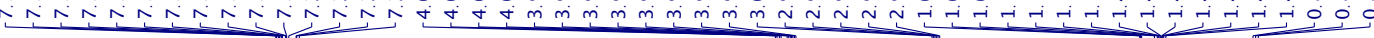

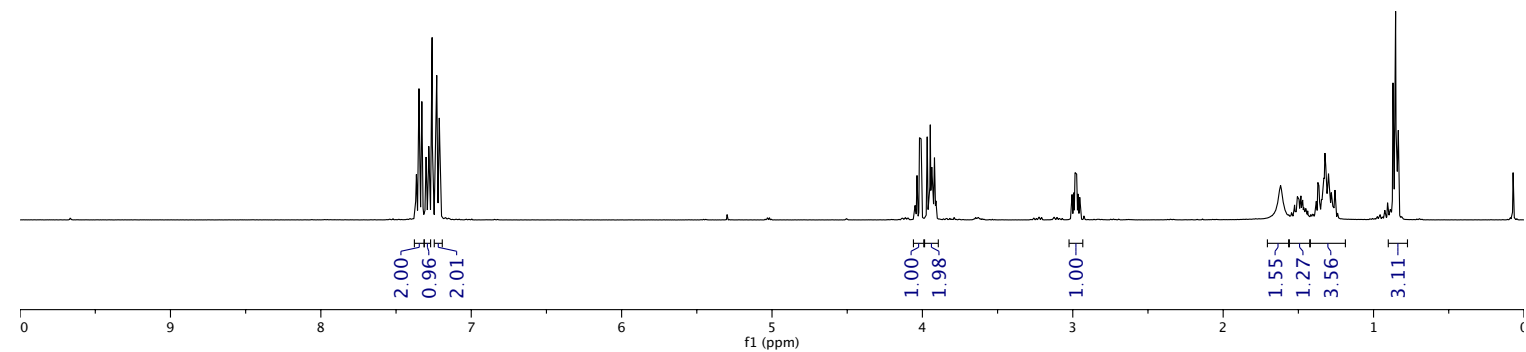

\begin{tabular}{|c|c|c|c|c|}
\hline 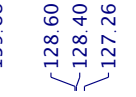 & $\stackrel{\substack{N \\
N}}{i}$ & $\begin{array}{l}\stackrel{\bullet}{N} \\
\stackrel{\sim}{\sim} \\
\text { । }\end{array}$ & 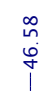 & $\stackrel{2}{2}$ \\
\hline
\end{tabular}

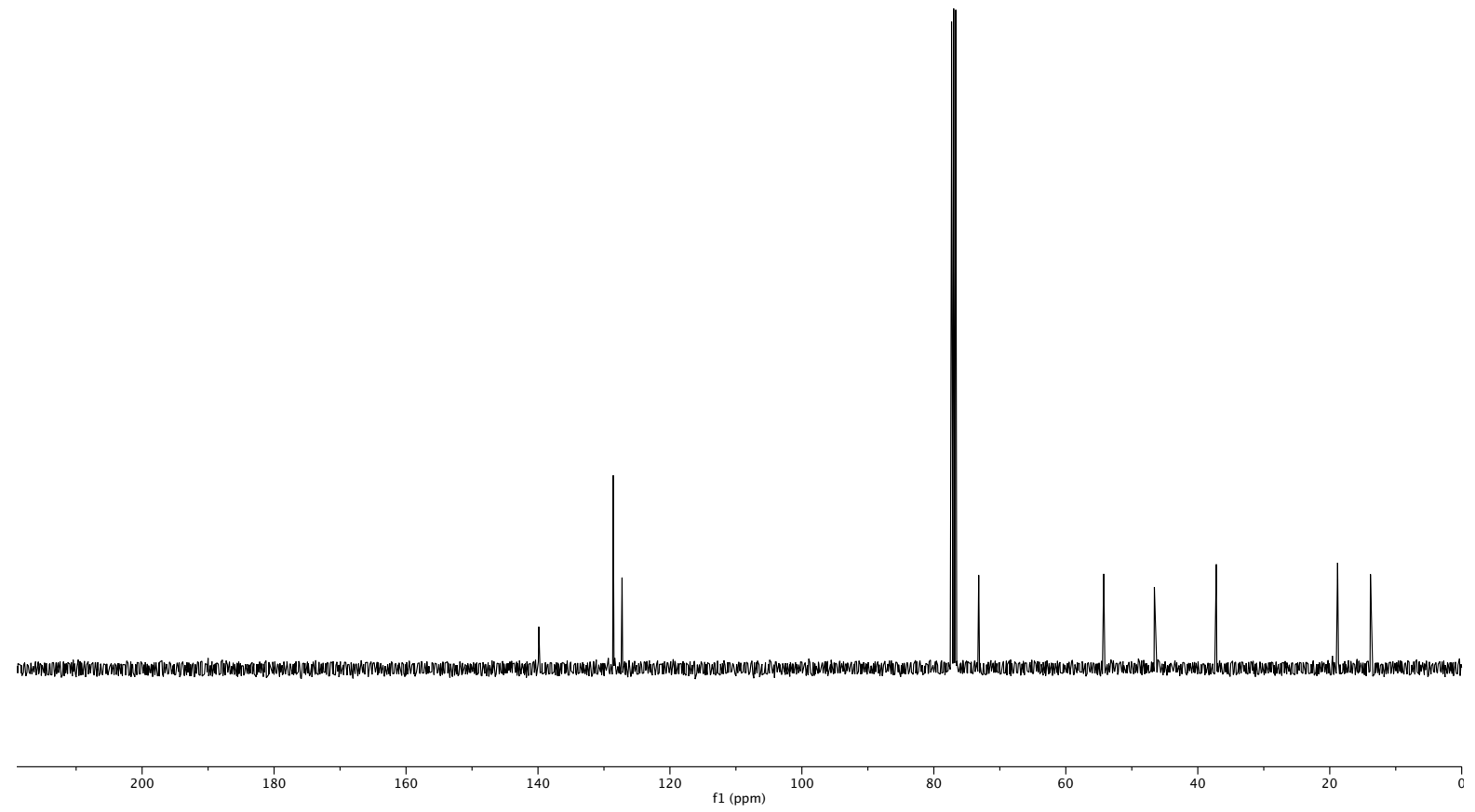


<smiles>CCCC(O)[C@H](CCl)c1ccc2c(ccn2[AsH2])c1</smiles>

$\left(2 R^{*}, 3 R^{*}\right)$-1-Chloro-2-(1-tosyl-1H-indol-5-yl)hexan-3-ol ( \pm )-7g

General Procedure D: Epoxide $( \pm)-5 \mathrm{~g}(55 \mathrm{mg}, 0.15 \mathrm{mmol})$ was employed. Purification of the residue by FCC (15:1, hexane-EtOAc) afforded the title compound ( $45 \mathrm{mg}, 81 \%)$ as a brown oil.

${ }^{1} \mathrm{H}$ NMR $\left(500 \mathrm{MHz} \mathrm{CDCl}_{3}\right) \delta 7.94(\mathrm{~d}, J=8.6 \mathrm{~Hz}, 1 \mathrm{H}), 7.79(\mathrm{~d}, J=8.1 \mathrm{~Hz}, 2 \mathrm{H}), 7.56(\mathrm{~d}, J=3.6$ $\mathrm{Hz}, 1 \mathrm{H}), 7.39(\mathrm{~d}, J=1.7 \mathrm{~Hz}, 1 \mathrm{H}), 7.24(\mathrm{~d}, J=8.3 \mathrm{~Hz}, 2 \mathrm{H}), 7.16(\mathrm{dd}, J=8.6,1.7 \mathrm{~Hz}, 1 \mathrm{H}), 6.63(\mathrm{~d}$, $J=3.5 \mathrm{~Hz}, 1 \mathrm{H}), 4.02(\mathrm{dd}, J=11.0,4.2 \mathrm{~Hz}, 1 \mathrm{H}), 3.94(\mathrm{dd}, J=10.9,8.2 \mathrm{~Hz}, 1 \mathrm{H}), 3.95-3.91(\mathrm{~m}$, 1H), 3.04 (app. td, $J=8.0,4.2 \mathrm{~Hz}, 1 \mathrm{H}), 2.36$ (s, 3H), 1.62 (br. s, 1H), 1.52-1.44 (m, 1H), 1.34-1.24 (m, 3H), $0.82(\mathrm{t}, J=6.9 \mathrm{~Hz}, 3 \mathrm{H})$;

${ }^{13} \mathrm{C}$ NMR (100 MHz, $\left.\mathbf{C D C l}_{3}\right) \delta$ 145.0, 135.4, 135.0, 134.0, 130.9, 130.0, 126.9, 126.7, 125.0, 121.1, 113.6, 108.8, 73.4, 54.1, 46.8, 37.2, 21.6, 18.9, 13.9;

IR (NaCl, thin film) 3567, 3420, 2958, 2932, 2872, 1596, 1460, 1370, 1267, 1172, 1130, 1093, $997 \mathrm{~cm}^{-1}$;

HRMS (ESI-TOF) $\mathrm{m} / \mathrm{z}$ calcd for $\mathrm{C}_{21} \mathrm{H}_{24}{ }^{35} \mathrm{ClO}_{3} \mathrm{NSNa}^{+}(\mathrm{M}+\mathrm{Na})^{+}: 428.1063$, found 428.1066 . 


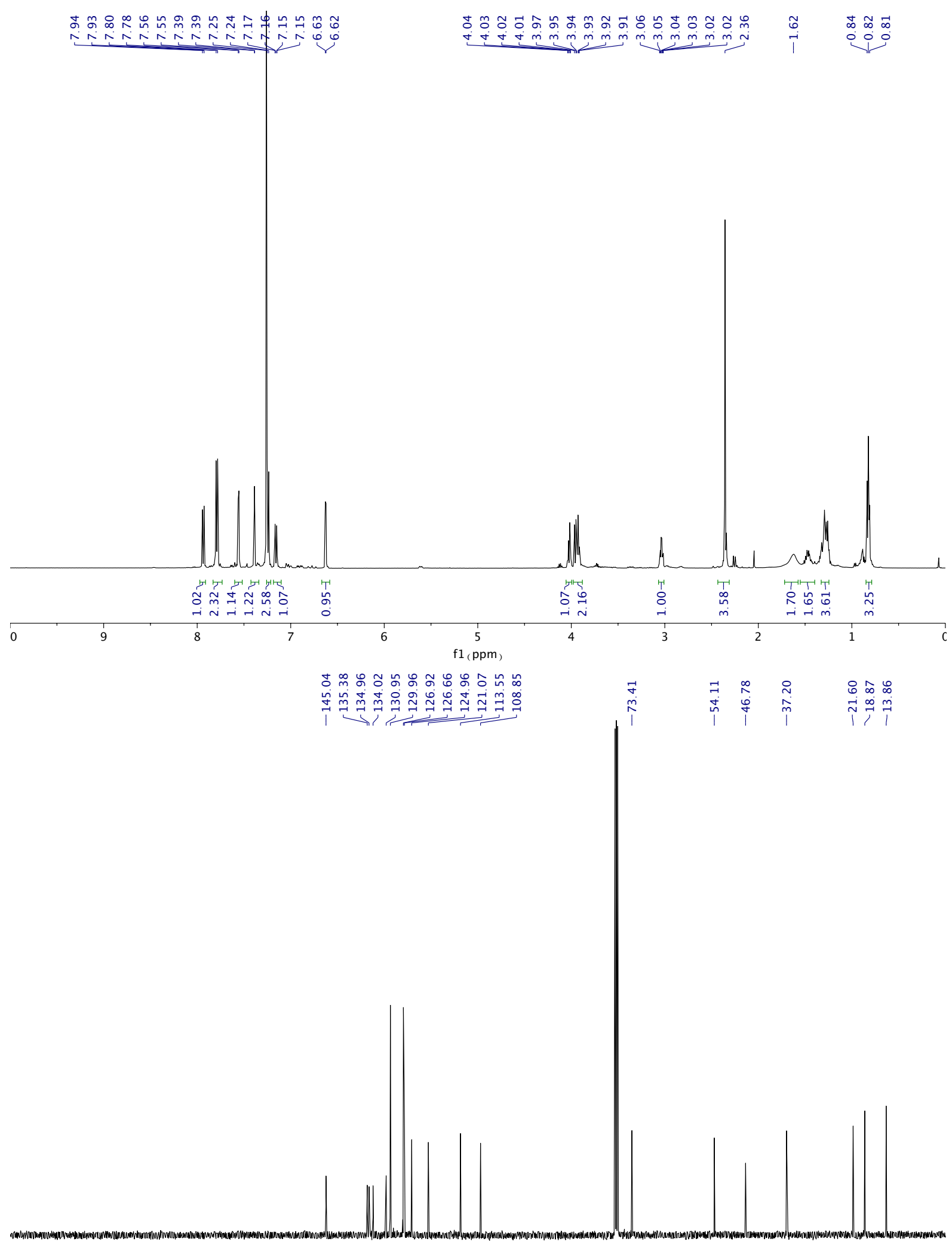

$200 \quad 12 \quad 10$

140

$120 \quad \mathrm{f} 1(\mathrm{ppm}) \quad 100$

80

60

40

$20 \quad c$ 
<smiles>CC(C)[C@@H](O)[C@H](CCl)c1ccc(F)cc1</smiles>

$\left(2 R^{*}, 3 R^{*}\right)-1-C h l o r o-2-(4-f l u o r o p h e n y l) h e x a n-3-o l ~( \pm)-7 \mathrm{~h}$

General Procedure D: Epoxide $( \pm)-5 \mathrm{~h}(48 \mathrm{mg}, 0.25 \mathrm{mmol})$ was employed. Purification of the residue by FCC (5:1, hexane-EtOAc) afforded the title compound (56 $\mathrm{mg}, 97 \%)$ as a pale yellow oil.

${ }^{1} \mathrm{H}$ NMR $\left(400 \mathrm{MHz}, \mathrm{CDCl}_{3}\right) \delta$ 7.21-7.17 (m, 2H), 7.06-7.01 (m, 2H), $4.00(\mathrm{dd}, J=11.0,4.1 \mathrm{~Hz}$, $1 \mathrm{H}), 3.91$ (dd, $J=11.0,8.3 \mathrm{~Hz}, 1 \mathrm{H}), 3.91-3.87(\mathrm{~m}, 1 \mathrm{H}), 2.96$ (app. td, $J=8.1,4.1 \mathrm{~Hz}, 1 \mathrm{H}), 1.63$ (br. s, 1H), 1.52-1.43 (m, 1H), 1.35-1.26 (m, 3H), $0.85(\mathrm{t}, J=6.8 \mathrm{~Hz}, 3 \mathrm{H})$;

${ }^{13} \mathrm{C}$ NMR (100 MHz, $\left.\mathrm{CDCl}_{3}\right) \delta 162.0$ (d, J = 245.7 Hz), 135.6 (d, J = 3.2 Hz), 129.9 (d, J = 7.9 Hz), $115.5(\mathrm{~d}, J=21.2 \mathrm{~Hz}), 73.1,53.5,46.7,37.2,18.8,13.8$;

${ }^{19}$ F NMR (471 MHz, $\left.\mathrm{CDCl}_{3}\right) \delta-115.4(\mathrm{~s})$;

IR ( $\mathrm{NaCl}$, thin film) 3387, 2960, 2935, 2873, 1511, 1457, 1225, 1160, 1015, $833 \mathrm{~cm}^{-1}$;

HRMS $\left(\mathrm{Cl}^{+}\right) \mathrm{m} / \mathrm{z}$ calcd for $\mathrm{C}_{12} \mathrm{H}_{16} \mathrm{~F}^{35} \mathrm{ClONH}_{4}{ }^{+}\left(\mathrm{M}+\mathrm{NH}_{4}\right)^{+}:$248.1212, found 248.1212. 


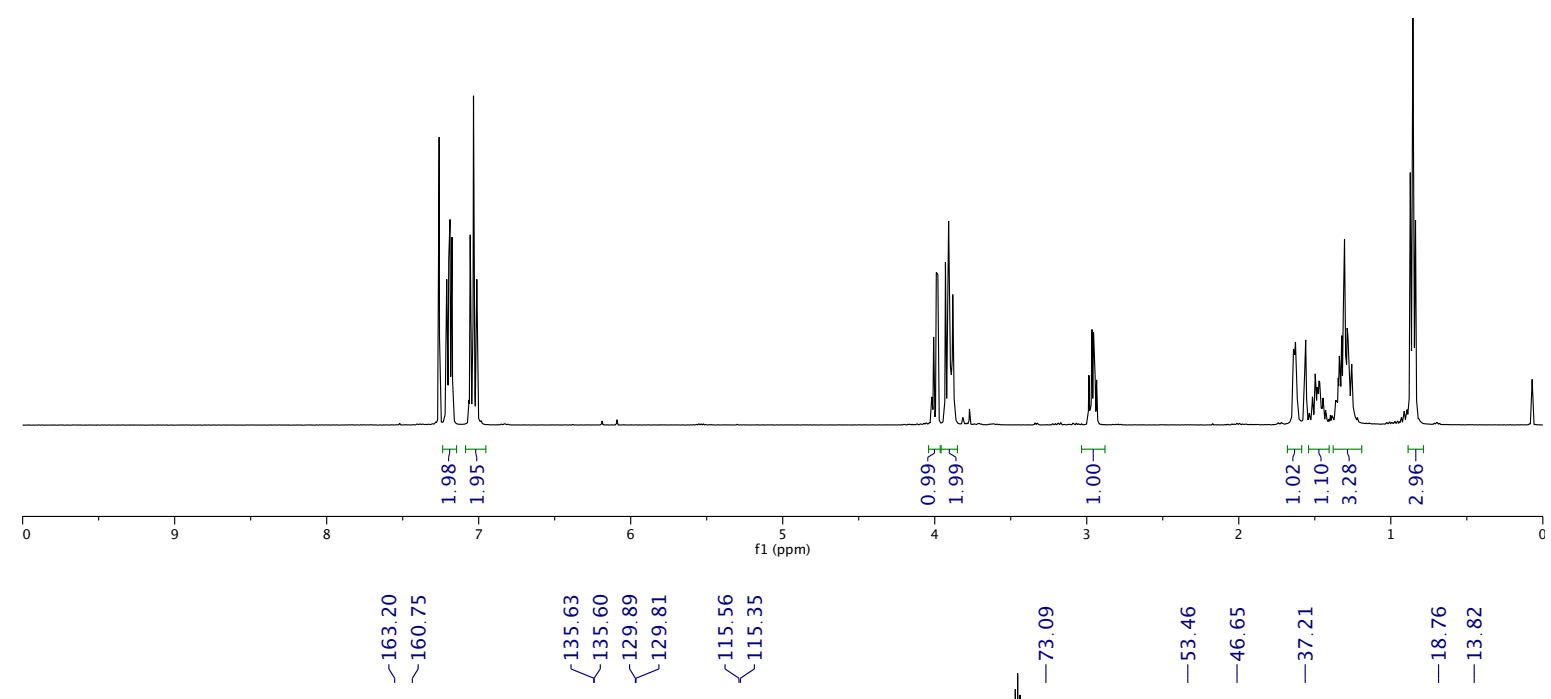


<smiles>CCCC(O)[C@H](CCl)c1ccc(Cl)cc1</smiles>

$\left(2 R^{*}, 3 R^{*}\right)-1-C h l o r o-2-(4-c h l o r o p h e n y l) h e x a n-3-o l( \pm)-7 i$

General Procedure D: Epoxide ( \pm )-5i $(53 \mathrm{mg}, 0.25 \mathrm{mmol})$ was employed. Purification of the residue by FCC (5:1, hexane-EtOAc) afforded the title compound (55 mg, 89\%) as a colorless oil.

${ }^{1} \mathrm{H}$ NMR $\left(400 \mathrm{MHz}, \mathrm{CDCl}_{3}\right) \delta 7.32(\mathrm{~d}, J=8.4 \mathrm{~Hz}, 2 \mathrm{H}), 7.16(\mathrm{~d}, J=8.5 \mathrm{~Hz}, 2 \mathrm{H}), 4.00$ (dd, $J=11.0$, $4.0 \mathrm{~Hz}, 1 \mathrm{H}), 3.90$ (dd, $J=11.0,8.3 \mathrm{~Hz}, 1 \mathrm{H}), 3.90-3.87(\mathrm{~m}, 1 \mathrm{H}), 2.95$ (app. td, $J=8.2,4.0 \mathrm{~Hz}, 1 \mathrm{H}$ ), 1.61 (br. s, $1 \mathrm{H}), 1.52-1.43(\mathrm{~m}, 1 \mathrm{H}), 1.38-1.28(\mathrm{~m}, 3 \mathrm{H}), 0.86(\mathrm{t}, J=6.9 \mathrm{~Hz}, 3 \mathrm{H})$;

${ }^{13} \mathrm{C}$ NMR $\left(100 \mathrm{MHz}, \mathrm{CDCl}_{3}\right) \delta$ 138.4, 133.1, 129.8, 128.8, 73.0, 53.7, 46.6, 37.3, 18.8, 13.8;

IR ( $\mathrm{NaCl}$, thin film) 3395, 2959, 2934, 2873, 1493, 1456, 1411, 1287, 1092, 1015, 945, $825 \mathrm{~cm}^{-1}$; HRMS $\left(\mathrm{Cl}^{+}\right) \mathrm{m} / \mathrm{z}$ calcd for $\mathrm{C}_{12} \mathrm{H}_{16}{ }^{35} \mathrm{Cl}_{2} \mathrm{ONH}_{4}{ }^{+}\left(\mathrm{M}+\mathrm{NH}_{4}\right)^{+}:$264.0916, found 264.0915. 


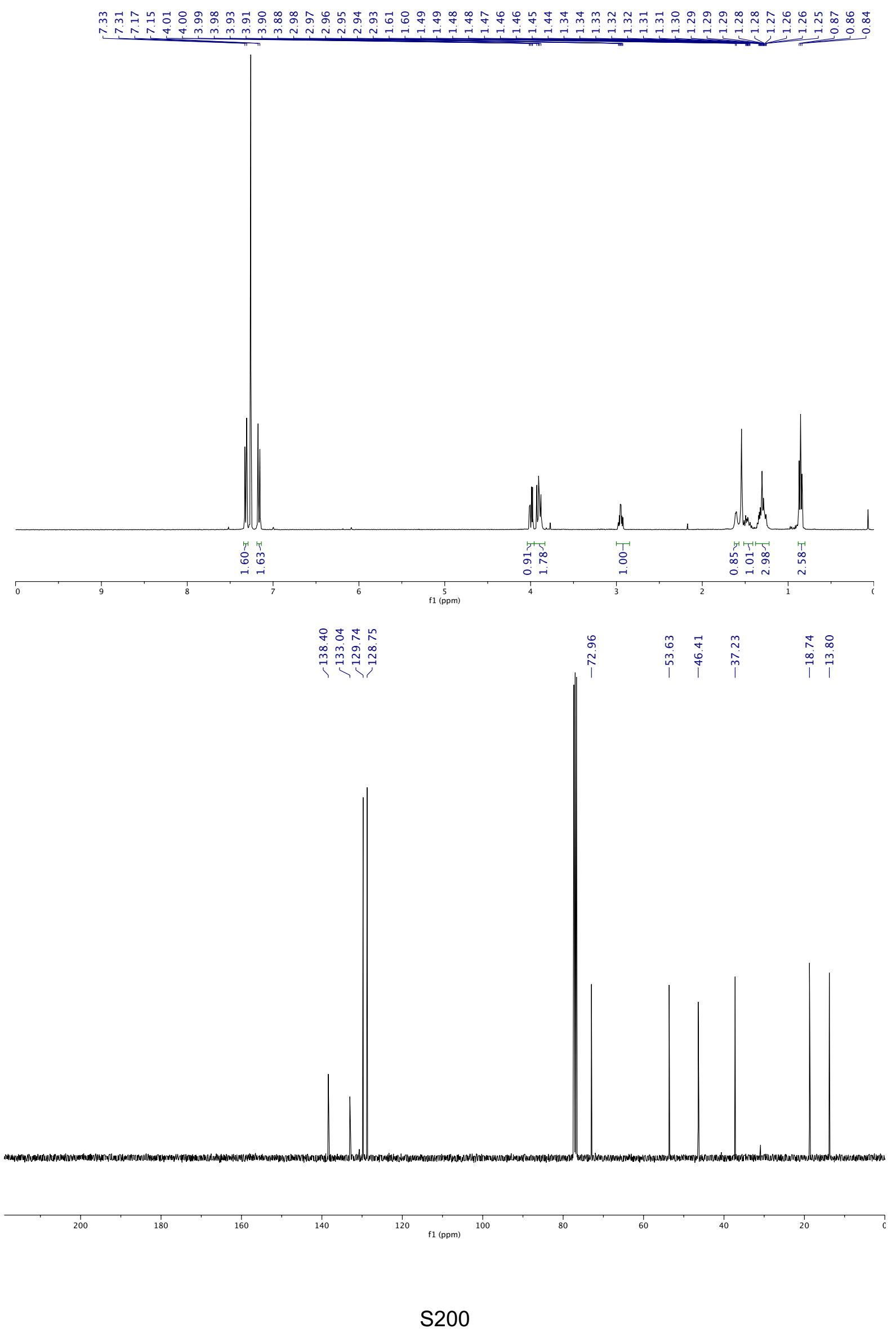


<smiles>CC(C)C(O)[C@@H](CCl)c1ccc(Br)cc1</smiles>

$\left(2 R^{*}, 3 R^{*}\right)-2-(4-B r . o m o p h e n y l)-1-c h l o r o h e x a n-3-o l( \pm)-7 \mathrm{j}$

General Procedure D: Epoxide $( \pm)-5 \mathrm{j}(64 \mathrm{mg}, 0.25 \mathrm{mmol})$ was employed. Purification of the residue by FCC (5:1, hexane-EtOAc) afforded the title compound (54 $\mathrm{mg}, 74 \%)$ as a pale yellow oil.

${ }^{1} \mathrm{H}$ NMR $\left(400 \mathrm{MHz}, \mathrm{CDCl}_{3}\right) \delta 7.47(\mathrm{~d}, J=8.4 \mathrm{~Hz}, 2 \mathrm{H}), 7.11(\mathrm{~d}, J=8.4 \mathrm{~Hz}, 2 \mathrm{H}), 4.00$ (dd, $J=11.0$, $4.1 \mathrm{~Hz}, 1 \mathrm{H}), 3.90(\mathrm{dd}, J=11.0,8.4 \mathrm{~Hz}, 1 \mathrm{H}), 3.91-3.86(\mathrm{~m}, 1 \mathrm{H}), 2.94$ (app. td, $J=8.2,4.0 \mathrm{~Hz}, 1 \mathrm{H})$, 1.70 (br. s, $1 \mathrm{H}), 1.53-1.42(\mathrm{~m}, 1 \mathrm{H}), 1.36-1.24(\mathrm{~m}, 3 \mathrm{H}), 0.85(\mathrm{t}, J=6.8 \mathrm{~Hz}, 3 \mathrm{H})$;

${ }^{13} \mathrm{C}$ NMR $\left(125 \mathrm{MHz}, \mathrm{CDCl}_{3}\right) \delta$ 138.9, 131.7, 130.1, 121.2, 72.9, 53.7, 46.3, 37.3, 18.8, 13.8;

IR ( $\mathrm{NaCl}$, thin film) 3387, 2959, 2933, 2872, 1489, 1457, 1408, 1285, 1121, 1074, 1011, 946, 821 $\mathrm{cm}^{-1}$;

HRMS $\left(\mathrm{Cl}^{+}\right) \mathrm{m} / \mathrm{z}$ calcd for $\mathrm{C}_{12} \mathrm{H}_{16}{ }^{35} \mathrm{Cl}^{79} \mathrm{BrONH}_{4}{ }^{+}\left(\mathrm{M}+\mathrm{NH}_{4}\right)^{+}: 308.0411$, found 308.0403 . 


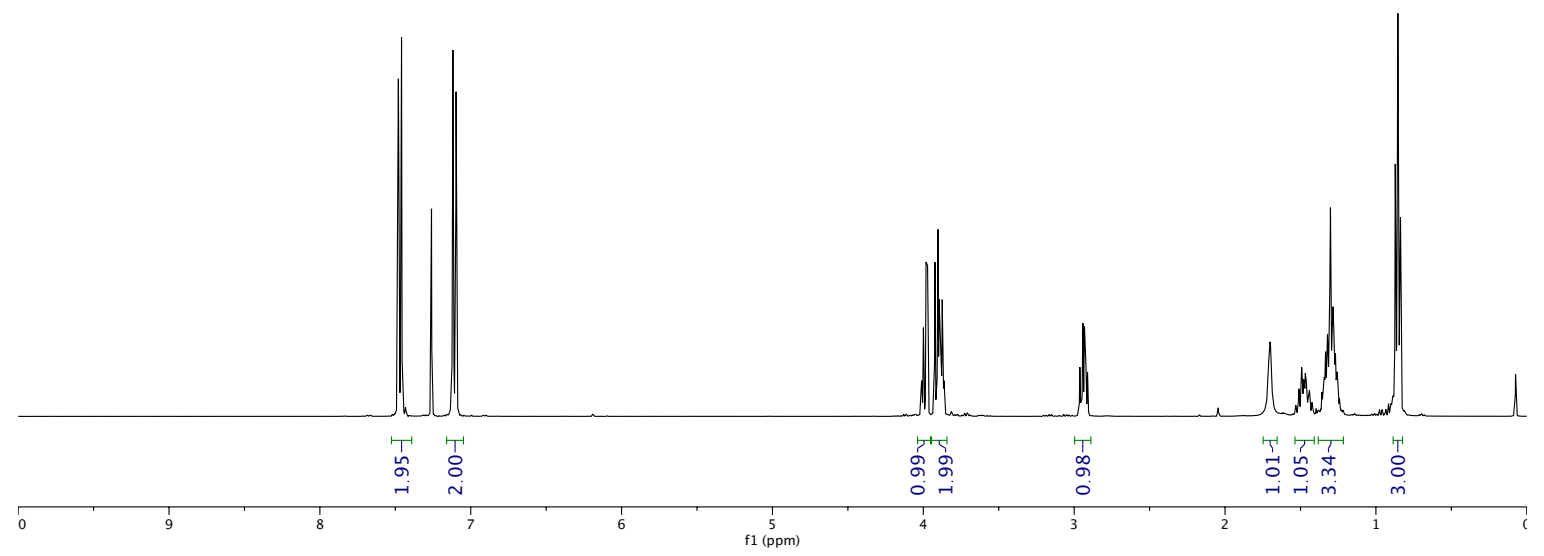

$\tilde{N} \sim \mathcal{2}$

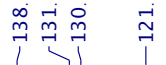

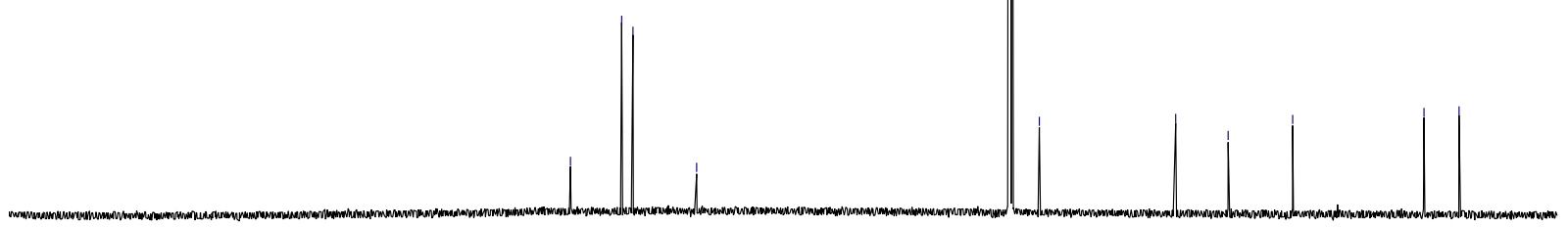

200

180

160

140

$120 \quad \mathrm{f} 1(\mathrm{ppm}) \quad 100$

80

60

40

20 
<smiles>CCC[C@H](Cl)[C@H](O)Cc1ccc(C(F)(F)F)cc1</smiles>

$\left(2 R^{*}, 3 R^{*}\right)$-1-Chloro-2-(4-(trifluoromethyl)phenyl)hexan-3-ol $( \pm)-7 \mathrm{k}$ $\left(2 R^{*}, 3 S^{*}\right)$-3-Chloro-1-(4-(trifluoromethyl)phenyl)hexan-2-ol ( \pm )-9k

General Procedure D: Epoxide $( \pm)-5 k(61 \mathrm{mg}, 0.25 \mathrm{mmol})$ was employed. Purification of the residue by FCC (15:1, hexane-EtOAc) afforded chlorohydrin ( \pm )-9k $(29 \mathrm{mg}, 42 \%)$ as a colorless, low melting solid. Continued elution (5:1, hexane-EtOAc) afforded rearranged product $( \pm)-7 \mathbf{k}(30$ $\mathrm{mg}, 43 \%)$ as a colorless solid.

Data for $( \pm)-7 k$

${ }^{1} \mathrm{H}$ NMR $\left(400 \mathrm{MHz}, \mathrm{CDCl}_{3}\right) \delta 7.60(\mathrm{~d}, J=8.2 \mathrm{~Hz}, 2 \mathrm{H}), 7.36(\mathrm{~d}, J=8.2 \mathrm{~Hz}, 2 \mathrm{H}), 4.02$ (dd, $J=11.1$, $4.0 \mathrm{~Hz}, 1 \mathrm{H}), 3.95(\mathrm{dd}, J=11.0,8.4 \mathrm{~Hz}, 1 \mathrm{H}), 3.97-3.92(\mathrm{~m}, 1 \mathrm{H}), 3.05(\operatorname{app} . \mathrm{td}, J=8.2,4.1 \mathrm{~Hz}, 1 \mathrm{H})$, 1.56 (br. s, 1H), 1.53-1.44 (m, 1H), 1.35-1.25 (m, 3H), $0.86(\mathrm{t}, J=7.0 \mathrm{~Hz}, 3 \mathrm{H})$;

${ }^{13} \mathrm{C}$ NMR $\left(125 \mathrm{MHz}, \mathrm{CDCl}_{3}\right) \delta 144.1,129.6$ (q, $\left.J=32.6 \mathrm{~Hz}\right), 128.8,125.5(\mathrm{q}, J=3.8 \mathrm{~Hz}), 124.1$ (q, $J=272.0 \mathrm{~Hz}$ ), 72.9, 54.1, 46.1, 37.3, 18.8, 13.8;

${ }^{19} \mathrm{~F}\left\{{ }^{1} \mathrm{H}\right\}$ NMR $\left(376 \mathrm{MHz}, \mathrm{CDCl}_{3}\right) \delta-62.5(\mathrm{~s})$;

IR ( $\mathrm{NaCl}$, thin film) 3385, 2961, 2937, 2875, 1620, 1420, 1327, 1166, 1126, 1069, 1019, $837 \mathrm{~cm}^{-1}$; HRMS $\left(\mathrm{Cl}^{+}\right) \mathrm{m} / \mathrm{z}$ calcd for $\mathrm{C}_{13} \mathrm{H}_{16} \mathrm{~F}_{3}{ }^{35} \mathrm{ClONH}_{4}{ }^{+}\left(\mathrm{M}+\mathrm{NH}_{4}\right)^{+}$: 298.1180, found 298.1176;

M pt $56-58^{\circ} \mathrm{C}$.

Data for chlorohydrin ( \pm )-9k

${ }^{1} \mathrm{H}$ NMR $\left(400 \mathrm{MHz}, \mathrm{CDCl}_{3}\right) \delta 7.58(\mathrm{~d}, J=8.0 \mathrm{~Hz}, 2 \mathrm{H}), 7.38(\mathrm{~d}, J=8.0 \mathrm{~Hz}, 2 \mathrm{H}), 4.03-3.95(\mathrm{~m}, 2 \mathrm{H})$, 3.01 (dd, $J=14.1,3.2 \mathrm{~Hz}, 1 \mathrm{H}$ ), 2.88 (dd, $J=14.0,8.7 \mathrm{~Hz}, 1 \mathrm{H}), 1.96$ (br. s, 1H), 1.88-1.75 (m, $2 \mathrm{H}), 1.73-1.61(\mathrm{~m}, 1 \mathrm{H}), 1.50-1.39(\mathrm{~m}, 1 \mathrm{H}), 0.96(\mathrm{t}, \mathrm{J}=7.3 \mathrm{~Hz}, 3 \mathrm{H})$;

${ }^{13} \mathrm{C}$ NMR (125 MHz, $\left.\mathrm{CDCl}_{3}\right) \delta$ 142.2, 129.7, 128.9 (q, $\left.J=32 \mathrm{~Hz}\right), 125.5$ (q, J = 3.7 Hz), 124.2 (q, $J=272.6 \mathrm{~Hz}), 75.4,67.1,39.0,35.0,19.8,13.5$;

${ }^{19} \mathrm{~F}\left\{{ }^{1} \mathrm{H}\right\}$ NMR $\left(376 \mathrm{MHz}, \mathrm{CDCl}_{3}\right) \delta-62.5(\mathrm{~s})$;

IR ( $\mathrm{NaCl}$, thin film) 3449, 2963, 1417, 1325, 1165, 1124, 1068, 1020, $820 \mathrm{~cm}^{-1}$;

HRMS $\left(\mathrm{Cl}^{+}\right) \mathrm{m} / \mathrm{z}$ calcd for $\mathrm{C}_{13} \mathrm{H}_{16} \mathrm{~F}_{3}{ }^{35} \mathrm{ClONH}_{4}{ }^{+}\left(\mathrm{M}+\mathrm{NH}_{4}\right)^{+}:$298.1180, found 298.1178. 
Data for $( \pm)-7 k$

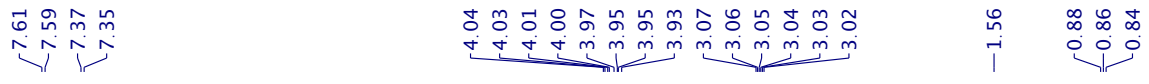
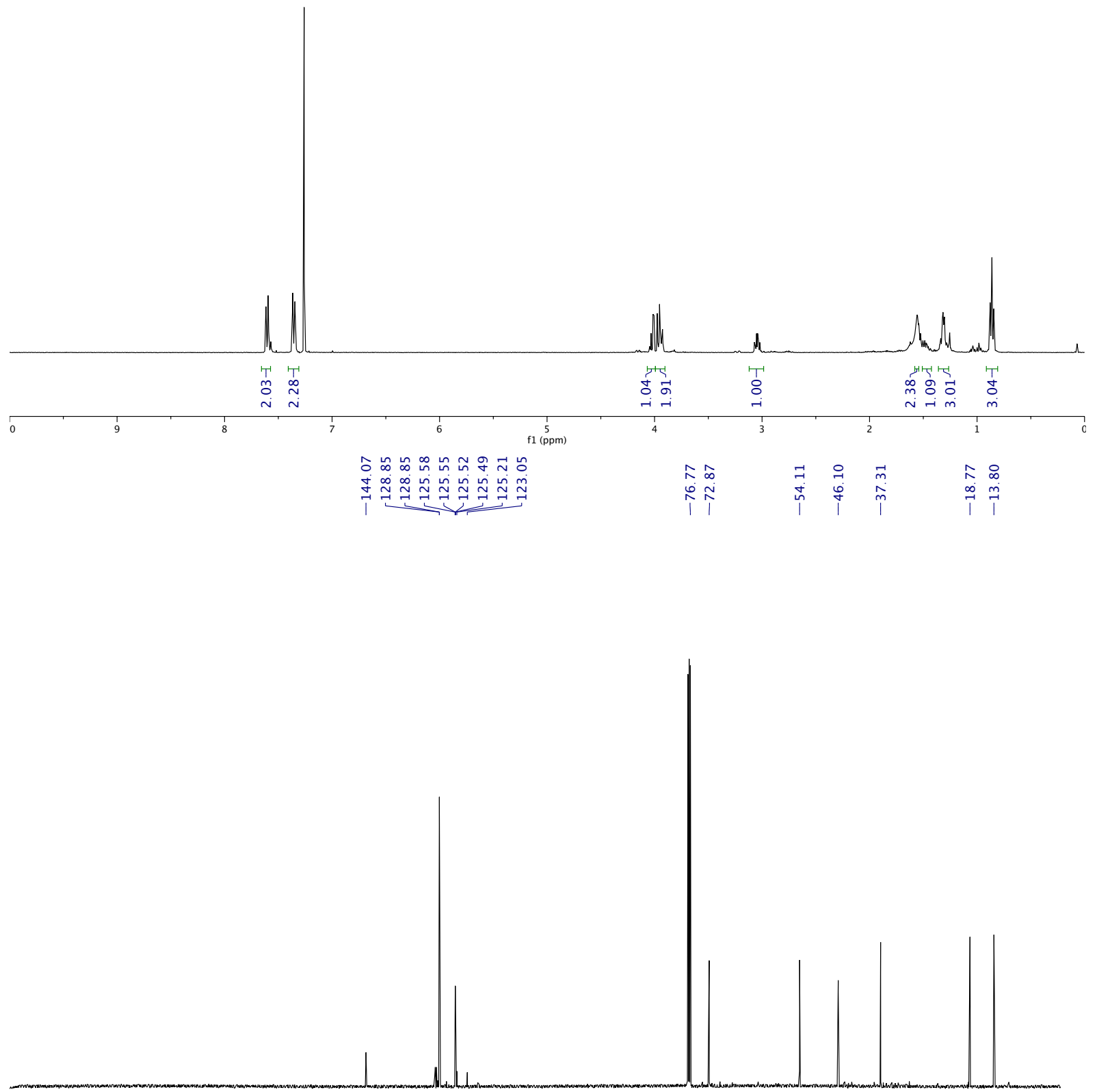

$\underset{180}{1}+\frac{1}{160}$

$140 \quad \frac{1}{120} \quad 100$

80

$60 \quad 40$

$20+0$ 
Data for $( \pm)-9 k$

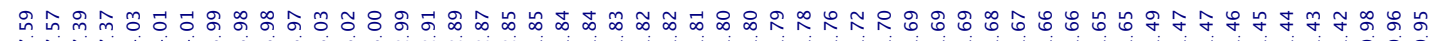

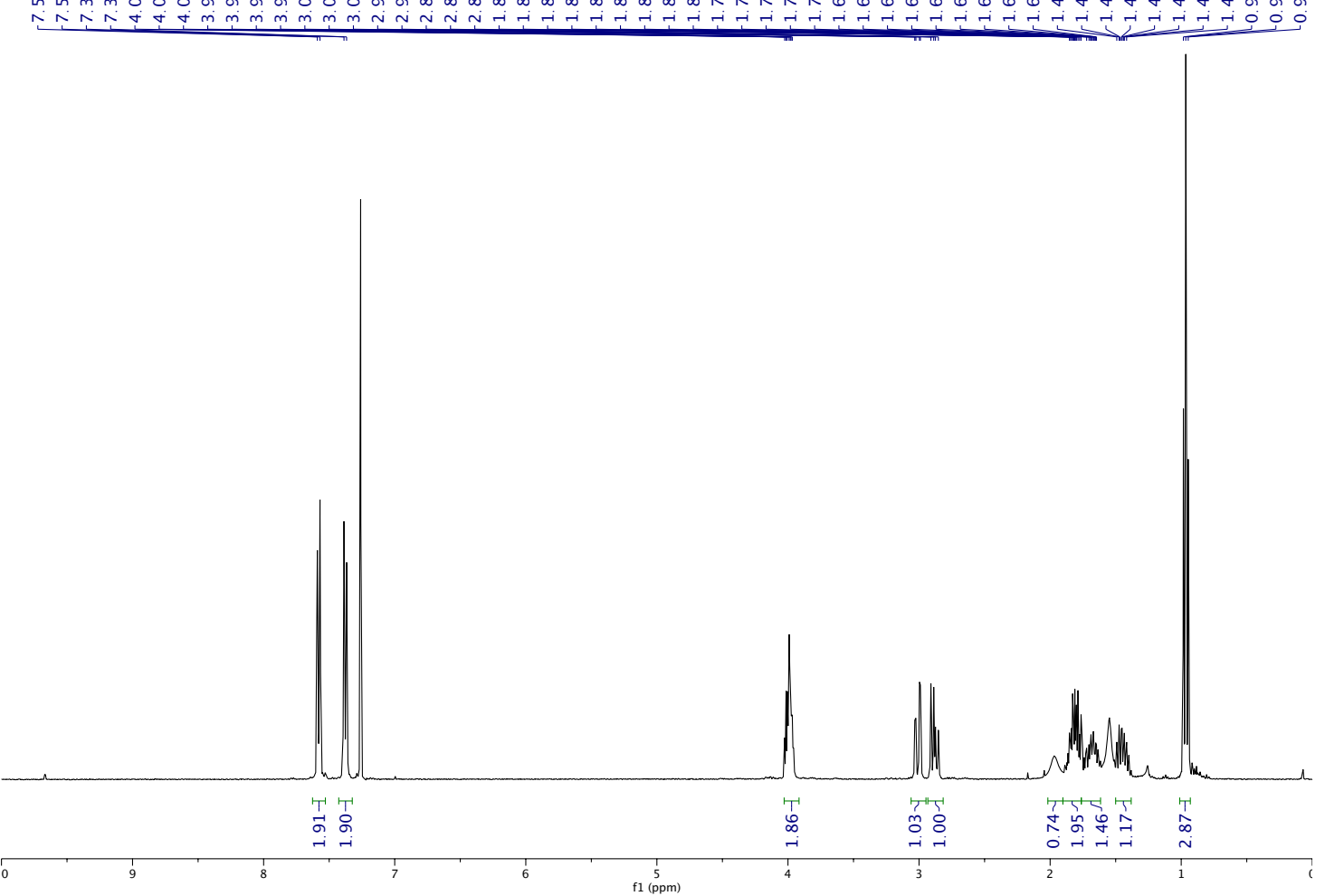

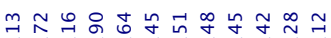

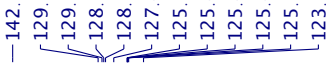

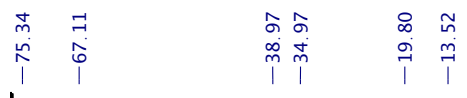

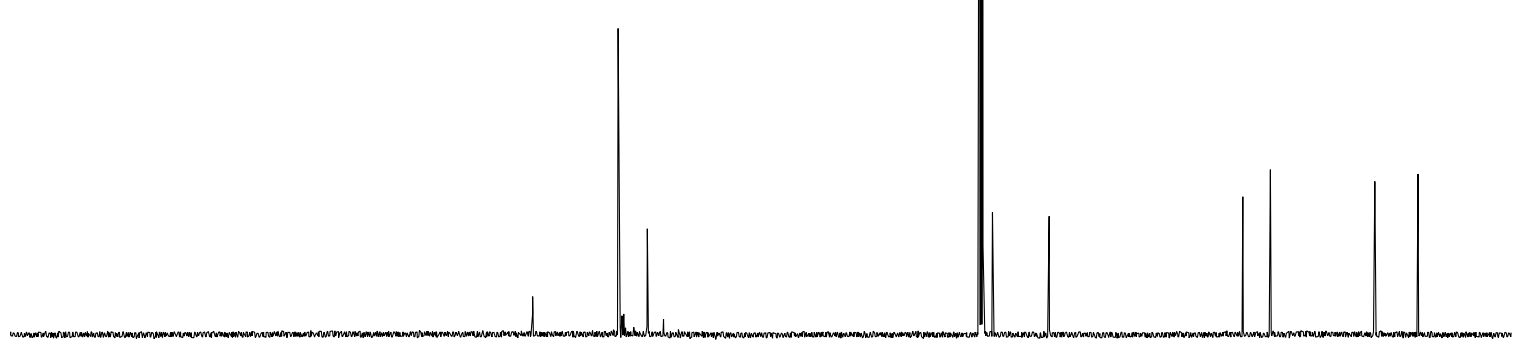

200

180

160

140

$120 \quad \frac{1}{f 1(p p m)} 100$

80

60

40

20 
<smiles>CC[C@@H](O)[C@H](CCl)c1cccc(F)c1</smiles>

$\left(2 R^{*}, 3 R^{*}\right)-1-C h l o r o-2-(3-f l u o r o p h e n y l) h e x a n-3-o l ~( \pm)-7 I$

General Procedure D: Epoxide $( \pm)-5 \mathrm{l}(48 \mathrm{mg}, 0.25 \mathrm{mmol})$ was employed. Purification of the residue by FCC (5:1, hexane-EtOAc) afforded the title compound (41 mg, 71\%) as a colorless oil.

${ }^{1} \mathbf{H}$ NMR $\left(400 \mathrm{MHz} \mathrm{CDCl}_{3}\right) \delta$ 7.34-7.28 (m, 1H), 7.02-6.93 (m, 3H), $4.01(\mathrm{dd}, J=11.1,4.0 \mathrm{~Hz}$, $1 \mathrm{H}), 3.91$ (dd, $J=11.0,8.4 \mathrm{~Hz}, 1 \mathrm{H}), 3.93-3.88(\mathrm{~m}, 1 \mathrm{H}), 2.97$ (app. td, $J=8.2,4.0 \mathrm{~Hz}, 1 \mathrm{H}), 1.63$ (br. s, 1H), 1.52-1.44 (m, 1H), 1.38-1.24 (m, 3H), $0.86(\mathrm{t}, J=6.9 \mathrm{~Hz}, 3 \mathrm{H})$;

${ }^{13} \mathrm{C} \mathrm{NMR}\left(125 \mathrm{MHz}, \mathrm{CDCl}_{3}\right) \delta 162.9(\mathrm{~d}, J=245.9 \mathrm{~Hz}), 142.5(\mathrm{~d}, J=7.0 \mathrm{~Hz}), 130.0$ (d, J = 8.2 Hz), 124.2 (d, $J=2.8 \mathrm{~Hz}), 115.3$ (d, $J=21.4 \mathrm{~Hz}), 114.2$ (d, $J=21.0 \mathrm{~Hz}), 73.0,54.0$ (d, $J=1.7 \mathrm{~Hz})$, 46.3, 37.3, 18.8, 13.8;

${ }^{19} \mathrm{~F}\left\{{ }^{1} \mathrm{H}\right\}$ NMR $\left(376 \mathrm{MHz}, \mathrm{CDCl}_{3}\right) \delta-112.7(\mathrm{~s})$;

IR ( $\mathrm{NaCl}$, thin film) 3392, 2960, 2935, 2873, 1610, 1590, 1490, 1450, 1272, 1243, 1146, 1020, $885,785,607 \mathrm{~cm}^{-1}$;

HRMS $\left(\mathrm{Cl}^{+}\right) \mathrm{m} / \mathrm{z}$ calcd for $\mathrm{C}_{12} \mathrm{H}_{16} \mathrm{~F}^{35} \mathrm{ClONH}_{4}{ }^{+}\left(\mathrm{M}+\mathrm{NH}_{4}\right)^{+}:$248.1212, found 248.1213 

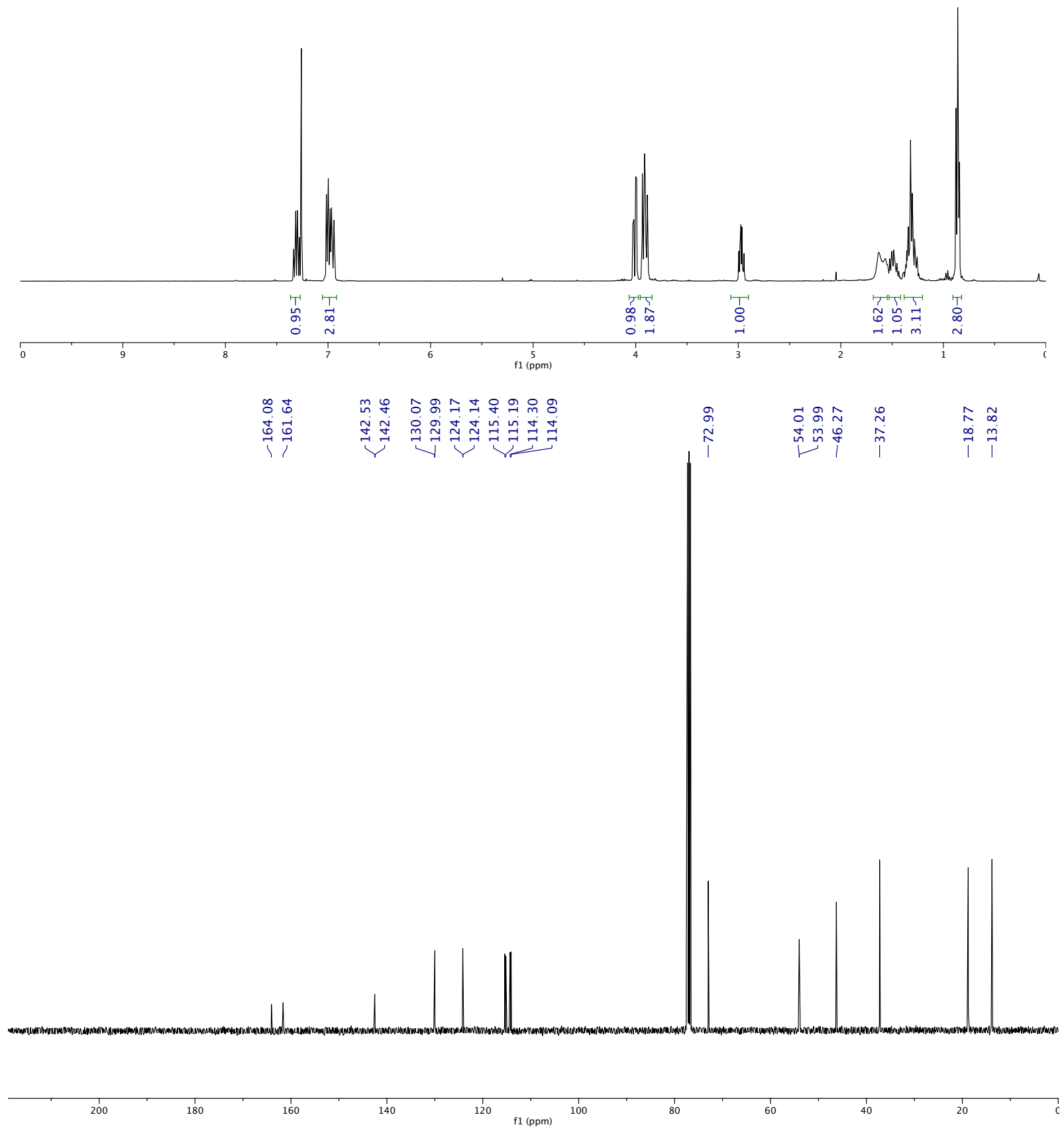


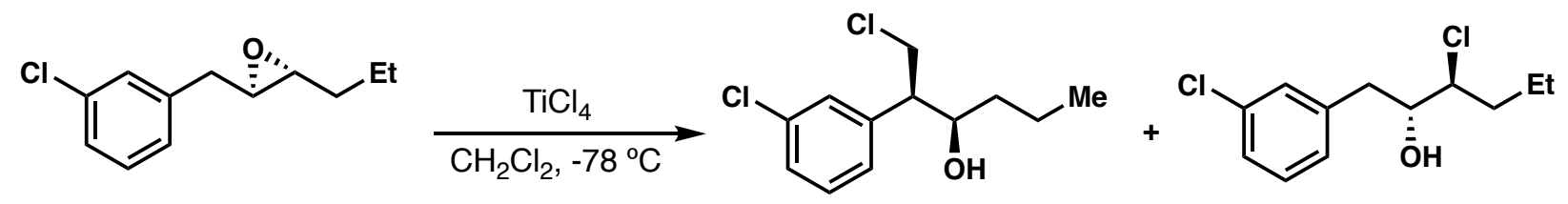

$\left(2 R^{*}, 3 R^{*}\right)$-1-Chloro-2-(3-chlorophenyl)hexan-3-ol ( \pm )-7m

$\left(2 R^{*}, 3 S^{*}\right)-3-C h l o r o-1-(3-c h l o r o p h e n y l) h e x a n-2-o l ~( \pm)-9 m$

General Procedure D: Epoxide $( \pm)-5 \mathrm{~m}(53 \mathrm{mg}, 0.25 \mathrm{mmol})$ was employed. Purification of the residue by FCC (15:1, hexane-EtOAc) afforded chlorohydrin $( \pm)-9 \mathrm{~m}(11 \mathrm{mg}, 18 \%)$ as a colorless oil. Continued elution (5:1, hexane-EtOAc) afforded rearranged product $( \pm)-7 \mathrm{~m}(48 \mathrm{mg}, 78 \%)$ as a yellow oil.

Data for $( \pm)-7 \mathrm{~m}$

${ }^{1} \mathbf{H}$ NMR $\left(400 \mathrm{MHz}, \mathrm{CDCl}_{3}\right) \delta$ 7.30-7.22 (m, 3H), 7.12-7.10 (m, 1H), $4.00(\mathrm{dd}, J=11.1,4.0 \mathrm{~Hz}$, $1 \mathrm{H}), 3.90(\mathrm{dd}, J=10.9,8.6 \mathrm{~Hz}, 1 \mathrm{H}), 3.92-3.87(\mathrm{~m}, 1 \mathrm{H}), 2.94$ (app. td, $J=8.2,4.0 \mathrm{~Hz}, 1 \mathrm{H}), 1.62$ (br. s, $1 \mathrm{H}), 1.53-1.43(\mathrm{~m}, 1 \mathrm{H}), 1.37-1.25(\mathrm{~m}, 3 \mathrm{H}), 0.86(\mathrm{t}, J=7.0 \mathrm{~Hz}, 3 \mathrm{H})$;

${ }^{13} \mathrm{C}$ NMR $\left(100 \mathrm{MHz}, \mathrm{CDCl}_{3}\right) \delta 142.0,134.4,129.8,128.5,127.5,126.6,73.0,54.0,46.2,37.3$, 18.8, 13.8;

IR ( $\mathrm{NaCl}$, thin film) 3406, 2960, 2933, 2873, 1597, 1572, 1431, 1330, 1164, 1123, 1075, 1021, $758,698 \mathrm{~cm}^{-1}$;

HRMS $\left(\mathrm{Cl}^{+}\right) \mathrm{m} / \mathrm{z}$ calcd for $\mathrm{C}_{12} \mathrm{H}_{16}{ }^{35} \mathrm{Cl}_{2} \mathrm{ONH}_{4}{ }^{+}\left(\mathrm{M}+\mathrm{NH}_{4}\right)^{+}:$264.0916, found 264.0911.

Data for chlorohydrin $( \pm)-9 m$

${ }^{1} \mathrm{H}$ NMR $\left(400 \mathrm{MHz}, \mathrm{CDCl}_{3}\right)$ ठ 7.27-7.22 (m, 3H), 7.15-7.12 (m, 1H), 4.01-3.94 (m, 2H), 2.94 (dd, $J=14.1,3.4 \mathrm{~Hz}, 1 \mathrm{H}), 2.79(\mathrm{dd}, J=14.0,8.8 \mathrm{~Hz}, 1 \mathrm{H}), 1.97$ (br. $\mathrm{s}, 1 \mathrm{H}), 1.88-1.74(\mathrm{~m}, 2 \mathrm{H}), 1.71-$ $1.61(\mathrm{~m}, 1 \mathrm{H}), 1.50-1.38(\mathrm{~m}, 1 \mathrm{H}), 0.96(\mathrm{t}, J=7.3 \mathrm{~Hz}, 3 \mathrm{H})$;

${ }^{13} \mathrm{C}$ NMR $\left(100 \mathrm{MHz}_{\mathrm{CDCl}}\right) \delta$ 140.0, 134.4, 129.8, 129.5, 127.6, 126.9, 75.4, 67.0, 38.9, 35.0, 19.8, 13.6;

IR $(\mathrm{NaCl}$, thin film) 3415, 1960, 1933, 2874, 1598, 1574, 1478, 1431, 1262, 1209, 1080, 1046, $781 \mathrm{~cm}^{-1}$

HRMS $\left(\mathrm{Cl}^{+}\right) \mathrm{m} / \mathrm{z}$ calcd for $\mathrm{C}_{12} \mathrm{H}_{16}{ }^{35} \mathrm{Cl}_{2} \mathrm{ONH}_{4}{ }^{+}\left(\mathrm{M}+\mathrm{NH}_{4}\right)^{+}:$264.0916, found 264.0911. 
NMR for ( \pm )-7m

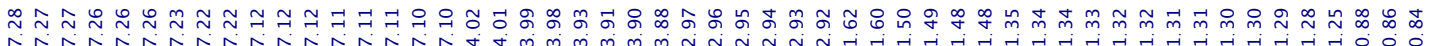

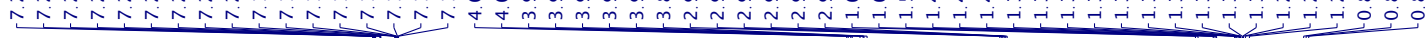
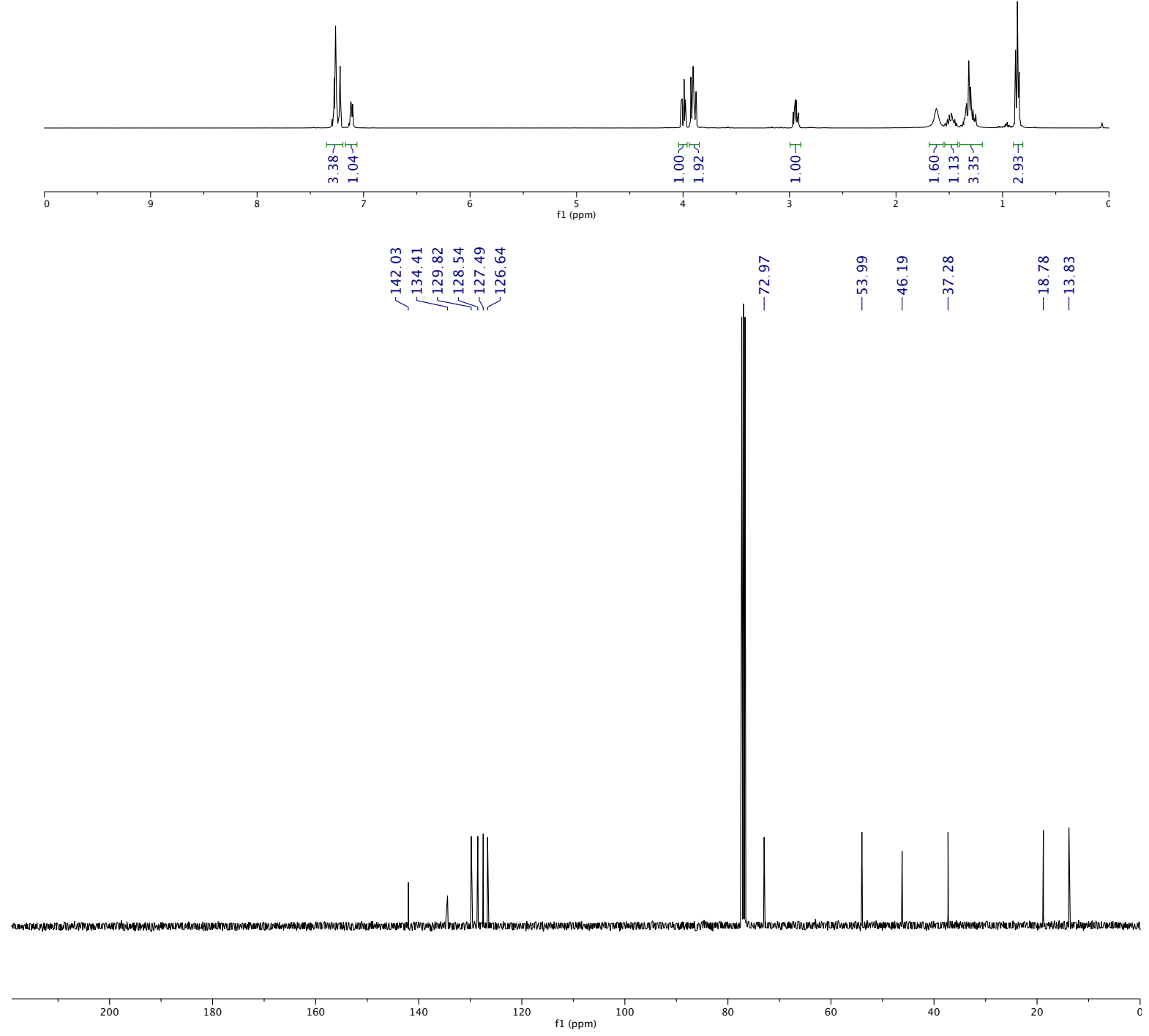
NMR for chlorohydrin ( \pm )-9m

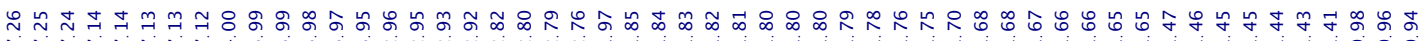

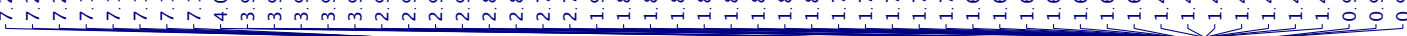
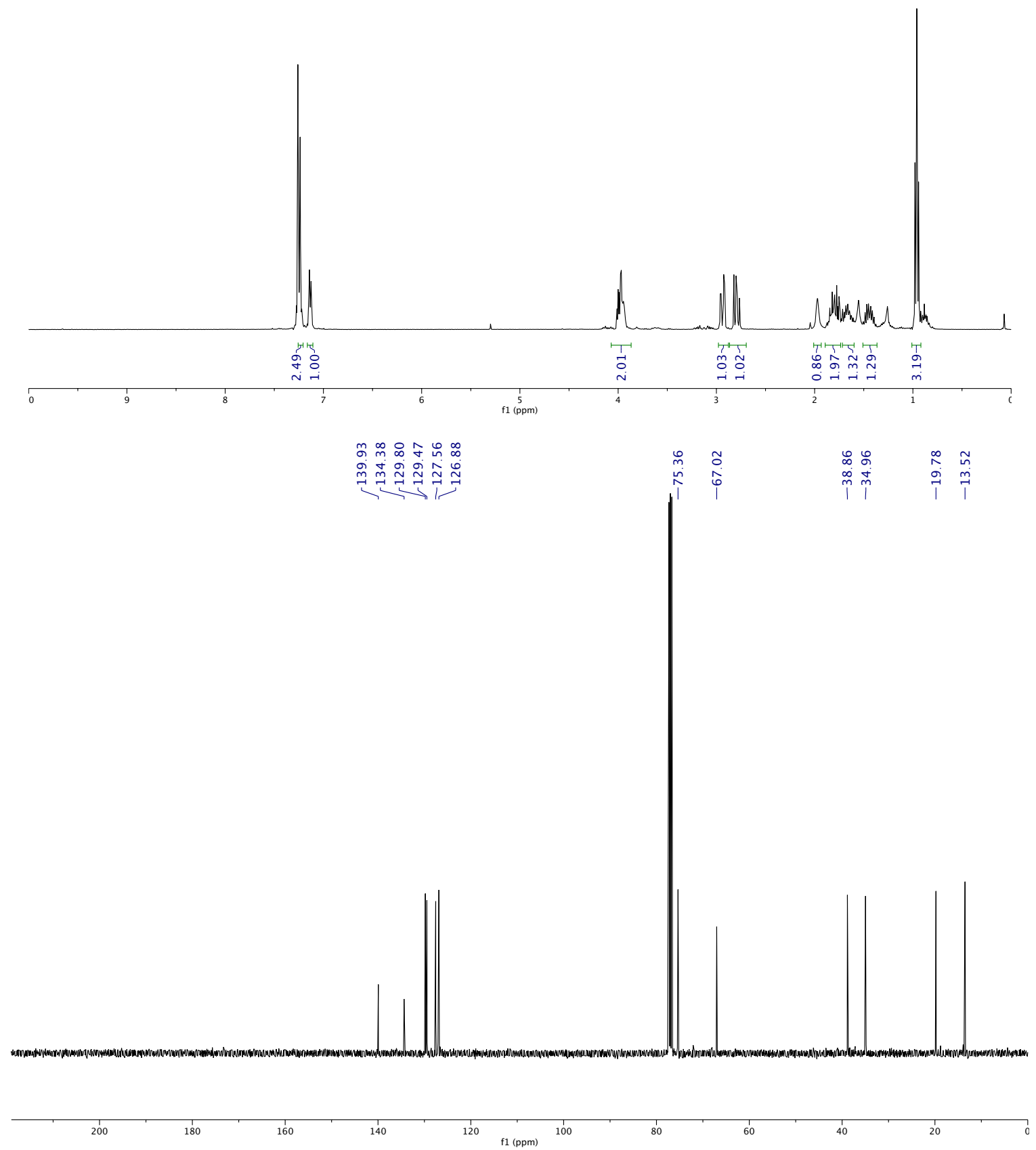
<smiles>COc1cccc([C@H](CCl)[C@H](O)C(C)C)c1</smiles>

$\left(2 R^{*}, 3 R^{*}\right)-1-C h l o r o-2-(3-m e t h o x y p h e n y l) h e x a n-3-o l ~( \pm)-7 \mathrm{n}$

General Procedure D: Epoxide $( \pm)-5 \mathrm{n}(52 \mathrm{mg}, 0.25 \mathrm{mmol})$ was employed. Purification of the residue by FCC (5:1, hexane-EtOAc) afforded the title compound (54 $\mathrm{mg}, 88 \%)$ as a pale orange oil.

${ }^{1} \mathrm{H}$ NMR (400 MHz, $\left.\mathrm{CDCl}_{3}\right) \delta$ 7.28-7.24 (m, 1H), 6.84-6.77 (m, 3H), $4.01(\mathrm{dd}, J=11.0,4.2 \mathrm{~Hz}$, $1 \mathrm{H}), 3.92(\mathrm{dd}, J=11.0,8.4 \mathrm{~Hz}, 1 \mathrm{H}), 3.93-3.89(\mathrm{~m}, 1 \mathrm{H}), 3.81(\mathrm{~s}, 3 \mathrm{H}), 2.94$ (app. td, $J=8.1,4.2$ $\mathrm{Hz}, 1 \mathrm{H}), 1.62$ (br. s, 1H), 1.53-1.46 (m, 1H), 1.40-1.24 (m, 3H), $0.86(\mathrm{t}, J=7.1 \mathrm{~Hz}, 3 \mathrm{H})$;

${ }^{13} \mathrm{C}$ NMR (100 MHz, $\left.\mathrm{CDCl}_{3}\right) \delta 159.7,141.5,129.6,120.7,114.6,112.1,73.2,55.2,54.3,46.5$, 37.2, 18.8, 13.8;

IR ( $\mathrm{NaCl}$, thin film) 3420, 2958, 1585, 1489, 1456, 1256, 1156, 1048, 783, $700 \mathrm{~cm}^{-1}$;

HRMS (ESI-TOF) $\mathrm{m} / \mathrm{z}$ calcd for $\mathrm{C}_{13} \mathrm{H}_{19}{ }^{35} \mathrm{ClO}_{2} \mathrm{Na}(\mathrm{M}+\mathrm{Na})^{+}:$: 265.0971, found 265.0971. 

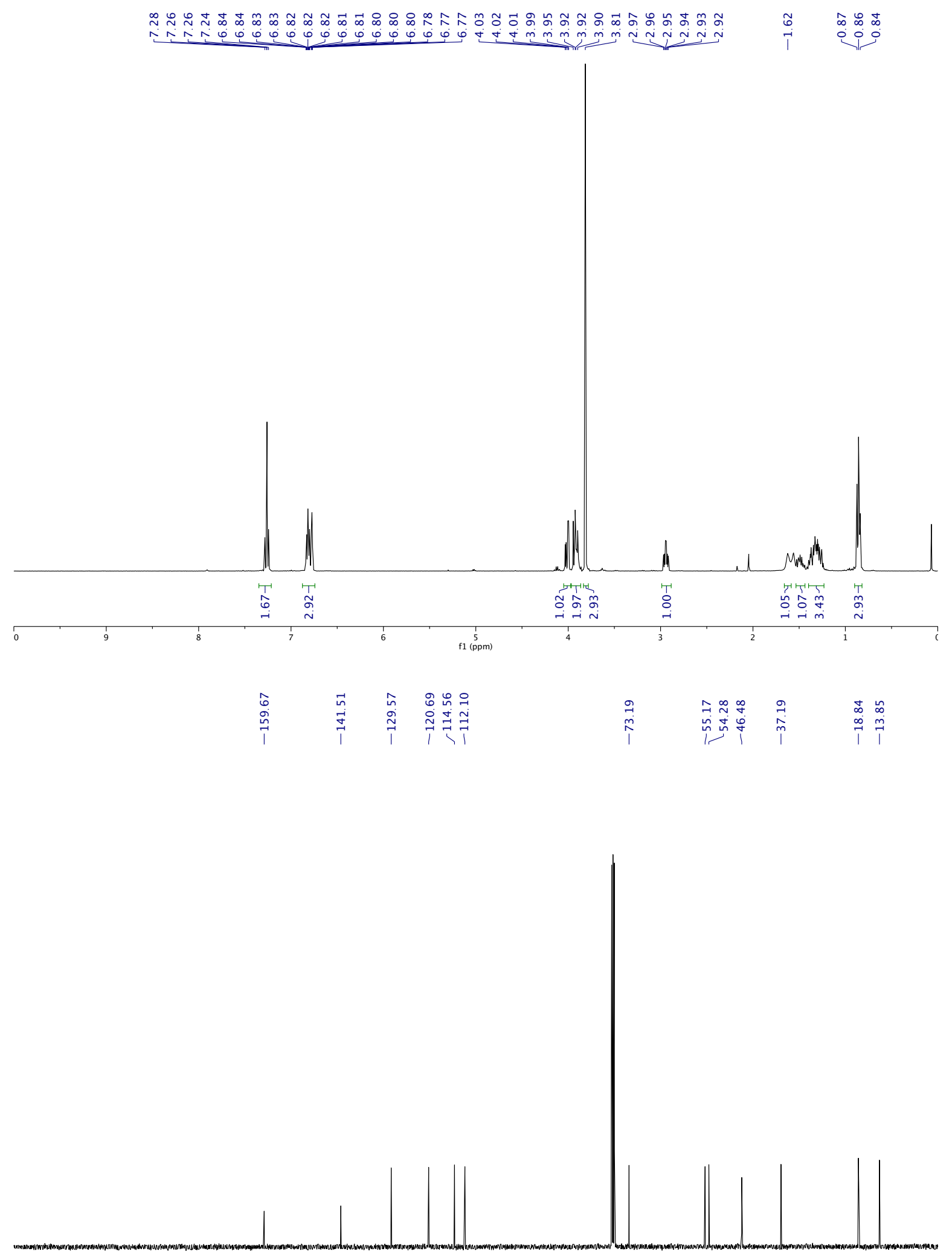

$200 \quad 180+160$

$\underset{140}{120} \underset{\mathrm{f} 1(\mathrm{ppm})}{ } 100$ 80 60 40 $20 \quad c$ 
<smiles>CCC1OC1Cc1cccc(C(F)(F)F)c1</smiles>

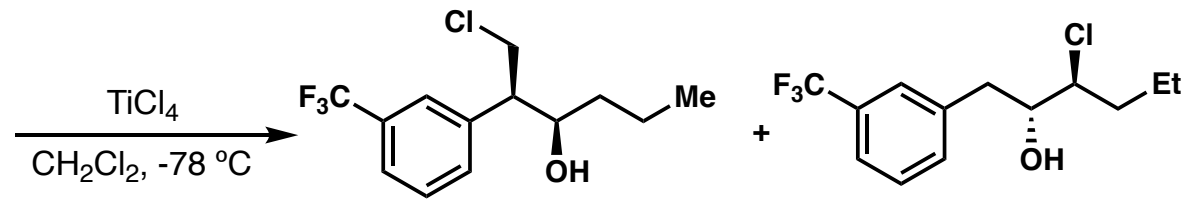

$\left(2 R^{*}, 3 R^{*}\right)$-1-Chloro-2-(3-(trifluoromethyl)phenyl)hexan-3-ol ( \pm )-7o

$\left(2 R^{*}, 3 S^{*}\right)-3-C h l o r o-1-(3-(t r i f l u o r o m e t h y l) p h e n y l) h e x a n-2-o l ~( \pm)-90$

General Procedure D: Epoxide $( \pm)-50(61 \mathrm{mg}, 0.25 \mathrm{mmol})$ was employed. Purification of the residue by FCC (15:1, hexane-EtOAc) afforded chlorohydrin ( \pm )-9o (30 mg, 43\%) as a colorless oil. Continued elution (5:1, hexane-EtOAc) afforded rearranged product $( \pm)-70(30 \mathrm{mg}, 43 \%)$ as a pale yellow oil.

Data for $( \pm)-70$

${ }^{1} \mathrm{H}$ NMR $\left(400 \mathrm{MHz}, \mathrm{CDCl}_{3}\right) \delta 7.56-7.42(\mathrm{~m}, 4 \mathrm{H}), 4.00(\mathrm{dd}, J=11.1,4.1 \mathrm{~Hz}, 1 \mathrm{H}), 3.95(\mathrm{dd}, J=$ 11.1, 8.2 Hz, 1H), 3.96-3.93 (m, 1H), 3.04 (app. td, $J=8.0,4.1 \mathrm{~Hz}, 1 \mathrm{H}$ ), 1.60 (br. s, 1H), 1.53$1.43(\mathrm{~m}, 1 \mathrm{H}), 1.35-1.27(\mathrm{~m}, 3 \mathrm{H}), 0.86(\mathrm{t}, J=7.0 \mathrm{~Hz}, 3 \mathrm{H})$;

${ }^{13} \mathrm{C}$ NMR (125 MHz, $\left.\mathrm{CDCl}_{3}\right) \delta 141.0,131.8,130.9(\mathrm{q}, J=32.1 \mathrm{~Hz}), 129.0,125.2(\mathrm{q}, J=3.8 \mathrm{~Hz})$, 124.2 (q, $J=3.8 \mathrm{~Hz}$ ), 124.1 (q, $J=273.9 \mathrm{~Hz}), 72.8,54.0,46.1$, 37.3, 18.8, 13.8;

${ }^{19} \mathrm{~F}\left\{{ }^{1} \mathrm{H}\right\}$ NMR (376 MHz, $\left.\mathrm{CDCl}_{3}\right) \delta-62.5(\mathrm{~s})$;

IR ( $\mathrm{NaCl}$, thin film) 3406, 2962, 2936, 2875, 1449, 1329, 1262, 1166, 1127, 1075, 1024, 803, 704 $\mathrm{cm}^{-1}$;

HRMS $\left(\mathrm{Cl}^{+}\right) \mathrm{m} / \mathrm{z}$ calcd for $\mathrm{C}_{13} \mathrm{H}_{16} \mathrm{~F}_{3}{ }^{35} \mathrm{ClONH}_{4}{ }^{+}\left(\mathrm{M}+\mathrm{NH}_{4}\right)^{+}:$298.1180, found 298.1173.

Data for chlorohydrin ( \pm -90

${ }^{1} \mathrm{H}$ NMR (400 MHz, CDCl 3 ) ס 7.53-7.49 (m, 2H), 7.46-7.43 (m, 2H), 4.03-3.95 (m, 2H), 3.01 (dd, $J=14.1,3.2 \mathrm{~Hz}, 1 \mathrm{H}), 2.88$ (dd, $J=14.1,8.9 \mathrm{~Hz}, 1 \mathrm{H}), 1.98$ (br. s, 1H), 1.90-1.75 (m, 2H), 1.74$1.62(\mathrm{~m}, 1 \mathrm{H}), 1.49-1.40(\mathrm{~m}, 1 \mathrm{H}), 0.97(\mathrm{t}, J=7.3 \mathrm{~Hz}, 3 \mathrm{H})$;

${ }^{13} \mathrm{C}$ NMR $\left(100 \mathrm{MHz}, \mathrm{CDCl}_{3}\right) \delta 139.0,132.8,130.9$ (q, $\left.J=32.1 \mathrm{~Hz}\right), 129.0,126.1$ (q, $\left.J=3.8 \mathrm{~Hz}\right)$, 124.1 (q, $J=272.2 \mathrm{~Hz}$ ), 123.6 (q, $J=3.9 \mathrm{~Hz}$ ), 75.4, 67.2, 39.0, 35.0, 19.8, 13.5;

${ }^{19} \mathrm{~F}\left\{{ }^{1} \mathrm{H}\right\}$ NMR (376 MHz, $\left.\mathrm{CDCl}_{3}\right) \delta-62.6(\mathrm{~s})$;

IR ( $\mathrm{NaCl}$, thin film) 3429, 2962, 2876, 1451, 1332, 1165, 1124, 1074, 798, $704 \mathrm{~cm}^{-1}$;

HRMS $\left(\mathrm{Cl}^{+}\right) \mathrm{m} / \mathrm{z}$ calcd for $\mathrm{C}_{13} \mathrm{H}_{16} \mathrm{~F}_{3}{ }^{35} \mathrm{ClONH}_{4}{ }^{+}\left(\mathrm{M}+\mathrm{NH}_{4}\right)^{+}:$298.1180, found 298.1180. 
Data for $( \pm)-70$
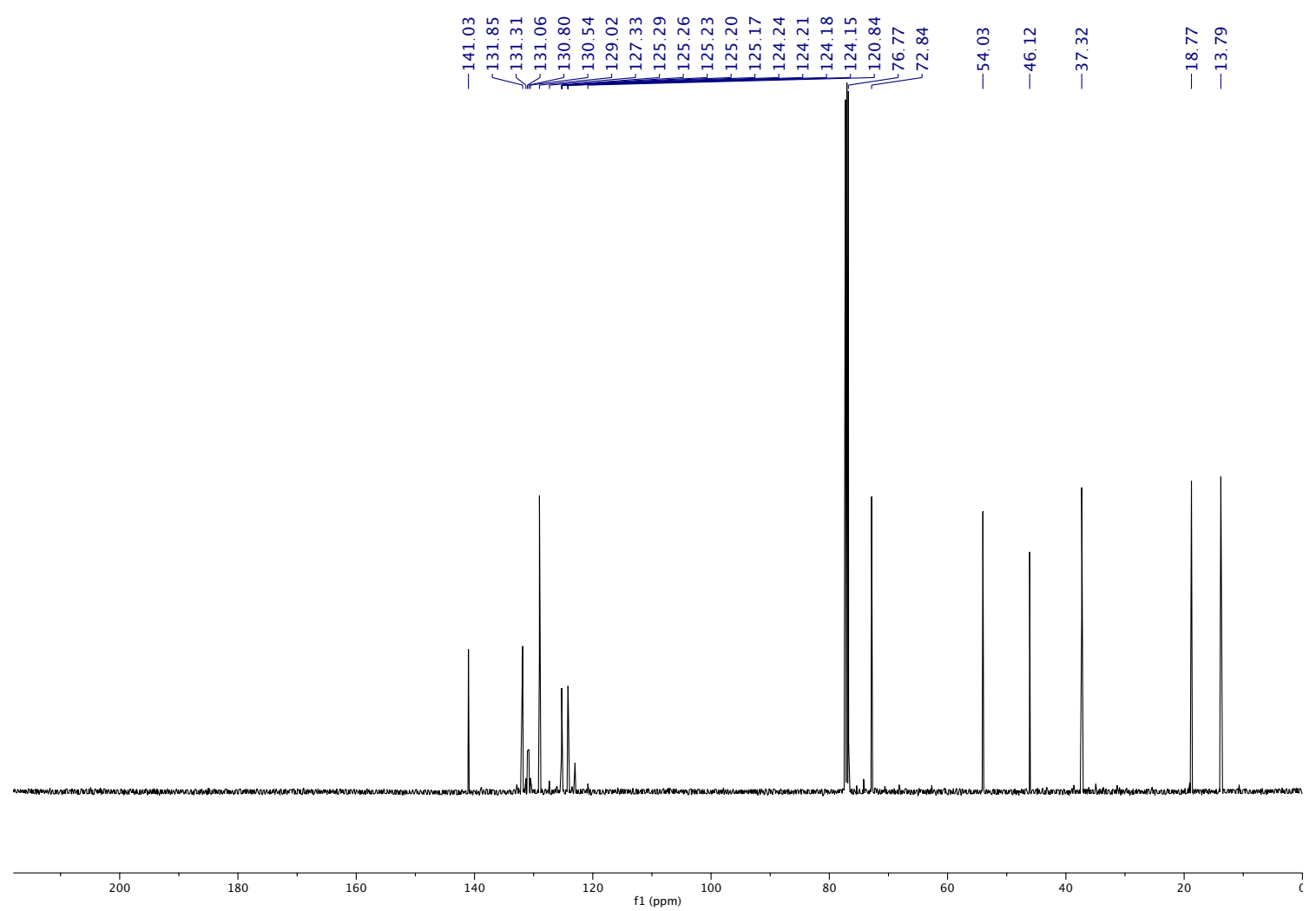

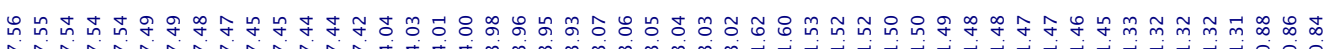

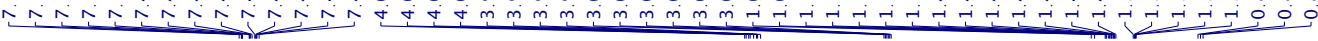

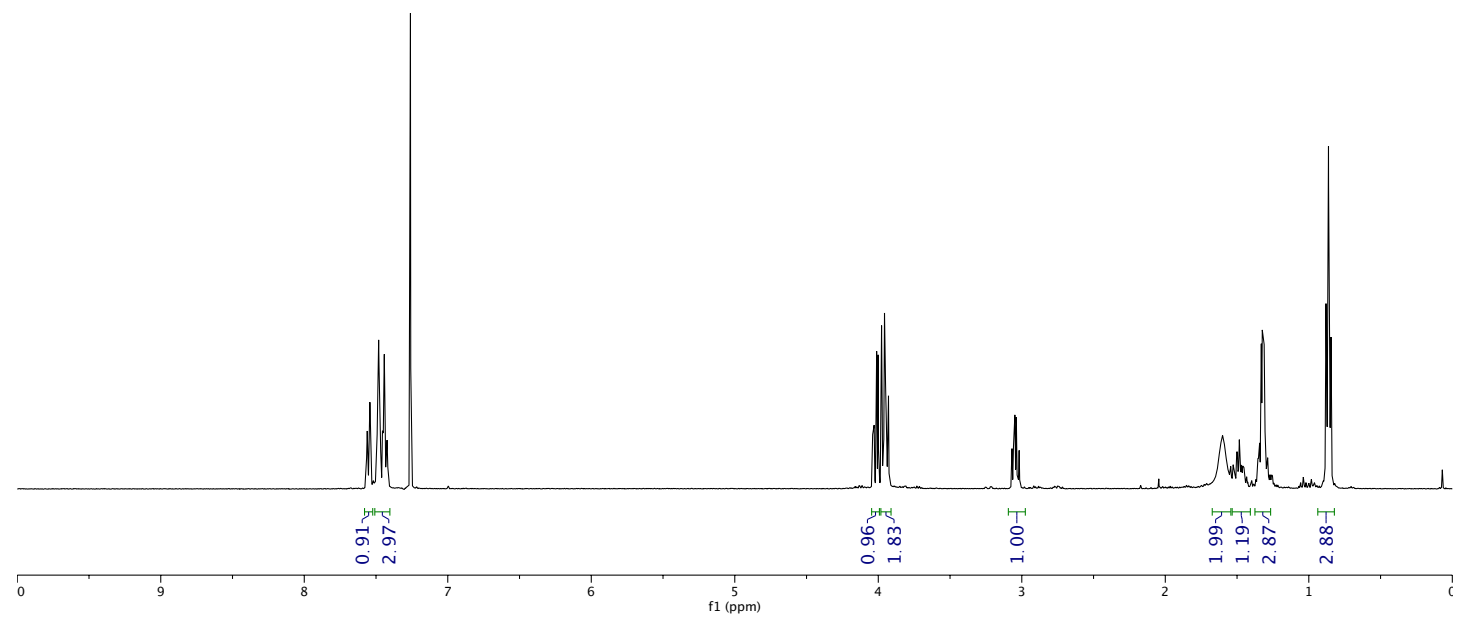




\section{Data for chlorohydrin $( \pm)-90$}

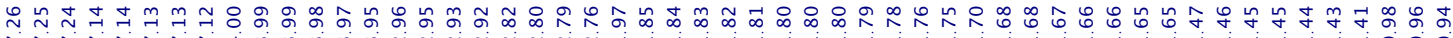

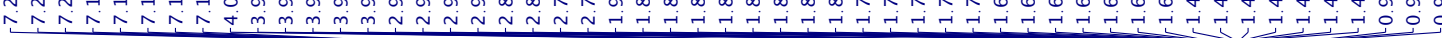
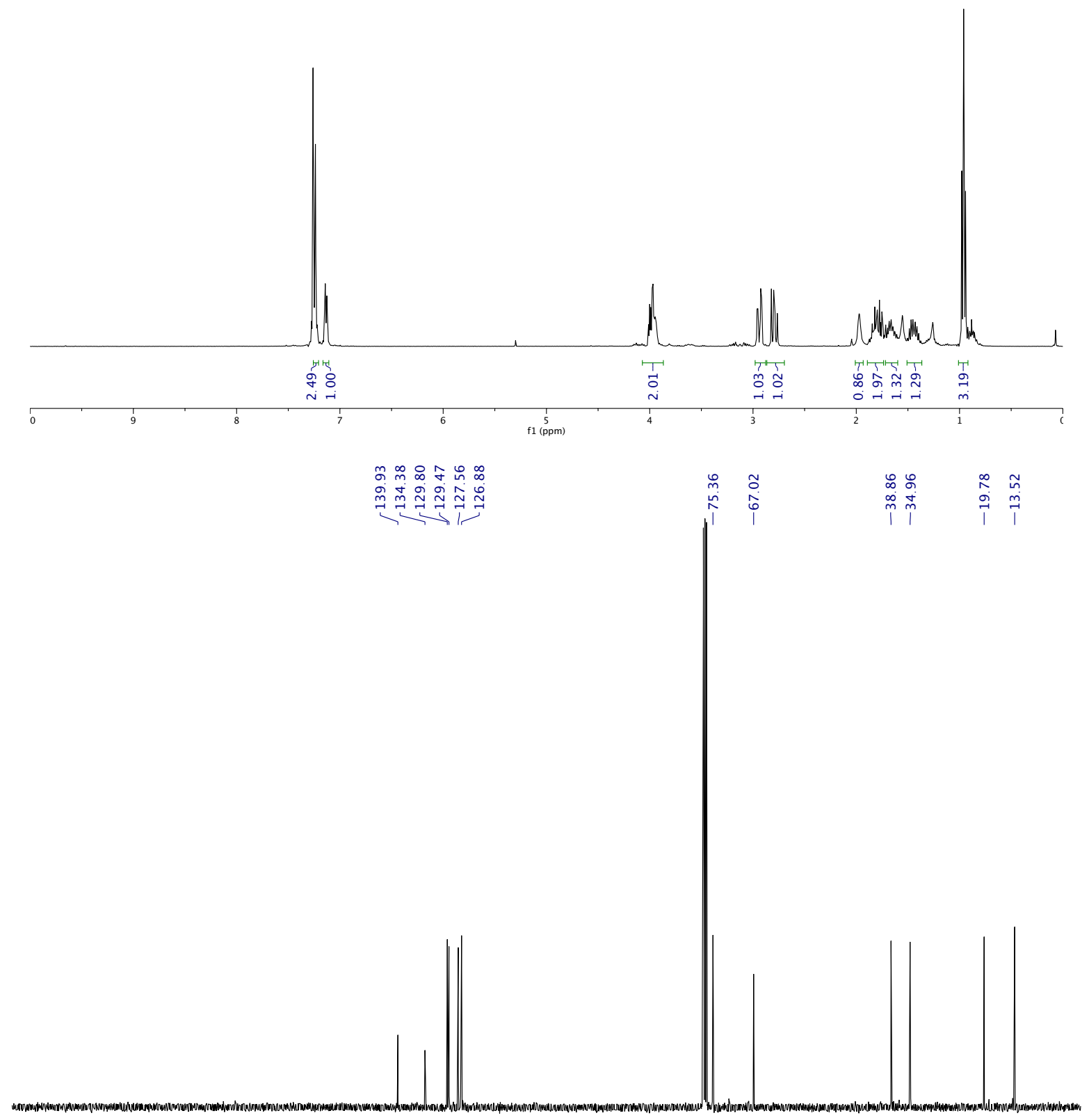

$200+180$

$120 \quad \mathrm{f} 1(\mathrm{ppm})$

100

80

60

40 
<smiles>CCC1O[C@@H]1Cc1ccc(F)c(F)c1</smiles>

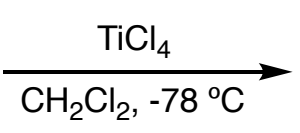<smiles>CCC[C@H](O)[C@H](CCl)c1ccc(F)c(F)c1</smiles><smiles>CC[C@H](Cl)[C@H](O)Cc1ccc(F)c(F)c1</smiles>

$\left(2 R^{*}, 3 R^{*}\right)-1-C h l o r o-2-(3,4-d i f l u o r o p h e n y l) h e x a n-3-o l( \pm)-7 \mathrm{p}$

$\left(2 R^{\star}, 3 S^{\star}\right)-3-C h l o r o-1-(3,4-d i f l u o r o p h e n y l) h e x a n-2-o l( \pm)-9 p$

General Procedure D: Epoxide $( \pm)-5 p(53 \mathrm{mg}, 0.25 \mathrm{mmol})$ was employed. Purification of the residue by FCC (15:1, hexane-EtOAc) afforded chlorohydrin ( \pm )-9p (16 mg, 26\%) as a colorless oil. Continued elution (5:1, hexane-EtOAc) afforded rearranged product $( \pm)-7 \mathrm{p}(40 \mathrm{mg}, 64 \%)$ as a pale yellow oil.

Data for $( \pm)-7 p$

${ }^{1} \mathbf{H}$ NMR $\left(400 \mathrm{MHz}, \mathrm{CDCl}_{3}\right) \delta$ 7.16-7.05 (m, 2H), 6.97-6.93 (m, 1H), $3.98(\mathrm{dd}, J=11.1,3.9 \mathrm{~Hz}$, $1 \mathrm{H}), 3.88$ (dd, $J=11.0,8.5 \mathrm{~Hz}, 1 \mathrm{H}$ ), 3.89-3.85 (m, 1H), 2.93 (app. td, $J=8.2,4.0 \mathrm{~Hz}, 1 \mathrm{H}$ ), 1.62 (br. s, $1 \mathrm{H}), 1.54-1.43(\mathrm{~m}, 1 \mathrm{H}), 1.37-1.25(\mathrm{~m}, 3 \mathrm{H}), 0.87(\mathrm{t}, J=6.9 \mathrm{~Hz}, 3 \mathrm{H})$;

${ }^{13} \mathrm{C}$ NMR $\left(100 \mathrm{MHz}, \mathrm{CDCl}_{3}\right) \delta 150.3(\mathrm{dd}, J=249.2,12.7 \mathrm{~Hz}), 149.6(\mathrm{dd}, J=247.7,12.7 \mathrm{~Hz}), 137.0$ (dd, $J=5.4,4.0 \mathrm{~Hz}$ ), 124.5 (dd, $J=6.2,3.6 \mathrm{~Hz}), 117.3$ (d, $J=10.1 \mathrm{~Hz}), 117.2$ (d, $J=10.3 \mathrm{~Hz}$ ), 72.9, 53.4, 46.3, 37.3, 18.7, 13.8;

${ }^{19}$ F NMR (376 MHz, $\left.\mathrm{CDCl}_{3}\right) \delta-137.2(\mathrm{~d}, J=21.3 \mathrm{~Hz}),-139.8(\mathrm{~d}, J=21.3 \mathrm{~Hz})$;

IR ( $\mathrm{NaCl}$, thin film) 3391, 2961, 2935, 2874, 1610, 1521, 1434, 1288, 1212, 1118, $778 \mathrm{~cm}^{-1}$;

HRMS $\left(\mathrm{Cl}^{+}\right) \mathrm{m} / \mathrm{z}$ calcd for $\mathrm{C}_{12} \mathrm{H}_{15} \mathrm{~F}_{2}{ }^{35} \mathrm{ClONH}_{4}{ }^{+}\left(\mathrm{M}+\mathrm{NH}_{4}\right)^{+}:$266.1118, found 266.1113 .

Data for $( \pm)-9 p$

${ }^{1} \mathrm{H}$ NMR $\left(400 \mathrm{MHz}, \mathrm{CDCl}_{3}\right)$ ठ 7.13-7.06 (m, 2H), 6.98-6.94 (m, 1H), 4.00-3.95 (m, 1H), 3.94-3.89 $(\mathrm{m}, 1 \mathrm{H}), 2.91(\mathrm{dd}, J=14.2,3.5 \mathrm{~Hz}, 1 \mathrm{H}), 2.78(\mathrm{dd}, J=14.2,8.9 \mathrm{~Hz}, 1 \mathrm{H}), 1.98(\mathrm{~d}, J=5.7 \mathrm{~Hz}, 1 \mathrm{H})$, 1.86-1.73 (m, 2H), 1.71-1.62 (m, 1H), 1.50-1.39 (m, 1H), $0.96(\mathrm{t}, J=7.3 \mathrm{~Hz}, 3 \mathrm{H})$;

${ }^{13} \mathrm{C}$ NMR $\left(100 \mathrm{MHz}, \mathrm{CDCl}_{3}\right) \delta 150.2(\mathrm{dd}, J=248.4,12.9 \mathrm{~Hz}), 149.3(\mathrm{dd}, J=246.8,12.9 \mathrm{~Hz}), 134.9$ (dd, $J=5.7,4.1 \mathrm{~Hz}$ ), 125.8 (dd, $J=6.1,3.6 \mathrm{~Hz}), 118.2$ (d, $J=16.9 \mathrm{~Hz}), 117.2$ (d, $J=17.0 \mathrm{~Hz}$ ), $75.4,67.0,38.4(\mathrm{~d}, J=1.3 \mathrm{~Hz}), 35.0,19.8,13.5$;

${ }^{19}$ F NMR (376 MHz, $\left.\mathrm{CDCl}_{3}\right)-137.8(\mathrm{~d}, \mathrm{~J}=21.6 \mathrm{~Hz}),-141.0(\mathrm{~d}, \mathrm{~J}=21.1 \mathrm{~Hz})$;

IR $\left(\mathrm{NaCl}\right.$, thin film) 3437, 2962, 2875, 1610, 1518, 1435, 1282, 1213, 1118, 1053, 816, $783 \mathrm{~cm}^{-1}$; HRMS $\left(\mathrm{Cl}^{+}\right) \mathrm{m} / \mathrm{z}$ calcd for $\mathrm{C}_{12} \mathrm{H}_{15} \mathrm{~F}_{2}{ }^{35} \mathrm{ClONH}_{4}{ }^{+}\left(\mathrm{M}+\mathrm{NH}_{4}\right)^{+}:$266.1118, found 266.1115. 
Data for $( \pm)-7 p$

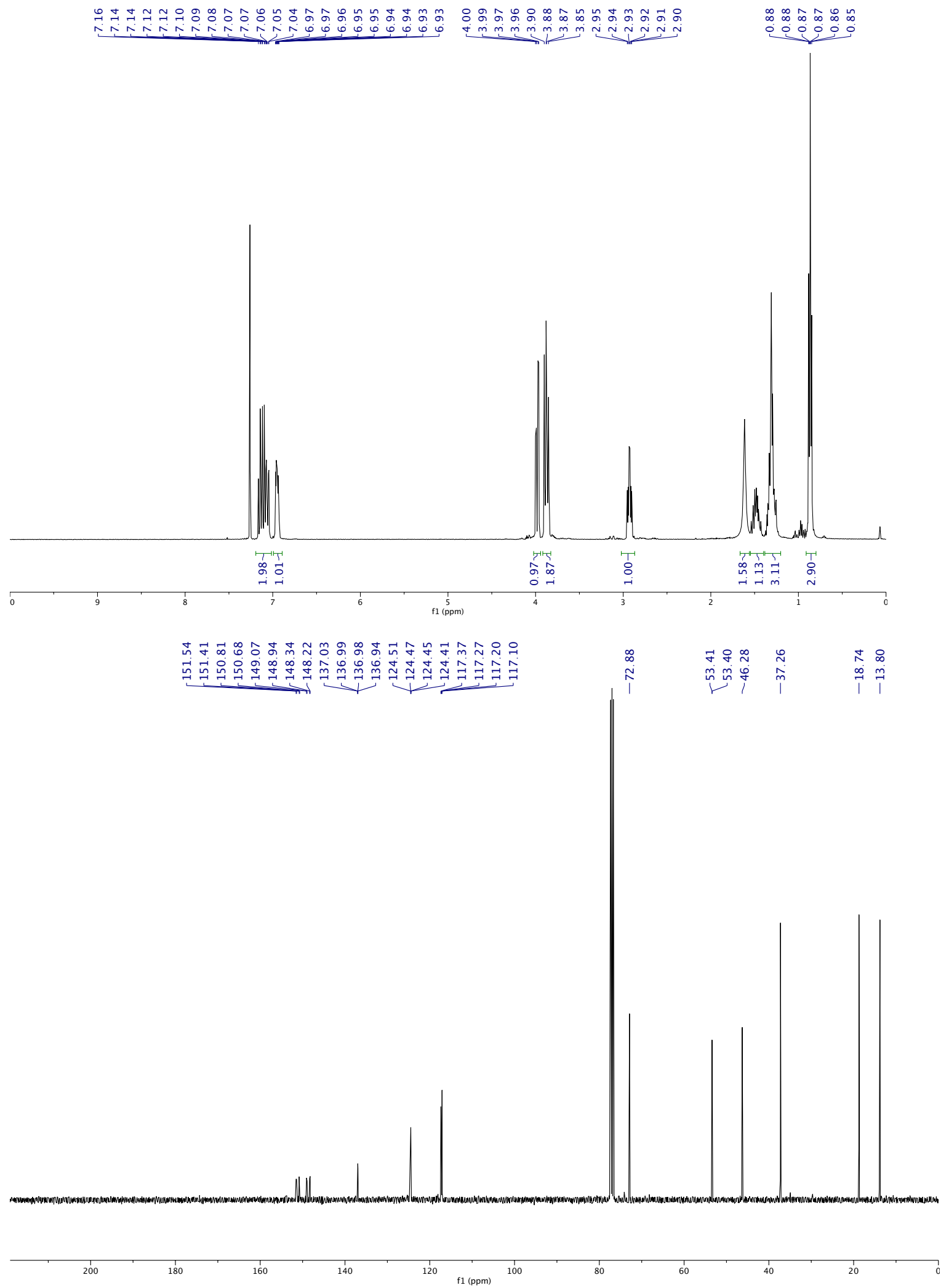




\section{Data for chlorohydrin $( \pm)-9 p$}

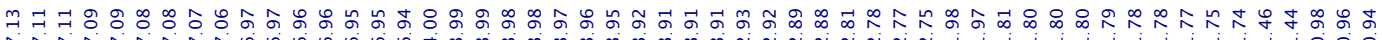

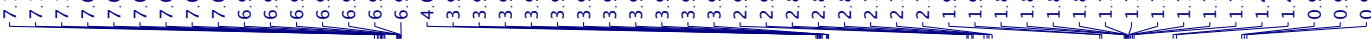

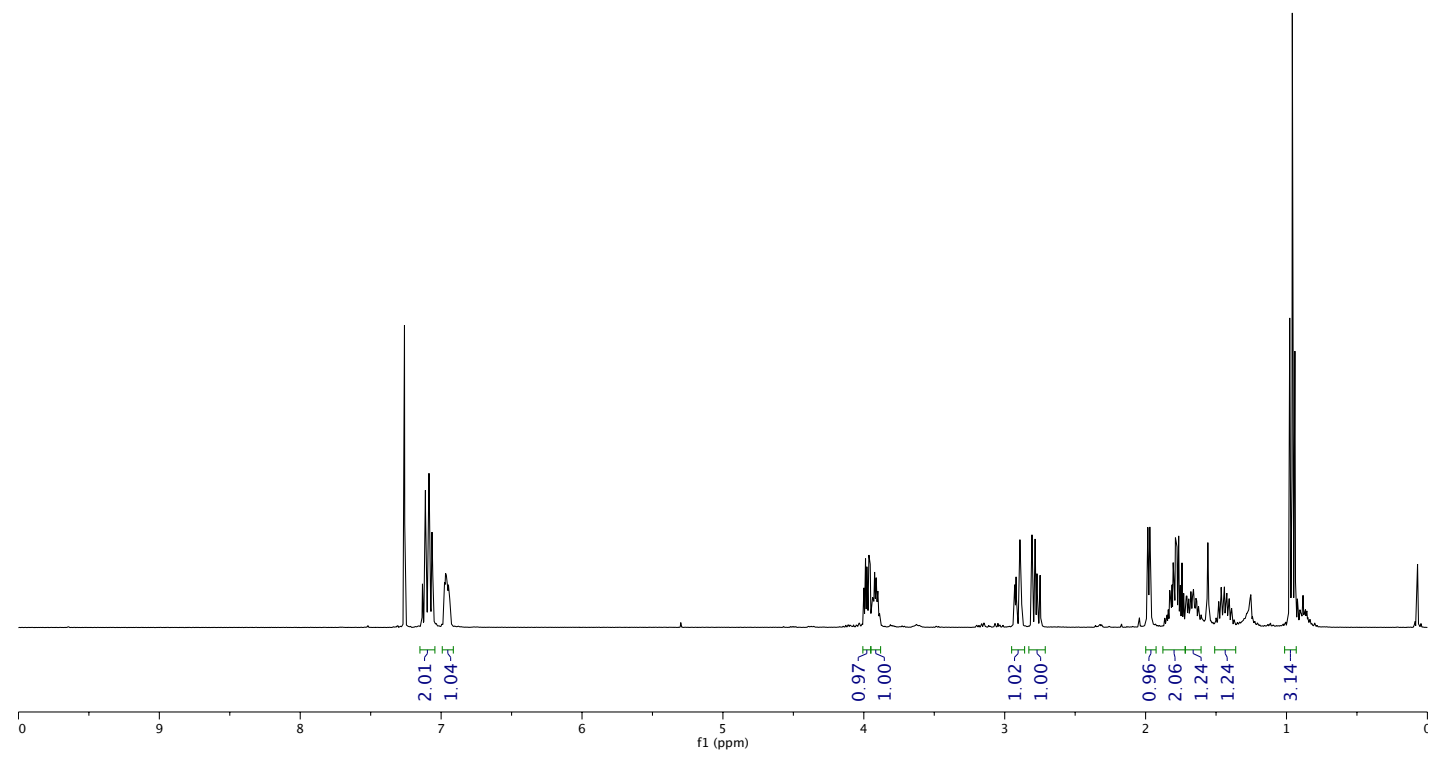

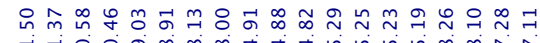

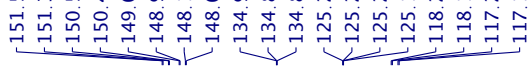

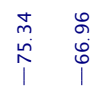

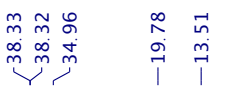

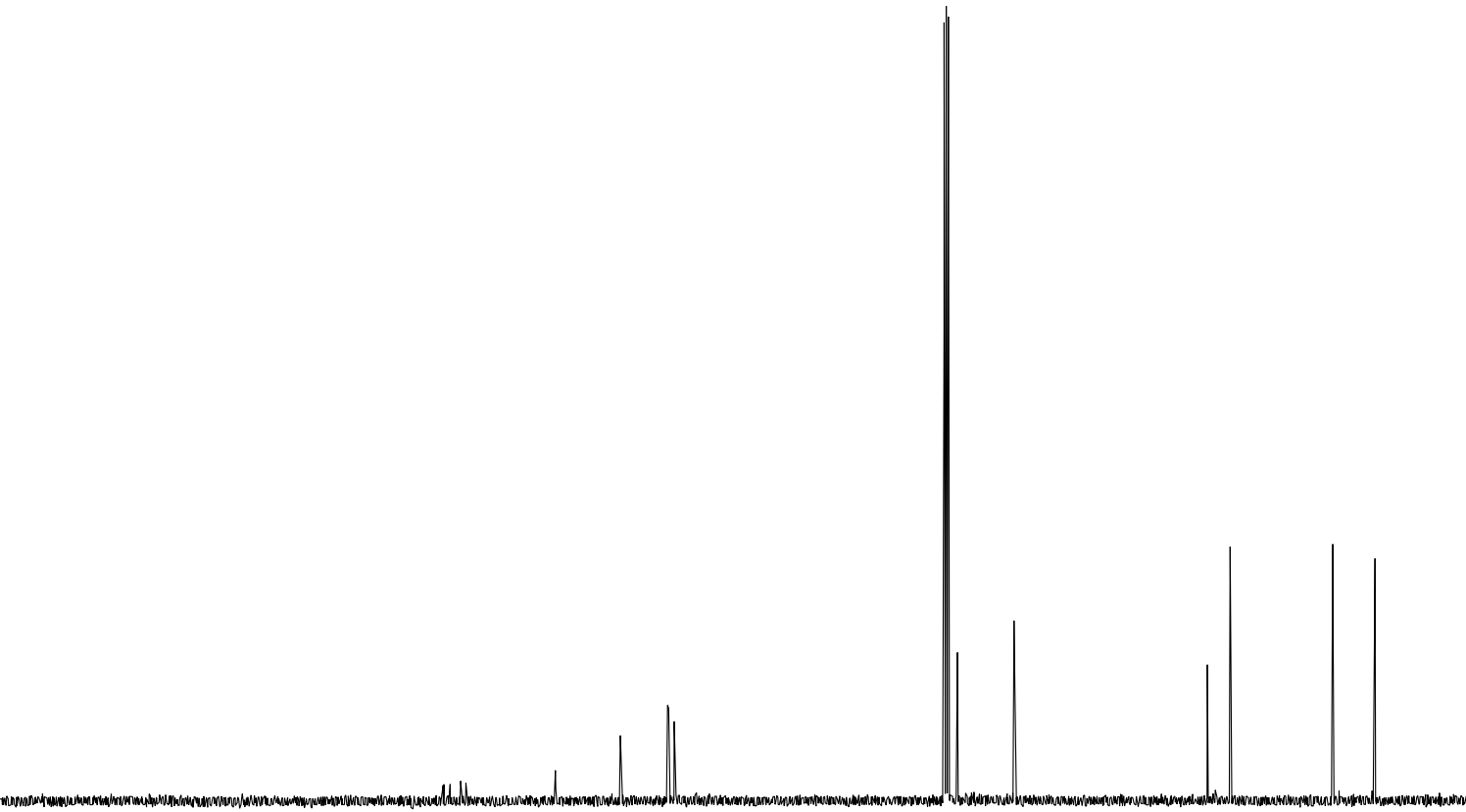

$200+180+160$

140

120

100

80

60

40

20 
<smiles>CCC1O[C@@H]1Cc1cc(F)c(F)c(F)c1</smiles>

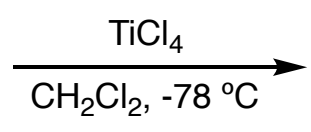<smiles>CCC[C@H](Cl)[C@H](O)Cc1cc(F)c(F)c(F)c1</smiles>

$\left(2 R^{*}, 3 S^{*}\right)-3-C h l o r o-1-(3,4,5-t r i f l u o r o p h e n y l) h e x a n-2-o l( \pm)-9$ ac

General Procedure D: Epoxide $( \pm)-5 \mathrm{ac}(57 \mathrm{mg}, 0.25 \mathrm{mmol})$ were employed. Purification of the residue by FCC (15:1, hexane-EtOAc) afforded chlorohydrin ( \pm )-9ac (47 $\mathrm{mg}, 73 \%)$ as a colorless solid.

${ }^{1} \mathrm{H}$ NMR (400 MHz, $\left.\mathrm{CDCl}_{3}\right) \delta$ 6.91-6.87 (m, 2H), 3.97 (app. dt, J = 9.1, 4.2 Hz, 1H), 3.92-3.87 (m, 1H), 2.87 (dd, $J=14.3,3.2 \mathrm{~Hz}, 1 \mathrm{H}), 2.75$ (dd, $J=14.3,9.1 \mathrm{~Hz}, 1 \mathrm{H}), 2.02$ (br. s, 1H), 1.83-1.62 $(\mathrm{m}, 3 \mathrm{H}), 3.49-3.39(\mathrm{~m}, 1 \mathrm{H}), 0.96(\mathrm{t}, J=7.3 \mathrm{~Hz}, 3 \mathrm{H})$;

${ }^{13} \mathrm{C}$ NMR (125 MHz, $\left.\mathrm{CDCl}_{3}\right) \delta 151.1$ (ddd, $\left.J=249.6,9.9,4.2 \mathrm{~Hz}\right), 136.6$ (dt, $J=250.1,15.2 \mathrm{~Hz}$ ), 134.3 (app. td, $J=7.4,4.7 \mathrm{~Hz}$ ), 113.3 (dd, $J=16.0,4.8 \mathrm{~Hz}$ ), 75.1, 66.9, 38.4, 35.0, 19.8, 13.5;

${ }^{19} \mathrm{~F}\left\{{ }^{1} \mathrm{H}\right\}$ NMR $\left(376 \mathrm{MHz}, \mathrm{CDCl}_{3}\right) \delta-134.7(\mathrm{~d}, \mathrm{~J}=20.7 \mathrm{~Hz}),-163.5(\mathrm{t}, \mathrm{J}=20.6 \mathrm{~Hz})$;

IR ( $\mathrm{NaCl}$, thin film) 3443, 2963, 2936, 2876, 1621, 1532, 1450, 1351, 1236, 1049, 834, $792 \mathrm{~cm}^{-1}$; HRMS $\left(\mathrm{Cl}^{+}\right) \mathrm{m} / \mathrm{z}$ calcd for $\mathrm{C}_{12} \mathrm{H}_{14} \mathrm{~F}_{3}{ }^{35} \mathrm{ClONH}_{4}{ }^{+}\left(\mathrm{M}+\mathrm{NH}_{4}\right)^{+}:$284.1024, found 284.1020;

M pt $43-44^{\circ} \mathrm{C}$. 

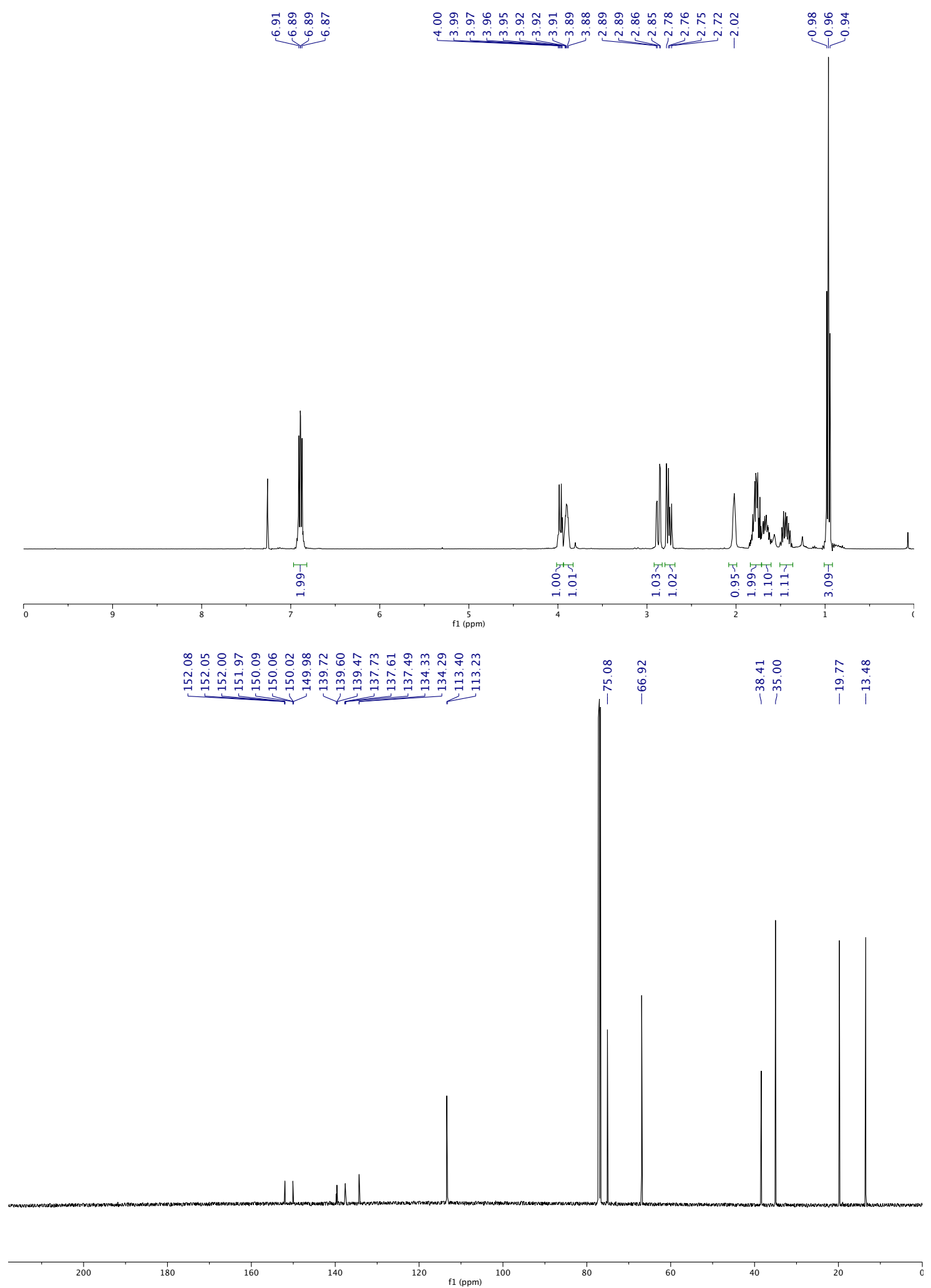
<smiles>COc1ccc([C@@H](CCl)[C@H](O)CCCCCl)cc1</smiles>

\section{$\left(2 S^{*}, 3 S^{*}\right)-1,7-D i c h l o r o-2-(4-m e t h o x y p h e n y l) h e p t a n-3-o l ~( \pm)-7 q$}

General Procedure D: Epoxide $( \pm)-5 q(38 \mathrm{mg}, 0.15 \mathrm{mmol})$ was employed. Purification of the residue by FCC (3:1, hexane-EtOAc) afforded the title compound (42 $\mathrm{mg}, 96 \%)$ as a colorless solid.

${ }^{1} \mathrm{H}$ NMR $\left(400 \mathrm{MHz}, \mathrm{CDCl}_{3}\right) \delta 7.13(\mathrm{~d}, J=8.7 \mathrm{~Hz}, 2 \mathrm{H}), 6.88(\mathrm{~d}, J=8.7 \mathrm{~Hz} 2 \mathrm{H}), 3.98$ (dd, $J=10.9$, $4.4 \mathrm{~Hz}, 1 \mathrm{H}), 3.94-3.88(\mathrm{~m}, 2 \mathrm{H}), 3.80(3 \mathrm{H}, \mathrm{s}), 3.47(\mathrm{t}, J=6.5 \mathrm{~Hz}, 2 \mathrm{H}), 2.93$ (ddd, $J=4.4,3.9,3.9$ $\mathrm{Hz}, 1 \mathrm{H}), 1.79-1.56(\mathrm{~m}, 4 \mathrm{H}), 1.49-1.36(\mathrm{~m}, 2 \mathrm{H}), 1.35-1.24(\mathrm{~m}, 1 \mathrm{H})$;

${ }^{13} \mathrm{C}$ NMR $\left(100 \mathrm{MHz}, \mathrm{CDCl}_{3}\right) \delta 158.8,131.6,129.3$ 114.1, 73.2, 55.2, 53.2, 46.9, 44.8, 34.1, 32.3, 23.0;

IR $\left(\mathrm{NaCl}\right.$, thin film) 3446, 2953, 1612, 1514, 1444, 1299, 1248, 1180, 1035, $829 \mathrm{~cm}^{-1}$;

HRMS $\left(\mathrm{Cl}^{+}\right) \mathrm{m} / \mathrm{z}$ calcd for $\mathrm{C}_{14} \mathrm{H}_{20}{ }^{35} \mathrm{Cl}_{2} \mathrm{O}_{2} \mathrm{NH}_{4}{ }^{+}\left(\mathrm{M}+\mathrm{NH}_{4}\right)^{+}: 308.1179$, found 308.1177. 

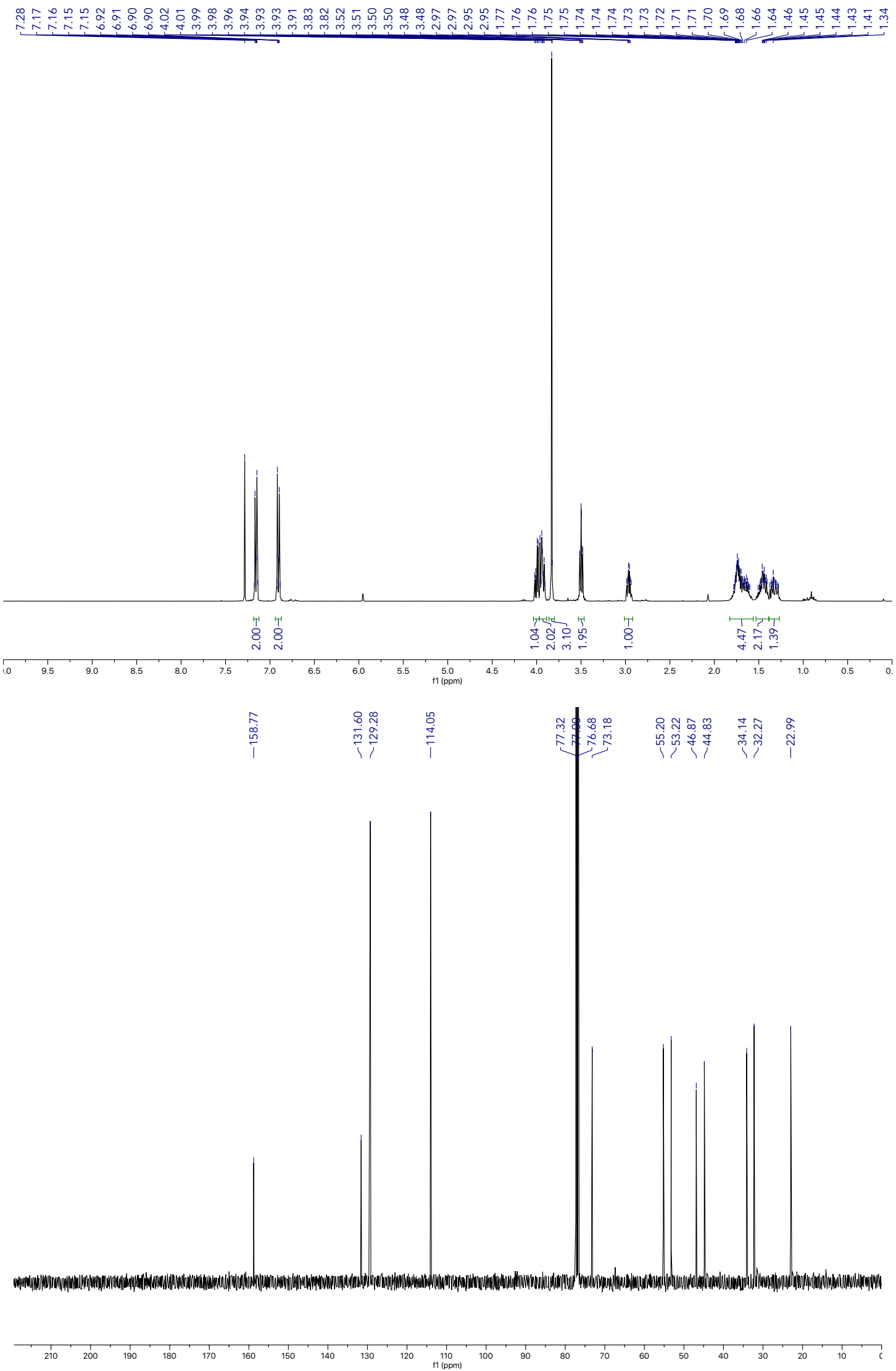
<smiles>COc1ccc([C@@H](CCl)[C@H](O)CCCCC#N)cc1</smiles>

$\left(6 R^{*}, 7 R^{*}\right)-8-C h l o r o-6-h y d r o x y-7-(4-m e t h o x y p h e n y l) o c t a n e n i t r i l e ~( \pm)-7 r$

General Procedure D: Epoxide $( \pm)-5 r(37 \mathrm{mg}, 0.15 \mathrm{mmol})$ was employed. Purification of the residue by FCC (5:1, hexane-EtOAc) afforded the title compound ( $40 \mathrm{mg}, 96 \%)$ as a yellow oil.

${ }^{1} \mathrm{H}$ NMR $\left(400 \mathrm{MHz}, \mathrm{CDCl}_{3}\right) \delta 7.13(\mathrm{~d}, J=8.7 \mathrm{~Hz}, 2 \mathrm{H}), 6.88(\mathrm{~d}, J=8.7 \mathrm{~Hz}, 2 \mathrm{H}), 3.98$ (dd, $J=10.9$, $4.4 \mathrm{~Hz}, 1 \mathrm{H}), 3.94-3.88(\mathrm{~m}, 2 \mathrm{H}), 3.80(\mathrm{~s}, 3 \mathrm{H}), 3.47(\mathrm{t}, J=6.5 \mathrm{~Hz}, 2 \mathrm{H}), 2.93$ (ddd, J = 4.4, 3.9, 3.9 $\mathrm{Hz}, 1 \mathrm{H}), 1.79-1.56(\mathrm{~m}, 4 \mathrm{H}), 1.49-1.36(\mathrm{~m}, 2 \mathrm{H}), 1.35-1.24(\mathrm{~m}, 1 \mathrm{H})$;

${ }^{13} \mathrm{C}$ NMR $\left(100 \mathrm{MHz}, \mathrm{CDCl}_{3}\right) \delta 158.8,131.6,129.3,114.1,73.2,55.2,53.2,46.9,44.8,34.1$, 32.3, 23.0;

IR ( $\mathrm{NaCl}$, thin film) 3477, 3003, 2942, 2868, 2248, 1612, 1513, 1463, 1300, 1252, 1179, 1033, $944,831 \mathrm{~cm}^{-1}$;

HRMS (ESI-TOF) $m / z$ calcd for $\mathrm{C}_{15} \mathrm{H}_{20} \mathrm{CINNaO}_{2}(\mathrm{M}+\mathrm{Na})^{+}: 304.1075$, found 304.1073. 

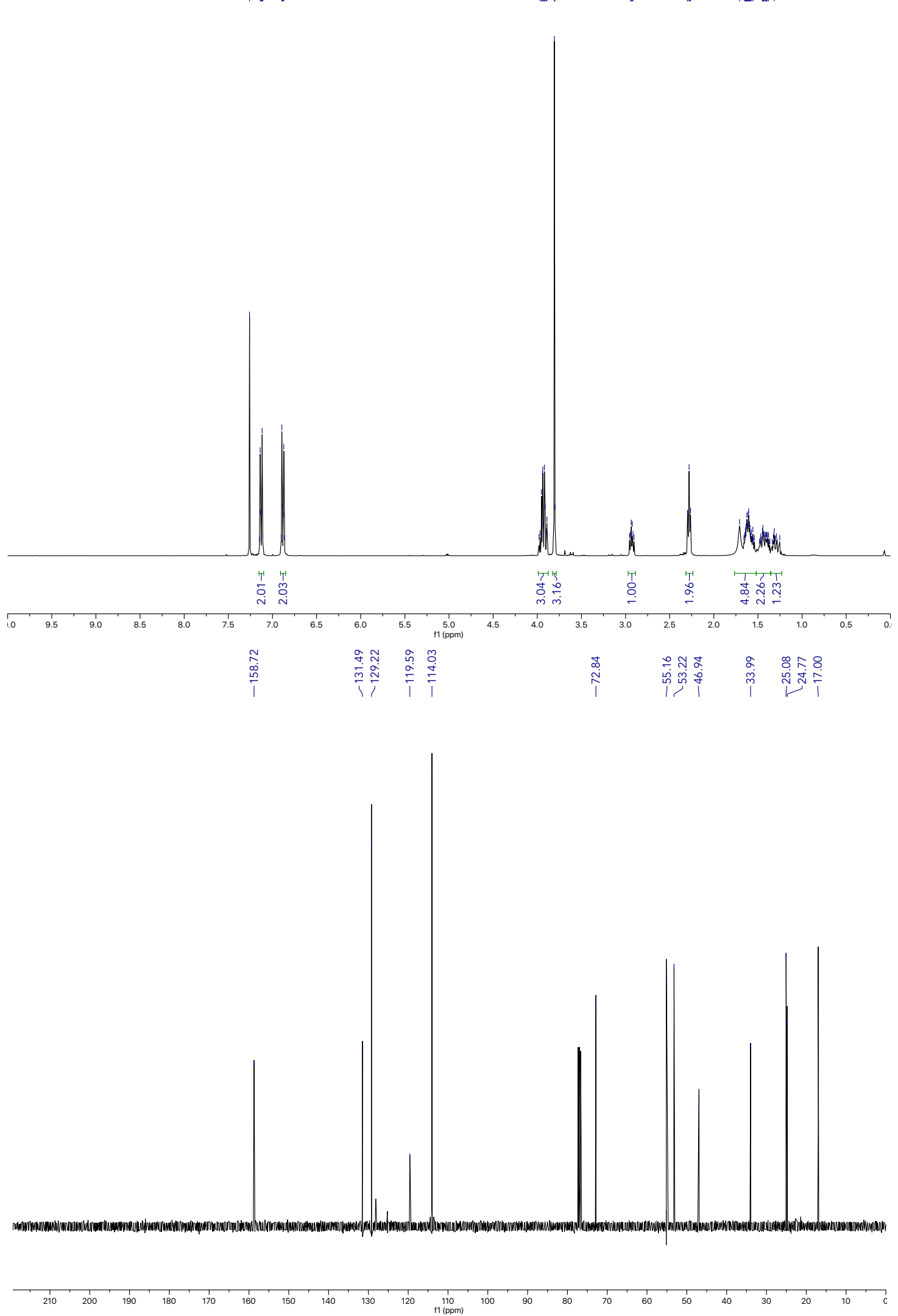
<smiles>COc1ccc([C@@H](CCl)[C@H](O)CCCCOCc2ccccc2)cc1</smiles>

$\left(2 R^{*}, 3 R^{*}\right)-7-(B e n z y l o x y)-1-c h l o r o-2-(4-m e t h o x y p h e n y l) h e p t a n-3-o l ~( \pm)-7 t$

General Procedure D: Epoxide $( \pm)-5 t(48 \mathrm{mg}, 0.15 \mathrm{mmol})$ was employed. Purification of the residue by FCC (5:1, hexane-EtOAc) afforded the title compound ( $45 \mathrm{mg}, 82 \%)$ as a yellow oil. This material was contaminated with approx. $5 \%$ of an unknown impurity.

${ }^{1} \mathrm{H}$ NMR $\left(400 \mathrm{MHz}, \mathrm{CDCl}_{3}\right) \delta$ 7.40-7.28 (m, 5H), $7.12(\mathrm{~d}, \mathrm{~J}=8.7 \mathrm{~Hz}, 2 \mathrm{H}), 6.87(\mathrm{~d}, \mathrm{~J}=8.7 \mathrm{~Hz}, 2 \mathrm{H})$, 4.47 (s, 2H), 3.99 (dd, $J=10.9,4.2 \mathrm{~Hz}, 1 \mathrm{H}), 3.93-3.83(\mathrm{~m}, 2 \mathrm{H}), 3.80(\mathrm{~s}, 3 \mathrm{H}), 3.46-3.38(\mathrm{~m}, 2 \mathrm{H})$, 2.91 (app. td, $J=8.1,4.1 \mathrm{~Hz}, 1 \mathrm{H}), 1.73-1.13(\mathrm{~m}, 6 \mathrm{H})$;

${ }^{13} \mathrm{C}$ NMR $\left(100 \mathrm{MHz}, \mathrm{CDCl}_{3}\right) \delta 158.6,138.4,131.8,130.3,129.3,128.3,127.6,127.5,113.9$, 73.2, 72.9, 70.1, 55.1, 53.4, 47.0, 34.6, 29.3, 22.3;

IR ( $\mathrm{NaCl}$, thin film) 3429, 2937, 2860, 2096, 1611, 1513, 1453, 1248, 1179, 1036, 830, $739 \mathrm{~cm}^{-1}$; HRMS (El-Orbitrap) $\mathrm{m} / \mathrm{z}$ calcd for $\mathrm{C}_{21} \mathrm{H}_{28}{ }^{35} \mathrm{ClO}_{3}{ }^{+}(\mathrm{M}+\mathrm{H})^{+} 363.1721$, found 363.1711. 

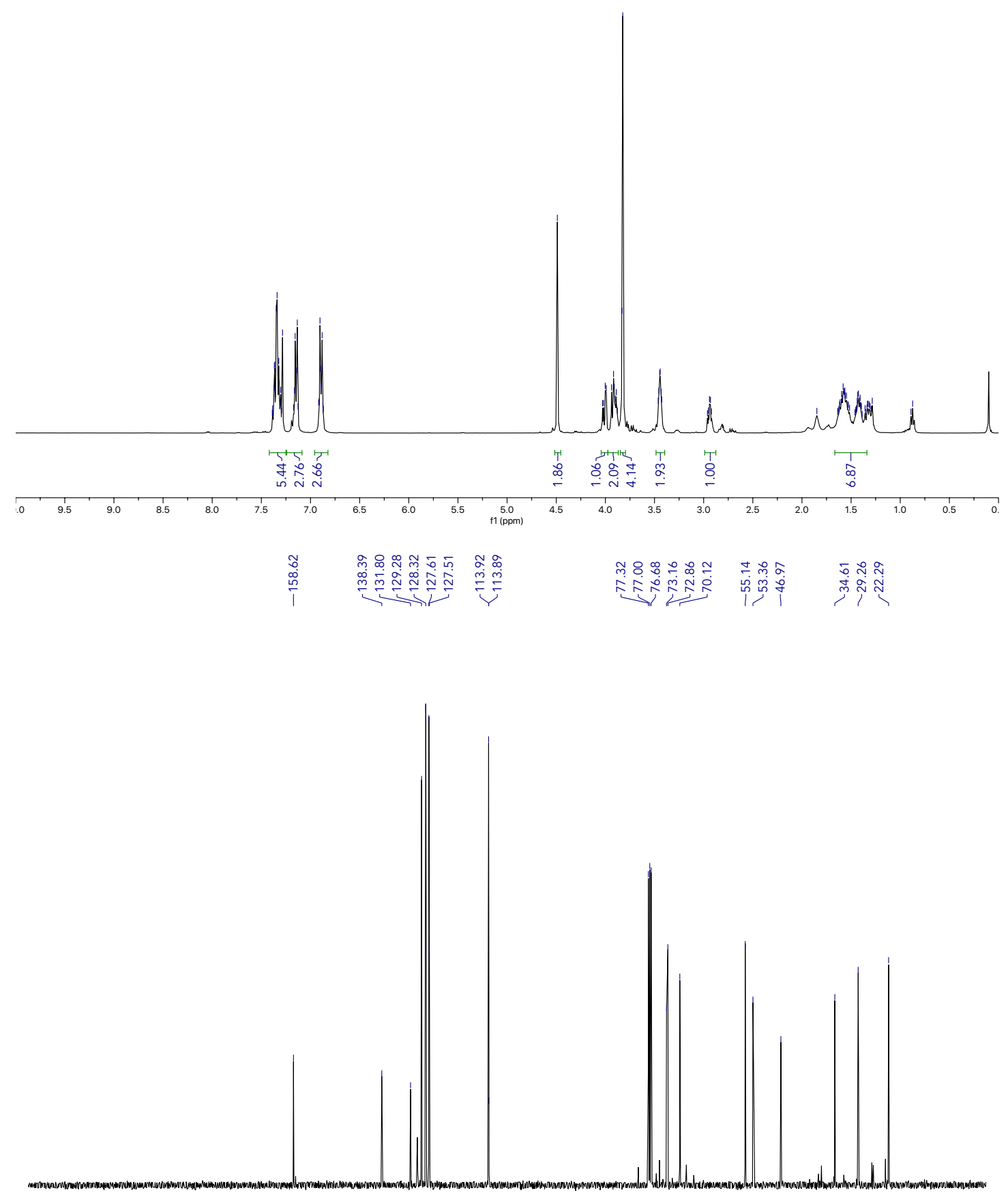
<smiles>COc1ccc([C@@H](CCl)[C@H](O)CCCCO)cc1</smiles>

$\left(4 R^{*}, 5 R^{*}\right)-6-C h l o r o-5-(4-m e t h o x y p h e n y l) h e x a n e-1,4-d i o l ~( \pm)-7 \mathrm{u}$

General Procedure D: Epoxide $( \pm)-5 \mathbf{u}(52 \mathrm{mg}, 0.15 \mathrm{mmol})$ was employed. Purification of the residue by FCC (1:1, hexane-EtOAc) afforded the title compound (32 mg, 78\%) as a colorless oil.

${ }^{1} \mathrm{H}$ NMR $\left(400 \mathrm{MHz}, \mathrm{CDCl}_{3}\right) \delta 7.13(\mathrm{~d}, J=8.7 \mathrm{~Hz}, 2 \mathrm{H}), 6.88(\mathrm{~d}, J=8.7 \mathrm{~Hz}, 2 \mathrm{H}), 3.99$ (dd, $J=10.9$, $4.2 \mathrm{~Hz}, 1 \mathrm{H}), 3.92(\mathrm{~d}, J=8.1 \mathrm{~Hz}, 1 \mathrm{H}), 3.89(\mathrm{~d}, J=8.1 \mathrm{~Hz}, 1 \mathrm{H}), 3.80(\mathrm{~s}, 3 \mathrm{H}), 3.60(\mathrm{t}, J=5.6 \mathrm{~Hz}$, 2H), 2.93 (app. td, $J=8.0,4.3 \mathrm{~Hz}, 1 \mathrm{H}), 1.58-1.21(\mathrm{~m}, 6 \mathrm{H})$.

${ }^{13} \mathrm{C}$ NMR $\left(100 \mathrm{MHz}, \mathrm{CDCl}_{3}\right) \delta 158.9,131.9,129.5,114.2,73.5,62.8,55.4,53.5,47.1,34.7,32.4$, 22.0;

IR ( $\mathrm{NaCl}$, thin film) 2912, 2848, 1748, 1652, 1507, 1457, 1249, 1009, $952 \mathrm{~cm}^{-1}$;

HRMS (El-TOF) $m / z$ calcd for $\mathrm{C}_{14} \mathrm{H}_{21}{ }^{35} \mathrm{ClO}_{3} \mathrm{Na}(\mathrm{M}+\mathrm{Na})^{+}:$295.1071, found 295.1064. 


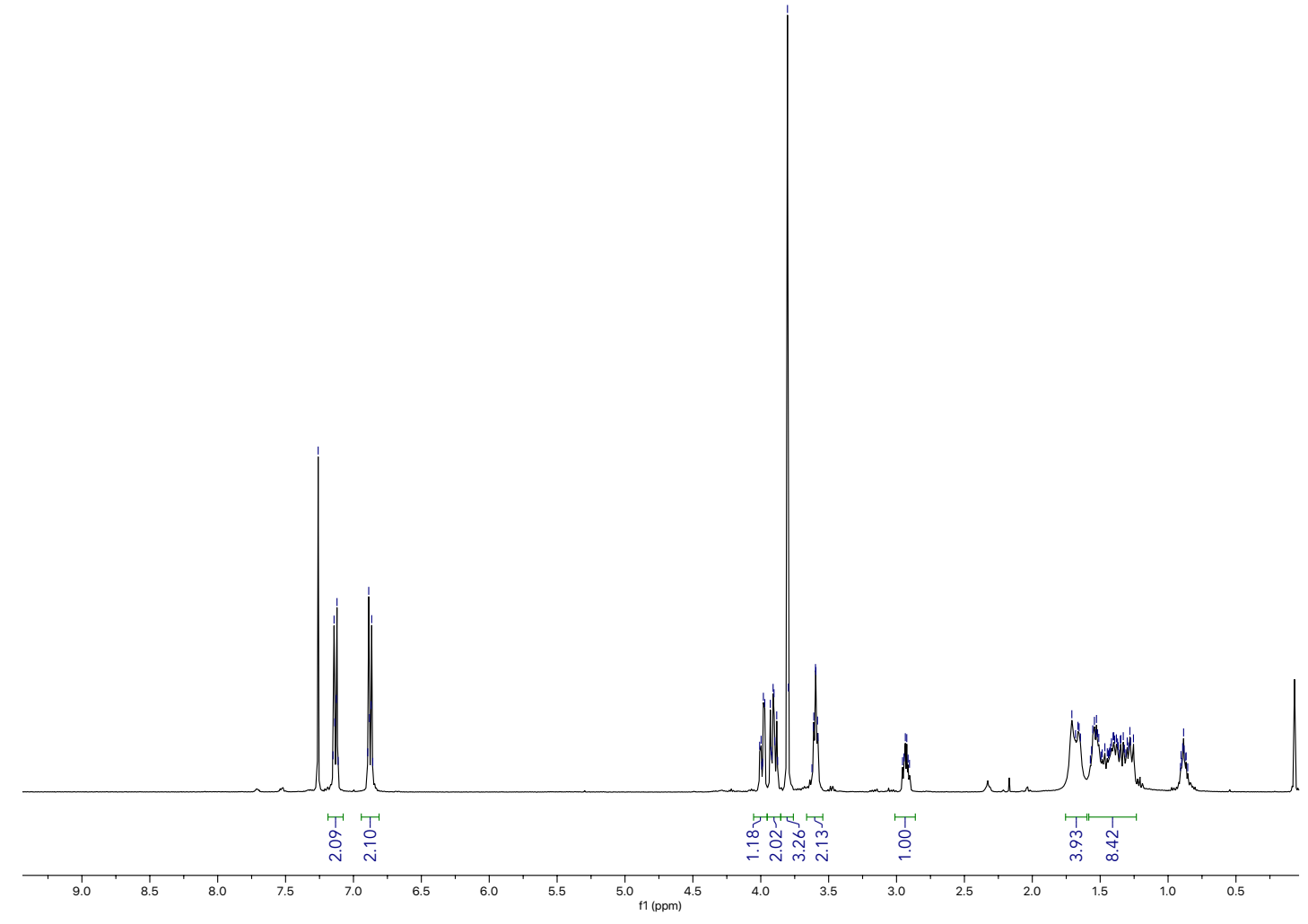

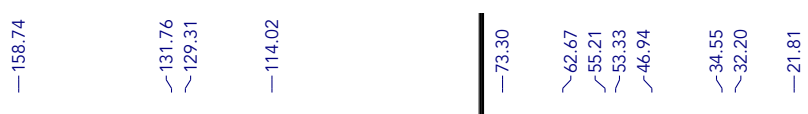

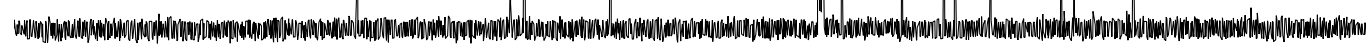

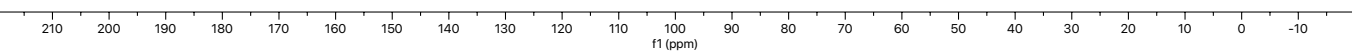


$C_{O H}^{C l}$

$\left(2 R^{*}, 3 R^{*}\right)-1-C h l o r o-2-(4-m e t h o x y p h e n y l)-7-p h e n o x y h e p t a n-3-o l ~( \pm)-7 v$

General Procedure D: Epoxide $( \pm)-5 v(47 \mathrm{mg}, 0.15 \mathrm{mmol})$ was employed. Purification of the residue by FCC (5:1, hexane-EtOAc) afforded the title compound (38 $\mathrm{mg}, 72 \%)$ as a yellow oil.

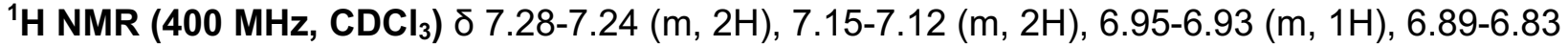
$(\mathrm{m}, 4 \mathrm{H}), 4.00$ (dd, $J=10.9,4.3 \mathrm{~Hz}, 1 \mathrm{H}), 3.95-3.87(\mathrm{~m}, 4 \mathrm{H}), 3.80(\mathrm{~s}, 3 \mathrm{H}), 2.95$ (app. td, $J=8.0 \mathrm{~Hz}$, $4.3 \mathrm{~Hz}, 1 \mathrm{H}), 1.77-1.59(\mathrm{~m}, 4 \mathrm{H}), 1.52-1.42(\mathrm{~m}, 2 \mathrm{H}), 1.40-1.33(\mathrm{~m}, 1 \mathrm{H})$;

${ }^{13} \mathrm{C}$ NMR (125 MHz, $\left.\mathrm{CDCl}_{3}\right) \delta$ 158.9, 158.8, 131.7, 129.4, 129.3, 120.6, 114.5, 114.1, 73.3, 67.6, 55.2, 53.3, 47.0, 34.6, 29.0, 22.3;

IR ( $\mathrm{NaCl}$, thin film) 3446, 2942, 2868, 1600, 1585, 1514, 1497, 1300, 1247, 1179, 1034, 830, 756 $\mathrm{cm}^{-1}$;

HRMS (ESI-TOF) $\mathrm{m} / \mathrm{z}$ calcd for $\mathrm{C}_{20} \mathrm{H}_{25}{ }^{35} \mathrm{ClO}_{3} \mathrm{Na}(\mathrm{M}+\mathrm{Na})^{+}: 371.1390$, found 371.1384 . 


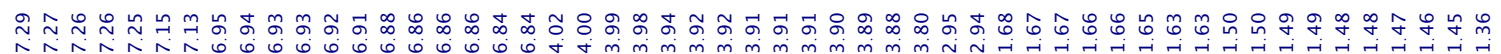
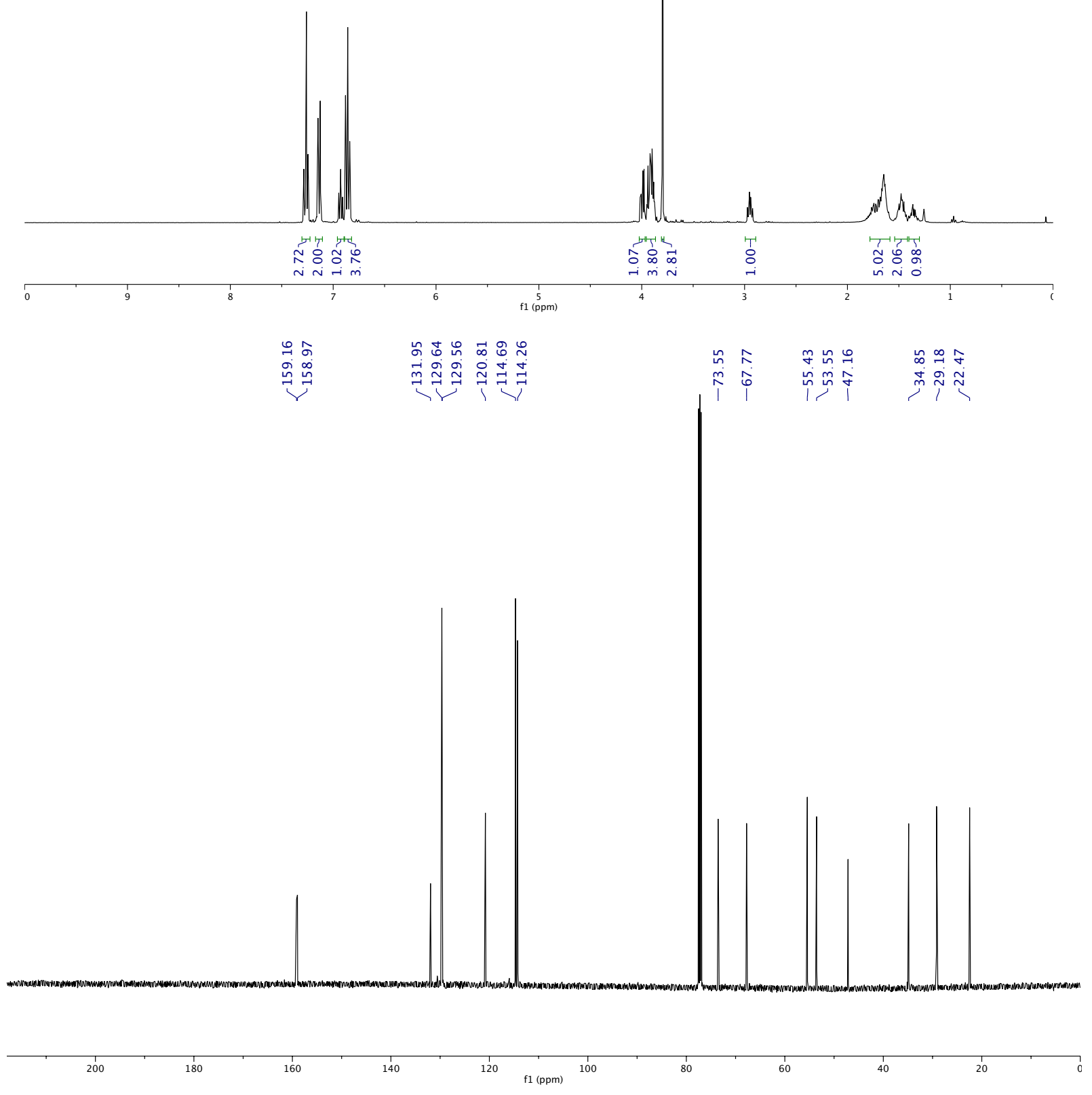

S230 
<smiles>COc1ccc([C@@H](CCl)[C@H](O)CCCCNc2ccccc2)cc1</smiles>

2-((5R*,6R*)-7-Chloro-5-hydroxy-6-(4-methoxyphenyl)heptyl)isoindoline-1,3-dione ( \pm )-7w

General Procedure D: Epoxide ( \pm )-5w (45 mg, $0.12 \mathrm{mmol})$ was employed. Purification of the residue by FCC (5:1, hexane-EtOAc) afforded the title compound ( $45 \mathrm{mg}, 93 \%)$ as a yellow oil.

${ }^{1} \mathrm{H}$ NMR $\left(400 \mathrm{MHz}, \mathrm{CDCl}_{3}\right) \delta$ 7.86-7.80 (m, 2H), 7.73-7.69 (m, 2H), 7.12 (d, J = 8.7 Hz, 2H), 6.86 (d, $J=8.7 \mathrm{~Hz}, 2 \mathrm{H}), 3.99(\mathrm{dd}, J=10.9,4.2 \mathrm{~Hz}, 1 \mathrm{H}), 3.92-3.85(\mathrm{~m}, 2 \mathrm{H}), 3.79(\mathrm{~s}, 3 \mathrm{H}), 3.63(\mathrm{t}, J=$ $6.9 \mathrm{~Hz}, 2 \mathrm{H}$ ), 2.91 (app. td, $J=8.2,4.1 \mathrm{~Hz}, 1 \mathrm{H}), 1.77-1.22(\mathrm{~m}, 6 \mathrm{H})$;

${ }^{13} \mathrm{C}$ NMR $\left(100 \mathrm{MHz}, \mathrm{CDCl}_{3}\right) \delta 168.5,158.7,133.9,132.1,131.7,129.3,123.2,114.0,73.1,55.2$, 53.4, 47.0, 37.6, 34.3, 28.3, 22.7;

IR $\left(\mathrm{NaCl}\right.$, thin film) 3469, 2942, 1770, 1710, 1612, 1514, 1398, 1248, $1034 \mathrm{~cm}^{-1}$;

HRMS (El-TOF) $m / z$ calcd for $\mathrm{C}_{22} \mathrm{H}_{24}{ }^{35} \mathrm{CINO}_{4} \mathrm{Na}[\mathrm{M}+\mathrm{Na}]^{+} 424.1292$, found 424.1262. 


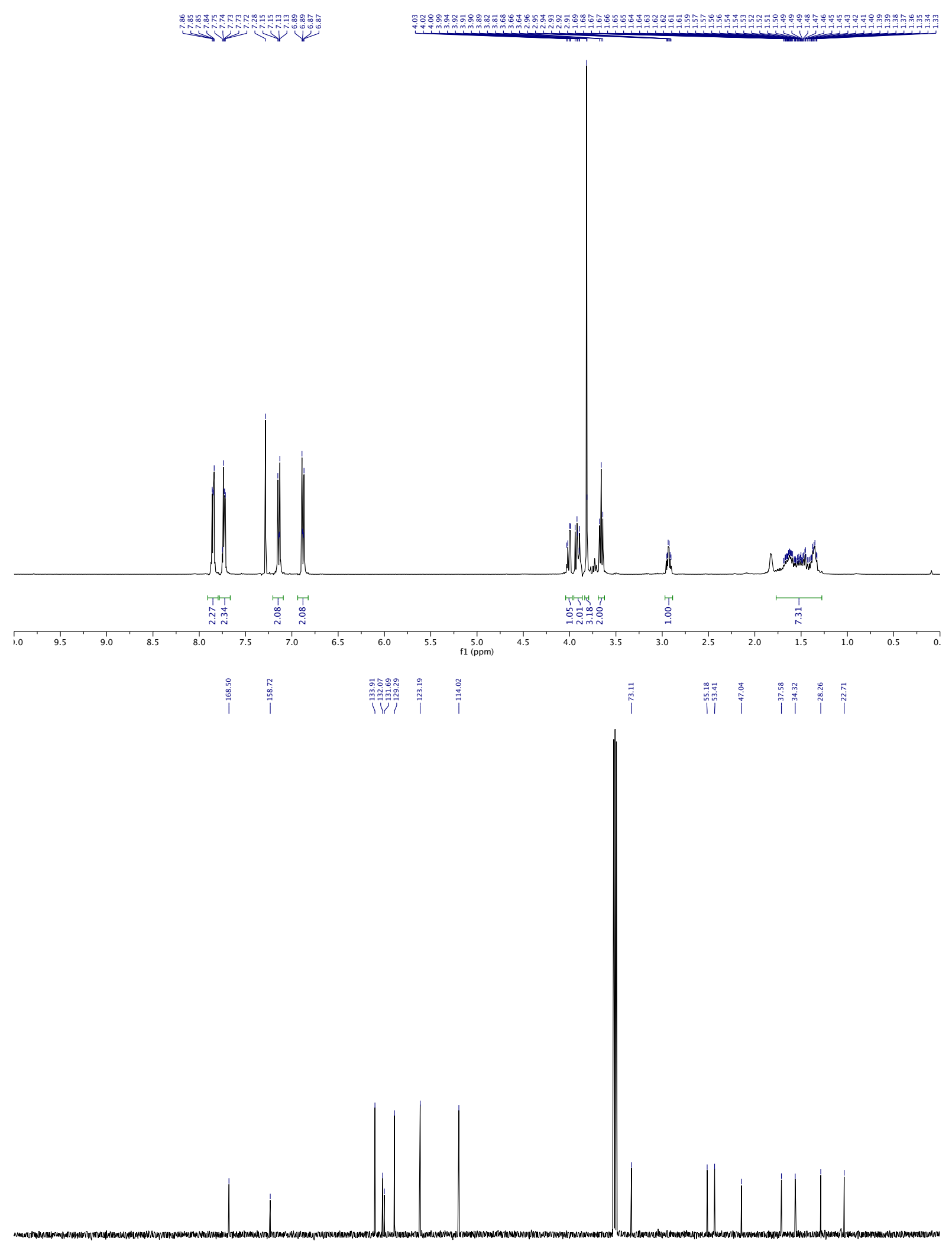


<smiles>COc1ccc([C@H](CCl)[C@H](O)CCCCS(=O)(=O)c2ccccc2)cc1</smiles>

$\left(2 R^{*}, 3 R^{*}\right)-1-C h l o r o-2-(4-m e t h o x y p h e n y l)-7-(p h e n y l s u l f o n y l) h e p t a n-3-o l ~( \pm)-7 x$

General Procedure D: Epoxide $( \pm)-5 x(64 \mathrm{mg}, 0.17 \mathrm{mmol})$ was employed. Purification of the residue by FCC (5:1, hexane-EtOAc) afforded the title compound (62 $\mathrm{mg}, 91 \%)$ as a yellow oil.

${ }^{1} \mathrm{H}$ NMR $\left(400 \mathrm{MHz}, \mathrm{CDCl}_{3}\right)$ ठ 7.96-7.77 (m, 2H), 7.67-7.63 (m, 1H), 7.58-7.54 (m, 2H), $7.09(\mathrm{~d}, \mathrm{~J}$ $=8.7 \mathrm{~Hz}, 2 \mathrm{H}), 6.87(\mathrm{~d}, J=8.7 \mathrm{~Hz}, 2 \mathrm{H}), 3.93(\mathrm{dd}, J=10.9,4.5 \mathrm{~Hz}, 1 \mathrm{H}), 3.91-3.84(\mathrm{~m}, 2 \mathrm{H}), 3.80$ (s, 3H), 3.01 (t, $J=7.8 \mathrm{~Hz}, 2 \mathrm{H}$ ), 2.88 (app. td, $J=7.8,4.5 \mathrm{~Hz}, 1 \mathrm{H}), 1.80(\mathrm{~d}, J=5.6 \mathrm{~Hz}, 1 \mathrm{H}), 1.76-$ $1.16(\mathrm{~m}, 6 \mathrm{H})$;

${ }^{13} \mathrm{C} \mathrm{NMR}\left(100 \mathrm{MHz}, \mathrm{CDCl}_{3}\right) \delta 158.8,139.1,133.7,131.5,129.3,129.2,128.0,114.1,72.8,56.0$, $55.2,53.2,46.9,34.2,24.4,22.4$;

IR ( $\mathrm{NaCl}$, thin film) 3505, 2946, 2867, 1611, 1514, 1248, 1148, 1086, $1032 \mathrm{~cm}^{-1}$;

HRMS (El-Orbitrap) $\mathrm{m} / \mathrm{z}$ calcd for $\mathrm{C}_{20} \mathrm{H}_{26}{ }^{35} \mathrm{ClO}_{4} \mathrm{~S}^{+}(\mathrm{M}+\mathrm{H})^{+} 397.1235$, found 397.1222. 

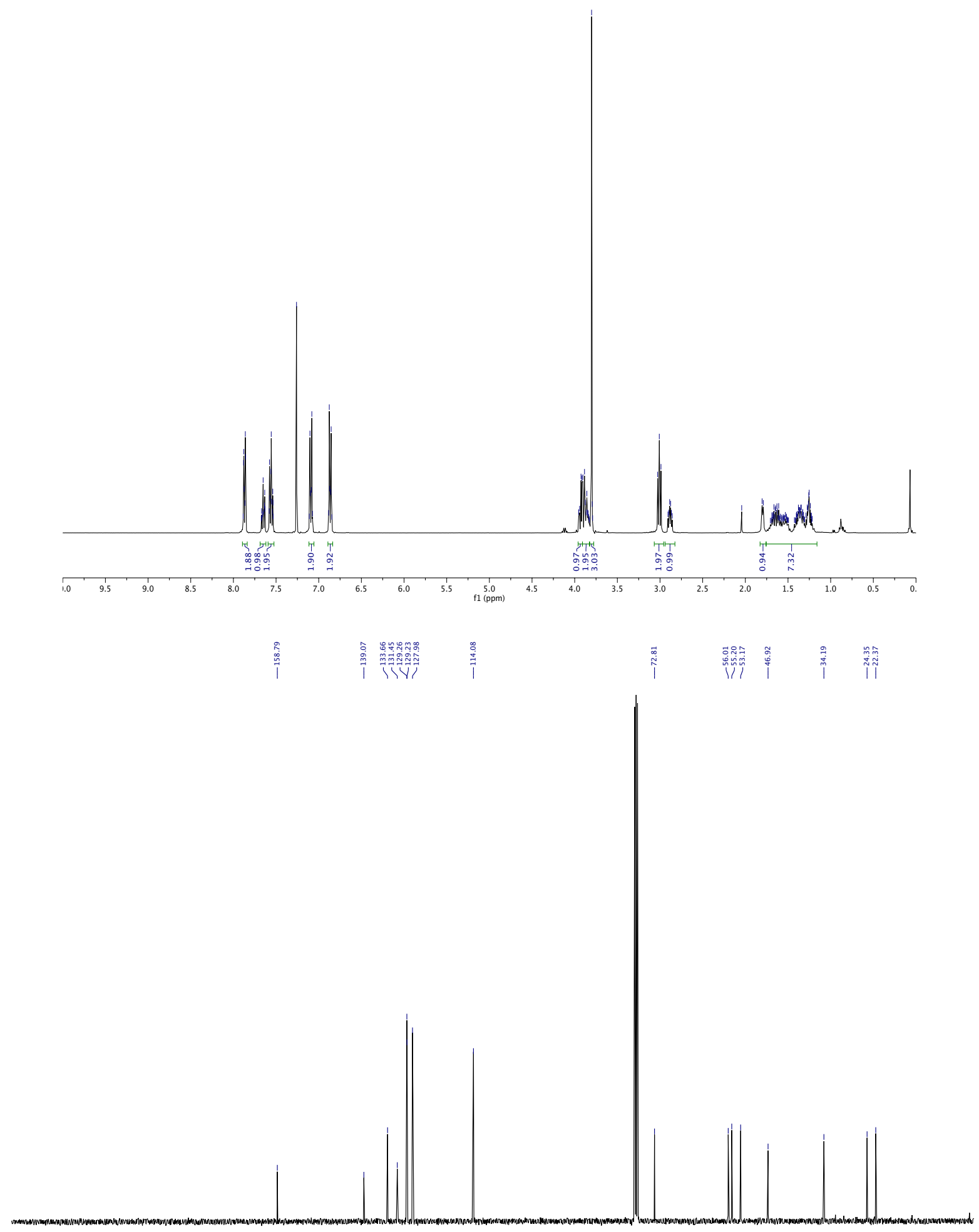
<smiles>COc1ccc([C@H](CCl)[C@H](O)C(C)=O)cc1</smiles>

\section{$\left(3 S^{*}, 4 R^{*}\right)-5$-Chloro-3-hydroxy-4-(4-methoxyphenyl)pentan-2-one ( \pm )-7y}

General Procedure D: Epoxide $( \pm)-5 y(31 \mathrm{mg}, 0.15 \mathrm{mmol})$ was employed. Purification of the residue by FCC (4:1, hexane-EtOAc) afforded the title compound (34 mg, 94\%) as a colorless oil.

${ }^{1} \mathrm{H}$ NMR $\left(400 \mathrm{MHz}, \mathrm{CDCl}_{3}\right) \delta 7.31(\mathrm{~d}, J=8.7 \mathrm{~Hz}, 2 \mathrm{H}), 6.90(\mathrm{~d}, J=8.7 \mathrm{~Hz}, 2 \mathrm{H}), 4.32(\mathrm{t}, J=4.2 \mathrm{~Hz}$, $1 \mathrm{H}), 3.97(\mathrm{dd}, J=11.0,7.7 \mathrm{~Hz}, 1 \mathrm{H}), 3.81(\mathrm{~s}, 3 \mathrm{H}), 3.78(\mathrm{~d}, J=4.6 \mathrm{~Hz}, 1 \mathrm{H}), 3.69(\mathrm{dd}, J=11.0,6.5$ $\mathrm{Hz}, 1 \mathrm{H}$ ), 3.39 (ddd, $J=7.8,6.5,3.9 \mathrm{~Hz}, 1 \mathrm{H}), 2.18(\mathrm{~s}, 3 \mathrm{H})$;

${ }^{13} \mathrm{C}$ NMR $\left(100 \mathrm{MHz}, \mathrm{CDCl}_{3}\right) \delta 208.6,159.2,131.6,129.2,114.3,79.2,55.3,50.5,44.4,26.3$;

IR ( $\mathrm{NaCl}$, thin film) 3449, 2961, 2837, 1712, 1611, 1514, 1251, 1181, 1032, $835 \mathrm{~cm}^{-1}$;

HRMS (El-Orbitrap) $\mathrm{m} / \mathrm{z}$ calcd for $\mathrm{C}_{12} \mathrm{H}_{16}{ }^{35} \mathrm{ClO}_{3}{ }^{+}(\mathrm{M}+\mathrm{H})^{+} 243.0782$, found 243.0772 

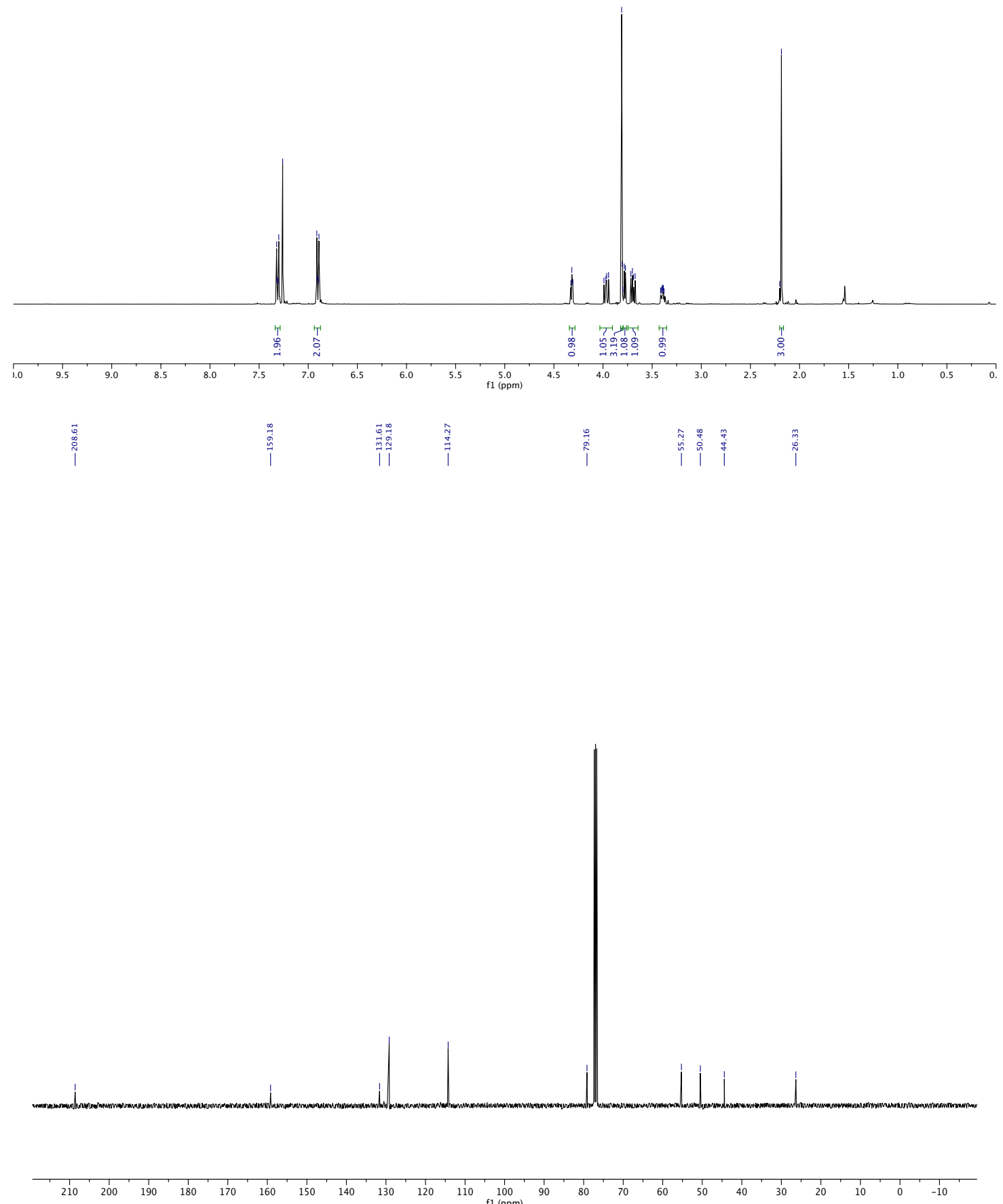
<smiles>COc1ccc([C@@H](CCl)[C@H](O)C2CCOCC2)cc1</smiles>

$\left(1 R^{*}, 2 R^{*}\right)-3-c h l o r o-2-(4-m e t h o x y p h e n y l)-1$-(tetrahydro-2H-pyran-4-yl)propan-1-ol ( \pm )-7z

General Procedure D: Epoxide $( \pm)-5 z(74 \mathrm{mg}, 0.30 \mathrm{mmol})$ was employed. Purification of the residue by FCC (5:1, hexane-EtOAc) afforded the title compound (66 mg, 77\%) as a colorless solid.

${ }^{1} \mathrm{H}$ NMR $\left(400 \mathrm{MHz}, \mathrm{CDCl}_{3}\right) \delta 7.15(\mathrm{~d}, J, 8.6 \mathrm{~Hz}, 2 \mathrm{H}), 6.89(\mathrm{~d}, J=8.6 \mathrm{~Hz}, 2 \mathrm{H}), 3.99-3.91(\mathrm{~m}, 4 \mathrm{H})$, 3.81 (s, 3H), 3.72, (dd, $J=8.2,3.2 \mathrm{~Hz}, 1 \mathrm{H}$ ), 3.29 (app. td, $J=11.8,2.3 \mathrm{~Hz}, 1 \mathrm{H}$ ), 3.20 (app. td, $J$ = 11.6, $2.4 \mathrm{~Hz}, 1 \mathrm{H}$ ), 3.07 (app. $\mathrm{td}, J=8.0,3.9 \mathrm{~Hz}, 1 \mathrm{H}), 1.66-1.45(\mathrm{~m}, 6 \mathrm{H})$;

${ }^{13} \mathrm{C}$ NMR $\left(125 \mathrm{MHz} \mathrm{CDCl}_{3}\right) \delta$ 158.8, 131.7, 129.2, 114.2, 67.7, 55.2, 49.4, 47.1, 37.4, 29.9, 26.1;

IR ( $\mathrm{NaCl}$, thin film) 3420, 2952, 2840, 1611, 1514, 1443, 1248, 1180, 1100, 1036, $830 \mathrm{~cm}^{-1}$;

HRMS $\left(\mathrm{Cl}^{+}\right) \mathrm{m} / \mathrm{z}$ calcd for $\mathrm{C}_{15} \mathrm{H}_{21}{ }^{35} \mathrm{ClO}_{3} \mathrm{NH}_{4}{ }^{+}\left(\mathrm{M}+\mathrm{NH}_{4}\right)^{+}: 302.1517$, found 302.1517.

M pt $123-125^{\circ} \mathrm{C}$ 


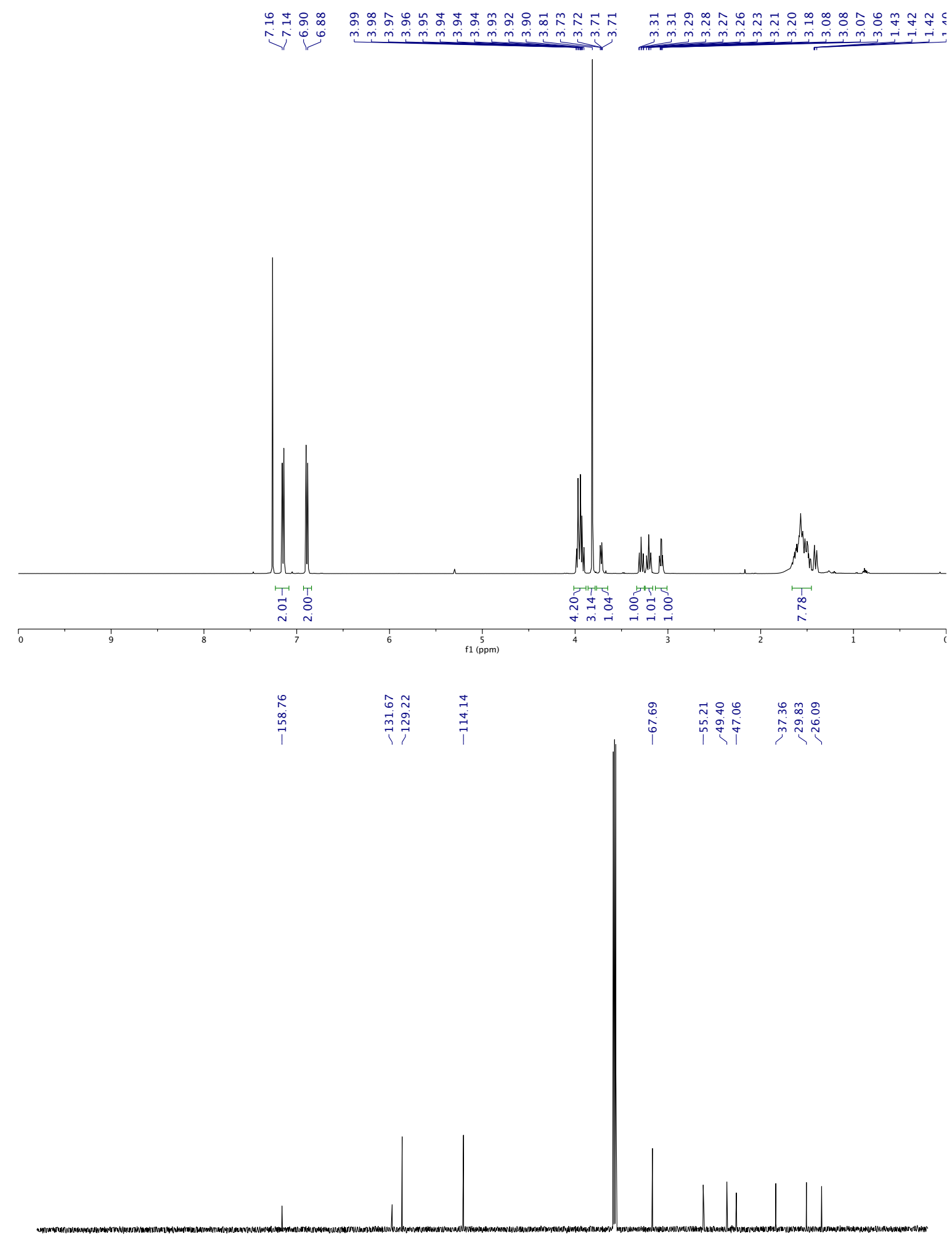

${ }_{200}^{1}+180$

160

140

$120 \quad \mathrm{f} 1(\mathrm{ppm}) \quad 100$

80

60

40 
<smiles>COc1ccc([C@H](CCl)[C@H](O)C2CCN(C(C)(C)C)CC2)cc1</smiles>

tert-Butyl 4-((1R*,2R*)-3-chloro-1-hydroxy-2-(4-methoxyphenyl)propyl)piperidine-1carboxylate $( \pm)-7$ aa

General Procedure D: Epoxide ( \pm )-5aa-Boc (34 mg, $0.1 \mathrm{mmol})$ was employed. Purification of the residue by FCC (5:1, hexane-EtOAc) afforded the title compound ( $25 \mathrm{mg}, 66 \%)$ as a yellow oil.

${ }^{1} \mathrm{H}$ NMR $\left(400 \mathrm{MHz}, \mathrm{CDCl}_{3}\right) \delta 7.14(\mathrm{~d}, J=8.8 \mathrm{~Hz}, 2 \mathrm{H}), 6.88(\mathrm{~d}, J=8.8 \mathrm{~Hz}, 2 \mathrm{H}), 4.10$ (br. s, 2H), 3.97 (dd, $J=10.9,3.9 \mathrm{~Hz}, 1 \mathrm{H}), 3.91(\mathrm{dd}, J=10.9,7.9 \mathrm{~Hz}, 1 \mathrm{H}), 3.81(\mathrm{~s}, 3 \mathrm{H}), 3.78-3.70(\mathrm{~m}, 1 \mathrm{H})$, 3.06 (app. td, $J=8.1,3.9 \mathrm{~Hz}, 1 \mathrm{H}$ ), 2.56-2.43 (br. m, 2H), 1.85 (br. s, 1H), 1.62 (br. m, 2H), 1.44 (s, 9H), 1.39-1.21 (m, 2H);

${ }^{13} \mathrm{C}$ NMR $\left(125 \mathrm{MHz}, \mathrm{CDCl}_{3}\right) \delta 158.8,154.7,131.6,129.2,114.2,79.4,76.5,55.2,49.7,47.3,43.7$ (broad), 38.3, 28.4; Missing $1 \times \mathrm{C}($ alkyl)

IR ( $\mathrm{NaCl}$, thin film) 3005, 2918, 2878, 2361, 1791, 1512, 1251,939, $746 \mathrm{~cm}^{-1}$;

HRMS (El-Orbitrap) $\mathrm{m} / \mathrm{z}$ calcd for $\mathrm{C}_{20} \mathrm{H}_{31}{ }^{35} \mathrm{CINO}_{4}{ }^{+}(\mathrm{M}+\mathrm{H})^{+} 384.1936$, found 384.1927. 

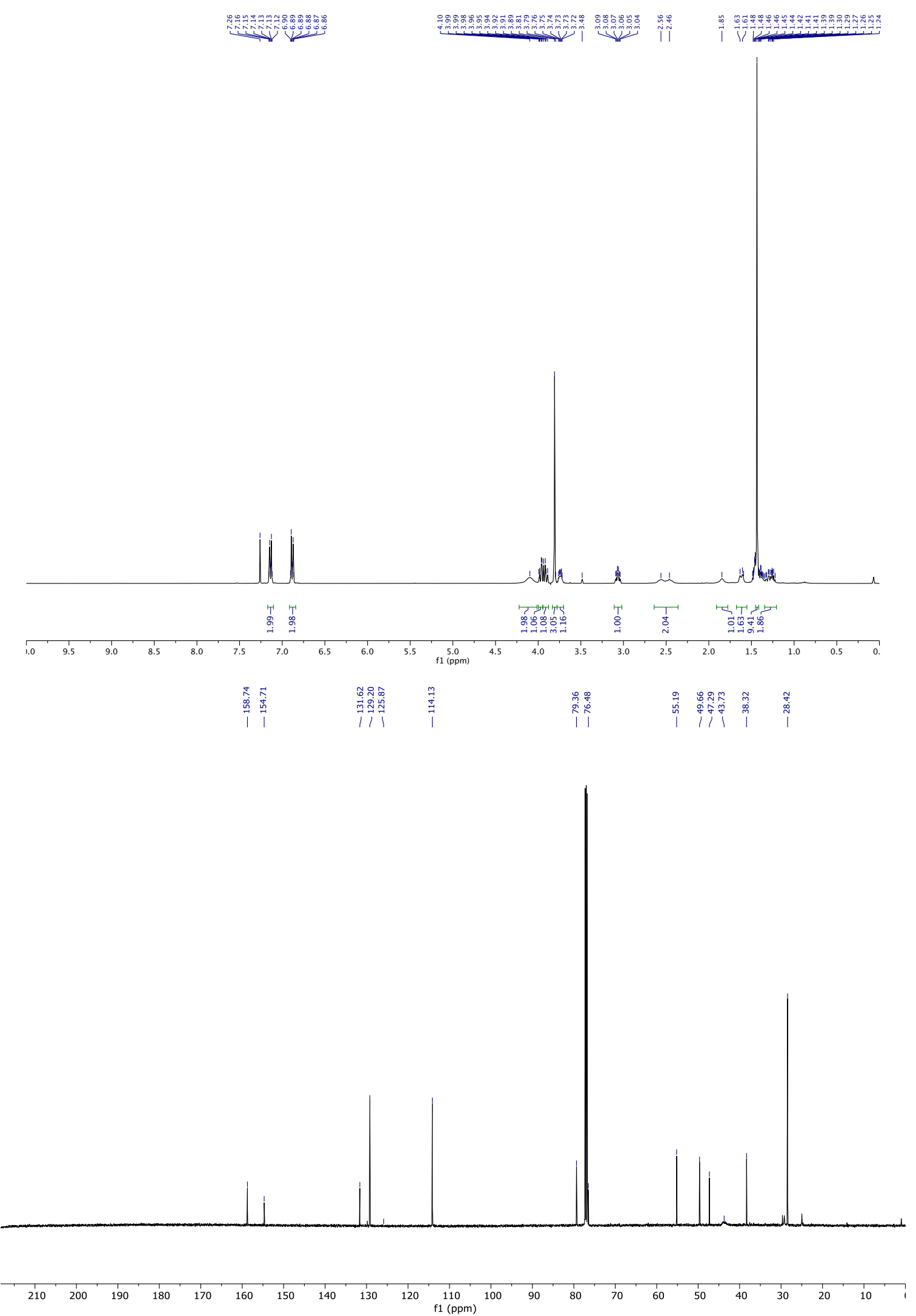
<smiles>COc1ccc([C@H](CCl)[C@H](O)C2CN(C(C)(C)C)C2)cc1</smiles>

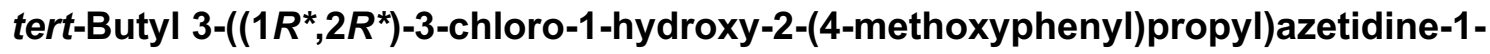
carboxylate $( \pm)-7 a b$

General Procedure D: Epoxide ( \pm )-5ab-Boc (48 mg, $0.15 \mathrm{mmol})$ was employed. Purification of the residue by FCC (5:1, hexane-EtOAc) afforded the title compound (32 $\mathrm{mg}, 60 \%)$ as a yellow oil.

${ }^{1} \mathrm{H}$ NMR $\left(400 \mathrm{MHz}, \mathrm{CDCl}_{3}\right) \delta 7.09(\mathrm{~d}, \mathrm{~J}=8.7 \mathrm{~Hz}, 2 \mathrm{H}), 6.86(\mathrm{~d}, \mathrm{~J}=8.7 \mathrm{~Hz}, 2 \mathrm{H}), 4.02-3.95(\mathrm{~m}, 2 \mathrm{H})$, 3.94-3.83 (m, 2H), 3.82-3.77 (m, 4H), $3.63(\mathrm{t}, J=8.6 \mathrm{~Hz}, 1 \mathrm{H}), 3.53(\mathrm{dd}, J=8.8,6.1 \mathrm{~Hz}, 1 \mathrm{H}), 2.85$ (td, $J=8.1,4.3 \mathrm{~Hz}, 1 \mathrm{H}), 2.56-2.48(\mathrm{~m}, 2 \mathrm{H}), 1.40(\mathrm{~s}, 9 \mathrm{H})$.

${ }^{13} \mathrm{C} \mathrm{NMR}\left(100 \mathrm{MHz}, \mathrm{CDCl}_{3}\right) \delta 159.0,156.3,130.9,129.1,114.2,79.4,73.7,55.2,51.8,46.6,32.5$, $28.4 ; 1 \times$ alkyl carbon too broad to peak pick due to the rotameric nature of Boc group.

IR ( $\mathrm{NaCl}$, thin film) 3401, 2975, 2756, 1673, 1514, 1427, 1250, $1146 \mathrm{~cm}^{-1}$;

HRMS (El-Orbitrap) $\mathrm{m} / \mathrm{z}$ calcd for $\mathrm{C}_{18} \mathrm{H}_{27}{ }^{35} \mathrm{CINO}_{4}{ }^{+}(\mathrm{M}+\mathrm{H})^{+} 356.1623$, found 356.1614. 


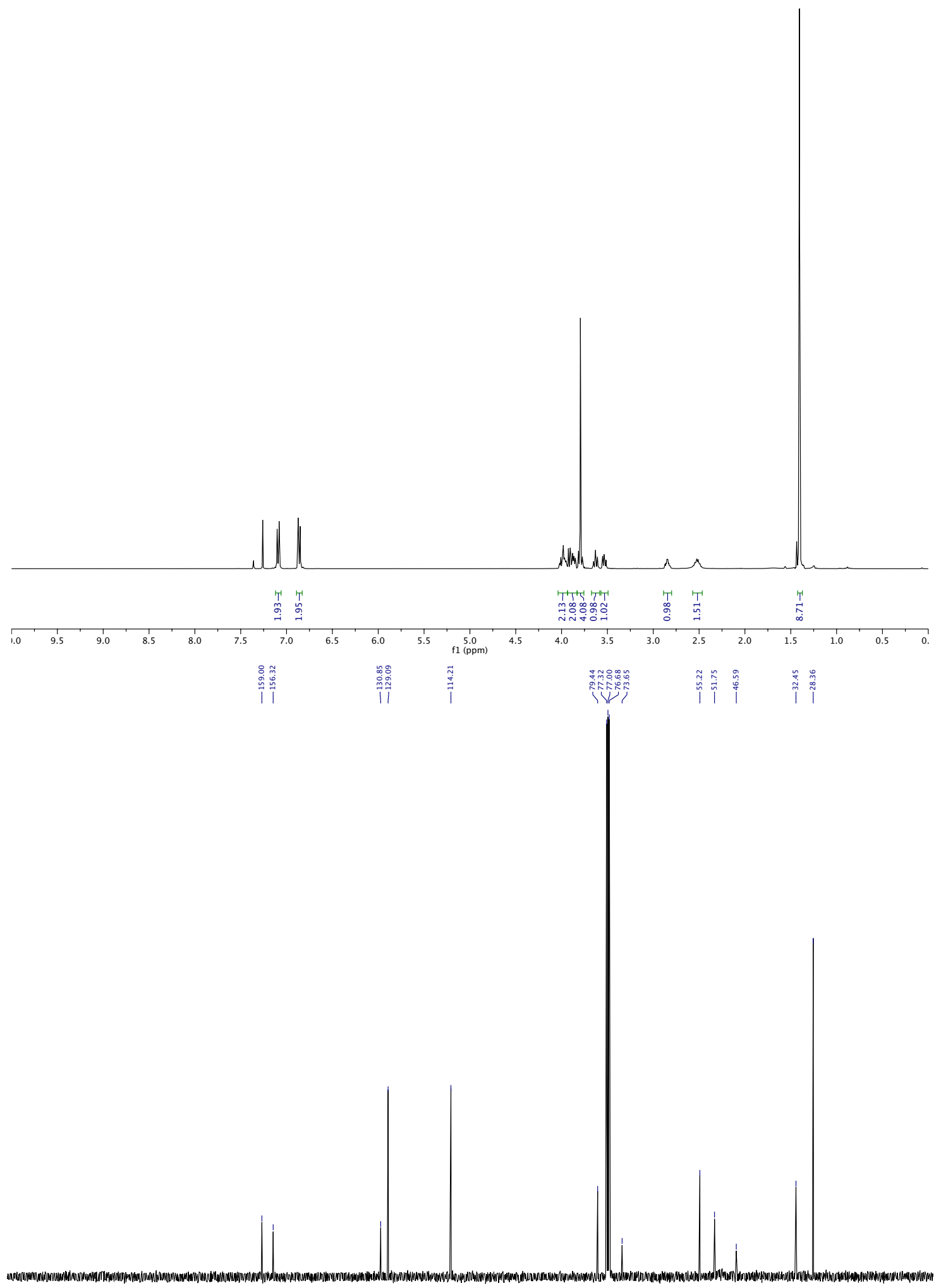

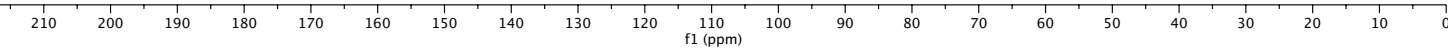




\section{Determining the enantiospecificity of $\mathrm{SnCl}_{4}$ and $\mathrm{TiCl}_{4}$ reactions}

Synthesis of enantioenriched epoxide 5a was carried out via Shi epoxidation following two reported procedures. ${ }^{11}$ Procedure A afforded epoxide $\mathbf{5 a}$ in 93:7 er and procedure B afforded epoxide $\mathbf{5 a}$ in 95:5 er. Both of these epoxides were used for the experiments outlined in Figures 4 and 5 in the main text. The enantiomeric ratio of the starting epoxide is indicated in each procedure below, where appropriate, to enable determination of enantiospecificity of the reaction.

\section{Procedure A}

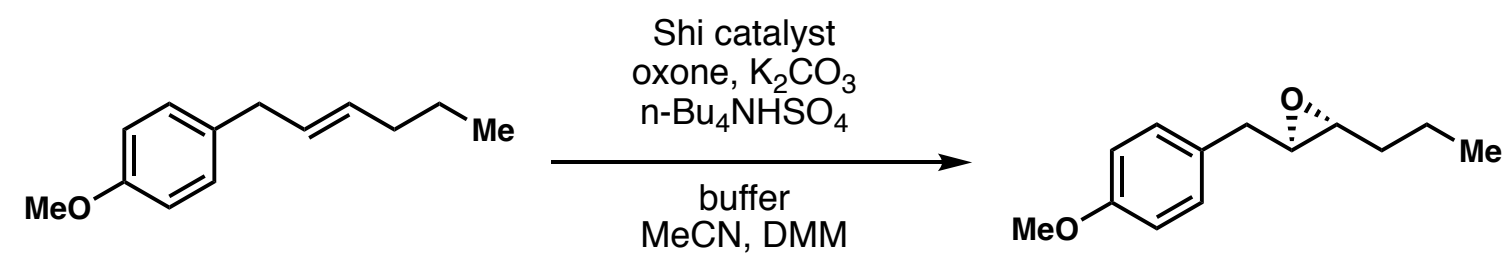

(E)-1-(Hex-2-en-1-yl)-4-methoxybenzene (190 mg, $1.0 \mathrm{mmol}$ ) was dissolved in acetonitrile $(5 \mathrm{~mL})$ and dimethoxymethane $(10 \mathrm{~mL})$. Subsequently were added buffer solution $(10 \mathrm{~mL}, 0.05 \mathrm{M}$ solution of $\mathrm{Na}_{2} \mathrm{~B}_{4} \mathrm{O}_{7} .10 \mathrm{H}_{2} \mathrm{O}$ in $4 \times 10^{-4} \mathrm{M}$ aqueous $\mathrm{Na}_{2}$ (EDTA)), tetrabutylammonium hydrogen sulfate (13.6 $\mathrm{mg}, 0.04 \mathrm{mmol})$, and Shi catalyst $(77.4 \mathrm{mg}, 0.3 \mathrm{mmol})$. A solution of Oxone ${ }^{\circledR}(0.85 \mathrm{~g}, 1.38 \mathrm{mmol})$ in aqueous $\mathrm{Na}_{2}\left(\right.$ EDTA) $\left(4 \times 10^{-4} \mathrm{M}, 6.5 \mathrm{~mL}\right)$ and a solution of $\mathrm{K}_{2} \mathrm{CO}_{3}(800 \mathrm{mg}, 5.8 \mathrm{mmol})$ in water $(6.5 \mathrm{~mL})$ were added simultaneously, but separately, via syringe pump over a period of 30 minutes at room temperature. After addition was complete, the reaction was quenched by addition of $\mathrm{H}_{2} \mathrm{O}$ $(30 \mathrm{~mL})$ and extracted with hexanes $(4 \times 40 \mathrm{~mL})$. The combined organic layers were washed with brine $(30 \mathrm{~mL})$, dried $\left(\mathrm{Na}_{2} \mathrm{SO}_{4}\right)$, filtered, and concentrated in vacuo. Purification of the residue by FCC (20:1, hexane-EtOAc) afforded $(R, R)-5 \mathrm{a}(160 \mathrm{mg}, 78 \%$ yield, $93: 7 \mathrm{er})$ as a colorless oil. The enantiomeric purity of this compound was determined by chiral HPLC (Chiralpak IA, isocratic hexanes- $-\mathrm{PrOH}$ 99:1, $0.5 \mathrm{~mL} / \mathrm{min}, 23{ }^{\circ} \mathrm{C}$ ); $\mathrm{t}_{\mathrm{R}}$ (major) $=8.0 \mathrm{~min}$ and $\mathrm{t}_{\mathrm{R}}(\mathrm{minor})=8.4 \mathrm{~min}$. The absolute stereochemistry was assigned by analogy to related compounds in the literature. ${ }^{11}$

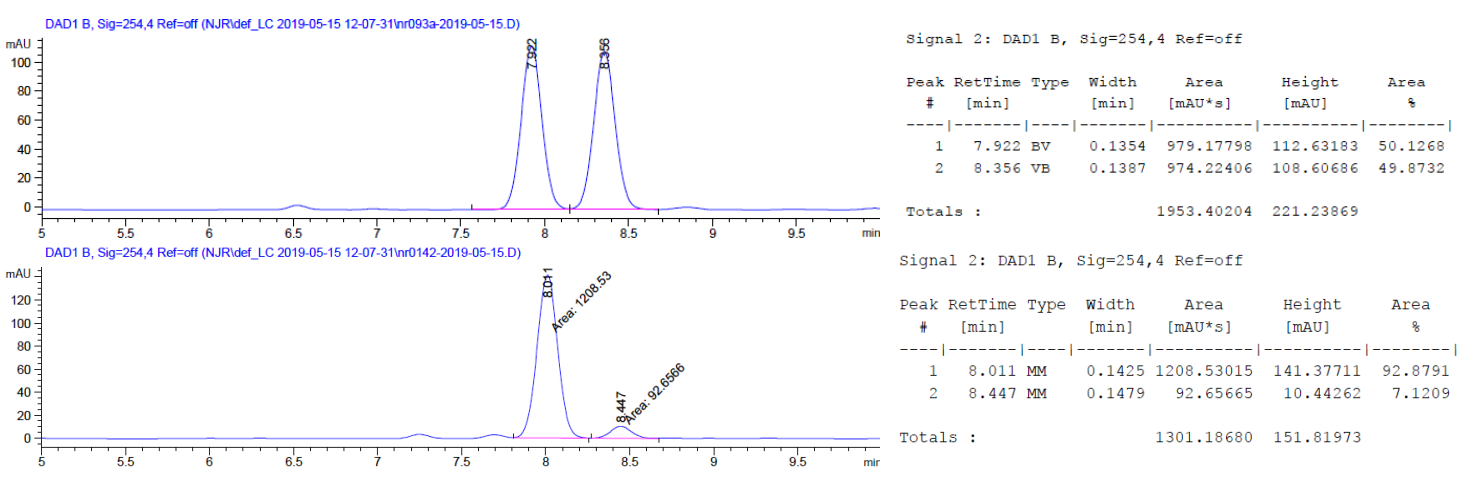




\section{Procedure B}

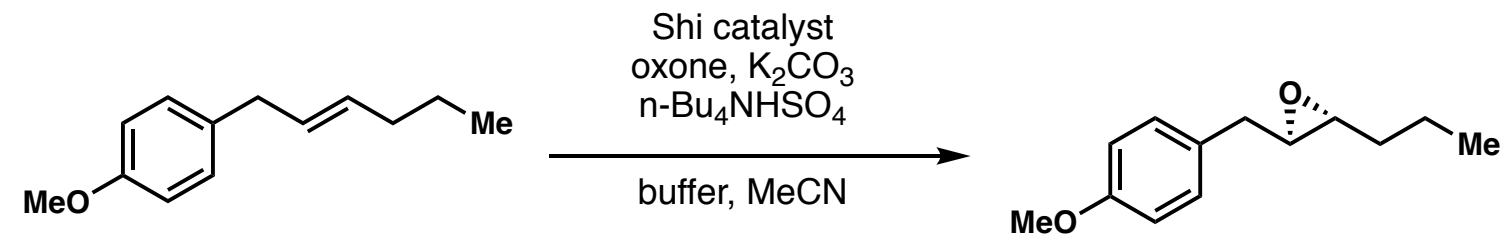

(E)-1-(Hex-2-en-1-yl)-4-methoxybenzene (475 mg, $2.50 \mathrm{mmol}$ ) was dissolved in acetonitrile (38 $\mathrm{mL}$ ). Subsequently were added buffer solution $\left(25 \mathrm{~mL}, 0.05 \mathrm{M}\right.$ solution of $\mathrm{Na}_{2} \mathrm{~B}_{4} \mathrm{O}_{7} .10 \mathrm{H}_{2} \mathrm{O}$ in $4 \times$ $10^{-4} \mathrm{M}$ aqueous $\mathrm{Na}_{2}(\mathrm{EDTA})$ ), tetrabutylammonium hydrogen sulfate (34 $\mathrm{mg}, 0.100 \mathrm{mmol}$ ), and Shi catalyst (194 mg, $0.750 \mathrm{mmol})$. A solution of Oxone ${ }^{\circ}(2.10 \mathrm{~g}, 3.45 \mathrm{mmol})$ in aqueous $\mathrm{Na}_{2}($ EDTA) $\left(4 \times 10^{-4} \mathrm{M}, 16 \mathrm{~mL}\right)$ and a solution of $\mathrm{K}_{2} \mathrm{CO}_{3}(2.00 \mathrm{~g}, 14.5 \mathrm{mmol})$ in water $(16 \mathrm{~mL})$ were added simultaneously, but separately, via syringe pump over a period of 1.5 hours at room temperature. After addition was complete, the reaction was quenched by addition of $\mathrm{H}_{2} \mathrm{O}(50 \mathrm{~mL})$ and extracted with hexanes $(3 \times 100 \mathrm{~mL})$. The combined organic layers were washed with brine $(50 \mathrm{~mL})$, dried $\left(\mathrm{Na}_{2} \mathrm{SO}_{4}\right)$, filtered, and concentrated in vacuo. Purification of the residue by FCC (20:1, hexaneEtOAc) afforded $(R, R)-5 a(309 \mathrm{mg}, 60 \%$ yield, 95:5 er) as a colorless oil. The enantiomeric purity of this compound was determined by chiral HPLC (Chiralpak IA, isocratic hexanes-i-PrOH 99:1, $\left.0.5 \mathrm{~mL} / \mathrm{min}, 23^{\circ} \mathrm{C}\right) ; \mathrm{t}_{\mathrm{R}}$ (major) $=8.0 \mathrm{~min}$ and $\mathrm{t}_{\mathrm{R}}$ (minor) $=8.4 \mathrm{~min}$. The absolute stereochemistry was assigned by analogy to related compounds in the literature. ${ }^{11}$

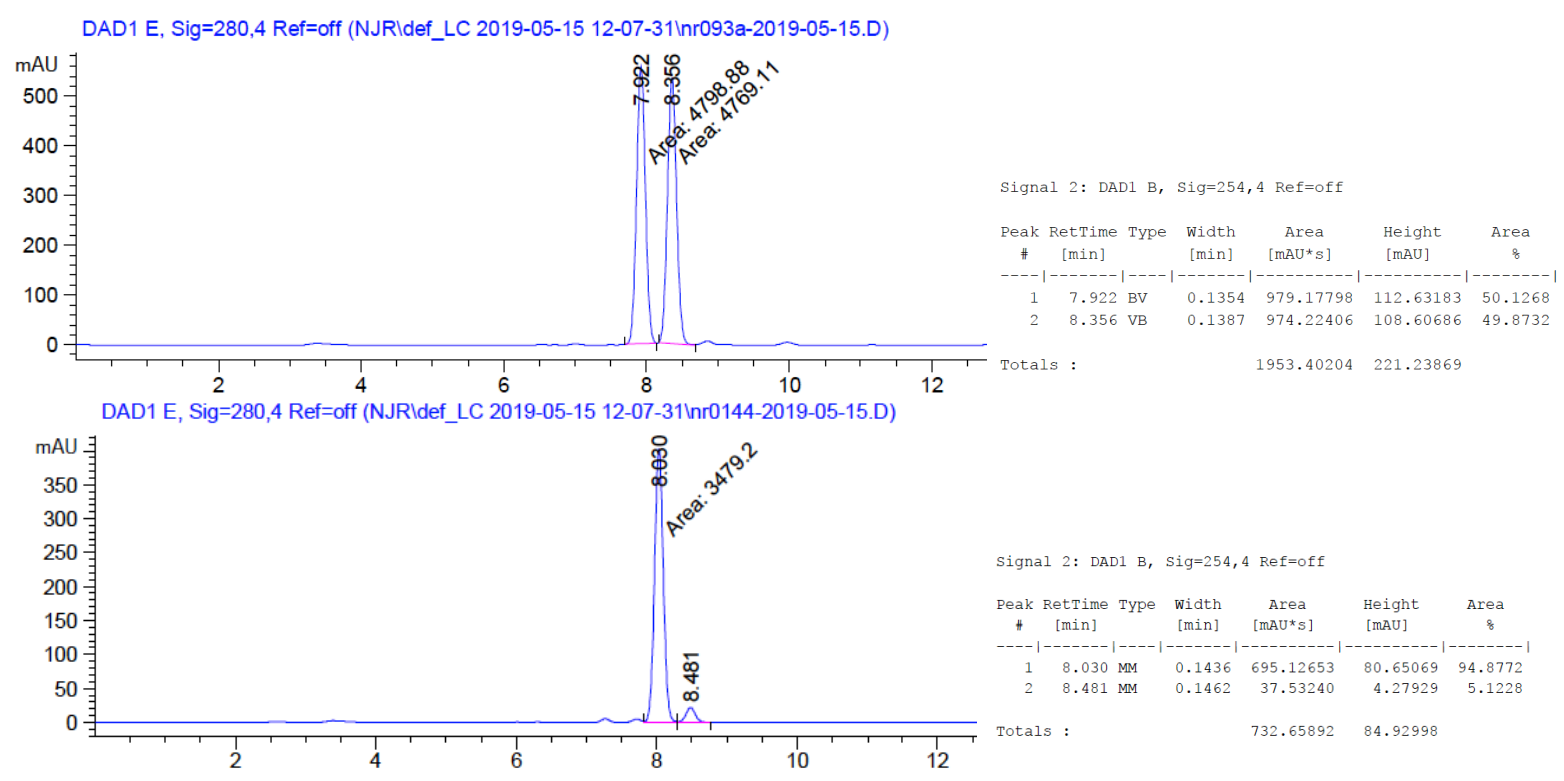


<smiles>CCCC1OC1Cc1ccc(OC)cc1</smiles>

93:7 er

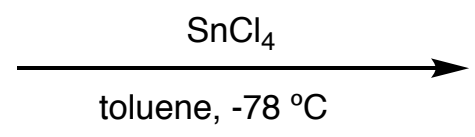

toluene, $-78^{\circ} \mathrm{C}$<smiles>CCC[C@H](O)[C@H](Cl)Cc1ccc(OC)cc1</smiles>

93:7 er

\section{(2R,3R)-2-Chloro-1-(4-methoxyphenyl)hexan-3-ol (R,R)-6a}

General Procedure C: Epoxide $(R, R)-5 a(42 \mathrm{mg}, 0.20 \mathrm{mmol}, 93: 7 \mathrm{er})$ was employed. Purification of the residue by FCC (10:1, hexane-EtOAc) afforded the title compound (44 $\mathrm{mg}, 90 \%, 93: 7 \mathrm{er}$ ) as a colorless oil.

The enantiomeric purity of this compound was determined by chiral HPLC (Chiralpak IA, isocratic hexanes-i-PrOH 97:3, $0.5 \mathrm{~mL} / \mathrm{min}, 23^{\circ} \mathrm{C}$ ); $t_{R}$ (minor) $=13.2 \mathrm{~min}$ and $t_{R}$ (major) $=13.8 \mathrm{~min}$.

The spectroscopic properties matched those of the racemic material.
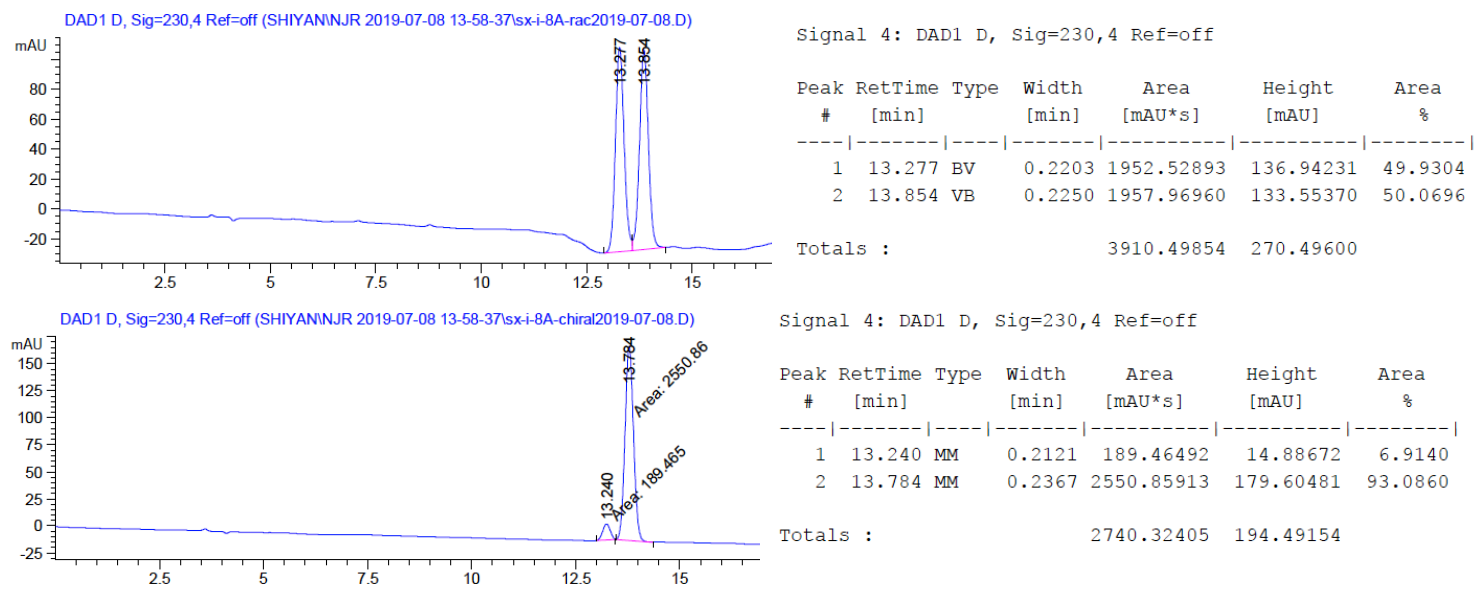
<smiles>CCCC1OC1Cc1ccc(OC)cc1</smiles>

95:5 er<smiles>COc1ccc([C@H](CCl)[C@H](O)C(C)C)cc1</smiles>

94.5:5.5 er

(2R,3R)-1-Chloro-2-(4-methoxyphenyl)hexan-3-ol $(R, R)$-7a

General Procedure D: Epoxide $(R, R)-5 a(250 \mathrm{mg}, 1.21 \mathrm{mmol}, 95: 5 \mathrm{er})$ was employed. Purification of the residue by FCC (3:1, hexane-EtOAc) afforded the title compound ( $281 \mathrm{mg}, 96 \%)$ as a pale yellow oil.

The enantiomeric excess of this material was inferred as 94.5:5.5 er after cyclization to oxetane 16 (see below). 
Procedures for Figure 3<smiles>CCC[C@H](O)[C@H](Cl)Cc1ccc(OC)cc1</smiles>

\section{(2S,3R)-2-Azido-1-(4-methoxyphenyl)hexan-3-ol (S,R)-10a}

To a solution of chlorohydrin $(R, R)-6 \mathrm{a}(24 \mathrm{mg}, 0.10 \mathrm{mmol})$ in DMF $(0.1 \mathrm{~mL})$ was added $\mathrm{NaN}_{3}(13$ $\mathrm{mg}, 0.20 \mathrm{mmol}$ ). The reaction mixture was stirred at $70^{\circ} \mathrm{C}$ for 12 hours. The solution was then quenched by pouring into saturated aq. $\mathrm{NH}_{4} \mathrm{Cl}(1 \mathrm{~mL})$. The solution was extracted with $\mathrm{Et}_{2} \mathrm{O}(4 \mathrm{x}$ $1 \mathrm{~mL})$ and washed with water $(3 \times 0.1 \mathrm{~mL})$, brine $(0.1 \mathrm{~mL})$. The organic layer was dried with $\mathrm{MgSO}_{4}$ and the solvent was evaporated under reduced pressure. Purification of the residue by FCC (10:1, hexane-EtOAc) afforded the title compound (22 mg, 93\% yield, 92.5:7.5 er) as a colorless oil. The enantiomeric purity of this compound was determined by chiral HPLC (Chiralpak $\mathrm{IA}$, isocratic hexanes-i-PrOH 95:5, $0.5 \mathrm{~mL} / \mathrm{min}, 23^{\circ} \mathrm{C}$ ); $t_{R}$ (minor) $=12.4$ min and $t_{R}$ (major) $=13.0$ $\min$.

${ }^{1} \mathrm{H}$ NMR $\left(400 \mathrm{MHz}, \mathrm{CDCl}_{3}\right) \delta 7.16(\mathrm{~d}, J=8.6 \mathrm{~Hz}, 2 \mathrm{H}), 6.87(\mathrm{~d}, J=8.6 \mathrm{~Hz}, 2 \mathrm{H}), 3.80(\mathrm{~s}, 3 \mathrm{H}), 3.70$ (d, $J=4.4 \mathrm{~Hz}, 1 \mathrm{H}), 3.57(\mathrm{dt}, J=9.5,4.3 \mathrm{~Hz}, 1 \mathrm{H}), 2.88(\mathrm{dd}, J=14.2,4.3 \mathrm{~Hz}, 1 \mathrm{H}), 2.74(\mathrm{dd}, J=$ 14.2, $9.6 \mathrm{~Hz}, 1 \mathrm{H}), 1.72(\mathrm{~d}, J=5.5 \mathrm{~Hz}, 1 \mathrm{H}), 1.57-1.52(\mathrm{~m}, 3 \mathrm{H}), 1.44-1.32(\mathrm{~m}, 1 \mathrm{H}), 0.97$ (t, $J=7.1$ $\mathrm{Hz}, 3 \mathrm{H})$;

${ }^{13} \mathrm{C}$ NMR $\left(101 \mathrm{MHz}, \mathrm{CDCl}_{3}\right) \delta$ 158.5, 130.2, 129.7, 114.1, 73.2, 69.0, 55.3, 35.2, 34.6, 19.0, 14.0;

IR $\left(\mathrm{NaCl}\right.$, thin film) 3444, 2925, 2103, 1614, 1584, 1514, 1446, 1250, 1181, 1033, $818 \mathrm{~cm}^{-1}$;

HRMS (ESI-TOF) $\mathrm{m} / \mathrm{z}$ calcd for $\mathrm{C}_{13} \mathrm{H}_{19} \mathrm{~N}_{3} \mathrm{NaO}_{2}{ }^{+}(\mathrm{M}+\mathrm{Na})^{+}:$272.1369, found 272.1366.
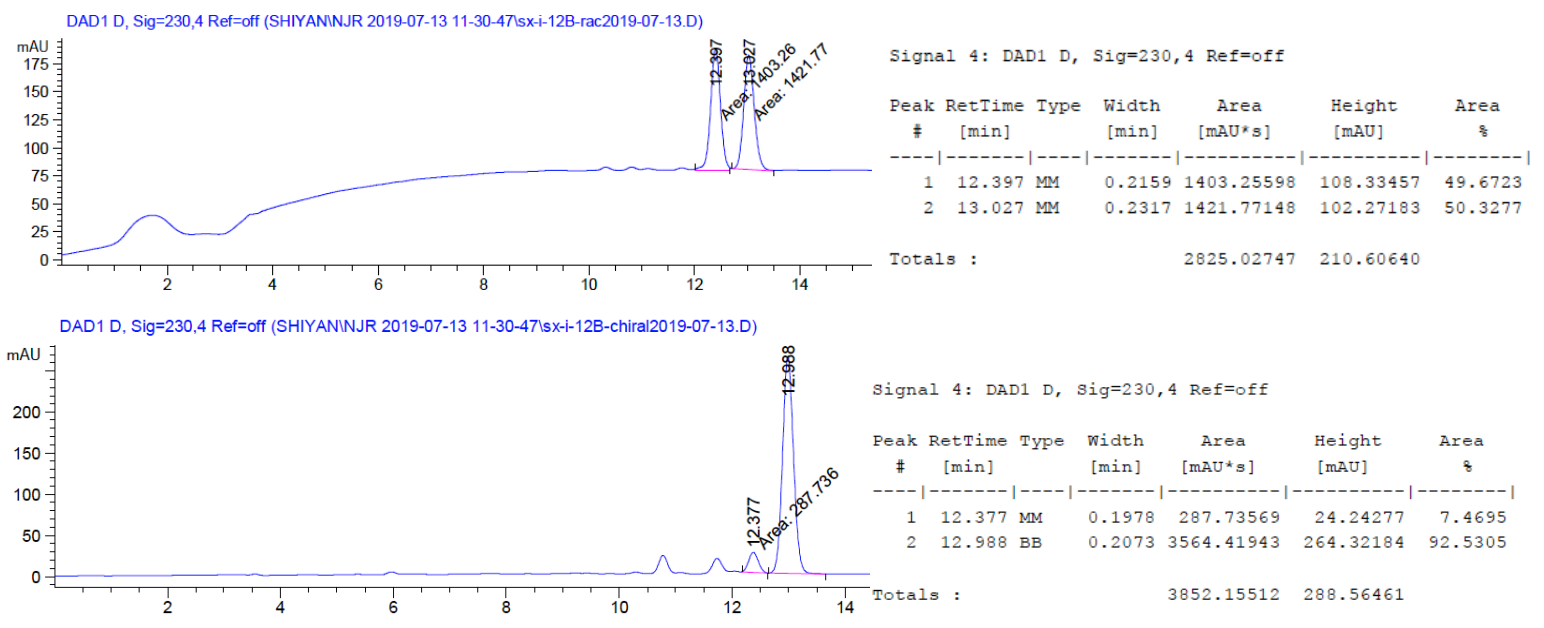


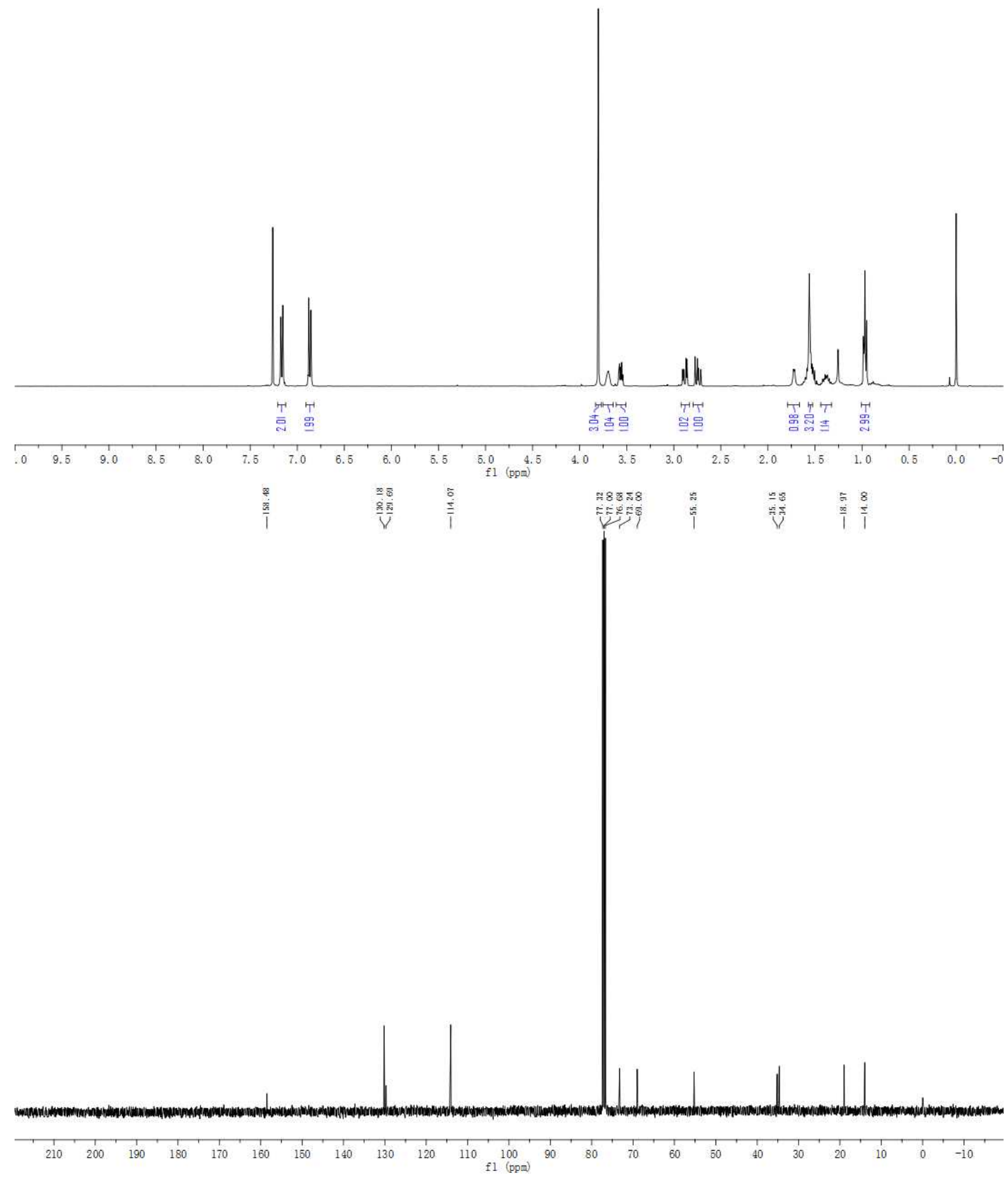



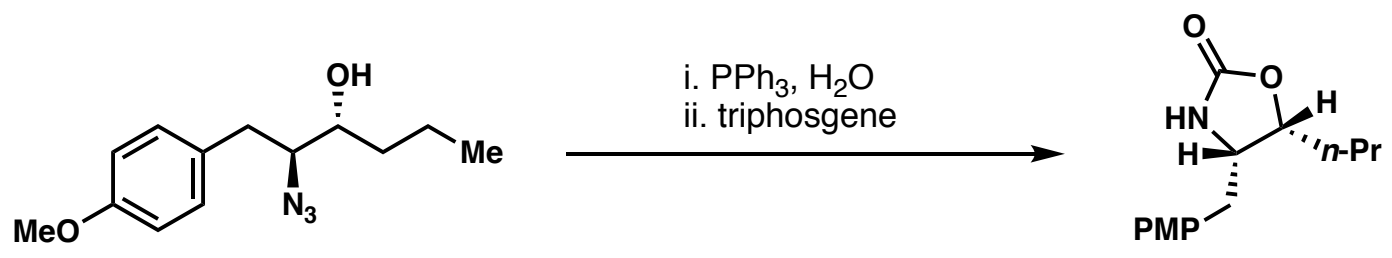

\section{$\left(4 S^{*}, 5 R^{*}\right)-4-(4-M e t h o x y b e n z y l)-5-p r o p y l o x a z o l i d i n-2-o n e$}

This reaction sequence was used to determine the relative stereochemistry of $( \pm)-10 a$. The spectroscopic properties of $(S, R)-10 \mathrm{a}$ were identical to $( \pm)-10 \mathrm{a}$ and therefore the relative stereochemistry is assigned by analogy.

To a solution of racemic azide $( \pm)-10 \mathrm{a}(25 \mathrm{mg}, 0.10 \mathrm{mmol})$ in THF $(1 \mathrm{~mL})$ were added $\mathrm{PPh}_{3}(53$ $\mathrm{mg}, 0.20 \mathrm{mmol})$ and $\mathrm{H}_{2} \mathrm{O}(18 \mathrm{mg}, 1.0 \mathrm{mmol})$ sequentially. The reaction mixture was stirred at 50 ${ }^{\circ} \mathrm{C}$ for 12 hours. After this time, the solvent was evaporated and crude mixture was redissolved in $\mathrm{CH}_{2} \mathrm{Cl}_{2}(1 \mathrm{~mL})$ and cooled to $0{ }^{\circ} \mathrm{C}$. Di-iso-propylethylamine $(43 \mu \mathrm{L}, 0.25 \mathrm{mmol})$ and triphosgene (15 mg, $0.05 \mathrm{mmol}$ ) were then added sequentially. The reaction was allowed to warm to room temperature and stirred for 2 hours. $\mathrm{H}_{2} \mathrm{O}(2.5 \mathrm{~mL})$ and $\mathrm{CH}_{2} \mathrm{Cl}_{2}(20 \mathrm{~mL})$ were added to the mixture, and the organic phase was separated, dried $\left(\mathrm{MgSO}_{4}\right)$, and concentrated in vacuo. Purification of the residue by FCC (1:1, hexane-EtOAc) gave the title compound as a colorless oil $(20 \mathrm{mg}, 82 \%$ yield).

${ }^{1} \mathrm{H}$ NMR $\left(500 \mathrm{MHz}, \mathrm{CDCl}_{3}\right) \delta 7.08(\mathrm{~d}, J=8.5 \mathrm{~Hz}, 2 \mathrm{H}), 6.87(\mathrm{~d}, J=8.5 \mathrm{~Hz}, 2 \mathrm{H}), 4.75(\mathrm{~s}, 1 \mathrm{H})$, 4.72-4.64 (m, 1H), 3.89 (ddd, $J=10.9,7.3,3.3 \mathrm{~Hz}, 1 \mathrm{H}$ ), 3.80 (s, 3H), 2.80 (dd, J = 13.5, $3.2 \mathrm{~Hz}$, $1 \mathrm{H}), 2.59(\mathrm{dd}, J=13.2,11.7 \mathrm{~Hz}, 1 \mathrm{H}), 1.85(\mathrm{dt}, J=17.0,6.8 \mathrm{~Hz}, 1 \mathrm{H}), 1.65$ (ddt, $J=13.1,11.1,5.8$ $\mathrm{Hz}, 2 \mathrm{H}), 1.50-1.42(\mathrm{~m}, 1 \mathrm{H}), 1.01(\mathrm{t}, J=7.1 \mathrm{~Hz}, 3 \mathrm{H})$;

${ }^{13} \mathrm{C}$ NMR $\left(126 \mathrm{MHz}, \mathrm{CDCl}_{3}\right) \delta 158.7,158.6,129.9,128.5,114.5,79.7,57.0,55.3,35.4,31.4,19.3$, 13.9 ;

IR ( $\mathrm{NaCl}$, thin film) 3282, 2960, 2924, 2873, 1748, 1613, 1514, 1464, 1393, 1249, 1180, 1033, $812 \mathrm{~cm}^{-1}$;

HRMS (ESI-TOF) $\mathrm{m} / \mathrm{z}$ calcd for $\mathrm{C}_{14} \mathrm{H}_{19} \mathrm{NNaO}_{3}{ }^{+}(\mathrm{M}+\mathrm{Na})^{+}: 272.1263$, found 272.1255 .

The syn relative stereochemistry was assigned by nOe enhancements (highlighted on the structure) and also the diagnostic $\mathrm{H}-\mathrm{H}$ coupling constant of $7.3 \mathrm{~Hz}$ between the adjacent hydrogens. ${ }^{12}$

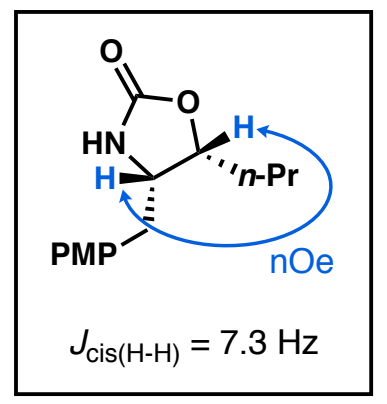



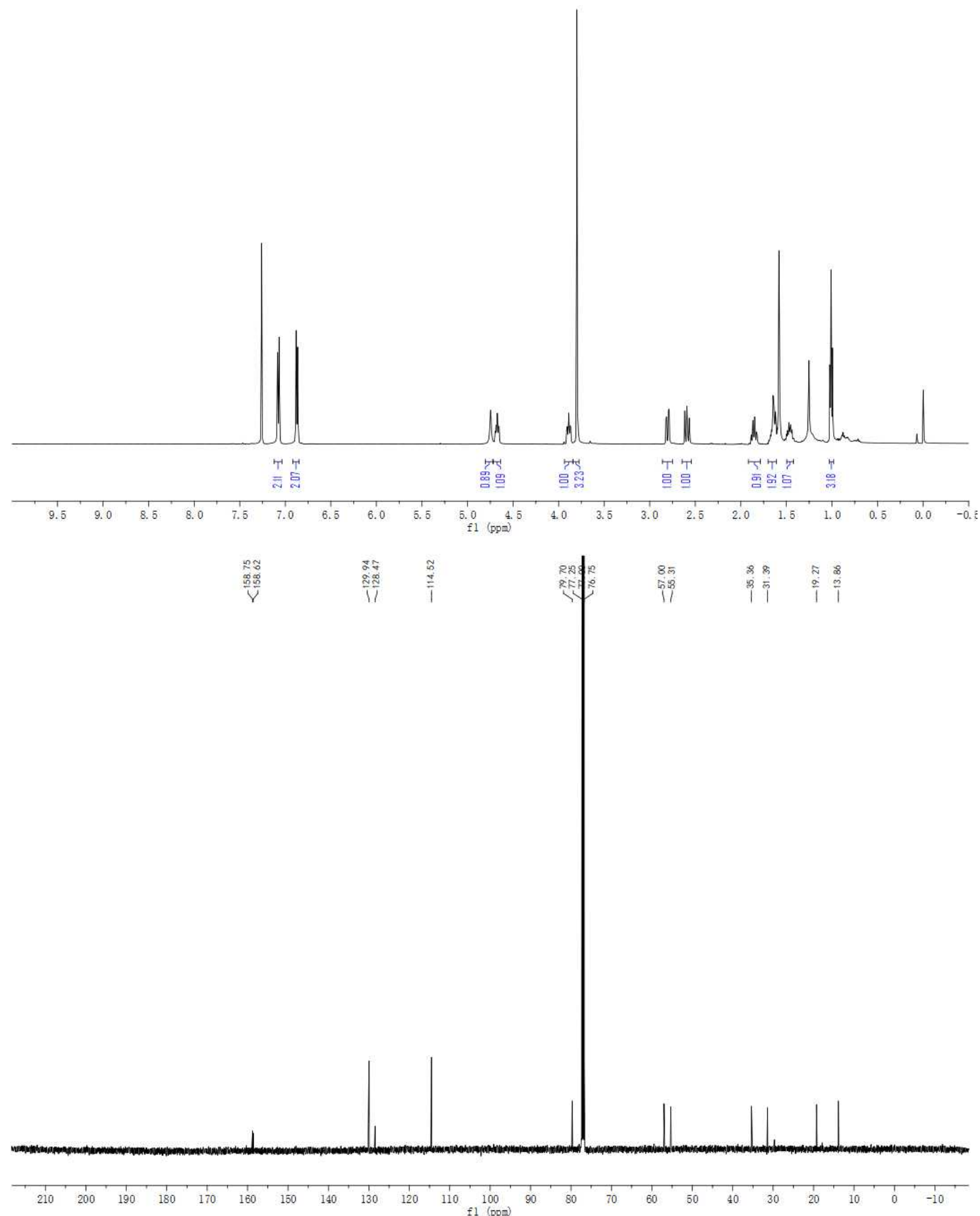
<smiles>CCC[C@@H](O)[C@H](Cl)Cc1ccc(Cl)cc1</smiles>

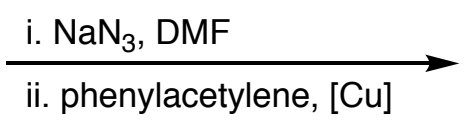<smiles>CCC[C@H](O)[C@@H](Cc1ccc(Cl)cc1)n1cc(-c2ccccc2)nn1</smiles>

\section{$\left(2 S^{*}, 3 R^{*}\right)-1-(4-C h l o r o p h e n y l)-2-(4-p h e n y l-1 H-1,2,3-t r i a z o l-1-y l) h e x a n-3-o l ~ 11$}

To a solution of chlorohydrin $( \pm)-6 \mathbf{i}(25 \mathrm{mg}, 0.10 \mathrm{mmol})$ in DMF $(0.10 \mathrm{~mL})$ was added $\mathrm{NaN}_{3}(13$ $\mathrm{mg}, 0.020 \mathrm{mmol})$. The reaction mixture was stirred at $70{ }^{\circ} \mathrm{C}$ for 12 hours. After this time, the reaction was cooled to room temperature and quenched by pouring into saturated $\mathrm{NH}_{4} \mathrm{Cl}$ aqueous solution $(1 \mathrm{~mL})$. The mixture was extracted with $\mathrm{Et}_{2} \mathrm{O}(4 \times 1 \mathrm{~mL})$ and washed with water $(3 \times 0.1$ $\mathrm{mL})$, brine $(0.1 \mathrm{~mL})$. The organic layer was dried with $\mathrm{MgSO}_{4}$ and concentrated in vacuo. The crude azide was used in the next step without further purification.

A vial was charged with copper iodide (1.9 mg, $0.010 \mathrm{mmol}), 2,2$ '-bipyridine (1.6 $\mathrm{mg}, 0.010 \mathrm{mmol})$, and $\mathrm{CH}_{2} \mathrm{Cl}_{2}(0.25 \mathrm{~mL})$. The resulting solution was stirred at ambient temperature for $5 \mathrm{~min}$. A separate vial was charged with crude azide, phenylacetylene (12 $\mathrm{mg}, 0.12 \mathrm{mmol}$ ), and $\mathrm{CH}_{2} \mathrm{Cl}_{2}$ $(0.75 \mathrm{~mL})$. The substrate solution was added to the solution of copper catalyst. The vial was sealed and heated to $40{ }^{\circ} \mathrm{C}$. After 16 hours, the reaction was cooled and quenched by addition of silver nitrate solution (50 $\mu \mathrm{L}, 0.1 \mathrm{M}$ in 9:1 DME:water). The resulting mixture was concentrated in vacuo. Purification by of the residue by FCC (2:1, hexane-EtOAc) afforded the title compound (30 $\mathrm{mg}, 85 \%$ yield over 2 steps) as a colorless oil.

${ }^{1} \mathrm{H}$ NMR $\left(500 \mathrm{MHz}, \mathrm{CDCl}_{3}\right) \delta 7.72(\mathrm{~d}, J=7.6 \mathrm{~Hz}, 2 \mathrm{H}), 7.43(\mathrm{~s}, 1 \mathrm{H}), 7.40(\mathrm{t}, J=7.5 \mathrm{~Hz}, 2 \mathrm{H}), 7.32$ $(\mathrm{t}, J=7.3 \mathrm{~Hz}, 1 \mathrm{H}), 7.16(\mathrm{~d}, J=7.8 \mathrm{~Hz}, 2 \mathrm{H}), 6.89(\mathrm{~d}, J=7.8 \mathrm{~Hz}, 2 \mathrm{H}), 4.50$ (dd, $J=6.2,4.5 \mathrm{~Hz}$, $1 \mathrm{H}), 4.20-4.10(\mathrm{~m}, 1 \mathrm{H}), 3.47-3.26(\mathrm{~m}, 2 \mathrm{H}), 1.66-1.54(\mathrm{~m}, 2 \mathrm{H}), 1.54-1.37(\mathrm{~m}, 2 \mathrm{H}), 0.95(\mathrm{t}, J=7.0$ $\mathrm{Hz}, 3 \mathrm{H})$;

${ }^{13} \mathrm{C}$ NMR $\left(126 \mathrm{MHz}, \mathrm{CDCl}_{3}\right) \delta 146.9,135.6,132.8,130.2(2 \mathrm{C}), 128.8(2 \mathrm{C}), 128.2,125.6,120.5$, 73.2, 68.0, 35.6, 34.9, 19.1, 13.9;

IR ( $\mathrm{NaCl}$, thin film) 3337, 2959, 2361, 2336, 1653, 1558, 1506, 1490, 1457, 1249, 1180, 1033, $812 \mathrm{~cm}^{-1}$;

HRMS (ESI-TOF) $\mathrm{m} / \mathrm{z}$ calcd for $\mathrm{C}_{14} \mathrm{H}_{19} \mathrm{NNaO}_{3}{ }^{+}(\mathrm{M}+\mathrm{Na})^{+}: 378.1344$, found 378.1347 . 

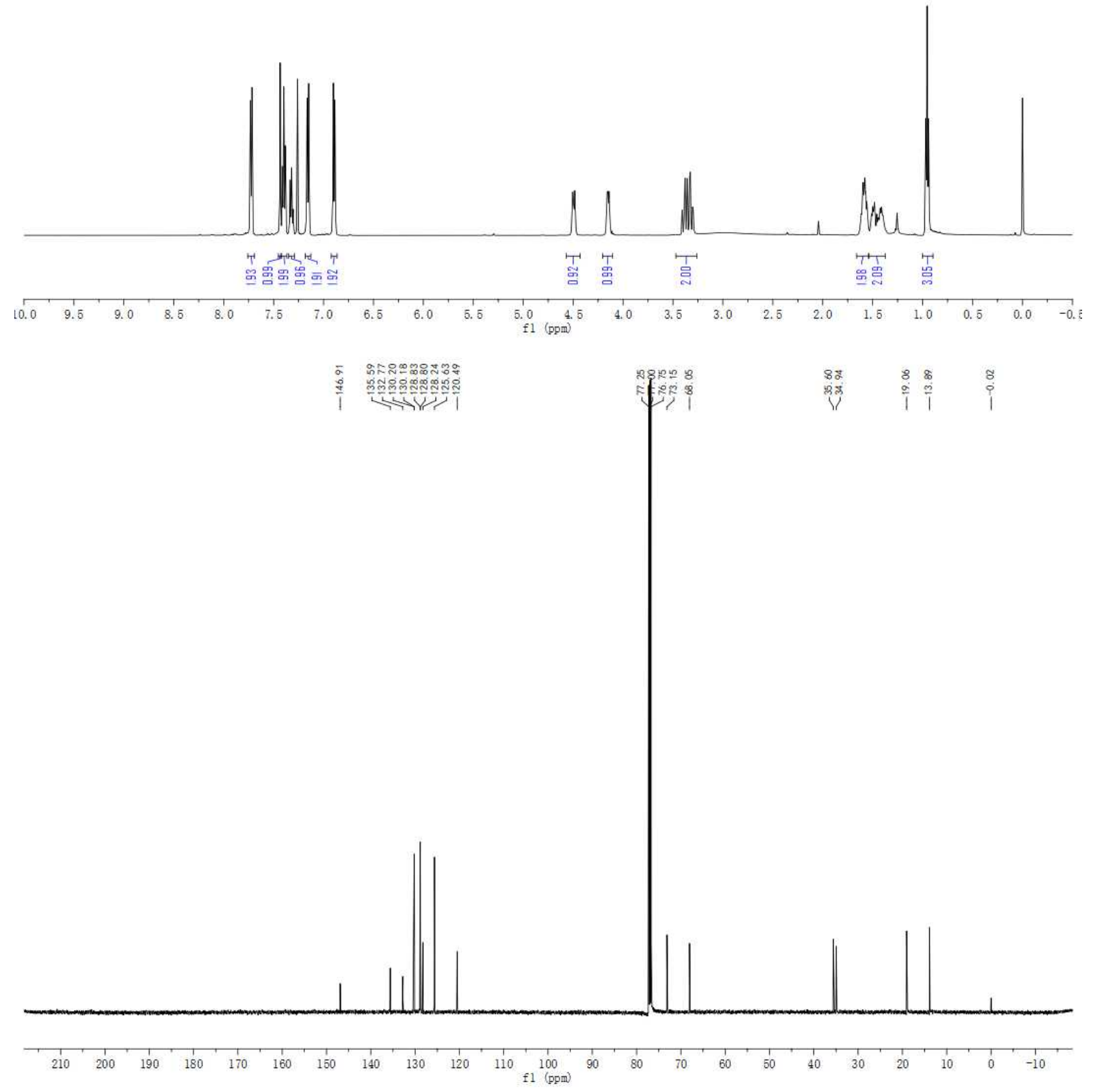
Procedures for Figure 4<smiles>CCCC(O)C(CC#N)c1ccc(OC)cc1</smiles>

$\left(3 R^{*}, 4 R^{*}\right)$-4-Hydroxy-3-(4-methoxyphenyl)heptanenitrile $( \pm)$-12

To a solution of alcohol $( \pm)-7 \mathrm{a}(20 \mathrm{mg}, 0.083 \mathrm{mmol})$ in DMF $(2 \mathrm{~mL})$ was added sodium cyanide $(8.1 \mathrm{mg}, 0.17 \mathrm{mmol})$ and sodium iodide $(2.5 \mathrm{mg}, 0.016 \mathrm{mmol})$. The reaction mixture was stirred at $80^{\circ} \mathrm{C}$ until consumption of starting material occurred, as determined by TLC analysis. The reaction was then diluted with ether $(3 \mathrm{~mL})$, transferred to a separatory funnel and washed with brine $(3 \times 5 \mathrm{~mL})$. The organic phase was dried $\left(\mathrm{MgSO}_{4}\right)$, filtered, and concentrated in vacuo. Purification of the residue by FCC (5:1, hexane-EtOAc) afforded the title compound ( $16 \mathrm{mg}, 82 \%)$ as a yellow oil

${ }^{1} \mathrm{H}$ NMR $\left(400 \mathrm{MHz}, \mathrm{CDCl}_{3}\right) \delta 7.15(\mathrm{~d}, J=8.7 \mathrm{~Hz}, 2 \mathrm{H}), 6.89(\mathrm{~d}, J=8.7 \mathrm{~Hz}, 2 \mathrm{H}), 3.84-3.79(\mathrm{~m}, 1 \mathrm{H})$, $3.81(\mathrm{~s}, 3 \mathrm{H}), 2.91-2.84(\mathrm{~m}, 2 \mathrm{H}), 2.79-2.72(\mathrm{~m}, 1 \mathrm{H}), 1.69(\mathrm{br} . \mathrm{s}, 1 \mathrm{H}), 1.50-1.41(\mathrm{~m}, 1 \mathrm{H}), 1.38-1.25$ $(\mathrm{m}, 3 \mathrm{H}), 0.86(\mathrm{t}, J=7.0 \mathrm{~Hz}, 3 \mathrm{H})$;

${ }^{13} \mathrm{C}$ NMR $\left(100 \mathrm{MHz}, \mathrm{CDCl}_{3}\right) \delta 159.0,131.7,128.8,119.3,114.4,73.9,55.2,47.8,37.2,20.9,18.7$, 13.8;

IR ( $\mathrm{NaCl}$, thin film) 3456, 2958, 2872, 2223, 1612, 1514, 1304, 1251, 1034, $830 \mathrm{~cm}^{-1}$;

HRMS (El-Orbitrap) $\mathrm{m} / \mathrm{z}$ calcd for $\mathrm{C}_{14} \mathrm{H}_{20} \mathrm{NO}_{2}{ }^{+}(\mathrm{M}+\mathrm{H})^{+} 234.1489$, found 234.1484 . 

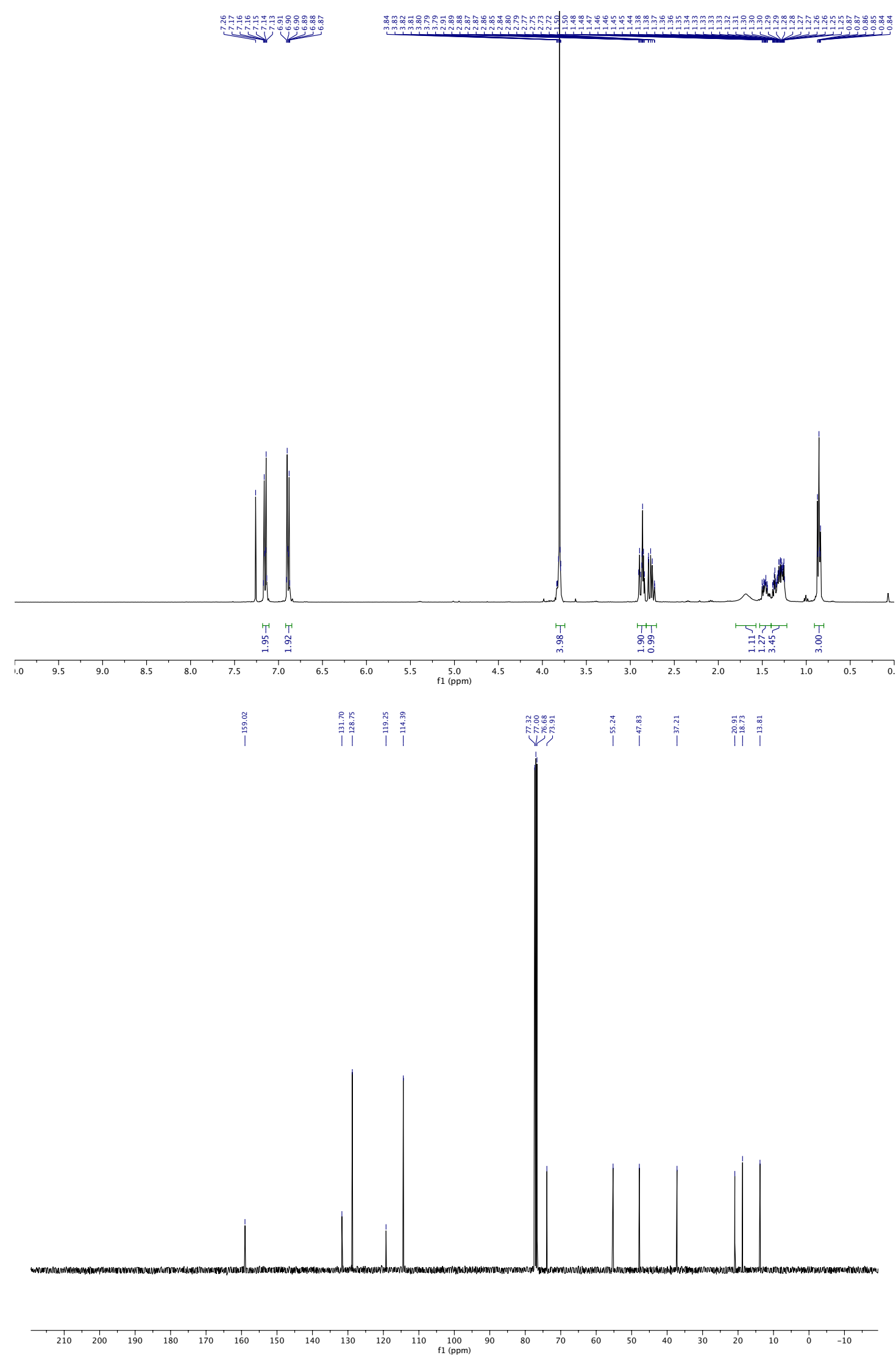
<smiles>CCC[C@@H](O)[C@H](CN)c1ccc(OC)cc1</smiles>

\section{$\left(2 S^{*}, 3 R^{*}\right)$-1-Azido-2-(4-methoxyphenyl)hexan-3-ol ( \pm )-13}

To a solution of alcohol $( \pm)-7 a(20 \mathrm{mg}, 0.083 \mathrm{mmol})$ in DMF $(2 \mathrm{~mL})$ was added sodium azide (38 $\mathrm{mg}, 0.58 \mathrm{mmol}$ ). The reaction mixture was stirred at $70^{\circ} \mathrm{C}$ until consumption of starting material occurred, as determined by TLC analysis. The reaction was then quenched by addition of saturated aq. $\mathrm{Na}_{2} \mathrm{CO}_{3}(1 \mathrm{~mL})$, diluted with ether $(3 \mathrm{~mL})$ and was transferred to a separatory funnel. The layers were separated and the organic phase was then washed with brine $(2 \times 5 \mathrm{~mL})$, dried $\left(\mathrm{MgSO}_{4}\right)$, filtered, and concentrated in vacuo. Purification of the residue by FCC $(5: 1$, hexaneEtOAc) afforded the title compound (16 mg, 77\%) as a yellow oil.

${ }^{1} \mathrm{H}$ NMR $\left(400 \mathrm{MHz}, \mathrm{CDCl}_{3}\right) \delta 7.13(\mathrm{~d}, J=8.7 \mathrm{~Hz}, 2 \mathrm{H}), 6.88(\mathrm{~d}, J=8.7 \mathrm{~Hz}, 2 \mathrm{H}), 3.86-3.81(\mathrm{~m}, 1 \mathrm{H})$ $3.80(\mathrm{~s}, 3 \mathrm{H}), 3.76(\mathrm{dd}, J=12.2,5.0 \mathrm{~Hz}, 1 \mathrm{H}), 3.69(\mathrm{dd}, J=12.2,8.1 \mathrm{~Hz}, 1 \mathrm{H}), 2.79$ (td, $J=8.0,5.1$ $\mathrm{Hz}, 1 \mathrm{H}), 1.79(\mathrm{~s}, 1 \mathrm{H}) \mathrm{z} 1.34-1.25(\mathrm{~m}, 4 \mathrm{H}), 0.85(\mathrm{t}, J=7.0 \mathrm{~Hz}, 3 \mathrm{H})$;

${ }^{13} \mathrm{C}$ NMR $\left(100 \mathrm{MHz}, \mathrm{CDCl}_{3}\right) \delta$ 158.9, 132.2, 129.4, 114.3, 73.7, 55.4, 53.9, 51.2, 37.5, 18.9, 14.0;

IR ( $\mathrm{NaCl}$, thin film) 3428, 2929, 2096, 1716, 1513, 1463, 1249, 1179, 1124, 1036, $829 \mathrm{~cm}^{-1}$;

HRMS (El-TOF) $\mathrm{m} / \mathrm{z}$ calcd for $\mathrm{C}_{13} \mathrm{H}_{19} \mathrm{~N}_{3} \mathrm{O}_{2} \mathrm{Na}^{+}(\mathrm{M}+\mathrm{Na})^{+} 272.1369$, found 272.1360. 

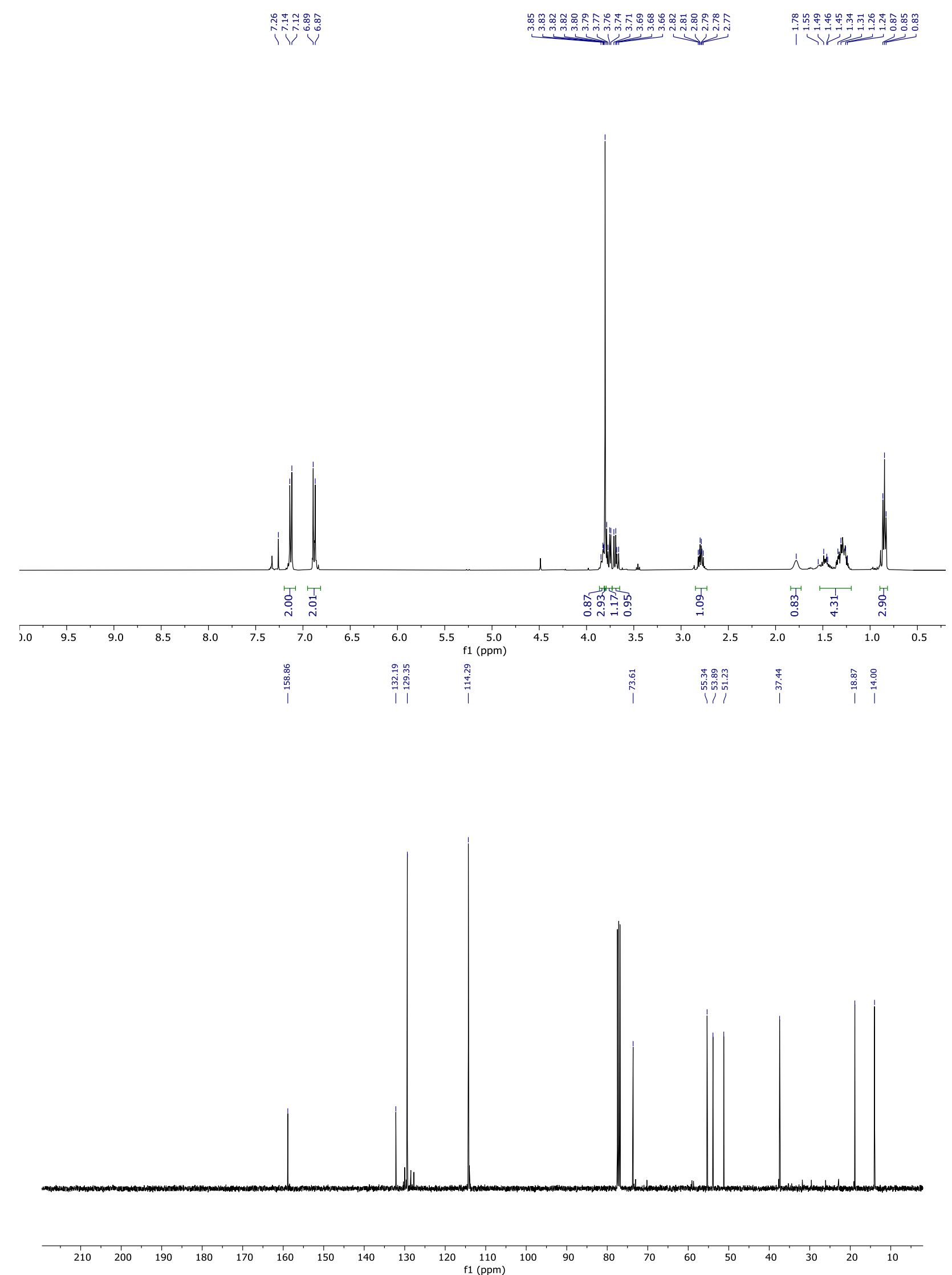
<smiles>CCC[C@@H](O)[C@H](CSc1ccc(Br)cc1)c1ccc(OC)cc1</smiles>

\section{$\left(2 R^{\star}, 3 R^{\star}\right)-1-((4-B r o m o p h e n y l) t h i o)-2-(4-m e t h o x y p h e n y l) h e x a n-3-o l ~( \pm)-14$}

To an oven dried reaction tube, charged with a stir bar, was added ( \pm )-7a (34 mg, $0.14 \mathrm{mmol}$ ) and 4-bromothiophenol $(76 \mathrm{mg}, 0.40 \mathrm{mmol})$. The tube was sealed with a septum and purged with argon. DMF $(1 \mathrm{~mL})$ and $\mathrm{Cs}_{2} \mathrm{CO}_{3}(195 \mathrm{mg}, 0.60 \mathrm{mmol})$ were added and the resultant mixture was stirred at $80^{\circ} \mathrm{C}$ for 20 hours. After this time, the reaction was cooled to room temperature, diluted with $\mathrm{Et}_{2} \mathrm{O}(5 \mathrm{~mL})$ and washed with $\mathrm{H}_{2} \mathrm{O}(2 \times 5 \mathrm{~mL})$. The organic phase was dried over $\mathrm{MgSO}_{4}$, filtered, and concentrated in vacuo. Purification of the residue by FCC (20:1 - 5:1, hexane-EtOAc) afforded the title compound (39 $\mathrm{mg}, 70 \%$ ) as a yellow oil.

${ }^{1} \mathrm{H}$ NMR $\left(400 \mathrm{MHz}, \mathrm{CDCl}_{3}\right) \delta 7.35(\mathrm{~d}, J=8.5 \mathrm{~Hz}, 2 \mathrm{H}), 7.13(\mathrm{~d}, J=8.5 \mathrm{~Hz}, 2 \mathrm{H}), 7.07(\mathrm{~d}, J=8.6$ $\mathrm{Hz}, 2 \mathrm{H}), 6.85(\mathrm{~d}, J=8.6 \mathrm{~Hz}, 2 \mathrm{H}), 3.80(\mathrm{~s}, 3 \mathrm{H}), 3.80-3.76(\mathrm{~m}, 1 \mathrm{H}), 3.57$ (dd, $J=12.7,4.5 \mathrm{~Hz}, 1 \mathrm{H}$ ), 3.18 (dd, $J=12.7,10.0 \mathrm{~Hz}, 1 \mathrm{H}$ ), 2.81 (ddd, $J=9.9,7.3,4.4 \mathrm{~Hz}, 1 \mathrm{H}$ ), 1.57 (br. s, 1H), 1.50-1.40 $(\mathrm{m}, 1 \mathrm{H}), 1.36-1.22(\mathrm{~m}, 3 \mathrm{H}), 0.84(\mathrm{t}, \mathrm{J}=7.0 \mathrm{~Hz}, 3 \mathrm{H})$;

${ }^{13} \mathrm{C}$ NMR $\left(100 \mathrm{MHz}, \mathrm{CDCl}_{3}\right) \delta 158.6,136.2,132.8,131.8,130.5,129.3,119.5,114.0,75.1,55.2$, 51.0, 37.2, 36.3, 18.9, 13.9;

IR ( $\mathrm{NaCl}$, thin film) 3448, 2956, 2932, 2871, 1611, 1513, 1473, 1247, 1179, 1092, 1008, 828, 808 $\mathrm{cm}^{-1}$;

HRMS $\left(\mathrm{Cl}^{+}\right) \mathrm{m} / \mathrm{z}$ calcd for $\mathrm{C}_{19} \mathrm{H}_{23}{ }^{79} \mathrm{BrO}_{2} \mathrm{SNH}_{4}{ }^{+}\left(\mathrm{M}+\mathrm{NH}_{4}\right)^{+}:$412.0940, found 412.0937 . 


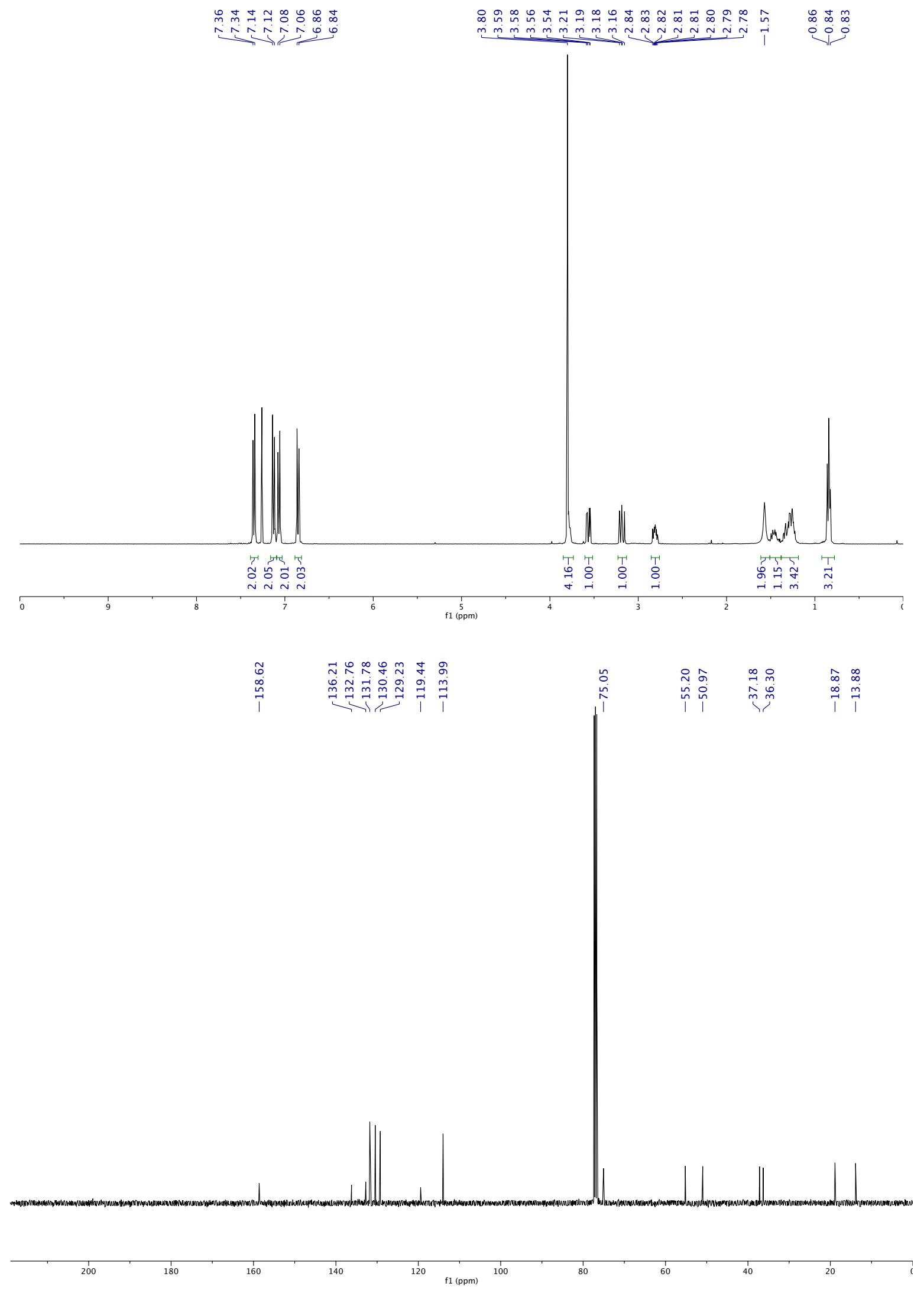


<smiles>C=CCC[C@H](c1ccc(OC)cc1)[C@H](O)CCC</smiles>

$\left(4 R^{*}, 5 R^{*}\right)-5-(4-M e t h o x y p h e n y l)$ non-8-en-4-ol $( \pm)-15$

To a solution of alcohol $( \pm)-7 a(29 \mathrm{mg}, 0.10 \mathrm{mmol})$ in THF $(1 \mathrm{~mL})$ was added $\mathrm{MeMgBr}(0.22 \mathrm{~mL}$, $1 \mathrm{M}, 0.22 \mathrm{mmol})$. The mixture was stirred at room temperature for 3 hours after which time the reaction was quenched with saturated $\mathrm{NH}_{4} \mathrm{Cl}$ aq. solution $(0.1 \mathrm{~mL})$. The solution was filtrated through celite ${ }^{\circledR}$ and concentrated in vacuo. Purification of the residue by $\operatorname{FCC}(5: 1$, hexaneEtOAc) afforded the title compound ( $24 \mathrm{mg}, 91 \%$ yield) as a colorless oil.

${ }^{1} \mathrm{H}$ NMR $\left(500 \mathrm{MHz}, \mathrm{CD}_{2} \mathrm{Cl}_{2}\right) \delta 7.08(\mathrm{~d}, J=8.4 \mathrm{~Hz}, 2 \mathrm{H}), 6.85(\mathrm{~d}, J=8.5 \mathrm{~Hz}, 2 \mathrm{H}), 5.79$ (dq, $J=10.1$, $6.8 \mathrm{~Hz}, 1 \mathrm{H}), 5.01-4.82(\mathrm{~m}, 2 \mathrm{H}), 3.79(\mathrm{~s}, 3 \mathrm{H}), 3.62(\mathrm{~s}, 1 \mathrm{H}), 2.57-2.50(\mathrm{~m}, 1 \mathrm{H}), 1.98(\mathrm{dd}, J=9.8$, $6.4 \mathrm{~Hz}, 1 \mathrm{H}), 1.90(\mathrm{~d}, J=14.4 \mathrm{~Hz}, 1 \mathrm{H}), 1.81(\mathrm{dd}, J=14.5,7.5 \mathrm{~Hz}, 1 \mathrm{H}), 1.70(\mathrm{dd}, J=16.3,8.0 \mathrm{~Hz}$, $1 \mathrm{H}), 1.46(\mathrm{~d}, J=14.4 \mathrm{~Hz}, 2 \mathrm{H}), 1.33-1.25(\mathrm{~m}, 2 \mathrm{H}), 1.20(\mathrm{dd}, J=14.6,6.8 \mathrm{~Hz}, 1 \mathrm{H}), 0.84(\mathrm{t}, J=7.0$ $\mathrm{Hz}, 3 \mathrm{H})$;

${ }^{13} \mathrm{C}$ NMR $\left(126 \mathrm{MHz}, \mathrm{CD}_{2} \mathrm{Cl}_{2}\right) \delta 159.0,139.7,135.2,130.2,114.9,114.5,76.2,55.9,51.9,37.9$, $32.5,30.9,19.9,14.6$;

IR (thin film) 3410, 2957, 2932, 2871, 2836, 2358, 2337, 1611, 1512, 1457, 1247, 1177, 1038, $827,648 \mathrm{~cm}^{-1}$;

HRMS (ESI-TOF) $\mathrm{m} / z$ calcd for $\mathrm{C}_{16} \mathrm{H}_{24} \mathrm{NaO}_{2}{ }^{+}(\mathrm{M}+\mathrm{Na})^{+}: 271.1669$, found 271.1662 . 

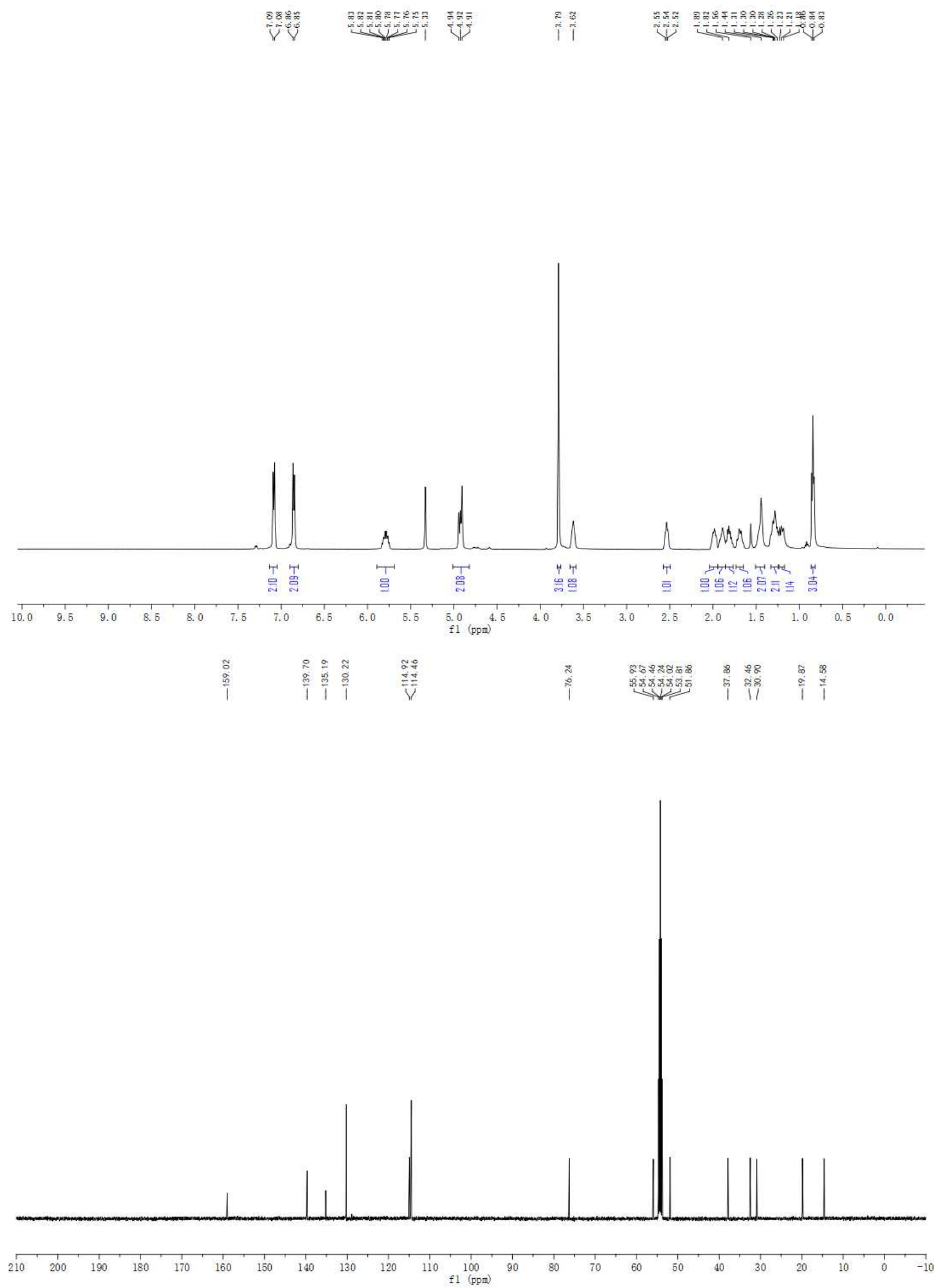

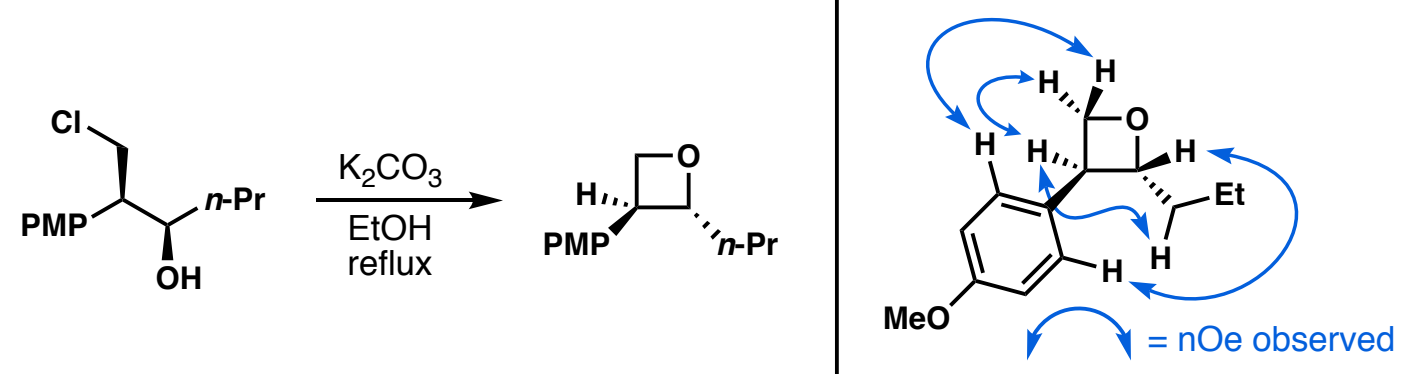

\section{(2R,3S)-3-(4-Methoxyphenyl)-2-propyloxetane 16}

To a solution of alcohol $(R, R)-7 \mathrm{a}(50 \mathrm{mg}, 0.21 \mathrm{mmol})$ in $\mathrm{EtOH}(2 \mathrm{~mL})$ was added $\mathrm{K}_{2} \mathrm{CO}_{3}(116 \mathrm{mg}$, $0.84 \mathrm{mmol}$ ). The mixture was stirred at $80{ }^{\circ} \mathrm{C}$ for 3 days after which time the reaction mixture was diluted with $\mathrm{Et}_{2} \mathrm{O}(5 \mathrm{~mL})$. The solution was washed with water $(2 \times 5 \mathrm{~mL})$ and brine $(1 \times 5 \mathrm{~mL})$, dried with $\mathrm{MgSO}_{4}$, and concentrated in vacuo. Purification of the residue by FCC (5:1, hexaneEtOAc) afforded the title compound (38 $\mathrm{mg}, 89 \%$ yield, 94.5:5.5 er) as a colorless oil.

${ }^{1} \mathrm{H}$ NMR $\left(400 \mathrm{MHz}, \mathrm{CDCl}_{3}\right) \delta 7.28(\mathrm{~d}, J=8.7 \mathrm{~Hz}, 2 \mathrm{H}), 6.90$ (d, $\left.J=8.7 \mathrm{~Hz}, 2 \mathrm{H}\right), 4.81$ (app. dd, $J=$ 8.7, $5.99 \mathrm{~Hz}, 1 \mathrm{H}$ ), 4.78 (app. dd, $J=13.16 .6 \mathrm{~Hz}, 1 \mathrm{H}$ ), 4.65 (dd, J=7.6, $6.0 \mathrm{~Hz}, 1 \mathrm{H}$ ), 3.81 (s, 3H), 3.73 (app. dt, $J=8.5,7.1 \mathrm{~Hz}, 1 \mathrm{H}$ ), 1.88 (dddd, $J=13.1,9.6,7.1,5.8 \mathrm{~Hz}, 1 \mathrm{H}$ ), 1.74 (app. ddt, $J=$ $13.1,9.6,6.1 \mathrm{~Hz}, 1 \mathrm{H}), 1.45-1.28(\mathrm{~m}, 2 \mathrm{H}), 0.92(\mathrm{t}, J=0.92,3 \mathrm{H})$;

${ }^{13} \mathrm{C}$ NMR $\left(100 \mathrm{MHz}, \mathrm{CDCl}_{3}\right) \delta 158.4,133.0,128.1,114.0,90.0,74.6,55.3,46.0,39.7,17.4,14.0$;

IR ( $\mathrm{NaCl}$, thin film) 2961, 2932, 2879, 1612, 1511, 1463, 1245, 1179, 1033, $831 \mathrm{~cm}^{-1}$;

HRMS $\left(\mathrm{Cl}^{+}\right) \mathrm{m} / \mathrm{z}$ calcd for $\mathrm{C}_{13} \mathrm{H}_{18} \mathrm{O}_{2} \mathrm{H}^{+}(\mathrm{M}+\mathrm{H})^{+}: 207.1380$, found 207.1376

The enantiomeric purity of this compound was determined by chiral HPLC (WhelkO-1, isocratic hexanes-i-PrOH 95:5, $1.0 \mathrm{~mL} / \mathrm{min}, 23^{\circ} \mathrm{C}$ ); $t_{R}$ (minor) $=10.7$ min and $t_{R}$ (major) $=14.4 \mathrm{~min}$.

The relative stereochemistry was determined through nOe analysis, indicated above.
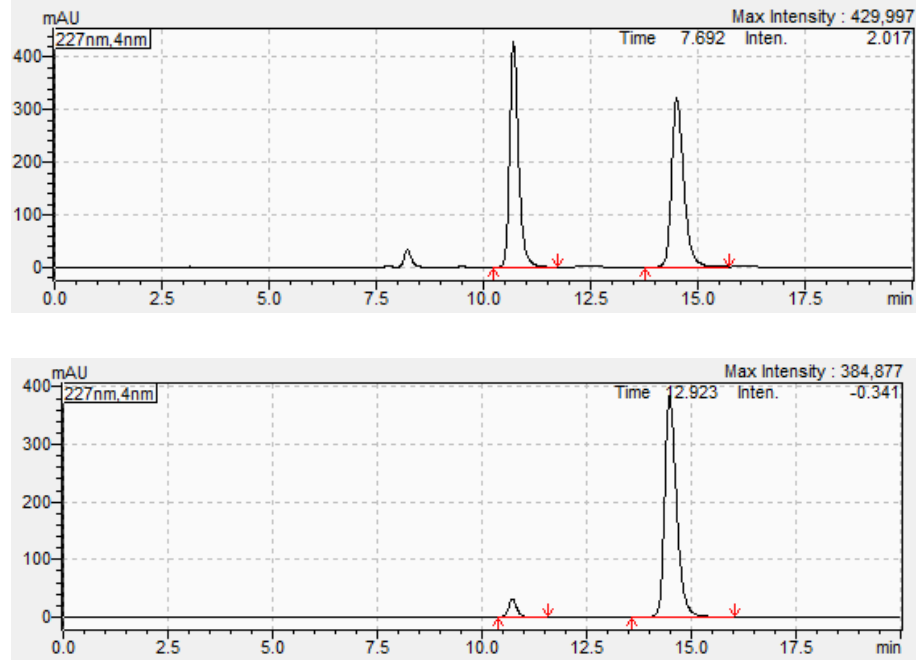

\begin{tabular}{|l|r|r|r|}
\hline Peak\# & Ret. Time & \multicolumn{1}{|c|}{ Area } & \multicolumn{1}{|c|}{ Area\% } \\
\hline 1 & 10.700 & 6301397 & 49.646 \\
\hline 2 & 14.507 & 6391238 & 50.354 \\
\hline Total & & 12692636 & 100.000 \\
\hline
\end{tabular}

\begin{tabular}{|l|r|r|r|}
\hline Peak\# & Ret. Time & \multicolumn{1}{|c|}{ Area } & \multicolumn{1}{|c|}{ Area\% } \\
\hline 1 & 10.725 & 446164 & 5.526 \\
\hline 2 & 14.480 & 7627594 & 94.474 \\
\hline Total & & 8073759 & 100.000 \\
\hline
\end{tabular}




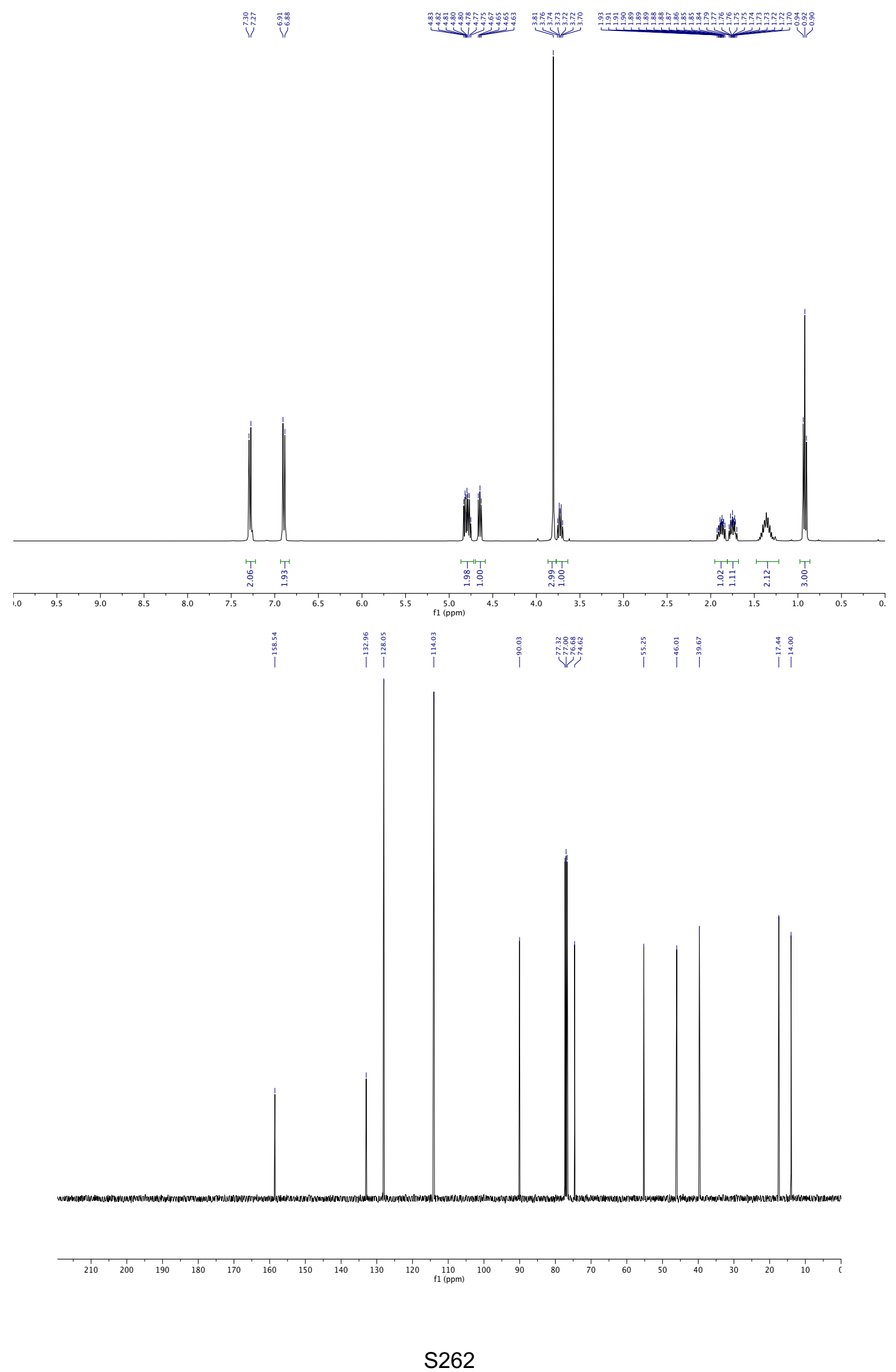




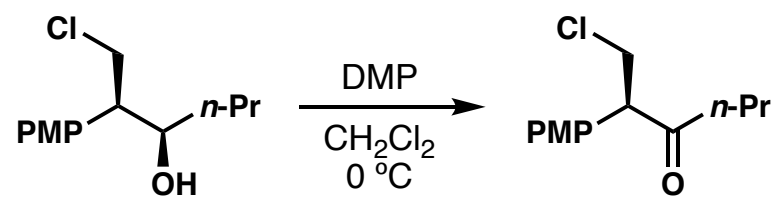

(R)-1-Chloro-2-(4-methoxyphenyl)hexan-3-one 17

To a solution of $7 \mathrm{a}(321 \mathrm{mg}, 1.32 \mathrm{mmol})$ in $\mathrm{CH}_{2} \mathrm{Cl}_{2}(5.3 \mathrm{~mL})$ was added Dess-Martin periodinane $(3.5 \mathrm{~g}, 8.26 \mathrm{mmol})$. The reaction mixture was stirred at room temperature until consumption of starting material occurred, as determined by TLC analysis. The reaction was then quenched by addition of saturated aq. $\mathrm{NaHSO}_{3}(10 \mathrm{~mL})$. The mixture was filtered through celite ${ }$, washing with $\mathrm{Et}_{2} \mathrm{O}$. The filtrate was transferred to a separatory funnel, washed with saturated aq. $\mathrm{NaHCO}_{3}(2 \times$ $10 \mathrm{~mL})$, brine $(10 \mathrm{~mL})$, dried $\left(\mathrm{MgSO}_{4}\right)$, filtered, and concentrated in vacuo. Purification of the residue by FCC, (3:1, hexane-EtOAc) afforded the title compound (305 $\mathrm{mg}, 96 \%)$ as a yellow oil.

${ }^{1} \mathrm{H}$ NMR $\left(400 \mathrm{MHz}, \mathrm{CDCl}_{3}\right) \delta 7.13(\mathrm{~d}, J=8.8 \mathrm{~Hz}, 2 \mathrm{H}), 6.87(\mathrm{~d}, J=8.8 \mathrm{~Hz}, 2 \mathrm{H}), 4.12$ (dd, $J=10.7$, $8.8 \mathrm{~Hz}, 1 \mathrm{H}$ ), 3.98 (dd, $J=8.8,5.7 \mathrm{~Hz}, 1 \mathrm{H}), 3.80(\mathrm{~s}, 3 \mathrm{H}), 3.55(\mathrm{dd}, J=10.7,5.7 \mathrm{~Hz}, 1 \mathrm{H}), 2.38$ (t, $J$ $=7.3 \mathrm{~Hz}, 2 \mathrm{H}), 1.72-1.43(\mathrm{~m}, 2 \mathrm{H}), 0.81(\mathrm{t}, J=7.4 \mathrm{~Hz}, 3 \mathrm{H})$;

${ }^{13} \mathrm{C}$ NMR $\left(100 \mathrm{MHz}, \mathrm{CDCl}_{3}\right) \delta 207.8,159.5,129.3,127.6,114.5,59.8,55.3,44.4,44.3,17.0,13.5$; IR (NaCl, thin film) 2936, 2934, 2352, 1717, 1512, 1252, 1035, $898 \mathrm{~cm}^{-1}$;

HRMS (El-TOF) $\mathrm{m} / \mathrm{z}$ calcd for $\mathrm{m} / \mathrm{z}$ calcd for $\mathrm{C}_{13} \mathrm{H}_{17} \mathrm{ClO}_{2} \mathrm{Na}(\mathrm{M}+\mathrm{Na})^{+}:$:263.0809, found 263.0786.

The enantiomeric purity of this compound is be inferred to be approximately 95:5 er from analysis of enantiopurity of oxetanes 18 and 19 below. 

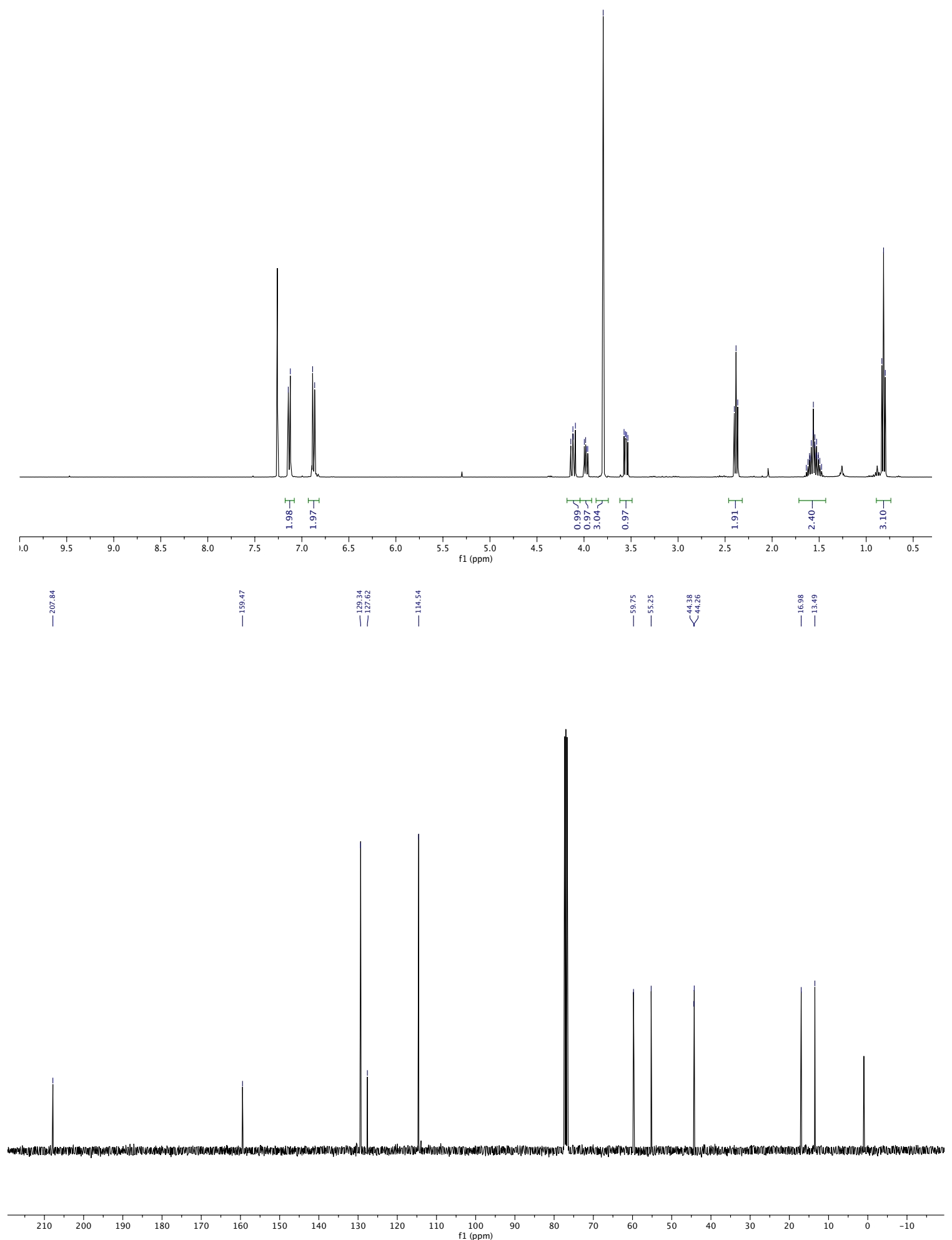

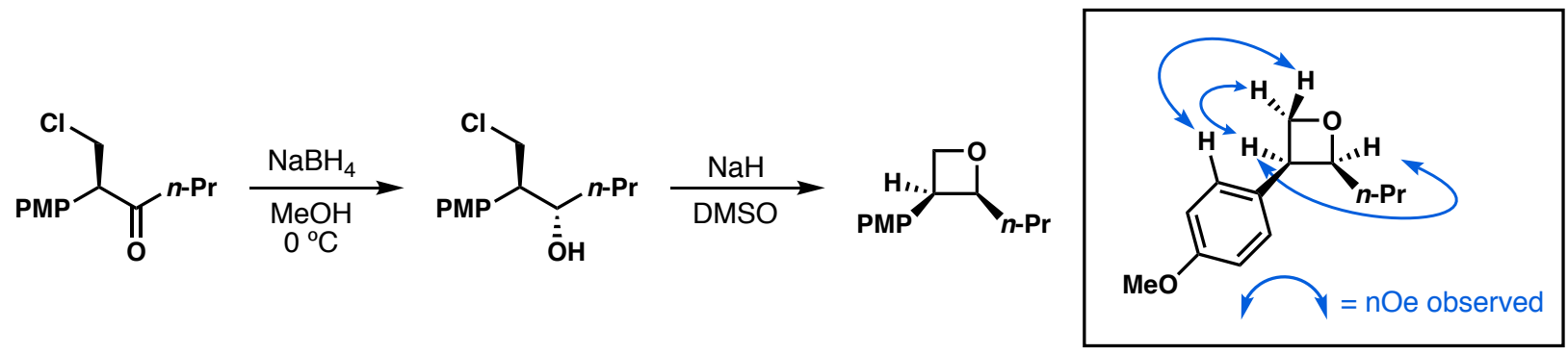

\section{(2S,3S)-3-(4-Methoxyphenyl)-2-propyloxetane 18}

To a mixture of ketone $17(20 \mathrm{mg}, 0.083 \mathrm{mmol})$ in $\mathrm{MeOH}(1 \mathrm{~mL})$ at $0{ }^{\circ} \mathrm{C}$ was added $\mathrm{NaBH}_{4}(6.3$ $\mathrm{mg}, 0.17 \mathrm{mmol})$. The reaction mixture was stirred at this temperature for 4 hours. The reaction was then quenched by addition of saturated aq. $\mathrm{NH}_{4} \mathrm{Cl}(5 \mathrm{~mL})$. The mixture was extracted with $\mathrm{CH}_{2} \mathrm{Cl}_{2}(3 \times 5 \mathrm{~mL})$, and the combined organic layers were washed with brine $(5 \mathrm{~mL})$, dried $\left(\mathrm{MgSO}_{4}\right)$, filtered, and concentrated in vacuo to give crude alcohol. This material was dissolved in DMSO $(0.5 \mathrm{~mL})$ and $\mathrm{NaH}(60 \%, 6.6 \mathrm{mg}, 0.17 \mathrm{mmol})$ was added. The reaction was stirred at room temperature until consumption of the starting material was determined by TLC analysis. The reaction mixture was then cooled to $0^{\circ} \mathrm{C}$ and quenched by addition of saturated aq. $\mathrm{NaHCO}_{3}(1$ $\mathrm{mL})$ and extracted with ether $(2 \times 2 \mathrm{~mL})$. The combined organic extracts were washed with $\mathrm{NaHCO}_{3}(2 \mathrm{~mL})$ and brine $(2 \mathrm{~mL})$. Purification of the residue by FCC (5:1, hexane-EtOAc) afforded the title compound (10 mg, 60\% yield over two steps, 96:4 er) as a yellow oil.

${ }^{1} \mathrm{H}$ NMR $\left(400 \mathrm{MHz}, \mathrm{CDCl}_{3}\right) \delta 7.26(\mathrm{~d}, J=8.6 \mathrm{~Hz}, 2 \mathrm{H}), 6.90(\mathrm{~d}, J=8.7 \mathrm{~Hz}, 2 \mathrm{H}), 5.06(\mathrm{td}, J=8.1$, $5.2 \mathrm{~Hz}, 1 \mathrm{H}), 4.99$ (dd, J = 8.1, $6.5 \mathrm{~Hz}, 1 \mathrm{H}), 4.68$ (app. t, $J=6.2 \mathrm{~Hz}, 1 \mathrm{H}), 4.13(\mathrm{td}, J=8.0,5.9 \mathrm{~Hz}$, $1 \mathrm{H}), 3.82(\mathrm{~s}, 3 \mathrm{H}), 1.53-1.45(\mathrm{~m}, 1 \mathrm{H}), 1.24-1.12(\mathrm{~m}, 2 \mathrm{H}), 1.06-0.99(\mathrm{~m}, 1 \mathrm{H}), 0.76(\mathrm{t}, J=7.2 \mathrm{~Hz}$, $3 \mathrm{H})$;

${ }^{13} \mathrm{C}$ NMR $\left(100 \mathrm{MHz}, \mathrm{CDCl}_{3}\right) \delta$ 158.5, 130.0, 129.4, 113.7, 85.7, 73.3, 55.2, 42.8, 34.8, 17.5, 13.9, IR ( $\mathrm{NaCl}$, thin film) 2958, 2933, 2871, 1611, 1513, 1463, 1248, 1178, 1034, $828 \mathrm{~cm}^{-1}$;

HRMS (El-Orbitrap) $\mathrm{m} / \mathrm{z}$ calcd for $\mathrm{C}_{13} \mathrm{H}_{19} \mathrm{O}_{2}{ }^{+} 207.1380$, found 207.1373.

The enantiomeric purity of this compound was determined by chiral HPLC (WhelkO-1, isocratic hexanes-i-PrOH 95:5, $\left.1.0 \mathrm{~mL} / \mathrm{min}, 23^{\circ} \mathrm{C}\right) ; \mathrm{t}_{\mathrm{R}}$ (major) $=10.7 \mathrm{~min}$ and $\mathrm{t}_{\mathrm{R}}($ minor $)=12.1 \mathrm{~min}$.

The relative stereochemistry was determined through nOe analysis, indicated above.

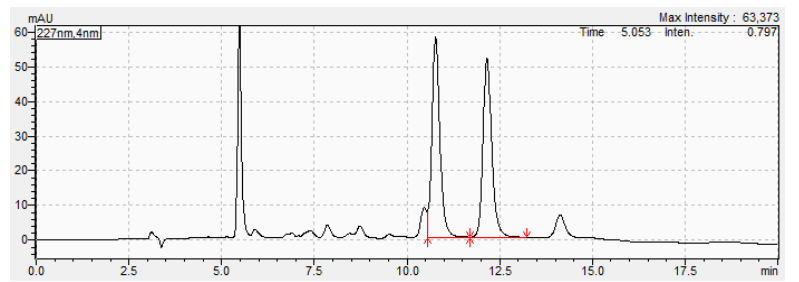

\begin{tabular}{|l|r|r|r|}
\hline Peak\# & Ret. Time & \multicolumn{1}{|c|}{ Area } & \multicolumn{1}{c|}{ Area\% } \\
\hline 1 & 10.771 & 868388 & 50.248 \\
\hline 2 & 12.158 & 859831 & 49.752 \\
\hline Total & & 1728219 & 100.000 \\
\hline
\end{tabular}

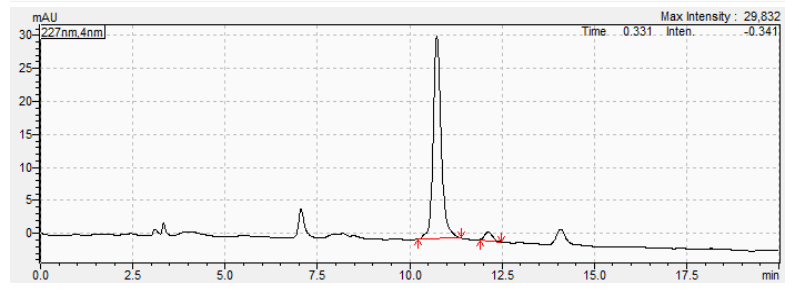

\begin{tabular}{|l|r|r|r|}
\hline Peak\# & Ret. Time & \multicolumn{1}{|c|}{ Area } & \multicolumn{1}{|c|}{ Area\% } \\
\hline 1 & 10.735 & 468889 & 96.284 \\
\hline 2 & 12.128 & 18097 & 3.716 \\
\hline Total & & 486986 & 100.000 \\
\hline
\end{tabular}




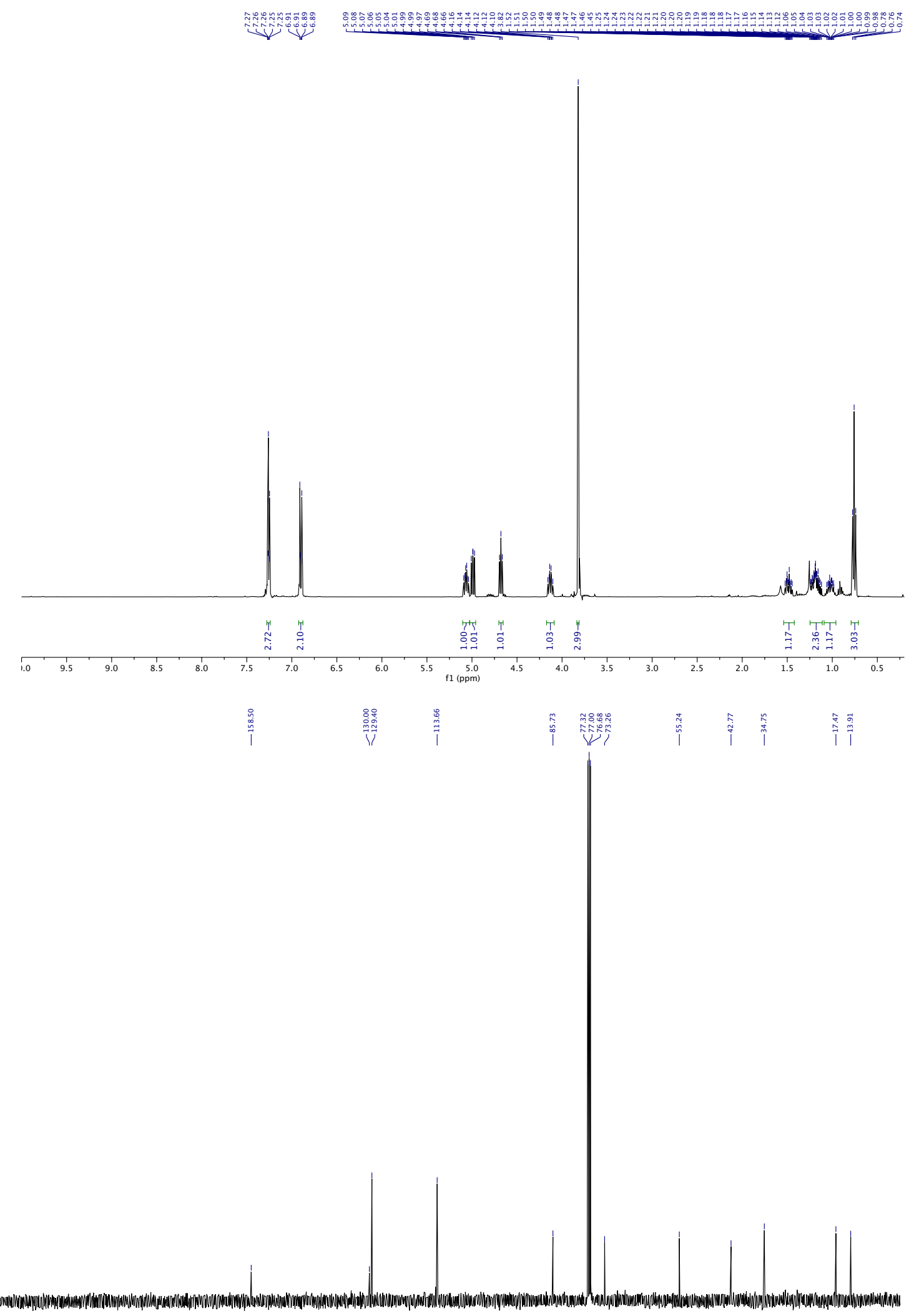

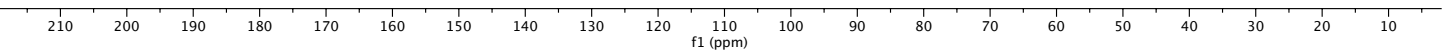



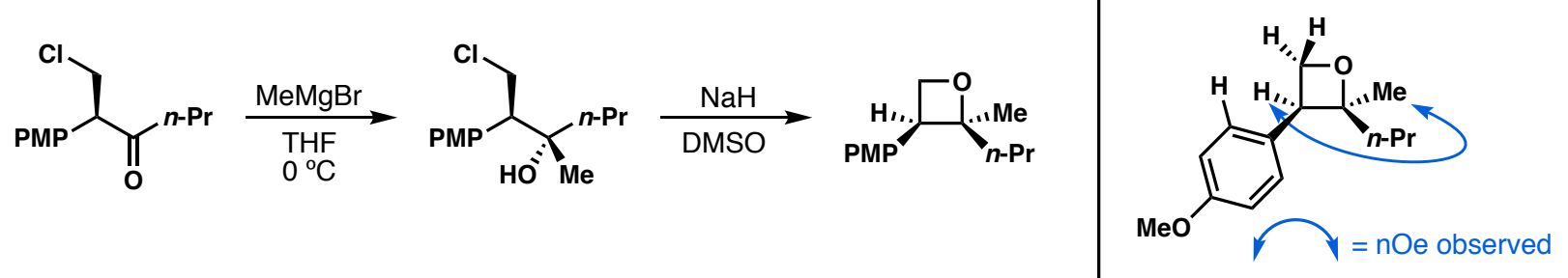

\section{(2S,3S)-3-(4-Methoxyphenyl)-2-methyl-2-propyloxetane 19}

To a solution of ketone $17(24 \mathrm{mg}, 0.10 \mathrm{mmol})$ in THF $(1 \mathrm{~mL})$ at $-20^{\circ} \mathrm{C}$ was added with $\mathrm{MeMgBr}$ $(0.20 \mathrm{~mL}, 1.0 \mathrm{M}$ in THF). The mixture was stirred at room temperature for 3 hours, after which time it was quenched with saturated aq. $\mathrm{NH}_{4} \mathrm{Cl}(0.1 \mathrm{~mL})$. The solution was filtered through celite ${ }^{\circ}$ and concentrated in vacuo to give the crude alcohol. This crude material was re-dissolved in ethanol $(1 \mathrm{~mL})$ and $\mathrm{K}_{2} \mathrm{CO}_{3}(28 \mathrm{mg}, 0.20 \mathrm{mmol})$ was added. The reaction mixture was stirred at 70 ${ }^{\circ} \mathrm{C}$ for 12 hours. The solution was filtrated through celite $\AA$ and concentrated in vacuo. Purification of the residue by FCC (5:1, hexane-EtOAc) afforded the title compound (16 $\mathrm{mg}, 72 \%$ yield over 2 steps, 94.5:5.5 er) as a colorless oil.

${ }^{1} \mathrm{H}$ NMR $\left(500 \mathrm{MHz}, \mathrm{CDCl}_{3}\right) \delta$ 7.20-7.15 (m, 2H), 6.93-6.81 (m, 2H), $4.73(\mathrm{dt}, \mathrm{J}=14.6,6.5 \mathrm{~Hz}, 2 \mathrm{H})$, $3.89(\mathrm{t}, J=8.1 \mathrm{~Hz}, 1 \mathrm{H}), 3.82(\mathrm{~s}, 3 \mathrm{H}), 1.54(\mathrm{~s}, 3 \mathrm{H}), 1.49(\mathrm{dt}, J=11.6,3.4 \mathrm{~Hz}, 1 \mathrm{H}), 1.26-1.14(\mathrm{~m}$, $2 \mathrm{H}), 0.96-0.87(\mathrm{~m}, 1 \mathrm{H}), 0.74-0.66(\mathrm{~m}, 3 \mathrm{H})$.

${ }^{13} \mathrm{C} \mathrm{NMR}\left(126 \mathrm{MHz}, \mathrm{CDCl}_{3}\right) \delta 158.4,129.9,129.1,113.6,89.6,68.5,55.2,49.6,39.3,27.7,16.0$, 14.6.

IR (thin film): 2958, 2934, 2872, 2363, 1162, 1514, 1504, 1462, 1249, 1177, 1038, 978, $847 \mathrm{~cm}^{-1}$.

HRMS (ESI-TOF) $\mathrm{m} / \mathrm{z}$ calcd for $\mathrm{C}_{14} \mathrm{H}_{20} \mathrm{NaO}_{2}{ }^{+}(\mathrm{M}+\mathrm{Na})^{+}:$243.1356, found 243.1350.

The enantiomeric purity of this compound was determined by chiral SFC (Trefoil CEL2, isocratic $\left.\mathrm{CO}_{2}-\mathrm{MeOH} 95: 5,1 \mathrm{~mL} / \mathrm{min}, 40^{\circ} \mathrm{C}\right) ; t_{R}($ minor $)=1.52 \mathrm{~min}$ and $t_{R}$ (major) $=1.68 \mathrm{~min}$.

The relative stereochemistry was determined through nOe analysis, indicated above.
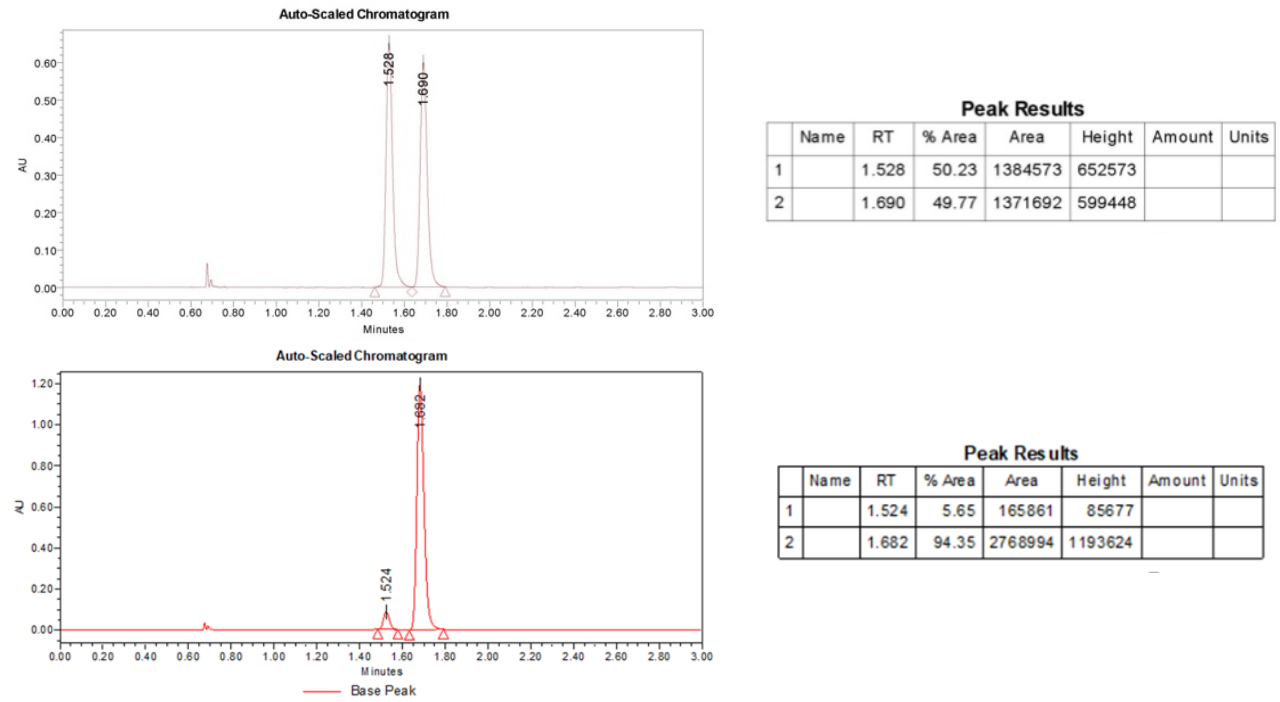

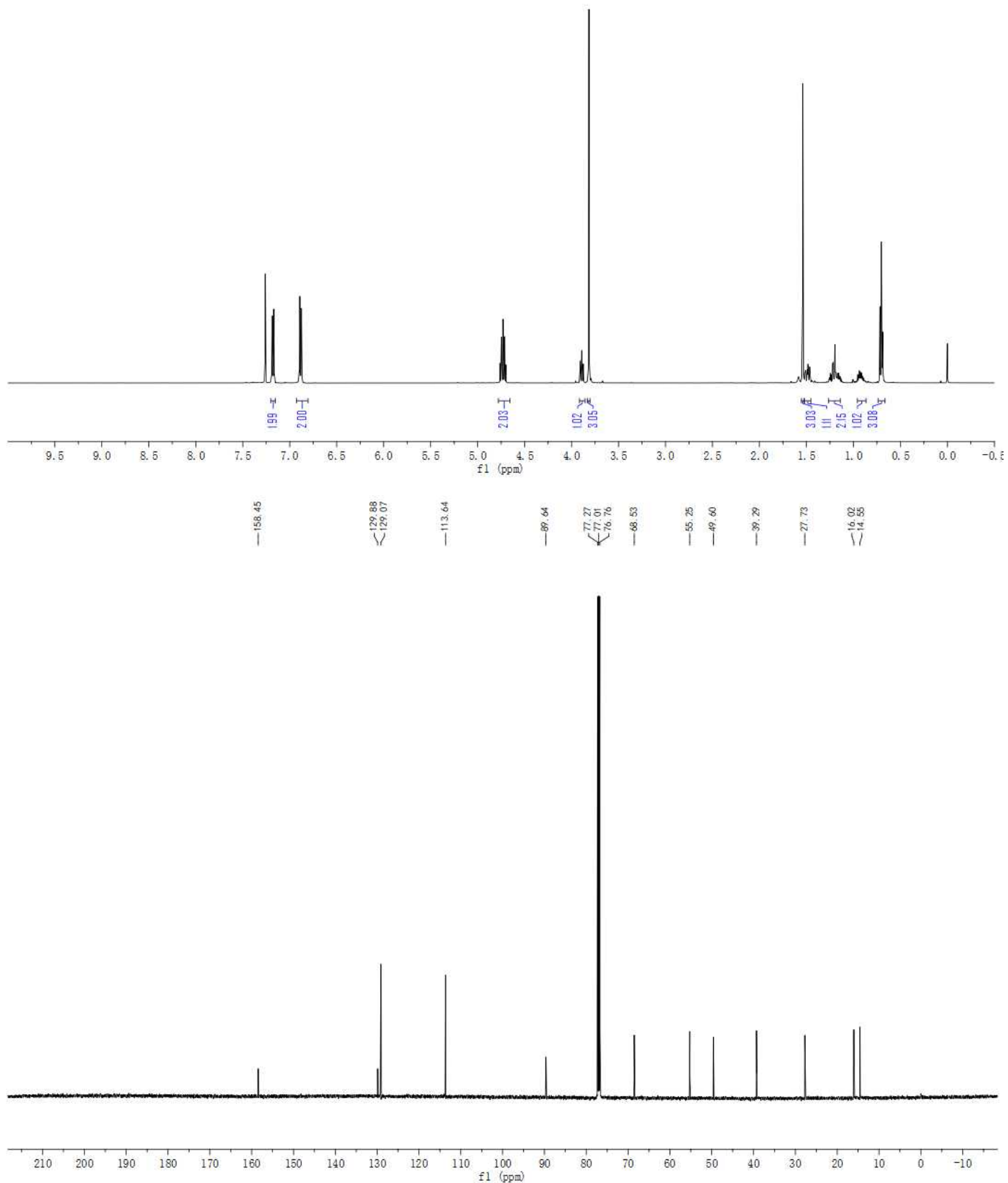


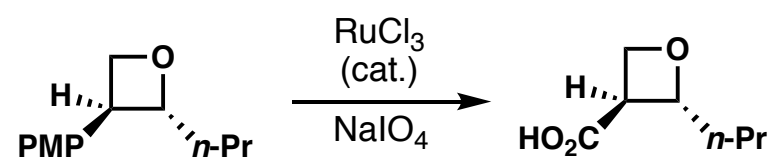

\section{$\left(2 R^{*}, 3 S^{*}\right)$-2-Propyloxetane-3-carboxylic acid $( \pm)-20$}

Sodium periodate $(569 \mathrm{mg}, 2.66 \mathrm{mmol})$, and ruthenium trichloride hydrate $(8 \mathrm{mg}, 0.04 \mathrm{mmol})$ were added to a solution of $( \pm)-16(40 \mathrm{mg}, 0.19 \mathrm{mmol})$ in water $(2 \mathrm{~mL})$ and acetonitrile $(1 \mathrm{~mL})$. The mixture was stirred for 20 hours at room temperature by which time consumption of starting material was determined by TLC analysis. The mixture was cooled to $0^{\circ} \mathrm{C}$ and diluted with EtOAC $(10 \mathrm{~mL})$. The solution was filtered through celite ${ }^{\circledR}$, the phases were separated, and the aqueous layer was extracted with EtOAc $(3 \times 5 \mathrm{~mL})$. The combined organic layers were washed with brine $(5 \mathrm{~mL})$, dried over $\mathrm{MgSO}_{4}$, filtered, and concentrated in vacuo. Purification by FCC (100:1:0.1, $\mathrm{CH}_{2} \mathrm{Cl}_{2}-\mathrm{MeOH}$-acetic acid) afforded the title compound (17 mg, 62\%) as a pale orange oil.

${ }^{1} \mathrm{H}$ NMR (400 MHz, $\mathrm{CDCl}_{3}$ ) $\delta 4.92$ (app. q, $\left.J=6.6 \mathrm{~Hz}, 1 \mathrm{H}\right), 4.79$ (dd, $\left.J=7.3,6.2 \mathrm{~Hz}, 1 \mathrm{H}\right), 4.62$ (dd, $J=8.8,6.2 \mathrm{~Hz}, 1 \mathrm{H}), 3.48(\mathrm{dt}, J=8.8,7.0 \mathrm{~Hz}, 1 \mathrm{H}), 1.90-1.81(\mathrm{~m}, 1 \mathrm{H}), 1.78-1.69(\mathrm{~m}, 1 \mathrm{H}), 1.44-$ $1.34(\mathrm{~m}, 2 \mathrm{H}), 0.95$ (t, $J=7.4 \mathrm{~Hz}, 3 \mathrm{H})$;

${ }^{13} \mathrm{C}$ NMR $\left(100 \mathrm{MHz}, \mathrm{CDCl}_{3}\right) \delta 177.2,84.0,68.6,44.0,39.2,17.0,13.4$;

IR ( $\mathrm{NaCl}$, thin film) 3500, 2961, 1735, 1231, $937 \mathrm{~cm}^{-1}$;

HRMS (APCl) $\mathrm{m} / \mathrm{z}$ calcd for $\mathrm{C}_{7} \mathrm{H}_{13} \mathrm{O}_{3}{ }^{+}(\mathrm{M}+\mathrm{H})^{+}:$145.0865, found 145.0851 .

The relative stereochemistry was determined through nOe analysis, indicated below.

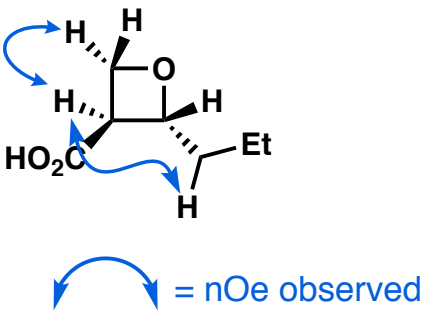




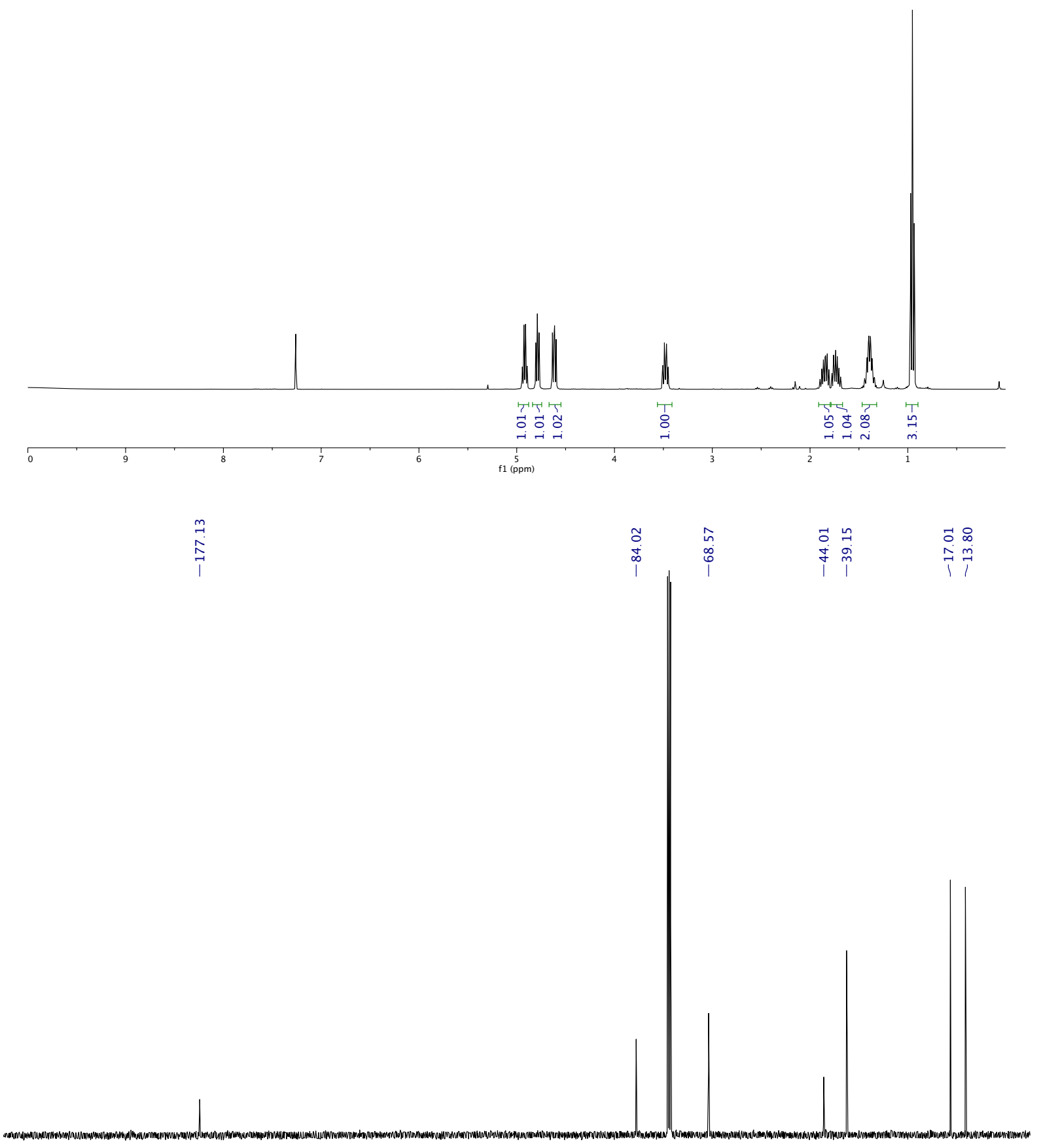

$\left.\right|_{180} ^{1}$

140

120

f1 (ppm) 1

$100+80$

60

40 


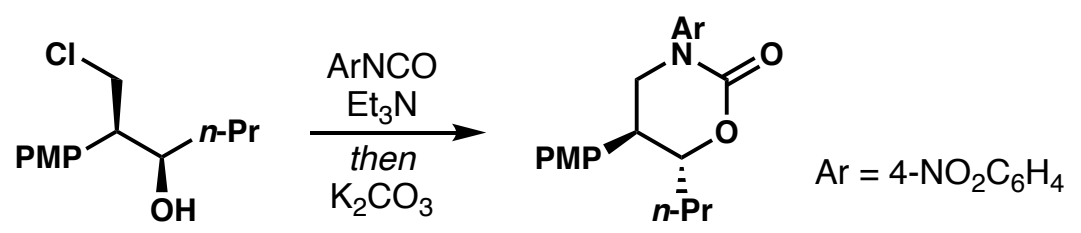

$\left(5 S^{*}, 6 R^{*}\right)-5-(4-M e t h o x y p h e n y l)-3-(4-n i t r o p h e n y l)-6-p r o p y l-1,3-o x a z i n a n-2-o n e( \pm)-21$

To an oven dried reaction tube, charged with a stir bar, was added $( \pm)-7 a(49 \mathrm{mg}, 0.20 \mathrm{mmol})$. The tube was sealed with a septum and purged with argon. Triethylamine $(0.5 \mathrm{~mL}, 0.02 \mathrm{M}$ in $\mathrm{CH}_{2} \mathrm{Cl}_{2}, 0.01 \mathrm{mmol}$ ) and 4-nitrophenyl isocyanate (39 $\mathrm{mg}, 0.24 \mathrm{mmol}$ ) were added. The mixture was stirred at $80^{\circ} \mathrm{C}$ for 24 hours then concentrated in vacuo. The crude residue was re-dissolved in acetonitrile $(0.5 \mathrm{~mL})$ and potassium carbonate $(41 \mathrm{mg}, 0.30 \mathrm{mmol})$ was added. The mixture was stirred at $80^{\circ} \mathrm{C}$ for 30 hours then diluted with diethyl ether $(5 \mathrm{~mL})$, washed with water $(2 \times 5$ $\mathrm{mL}$ ), dried with $\mathrm{MgSO}_{4}$, filtered, and concentrated under reduced pressure. Purification of the residue by FCC (10:1, toluene-acetone) afforded the title compound (50 mg, 67\%) as a yellow oil.

${ }^{1} \mathrm{H}$ NMR $\left(400 \mathrm{MHz}, \mathrm{CDCl}_{3}\right) \delta 8.22(\mathrm{~d}, J=9.0 \mathrm{~Hz}, 2 \mathrm{H}), 7.58(\mathrm{~d}, J=9.1 \mathrm{~Hz}, 2 \mathrm{H}), 7.15(\mathrm{~d}, J=8.7$ $\mathrm{Hz}, 2 \mathrm{H}$ ), $6.92(\mathrm{~d}, J=8.7 \mathrm{~Hz}, 2 \mathrm{H}$ ), 4.59 (ddd, $J=10.6,7.5,3.2 \mathrm{~Hz}, 1 \mathrm{H}), 3.93$ (app. t, J = $11.5 \mathrm{~Hz}$, $1 \mathrm{H}), 3.81(\mathrm{~s}, 3 \mathrm{H}), 3.78$ (dd, $J=11.5,5.3 \mathrm{~Hz}, 1 \mathrm{H}), 3.19$ (app. td, $J=10.9,5.2 \mathrm{~Hz}, 1 \mathrm{H}) .1 .66-1.57$ $(\mathrm{m}, 1 \mathrm{H}), 1.54-1.47(\mathrm{~m}, 2 \mathrm{H}), 1.45-1.33(\mathrm{~m}, 1 \mathrm{H}), 0.86(\mathrm{t}, J=7.2 \mathrm{~Hz}, 3 \mathrm{H})$;

${ }^{13} \mathrm{C}$ NMR $\left(100 \mathrm{MHz}, \mathrm{CDCl}_{3}\right) \delta 159.5,152.0,148.2,144.9,128.9,128.3,124.6,124.4,114.8,82.1$, 55.4, 54.1, 43.4, 34.9, 17.7, 13.7;

IR ( $\mathrm{NaCl}$, thin film) 2960, 1706, 1515, 1407, 1345, 1249, 1168, 1111, 1032, 856, $756 \mathrm{~cm}^{-1}$;

HRMS (APCl) $\mathrm{m} / z$ calcd for $\mathrm{C}_{20} \mathrm{H}_{23} \mathrm{~N}_{2} \mathrm{O}_{5}{ }^{+}(\mathrm{M}+\mathrm{H})^{+}: 371.1607$, found 371.1603. 

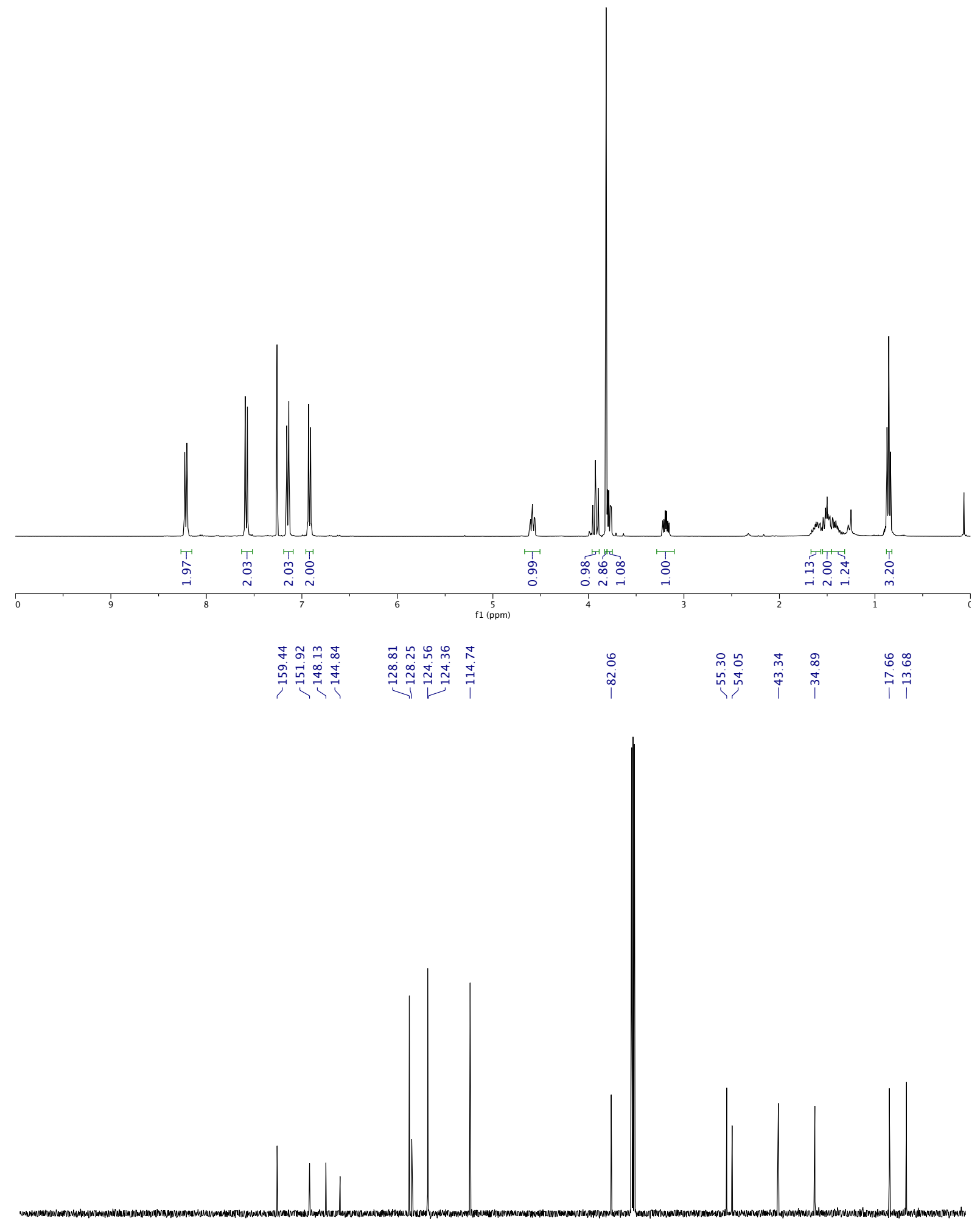


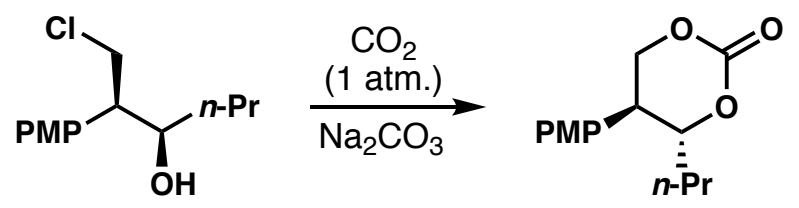

$\left(4 R^{*}, 5 S^{*}\right)-5-(4-M e t h o x y p h e n y l)-4-p r o p y l-1,3-d i o x a n-2-o n e( \pm)-22$

To an oven dried flask, charged with a stir bar and purged with argon, was added ( \pm )-7a (49 mg, $0.20 \mathrm{mmol})$. The residue was dissolved in DMF $(1 \mathrm{~mL})$ and treated with sodium carbonate $(42$ $\mathrm{mg}, 0.40 \mathrm{mmol}$ ). The mixture was purged with $\mathrm{CO}_{2}$ (balloon) for 10 minutes then heated to $100^{\circ} \mathrm{C}$ under a $1 \mathrm{~atm} \mathrm{CO}_{2}$ atmosphere for 7 days. The mixture was diluted with diethyl ether $(5 \mathrm{~mL})$, washed with water $(3 \times 5 \mathrm{~mL})$ and brine $(5 \mathrm{~mL})$, dried with $\mathrm{MgSO}_{4}$, filtered, and concentrated in vacuo. ${ }^{1} \mathrm{H}$ analysis of the crude reaction mixture, using 1,3,5-trimethoxybenzene as an internal standard, revealed a $67 \%$ in situ yield of the title compound. Purification of the residue by FCC (100:1, toluene-acetone) afforded the title compound $(21 \mathrm{mg}, 42 \%)$ as a yellow oil.

${ }^{1} \mathrm{H}$ NMR $\left(400 \mathrm{MHz}_{\mathrm{CDCl}}\right) \delta 7.10(\mathrm{~d}, J=8.7 \mathrm{~Hz}, 2 \mathrm{H}), 6.91(\mathrm{~d}, J=8.7 \mathrm{~Hz}, 2 \mathrm{H}), 4.56$ (ddd, $J=$ 10.8, 7.1, 3.9 Hz, 1H), 4.38-4.36 (m, 2H), 3.81 (s, 3H), 3.05 (app. td, $J=10.0,7.2 \mathrm{~Hz}, 1 \mathrm{H}$ ), 1.62$1.47(\mathrm{~m}, 3 \mathrm{H}), 1.41-1.31(\mathrm{~m}, 1 \mathrm{H}), 0.84(\mathrm{t}, J=7.2 \mathrm{~Hz}, 3 \mathrm{H})$;

${ }^{13} \mathrm{C} \mathrm{NMR}\left(100 \mathrm{MHz}, \mathrm{CDCl}_{3}\right) \delta 159.6,149.0,129.0,126.3,114.8,83.6,71.6,55.3,42.7,35.1,17.6$, 13.6;

IR ( $\mathrm{NaCl}$, thin film) 2960, 1752, 1515, 1405, 1251, 1214, 1119, 1098, $1031 \mathrm{~cm}^{-1}$;

HRMS (APCI) $m / z$ calcd for $\mathrm{C}_{14} \mathrm{H}_{19} \mathrm{O}_{4}{ }^{+}(\mathrm{M}+\mathrm{H})^{+}:$251.1283, found 251.1278. 

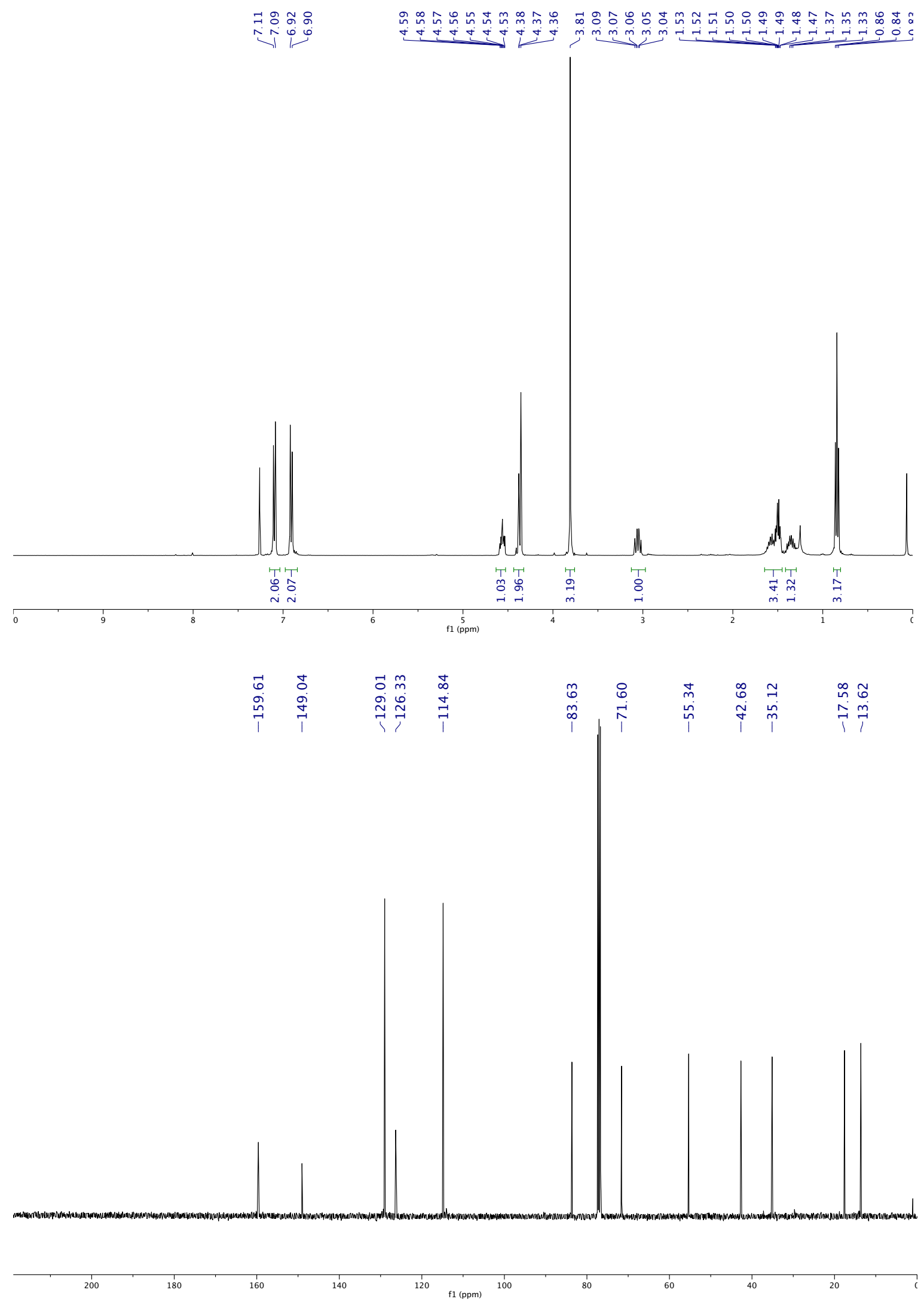


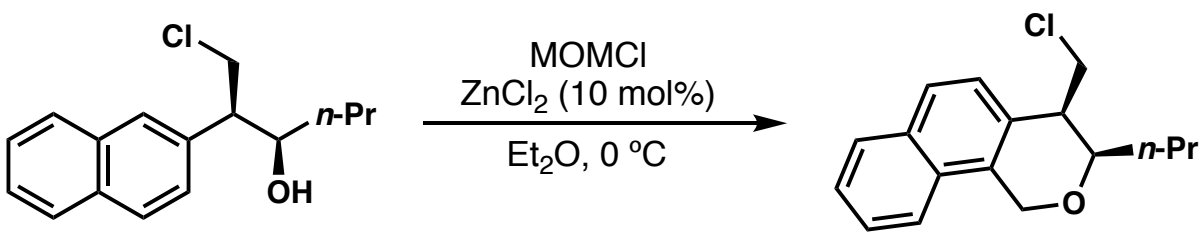

$\left(3 R^{\star}, 4 R^{\star}\right)$-4-(Chloromethyl)-3-propyl-3,4-dihydro-1 H-benzo[ $\left.h\right]$ isochromene $( \pm)$-23

An oven-dried reaction tube was charged with a magnetic stir bar and $\mathrm{ZnCl}_{2}(8.0 \mathrm{mg}, 0.06 \mathrm{mmol})$. The atmosphere was purged with argon and dimethoxymethane $(51 \mu \mathrm{L}, 0.57 \mathrm{mmol})$ was added via syringe. The mixture was cooled to $0{ }^{\circ} \mathrm{C}$ and freshly-distilled acetyl chloride $(41 \mu \mathrm{L}, 0.57 \mathrm{mmol})$ was added. The reaction was stirred at room temperature for 20 minutes, after which time it was diluted with $\mathrm{Et}_{2} \mathrm{O}(2 \mathrm{~mL})$ and cooled to $0{ }^{\circ} \mathrm{C}$. Alcohol $( \pm)-7 \mathrm{~d}(50 \mathrm{mg}, 0.19 \mathrm{mmol})$ was added as a solution in $\mathrm{Et}_{2} \mathrm{O}(1 \mathrm{~mL})$ and the resulting mixture was warmed to room temperature. After two hours the reaction was quenched by addition of $\mathrm{H}_{2} \mathrm{O}(2 \mathrm{~mL})$ and extracted with EtOAc $(3 \times 5 \mathrm{~mL})$. The combined organic layers were washed with saturated aq. $\mathrm{NaHCO}_{3}(2 \mathrm{~mL}), \mathrm{H}_{2} \mathrm{O}(2 \mathrm{~mL})$, brine $(2 \mathrm{~mL})$, dried $\left(\mathrm{Na}_{2} \mathrm{SO}_{4}\right)$, filtered and concentrated in vacuo. Purification of the residue by FCC (20:1, hexane-EtOAc) afforded the title compound (31 $\mathrm{mg}, 60 \%)$ as a pale yellow oil.

${ }^{1} \mathrm{H}$ NMR $\left(400 \mathrm{MHz}, \mathrm{CDCl}_{3}\right) \delta$ 7.87-7.83 (m, 1H), $7.73(\mathrm{~d}, J=8.5 \mathrm{~Hz}, 1 \mathrm{H}), 7.69-7.66(\mathrm{~m}, 1 \mathrm{H})$, 7.54$7.47(\mathrm{~m}, 2 \mathrm{H}), 7.41(\mathrm{~d}, J=8.5 \mathrm{~Hz}, 1 \mathrm{H}), 5.42(\mathrm{~d}, J=15.6 \mathrm{~Hz}, 1 \mathrm{H}), 5.20(\mathrm{~d}, J=15.6 \mathrm{~Hz}, 1 \mathrm{H}), 4.05$ (dd, $J=11.3,6.2 \mathrm{~Hz}, 1 \mathrm{H}$ ), 3.85 (ddd, $J=8.8,4.9,2.4 \mathrm{~Hz}, 1 \mathrm{H}), 3.70$ (dd, $J=11.3,6.2 \mathrm{~Hz}, 1 \mathrm{H}$ ), $1.90-1.45(\mathrm{~m}, 4 \mathrm{H}), 1.03(\mathrm{t}, J=7.3 \mathrm{~Hz}, 3 \mathrm{H})$;

${ }^{13} \mathrm{C}$ NMR $\left(100 \mathrm{MHz}, \mathrm{CDCl}_{3}\right) \delta 133.3,132.4,129.5,129.0,128.7,128.2,126.6,126.4,125.8$, $121.7,76.5,67.2,44.7,44.1,34.8,19.6,14.1$;

IR $(\mathrm{NaCl}$, thin film)

HRMS $\left(\mathrm{Cl}^{+}\right) \mathrm{m} / \mathrm{z}$ calcd for $\mathrm{C}_{17} \mathrm{H}_{19}{ }^{35} \mathrm{ClOH}^{+}(\mathrm{M}+\mathrm{H})^{+}:$: 275.1197, found 275.1200. 

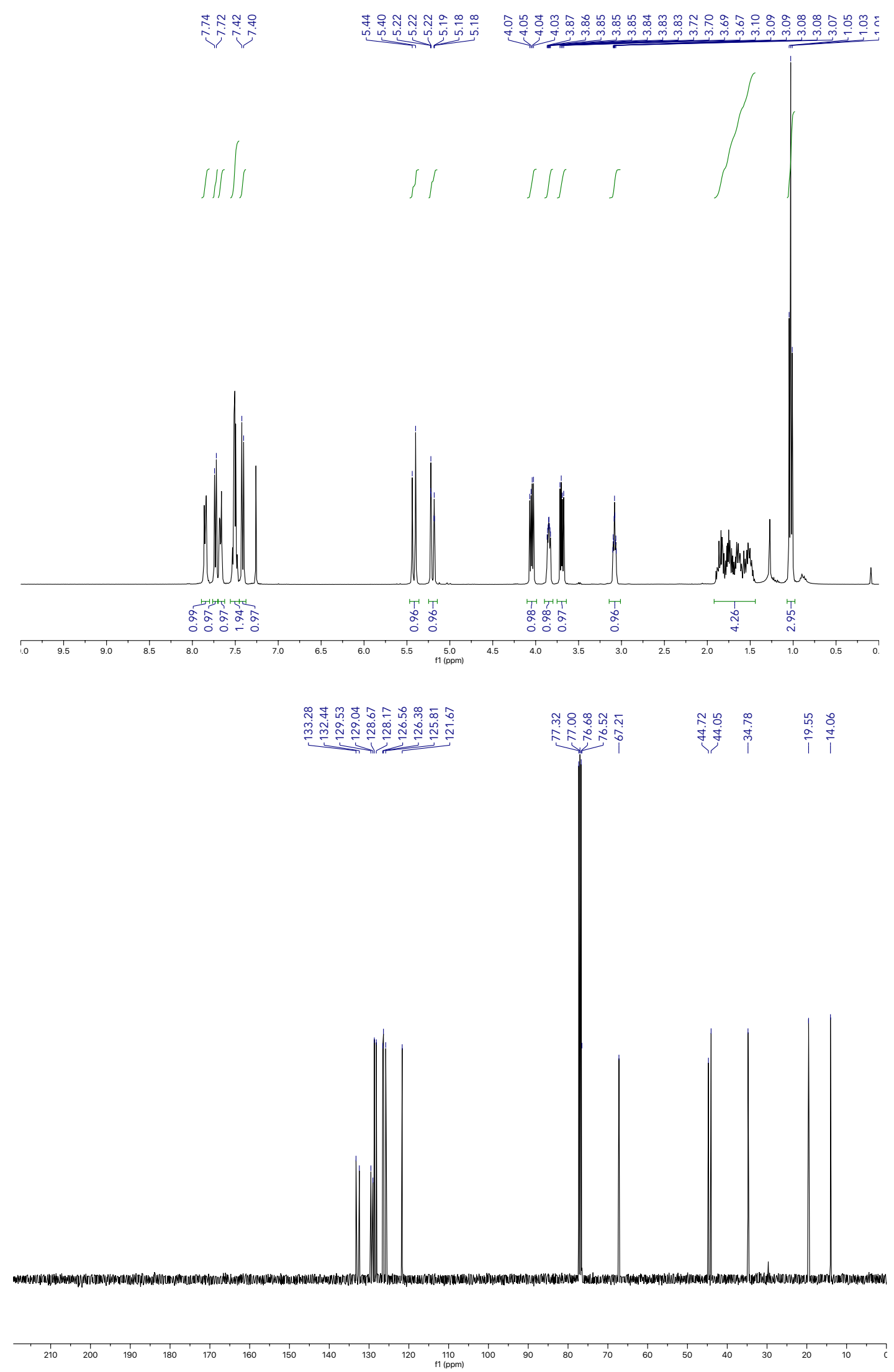


\section{Single Crystal X-ray Analysis of $6 z$}

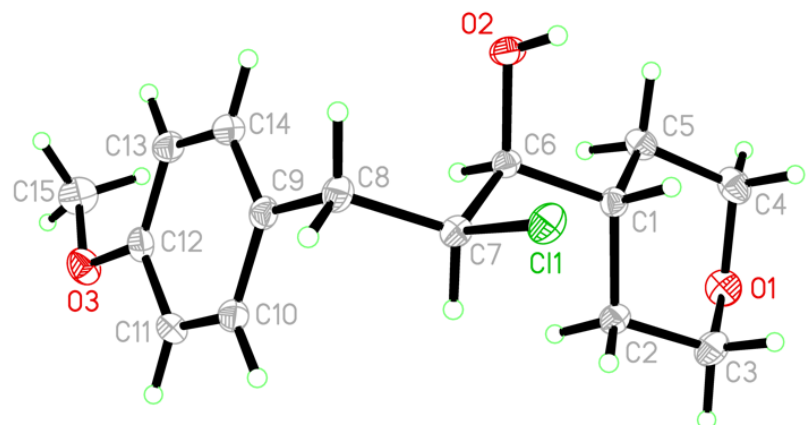

Table S1. Crystal data and structure refinement for $\mathbf{6 z}$

\begin{tabular}{|c|c|c|}
\hline CCDC entry & \multicolumn{2}{|l|}{1956422} \\
\hline Empirical formula & \multicolumn{2}{|l|}{$\mathrm{C}_{15} \mathrm{H}_{2}{ }_{1} \mathrm{ClO}_{3}$} \\
\hline Formula weight & \multicolumn{2}{|l|}{284.77} \\
\hline Temperature & \multicolumn{2}{|l|}{$100(2) \mathrm{K}$} \\
\hline Wavelength & \multicolumn{2}{|l|}{$0.71073 \AA$} \\
\hline Crystal system & \multicolumn{2}{|l|}{ Orthorhombic } \\
\hline Space group & \multicolumn{2}{|l|}{$P 2{ }_{1} 2_{1}{ }_{1}$} \\
\hline \multirow[t]{3}{*}{ Unit cell dimensions } & $a=8.9476(11) \AA$ & $\alpha=90^{\circ}$ \\
\hline & $b=10.1905(14) \AA$ & $\beta=90^{\circ}$ \\
\hline & $c=15.587(2) \AA$ & $\gamma=90^{\circ}$ \\
\hline Volume & \multicolumn{2}{|l|}{$1421.2(3) \AA^{3}$} \\
\hline$z$ & \multicolumn{2}{|l|}{4} \\
\hline Density (calculated) & \multicolumn{2}{|l|}{$1.331 \mathrm{Mg} / \mathrm{m}^{3}$} \\
\hline Absorption coefficient & \multicolumn{2}{|l|}{$0.270 \mathrm{~mm}^{-1}$} \\
\hline$F(000)$ & \multicolumn{2}{|l|}{608} \\
\hline Crystal color, morphology & \multicolumn{2}{|l|}{ Colorless, block } \\
\hline Crystal size & \multicolumn{2}{|c|}{$0.230 \times 0.210 \times 0.200 \mathrm{~mm}^{3}$} \\
\hline Theta range for data collection & \multicolumn{2}{|l|}{2.388 to $30.519^{\circ}$} \\
\hline Index ranges & \multicolumn{2}{|c|}{$-12 \leq h \leq 12,-14 \leq k \leq 14,-22 \leq I \leq 22$} \\
\hline Reflections collected & \multicolumn{2}{|l|}{69067} \\
\hline Independent reflections & \multicolumn{2}{|c|}{$4351[R$ (int) $=0.0589]$} \\
\hline Observed reflections & \multicolumn{2}{|l|}{4174} \\
\hline Completeness to theta $=25.242^{\circ}$ & \multicolumn{2}{|l|}{$100.0 \%$} \\
\hline Absorption correction & \multicolumn{2}{|l|}{ Multi-scans } \\
\hline Max. and min. transmission & \multicolumn{2}{|l|}{0.6478 and 0.6124} \\
\hline Refinement method & \multicolumn{2}{|c|}{ Full-matrix least-squares on $F^{2}$} \\
\hline Data / restraints / parameters & \multicolumn{2}{|l|}{$4351 / 0 / 174$} \\
\hline Goodness-of-fit on $F^{2}$ & \multicolumn{2}{|l|}{1.076} \\
\hline Final $R$ indices $[/>2 \operatorname{sigma}(/)]$ & \multicolumn{2}{|c|}{$R 1=0.0250, w R 2=0.0618$} \\
\hline$R$ indices (all data) & \multicolumn{2}{|c|}{$R 1=0.0268, w R 2=0.0633$} \\
\hline Absolute structure parameter & \multicolumn{2}{|l|}{$-0.028(15)$} \\
\hline Largest diff. peak and hole & \multicolumn{2}{|c|}{0.257 and -0.144 e. $\AA^{-3}$} \\
\hline
\end{tabular}

$$
R_{1}=\Sigma|| F_{\mathrm{O}}|-| F_{\mathrm{C}} \| / \Sigma\left|F_{\mathrm{o}}\right| ; w R 2=\left[\Sigma\left[w\left(F_{\mathrm{o}}{ }^{2}-F_{\mathrm{C}}{ }^{2}\right)^{2}\right] / \Sigma\left[w\left(F_{\mathrm{o}}{ }^{2}\right)^{2}\right]\right]^{1 / 2}
$$




\section{Single Crystal X-ray Analysis of 7c}

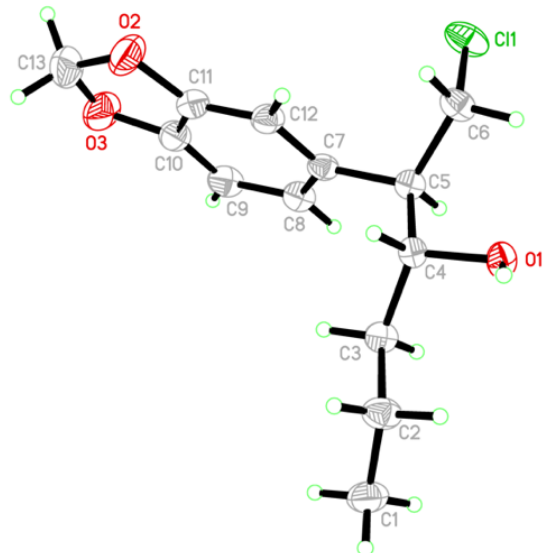

Table S2. Crystal data and structure refinement for 7c

\begin{tabular}{|c|c|c|}
\hline CCDC entry & \multicolumn{2}{|l|}{1978603} \\
\hline Empirical formula & \multicolumn{2}{|l|}{$\mathrm{C}_{13} \mathrm{H}_{17} \mathrm{ClO}_{3}$} \\
\hline Formula weight & \multicolumn{2}{|l|}{256.71} \\
\hline Temperature & \multicolumn{2}{|l|}{$125(2) \mathrm{K}$} \\
\hline Wavelength & \multicolumn{2}{|l|}{$0.71073 \AA$} \\
\hline Crystal system & \multicolumn{2}{|l|}{ Monoclinic } \\
\hline Space group & \multicolumn{2}{|l|}{$\mathrm{P} 2{ }_{1} / \mathrm{c}$} \\
\hline \multirow[t]{3}{*}{ Unit cell dimensions } & $a=9.388(3) \AA$ & $\alpha=90^{\circ}$ \\
\hline & $b=5.204(2) \AA$ & $\beta=98.911(15)^{\circ}$ \\
\hline & $c=26.465(9) \AA$ & $\gamma=90^{\circ}$ \\
\hline Volume & \multicolumn{2}{|l|}{$1277.4(8) \AA^{3}$} \\
\hline$z$ & \multicolumn{2}{|l|}{4} \\
\hline Density (calculated) & \multicolumn{2}{|l|}{$1.335 \mathrm{Mg} / \mathrm{m}^{3}$} \\
\hline Absorption coefficient & \multicolumn{2}{|l|}{$0.293 \mathrm{~mm}^{-1}$} \\
\hline$F(000)$ & \multicolumn{2}{|l|}{544} \\
\hline Crystal color, morphology & \multicolumn{2}{|l|}{ Colourless, Plate } \\
\hline Crystal size & \multicolumn{2}{|c|}{$0.220 \times 0.110 \times 0.060 \mathrm{~mm}^{3}$} \\
\hline Theta range for data collection & \multicolumn{2}{|l|}{2.488 to $28.266^{\circ}$} \\
\hline Index ranges & \multicolumn{2}{|c|}{$-12 \leq h \leq 12,-6 \leq k \leq 6,-35 \leq I \leq 34$} \\
\hline Reflections collected & \multicolumn{2}{|l|}{11991} \\
\hline Independent reflections & \multicolumn{2}{|c|}{$3140[R($ int $)=0.0375]$} \\
\hline Observed reflections & \multicolumn{2}{|l|}{2410} \\
\hline Completeness to theta $=25.242^{\circ}$ & \multicolumn{2}{|l|}{$99.7 \%$} \\
\hline Absorption correction & \multicolumn{2}{|l|}{ Multi-scan } \\
\hline Max. and min. transmission & \multicolumn{2}{|c|}{0.7457 and 0.6484} \\
\hline Refinement method & \multicolumn{2}{|c|}{ Full-matrix least-squares on $F^{2}$} \\
\hline Data / restraints / parameters & \multicolumn{2}{|l|}{$3140 / 42$ / 208} \\
\hline Goodness-of-fit on $F^{2}$ & \multicolumn{2}{|l|}{1.004} \\
\hline Final $R$ indices $[/>2 \operatorname{sigma}(/)]$ & \multicolumn{2}{|c|}{$R 1=0.0440, w R 2=0.1043$} \\
\hline$R$ indices (all data) & \multicolumn{2}{|c|}{$R 1=0.0620, w R 2=0.1145$} \\
\hline Largest diff. peak and hole & \multicolumn{2}{|c|}{0.263 and -0.226 e. $\AA^{-3}$} \\
\hline
\end{tabular}

$$
R_{1}=\Sigma|| F_{\mathrm{o}}|-| F_{\mathrm{C}} \| / \Sigma\left|F_{\mathrm{o}}\right| ; w R 2=\left[\Sigma\left[w\left(F_{\mathrm{O}}{ }^{2}-F_{\mathrm{C}}{ }^{2}\right)^{2}\right] / \Sigma\left[w\left(F_{\mathrm{O}}{ }^{2}\right)^{2}\right]\right]^{1 / 2}
$$




\section{Single Crystal X-ray Analysis of 9ac}

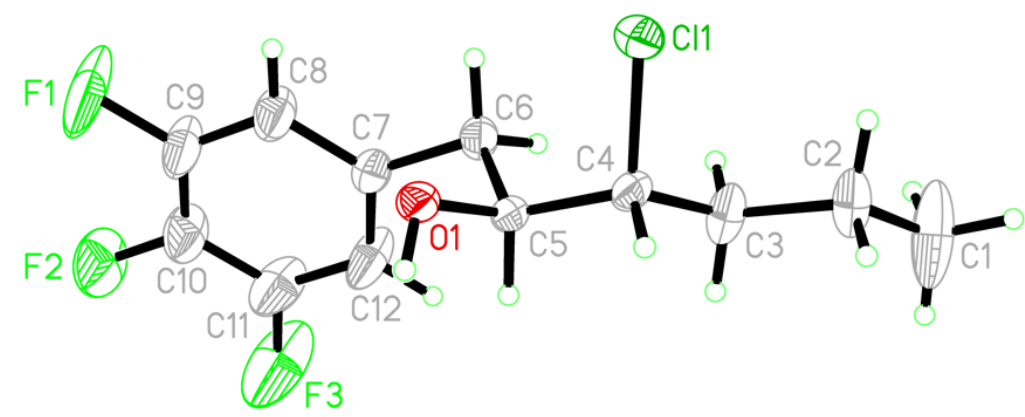

Table S3. Crystal data and structure refinement for 9ac

\begin{tabular}{|c|c|c|}
\hline CCDC entry & 1978273 & \\
\hline Empirical formula & $\mathrm{C}_{12} \mathrm{H}_{14} \mathrm{ClF}_{3} \mathrm{O}$ & \\
\hline Formula weight & 266.68 & \\
\hline Temperature & $125(2) \mathrm{K}$ & \\
\hline Wavelength & $0.71073 \AA$ & \\
\hline Crystal system & Orthorhombic & \\
\hline Space group & Pna2 1 & \\
\hline Unit cell dimensions & $a=20.0407(10) \AA$ & $\alpha=90^{\circ}$ \\
\hline & $b=12.4313(7) \AA$ & $\beta=90^{\circ}$ \\
\hline & $c=5.1226(2) \AA$ & $\gamma=90^{\circ}$ \\
\hline Volume & $1276.20(11) \AA^{3}$ & \\
\hline Z & 4 & \\
\hline Density (calculated) & $1.388 \mathrm{Mg} / \mathrm{m}^{3}$ & \\
\hline Absorption coefficient & $0.317 \mathrm{~mm}^{-1}$ & \\
\hline$F(000)$ & 552 & \\
\hline Crystal color, morphology & Colourless, Needle & \\
\hline Crystal size & $0.500 \times 0.110 \times 0.0$ & \\
\hline Theta range for data collection & 2.611 to $28.393^{\circ}$ & \\
\hline Index ranges & $-26 \leq h \leq 26,-16 \leq h$ & $I \leq 6$ \\
\hline Reflections collected & 11767 & \\
\hline Independent reflections & $3189[R(\mathrm{int})=0.027$ & \\
\hline Observed reflections & 2861 & \\
\hline Completeness to theta $=25.242^{\circ}$ & $99.8 \%$ & \\
\hline Absorption correction & Multi-scan & \\
\hline Max. and min. transmission & 0.7457 and 0.6274 & \\
\hline Refinement method & Full-matrix least-sq & \\
\hline Data / restraints / parameters & $3189 / 1 / 156$ & \\
\hline Goodness-of-fit on $F^{2}$ & 1.021 & \\
\hline Final $R$ indices $[/>2 \operatorname{sigma}(I)]$ & $R 1=0.0355, w R 2=$ & \\
\hline$R$ indices (all data) & $R 1=0.0416, w R 2=$ & \\
\hline Absolute structure parameter & $0.12(3)$ & \\
\hline Largest diff. peak and hole & 0.262 and -0.268 e. & \\
\hline
\end{tabular}

$$
R_{1}=\Sigma|| F_{\mathrm{O}}|-| F_{\mathrm{C}} \| / \Sigma\left|F_{\mathrm{O}}\right| ; w R 2=\left[\Sigma\left[w\left(F_{\mathrm{O}}{ }^{2}-F_{\mathrm{C}}{ }^{2}\right)^{2}\right] / \Sigma\left[w\left(F_{\mathrm{O}}{ }^{2}\right)^{2}\right]\right]^{1 / 2}
$$




\section{References}

(1) Ahlsten, N.; Bermejo Gómez, A.; Martín-Matute, B. Iridium-Catalyzed 1,3-Hydrogen Shift/Chlorination of Allylic Alcohols, Angew. Chem. Int. Ed. 2013, 52, 6273-6276.

(2) Lipp, B.; Lipp, A.; Detert, H.; Opatz, T. Light-Induced Alkylation of (Hetero)aromatic Nitriles in a Transition-Metal-Free C-C-Bond Metathesis, Org. Lett. 2017, 19, 2054-2057.

(3) Drew, S. L.; Lawrence, A. L.; Sherburn, M. S. Total Synthesis of Kingianins A, D, and F, Angew. Chem. Int. Ed. 2013, 52, 4221-4224.

(4) Atkinson, S. J.; Demont, E. H.; Harrison, L. A.; Liwicki, G. M.; Lucas, S. C.; Preston, A. G.; Seal, J. T.; Wall, I. D.; Watson, R. J. Pyrazole Derivatives as Bromodomain Inhibitors. International Patent WO 2018/158212 (A1), 2018.

(5) Kikuchi, K.; Tatewaki, Y.; Okada, S. Self-Assembly and Solid-State Polymerization of Butadiyne Derivatives with Amide and Trialkoxyphenyl Groups, Bull. Chem. Soc. Jpn. 2016, 90, 298-305.

(6) Ma, X.; Hazelden, I. R.; Langer, T.; Munday, R. H.; Bower, J. F. Enantioselective Aza-Heck Cyclizations of N-(Tosyloxy)carbamates: Synthesis of Pyrrolidines and Piperidines, J. Am. Chem. Soc. 2019, 141, 3356-3360.

(7) Simon, M.-O.; Girard, S. A.; Li, C.-J. Catalytic Aerobic Synthesis of Aromatic Ethers from NonAromatic Precursors, Angew. Chem. Int. Ed. 2012, 51, 7537-7540.

(8) Cheung, C. W.; Ren, P.; Hu, X. Mild and Phosphine-Free Iron-Catalyzed Cross-Coupling of Nonactivated Secondary Alkyl Halides with Alkynyl Grignard Reagents, Org. Lett. 2014, 16, 25662569.

(9) Braisted, A. C.; Oslob, J. D.; Delano, W. L.; Hyde, J.; McDowell, R. S.; Waal, N.; Yu, C.; Arkin, M. R.; Raimundo, B. C. Discovery of a Potent Small Molecule IL-2 Inhibitor through Fragment Assembly, J. Am. Chem. Soc. 2003, 125, 3714-3715.

(10) Kohler, P.; Schwaninger, M.; Stutz, A.; Karge, R.; Abele, S. Scalable Process for the Production of a Highly Energetic Bromoacetylene Building Block, Organic Process Research \& Development 2018, 22, 1409-1418.

(11) Wang, Z.-X.; Tu, Y.; Frohn, M.; Zhang, J.-R.; Shi, Y. An Efficient Catalytic Asymmetric Epoxidation Method, J. Am. Chem. Soc. 1997, 119, 11224-11235.

(12) Andrés, J. M.; Barrio, R.; Martínez, M. A.; Pedrosa, R.; Pérez-Encabo, A. Synthesis of Enantiopure syn- $\beta$-Amino Alcohols. A Simple Case of Chelation-Controlled Additions of Diethylzinc to $\alpha$-(Dibenzylamino) Aldehydes, J. Org. Chem. 1996, 61, 4210-4213. 